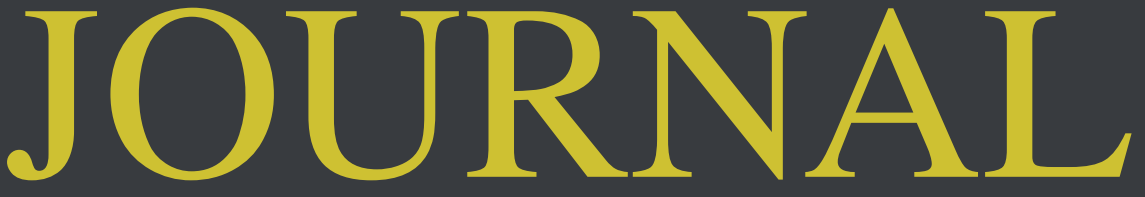

for Research and

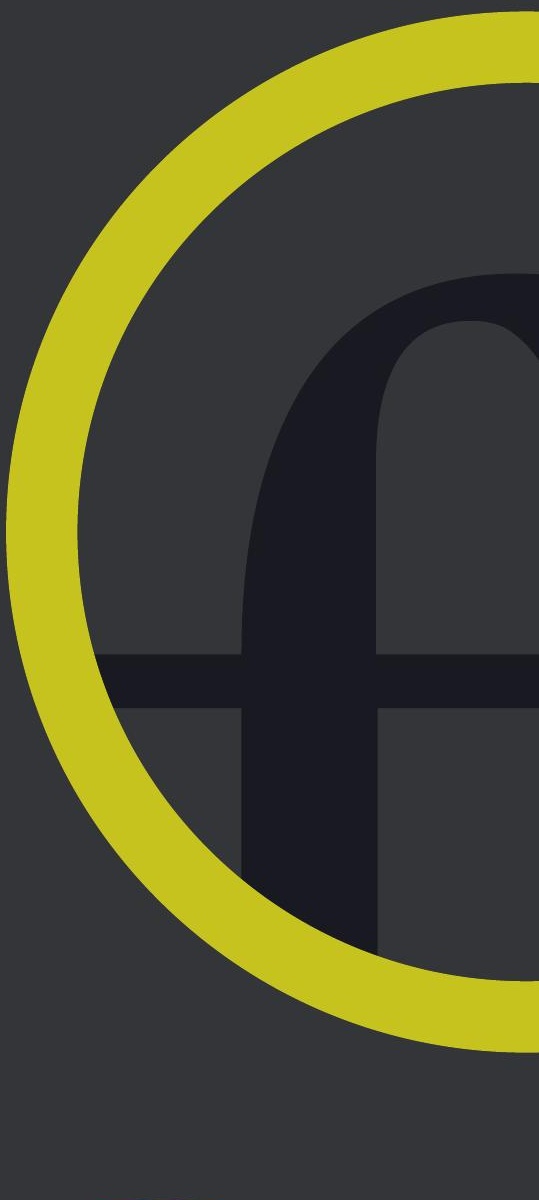

Technology Policy

Evaluation

sancumanem
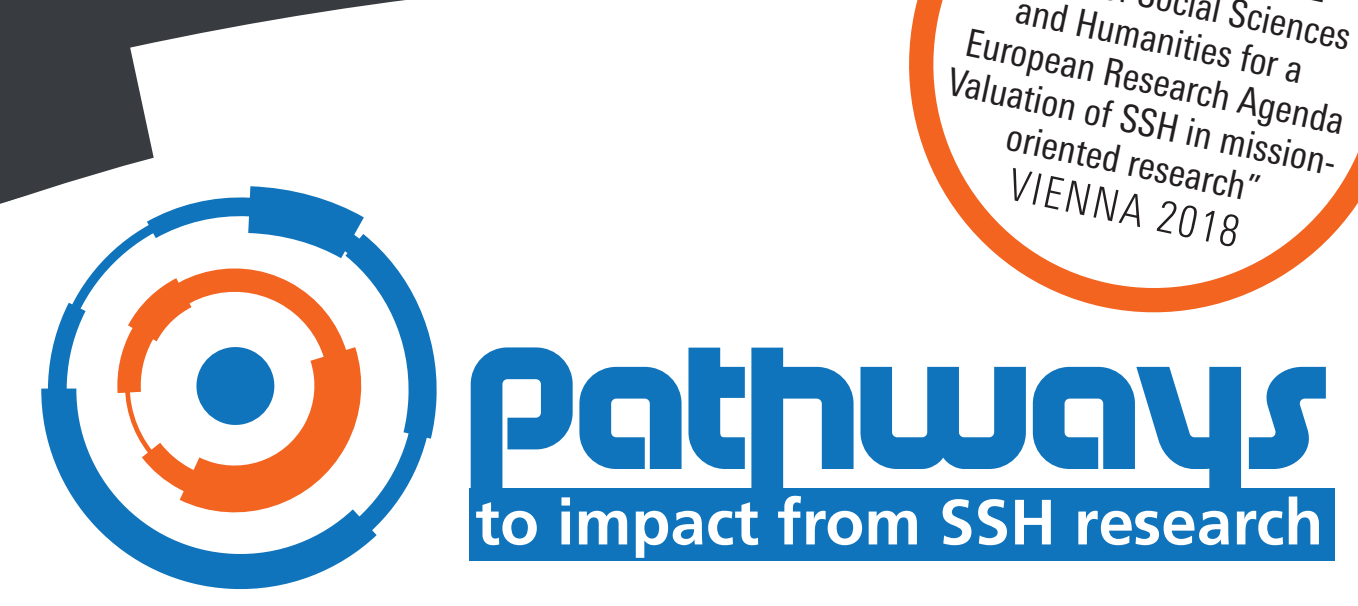

PETER VAN DEN BESSELAAR AND ULF SANDSTRÖM

PANEL COMPOSITION AS

PATHWAY TO IMPACT:

DO WE NEED STAKEHOLDER

EXPERTISE TO SELECT

RELEVANT MISSION-

ORIENTED PROJECTS?
JÜRGEN HOWALDT

NEW PATHWAYS TO

SOCIAL CHANGE -

CREATING IMPACT

THROUGH SOCIAL

INNOVATION RESEARCH
SARAH DE RIJCKE ET AL.

EVALUATIVE INQUIRY:

ENGAGING RESEARCH

EVALUATION ANALYTICALLY

AND STRATEGICALLY 
FEDERAL MINISTRY OF EDUCATION SCIENCE AND RESEARCH

Minoritenplatz 5, 1014 Vienna

Mag. Irene Danler

E: irene.danler@bmbwf.gv.at

Mag. ${ }^{\text {a Simone Mesner }}$

E: simone.mesner@bmbwf.gv.at

FEDERAL MINISTRY FOR DIGITAL AND ECONOMIC AFFAIRS

Stubenring 1, 1014 Vienna

Mag. ${ }^{\text {a Sabine Pohoryles-Drexel }}$

E: sabine.pohoryles-drexel@bmwtw.gv.at

FEDERAL MINISTRY FOR

TRANSPORT, INNOVATION AND

TECHNOLOGY

Radetzkystraße 2, 1030 Vienna

Dr. Rupert Pichler

E: rupert.pichler@bmvit.gv.at

Dr. Mario Steyer

E: mario.steyer@bmvit.gv.at

ACR - AUSTRIAN COOPERATIVE

RESEARCH

Sensengasse 1, 1010 Vienna

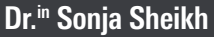

E: sheikh@acr.ac.at

AUSTRIAN COUNCIL FOR RESEARCH

AND TECHNOLOGY DEVELOPMENT

Pestalozzigasse 4/DG 1, 1010 Vienna

Dr. Johannes Gadner

E: j.gadner@rat-fte.at

AIT - AUSTRIAN INSTITUTE OF

TECHNOLOGY

Giefinggasse 4, 1210 Vienna

Mag. Michael Dinges

E: michael.dinges@ait.ac.at

Mag. ${ }^{a}$ Barbara Heller-Schuh, MAS

E: barbara.heller-schuh@ait.ac.at

AO AUSTRIA - AGENCY FOR OUALITY

ASSURANCE AND ACCREDITATION

AUSTRIA

Franz-Klein-Gasse 5, 1190 Vienna

Dr. in Elisabeth Froschauer-Neuhauser

E: elisabeth.froschauer-neuhauser@aq.ac.at

Dr. ${ }^{\text {in }}$ Eva Maria Freiberger

E: eva.maria.freiberger@aq.ac.at

AWS - AUSTRIA

WIRTSCHAFTSSERVICE GMBH

Walcherstraße 11A, 1020 Vienna

Mag. ${ }^{a}$ Marlis Baurecht

E: M.Baurecht@aws.at

Mag. Norbert Knoll

E: n.knoll@awsg.at
CDG - CHRISTIAN DOPPLER

RESEARCH ASSOCIATION

Boltzmanngasse 20, 1090 Vienna

DI ${ }^{\text {in }}$ Mag ${ }^{\text {a }}$ Brigitte Müller

E: brigitte.mueller@cdg.ac.at

CONVELOP - COOPERATIVE

KNOWLEDGE DESIGN GMBH

Bürgergasse 8-10/l, 8010 Graz

D $^{\text {in }}$ Drin. Karin Grasenick

E: karin.grasenick@convelop.at

Erdbergstraße 82/4, 1030 Wien

Mag. Thomas Jud

E: thomas.jud@convelop.at

FFG - AUSTRIAN RESEARCH

PROMOTION AGENCY

Haus der Forschung

Sensengasse 1, 1090 Vienna

D| ${ }^{i n}$ Dr.in Sabine Mayer

E: sabine.mayer@ffg.at

Mag. Leonhard Jörg

E: leonhard.joerg@ffg.at

FWF - AUSTRIAN SCIENCE FUND

Haus der Forschung

Sensengasse 1, 1090 Vienna

Dr. Falk Reckling

E: falk.reckling@fwf.ac.at

Dr. Thomas Völker

E: thomas.voelker@fwf.ac.at

IHS - INSTITUTE FOR ADVANCED

STUDIES

Josefstädter Straße 39, 1080 Vienna

Dr. ${ }^{\text {in }}$ Angela Wroblewski

E: wroblews@ihs.ac.at

Mag. Richard Sellner

E: richard.sellner@ihs.ac.at

INDUSTRIEWISSENSCHAFTLICHES

INSTITUT - IWI

Mittersteig 10, 1050 Wien

FH-Hon.Prof. Dr. Dr. Herwig W. Schneider

E: schneider@iwi.ac.at

Mag. Philipp Brunner

E: brunner@iwi.ac.at

JOANNEUM RESEARCH

FORSCHUNGSGESELLSCHAFT MBH

Haus der Forschung

Sensengasse 1, 1090 Vienna

Mag. Wolfgang Polt

E: wolfgang.polt@joanneum.at

Mag. Jürgen Streicher

E: juergen.streicher@joanneum.at
KMU FORSCHUNG AUSTRIA

AUSTRIAN INSTITUTE FOR SME

RESEARCH

Gusshausstraße 8, 1040 Vienna

Mag. ${ }^{a}$ Iris Fischl

E: i.fischl@kmuforschung.ac.at

Mag. Peter Kaufmann

E: p.kaufmann@kmuforschung.ac.at

LUDWIG BOLTZMANN GESELLSCHAFT Nußdorfer Straße 64, 1090 Vienna

Mag. Patrick Lehner

E: patrick.lehner@lbg.ac.at

OEAW - AUSTRIAN ACADEMY OF

SCIENCE

Dr. Ignaz Seipel-Platz 2, 1010 Vienna

Nikolaus Göth, MSc

E: nikolaus.goeth@oeaw.ac.at

TECHNOLPOLIS GROUP

AUSTRIA

Rudolfsplatz 12/11, 1010 Vienna

Mag. Katharina Warta

E: warta@technopolis-group.com

VIENNA BUSINESS AGENCY.

A SERVICE OFFERED BY THE CITY OF

VIENNA

Mariahilfer Straße 20, 1070 Vienna

Robert Mayer-Unterholzer

E: mayer-unterholzner@wirtschaftsagentur.at

WIFO - AUSTRIAN INSTITUTE OF

ECONOMIC RESEARCH

Arsenal, Objekt 20

Postfach 91, 1103 Vienna

Dr. Jürgen Janger

E: Juergen.Janger@wifo.ac.at

Dr. Andreas Reinstaller

E: andreas.reinstaller@wifo.ac.at

WPZ RESEARCH GMBH

Mariahilfer Straße 115/16, 1060 Vienna

Dr. in Brigitte Ecker

E: brigitte.ecker@wpz-research.com

WWTF - VIENNA SCIENCE AND

TECHNOLOGY FUND

Schlickgasse 3/12, 1090 Vienna

Dr. Michael Stampfer

E: michael.stampfer@wwtf.at

Dr. Michael Strassnig

E: michael.strassnig@wwtf.at

ZSI - CENTRE FOR SOCIAL INNOVATION

Linke Wienzeile 246, 1150 Vienna

Dr. Klaus Schuch

E: schuch@zsi.at 


\section{CONTENT}

5 PREFACE

HEINZ FASSMANN, AUSTRIAN FEDERAL MINSTER OF EDUCATION, SCIENCE AND RESEARCH

6 FOREWORD

MATTHIAS REITER-PÁZMÁNDY

8 IMPACT RE-LOADED

THOMAS KÖNIG, HELGA NOWOTNY AND KLAUS SCHUCH

10 SSH-IMPACT PATHWAYS AND SSH-INTEGRATION IN EU RESEARCH FRAMEWORK PROGRAMMES.

THOMAS KÖNIG

26 SOCIAL SCIENCES AND HUMANITIES RESEARCH MATTERS. GUIDELINES ON HOW TO SUCCESSFULLY DESIGN, AND IMPLEMENT, MISSION-ORIENTED RESEARCH PROGRAMMES THOMAS KÖNIG

29 THE PUBLIC VALUE OF THE SOCIAL SCIENCES JOHN D. BREWER

33 BRINGING CONCEPTS TOGETHER: INTERDISCIPLINARITY, TRANSDISCIPLINARITY, AND SSH INTEGRATION JOËL GRAF

37 NEW PATHWAYS TO SOCIAL CHANGE CREATING IMPACT THROUGH SOCIAL INNOVATION RESEARCH JÜRGEN HOWALDT

49 THE IMPORTANCE OF SSH RESEARCH IN HORIZON EUROPE JOHN STEPHEN BELL.

53 INNOVATION FOR THE REFLECTIVE SOCIETY RICCARDO POZZO

56 STRIVING FOR REFLEXIVE SCIENCE ROGER STRAND 
62 SSHA-DRIVEN KNOWLEDGE TRANSFER WITHIN THE THIRD MISSION OF UNIVERSITIES GEORG RUSSEGGER

66 PANEL COMPOSITION AS PATHWAY TO IMPACT:

DO WE NEED STAKEHOLDER EXPERTISE TO SELECT RELEVANT MISSION-ORIENTED PROJECTS?

PETER VAN DEN BESSELAAR AND ULF SANDSTRÖM

72 SENIOR ACADEMICS AS KEY NEGOTIATORS IN THE IMPLEMEN TATION OF IMPACT POLICIES IN THE SOCIAL SCIENCES AND HUMANITIES

MARC VANHOLSBEECK, THEODOSIA DEMETRIOU, AGNE GIRKONTAITE, ANDREJA ISTENIC STARCIC, VILLE KEISKI, EMANUEL KULCZYCKI, ELENA PAPANASTASIOU, JANNE PÖLÖNEN, HULDA PROPPE AND MAJA VEHOVEC

80 INCLUSIVE FUNDING FOR ENHANCED IMPACT OF SOCIAL SCIENCES AND HUMANITIES

MALIN LINDBERG, SVETLANA GROSS, MILDA RÖNN, LISSA NORDIN, JAN SANDRED, LARS WÄRNGÅRD AND CATHARINA NORBERG

86 THE OULU WAY OF STRENGTHENING SOCIAL IMPACT OF SSH SCIENCES: FROM LINEAR MODELS OF IMPACT TO PRODUCTIVE INTERACTIONS AND BEYOND

JUHA TUUNAINEN, RAULI SVENTO, PENTTI HADDINGTON, KIRSI OJUTKANGAS AND SIRPA AALTO

91 ORIH: TOWARDS A FITTING SYSTEM FOR HUMANITIES RESEARCH EVALUATION

AD PRINS, JACK SPAAPEN, THED VAN LEEUWEN AND NELLEKE VAN DEN BROEK-HONINGH

99 ETA PROGRAMME ARTS, SOCIAL SCIENCES AND HUMANITIES AS AN INTEGRAL PART OF THE INNOVATION ECOSYSTEM OF THE 21 ${ }^{\text {ST }}$ CENTURY MARCEL KRAUS 
105 THE "6I RESEARCH MODEL": EVOLUTION OF AN INNOVATIVE INSTITUTIONAL STI POLICY FRAMEWORK AT THE UNIVERSITY OF DEUSTO

ANTONIA CARO-GONZALEZ

114 EVIDENCE-BASED PRACTICE AND POLICES FOR IMPACT ON MENTAL HEALTH OF CHILDREN AND ADOLESCENTS RAPHAELA E. KAISLER AND JEAN L. PAUL

120 IMPACT OF SOCIAL SCIENCES AND HUMANITIES FOR A EUROPEAN RESEARCH AGENDA - VALUATION OF SSH IN MISSION-ORIENTED RESEARCH BETTINA UHRIG

125 THE CHANGING PATTERN OF SOCIAL SCIENCES AND HUMANITIES IN THE EU FRAMEWORK PROGRAMMES RETHINKING SOCIETAL IMPACT - COLLABORATION WITH STAKEHOLDERS ÜLLE MUST

129 SPECIFIC SSH RESEARCH AND GENERAL RESEARCH POLICY INSTRUMENTS. THE CASE OF THE NATIONAL RESEARCH PROGRAMMES AND "NEED DRIVEN SSH RESEARCH" IN LITHUANIA RÜTA PETRAUSKAITÉ

133 GHENT UNIVERSITY'S INTERDISCIPLINARY SSH-CONSORTIA A STRATEGY TO ENHANCE THE SOCIETAL IMPACT OF RESEARCH

NOËL KLIMA, STEFAN MEYSMAN, JULIE CARLIER, ALEXIS DEWAELE AND ESTHER DE SMET EVALUATION OF SOCIETAL IMPACT IN NORWEGIAN SSH

139 EVALUATIONS OF SOCIETAL IMPACT IN NORWEGIAN SSH EVALUATIONS

JON HOLM AND ANETTE ASKEDAL 
143 STAKEHOLDERS' ROLE TO PRODUCE IMPACT FROM SOCIAL SCIENCE RESEARCH: WHAT LESSONS FOR EVALUATION? EMANUELA REALE, SERENA FABRIZIO AND LUCIO MORETTINI

152 ASSESSING THE IMPACT OF SSH-RRI APPROACH ON ICT RESEARCH \& INNOVATION: THE HUBIT PROJECT

TAL SOFFER, RUTH ZUZOVSKY, OLENA NEDOZHOGINA AND EMANUELE BARDONE

159 RETHINKING RESEARCH IMPACT ASSESSMENT: A MULTIDIMENSIONAL APPROACH SERGIO MANRIQUE, MARTA NATALIA WRÓBLEWSKA AND BRADLEY GOOD

176 EVALUATIVE INQUIRY: ENGAGING RESEARCH EVALUATION ANALYTICALLY AND STRATEGICALLY

SARAH DE RIJCKE, TIITSKE HOLTROP, WOLFGANG KALTENBRUNNER, JOCHEM ZUIJDERWIJK, ANNE BEAULIEU, THOMAS FRANSSEN, THED VAN LEEUWEN, PHILIPPE MONGEON, CLIFFORD TATUM, GOVERT VALKENBURG AND PAUL WOUTERS

183 VALUATION OF SSH RESEARCH FOR A TRANSFORMATIVE EUROPEAN RESEARCH AGENDA - A FEW CLOSING WORDS KLAUS SCHUCH

188 SCIENTIFIC COMMITTEE OF THE CONFERENCE

189 POSTERS 


\section{PREFACE}

HEINZ FASSMANN, AUSTRIAN FEDERAL MINSTER OF EDUCATION, SCIENCE AND RESEARCH

\section{LADIES AND GENTLEMEN,}

$\mathrm{T}$

The conference proceedings you are about to read are the result of the conference "Impact of Social Sciences and Humanities for a European Research Agenda" during the Austrian Presidency of the European Council in the second half of 2018. The conference was part of the Austrian presidency's activities in the field of Research \& Innovation policy and served as a forum for policy makers and researchers to discuss matters of social sciences and humanities (SSH) research in the context of the evolving European research framework programme Horizon Europe.

The papers in these proceedings reflect the rich and broad debate at the conference. They give a most valuable overview about issues of the integration of social sciences and humanities in research programmes and about the impact of SSH.

As Minister responsible for science and research policy let me underline the necessity of the social sciences and humanities from a policy perspective. SSH-research is of high importance for Europe and of high importance for European research framework programmes. SSH-disciplines produce fundamental knowledge about us as individuals and as a society. The social sciences and humanities show us where we come from and help us better understand where we are going. Expertise in the fields of history, society, law, languages, arts and culture, to name only a few, provide an important contribution to economic and societal progress in Europe. The challenges of our time - climate change, sustainability, violent conflicts, an ageing society, artificial intelligence and its consequences for society - cannot be solved by contributions from the natural sciences and engineering alone. We need contributions from SSH to better understand human behavior and to find solutions for new technologies, social innovations or political decision making.

Social sciences and humanities are scientific fields that have a strong trait of self-reflexivity. They have intensive debates about their theories and methods. Their critical perspective is constantly challenging established patterns of thinking and behaving. This perspective should be used for research and innovation in general in a productive way. SSH also have a specific role, when it comes to the question of impact. They look at themselves and their own impact. But they are also deeply involved in developing the concept of impact in general. These disciplines have much contributed to the debate of re-defining impact that has developed from looking merely at research impact measured by h-factors, citations, and the amount of publications towards looking more broadly at societal impact.

Another important feature of the social sciences and humanities is their role in contributing to the implementation of scientific results into reality. One pathway to bring scientific results into reality is, by contributing to sectoral policies. Research and innovation play an ever more important role in sectoral policies. R\&l policy should reflect this more strongly. The challenges of our time need modern governance, that is, more cooperation between all policy areas. SSH-research plays an important role in facilitating this exchange between research policy and sectoral policies.

In Horizon Europe social sciences and humanities will play a strong role. All three political institutions involved in its negotiation, the European Commission, the European Parliament and the European Member States agree that SSH will be key to turn Horizon Europe into a success.

Social sciences and humanities will play an important role in the first pillar of Horizon Europe, in the European Research Council. In that part of the programme SSH has always been a strong component. SSH will also play a very important role in pillar two, Global Challenges and Industrial Competitiveness. The challenges of our time cannot be solved by contributions from the natural sciences and engineering alone. In each cluster of the second pillar, SSH-integration will bring much needed expertise to advisory groups, calls and subsequent research projects. Cluster 2 will have a particular focus on research questions in the fields of democracy, cultural heritage and creativity as well as societal transformation. All of them are highly relevant for Europe and its future and all of these fields will generate SSH-intensive research. Last but certainly not least, SSH will also be essential for the new and promising instrument of Missions in Horizon Europe.

The Vienna conference focused on SSH-integration and the impact of SSH-research in Horizon Europe and beyond. The research framework programme, now Horizon 2020, very soon Horizon Europe, is unique in the world. Just alike SSH-Integration as a broad, cross-cutting issue and a genuine strategy in such a large programme is a unique feature. It really shows the importance SSH has in European society and European science.

I wish you much inspiration, but maybe even more important stimulus for action when reading the conference proceedings.

\author{
Heinz Fassmann \\ Austrian Federal Minster of Education, Science and Research
}




\section{FOREWORD}

\author{
MATTHIAS REITER-PÁZMÁNDY
}

DOI: $10.22163 /$ fteval.2019.360

\section{LADIES AND GENTLEMEN, DEAR COLLEAGUES, DEAR FRIENDS,}

$\mathrm{T}$ his is the moment to look back on a very successful conference on the impact of social sciences and humanities and their integration in research framework programmes.

The conference had 30 sessions, 130 speakers, 20 papers and on the two days 340 people (55\% women) from over 30 countries attended the event. The conference did not only induce real life debates, but also lively interactions online. During the conference the dedicated Twitter hashtag \#SSHimpact was a huge trend generating more than 1.900 tweets. The conference video and the livestreamed sessions were accessed more than 200 times each. The photos and videos about the conference were downloaded several hundred times.

For me the conference was a very impressing experience. I like to think back to the very intriguing start with pointed statements by Klaus Schuch and Ulrike Felt. In his opening words Austrian Federal Minister for Education, Science and Research Heinz Fassmann put great emphasis on the importance of SSH for research in general and the European Research Area in particular. Deputy Director General Wolfgang Burtscher lined out how the European Commission attaches great importance to SSH for their transformational power and the power to help solving the social challenges of our time. The keynote speech by John Brewer put forth the necessity that the social science and humanities engage in the impact discussion because they are already very good at impact. The very spirited afternoon keynote from Ineke Sluiter discussed the role of the humanities in innovation, the humanities' impact in teaching and she provided the audience with insights about the roots of particular innovations in ancient Greece. An afternoon full of hands-on interaction with policy officers from a number of Directorate Generals of the European Commission, DG Research \& Innovation, DG Migration \& Home Affairs, DG Health and DG Connect, showed that SSH experts already engage now with all scientific and policy fields where society is faced with challenges. But they also showed that there is still much potential to broaden and deepen this exchange across disciplines and across sectors. I particularly like to remember the late afternoon session about artificial intelligence and SSH together with industry representatives that resulted in demands for more interdisciplinary curricula and more practical guideline in ethics and Al. The second day started with James Wilsdon giving a broad overview about impact debates and SSH. The main focus of that day was on the paper sessions. You find their output largely reflected in the papers published in this issue. I listed some of my personal highlights, but of course there were many more. The conference ended with an emphatic contribution from Head of Unit Harald Hartung from the European Commission, DG Research \& Innovation and the clear invitation towards the SSH-community to work more closely together.

The event joined the choir of positions that led to the role of SSH in the current version of the Horizon Europe regulation. SSH will be much stronger rooted in the regulation for Horizon Europe ${ }^{1}$ than it was in the regulation for Horizon 2020. Article 4 on the programme structure regulates that social sciences and humanities (SSH) shall play an important role across all clusters of Horizon Europe. Article 6a on the principles of the programme regulates that Horizon Europe shall ensure a multidisciplinary approach as well as the integration of SSH across all clusters and activities developed under the programme. Article 7 on the Missions defines that Missions shall stimulate activity across disciplines including SSH. The specific programme mentions SSH also several times explicitly and regulates the research areas in a more detailed way. ${ }^{2}$ All these concrete references are a substantial improvement and upgrading of the role of $\mathrm{SSH}$ in the legal basis of the future framework programme in comparison to Horizon 2020.

The conference was not only designed as a forum for exchange between researchers and policy makers, but also as an undertaking that produces tangible outcomes supporting researchers and policy makers in their work. The first publication was a four-pager with the title "Social Sciences and Humanities Research Matters. Guidelines on how to successfully design, and implement, mission-oriented research programmes", in short "SSH-guidelines". The content was intensively discussed, in a dedicated workshop during the conference, with experts from research and policy making, including the European Commission. The aim of the hands-on document is to provide policy makers who design research programmes, but also research managers, reviewers and evaluators with recommendations for their work. Ever since it was published in February 2019 it has sparked debate and action on European, but also on national level.

\footnotetext{
1 In the current result of negotiations as a Proposal for a Regulation of the European Parliament and of the Council establishing Horizon Europe - the Framework Programme for Research and Innovation, laying down its rules for participation and dissemination, 22.03.2019, 2018/0224(C0D), https://www. consilium.europa.eu/media/38902/st07942-en19.pdf In the current result of negotiations as a Proposal for a Decision of the Council on establishing the specific programme implementing Horizon Europe - the
Framework Programme for Research and Innovation - Partial General Approach, 15.04.2019, 2018/0225(COD), https://data.consilium.europa.eu/doc/document/ST-8550-2019-INIT/en/pdf
} 
The SSH-guidelines were followed by the Working Paper "SSH-Impact Pathways and SSH-Integration in EU Research Framework Programmes" by Thomas König that will broaden the perspective of the practical recommendations in scientific and policy debates. It builds on the scientific discourse on valuation of SSH research as well as SSH-integration in EU framework programmes. It discusses recent trends in research funding, provides a brief historical overview of the efforts of integrating SSH into the EU research framework programmes and concludes with suggestions for SSH-researchers. The Working Paper is included in this issue.

One of the specific recommendations is to organize workshops for SSH researchers and policy makers on the national level. In Austria we started with a pilot workshop in March 2019. SSH researchers from different disciplines and research fields, from universities and non-university research institutions met up with national Programme Committee delegates from different sectoral Ministries as well as the National Contact Points (NCPs) in Austria. The workshop was a great start of an initiative that needs to grow further as Horizon Europe will come closer.

I would like to thank once again all who contributed to the conference. First of all, a thank you goes to the Local Advisory Board of the conference who discussed the earliest drafts of the concept: Tara Andrews, Christian Fleck, Michaela Glanz, Barbara Horejs, Monika Mokre, Stephanie Rammel, and Frank Welz. A particular thank you goes to Thomas König and Katja Mayer who worked closely with us throughout the preparatory phase. I would like to thank the international Scientific Committee that did all the hard work of reviewing the papers of the Call to this conference: Paul Benneworth, Olivier Bouin, Ulrike Felt, Yves Gingras, Poul Holm, Jürgen Howaldt, Carina Keskitalo, Kirsten Langkilde, Stefania Milan, Andrea Pető, Claudio Radaelli, Emanuela Reale, Sarah de Rijcke, Evelyn Ruppert, Marta Soler, Jack Spaapen, Tereza Stöckelova, Johannes Vogel, and Milena Zic-Fuchs. I also want to thank the committee's chair Helga Nowotny, who was giving us the most valuable input early on and drafted the "Impact Re-loaded" input paper. I would also like to thank the European Commission for their financial support and endorsement. A special thank you goes to the staff at the Unit B6 in DG Research \& Innovation, who were extremely helpful throughout the preparation: Head of Unit Harald Hartung and Project Officer Monica Menapace. A very special thank you goes to Project Officer Basudeb Chaudhari, who was putting that extra effort in our common preparatory work. I also would like to thank for the support within my own Ministry, especially from our Director General Barbara Weitgruber, the Head of EU-Department Christian Naczinsky and my Head of Department Ursula Brustmann. I also have to thank all those invaluable helping hands that created such a good environment at the conference: Philipp Brugner, Patrik Cunat, Alexander Degelsegger-Márquez, Isabell Duscher, Tanja Feiler, Robert Frühstückl, Maximilian Jäger, Doris Kaiserreiner, Elisabeth Koller, Alexandra Krammer, Silvia Kraml, Martina Lindorfer, Gottfried Prinz, Peter Seitz, and Gorazd Weiss. A very special thank you goes to Elke Dall, and an extra special thanks to Bettina Glaser and Berenicke Ecker who went the metaphoric extra mile - and at the conference they did so in the real sense of the saying. Last but not least, a special thank you goes to Klaus Schuch, the director of the ZSI, Centre for Social Innovation, who did a beautiful job both on the organisational as well as on the intellectual level of the conference preparations.
The conference is over, the publications are published, but the work will continue. We need to cooperate to unfold the full potential of SSH in Horizon Europe, its Clusters and Missions. More exchange between policy makers and researchers is still needed. SSH researchers need not to shy away from approaching their policy makers. Policy makers should seek to exchange more with SSH researchers, their projects and their findings. This conference aimed at providing a forum for this exchange. I do wish that the conference publications - the SSH-Guidelines, the Working Paper and these proceedings - will help to carry on this exchange. Have an interesting read!

\author{
Matthias Reiter-Pázmándy \\ Austrian Federal Ministry of Education, Science and Research
}




\section{IMPACT RE-LOADED}

\author{
THOMAS KÖNIG, HELGA NOWOTNY AND KLAUS SCHUCH \\ D0I: 10.22163/fteval.2019.361
}

$\mathrm{T}$ here are various attempts to circumscribe and catch the meaning of "impact" related to and resulting from scholarly research from the social sciences and humanities. 1 For all their commendable efforts, these definitions cannot remove the impression that the initial need to come up with a definition is driven by political motives. As a result, the use of the term "impact" has often acquired a defensive tone. The political motives spring largely from increasing demands for accountability; and the defensiveness can be detected in the way "impact" is set up to prove the relevance to society.

We argue that time has come to move beyond a purely defensive stance on the part of the social sciences and humanities. There is a more substantial issue involved, namely, to re-think the transformative relationship between science and society. Scientific research is about transformation - how to enable it, or how to avoid it. It is about the transformation that society is undergoing as much as about the transformative power inherent in knowledge and policies based on social science knowledge. The social sciences and humanities are deeply involved in the processes that use scientific and scholarly approaches to bring about a better society, difficult as it may be to define it. Arguably, their societal and political relevance has always been more present in the political arena than that of the natural sciences. This should be acknowledged and not denied.

Social sciences and humanities have to look at "impact" in a different way - the term needs to be "re-loaded" with a renewed sense of responsibility and reflecting a different self-image of their role and position in society. Instead of using "impact" solely as a defensive instrument to preserve the status quo of the social sciences and humanities, the contemporary focus on "impact" offers a unique window of opportunity for the social sciences and humanities to reflect upon and redefine their role and redefine their societal relevance. This understanding of "impact" is not limited to the instrumental "use value" that SSH research may provide for certain user groups, but is wide-ranging through the implicit embeddedness of SSH within society, provided that it remains open to society, and its power to analyse and explain social phenomena and to contribute to overcoming societal drawbacks through a diversity of discourse and exchange levels and formats. These aspects can be dealt with distinctively, albeit they are interrelated.

a. With "impact" becoming the driving force for assessing relevance of scientific endeavors the social sciences and humanities are in a position to contribute to, and shape the concept. The reason is that they study impact, they reflect impact, and they assess impact. It is not by chance that a social scientist has elevated the notion of "unintended consequences" to prominence and that assessing these consequences has become one of the main rationales of applied research in the social sciences and humanities.

b. The social sciences and humanities have made tremendous progress in the past two decades, in terms of expanding their methodology and conceptual approaches. While there is still much disagreement among disciplines, schools of thought, and epistemic communities, much of this is due to the logic of how academia is organised. Here, "impact" may offer a powerful leverage to address inconsistencies and to come up with a more collaborative understanding of what is at stake, thereby ironing out many of the rather frustrating internal academic struggles.

c. Finally, the rise and productivity of the social sciences and humanities have been strongly connected and inevitably shaped by the process of modernity. While this interdependence has been acknowledged, the repercussions have not fully been absorbed. Transformative science must be transformative in a double sense: wanting to exert influence in society but also open to be influenced by society and its needs. In other words, only if open two-way mutual communication channels are established, societal needs, regardless of their origins and the ways in which they are expressed, the transformative power inherent in SSH research can unfold in society. Among other, this necessitates greater openness and readiness as well as capability to involve heterogeneous groups in society, rather than remaining fixated on policy-makers.

The conference offers a unique setting to take on these various aspects, and to rethink the vital role the social sciences and humanities can play in facing many of the challenges European societies are confronted with. Policy issues range: from environmental issues and behaviour, agricultural policy and consumption, technology and innovation, security, foreign and defence, public finances, culture and media, health, judiciary, to transport and economic sustainability. To stimulate a process of re-loading, we invite for papers from different perspectives of impact. In particular, we would like to move "impact" from a mostly defensive, albeit policy-relevant instrument to something that will become a transformative element towards a more inclusive society. 


\section{AUTHORS}

\section{THOMAS KÖNIG}

Institut für Höhere Studien - Institute for Advanced Studies (IHS)

Josefstädter Straße 39, Vienna, 1080 (Austria)

E: koenig@ihs.ac.at

\section{HELGA NOWOTNY}

Wiener Wissenschafts-, Forschungs- und Technologiefonds

(WWTF), Vienna Science and Technology Fund

Schlickgasse 3/8, Vienna, 1090 (Austria)

E: helga.nowotny@wwtf.at

\section{KLAUS SCHUCH}

Zentrum für Soziale Innovation GmbH - Centre for Social Innovation (ZSI)

Linke Wienzeile 246, Vienna, 1150 (Austria)

E: $\underline{\text { schuch@zsi.at }}$ 


\title{
SSH-IMPACT PATHWAYS AND SSH- INTEGRATION IN EU RESEARCH FRAMEWORK PROGRAMMES.
}

\author{
THOMAS KÖNIG \\ DOI: 10.22163/fteval.2019.362
}

\author{
IN REMEMBRANCE OF \\ PHILIPPE KERAUDREN (1963-2017)
}

\section{ABSTRACT}

$\mathrm{T}$ This Working Paper builds on the scientific discourse on valuation of SSH research as well as SSH-integration in EU framework programmes and aims at summarising the key findings from the November 2018 Austrian EU Presidency Conference "Impact of Social Sciences and Humanities for a European Research Agenda - Valuation of SSH in mission-oriented research". It deals with the topic in three instalments. First, it will discuss recent trends in research funding. Second, it provides a brief historical overview of the efforts of integrating SSH into the EU Research Framework Programme. It then adds some observations about continued challenges in SSH. Finally, it will conclude with some suggestions for SSH scholars, based on the discussions from the conference. In that regard the Working Paper is also a document for further reading for those who have read earlier, shorter texts that were published in preparation of that conference.

\section{TABLE OF CONTENT}

SSH-Impact Pathways and SSH-Integration in EU Research Framework Programmes.

Abstract

1. Introduction

2. Recent developments in research funding

3. Historical assessment of integrating SSH

4. Challenges in SSH

5. Impact re-loaded in Horizon Europe

Annex: Summary of Meeting "Social Sciences and Humanities in Horizon Europe"

Bibliography

Keywords

\section{INTRODUCTION}

7 his Working Paper reflects the current status of research in the social sciences and humanities (in the following: SSH research) in the context of European research policy. ${ }^{1}$ It examines three seemingly separate issues: the recent development of research policy, both in terms of actual funding as well as its rhetoric; the actual history of SSH research within the European Union research funding instruments; and the epistemological characteristics of SSH research. Tying these issues together will provide a better understanding of where the social sciences and humanities stand, what their capacities are, and what they can provide to tackle societal challenges that we, as humankind, face today. Based on this background, the ambition of this Working Paper is to discuss how to enhance the role of SSH research in current and future research funding policies.

This Working Paper follows up on two shorter policy papers. The first, called "impact re-loaded", was written in spring 2018 by three co-organisers of the SSH impact conference in Vienna in November of the same year, making the case to their colleagues in the SSH community to "reflect upon and redefine their role and redefine their societal relevance". Specifically, the paper wanted to achieve three things: "to contribute to, and shape the concept" of impact; to shed away academic struggles in order "to come up with a more collaborative understanding of what is at stake"; and finally, while "wanting to exert influence in society", also being "open to be influenced by society and its needs." (König, Nowotny, and Schuch 2018) While this Working Paper hopes to provide additional insights into all three of these aspects, it is clearly focused on the second aspect, that is, to contribute to enhancing the conditions for SSH to provide robust, and lasting, contributions to solving societal challenges. The other paper, shortly SSH Guidelines, summarises recommendations for R\&D programme authorities, reviewers and programme evaluators (König 2019). Since this second policy paper could only make claims, the Working Paper also aims at substantiating the role, and characteristics, of SSH research (for more details on the SSH Guidelines, see section 5).

Given the perspective and supplemental role of this Working Paper, there are three important restrictions to announce right away. (1) The ambition of this document is not to lay out in detail what kind of SSH 
research is relevant for cooperative, interdisciplinary research tackling societal challenges, and to what end. Other reports have already provided substantial input to this, and interested readers are explicitly invited to read them with great attention (see Atkinson et al. 2009; Drotner 2013; Daston et al. 2018). Rather, this Working Paper is to discuss the context, constraints, and potentials of SSH research. It is much more concerned with questions related to science policy and, more specifically, research policy.

(2) For the remainder of this Working Paper, SSH research means primarily research carried out along project-based funding. At European level, this is mostly done under the EU Research and Innovation Framework Programme (aka FP, currently in its eighth edition, called Horizon 2020 and from 2021 onwards in its ninth edition called Horizon Europe). Obviously, there is a wide array of contributions of social sciences and humanities in other areas of the European polity - providing crucial social and economic data (like EUROSTAT), building up transnational infrastructure (such as CESSDA, CLARIN, DARIAH, ESS, SHARE), or providing intellectual reflection and independent analysis of the European integration process (by institutes such as EUI, but also in academic conferences, etc.). ${ }^{2}$

(3) Even the focus on the FP and its sprawling set of funding instruments requires further restriction, as this paper is interested mainly in programmes dealing with mission-oriented research funding. Again, there are other funding opportunities within the FP that enable researchers from SSH to conduct academic research. ${ }^{3}$ The restriction is justified because the question at hand is about the potential role of SSH in contributing to producing new knowledge specifically to solve problems that are generally perceived to be worrying risks to individuals, peoples, societies and humanity. These problems are not defined in a purely scientific manner, albeit scientific research may have contributed to their existence in the first place and usually also provides the toolkits to recognise and understand them. For example, the list of "Sustainable Development Goals" (SDG), as adopted by the United Nations General Assembly in 2015 , consists of a number of problems that have been identified, acknowledged, and also negotiated in an intricate policy process involving all UN member states.

Whatever their denomination in the specific policy context: the emphasis on "challenges", goals" and "missions" recognises that there are problems so wicked that we require particular efforts to cope with them. Obviously, science - and new scientific knowledge - is key to understanding those problems, to alleviating them and also to preparing for potential fallouts. At the same time, this added a new layer to the ambitions of research funding policy. It has also renewed the quest to increase cooperation between different fields of science and scholarship, and has reinforced the growing demand for "impact".

\section{RECENT DEVELOPMENTS IN RESEARCH FUNDING}

From a scholarly perspective, "science" can be described as a selfregulatory, multi-faceted, highly specialised institution whose numerous factions and divisions nonetheless share some important informal norms (Merton 1957, 537-61), and research (or, in economical terms: knowledge production) is one of the key components of this institution. 4 Yet science is also regulated by policy, and money has emerged as a defining ingredient in this relationship (Greenberg 2001). Historically, public policy attempts to guarantee public benefits while maintaining scientific independence can be differentiated into periods.5 Against this backdrop, the relationship of "scientific research" and public policy has been coined by three interlaced trends over the last two decades. The first is the innovation policy narrative; the second is about interdisciplinary cooperation; the third is about impact. All three have consequences for SSH research in the European research funding landscape at large, and in the missionoriented research funding parts of the FP specifically. In the following three instalments, a closer look at each of those trends is provided.

\section{THE INNOVATION POLICY NARRATIVE}

The narrative of innovation policy stresses the importance of scientific research for innovation, and thus, for the well-being of individuals and our societies. If economic growth is the bedrock of democracy, then innovation is the best guarantee for economic growth. But because investment in scientific research is broadly accepted to be a common good (Stephan 2012), innovation must be stimulated through public spending in research and development (R\&D). In the European Union, this narrative emerged in the 1990s (Ulnicane 2015), solidified into a new, additional European "governance architecture" (Borrás and Radaelli 2011), and, with its flagship "innovation union" (European Commission 2010) has become one of the latest hopeful driving forces for further integration amidst an EU that otherwise is often described as being in crisis. ${ }^{6}$ The current debate about the future EU-Budget, the next multiannual financial framework (MFF) from 2021-2027, vividly continues this narrative.

The innovation policy narrative (see Figure 1) shares some similarities with what is usually known as the linear model of innovation, the assumption that there is a sequence of steps from "basic research" through applied research to development and marketisation of new products. As has been convincingly argued, while the linear model of innovation is often thought of as too simple by experts", it remains a "social fact" partly

2 For useful reflections of the relationship between social sciences and European integration, see Rosamond (2007), also Anderson (2009).

3 Most prominently, this is the European Research Council (ERC), which offers generous funding to individual researchers in a highly competitive manner (König 2016).

$4 \quad$ Other components are training in scientific methods and teaching of theories, and dissemination of research results. Merton, in the book referred to, also points out that "science is a deceptively inclusive word", and restricts his own analysis to "science as an institution" (551). This is true also for the way the term is used here, except that it explicitly includes the social sciences and humanities.

$5 \quad$ The most basic, and best known, differentiation is the one between "Mode 1" and "Mode 2" periods; cf. Gibbons et al. (1994; see also Braun 2003 for further elaboration). Elzinga (2012) suggests a periodisation that better aligns to historical developments since the end of WWII; he distinguishes between the consecutive periods of "legitimation", "professionalisation", and "accountability".

6 See, for the European Union, a short analysis in König $(2017,123-27)$

7 Alternative approaches include the "Mode 2" and various "helix" models; for a good overview, cf. Hessels and van Lente (2008). 
because it is so entrenched in statistics (Godin 2009, 27). Similarly, while there are reasonable doubts about the underlying assumptions of the narrative (Wladawsky-Berger 2018), and attempts to come up with alternatives (Nowotny 2016), it seems fair to say that the innovation policy narrative remains convincing for policy makers thus far.
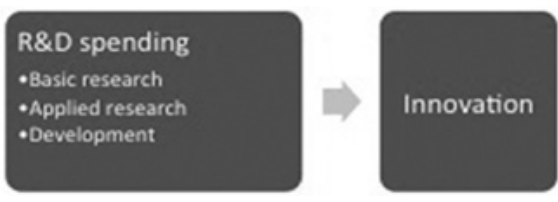
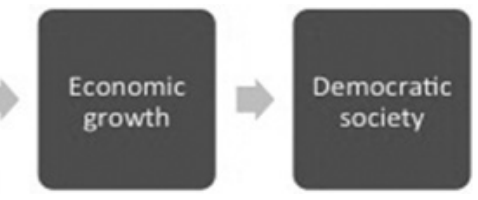

Fig. 1: Schematic depiction of the innovation policy narrative (Drawn by the author)

Why this persistence? The rise of the innovation policy narrative is not purely a discursive phenomenon, as it has increased attention towards creating opportunities for new knowledge (Flink and Kaldewey 2018). Policy makers and the public have been willing to pour more money into scientific research with the expectation of increased social benefit. But this is coming with strings attached, and potential ramifications for SSH research in particular. One consequence is that "innovation" is usually thought of in a narrow sense: everything that leads to commodification, marketisation of products. ${ }^{8}$ Such expectations are also somewhat predetermining the type of research that is to be supported in the first place. Also, there seems to be a preference for spending additional means in the form of competitive project-based research funding. Finally, there is an increased demand to prove the value of research funded by public domain, the (perceived) pressure on policy makers to show accountability to the tax payer, and to objectively control the usage of public funding in a new bureaucratic fashion ("audit culture").

\section{INTERDISCIPLINARY COOPERATION}

Debate about the illnesses of academically organised, disciplinary research is ongoing. ${ }^{9}$ One of the oldest tropes of science policy has been the notion of "interdisciplinarity" - the idea of overcoming the "epistemic rent-seeking" of scientific disciplines (Fuller 2016) by integrating the strengths of various disciplines towards one research goal (Frodeman, Klein, and Pacheco 2017). In the words of one of the leading scholars on the topic, the notion of interdisciplinarity is more about "expressing our dissatisfaction with current modes of knowledge production" than providing a concept of what it actually is (or could be) (Frodeman 2010). At the same time, this combination of emptiness and promise might easily be one major reason for its continued success.

This does not mean that interdisciplinary research is not taking place. Yet the innovation policy narrative and its aforementioned ramifications for research (and SSH research specifically) bring a new dynamic to the age-old idea of interdisciplinarity. The increase of project-based research funding and the new emphasis on tackling societal challenges mean that interdisciplinary research is often expected in terms of temporary, contractual cooperation - with all the problems and opportunities that go along with it (Lyall et al. 2013; König and Gorman 2016). But it is necessary to think of different "modes" of interdisciplinary cooperation that result from the specific questions to be tackled as much as from the broader circumstances that drive research. Indeed, one can distinguish between an integrative, a subordinate, and an agonistic mode of interdisciplinary cooperation (Barry, Born, and Weszkalnys 2008, 28-29). It is easy to see how this is of particular importance for SSH research: on the one hand, the tendency to bring scholarly research in the social sciences and humanities under an all-encompassing funding regime, together with the natural and life sciences and engineering, is an opportunity to make better use of SSH research and to open up the field. Yet there is also a considerable pressure to align research on intricate and complex relations of societal ailments to the formal requirements of those temporary combinations of researchers. Also, there is a tendency to delegate certain aspects (like participation, communication or ethics) of a large cooperative research project to partners from SSH fields, which does not necessarily do justice to the potential input that could be provided.

\section{IMPACT ASSESSMENT}

Public funding bodies have established and fine-tuned administrative procedures to make sure that taxpayers' money is well-used. As in many other areas of performance measurement (Muller 2018), the New Public Management style has found its expression in a "metric tide" at universities (Wilsdon et al. 2015) with the attempt to assess input, output, and impact of scientific research (de Rijcke and Rushforth 2015). While in the late 1990s and early 2000s, the main focus of assessing the quality of research and scientific conduct has been on academic relevance (often under the term "excellence"), recently there has been a shift towards the broader notion of impact. ${ }^{10}$

Impact of research means at least three different dimensions of newly produced knowledge; besides academic impact it also includes impact on the political realm and on the public, or society, by and large. Academic impact of knowledge production is rather easily assessed, usually

8 A historiographical analysis has revealed the complex history of the term "innovation", see Godin (2015)

$9 \quad$ For a powerful, recent argument in that context see the essay by Dan Sarewitz (2016). A good summary of "malfunctions" of science is provided by Fischer (2008).

10 For a recent, powerful critique on the notion of excellence in research see Moore et al. (2017). 
through citations; it relies on a decade-old field called "scientometrics" (Mingers and Leydesdorff 2015; Gingras 2016). Broadening the meaning of impact has opened the door to a wider variety of tools of assessment, some of which rely on exciting new techniques; ${ }^{11}$ yet it also brought in considerable difficulties, or ambiguities. ${ }^{12}$ To start with, there are different types of impact along two dimensions (expected vs. unexpected, and intended vs unintended) (Reale et al. 2014, 37). Also, there are at least four problems when assessing, or measuring, impact of research: the problem of causality, the problem of attribution, the problem of internationality, and the problem of the observation period (Felt and Fochler 2018, 9-10). These difficulties apply not solely to SSH research. Given the difficulties that come along with it, the broadening of the concept of impact has specific ramifications for the social sciences and humanities. (Reale et al. 2017)

\section{WHAT THESE TRENDS MEAN FOR SSH RESEARCH}

Based on this tour de force, we can briefly summarise the constraints that current trends in research funding pose on SSH research specifically. One is that the narrow concept of innovation seems to exclude broader notions of societal innovation. Another is the urge to collaborate temporarily and the tendency of being delegated a specific role in the interdisciplinary machinery. And yet another one relates to the inherent difficulties of proving its value under the current audit culture regime. At the same time, one must also emphasise the opportunities that are created here for SSH research to actually play a more important role in the production of knowledge that is relevant for society. We can see within the continued paradigm of innovation policy that a dual shift is taking place. On the one hand, this shift is moving away from the excellence rhetoric that was behind the drive to reinvigorate the European Research Area, aiming at broader impact; on the other hand, the new focus on tackling societal challenges through mission-oriented research funding instruments also means that the narrow understanding of "innovation" may be prone to some conceptual adaptation.

A critical issue of this summary is that much depends on the SSH communities themselves: it is up to them to get involved and to make sure their considerable amount of expertise is better heard. This call for active involvement is not new. The next section aims to take a look at the history of SSH involvement and the achievements that have been made so far.

\section{HISTORICAL ASSESSMENT OF INTEGRATING SSH}

Against the backdrop of the general context of recent developments in research funding mentioned in the previous section, it is now necessary to assess the development of dealing with social sciences and humanities under the latest editions of the EU Research Framework Programme (FP). The 2009 Lisbon "Treaty on European Union" put science and research officially at the European stage (TEU Art 3(3), and TFEU Art 179-190), but as a matter of facts, research policy had been there for a long time already (Banchoff 2002, 7-8; also Guzzetti 2000, 2009). Social Sciences had their own targeted programme from 1994 on, immediately following the Maastricht Treaty (Kastrinos 2010, 300). Since the sixth edition of the FP, the humanities were also officially included (Smith 2003). For SSH research as a whole, therefore, the role of European funding, and the European Commission's FP specifically (Schögler 2013; Schögler and König 2017), has increased over the past two decades, and with regards to two aspects.

In his analysis of SSH in Europe from 2010, Nikos Kastrinos (2010) found that, despite the emphasis of research priorities and thematic orientations, European research funding then was moving more and more towards a "diffusion-oriented model", emphasising capacity building over fulfilling a distinct mission (301). This would also remain the case with the eighth edition of the Framework Programme, Horizon 2020, even though the missions-approach would soon make a comeback. The second observation was that the EU research programmes had emerged as points-of-reference for the member states, both in terms of themes (such as the challenges) and in the orientation (diffusion instead of mission): ${ }^{13}$ in some respect they had even outpaced funding opportunities at national level. The third observation referred to the fact that, despite of its limited size within the overall FP budget, and despite several national funding schemes targeting research in the social sciences and humanities, "in comparative terms" the FP's own dedicated research funding for SSH "has been the largest targeted programme in Europe" that was available for research in social sciences and humanities (304).

\section{RUNNING UP TO HORIZON 2020}

Kastrinos article summarised the state of development for SSH research shortly before negotiations of the eighth edition of the FP (Horizon 2020, which was scheduled to begin with 2014), and the role of SSH research in it, started. However, to understand the debate that followed, it is important to also take into consideration the broader context of that time. The diffusion-oriented approach of defining broad thematic challenges, the growing importance of coordination of research policy at European level, and the fact that the latest editions of the FP also included large programmes funding SSH research already put pressure to fit in on those communities that perceive themselves as part of the label "SSH". This only intensified in 2008 and the following years, when

\footnotetext{
11 See, for example, the topic analysis in the UK report on arts and humanities by Draux and Szomszor (2017)

12 For a somewhat different debate on impact assessment in the US, see Kamenetzky (2013).

13 Similarly, Lebeau and Papatsiba (2016).
} 
researchers and universities alike experienced that, in numerous member states, national budgets were concentrated and cut due to financial constraints. When, in 2010, the directorate dedicated to social sciences and humanities research in the Directorate General for Research and Innovation was abolished, this experience was now also projected onto the EU research framework.

In response to this, members of the SSH communities began to rally. In December 2010, researchers from HU Berlin mobilised against what they perceived as the "thematic and financial" "downsizing of Social Sciences in the EU". (Börzel, Risse, and Sprungk 2010) This was followed by an Open Letter to the European Commission by the newly created "European Alliance for Social Sciences and Humanities" (EASH 2011; Klein 2011). In those and other comments and interventions, the core arguments can be extrapolated in the following way: (1) To express fear about the "downsizing" of SSH in Horizon 2020. (2) To emphasise the need for specific topics and "Social Sciences and Humanities (SSH)-centred challenges" (EASH 2011) that serve the purpose of the SSH community. (3) To question the reasoning behind the societal challenges, pointing out the narrow definition of "innovation". (4) To highlight the importance of SSH research for Europe, and more specifically, for fulfilment of the successful solution of the Societal Challenges.

While this spray of arguments hardly represented a stringent lobbying campaign, it represented the various concerns and beliefs from within the wider SSH communities. The initiative was successful insofar as the Open Letter was signed by almost 26,000 people, and the EU research ministers were successfully mobilised to express their concerns "whether the role of social science and humanities will be adequately reflected in the tackling of the grand societal challenges" (Myklebust 2012). In response, the European Commission launched an information campaign on its own. The then Commissioner Máire Geoghegan-Quinn and the leading management of the Directorate General for Research and Innovation, headed by Robert Jan Smits, went long distances to present the Commission's ideas of Horizon 2020 to associations, learned societies, and so on. To alleviate the concerns expressed by the SSH communities, they settled on two arguments: one was that, in the socalled first pillar of Horizon 2020, SSH would be continued to be served by funding from the more academically driven instruments, such as the ERC and the Marie Skłodowska-Curie Actions; the second was that, in the second pillar dealing with "Grand Challenges", SSH would have to be meaningfully integrated.

In November 2011, Geoghegan-Quinn addressed the issue at a gathering at the British Academy. She reassured the participants "that future funding at the European level will provide significant space for social sciences and humanities research". This should be reached through adding another dedicated challenge, and through "embedding" the social sciences and humanities into all societal challenges "to work beyond the 'silos' of different disciplines" (Geoghegan-Quinn 2011b; see also Young 2015). In other words, the Commissioner accepted the instalment of an additional "challenge" which was perceived as the one dedicated to SSH. She also continued to argue for a broadening of the term "innovation" and emphasising the crucial role of SSH to the successful completion of all (now seven) challenges.

The strategy of the Commissioner - to embrace the critics - is understandable only if seen in the context. At that time, it was all but clear if the Horizon 2020 programme would stand the brisk austerity ambitions of European Union member states. Besides the fact that it was foreseen to substantially increase the budget for this programme, its creators perceived Horizon 2020 as "a clear departure from business as usual", as the Commissioner stated in an earlier speech (Geoghegan-Quinn 2011a). It is therefore tempting to assume that the Commission did not want to have additional political disturbances in getting their ambitious programme through. It aimed at not having to overthrow the conception behind the Horizon 2020 programme, and therefore remained conciliatory but firm.

This approach had several consequences that would dominate the second part of the discursive controversy, mostly constituted through reports and statements by interest groups (van den Doel 2012; Science Europe 2013): First, the overall structure of Horizon 2020 was not touched; instead, another challenge was added. The discussion now focused on how this new (additional) challenge should be named, and how much resources it would get. Second, it reluctantly broadened the notion of "innovation" that is the core of the Commission's political agenda (European Commission 2009; Paraskevopoulou 2012). The discussion focused on what "social innovation" actually should be, and whether this meant an "instrumentalisation" of SSH or its useful application. Third, it sought to encourage SSH researchers to think out of the box and to cooperate with colleagues from the natural sciences. Thus, the pros and cons of "interdisciplinarity" and "integration" were at the centre of the discussion, and how SSH would fare within the remaining six challenges.

This was also the context of the Vilnius Conference that marked the final phase of negotiating the structure of the Horizon 2020 programme and its underlying principles, and transferred the discussion into the operational details of Working Programmes, membership in Advisory Groups and so on. The conference in Vilnius under the Lithuanian Presidency in the second half of 2013 (Mayer, König, and Nowotny 2013) crystallised into an important one-time event in which the Commission would be able to show its good-will while members of the SSH communities could express their hope for a better future while venting their frustrations with the current setup.

\section{RESULTS OF THE INTEGRATION EFFORTS UNDER HO- RIZON 2020}

Overall, the efforts in the early years of the 2010s resulted in a good compromise. On the one hand, one Societal Challenge (SC) was dedicated, as in previous editions of the Framework Programme, to topics at the heart of research from social sciences and humanities (the so-called SC6, named "Inclusive, Innovative and Reflective Societies"). While there was less funding reserved for the SSH-labelled "challenge" than in the previous editions of the FP (in share), ${ }_{1}^{14}$ at least the very issue has been successfully retained..$^{15}$ On the other hand, the idea of integrating SSH into other parts ("challenges") of the policy-oriented research funding part of the next edition of the FP allowed for some vague promise that some new forms of cooperative research might emerge.

The crucial question, of course, is how well this played out. The European Commission holds significant sway in the implementation of policies. There should be no doubt that, once formally put in the legal text of Horizon 2020 (European Parliament and Council of the European Union 
2013), the Commission - as the executive arm of the European Union - took the task of integration very seriously. SSH integration became one of several "cross-cutting issues" running across the entire FP. The Commission set up measures for better integrating SSH into the other six Societal Challenges as well as into other parts of Horizon 2020, meaning that its routines and procedures were amended in a way that funding calls could require participation of SSH partners. Such calls would be "flagged" and participation of one (or more) SSH partners would be rewarded through better evaluation scores. ${ }^{16}$ The Commission's efforts also resulted in substantial annual analyses of the extent to which the integration exercise was successful (Hetel, Møller, and Stamm 2015; Birnbaum et al. 2017; Strom et al. 2018; Swinnen, Lemaire, and Kania 2019).

Given those efforts, it is therefore worth assessing briefly to what degree the Commission's efforts bore fruit. The Vilnius Declaration from 2013 (Mayer, König, and Nowotny 2013) defined four "conditions for the successful integration of Social Sciences and Humanities in Horizon 2020": "recognising knowledge diversity"; "collaborating effectively"; "fostering interdisciplinary training and research"; and "connecting social values and research evaluation". It is difficult to identify indicators for each of these conditions; however, some data can be gathered to assess the interim results. One indicator is the composition of the advisory boards established for each Societal Challenge (1). Another is the share of topics actually flagged for SSH integration (2), and yet another one concerns the actual overall distribution to SSH research (3).

To understand the significance and context of those indicators, it is important to briefly reiterate the processes from developing a funding call for research to the actual funding decision. Typically, within a so- called "Specific Programme" (which is in fact a sub-programme within the overall Framework Programme; hence the name of the latter), annual or bi-annual Work Programmes define the calls that will be announced. The Work Programmes themselves are drafted by the European Commission, based on input from the advisory groups consisting of experts in the field. The draft Work Programme is amended along input from the so-called Programme Committee, that is, a gathering of representatives from all EU member states (typically, those representatives are ministry officials). ${ }^{17}$ Research proposals, submitted on funding calls, are evaluated along evaluation criteria by independent reviewers; the funding decision is then made by the respective Commission service tasked with carrying out the funding call.

(1) Advisory panels play a crucial role in the Societal Challenges of Horizon 2020 insofar as they consist of experts that suggest fields of research and therefore often help shaping the Work Programmes and funding calls. The advisory groups are put together by the Commission services and meet on average two to three times every year. The size of each panel varies, and in some groups there are not only individual experts but also public entities represented. While the mechanism of selecting members is not disclosed, and overall composition may change over the course of the edition of the FP, it seems clear that each group is expected to follow some basic rules concerning diversity in terms of gender, country of origin, and also disciplinary background (as seen relevant for the respective SC). The latter is interesting to our case; as can be seen from Figure 2, while each group holds at least one representative from SSH, the share is quite small, and, notably, consisting primarily of economists.

\begin{tabular}{|l|l|l|l|}
\hline Group & Title & Experts & SSH representatives \\
\hline E02942 & $\begin{array}{l}\text { Advisory group for Health, demographic } \\
\text { change and wellbeing (SC1) }\end{array}$ & 26 & 1 economist \\
\hline E03279 & Scientific Panel for Health (SPH) & 25 & 2 economists \\
\hline E02939 & $\begin{array}{l}\text { Advisory Group for Food Security, Sustainable Agriculture, } \\
\text { Marine and Maritime Research and the Bioeconomy (SC2) }\end{array}$ & 18 & 4 economists, 2 social scientists, 1 humanist \\
\hline E02981 & Advisory Group on Energy (SC3) & 23 & 5 economists, 3 social scientists \\
\hline E02969 & Advisory Group for Smart, green and integrated transport (SC4) & 23 & 2 economists, 1 social scientist \\
\hline E02924 & $\begin{array}{l}\text { Advisory Group for Climate Action, Environment, } \\
\text { Resource Efficiency and Raw Materials (SC5) }\end{array}$ & 10 & 3 economists, 1 social scientist \\
\hline
\end{tabular}

Fig. 2: Analysis of Horizon 2020 advisory panels of six challenges ${ }^{18}$

(Put together by the author)

\footnotetext{
16 For details, see https://ec.europa.eu/research/participants/docs/h2020-funding-guide/cross-cutting-issues/ssh_en.htm (last accessed: 2019-03-01)

17 For a meticulous process overview and analysis of how work programmes are developed and adopted, see Schögler (2013, 74-106)

18 The groups have been identified through the "Register of Commission expert groups" http://ec.europa.eu/transparency/regexpert/ (last accessed: 201808-15). Societal Challenge 1 seems to have two bodies advising on the Work Programme. No entry could be found for Societal Challenge 7 . The number of experts for each group refers exclusively to the "individual experts appointed in personal capacity".
} 
(2) Flagging of topics is taking place during the process of writing the Work Programme. It is obviously an important prerequisite for actually integrating SSH research; hence the interesting question is, how many topics per SC have been flagged? The number of topics varies widely between the Societal Challenges, and also between years (Work Programmes), from 15 to 50. Between 2014 and 2017, the share of topics flagged for SSH integration has not been lower than 20 per cent, and not higher than 55 per cent. However, given that topics have different budgets available, it may be more interesting to look at the actual accumulated funding share flagged for SSH integration per SC, and therefore, essentially, available to SSH research. Based on the Commission's annual SSH integration reports, the data indicate that the share is different in each Societal Challenge programme, as depicted in Figure 3, and that there are substantial annual fluctuations. Put together, the share has improved over time, 36 per cent in 2014 (€ 902 Million out of $€ 2.515$ Million) to 47 per cent in 2017 (€ 960 Million out of $€ 2.060$ Million).

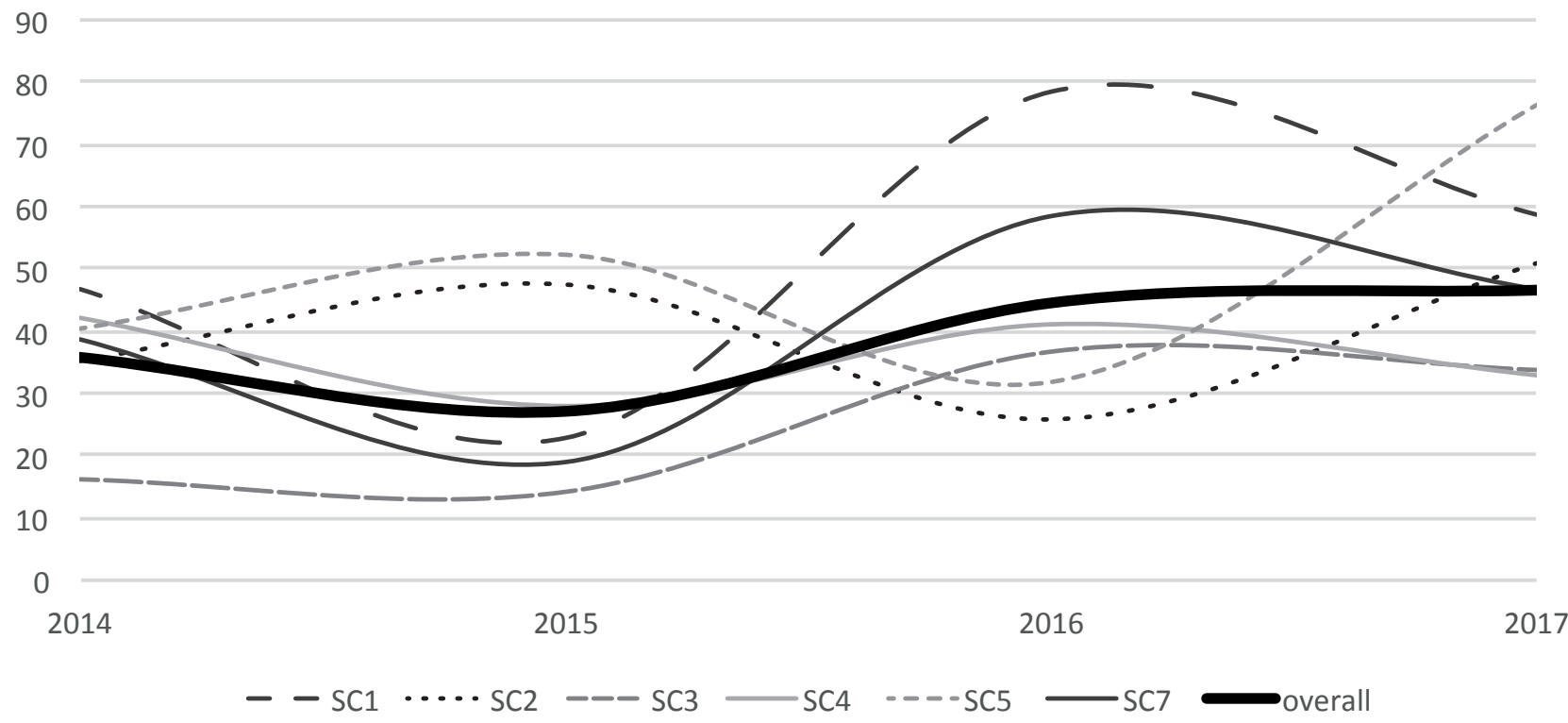

Fig. 3: Annual share of budget per Societal Challenge flagged for SSH integration ${ }^{19}$ (Put together by the author)

(3) While the previous paragraph was concerned with the question to what extent SSH integration has been enabled by applying the opportunity of "flagging" of specific topics (and, thereby, dedicated funding budgets), it is yet another story how much funding actually ended up in projects that had at least one SSH partner on board. To that end, the annual Commission reports have developed a useful composite indicator, which allows to better judge the actual SSH integration of each project. The indicator consists of four criteria: the share of SSH partners; the budget going to SSH; and the person-months by SSH partners all to be above the threshold of 10 per cent. In addition, the fourth criterion is about whether contributions in the project are coming from at least two SSH disciplines. A good integration of SSH is achieved when all four criteria are met; with three criteria met, it is "fair"; "weak" with two; and "none" with zero. According to the Commission's own assessment (the fine-tuned analysis on project level cannot be reproduced with the available data), the share of projects from within the flagged topics with good SSH integration has risen from 2014, with 40 per cent, to 56 per cent, in 2017 (Swinnen, Lemaire, and Kania 2019, 6-7). ${ }^{20}$ However, 21 per cent have no SSH research component whatsoever. 2019, 17). Numbers in this Figure, as well as in the corresponding paragraph, are solely on Societal Challenges 1-5 and 7. Societal Challenge 6 is not considered, because it is the designated "SSH" programme, and therefore not subject of the integration exercise. It should be added that, in terms of funding, SC6 is also by far the smallest programme of all Societal Challenges, with € 114 Million in 2014, € 127 Million in 2015, € 93 Million in 2016, and € 126 Million in 2017.

20 It is important to mention that, for the first three criteria of the composite indicator, the report actually defines two thresholds: one being 10 per cent, as mentioned above; the other being 20 per cent. If the latter threshold is applied, the share of projects achieving "good" SSH integration falls to 41 per cent. A methodological difficulty concerns the fact that the Commission also includes projects from the SC6 programme, which centre around SSH research by design. 


\section{Lessons to be learnt}

What can we learn from these assessments? Certainly, the Commission has put a lot of efforts into enabling, and achieving, integration of SSH research into the SC programmes of Horizon 2020 (and this is in addition to the funding for SSH research provided through other instruments of this edition of the FP). On a practical level, it seems to have been executed in a rather mechanistic way. Given the immense apparatus that has been set up to assure that the money spent through the Framework Programme is legally, financially, and politically accountable and legitimate, this may not be surprising. In any case, it comes with the danger of reifying some of the traditional roles that SSH have been ascribed to - most notably the tendency of delegating the public relations aspects of a cooperative project to SSH partners. ${ }^{21}$ As for the balance of SSH disciplines and fields, it is obvious not only that economics is much better represented in the advisory groups than the other social sciences, while humanities are barely in place at all, but also that the predominant share of funding from the SC programmes goes to social sciences, namely economics, political science, public administration and law, as well as education and communication. Together, these few fields accounted for 71 per cent of all funding going to SSH research partners in 2017 (Swinnen, Lemaire, and Kania 2019, 25).22

However, more substantial is the fact that the existing arrangement has mostly preserved from previous editions of the FP the overall funding that is actually going to SSH. Also, the discussion about integration of SSH has enabled important research projects that deal with the SSH at European (that is, transnational, comparative) level, providing thus much new insight and transnational expertise as well as networks in a field that is, by historical definition, rather drawn to the national context (an issue that will be discussed further in the next section). ${ }^{23}$

With the debate on the next edition of the FP, Horizon Europe, there is general agreement that integration is really taken from the heart, and considering all circles. This has also been emphasised by advisory documents, most notably the Lamy Report (Lamy et al. 2017). Another important aspect is that the representatives of the SSH communities by now seem to have more experience, in the sense that they now know better who the people are to address, know how the Framework Programme machinery is running in principle and thus have a better understanding when, and where, to intervene; and also know better how to argue with policy makers, shifting away from complaining to making constructive suggestions.

Most importantly, the efforts of learning from the past have come to fruition - among other initiatives, this holds true to the fact that there was another Conference (this time under the Austrian Presidency, in November 2018) dedicated to discussing the role of SSH in Horizon Europe, ${ }^{24}$ in a reinvigorated joint platform (now slightly rebranded as EASSH), ${ }^{25}$ and in the continued efforts by the network of National Contact Points Net4Society. ${ }^{26}$ The importance of integrating SSH research has also been exemplarily been realised by other, more technology-focused funding instruments in the Horizon 2020 portfolio (see for example Langer et al. 2016) Important questions remain open, however. We do not know the amount of money that will be spent. Given the reluctance of national policy makers to spend more money at European level, and the fact that the pie will not grow substantially, powerful lobbies will do their best to increase their share, which will leave the SSH community in perils.

\section{CHALLENGES IN SSH}

Seen from a historical perspective, the social sciences and humanities are deeply entangled with the history of the nation state and its agencies, with modernity and its cultural achievements (Wittrock 2000; Porter and Ross 2003; Wagner 2007; Raphael 2012). As a group, social sciences and humanities have proven to be useful by providing techniques and concepts that help to analyse, understand, and impact the social world. With the professionalisation and extensive growth of scientific (and scholarly) institutions, disciplines associated with social sciences and humanities have always also been part of the academic pecking order - and have been drawn into, or taken aback from, being counted as a social scientific discipline, or a humanities discipline.

Along the same line, the history of social sciences and humanities is full of attempts to describe the relationship within their own epistemic communities, as well as their relationship to science, in terms such as "nomothetic" vs. "ideographic", "descriptive" vs. "analytical", two or three worlds, etc. (Kagan 2009; Sala 2013). Today, the umbrella term SSH has been established, but while this may (or may not) help to overcome infights between disciplines and schools, it also disguises the differences - and resulting from this, different challenges - that the numerous disciplines, fields and schools are facing underneath.

However: one challenge remains the same, and that is the fact that, today, social sciences and humanities are increasingly treated the same way the STEM fields are. That this is the case may be argued normatively (to treat all the same way), but it does not necessarily make sense in terms of efficiency - since the social sciences and humanities arguably have a more complex relationship to truth, power, and knowledge than their siblings from the sciences. It may well have been useful to find different regimes of funding for different purposes; but this does not easily comply with fairness, and audits. Interestingly, SSH are treated differently in some respects when it comes to curricula, and application of their methods, concepts, and theories. Save to assume, however, that two complementary forces were at work. Available funds, and attached reputation is an attraction. Representatives from the social sciences and humanities quickly felt the urge to participate. At the same time, it was more convenient for policy-makers to set up funding in a way that mimiassessment report states that "although research in technologies can provide technical solutions to major challenges, Social Sciences and Humanities (SSH) can help making them accepted, understood and appropriated by the general public." (Strom et al. 2018, 6)

22 Again, note that the Commission report includes projects from SC6, which cannot be subtracted out due to lack of data.

23 Those projects are ACCOMPLISH, DANDELION, and, as a COST action, one could add the ENRESSH network.

24 See the programme of the conference "Impact of Social Sciences and Humanities for a European Research Agenda - Valuation of SSH in mission-oriented research" under https://www.ssh-impact.eu/programme

25 See the website of the European Alliance for social Sciences and Humanities, https://www.eassh.eu

26 See the website https://www.net4society.eu 
cked the established paths of sciences. The result is that social sciences and humanities have been increasingly caught up in receiving projectbased funding.

This is often seen as a problem, and at the level of individual research questions, this might be justified. However, SSH research fundamentally shares the same values as research from other fields, that is, to produce robust knowledge and to enhance human kind; and that is also true when it comes to the social contributions of research. Given this fact, it may be well worth to reassess briefly existing, highly instructive and reflective literature on the nature of social sciences and humanities to give credit to the diversity of SSH. By doing so this section also aims at establishing an argument why and how this diversity can serve as strength, rather than a weakness, for cooperative research that is tasked to contribute to solving societal challenges.

Methods, terms, and concepts have permeated the academic world and changed the way people look at their lives, societies, and polities. From this point of view, social sciences and humanities have been spectacularly successful at least at two levels. One is, that these techniques have become standard requirements for civil servants as well as aspiring members of the elite. And that the knowledge produced by these techniques and theoretical presumptions is critical for states, for bureaucracies, to govern. Demography, for example, enables governments to assess their populace and to perform one of their most basic tasks, namely redistribution (Desrosières 1998). Wolfgang Streeck has recently renewed this argument, namely that "the descriptive analysis of social reality by counting, measuring, observing might be of significant practical and societal use" (Streeck 2011, 8).

Just like the natural and life sciences, as well as in engineering, the social sciences and humanities have considerably contributed to the ways we understand and look at our social world. If it is true that what the natural and life sciences and engineering have contributed to our modern societies has become invisible (Shapin 2016), this is even more true for the social sciences and the humanities, simply because they have a much closer and direct relationship to society (Felt 2000). Due to the thematic orientation of SSH on matters of social relevance, the boundaries between academia and the rest of the world is even more blurred, which is why the academic social sciences in particular have established a way of abstract theorising that is not only often hiding a banality, but is also perceived as hermetic. ${ }^{27}$

The current epistemological debates about social sciences and humanities cannot be addressed in full detail here. But it is possible to point to the following issues. As mentioned before, SSH deals with contextualised knowledge, and is not so much about discovering universal laws or functional analysis, but rather about "intentional explanation" (Elster 1983). Not only do social sciences and humanities have a "performativity" on society of their own (MacKenzie, Muniesa, and Siu 2008; Boltanski, Esquerre, and Muniesa 2015), they also are inseparable from political goals, and it is often difficult, albeit important as an exercise in self-reflection, to separate analysis from value judgment (Weber 1968; Ringer 1997). SSH play an important role in what can be called "new knowledge relations" within the scientific disciplines, that is between the SSH and technosciences, but also regarding the relation of traditional actors in the innovation chain and societal actors (Felt 2014, 394).
Another, final important observation from the field has been the dynamic within the social sciences and humanities towards "fractal distinction" (Abbott 2001, 2015). Because of its complexity, there is an inherent tendency within the fields analysing the social world (or one of its aspects) to create ever new approaches, questions, focal points. What may be called paradigmatic theory according to Thomas Kuhn (Kuhn 1970) is, in many branches of the social sciences and humanities, most often only short-lived and quickly disputed internally. Instead of being desperate about this, this should be taken as a feature and dealt with as such. It does not make sense to try to stylise SSH in the manner of other branches of the scientific enterprise, but rather embrace, acknowledge the above-mentioned specificities and build on that. Also, it is important to understand that despite the fact that SSH communities often resort on the lower end of the pecking order, SSH bring along expertise that is urgently needed specifically for the task of tackling societal challenges.

\section{IMPACT RE-LOADED IN HORIZON EUROPE}

Facing the overall ambition of Horizon Europe towards impact generation, an argument for stronger cooperation with and within SSH is made here to shift the focus away from marginalisation experiences and lament of the past. It was not by chance that the scope paper for the conference in 2018 was called "impact re-loaded" (König, Nowotny, and Schuch 2018). Similarly, the conference aimed at practical SSH Guidelines directed at those who deal with research funding programmes, and specifically programmes that aim at tackling a societal problem through the means and opportunities provided by scientific and scholarly research.

\section{ABOUT THE SSH GUIDELINES}

The conference and this Working Paper, together with the SSH Guidelines "Social Sciences and Humanities Research Matters. Guidelines on how to successfully design, and implement, mission-oriented research programmes" (König 2019), intend to build on this position, and to push further for more and better integration in Horizon Europe. This also means that there has to be a substantial understanding what SSH research is about, and how it is properly treated and valued. To do so, the SSH Guidelines concentrate entirely on mission-oriented research programmes. It distinguishes four steps in the life-cycle of such a programme, namely design, implementation, evaluation and decision-making; and it addresses all those persons who play a role in either of those steps.

The idea of the SSH Guidelines is to provide a comprehensive, quickto-read set of arguments for why SSH should be central for mission-oriented research programmes, and how to value them properly at each of the steps of the programme's lifecycle. It provides a number of practical tips for bringing SSH-expertise to the design and implementation of Rylprogrammes. It builds, and extends, the extremely useful leaflet produced by Net4Society that has a similar ambition, albeit it was directed at 
the integration of SSH under Horizon 2020.28 Indeed, the SSH Guidelines intend to make sure the effectiveness of the idea behind the leaflet is taken on, and made use of, based on an elaborate consultation process, which started several months before the conference, resulting in a first draft version, which was then subject to further discussion, and scrutiny, at a specifically dedicated on drafting the SSH Guidelines. Two additional cycles of consultation with numerous experts resulted in the final version of the policy paper in mid-January 2019. ${ }^{29}$

\section{SUGGESTIONS FOR SSH SCHOLARS}

Complementary to the SSH Guidelines, this section is dedicated to some key suggestions for SSH scholars who set out to improve the depth and range of cooperation in the mission-oriented parts of Horizon Europe, as well as other research funding instruments at European, national, or local level. The SSH Guidelines define four specific strengths of SSH in designing research funding programmes:

- the expertise to calibrate missions

- the capacities of translating between academic disciplines, policy makers, and different publics

- the expertise in placing specific problems in broader contexts (combining local and global perspectives)

- and the capacity of methodological reflexivity. ${ }^{30}$

While these strengths aim at setting the tone for policy makers and managers, it also provides a good introduction to the concluding section of this Working Paper. It discusses some ideas for scholars and researchers from SSH communities in order to advance the role of SSH in Pillar 2 (with the title "Global Challenges and Industrial Competitiveness") of the next Framework Programme, as well as other (national) research funding programmes that are dedicated to fund mission-oriented research projects.

The most important, yet often overlooked aspect concerns the participation in the process of designing a research funding programme, or research funding instrument. As we have seen, the number of SSH scholars in the advisory boards of various Societal Challenges in Horizon 2020 has been low. This is a real problem: it is in this realm that the overall goals of the programme, or instrument, are defined; hence bringing SSH scholars to the table is crucial if interdisciplinary cooperation between SSH and STEM is really expected to lead to new, relevant knowledge.

Funding calls sometimes require SSH researchers to be creative and, when it comes to finding funding opportunities, to look at things dif- ferently. Three aspects should be emphasised here: One, it sometimes is worth to take the step and submit a proposal, even though this may appear to be risky. Along the same line, it is also important to be ready, and to muster capacity, to take over the consortium coordination, in order to stronger influence the tone for a project. On a related matter, it is crucial to ask for local support infrastructure. SSH sometimes have the disadvantage of not being supported the same way as their STEM colleagues are.

\section{A PRACTICAL WAY FORWARD - FOR SCHOLARS AND POLICY MAKERS}

As a practical next step, and taking up the many suggestions and ideas brought forward in various meetings such as the 2018 conference in Vienna, representatives of SSH research could set up meetings at national level with the respective Delegates in Programme Committees and National Contact Points (NCPs). As a pilot, such a meeting was organised in Austria in March 2019, with great success (see Annex). Despite those efforts, the necessary requirements to enable SSH scholars engaging in those two activities - designing funding calls and participating in project applications - are still far from being fully achieved. Yet by addressing the need and playing a more pro-active role, further improvement is to be expected, particularly given the positive developments at European level in preparation of "Horizon Europe". document by the FET Advisory Group also provides some important suggestions (Langer et al. 2016).

29 For feedback and comments during the productive consultation process, I am grateful to Paul Benneworth, Basudeb Chaudhuri, Alice Dijkstra, Martina Kadunc, Angela Liberatore, Gabi Lombardo, Stephanie Rammel, Angela Schindler-Daniels, Marc Vanholsbeeck, in addition to the colleagues already mentioned in footnote 1, as well as many others. The suggestions in the SSH Guidelines have been inspired by various documents that, in recent years, started to take a critical view on the metrics craze (Muller 2018), highlighting the "patina of precision" (Gingras 2016), the "different types of impact" (Reale et al. 2014) the "gatekeepers of high impact" (Hicks et al. 2015), the "ubiquity of excellence rhetoric" (Moore et al. 2017). 
ANNEX:

SUMMARY OF MEETING

"SOCIAL SCIENCES

AND HUMANITIES IN HORIZON EUROPE"

(by Thomas König, Stephanie Rammel, Matthias Reiter-Pázmándy, Klaus Schuch, Johannes Starkbaum)

On Friday, March 8, the meeting "Social Sciences and Humanities in Horizon Europe" took place on the premises of the Institute for Advanced Studies (IHS), Vienna. It was a follow-up of the Austrian EU Presidency Conference "Impact of Social Sciences and Humanities for a European Research Agenda - Valuation of SSH in mission-oriented research", ${ }^{31}$ which had taken place in Vienna on 28-29 November 2018. As the current EU Research Funding Programme, "Horizon 2020", 32 is coming to an end, and discussions for the next edition, called "Horizon Europe" ${ }^{33}$ have intensified, there is the need and opportunity to engage policy makers and SSH representatives at the national level, in order to open up space for discussion on how to better involve SSH expertise in the drafting process of the thematic clusters of "Horizon Europe". The clusters are gathered under the paramount title "Global Challenges and Industrial Competitiveness".

The follow-up meeting was organised by Thomas König (IHS), Stephanie Rammel (FFG), Matthias Reiter-Pázmándy (BMBWF), and Klaus Schuch (ZSI). It brought together about fifty people - representatives from social sciences and humanities in Austria, National Contact Points (NCPs) for the different thematic areas as well as policy makers and ministry officials.

\section{PURPOSE OF THE MEETING}

The initiative to the meeting was driven by two insights. One is that, as Stephanie Rammel made clear in her presentation, integration of SSH into the thematic research funding instruments of the current "Horizon 2020 " is an ambitious attempt, but still far from being satisfying. Another is that representatives from SSH repeatedly complained that they are not involved in the shaping, and designing, of funding calls and work programmes. Once the remit of a call is decided upon, it is difficult to bring specific SSH knowledge in - unless, maybe, as an add-on. Given the fact that the Framework Programmes have increasingly become also templates for research funding programmes in the member states, one cannot underestimate the role - both directly and indirectly - in shaping the status, and involvement, of SSH in European research funding generally.

The meeting kicked off with a keynote by Prof. Ulrike Felt who provided food for thought by talking about the role of SSH in coping with societal challenges. Other presentations were about providing key statistics on integration of SSH into the Clusters ("Societal Challenges") of "Horizon 2020", information on the state of negotiations regarding "Horizon Europe", and results from the November Conference. For the latter, Thomas König pointed towards the booklet "Social Sciences and Humanities Research Matters", a comprehensive set of guidelines addressing "research programmes that set out a specific goal to tackle a societal problem through the means and opportunities provided by scientific and scholarly research - both from SSH and STEM" ${ }^{34}$ All presenters agreed that SSH should be further integrated in future EU research funding.

Interdisciplinary and especially SSH-aspects have to be contributed throughout the whole development of a framework programme, said Matthias Reiter-Pázmándy, from the initial negotiations, to the Strategic Planning and the yearly Work Programmes. Special attention has to be paid to include SSH-researchers in the various Advisory Groups, in particular in the Mission Boards, but also in the evaluation panels of "Horizon 2020" and "Horizon Europe". Researchers from SSH also should register to be available as evaluators in order to provide enough choice for those who convene the panels. In addition to that, it is important to provide fora, where researchers and policy makers can meet and exchange across the boundaries of disciplines and the various sectoral policy areas. This event did exactly that.

The key element of the meeting, however, concerned the remaining 90 minutes which provided space for discussion among participants. To that end, participants were seated on one of six tables, each of which was dedicated to one of the (prospective) thematic clusters in Horizon Europe. ${ }^{35}$ The intention was to bring policy makers (the National Delegates to the specific programme committees in "Horizon 2020" and in the upcoming "Horizon Europe"), supporters (the NCPs) and SSH researchers together and discuss how to better take advantage of SSH expertise in designing and shaping the respective thematic cluster.

\section{SUMMARY OF CLUSTER TABLES}

Before a joint lunch buffet was served, the discussions were summarised and presented to the full audience, along two sets of questions:

a. In which of the cluster's topics is specific SSH expertise required?

b. What concrete measures can help Delegates and NCPs to facilitate integration of SSH in the cluster? Here are the summaries of each of the discussion tables:

\section{HEALTH}

a. All topics in this cluster are relevant for SSH expertise; much depends on the actual design. "Health systems" might be a focal point that works as a "catch all".

b. At EU level, more emphasis has to be on evaluation criteria, and 
the participant portal has to be made use of to identify potential partners. At Austrian level, thematic platforms should be made use of for cooperation (e.g., ÖPPM ${ }^{36}$, Netzwerk Altern ${ }^{37}$ ), policy makers should be stronger advocates for $\mathrm{SSH}$, and exchange at the level of the cluster should be intensified.

\section{CIVIL SECURITY FOR SOCIETY}

a. SSH is crucial for topics such as radicalisation, terrorism, prevention, and resilience.

b. Since topics are mostly identified by governments, SSH representatives should get in contact with NCPs and ministries. Also, with the national security research programme KIRAS, ${ }^{38}$ there is already a national model available for integrating SSH.

\section{DIGITAL, INDUSTRY AND SPACE}

a. All topics were considered important for SSH expertise; this is particularly the case for Al, Big Data, Next Generation Internet, and Digital Skills.

b. The Evaluation process is critical, both in terms of skills of reviewers and the evaluation criteria, the same is true for the work programmes, and the deliverables in the grant agreement. In relation to the "digital skills" topic, a sort of "meta-SSH" was emphasised, acting as a support-mechanism for a number of different research projects and dealing with their social impact, assessing also discriminatory aspects, exclusion and fears.

\section{CLIMATE AND ENERGY; MOBILITY}

a. All topics across this cluster are relevant for SSH.

b. Evaluation has to be organised in an interdisciplinary manner; move away from techno-economic, sector-specific solutions, towards integrating behavioural insights and sociocultural practices. SSH can serve as guidance for sectoral policies to implement R\&D-based solutions. Researchers and sectoral policy makers should step out of their bubbles and get together more often.

\section{BIOECONOMY, FOOD, NATURAL RESOURCES \& ENVI- RONMENT}

a. There are "areas of connectivity" ("bio economy", "food systems") and also areas that would require a stronger involvement of SSH (demand and supply problem in areas such as "environmental observation", "agriculture, forestry, and rural areas") - there are social impacts, conflict potentials, and more generally, a political economy to be analysed.

b. Technological "solutionism" approaches may not be enough, ${ }^{39}$ real problem solving requires integration of SSH in problem framing and analysis. A more holistic approach from strategy to calls is required! This also implies a cultural change, i.e. in the language used to describe a problem.

\section{NEXT STEPS}

The meeting was an experiment insofar as nothing similar has ever happened. Albeit there was little time for an exhaustive exchange, debates were initiated and the meeting was thus widely seen as a great success. There may be three reasons for that. One is that mission-oriented research funding demands exchange of SSH representatives with policy makers in order to align calls, proposals and research towards missions. Another is that Austrian Delegates and NCPs have an interest in increasing the share of funding that flows from the EU level to Austria. So even if they represent clusters that traditionally stand for a more technoscience orientation, they share the core interest of SSH representatives. Finally, all this happens in the context of a more positive attitude towards SSH in general, ${ }^{40}$ which provides the background for this initiative. Having said all this, there is still much to do, at national level as well as at European level.

\section{AT NATIONAL LEVEL}

One way forward would be for SSH experts, national delegates, and NCPs to meet regularly for further exchange. This would certainly support the uptake of SSH expertise on one hand, and awareness-raising and re-orientation on the side of SSH researchers on the other hand, which allows setting concrete action. It is now up to the respective Austrian institutes in their fields to take the lead and continue the work that was initiated in this meeting.

\section{AT EUROPEAN LEVEL}

It is important to highlight this meeting to SSH representatives in other EU member states, so that they can organise similar events. Also, a shared meeting in Brussels on presenting the SSH-Guidelines later in summer would provide a good opportunity to report about the progress made in Austria. 


\section{BIBLIOGRAPHY}

Abbott, A. (2001). Chaos of Disciplines. Chicago: University of Chicago Press.

Abbott, A. (2015). "Nach dem Chaos: Selbstähnlichkeiten in den Sozialwissenschaften." In Soziologiegeschichte: Wege und Ziele, edited by C. Daye and S. Moebius, 284-307. Berlin: Suhrkamp.

Anderson, P. (2009). The New Old World. London; New York: Verso.

Atkinson, T., Berkhout, F., De Grauwe, P., Floud, R., Hooimeijer, P., Honkapohja, S., Höpfinger, S. et al. (2009). "Vital Questions. The Contribution of European Social Scienc." Position Paper. Strasbourg: European Science Foundation.

Banchoff, T. (2002). "Institutions, Inertia and European Union Research Policy." Journal of Common Market Studies 40 (1): 1-21. https://doi. org/10.1111/1468-5965.00341.

Barry, A., Born, G. and Weszkalnys, G. (2008). "Logics of Interdisciplinarity." Economy and Society 37 (1): 20-49. https://doi. org/10.1080/03085140701760841.

Billig, M. (2013). Learn to Write Badly: How to Succeed in the Social Sciences. Cambridge: Cambridge University Press.

Birnbaum, B. I., Keraudren, P., Strom, T. and Vavikis, T. (2017). “Integration of Social Sciences and Humanities in Horizon 2020: Participants, Budget and Disciplines. 2nd Monitoring Report on SSH-Flagged Projects Funded in 2015 under the Societal Challenges and Industrial Leadership Priorities." Brussels: European Commission. http://ec.europa.eu/ research/social-sciences/index.cfm?pg=libraryglib=other_pub.

Boltanski, L., Esquerre, A. and Muniesa, F. (2015). "Grappling with the Economy of Enrichment." Valuation Studies 3 (1): 75-83. https://doi. org/10.3384/VS.2001-5592.153175.

Borrás, S. and Radaelli, C. M. (2011). "The Politics of Governance Architectures: Creation, Change and Effects of the EU Lisbon Strategy." Journal of European Public Policy 18 (4): 463-84. https://doi.org/10.1080 /13501763.2011.560069.

Börzel, T. A., Risse, T. and Sprungk, C. (2010). "Against the Downsizing of Social Sciences in the EU." December 9, 2010. http://www.polsoz. fu-berlin.de/en/v/transformeurope/news/allgemeines/2010_Against_ Downsizing_Social_Sciences.html.

Braun, D. (2003). "Lasting Tensions in Research Policy-Making - a Delegation Problem." Science and Public Policy 30 (5): 309-21. https://doi. org/10.3152/147154303781780353.

Daston, L., Gray-Little, B., Holt, R., Katznelson, I., King, G., Marrett, C. B., Nelson, A., Prewitt, K., Reed, J. S. and Zegart, A.( 2018). "To Secure Knowledge. Social Science Partnerships for the Common Good."
Report of the To Secure Knowledge Task Force of the SSRC. New York: Social Science Research Council. https://www.ssrc.org/to-secure-knowledge.

Desrosières, A. (1998). The Politics of Large Numbers: A History of Statistical Reasoning. Harvard University Press.

Doel, W. (2012). "Social Sciences and Humanities: Essential Fields for European Research and in Horizon 2020." 11. Advice Paper. Brussels: LERU.

Draux, H. and Szomszor, M. (2017). "Topic Modelling of Research in the Arts and Humanities. An Analysis of AHRC Grant Applications." Digital Research Reports. Arts and Humanities Research Council (AHRC). https://doi.org/10.6084/m9.figshare.5621260.v1.

Drotner, K. (2013). "Humanities in the Societal Challenges. 12 Compelling Cases for Policymakers." Brussels: Science Europe.

EASH. (2011). “Horizon 2020: Social Sciences and Humanities Research Provides Vital Insights for the Future of Europe." EASH. October 28, 2011. http://www.eash.eu/openletter2011/index.php?file=openletter.htm.

Elster, J. (1983). Explaining Technical Change: A Case Study in the Philosophy of Science. Cambridge University Press.

Elzinga, A. (2012). "Features of the Current Science Policy Regime: Viewed in Historical Perspective." Science and Public Policy 39 (4): 416-28. https://doi.org/10.1093/scipol/scs046.

European Commission. (2009). Communication on 'Reviewing Community Innovation Policy in a Changing World.' O.J. Vol. C 26/12.

European Commission.. 2010. Europe 2020 Flagship Initiative Innovation Union. O.J. Vol. C 121/53.

European Parliament and Council of the European Union. (2013). Regulation (EU) Establishing Horizon 2020 - the Framework Programme for Research and Innovation (2014-2020) and Repealing Decision No 1982/2006/EC. O.J. Vol. L 347/104.

Felt, U. (2000). “Die 'unsichtbaren' Sozialwissenschaften: Zur Problematik der Positionierung sozialwissenschaftlichen Wissens im öffentlichen Raum." In Soziologische und historische Analysen der Sozialwissenschaften, edited by C. Fleck, 177-212. Österreichische Zeitschrift für Soziologie, Sonderband 5. Opladen: Westdeutscher Verlag.

Felt, U. (2014). "Within, Across and Beyond: Reconsidering the Role of Social Sciences and Humanities in Europe." Science as Culture 23 (3): 384-96. https://doi.org/10.1080/09505431.2014.926146.

Felt, U. and Fochler, M. ( 2018). “Der gesellschaftliche Impact sozialwissenschaftlichen Wissens in Österreich: Wirkungswege, Messung, Potentiale. Eine explorative Studie." Vienna: Rat für Forschung und Technologieentwicklung.

Fischer, K. ( 2008). "Science and Its Malfunctions." Human Architecture: Journal of the Sociology of Self-Knowledge 6 (2): 1-22. 
Flink, T. and Kaldewey, D. (2018). "The Language of Science Policy in the Twenty-First Century. What Comes after Basic and Applied Research?" In Basic and Applied Research: The Language of Science Policy in the Twentieth Century, edited by D. Kaldewey and D. Schauz, 251-84. European Conceptual History. New York: Berghahn. https://www.berghahnbooks. com/downloads/OpenAccess/KaldeweyBasic/9781785338113_OA.pdf.

Frodeman, R. (2010). "Introduction." In The Oxford Handbook of Interdisciplinarity, edited by R. Frodeman, J. Thompson Klein, and C. Mitcham, xxix-xxxix. Oxford: Oxford University Press.

Frodeman, R., Thompson Klein, J. and Pacheco, R. C. S. eds.( 2017). The Oxford Handbook of Interdisciplinarity. Second Edition. Oxford Handbooks. Oxford, New York: Oxford University Press.

Fuller, S. (2016). "What Is the Problem for Which Interdisciplinarity Is the Solution?" Journal. Items. Insights from the Social Sciences. June 7, 2016. http://items.ssrc.org/what-is-the-problem-for-which-interdisciplinarity-is-the-solution/.

Geoghegan-Quinn, M. 2011a). "The Future of EU-Funded Research and Innovation Programmes: An Emerging Consensus.... and a New Name." Speech SPEECH/11/432. Brussels. EU Press releases database. http:// europa.eu/rapid/press-release_SPEECH-11-432_en.htm.

Geoghegan-Quinn, M. (2011b). "The Future of Social Sciences and Humanities in Horizon 2020." Speech SPEECH/11/741. London. EU Press releases database. http://europa.eu/rapid/press-release_SPEECH-11-741_ en.htm.

Gibbons, M., Limoges, C., Nowotny, H., Schwartzman, S., Scott, P. and Trow, M. (1994). The New Production of Knowledge: The Dynamics of Science and Research in Contemporary Societies. London ; Thousand Oaks, Calif: SAGE Publications Ltd.

Gingras, Y. (2016). Bibliometrics and Research Evaluation: Uses and Abuses.

Godin, B. (2009). The Making of Science, Technology and Innovation Policy: Conceptual Frameworks as Narratives, 1945-2005. Montréal: Centre Urbanisation Culture Société de I'INRS. http://www.csiic.ca/PDF/TheMakingOfScience.pdf.

Godin, B. (2015). Innovation Contested: The Idea of Innovation over the Centuries. 1. Ed. Routledge Studies in Social and Political Thought 98. New York, London: Routledge, Taylor and Francis Group.

Greenberg, D. S. (2001). Science, Money, and Politics: Political Triumph and Ethical Erosion. Chicago: University of Chicago Press.

Guzzetti, L., ed. (2000). Science and Power: The Historical Foundations of Research Policies in Europe. Luxembourg: Office for Official Publications of the European Communities.

Guzzetti, L., ed. (2009). "The 'European Research Area' Idea in the History of Community Policy-Making." In European Science and Technology Policy: Towards Integration Or Fragmentation?, edited by H. Delanghe, U.
Muldur and L. Soete, 64-77. Cheltenham: Edward Elgar Publishing.

Hessels, Laurens K. and van Lente, H. (2008). “Re-Thinking New Knowledge Production: A Literature Review and a Research Agenda." Research Policy 37 (4): 740-60. https://doi.org/10.1016/j.respol.2008.01.008.

Hetel, L., Møller, T-E. and Stamm, J. (2015). "Integration of Social Sciences and Humanities in Horizon 2020: Participants, Budget and Disciplines. Monitoring Report on SSH-Flagged Projects Funded in 2014 under the Societal Challenges and Industrial Leadership." Brussels: European Commission. https://ec.europa.eu/programmes/horizon2020/en/news/ integration-social-sciences-and-humanities-horizon-2020-participantsbudget-and-disciplines.

Hicks, D., Wouters, P., Waltman, L., de Rijcke, S. and Rafols, I. (2015). "Bibliometrics: The Leiden Manifesto for Research Metrics." Nature News 520 (7548): 429. https://doi.org/10.1038/520429a.

Kagan, J. (2009). The Three Cultures: Natural Sciences, Social Sciences, and the Humanities in the 21st Century. Cambridge University Press.

Kamenetzky, J. R. (2013). "Opportunities for Impact: Statistical Analysis of the National Science Foundation's Broader Impacts Criterion." Science and Public Policy 40 (1): 72-84. https://doi.org/10.1093/scipol/scs059.

Kastrinos, N. (2010). "Policies for Co-Ordination in the European Research Area: A View from the Social Sciences and Humanities." Science and Public Policy 37 (4): 297-310. https://doi.org/10.3152/030234210X496646.

Klein, R. (2011). "Understanding Europe in a Global Context: Transitions towards Innovation Societies." Non-paper. Brussels. http://www.eash. eu/openletter2011/docs/SSH_GrandChallenge_Draft_140411_olweb. pdf.

König, T. (2016). "Peer Review in the Social Sciences and Humanities at the European Level: The Experiences of the European Research Council." In Research Assessment in the Humanities, edited by M. Ochsner, S. E. Hug and H-D. Daniel, 151-63. Cham: Springer International Publishing. https://doi.org/10.1007/978-3-319-29016-4_12.

König, T. (2017). The European Research Council. Cambridge: Polity Press.

König, T. (2019). "Social Sciences and Humanities Research Matters - Guidelines on How to Successfully Design, and Implement, MissionOriented Research Programmes." Vienna: ZSI. DOI: 10.22163/ fteval.2019.305.

König, T. and Gorman, M. E. (2016). "The Challenge of Funding Interdisciplinary Research: A Look inside Public Research Funding Agencies." In The Oxford Handbook of Interdisciplinarity, edited by R. Frodeman, J. Thompson Klein and R. Pacheco, 2nd ed., 513-24. Oxford Handbooks. Oxford: Oxford University Press.

König, T., Nowotny, H. and Schuch, K. (2018). "Impact Re-Loaded." Non-paper. Vienna. https://www.ssh-impact.eu/impact-re-loaded/. Kuhn, T. S. (1970). The Structure of Scientific Revolutions. 2nd ed. Chicago: University of Chicago Press. 
Lamy, P., Brudermüller, M., Ferguson, M., Friis, L., Garmendia, C., Gray, I., Gulliksen, J. et al. (2017). "LAB-FAB-APP. Investing in the European Future We Want." Report of the independent High Level Group on maximising the impact of EU Research \& Innovation Programmes. Brussels: European Commission. doi:10.2777/477357.

Langer, J. M., Makarow, M., Teicher, M., Neves Amoroso, A. C., De Gelder, B., Diamond, D., Floreano, D. et al. (2016). "The Need to Integrate the Social Sciences and Humanities with Science and Engineering in Horizon 2020 and Beyond." Policy Document. Brussels: European Commission.

Lebeau, Y. and Papatsiba, V. (2016). "Conceptions and Expectations of Research Collaboration in the European Social Sciences: Research Policies, Institutional Contexts and the Autonomy of the Scientific Field." European Educational Research Journal 15 (4): 377-94. https://doi. org/10.1177/1474904116642777.

Lyall, C., Bruce, A., Marsden, W. and Meagher, L. (2013). "The Role of Funding Agencies in Creating Interdisciplinary Knowledge." Science and Public Policy 40 (1): 62-71. https://doi.org/10.1093/scipol/scs121.

MacKenzie, D. A., Muniesa, F. Siu, L. (2008). Do Economists Make Markets?: On the Performativity of Economics. Princeton, N.J.; Woodstock: Princeton University Press.

Mayer, K., König, T. and Nowotny, H. (2013). "Horizons for Social Sciences and Humanities." Conference Report. Vilnius: Mykolas Romeris University. http://horizons.mruni.eu/wp-content/uploads/2014/02/ssh_ mru_conference_report_final.pdf.

Mazzucato, M. (2018). "Mission-Oriented Research and Innovation in the European Union. A Problem-Solving Approach to Fuel Innovation-Led Growth." Brussels: European Commission. doi:10.2777/360325.

Merton, R. K. (1957). Social Theory and Social Structure; toward the Codification of Theory and Research. Revised and enlarged edition. Glencoe, III.: Free Press.

Mingers, J. and Leydesdorff, L. (2015). "A Review of Theory and Practice in Scientometrics." European Journal of Operational Research 246 (1): 1-19. https://doi.org/10.1016/j.ejor.2015.04.002.

Moore, S., Neylon, C., Eve, M. P., O'Donnell, D. P. and Pattinson, D. (2017). "'Excellence R Us': University Research and the Fetishisation of Excellence." Palgrave Communications 3 (January). https://doi. org/10.1057/palcomms.2016.105.

Muller, J. Z. (2018). The Tyranny of Metrics.

Myklebust, J. P. (2012). "Research Ministers Demand Key Role for Social Sciences in Horizon 2020." University World News, February 26, 2012, sec. Global Edition Issue 210. http://www.universityworldnews.com/article.php?story=20120223201123111 \&mode=print.

Nowotny, H. (2016). The Cunning of Uncertainty. Cambridge, UK; Malden, Mass.: Polity Press.
Paraskevopoulou, E. (2012). "Non-Technological Regulatory Effects: Implications for Innovation and Innovation Policy." Research Policy 41 (6): 1058-71. https://doi.org/10.1016/j.respol.2012.03.018.

Porter, T. M. and Ross, D. eds. (2003). The Modern Social Sciences. The Cambridge History of Science 7. Cambridge; New York; Melbourne [etc.]: Cambridge University Press.

Raphael, L. (2012). "Embedding the Human and Social Sciences in Western Societies, 1880-1980: Reflections on Trends and Methods of Current Research." In Engineering Society: The Role of the Human and Social Sciences in Modern Societies, 1880-1980, edited by K. Brückweh, D. Schumann, R. F. Wetzell, and B. Ziemann, 41-56. Palgrave Macmillan.

Reale, E., Avramov, D., Canhial, K., Donovan, C., Flecha, R., Holm, P., Larkin, C. et al. (2017). "A Review of Literature on Evaluating the Scientific, Social and Political Impact of Social Sciences and Humanities Research." Research Evaluation, no. Special Issue: 1-11. https://doi. org/10.1093/reseval/rvx025.

Reale, E., Nedeva, M., Thomas, D. A. and Primeri, E. (2014). “Evaluation through Impact: A Different Viewpoint." Fteval Journal for Research Technology Policy Evaluation, no. 39: 36-41.

Reiter-Pázmándy, M. (2017). "A Pumping Heart for European Research." Policy Document. Vienna. https://era.gv.at/object/document/3300.

Rijcke, S. de, and Rushforth, A. (2015). "To Intervene or Not to Intervene; Is That the Question? On the Role of Scientometrics in Research Evaluation." Journal of the Association for Information Science and Technology 66 (9): 1954-58. https://doi.org/10.1002/asi.23382.

Ringer, F. K. (1997). Max Weber's Methodology: The Unification of the Cultural and Social Sciences. Cambridge: Harvard University Press.

Rosamond, B. (2007). "European Integration and the Social Science of EU Studies: The Disciplinary Politics of a Subfield." International Affairs 83 (1): 231-52.

Sala, R. (2013). "One, Two, or Three Cultures? Humanities Versus the Natural and Social Sciences in Modern Germany." Journal of the Knowledge Economy 4 (1): 83-97. https://doi.org/10.1007/s13132-012-01245.

Sarewitz, D. (2016). "Saving Science." The New Atlantis 49: 1-40.

Schögler, R. Y. (2013). “European Union Research Funding. Priority Setting in the Social Sciences and Humanities." Graz: University Graz.

Schögler, R. Y., and König, T.( 2017). "Thematic Research Funding in the European Union: What Is Expected from Social Scientific Knowledge-Making?" Serendipities 2 (1): 107. https://doi.org/10.25364/11.2:2017.1.7.

Science Europe. (2013). "Embedding Social Sciences and Humanities in the Horizon 2020 Societal Challenges." Science Europe Position State- 
ment. Brussels: Science Europe.

Shapin, S. (2016). "Invisible Science." The Hedgehog Review 18 (3). http://www.iasc-culture.org/THR/THR_article_2016_Fall_Shapin.php.

Smith, J. (2003). "Implementation of the European Research Area in the Social and Human Sciences." Discussion Paper EUR 20601. RTD Info. European Commission. http://ec.europa.eu/research/social-sciences/pdf/ study-era-shs-03_en.pdf.

Stephan, P. E. (2012). How Economics Shapes Science. Cambridge, Mass.: Harvard University Press.

Streeck, W. (2011). "Man Weiß Es Nicht Genau: Vom Nutzen Der Sozialwissenschaften Für Die Politik." MPIfG Working Paper 09/11. http:// www.mpifg.de/pu/workpap/wp09-11.pdf

Strom, T., Lemaire, C., Zacna, J., Montanez, J. A., and Birnbaum, B. I. (2018). "Integration of Social Sciences and Humanities in Horizon 2020: Participants, Budget and Disciplines. 3rd Monitoring Report on SSH Flagged Projects Funded in 2016 under the Societal Challenges and Industrial Leadership Priorities." Brussels: European Commission. https:// publications.europa.eu/en/publication-detail/-/publication/4365f75a5efe-11e8-ab9c-01aa75ed71a1/language-en.

Swinnen, L., Lemaire, C. and Kania, K. (2019). "Integration of Social Sciences and Humanities in Horizon 2020. Participants, Budget and Disciplines : 4th Monitoring Report on SSH Flagged Projects Funded in 2017 under the Societal Challenges and Industrial Leadership Priorities." Brussels: European Commission. https://publications.europa.eu/en/publication-detail/-/publication/4365f75a-5efe-11e8-ab9c-01aa75ed71a1/ language-en.

Ulnicane, I. (2015). "Broadening Aims and Building Support in Science, Technology and Innovation Policy: The Case of the European Research Area." Journal of Contemporary European Research 11 (1): 31-49.

Wagner, P. (2007). "Public Policy, Social Sciences, and the State: An Historical Perspective." In Handbook of Public Policy Analysis: Theory, Politics, and Methods, edited by F. Fischer and G. J. Miller, 29-40. Boca Raton: CRC Press.

Weber, M. (1968). "Die 'Objektivität' sozialwissenschaftlicher und sozialpolitischer Erkenntnis (1904)." In Gesammelte Aufsätze zur Wissenschaftslehre, edited by J. Winckelmann, 3rd ed., 146-214. Tübingen: Mohr Siebeck.

Wilsdon, J., Allen, L., Belfiore, E., Campbell, P., Curry, S., Hill, S., Jones, R. et al. (2015). "The Metric Tide: Report of the Independent Review of the Role of Metrics in Research Assessment and Management." Report from an expert group. London: HEFCE. http://www.hefce.ac.uk/ pubs/rereports/Year/2015/metrictide/Title,104463,en.html.

Wittrock, B. (2000). "Modernity: One, None, or Many? European Origins and Modernity as a Global Condition." Daedalus 129 (1): 31-60.

Wladawsky-Berger, I. (2018). "In an Era of Tech Innovation, Whispers of Declining Research Productivity." Wall Street Journal, July 13, 2018, sec.
CIO Journal. https://blogs.wsj.com/cio/2018/07/13/in-an-era-of-techinnovation-whispers-of-declining-research-productivity/.

Young, M. (2015). "Shifting Policy Narratives in Horizon 2020." Journal of Contemporary European Research 11 (1): 16-30.

\section{AUTHOR}

\section{THOAMS KÖNIG}

Institut für Höhere Studien - Institute for Advanced Studies (IHS)

Josefstädter Straße 39, Vienna, 1080 (Austria)

E: koenig@ihs.ac.at

\section{KEYWORDS:}

SSH research; social sciences; humanities; research policy; Horizon 2020; Horizon Europe; European integration 


\section{SOCIAL SCIENCES AND HUMANITIES RESEARCH MATTERS.} GUIDELINES ON HOW TO SUCCESSFULLY DESIGN, AND IMPLEMENT, MISSIONORIENTED RESEARCH PROGRAMMES

THOMAS KÖNIG

DOI: 10.22163/fteval.2019.305
$\mathrm{D}$ espite tremendous progress that our societies have made in recent decades, equally challenging tasks remain. These societal challenges directly concern the way we interact with each other and our environment, the way we produce and consume, and the way in which we construct and perceive meaning in our actions or change our behaviour.

Scientific research is an important driver for economic and social well-being. It provides analytical capacity and lays the groundwork for creating relevant and evidence-based policy solutions. It is thus not surprising that many research funding programmes aim at putting value in excellent research for tackling societal challenges.

Cooperation across and beyond different disciplinary backgrounds and with different (methodological, technological, theoretical) knowledge provides nuanced, multi-layered analyses and enables mitigation of grand challenges. That's why research funding programmes often ask specifically for interdisciplinary approaches, and for experts to look at problems from different perspectives.

\section{SSH RESEARCH IS CRUCIAL FOR SUCCESS OF PROGRAMMES}

In fundamental aspects, research in social sciences and humanities (SSH) plays a crucial role in the success of any research funding programme that aims at tackling societal challenges:

- Almost all of our current societal problems are influenced by different aspects of politics, social and cultural norms, ethics and legal frameworks, production and consumption patterns, traditions and life styles, and historic trajectories. To correctly identify and address the problem, those deep-running connections have to be analysed and understood. One can find aspects of political economy and a diversity of social and cultural dimensions in each research cluster, be it health, energy transition, climate change, bio-economy, or transport.

Similarly, if scientific research is expected to provide real solutions for ailments of humans or societies at large, it is important to study the intended as well as unintended - impact of innovations and their potential rebound effects thoroughly. Innovation is anything new that creates some form of value - often economic, but not always. Value creation also happens by adopting innovations, which is basically a social process with various societal implications. Innovation is thus not just the business of business, but also the business of society, and, thus, also a line of action for SSH.

By now, the unique set of expertise, knowledge, and capacity that SSH holds for research in tackling societal challenges is fully acknowledged: better understanding of the social dimension to the challenges we face needs to be tackled at the same time as we seek to use technological advancement to solve problems. Europe has realised the untapped resource of SSH research and has the ambition to become a global pioneer of "integrating" SSH across its research funding programmes. The Lamy Report on "Horizon Europe" (starting 2021) emphatically states that "Missions ... will, by design, fully integrate social sciences and humanities (SSH)." The Competitiveness Council of the European Council agreed "that social sciences and humanities (SSH) shall play an important role across all clusters". 2 Similarly, SSH communities have worked tirelessly in recent years to make themselves usable for addressing the societal challenges. ${ }^{3}$ 


\section{FROM "INTEGRATION" TO COOPERATION}

Yet so far, existing programmes have not entirely managed to unleash the full potential of interdisciplinary cooperation between SSH research and research from the natural sciences, technology, engineering, and mathematics (STEM). Monitoring of funded projects aiming at "integration" provides evidence of mixed results. Serious efforts must be strengthened to create a basis where SSH and STEM address global challenges together and on an equal footing. Thus far, SSH is often only brought in once the respective research task has already been framed or even only added-on at the end of a project - as if it were a consultancy service to make publics love the technologies that are being developed. However, framing a specific problem or mission omitting insights from SSH may prove detrimental, thus integration from the very beginning is essential.

A programme which identifies the connected nature of technological and human and social dimensions will have gone a long way to overcoming the hurdle for being successful and effective. For researchers from SSH being enabled to truly cooperate with their STEM colleagues, the efforts for achieving an equal footing have to be increased - at two levels. One concerns the level of implementing research programmes, as has been done already. At this level, much insight can be drawn from recent experiences. The other concerns the level of designing research programmes - and this is where little experience exists thus far and where more active involvement of SSH communities is needed.

This suggests two necessary avenues for SSH research to have the best chance of maximising its contribution to tackling societal challenges.

\section{STIMULATING AND ENABLING COOPERATION WHEN DESIGNING A RESEARCH FUNDING PROGRAMME}

When designing a new research funding programme, or when refurbishing an existing one, it is of utmost importance to co-determine agendas and priorities with insights and expertise from SSH researchers in an atmosphere of mutual respect. This section is intended to speak directly to policy makers and managers of funding bodies who - together with external stakeholders - usually define the overall goals of funding programmes, and who set aside budgets for funding research to achieve the desired goals. Those managers setting up such programmes have to recognise the differences of the fields and their own potentials, and that they can make use of practical guidance for achieving terms under which successful cooperation will occur and increase.

What does SSH research bring to the table?

"SSH" covers a broad field of academic disciplines and scientific areas. Because SSH research is as diverse as our societies, cultures, and economies are, it frames and co-shapes transformative aspects of research and contributes to integrating complex cross-domain perspectives and standpoints, including those from other scientific disciplines and non-academic actors. From this breadth and diversity, we can identify the multi-dimensional strengths of research in the social sciences and humanities:

The expertise to calibrate missions, highlighting priority aspects to focus on "what matters"

The capacities of translating between academic disciplines, policymakers and different publics

- The expertise in placing specific problems in broader contexts, integrating both local and global perspectives

- The long-standing tradition of methodological reflexivity, recognising social and cultural influences on research itself

Practical tips for unleashing the full potential of interdisciplinary cooperation to tackle societal challenges

- Bring members of different scientific fields to your advisory bodies, and specifically those from SSH research fields, to codetermine the goals of the research funding programme you are about to establish.

- Regard SSH research not as a critical add-on, but as a vital contribution to correctly understanding the problem at hand, and for implementing the resulting solution appropriately. This way, cooperation with SSH research will automatically shift from being "mandatory" to being obvious and fruitful.

- Grant respect equally to scientists and researchers from SSH as from STEM; trust the discursive powers of interdisciplinary negotiations and the expertise of SSH research in processes of co-creation.

- Be generous with stipulations concerning interdisciplinary cooperation, as it requires time and space for researchers from different backgrounds to become acquainted.

\section{FOSTERING COOPERATION WHILE IMPLEMENTING A RESEARCH FUNDING PROGRAMME}

Once a research funding programme is implemented, it is mandatory to make sure that SSH research is taken into account. Thus, this section is directed specifically at

- officers and managers within funding agencies establishing and executing funding programmes

- panellists and reviewers providing expertise and judgement for decision-making in the execution of funding programmes

- independent evaluators of those funding programmes

If you are a programme officer

- Make funding calls inclusive! Throughout the text of a call, explain that the social dimensions of a specific challenge need to be addressed alongside other aspects.

- Define criteria that encourage jury panellists and reviewers to identify the right people - not necessarily those with the formally best track and publication record. Metrics, rankings, or 
indicators may serve as means for decision-making, but should not serve as a universal panacea. ${ }^{4}$

- Increase variation! Involve experts (plural!) from SSH in the evaluation procedures of your calls.

- Encourage SSH researchers proactively to compete for funding, and to lead projects and consortia.

- If you are a panellist, or a reviewer

- Take context into account! Local and/or contextualised expertise from SSH may be more valuable for a project than "global" recognition of any scholar. Don't fall for the ubiquity of excellence rhetoric!

- Allow for original proposals that include, or are led by, SSH researchers. Respect the autonomy of SSH researchers to bring in their own ways of working to projects they are leading.

- Academic disciplines have different sizes and express their hierarchies differently. Do not believe the patina of precision, projected by metrics, as they often suggest impact where there is none.

- Look beyond potential scientific impact and consider also potential transformative societal, economic, political, ecological or cultural impact.

If you are an independent programme evaluator

- Look out for difference! There are different types of impact, and that they may be long-term as well as immediate. Ideas and concepts take time to ripple out from initial academic communities into society.

- Make sure the programme scope and call texts consider the social dimensions of the societal challenge to be tackled, and compare with the call winning teams and their composition.

- Account for the reflexive dimension of the programme, and look out for what social values are inscribed into the programme.

- Check the types of cooperation that are projected and actually take place, and to what degree participation and communication across and beyond disciplines are made possible.

\section{TO WHOM ARE THESE GUIDELINES ADDRESSED?}

This document is directed at all people who deal - in one way or another - with research funding programmes. Specifically (but not exclusively), these guidelines address research programmes that set out a specific goal to tackle a societal problem through the means and opportunities provided by scientific and scholarly research - both from SSH and STEM. The expected research is often described as "mission-oriented", albeit usage of this term differs.

These guidelines have been drafted, consulted and compiled by Thomas König on behalf of the organisers of the Austrian Presidency of the Council of the European Union Conference on 'Impacts of Social Sciences and Humanities for a European Research Agenda - Valuation of SSH in Mission-Oriented Research'. The conference was supported by project funding from the European Union's Horizon 2020 research and innovation programme under grant agreement No 814729. The author likes to acknowledge with much appreciation the input of the consulted experts who contributed to these guidelines.

Citation: König, T. (2019). Social Sciences and Humanities research matters - Guidelines on how to successfully design, and implement, mission-oriented research programmes. Austrian Presidency of the Council of the European Union Conference on 'Impacts of Social Sciences and Humanities for a European Research Agenda - Valuation of SSH in Mission-Oriented Research' organised by Centre for Social Innovation, Vienna.

\section{AUTHOR}

\section{THOAMS KÖNIG}

Institut für Höhere Studien - Institute for Advanced Studies (IHS) Josefstädter Straße 39, Vienna, 1080 (Austria)

E: koenig@ihs.ac.at 


\title{
THE PUBLIC VALUE OF THE SOCIAL SCIENCES
}

\author{
JOHN D. BREWER \\ DOI: 10.22163/fteval.2019.363
}

$\mathrm{P}$ blic value is integral to the very nature of the social sciences, since they emerged as separate disciplines out of moral philosophy in the eighteenth century precisely in order to better diagnose and improve the social condition. Engagement with social and human progress and with improvement and betterment marks social science as a public good. Incidentally, I would say the same about the humanities.

Two contemporary threats exist to social science, however, which, again, apply equally to the humanities. The first is the global university crisis, epitomised by the intensification of the audit culture and marketisation in higher education. With respect to the social sciences, I suggest we see this threat simultaneously as an opportunity to empower the social sciences in a new form of "public social science". The second threat is the impact agenda, which is linked to the first but has developed dynamics of its own. I suggest social science can engage positively with the impact agenda since the process of impact is easy to demonstrate for the social sciences.

However, impact is also a deeply flawed approach to assess the public value of social science research. There are diverse views on the meaning, it is very difficult to measure, even within the policy evaluation tradition for which the idea of impact slips easily off the pen, and the hostility generated by the impact agenda, associated as it negatively with the audit culture, has turned the debate gangrenous and ruled out the possibility of reasoned argument. Difficulties over its measurement have resulted in prioritising certain forms of impact because they can be more easily measured, such that measurement drives the debate. Impact can thus be discriminatory. There is an inevitable - almost inherent - bias towards favouring research whose impact is more readily demonstrable; and this mostly because of its direct policy benefit or user engagement.

Furthermore, impact is reducible to activities not directly connected to the quality of the research, for impact is mediated by a large number of processes independent of its findings and their quality. These include the social networks researchers are embedded in for communicating their results and for engaging with users, especially powerful groups, researchers' communication skills and their prior relationships with those who take up the results, like policymakers, the media and other users, the extent to which the field is one where policy debate is settled or still live, and how sensitised users already are to the potential benefit of the research findings. Reducing impact to metrics, like citation counts, further reinforces the self-referential and coincidental nature of impact. Impact is serendipitous, conditional, involving huge elements of chance and luck.

Given this argument, it may seem paradoxical for me to say here that impact is a sheep in wolves' clothing; it is much more warm and cuddly and much less dangerous than it appears. Two dimensions of impact must be distinguished in order to demystify it: the process of impact and its assessment (see Figure 1). By process is meant the method of delivering impact, by assessment its measurement. The process of impact can be simplified by reducing it to three questions which all social scientists can ask themselves about their research, even where it is theoretical: Who are the users of our research? How do I engage with them? What has been/ could be the effects of this engagement? The assessment of impact revolves around one question. What is the evidence of these effects?

Answers to the fourth question, which define its measurement, are more difficult to conjure, especially evidence of effects which are independent of the effects themselves rather than duplicates of them. An effect of the research is the intended or unintended change, due directly or indirectly to an intervention, whereas impact is perceived as the intended or unintended effects on beneficiaries of the intervention, the impact on which is measured by its effects. This is circular argument and it is particularly tricky to accurately connect the research, its effects and the evidence of these effects. This repeats the observation that measurement is the most problematic part of impact. When systems impose the measurement of impact, impact can get reduced to the effects of the research, and when there is no independent evidence of impact separate from these effects, impact is its measures.

This produces one of the major paradoxes of the current impact debate: the meaning of impact is broad and inclusive to enhance its popularity, but its measurement is narrow and exclusive. The process of impact and its assessment operate in opposition to one another, with the inclusiveness of its meaning not resolving the complications of its measurement. A system that insists on its assessment thus ends up being heavily criticised and practitioners lose sight of the feasibility and desirability of dealing with the process of impact. The baby has been thrown out with the bathwater; social scientists have rejected impact because of the difficulties of its measurement.

Of course, the neo-liberal context in which impact has emerged contributes to the suspicion amongst social scientists that it is a wolf in sheep's clothing, deceptive, dangerous and devouring. The marketisation of social scientific knowledge, via ideas of 'impact', 'use', 'knowledge transfer' and 'benefit', combine with the privatisation of public university education and enhanced state regulation of universities through the audit culture, to reinforce mutual suspicion between governments, higher education managers and social scientists.

I believe the debate therefore needs to move on from the public impact of social science to its public value. Public value is a vocabulary easier around which to develop a common conversation in order to conduct reasoned debate. Thus, my argument is not one in support of the narrow impact agenda that is currently dominating social science and higher education managers. I want to broaden the debate. 
I advance five claims with respect to impact:

- social science is well equipped and readily capable of demonstrating the impact of social science research;

- impact, however, is a deeply flawed way of approaching the public value of social science;

- it is necessary to shift the terms of the debate away from the public impact of social science to its public value;

- value can be deconstructed into several types which show the diverse ways in which the social sciences have value;

- it is possible to develop a definition of public value that demonstrates social science to be a public good.

This involves deconstruction of the term 'value' (see Figure 2). There are at least three different meanings to the term: value as usefulness and utility; value as quality and worth; and value as judgement and evaluation. The first we might call use value, the second price value, the third normative value. They prompt further deconstruction. Use value can be direct or indirect, price value intrinsic or added (giving us the phrase 'value added') and normative value can be private or public.

Direct use value describes the level of usefulness of an item unmediated by other things, indirect is the utility accorded when used in combination with other things. Use value does not necessarily diminish when it is indirect. A single university has direct use value but its indirect use value can be enhanced when set in relation to all other universities within higher education as a whole. Intrinsic price value is the worth of the item inherent unto itself, such as the cost of running the single university or all the others in the higher education system. Added price value describes the worth of things when put to use indirectly, such as the price value attributed to a student's education that utilises the university or to universities' contribution to the local economy, all of which universities now feverishly estimate to head off criticism. Private normative value refers to the quality attributed to an item by an individual in terms of the status to them derived from possessing it, public normative value to the quality attributed to it more widely, such as its social status and cultural significance. Personal sentiment can attach immense normative value to an item which is of little meaning and status to other individuals or collectively, and vice versa.

Elements of use, price and normative value are run together in current debates about impact, where 'impact' is often narrowly reduced to use-value and where arguments about the defining purposes of subjects is often related exclusively to their economic benefits. The neo-liberal habit of attaching a price to everything in effect reduces value to pricevalue - what it costs. By developing an appropriate sense of the purpose of the social sciences, it is possible to establish a definition of their value that broadens it from economic usefulness and costs.

This conceptual vocabulary means that we have to assess the value of the social sciences across different dimensions of value, and that the assessment of their worth varies accordingly. For example, this conceptual deconstruction allows us to argue that the value of the social sciences is not to be found solely in direct use value (say, economic usefulness), as if this can be assessed in isolation from indirect use value (say, their economic usefulness when assessed in relation to other things, such as the economic usefulness of social science graduates across their working lives, or the indirect use value of social science research in combination with other scientific research, in the form of medical-social science research, biological and social sciences research, and climate change science and the sociology of climate change, and so on).
We can further argue that the price value of the social sciences (their cost to the public budget set against what they realise by their direct use value) is a very poor measure of value. If the focus is on price value, we should properly calculate both the indirect use value of the social sciences and their "value added" price value - the price value of the social sciences when measured by what they add to the use, price and normative value of other things. The price value of the social sciences, for example, should be set in the context of what they add to the price value derived from, say, student exchanges, intellectual tourism and social and cultural events, or the impact of social science research on transport policy, housing, the welfare state, 'race' relations, better hospital care for the dying, crime rates, and so on, and what added price value accrues from having people educated in the social sciences (in terms of, say, socially-informed citizenry, workforces, communities and the like). Social science research on inter-cultural and inter-ethnic relations, ageing and population demographics, sport, heritage and so on can be stressed as part of their added price value.

This multidimensional view of value also means that the normative value of the social sciences is an important dimension equal to their use and price value. This is not just meant in the narrow sense of what they add to the quality of life and status of individuals educated in the social sciences or to the lives of people affected by social science research, important as these are a measure of private normative value. It is that the value of the social sciences can be assessed by their contribution to the social values they help garner and disseminate in culture, the market and the state.

The public normative value of the social sciences, therefore, gives the social sciences two qualities against which their status should be evaluated: they not only generate information about society, they are a medium for society's reproduction. They are the way in which society can find out about itself and in so doing generate the idea of society itself. If it is thought that this sort of value is incalculable, it is no more so than the proper enumeration of the use and price value of the social sciences.

The language of 'public value', as distinct from 'public impact', is challenging precisely because it is not reducible to monetary calculation in the same way price and use value are, which is why establishing the public value of social science is so important for rescuing the debate back from the marketeers who reduce everything to use and price value.

My argument is thus simple and clear cut: making people aware of themselves as comprising a society helps in the development and dissemination of key social values that make society possible - cultural values like trust, empathy, altruism, tolerance, compromise, social solidarity and senses of belonging. These everyday virtues assist in society's ongoing betterment and improvement. The social sciences help us understand the conditions which both promote and undermine these values and identify the sorts of public policies, behaviours and relationships that are needed in culture, the market and the state to ameliorate their absence and restore and repair them. It is for these reasons that social science is a public good.

The public normative value of the social sciences lies in their direct engagement with the DNA of society - individuals, groups, social relations, civil society, culture, law, legal governance, the market and the state. They are modes for understanding the mechanisms through which we live socially and as such are essential for making social life possible. Social sciences dissect the DNA of society and the information this discloses helps them improve the quality of social life. As such, the social sciences exist within a moral and ethical framework and simultaneously 
help to consolidate it as the framework within which everyone exists as social beings.

This is not the only form of value, however. People who declare the social sciences as a public good also need to recognise that the notion of public value into which it fits is multi-dimensional. 'Economic benefits' have to form part of the value narrative and use and price values(?) are part of the debate about the public value of the social sciences. This means articulating that the social and cultural relevance of social science research has economic utility in addition to its other benefits. My argument is that the social sciences have both economic value and constitute a public good. Thank you.

\section{THE TWIN DIMENSIONS OF IMPACT.}

\section{THE PROCESS OF IMPACT}

\section{Who are the users of my research?}

Culture

NGOs, civil society (national and global), educated citizenry, cultural consumers, librarians, archivists, schools, media, public bodies, private organizations, charities, individuals, families, etc.

The state

Governments (local, devolved, national and regional), political parties, politicians, policymakers, civil servants, national and interna-

The market

tional strategists, etc.

business, industry, trade unions, consumers, workers, etc.

\section{How do I engage with them?}

Culture

mailing lists, newsletters, website, social media, public talks, seminars, publications, popular writings and journalism, radio, television, posters, brochures, conferences and presentations, etc.

The state

publications, briefing papers and reports, workshops, talks, popular writing, presentations, etc.

The market

same as the above

\section{What has been/could be the effects of this engagement?}

Culture

behaviour and pursuits, understanding, civic and humanitarian values, public debate, public benefits, shared beliefs, health and well-being, health promotion, school performance, family relations, etc.

The state

evidence-based policy, management and use of public resources, decision-making, strategic thinking, etc. The market

knowledge transfer, spin off companies, product development, evidence-based market behaviour and strategy, decision-making, management of economic and human resources, industrial relations, consumer behaviour and choice, dispute management, etc.

\section{THE ASSESSMENT OF IMPACT}

\section{What is the evidence of these effects?}

Culture

take-up of research, influence on behaviours, beliefs, values and civic practice, etc. The state

policy, practice, evaluations, improved public scrutiny and accountability, etc.

The market

Knowledge transfer, policy and practice in business and industry, strategic thinking, industrial relations, conflict prevention and dispute management, consumer evaluations, etc.

Figure 1. The twin dimensions of impact. 


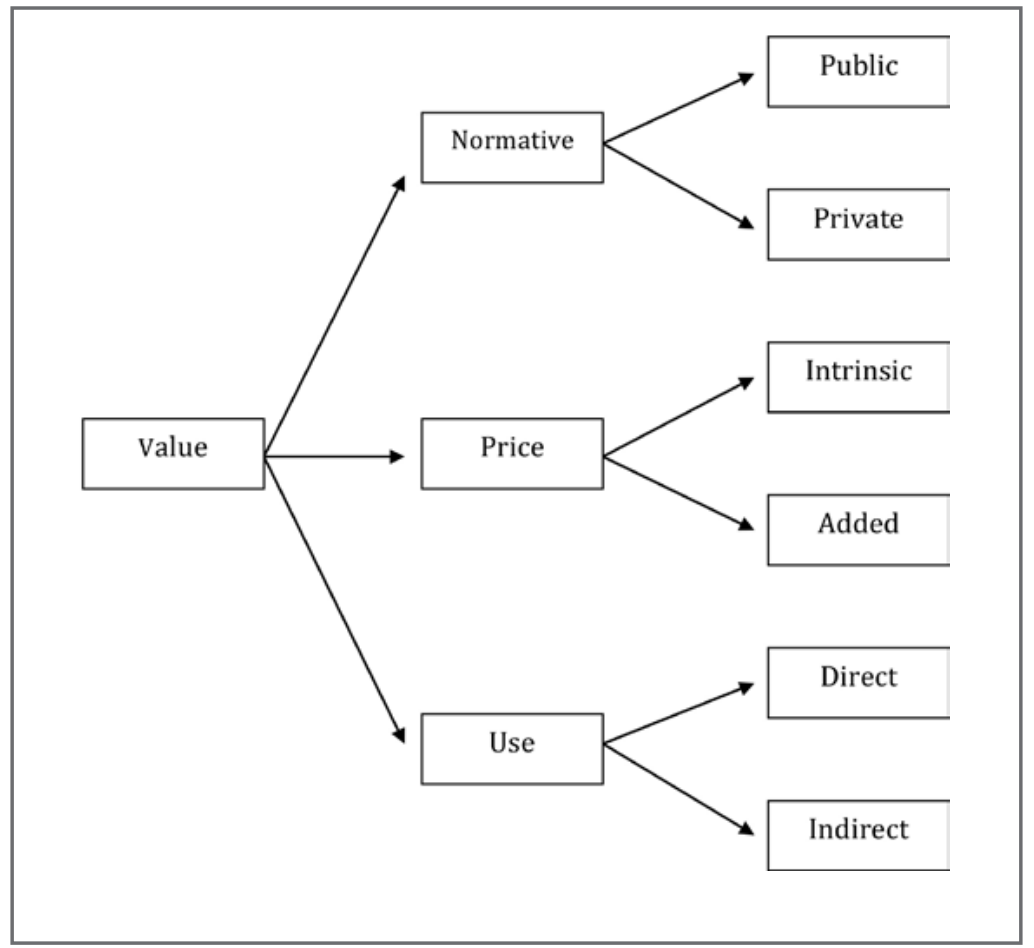

Figure 2. Types of value.

\section{AUTHOR}

\section{JOHN D. BREWER}

Senator George J Mitchell Institute for Global peace, Security and Justice,

Queen's University Belfast

University Road, Belfast BT7 1NN (UK)

E: j.brewer@qub.ac.uk 


\title{
BRINGING CONCEPTS TOGETHER: INTERDISCIPLINARITY, TRANSDISCIPLINARITY, AND SSH INTEGRATION
}

\author{
JOËL GRAF \\ DOI: $10.22163 /$ fteval.2019.364
}

\section{ABSTRACT}

$\mathrm{T}$ he European Commission has made a strong commitment to integrate the Social Sciences and Humanities (SSH) across Horizon 2020. The aim is to enhance the impact of activities tackling societal challenges. However, the question on how such an "SSH integration" should happen in practice still offers room for discussion. Therefore, this article focuses on the methodological challenges of bringing SSH into collaborative Horizon 2020 projects. It emphasises that $\mathrm{SSH}$ integration is a special case of inter- and transdisciplinarity, since different scientific disciplines as well as non-academic stakeholders are involved in the research process. Taking inter- and transdisciplinary expertise more systematically into account may thus contribute to both better proposals and improved project implementation for Horizon 2020 and the upcoming "Framework Programme Horizon Europe".

\section{INTRODUCTION}

The idea of SSH integration in Horizon 2020 goes back to the decision of the European Parliament and the Council stating that "social sciences and humanities will be mainstreamed as an essential element of the activities needed to tackle each of the societal challenges to enhance their impact."1 This statement sounds convincing and seems intuitively plausible. However, the past years have shown that such an "SSH integration" is far from trivial and requires sound methodological tools. The question of "how" to integrate SSH disciplines into consortia has not been in the focus of the European Commission (EC) so far and remains mainly up to the commitment and competence of the individual applicant. ${ }^{2}$ Neither has there been a broad reflection on how SSH integration relates to other concepts such as inter- and transdisciplinarity. ${ }^{3}$ Against this background, the present article highlights the potential of using theoretical and methodological expertise in the fields of inter- and transdisciplinarity in order to improve the impact generating processes in SSH research.

\section{INTERDISCIPLINARITY, TRANSDISCIPLINARITY AND SSH INTEGRATION}

When looking into Horizon 2020 topics one finds references to a broad range of collaborative research approaches, such as SSH integration, transdisciplinarity, interdisciplinarity, multidisciplinarity, crossdisciplinarity, multisectorality, co-creation and co-design. ${ }^{4}$ For a common ground of discussion, it is crucial to define those terms and clarify the relationships between each other's. In what follows, this will be done with interdisciplinarity, transdisciplinarity and SSH integration.

The FAO section of the participant portal defines interdisciplinarity as "the integration of information, data, techniques, tools, perspectives,

European Parliament and Council (2013). Regulation (EU) No 1291/2013 of 11 December 2013 establishing Horizon 2020, Annex I, 121 (URL: https://eur-lex. europa.eu/LexUriServ/LexUriServ.do?uri=0J:L:2013:347:0104:0173:EN:PDF, last access 31.10.2018).

2 The dedicated monitoring reports of the European Commission are limiting their assessment on how many SSH partners consortia involve and how much budget is dedicated to SSH researchers. These figures are of high relevance. However, it would be also important to assess the question on how SSH integration happened (common problem framing, workshops etc.). This would require a qualitative rather than a quantitative approach. B. I. Birnbaum et al. (Ed.). (2017). 2nd Monitoring report on SSH-flagged projects. Luxembourg: Publication Office of the European Union (URL: https://publications.europa.eu/ en/publication-detail/-/publication/acac40f5-e84b-11e6-ad7c-01aa75ed71a1, last access 31.10.2018).

3 It is significant that a 2018 topic in the Societal Challenge 6 asks for "lessons from the practices of interdisciplinarity" but explicitly excludes discussions on the "epistemology of interdisciplinarity".

European Commission 2018). Work Programme 2018-2020: Europe in a changing world - Inclusive, innovative and reflective societies, topic GOVERNANCE-15-2018: Taking lessons from the practices of interdisciplinarity in Europe, 54-55 (version of 24.07.2018. URL: http://ec.europa.eu/research/participants/data/ref/h2020/wp/2018-2020/main/h2020-wp1820-societies_en.pdf, last access 31.10.2018).

4 An example for the broad mix of concepts is to be found in the introduction of the Societal Challenge 5 Work Programme. It asks for: "a challenge-driven solutions-oriented, trans-disciplinary perspective that integrates technology, business models and economic organisation, finance, governance and regulation as well as skills and social innovation, and involves co-creation of knowledge and co-delivery of outcomes with economic, industrial and research actors, public authorities and/or civil society." (My italics, JG). European Commission (2018). Work Programme 2018-2020: Climate action, environment, resource efficiency and raw material, introduction, 6 (version of 24.07.2018. URL: http://ec.europa.eu/research/participants/data/ref/h2020/wp/2018-2020/main/ h2020-wp1820-climate_en.pdf, last access 31.10.2018). 
concepts or theories from two or more disciplines. Disciplines may be from the natural sciences, technology, engineering, economics, social sciences and humanities. "5 This definition focuses on the integration of knowledge from different academic disciplines. ${ }^{6}$

Even though explicit references to transdisciplinarity are to be found in various Horizon 2020 topic texts, it is quite difficult to find an official definition by the EC. A guidance document for evaluators published in 2014 states that "trans-disciplinarity [...] refers to approaches and methodologies that integrate as necessary (a) theories, concepts, knowledge, data, and techniques from two or more scientific disciplines, and (b) nonacademic and non-formalized knowledge. In this way, trans-disciplinarity contributes to advancing fundamental understanding or solving complex problems while fostering multi-actor engagement in the research and innovation process." "This definition goes beyond the collaboration between scientific disciplines by including the knowledge of non-academic stakeholders. ${ }^{8}$

The process of SSH integration is explained on the participant portal as follows: "Social Sciences and Humanities (SSH) are needed to tackle many of the complex societal challenges addressed in H2O2O, and contributions from one or more of these disciplines are frequently necessary for a successful proposal. These contributions are usually part of an interdisciplinary approach, involving either:

- collaboration between SSH disciplines and/or,

- collaboration between SSH disciplines and non-SSH disciplines such as natural sciences, medicine and technology. ${ }^{\text {"g }}$

SSH integration can therefore be considered as a specific form of interdisciplinarity. ${ }^{10}$ At the same time, SSH flagged topics are often requiring the involvement of non-academic stakeholders. In practice, integrating SSH researchers thus also touches issues of transdisciplinarity.

\section{STATE OF THE ART AND INVOLVEMENT OF EXPERTS}

SSH integration can only contribute to excellent science if the integration process itself is meeting state of the art principles. Therefore, existing expertise on inter- and transdisciplinary methodologies should to be taken into account by all stakeholders, i.e. the EC, applicants, "National Contact Points" (NCPs), and evaluators.

Such expertise is to be found at many different levels. Switzerland may serve as an example. The Swiss Academies of Arts and Sciences are hosting td-net, a dedicated contact point for researchers and funders in the field of inter- and transdisciplinary research and teaching. ${ }^{11}$ Amongst others, td-net provides a platform with concrete tools for the implementation of inter- and transdisciplinarity. Another institution dedicated to collaborative research is the Department of Environmental Systems Science at the ETH Zurich, which includes the transdisciplinary laboratory TdLab. TdLab aims at "integrating knowledge and values from different scientific perspectives, as well as from other societal actors". ${ }^{12}$

Europe has a lot of experts on inter- and transdisciplinarity. ${ }^{13}$ Bringing them together with Horizon 2020 applicants could lead to improved proposals and better project implementation.

$4 \quad$ An example for the broad mix of concepts is to be found in the introduction of the Societal Challenge 5 Work Programme. It asks for: "a challenge-driven, solutions-oriented, trans-disciplinary perspective that integrates technology, business models and economic organisation, finance, governance and regulation as well as skills and social innovation, and involves co-creation of knowledge and co-delivery of outcomes with economic, industrial and research actors, public authorities and/or civil society." (My italics, JG). European Commission (2018). Work Programme 2018-2020: Climate action, environment, resource efficiency and raw material, introduction, 6 (version of 24.07.2018. URL: http://ec.europa.eu/research/participants/data/ref/h2020/wp/2018-2020/main/ h2020-wp1820-climate_en.pdf, last access 31.10.2018).

$5 \quad$ "How should interdisciplinarity and stakeholder knowledge be addressed and evaluated in Horizon 2020 proposals?". European Commission (2016). FA0 Participant Portal, ID 935 (09-02-2016) (URL: https://ec.europa.eu/research/participants/portal/desktop/en/support/faqs/faq-935.html, last access 31.10.2018).

$6 \quad$ Such an understanding of interdisciplinarity corresponds with the state of the art of the relevant research literature. G. Hirsch Hadorn et al. (2008): The Emergence of Transdisciplinarity as a Form of Research. In G. Hirsch Hadorn et al. (Ed), Handbook of Transdisciplinary Research (Dordrecht: Springer), 19-39, here 28 .

$7 \quad$ European Commission (2014). "How should trans-disciplinarity be addressed and evaluated in proposals?". Guidance for evaluators of Horizon 2020 proposals: 6 (version 1.0, 15.07.2014, URL: http://ec.europa.eu/research/participants/data/ref/h2020/grants_manual/pse/h2020-evaluation-faq_en.pdf, last access 31.10 .2018 )

$8 \quad$ This is in line with the state of the art of the dedicated research community. G. Hirsch Hadorn et al. (2008): The Emergence of Transdisciplinarity as a Form of Research. In G. Hirsch Hadorn et al. (Ed), Handbook of Transdisciplinary Research (Dordrecht: Springer), 19-39, here 29.

$9 \quad$ European Commission (2018). "How should Social Sciences and Humanities (SSH) be addressed and evaluated in H2020 proposals?". FA0 Participant Portal, ID 938 (26.01.2018) (URL: https://ec.europa.eu/research/participants/portal/desktop/en/support/faqs/faq-938.html, last access 26.01.2018).

10 See also C. Schmaltz (2016). Multi- and Transdisciplinary Research in Horizon 2020. Presentation given at the National Network and Information Event 2016, NCP Life Sciences, Cologne, 01 June 2016, here 7. (URL: https://www.healthncp.net/sites/default/files/downloads/Plenar01_Health.pdf, last access 31.10.2018). Also note that transdisciplinarity is here defined as "creating a unity of intellectual frameworks beyond the disciplinary perspectives" (6). This understanding of the concept differs from the one referred to above (Guidance for evaluators" (2014)).

11 td-net: Network for Transdisciplinary Research (URL: http://www.transdisciplinarity.ch, last access 31.10.2018)

12 USYS TdLab (URL: http://www.tdlab.usys.ethz.ch, last access 31.10.2018).

13 See also the Horizon 2020 project ACCOMPLISSH (URL: https://www.accomplissh.eu, last access 04.12.2018). 


\section{EURESEARCH PILOT EVENT ON TRANSDISCIPLINARITY IN HORIZON 2020}

In April 2018, Euresearch, the Swiss advisory network on "European Research and Innovation", organised an event on transdisciplinarity in Horizon 2020.14 Applicants, coordinators, evaluators and experts discussed on how theoretical and methodological expertise in transdisciplinarity may contribute to both better proposals and improved implementation of projects.

Experts on transdisciplinary methodologies assessed the general design of collaborative projects in Horizon 2020. They especially mentioned the importance of the common problem framing by the consortium members. ${ }^{15}$ Against this backdrop, one of the main challenges concerns the implementation of transdisciplinary processes within top-down calls, as the latter are often strongly pre-defining the scope of the projects.

In a second step, evaluators and coordinators of Horizon 2020 discussed the concrete potential of transdisciplinarity within proposals and project implementation. It became obvious that in certain research fields (such as "Public Health and Sustainable Development") the use of transdisciplinary tools is daily business. In other fields, transdisciplinarity happens rather implicitly and by learning by doing. As for the evaluation, all panellists agreed on that an explicit consideration of transdisciplinarity would make proposals more credible. However, the involvement of transdisciplinary experts could also lead to conflict of aims as such experts generate additional costs for the consortium. The goal would therefore be to convince evaluators that the incorporation of transdisciplinary experts in consortia is an integral part of collaborative research and innovation.

\section{HORIZON EUROPE AND MISSION-ORIENTED RESEARCH}

In June 2018, the EC published its proposal on the "9th European Framework Programme Horizon Europe". One of the main recommendations is the preservation of the three pillar approach of Horizon 2020. For the present article, the pillar "Global Challenges and Industrial Competitiveness" is of special interest. It should "encourage cross-disciplinary, cross-sectoral, cross-policy and cross-border collaboration in pursuit of the UN SDGs and the competitiveness of the Union's industries therein." ${ }^{16} 0 \mathrm{n}$ top of the regular call for proposals "a limited set of highly visible missions will be introduced. [...] Missions, with ambitious but time-bound and achievable goals, should speak to the public and engage it where relevant. They will be co-designed with Member States, the European Parliament, stakeholders and citizens. ${ }^{17}$ On this basis, it seems very likely that SSH integration, as well as inter- and transdisciplinarity, will play an important role in the upcoming "Framework Programme".

However, there remain some open questions which have to be considered. The role of SSH is not explicitly addressed in the document. The most important programme for SSH Integration in Horizon 2020 was the "Societal Challenge" 6 on "Inclusive Societies". While there will be a similar cluster in Horizon Europe, the budget will be comparatively low. ${ }^{18} \mid t$ remains to be seen how much budget from the other clusters will be dedicated to SSH research. As for the question of inter- and transdisciplinarity, the idea of co-designing the newly introduced missions together with citizens is certainly a laudable initiative. There is, though, an obvious area of tension between the top-down approach of highly prescriptive topic texts and/or missions on the one hand and inter- and transdisciplinary processes on the other hand, as the latter require certain openness. ${ }^{19}$

\section{CONCLUSION AND RECOMMENDATIONS}

From a methodological point of view, SSH integration is a special case of interdisciplinarity. As SSH flagged topics often involve nonacademic stakeholders, transdisciplinarity is also of high relevance. The relation between SSH integration, interdisciplinarity and transdisciplinarity should therefore be thoroughly discussed and the results should be made available for the Horizon 2020 stakeholders.

The EC should reconsider the methodological terminology for collaborative projects, especially regarding the topic texts. One possibility would be to include basic concepts in the glossary of the "Funding and Tenders Portal". Some definitions are already provided on the Horizon 2020 FA0 section. They are, however, somewhat hidden and incomplete (e.g. no reference to transdisciplinarity is given). As for the "9th European Framework Programme Horizon Europe", the EC should include experts on inter- and transdisciplinarity. The latter could give valuable inputs on

14 Euresearch event on "Transdisciplinarity in Horizon 2020. Challenges and Approaches". Bern, 24 April 2018 (URL: https://www.euresearch.ch/en/events/ event-detail/showUid/746/, last access 31.10.2018)

15 C. Pohl et al. (2017). Ten Reflective Steps for Rendering Research Societally Relevant. GAIA 26/1 (2017), 43-51.

16 European Commission (2018). Proposal for a Regulation of the European Parliament and of the Council Establishing Horizon Europe (COM(2018) 435 final, 2018/0224 (COD)), 17 (URL: https://ec.europa.eu/commission/sites/beta-political/files/budget-may2018-horizon-europe-regulation_en.pdf, last access 31.10.2018)

17 European Commission (2018). Proposal for a Regulation of the European Parliament and of the Council Establishing Horizon Europe (COM(2018) 435 final, 2018/0224 (COD)), 10 (URL: https://ec.europa.eu/commission/sites/beta-political/files/budget-may2018-horizon-europe-regulation_en.pdf, last access 31.10.2018).

18 The proposal suggests around 3 billion Euro for a cluster called Inclusive and Secure Societies. European Commission (2018). Proposal for a Regulation of the European Parliament and of the Council Establishing Horizon Europe (COM(2018) 435 final, 2018/0224 (COD)), 32 (URL: https://ec.europa.eu/commission/ sites/beta-political/files/budget-may2018-horizon-europe-regulation_en.pdf, last access 31.10.2018).

19 C. Pohl et al. (2017). Ten Reflective Steps for Rendering Research Societally Relevant. GAIA 26/1 (2017), 43-51; L. van Drooge and J. Spaapen (2017). Evaluation and Monitoring of Transdisciplinary Collaborations. The Journal of Technology Transfer (2017) (URL: https://doi.org/10.1007/s10961-017-9607-7, last access 31.10.2018). 
do's and don'ts regarding the framing of collaborative projects (e.g. regarding the top-down approach, common problem framing, co-design etc.).

Collaborative Horizon 2020 projects often involve different academic disciplines and non-academic stakeholders. In these cases, applicants should explicitly address methodological issues regarding the integration of knowledge and the elaboration of common research and innovation results. They should take into account the state of the art research on corresponding concepts and - if necessary - involve experts on interand transdisciplinarity both for the proposal writing and the project implementation

Evaluators should be thoroughly briefed about SSH integration, not only regarding the numerical involvement of SSH researchers but also regarding the actual process of how the specific competences are integrated into the consortium. In general, evaluators should be systematically looking at inter- and transdisciplinary methodologies.

"National Contact Points" dealing with SSH integration should advise applicants about the basic challenges of integrating the knowledge of different stakeholders within collaborative projects. Dedicated tools such as webinars, factsheets etc. - do already exist in some case ${ }^{20}$ and should further be developed, also by the NCP networks. Specific events as the one described above may also contribute to the discussion.

\section{REFERENCES}

Birnbaum, B. I. et al. (Ed.). (2017). 2nd Monitoring report on SSHflagged projects. Luxembourg: Publication Office of the European Union (URL: https://publications.europa.eu/en/publication-detail/-/publication/acac40f5-e84b-11e6-ad7c-01aa75ed71a1, last access 31.10.2018).

European Commission (2018). Horizon 2020 Work Programme 20182020: Climate action, environment, resource efficiency and raw material, introduction (version of 24.07.2018. URL: http://ec.europa.eu/research/ participants/data/ref/h2020/wp/2018-2020/main/h2020-wp1820-climate_en.pdf, last access 31.10.2018).

European Commission (2018). Horizon 2020 Work Programme 20182020: Europe in a changing world - Inclusive, innovative and reflective societies (version of 24.07.2018. URL: http://ec.europa.eu/research/ participants/data/ref/h2020/wp/2018-2020/main/h2020-wp1820-societies_en.pdf, last access 31.10.2018).

European Commission (2016). "How should interdisciplinarity and stakeholder knowledge be addressed and evaluated in Horizon 2020 proposals?". FAO Participant Portal, ID 935 (09-02-2016) (URL: https:// ec.europa.eu/research/participants/portal/desktop/en/support/faqs/ faq-935.html, last access 31.10.2018).

European Commission (2018). "How should Social Sciences and Humanities (SSH) be addressed and evaluated in H2020 proposals?". FA0 Participant Portal, ID 938 (26.01.2018) (URL: https://ec.europa.eu/research/ participants/portal/desktop/en/support/faqs/faq-938.html, last access
26.01.2018).

European Commission (2014). "How should trans-disciplinarity be addressed and evaluated in proposals?" In Guidance for evaluators of Horizon 2020 proposals: 6 (version 1.0, 15.07.2014, URL: http://ec.europa. eu/research/participants/data/ref/h2020/grants_manual/pse/h2020evaluation-faq_en.pdf, last access 31.10.2018).

European Commission (2018). Proposal for a Regulation of the European Parliament and of the Council Establishing Horizon Europe (COM(2018) 435 final, 2018/0224 (COD)) (URL: https://ec.europa.eu/commission/ sites/beta-political/files/budget-may2018-horizon-europe-regulation_ en.pdf, last access 31.10.2018).

European Parliament and Council (2013). Regulation (EU) No 1291/2013 of 11 December 2013 establishing Horizon 2020, Annex I (URL: https:// eur-lex.europa.eu/LexUriServ/LexUriServ.do?uri=0J:L:2013:347:0104:01 73:EN:PDF, last access 31.10.2018).

Hirsch Hadorn, G. et al. (2008). The Emergence of Transdisciplinarity as a Form of Research. In G. Hirsch Hadorn et al. (Ed), Handbook of Transdisciplinary Research (Dordrecht: Springer), 19-39.

Net4Society4 (n.d.). Factsheet on "Keys to successful integration of Social Sciences and Humanities in Horizon 2020" (URL: https://www. net4society.eu/_media/170110_Factsheet_Expert\%20meeting_INTEGRATION_def.pdf, last access 31.10.2018).

Pohl, C. et al. (2017). Ten Reflective Steps for Rendering Research Societally Relevant. GAIA 26/1 (2017), 43-51.

Schmaltz, C. (2016). Multi- and Transdisciplinary Research in Horizon 2020. Presentation given at the National Network and Information Event 2016, NCP Life Sciences, Cologne, 01 June 2016

van Drooge, L. and Spaapen, J. (2017). Evaluation and Monitoring of Transdisciplinary Collaborations. The Journal of Technology Transfer (2017) (URL: https://doi.org/10.1007/s10961-017-9607-7, last access 31.10.2018).

\section{AUTHOR}

\section{JOËL GRAF}

Euresearch

Effingerstrasse 19, Bern, 3008 (Switzerland)

E: joel.graf@euresearch.ch

\section{KEYWORDS:}

Interdisciplinarity, Transdisciplinarity, SSH Integration

20 See, for instance Net4Society4 (n.d.). Factsheet on "Keys to successful integration of Social Sciences and Humanities in Horizon 2020" (URL: https://www. net4society.eu/_media/170110_Factsheet_Expert\%20meeting_INTEGRATION_def.pdf, last access 31.10.2018). 


\title{
NEW PATHWAYS TO SOCIAL CHANGE - CREATING IMPACT THROUGH SOCIAL INNOVATION RESEARCH
}

\author{
JÜRGEN HOWALDT \\ D0I: 10.22163/fteval.2019.365
}

The social sciences and humanities are deeply involved in the processes that use scientific and scholarly approaches to bring about a better society, difficult as it may be to define it.

(König et al. 2018)

\section{ABSTRACT}

$\mathrm{T}$ he paper emphasises the crucial role of social innovation in successfully addressing social, economic, political and environmental challenges of the 21st century. In this context, the global mapping of the international research project SI-DRIVE reveals the capacities of social innovations to modify or even re-direct social change and to empower people -i.e. to address a wide variety of stakeholder groups, as well as the broader public, in order to improve social cohesion and to allow for smart, sustainable and inclusive growth. Like technological innovations, successful social innovations are based on numerous presuppositions and require appropriate infrastructures and resources. This includes a new role of public policy and government for creating suitable framework and support structures, the integration of the economy and civil society as well as supporting measures by science and universities (e.g. education for social innovation performance, know-how transfer).

This also raises the question of the role of universities in general and of social sciences in particular in social innovation processes. It will be a major challenge for the development of social innovation to ensure a much higher involvement of research and education facilities. In these processes social sciences will be challenged to redefine their functions with regard to innovation. In the past, innovation research in the context of social sciences has contributed to explain the social dimensions, the complexity and paradoxes of innovation processes. Henceforth, much will depend on realigning the range of competencies of social science as well as social scientists by contributing actively to the development of social innovation. Against this background participatory approaches that promote involvement and empowerment of civil-society actors are indispensable.

\section{INTRODUCTION}

The importance of social innovation in successfully addressing social, economic, political and environmental challenges of the 21st century is recognised not only within the Europe 2020 strategy, but also on a global scale. As a novel approach to address complex problems in global health, social care, education, energy, and environmental policies, social innovation has been embraced by stakeholders and communities on the local, regional and even national level (Franz et al. 2012; Hochgerner 2013. Moulaert et al. 2013; Nicholls et al. 2015).

The term "Social Innovation" can be traced back to the early 19th century, long before technological-economic connotations determined the common understanding of innovation (Godin 2012, pp. 21). Nevertheless, there is no shared understanding of social innovation in the sense of a clear differentiation from other concepts such as social entrepreneurship or business innovation based on new technologies, organisational features and marketing models. Likewise, there is no integration of social innovation in a comprehensive innovation policy (Howaldt et al. 2014).

We define social innovation as a new combination and/or new configuration of social practices in certain areas of action or social contexts, prompted by certain actors or constellations of actors in an intentional targeted manner with the goal of better satisfying or answering needs and problems than is possible on the basis of established practices. An innovation is therefore social to the extent that it, conveyed by the market or "non/without profit", is socially accepted and diffused throughout society or in certain societal sub-areas, may become transformed depending on societal circumstances (context) and ultimately institutionalised as a new social practice or made routine. As every other innovation, "new" does not require absolute or genuine novelty: Most innovations are new in relative terms, i.e. transferred or disseminated to another region, city or social grouping, other sectors and policy fields. Moreover, an innovation termed social innovation does not necessarily provide impact that is "good" for all or "socially desirable" in an extensive and normative sense. Accordingly, the actors' practical rationale, social attributions for social innovations are generally uncertain (Howaldt/Schwarz 2010).

With a focus on social practices, their reproduction and change as the central element of sociality, "Social Practice Theories" (SPT) allow for identifying the social dynamics of change processes. This modified understanding of the social as social practices opens the view on their reconfiguration as a core element of social innovation and social change (Shove et al. 2012). The social world is therefore composed of very spe- 
cifically nameable, individual, although interdependent practices of governance and organising; partnership; negotiations; self (cf. Reckwitz 2003); comfort, cleanliness and convenience (Shove 2003); working and nurturing (Hargreaves et al. 2013), and; consumption (Brand 2010).

Therefore it does not surprise that in the context of the broad social debate surrounding sustainable development and necessary social transformation processes (Loorbach/Rotmans 2010), the question of the relationship between social innovations and social change becomes important (Avelino et al. 2014): How can processes of social change be initiated which go beyond the illusion of centralist management concepts to link social innovations from the mainstream of society to the intended social transformation processes (McGowan/Westley 2015)?

But what are the conditions under which social innovations flourish and create impact? Who are the stakeholders? How do social innovations diffuse and lead to social change? Against this background, a new generation of EU-funded projects worked on a sound theoretical understanding of social innovation and its relation to (transformative) social change to contribute to a better understanding of the conditions under which social innovations develop, flourish and finally increase their societal impact (chapter 2).

This also raises the question of the role of universities in general and of social sciences in particular in social innovation processes. It will be a major challenge for the development of social innovation to ensure a much higher involvement of research and education facilities (chapter 3). In these processes social sciences will be challenged to redefine their functions with regard to innovation. Against that background participatory approaches that promote participation and empowerment of civilsociety actors are indispensable to increase impact (Howaldt/Schwarz 2010) (chapter 4).

\section{THE GROWING IMPORTANCE OF SOCIAL INNOVATION - "A GLOBAL MAPPING OF SOCIAL INNOVATION INITIATIVES"}

Recent years have witnessed the emergence of this new type of innovation, as an object of research and development appearing in a variety of forms and influencing our lives. There is a growing consensus among practitioners, policy makers and the research community that technological innovations alone are not capable of overcoming the social and economic challenges modern societies are facing. This is why a vast number of social innovation initiatives in different world regions providing new levers for solving problems and contributing to social change, can be identified.

The first global mapping of social innovation initiatives, which was conducted in the SI-DRIVE (Social Innovation: Driving Force of Social Change) project, revealed the importance of social innovation in addressing social, economic, political and environmental challenges of the 21st century on a global scale. It demonstrates the need for social innovation to overcome the (policy field related) societal challenges and social demands. In many policy fields we find a variety of social innovation initiatives (see figure 1).

Social innovations change the manner in which we live together (shared housing), work (telework), consume (car-sharing), distribute wealth (unconditional basic income) or deal with economic crises (short time work instead of termination). Social innovations provide new forms of collaboration between people (co-working spaces), organisations (private-public-partnerships) and states (agreement on the free movement of labour). Social innovations can emerge within different sectors: in civil society (urban farming), politics (parental leave), and economy

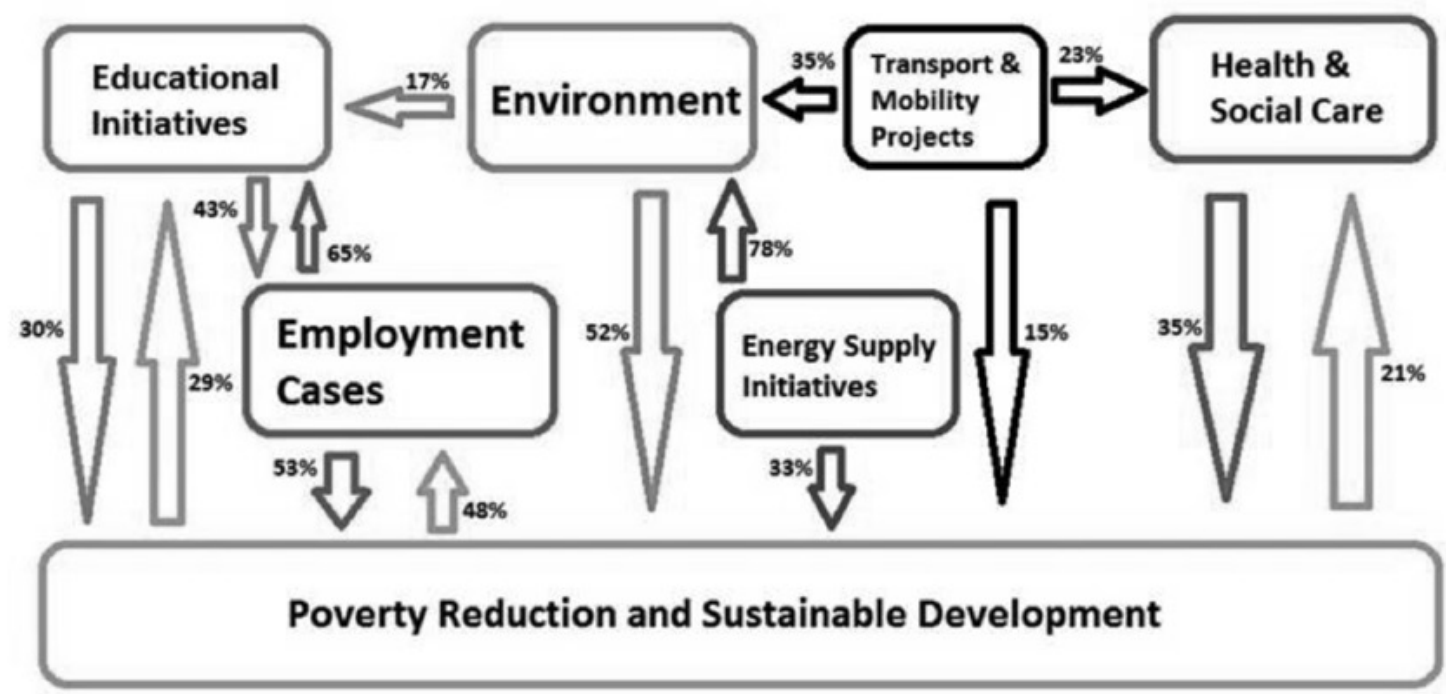

Figure 1. Social innovations cross policy fields. 
(micro credits). In short: social innovations in a sense of new practices are omnipresent and contribute to social change. The establishment of new social practices does play a prominent role in making mobility more environmentally friendly, diseases less scary or the energy turn around more successful. The high diversity of social needs and societal challenges addressed by the initiatives are not limited to one but often work across several policy fields. Social innovation has become a ubiquitous concept (Howaldt et al. 2016).

At the same time the global mapping demonstrated the capacities of social innovations to modify or even re-direct social change and to empower people -i.e. to address a wide variety of stakeholder groups, as well as the broader public, in order to improve social cohesion and to allow for smart, sustainable and inclusive growth (Howaldt et al. 2018). The mapping empirically shows that the societal and governance systems in which the social innovations are embedded are complex, the problems addressed are deeply rooted in established practices and institutions and that many initiatives are small in scale. Therefore, to better understand this relationship between social innovation and social change, the social embeddedness of any innovation in a dense network of existing practices, routines, institutions and context conditions, on the one hand, and innovation streams, on the other hand, has to be analysed. Any social innovation results in an outcome for those involved, yet to disseminate an impact further into society depends on specific conditions and mutual resonance between various social innovations. Growing social numbers and the range of social innovations may be likely to affect pace and perhaps directions of social change. Thus, social innovation in general has an impact on societal development, just as innovations in business are meant to have an impact on economic development and growth. The impact of social innovations varies (in every case) from raising awareness, which is essential in the ideation phase and the starting point of initiatives to create and implement an innovation, up to the formation of institutions (which is not necessarily the same as institutionalisation of new innovative practices, but often required to ensure the sustainability of social innovation). The mapping shed light on the great many, often nameless but still important, social innovations responding to specific and every-day social demands or incremental innovations (Howaldt et al. 2016).

Social innovative projects and initiatives aim to address social needs and societal challenges rather than focusing primarily on economic success and profit. Referring to a distinction introduced by BEPA ("Bureau of European Policy Advisers") who suggests that "the output dimension refers to the kind of value or output that social innovation is expected to deliver: a value that is less concerned with mere profit, and including multiple dimensions of output measurement" $(2010$, p. 26) there are three societal levels on which output may take place. In this understanding, social innovations

- "respond to social demands that are traditionally not addressed by the market or existing institutions and are directed towards vulnerable groups in society [...],

- tackle 'societal challenges' through new forms of relations between social actors, [...] respond to those societal challenges in which the boundary between social and economic blurs, and are directed towards society as a whole [...],

- or contribute to the reform of society in the direction of a more participative arena where empowerment and learning are both sources and outcomes of well-being" (ibid., p. 29).

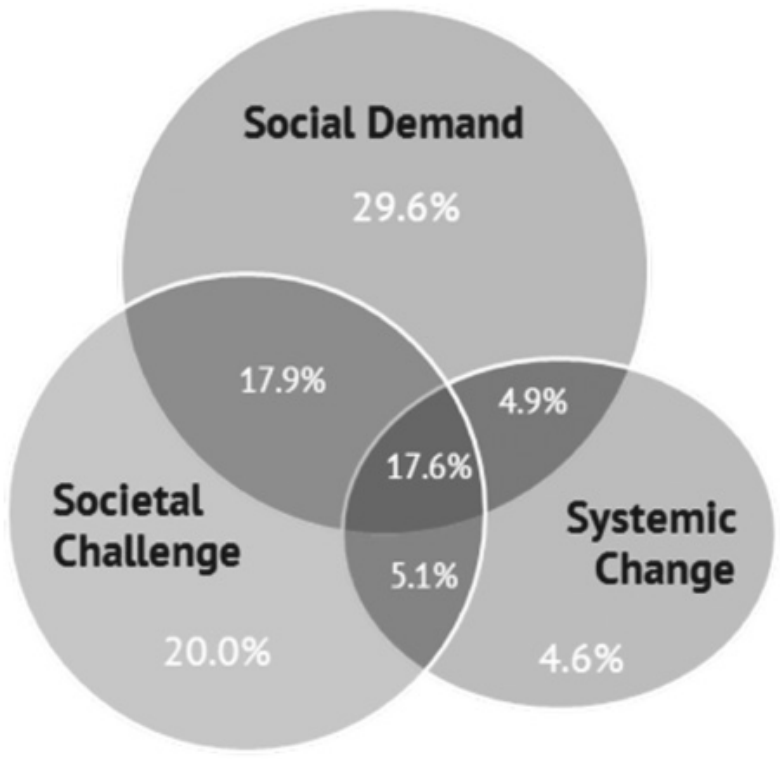

Figure 2. Societal level the initiative is addressing.

As figure 2 illustrates, most initiatives do not address one societal level alone, but rather different combinations. At the same time, the societal level addressed by the initiatives is varying in the different policy fields with a strong focus on social needs in most of the policy fields, except for "Transport and Mobility" and "Energy Supply", which both have a stronger orientation towards societal challenges. This result is also reflected in the feedback from policy workshops which highlights the dominant practice fields: cooperatives and wellconnected neighbourhood initiatives in the field of "Energy Supply" are mostly working on an agenda which goes beyond concrete and local social demands, and so do mobility clusters of inclusiveness/access dimension and greening mobility in the field of "Transport and Mobility". Global developments such as oil prices, environmental change and standard of living are considered a central driver in both policy fields.

At the same time, the global mapping revealed the diversity of the challenges modern societies are facing and the complexity of innovation processes. The mapping demonstrated that, like technological innovations, successful social innovations are based on numerous presuppositions and require appropriate infrastructures and resources. Moreover, social innovations require specific conditions because they aim at activating, fostering, and utilising the innovation potential of the whole society (BEPA 2010). Therefore, new ways of developing and diffusing social innovations are necessary (e.g. design thinking, innovation labs etc.) as well as additional far reaching resources, in order to unlock the potential of social innovation in society and to enable participation of the relevant actors and civil society.

This is not only a matter of appropriate funding but also of new participation and collaboration structures, co-creation and user involvement, empowerment and human resources development (see figure 3). Attention has to be paid to the invention and its development as well as its diffusion and imitation. From this innovation process and development perspective, resources, capabilities and constraints, drivers and barriers are not only relevant for the invention and implementation, but also for scaling and diffusion of successful innovations. 


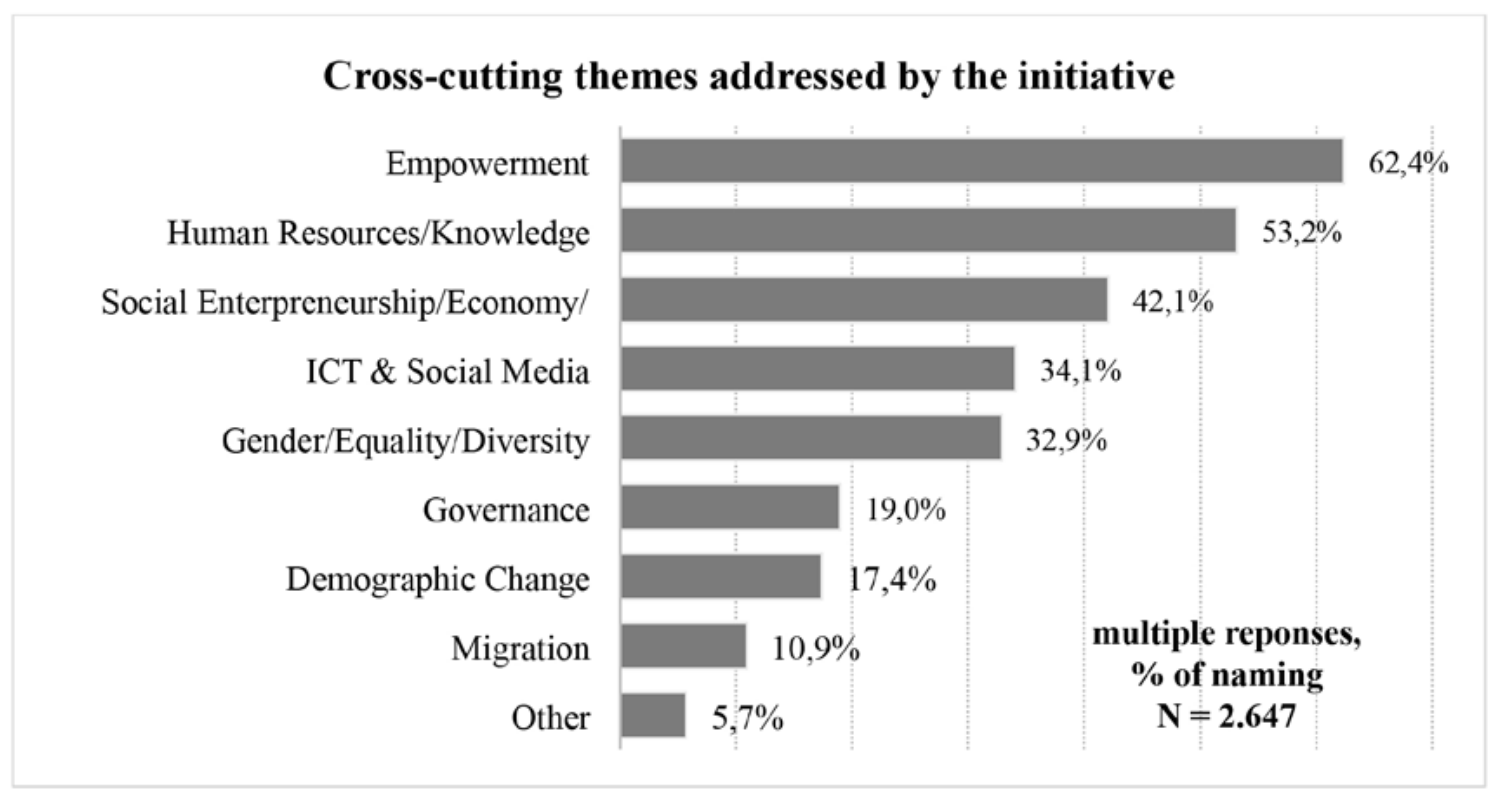

Figure 3. Cross-cutting themes addressed by the initiative.

To overcome societal challenges, cross-sector collaboration is crucial, actively involving public, economic and civil society partners - including active user or beneficiary involvement in almost half of the social innovation initiatives. This shows that most of the initiatives develop new alliances, guarantee cross-sector fertilisation and mobilise civil society (also proved by the high number of volunteers supporting the initiatives).

Such collaborations are picked up by at least two different heuristic models, the quadruple helix (Wallin 2010) on the one hand, where government, industry, academia and civil society work together to cocreate the future and drive specific structural changes, and the social innovation ecosystem (Sgaragli 2014) on the other hand (see figure 4), which also asks for interactions between the helix actors, adds the notion of systemic complexity and looks at both the serendipity and absorptive capacity of a system as a whole. Academic knowledge on social innovation ecosystems is very scarce and the concept is still fuzzy.

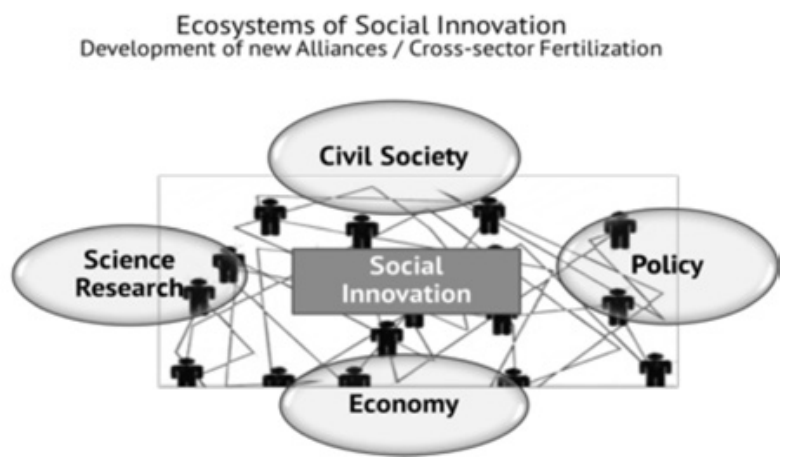

Figure 4. Social innovation ecosystem.

The results of the global mapping of the SI-DRIVE project demonstrated that social innovation processes and the underlying resources, capabilities and constraints are related to the actors of the different sectors of the social innovation ecosystem. This includes a new role of public policy and government for creating suitable framework and support structures, the integration of resources of the economy and civil society as well as supporting measures by science and universities (e.g. education for social innovation performance, know-how transfer).

While private companies, public bodies and Non-Governmental Organisations/Non-Profit-Organisations NGOs/NPOs are involved in the majority of initiatives, surprisingly, social enterprises are engaged only in minor parts of the initiatives. Additionally, academia is only a partner in some of the social innovation initiatives (see figure 5).

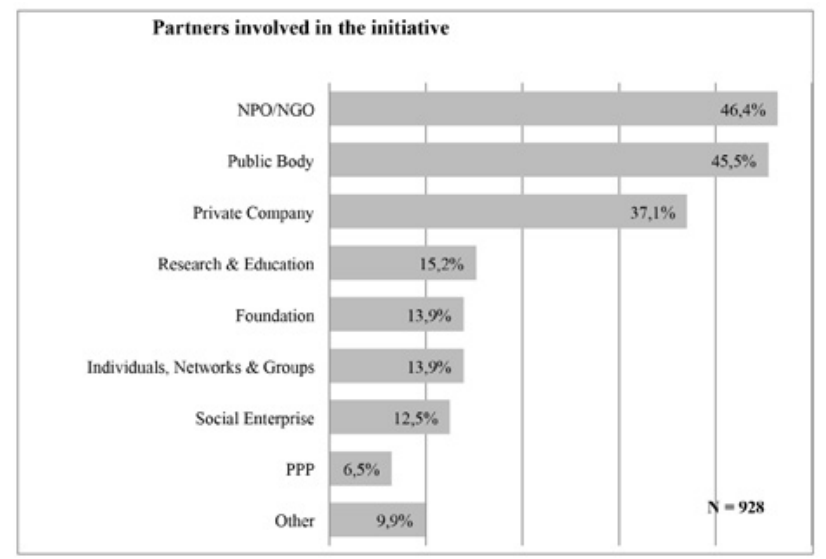

Figure 5. Partners involved in the initiative.

The marginal engagement of research and education facilities is in strong contrast to their essential role as knowledge providers in classical innovation processes (Mowery/Sampay 2005) and as one actor of the triple helix model. 
3. THE MISSING LINK THE ROLE OF "HIGHER EDUCATION INSTITUTES" (HEIS) IN SI-PROCESSES

This raises the question of the role of universities in general and of social sciences in particular in social innovation processes (see chapter 4). The marginal engagement of research and education facilities shown in the mapping, is in strong contrast to their essential role as knowledge providers in classical innovation processes and as one actor of the triple helix model. That means that at this time we find an uncompleted ecosystem of social innovation (quadruple helix) with one important pillar missing. It will be a major challenge for the development of social innovation to ensure a much higher involvement of research and education facilities.

The shift in focus towards social innovation means more than just taking new or other phenomena into account. To the extent that something new occurs at the level of social practices and not in the medium of technical artefact, a fundamental conceptual realignment in innovation research is necessary. It relates "to living together in communities and society" and concretely means "new forms of participation and social integration, of reconciling interest and social justice as well as individuality and solidarity" (Rammert 2010, p. 43).

Against that background the role that HEls are playing in social innovation has evolved in recent years. Besides researching transformation processes, more approaches in which science itself is considered as an active participant in processes of social innovation are increasingly coming to the fore. Concepts such as "Design Thinking" or "Transformative Research" with focus on active participation of stakeholders are becoming more important for the work of HEls with their environments (Schneidewind/Singer-Brodowski 2013). Through transformative research, science seeks to solve societal problems by activating processes of societal change. Against this background, the creation of appropriate structures ("Living Labs" and other spaces for exploration and learning) that help to develop knowledge based on experience in order to establish new social practices has received growing attention and needs to be further promoted. Only by sensitising people about societal problems and possible solutions, HEls can advance the development of social innovation with community members. Through concepts, such as "Service Learning" or "Explorative Learning", knowledge and experience of students are taken on and links between academia and society are developed, with the latter becoming an important partner in addition to economy. This also includes the question of new modes of knowledge production and scientific co-creation of knowledge aiming at an integration of practitioners and social innovators in the innovation processes (Nowotny et al. 2001).

Nevertheless, there are several challenges that HEls need to meet in order to advance in the area of social innovation. First, they need to understand better what social innovation is: while more and more HEls recognise the importance of social innovation for societal development and the need to engage in this area, they do not necessarily understand what social innovation is exactly about (e.g., it is often confused with the area of "University's Social Responsibility", which does not necessarily refer to (social) innovations). Hence, as long as those who work in this area and aim at introducing change have no clear concept and understanding of social innovation, it will be difficult to succeed.

This leads me to a second challenge. The topic of social innovation should be integrated along the three missions. On the one hand, social innovation is appearing on a growing number of universities' agendas, sometimes even becoming an important part of their development strategies. Some universities offer classes and degrees, such as Master or Bachelor. Others focus on research in social innovation. Probably the most common way for universities to engage in this topic that we can observe is related to manifold activities within what is usually referred to as the third mission (here mainly understood as social responsibility, outreach and engagement). On the other hand, we can rarely see a university where social innovation is major part of the strategy and integrated in all three missions (McKelvey/Zaring 2017). Therefore, the challenge is not only to develop activities in teaching, research and the third mission. It is the issue of integrating social innovation along the three missions in a comprehensive way: the work in every "mission" needs to be connected to the work in other missions, so that it can benefit from the others.

Third, there are two interrelated, fundamental characteristics of university support for social innovation that need to change: i) social innovation support activities tend to be ad hoc and largely altruistic, ii) as a result, while commercial innovation is recognised and institutionally supported by well-established knowledge transfer offices, there is no professional support function within universities for supporting social innovation. Until now, neither the infrastructure nor the funding has existed to make this possible, largely because governments and even university executives have been resistant to the notion of social innovation as an effective socioeconomic instrument. The adoption of social innovation at a policy level by governments throughout the world is creating an environment in which institutional support for this area becomes increasingly prevalent with funders willing to invest in projects.

Fourth, there is a challenge of integrating both the top-down and the bottom-up perspective. Usually, when universities assume their role as socially responsible institutions regarding their environment, they start developing initiatives, which are supposed to favour different target groups (e.g. communities). However, such initiatives tend to be designed and implemented from the university's perspective, missing to involve the target group right from the start. It is not surprising then that projects developed by HEls do not necessarily respond to the needs, the ideas and the visions of communities and other target groups. HEls have to learn how to work with target groups on equal footing and how to integrate their own perspective with the latter's perspective (Anderson et al. 2018)

\section{PARTICIPATORY APPROACHES IN SOCIAL INNOVATION RESEARCH}

While the future engagement of HEls in social innovation is crucial with regard to the impact of social innovation for societies, particular attention should be paid to the role of social sciences. It will be necessary to overcome the traditional "division of labour" in innovation processes between natural and engineering sciences, on the one hand, and social 
sciences and the humanities, on the other hand: "Natural and engineering sciences are different from social sciences and the arts primarily in that the former produce innovations or the prerequisites for innovations while the latter reflects on the emergence, the implementation and the success of innovation or also seek to explain the process" (Blättl-Mink 2006, p. 31). In the past, innovation research in the context of social sciences has helped to explain the social dimensions, the complexity and paradoxes of innovation processes.

Specifically in its analytical function, research in the social sciences contributed greatly to conceptually processing the social prerequisites for innovation and the social character of innovation processes. Its strengths rest in the analysis of innovation processes and their contextual circumstances. The findings picked up here have permeated social consciousness deeply, have determined the thinking and action of social actors and have contributed significantly to establishing a new sociologically enlightened innovation paradigm.

Shifting the perspective on innovation from technical to social innovation as an independent type of innovation, the present self-limitation of the social sciences to the concomitant research associated with a reference to the complexity and paradoxically loaded nature of innovation proves to be insufficient. For it is here that the subject matter of innovation itself rests immediately in the disciplinary perspective and the affiliated capacity for action and formation.

In the classical process of social science production, research takes place in research institutions society being an excursion for mining, an empirical source of data and information but not a partner, in the best case, also the address of transfer activities once research is concluded. But social science production can be seen as a social production of science. Social actors from the fields of social action relevant to the research theme or project participate in the whole process of research. Social scientists are social actors among others with the special task and role of driving the process towards the production of knowledge, knowledge achieving varying scopes of relevance: from "simple" problem solving with and for individual partners to general problem solutions in processes of societal transformation. Practitioners from civil society, companies and institutions - all these groups work together creating new knowledge. So the different forms of knowledge created have to be combined and tested to evolve into socially robust knowledge (Nowotny et al. 2001).

Purely analytical concepts fall short precisely in relation to the specific content of social innovations. After all, as mentioned previously, social innovations (in contrast to technological innovations) are a natural subject of the social sciences in terms of content, and as such social innovation can be not only analysed and indicated from a level of comprehension, but also be engendered and (co)shaped in terms of its (social and societal) preconditions, repercussions, etc.. Thus, it is hardly surprising that the role of the social sciences in examining and shaping social innovation is an important issue in the international scientific discussion on social innovation with a strong focus on participatory approaches that promote participation and empowerment of civil-society actors (Howaldt/ Schwarz 2010)'.

\subsection{THE CONCEPT OF WOLFGANG ZAPF}

Already Wolfgang Zapf connected the analysis of the meaning and specifics of social innovations with the question about the role and possibilities of the social sciences in researching social innovations (ZAPF 1989, p. 182 et seq.). Zapf emphasises that it is precisely the applicationoriented "tools for making decisions [delivered by the social sciences] forecasts, incremental planning, social experiments, evaluation, practices for mobilisation and motivation - (...) that [can] indeed enhance the ability of modern societies to solve problems and direct themselves" (ibid., p. 183). Zapf distinguishes potential contributions the social sciences can make to social innovation:

- decision-making support (survey research, personality tests, risk assessment and technology impact, human resources planning, etc.),

- sources of social technologies (quality management, co-determination model, group therapy),

- approaches to general theory in order to better understand innovation and productivity (1989.);

This sort of understanding of innovation processes requires developing appropriate forms of co-operation between science and practice that are not centrally focused on the transfer of expert knowledge into social practice. The aim of the conception of co-operation is to organise the process of change itself as a learning process that fosters the development and skills of every actor involved and enhances their ability to determine and reflect.

\subsection{SOCIAL INNOVATION IN LOCAL AND REGIONAL DEVELOPMENT}

One of the most prominent areas in which the concept of social innovation has increasingly become a research focus in the social sciences is local and regional development. It is the urban context in which challenges such as the effects of the economic crisis, demographic or climate change become directly visible as pressing social demands. And it is the cities where unlikely collaborations emerge to tackle problems when new competences are handed down from national or regional levels without corresponding budget allocations (Moulaert et al. 2013).

In Europe, a series of research projects delivered important findings on the role of the local level for social innovation; the latter mainly viewed under the perspective of the social economy. For example, the project "Integrated Area Development" (IAD) dealt with challenges faced by neighbourhoods and provided "an alternative to the more prevalent forms of market-led economic development" (Moulaert et al. 2013b, p. 19). Another important project in order to better understand the role of social innovation in community building was SINGOCOM (Social Innovation, Governance and Community Building). Findings from SINGOCOM also essentially contributed to the understanding of governance processes on the local level. For example, by focusing on the governance structures of neighbourhood management, it was possible to describe and analyse

1 Social innovation research can thereby build up on the long tradition of participatory approaches in social sciences (e.g. action research etc.) (Gustavsen 2012) 
how a direct link between the needs and demands of excluded groups and the resources to tackle them can be established (Moulaert et al. 2005). It showed that social innovations involve different dimensions such as the relation to culture, social connection and identity - going beyond material and economic issues (Moulaert et al. 2013b, p. 9).

The "International Handbook on Social Innovation", published by a group led by Frank Moulaert, presents a research perspective on social innovation that has been developed cooperatively over the last thirty years and which is intended to be a coherent methodological perspective that deals both conceptually and practically with structural, political and cultural forces that generate social exclusion. Furthermore, it has the potential for social change and socially innovative initiatives, and combines societal well-being with the shaping and organisation of society (Moulaert et al. 2013). The approach centres on a three-dimensional frame of reference that consists of the mutually associated defining characteristics of social innovation: satisfying needs in the sense of human development, reconfiguration of social relationships, and empowerment or political mobilisation. At the same time, the aim is to develop and demonstrate a specific type of social innovation research that seeks to find the right balance between "research on action", "action in research" and "research through and by action" (Moulaert et al. 2013a, p. 6), and that illustrates the extraordinary importance of social innovation as a field both of research and of action and social change (cf. ibid., p. 5).

According to the authors, social innovation is about a completely new ontology, which has to do with socialised change practices instead of organisational efficiency and an optimised use of knowledge. This notion of a different ontological perspective and an orientation towards a constitutive, performative role of social practices and their transformative potential is an interesting idea which would be worth further development.

In this sense, social innovation is an arena for a deliberating kind of decision-making with a transformative power, based on political negotiation at local/regional level by publics created by the political power of social movements. In this arena, social innovation researchers can be active actors: Social innovation research becomes an interactive process of research and action, starting from a collective discussion and decision by a transdisciplinary group regarding the problems of human development that should be addressed and which questions explored, what the composition of the team should be, and what the meta-theoretical frame of reference should look like.

\subsection{EMPOWERMENT AND DESIGN-THINKING}

The BEPA report supports this view when emphasising that social innovations have the function of mobilising citizens to take an active part in innovation processes and thereby enhance society's generic innovative capacity (BEPA 2010). Here, new models of governance in favour of self-organisation and political participation are required, allowing unexpected results through the involvement of stakeholders. ${ }^{2}$ If social innovation also has to do with innovation in social relations (Moulaert et al. 2013b), then it can be expected to become what former EC-President Barroso referred to as part of a new culture of empowerment (Franz et al. 2012). This notion of culture becomes important when the conditions for social innovations are not restricted to the level of actors, but understood as an ecosystem, a "complex environment in which social innovations are created, develop and flourish, on the one hand, and take effect or perish, on the other hand" (Eckhardt et al. 2017, p. 73).

Against this background different concepts of design thinking and related approaches have gained attention over the past years in a wide range of contexts beyond the communities of designers and design researchers including the discussion of social innovation. "The core idea is that the ways professional designers solve problems is useful in different contexts where individuals and groups in economy and society try to innovate and make change happen. This section reviews the core ideas of the concept of design thinking with regard to social innovation and social change" (Schaper Rinkel/Wagner-Luptacik 2014, p. 97).

In the Critical Literature Report of the SI-DRIVE project the role of design thinking in innovation processes summarised:

"Design thinking has become a dominant issue in contemporary design discourse and rhetoric, especially with the design thinking practice of the design and innovation firm IDEO, and with the application of its concept to design education at prestigious d.school, the Institute of Design at Stanford University (Bjogvinsson et al., 2012). The main characteristic of design thinking is its approach to think beyond the omnipotent designer and to overcome the obsession with artefacts, products, and things (Bjogvinsson et al., 2012). This is one of the interfaces between design thinking and social innovation approaches. Design thinking as part of design studies includes the complex social context of design to highlight the contradiction between uniqueness of design and designer as basis of business models in traditional design and the concept of transferable solutions as in social innovation concepts.

From this perspective, design thinking is closely connected with traditions such as "participatory design", "design for change" (Bjogvinsson et al., 2012, p. 101) and socially responsible design (Melles et al., 2011)". (Schaper Rinkel/Wagner-Luptacik 2014, p. 97)

As Deserti and others demonstrated, different approaches of design thinking have been developed to promote processes of social innovation by involving stakeholders in different contexts (Deserti et al. 2018, pp. 66 et seq.).

\subsection{TRANSITION RESEARCH AND DESIGN}

Social innovation research that addresses system transformation or embraces a transition perspective lays a strong focus on the reorganisation of society via participation, empowerment and social learning (cf.

2 Klein, Fontan, Harrisson, and Lévesque (2013) describe the development of the Québec Model as social innovation linked to social transformation. "From this standpoint, participative governance, co-production of services or activities, co-construction of public policies, as well as the plural character of the economy [...] represent important dimensions of social innovation" (Klein, Fontan, Harrisson, and Lévesque, 2013, p. 382). Thereby they identify the "economic turn" - "the fact that social movements have switched from merely demanding actions from other to proactive actions at the economic level" (Klein et al., 2013, p. 382) - as an important source for social innovation (Klein et al., 2013, p. 371). 
BEPA 2010; Elsen/Lorenz 2014). A whole series of more or less theoretically informed approaches that conceptually and programmatically focus on the shaping or shapeability of transformation processes in terms of sustainable and human development has been developed. Transformative social change here is not understood to be a largely uncontrolled outcome of gradual evolutionary developments (cf. Osterhammel 2011), but rather as something which can in principle be shaped by society, i.e. "by the actors and their innovations" (Schneidewind 2013, p. 123). Thus heterogeneous, more or less theoretically informed approaches (to shaping) change come to the fore, which elevate investigating and shaping the transformation process itself as well as the increasing importance of social innovations in this connection to the status of the actually relevant theme.

However, the "varied use of the term 'transformation'" (Aderhold et al. 2015, p. 135) - as can be seen in approaches such as transition management, transition design, transformation design, social design, and the Great Transformation - leads "to a conceptual uncertainty" (ibid., p. 135) rather than to a theoretically grounded, practicable model of transfor- mation (cf. Howaldt/Schwarz 2016, p.43 et seq.). Given the importance of social innovation in these discourses, as mentioned earlier, our view is that the lack of a well-developed and workable concept of social innovation that goes beyond a metaphorical description of certain phenomena and initiatives is one of the main reasons for this unsatisfactory situation ${ }^{3}$.

One transition approach which with a view to sustainable development directly aims at transforming social practices and at the same time explicitly aims to include and develop theories of change in order to better understand the dynamics of change in the social and natural world, is transition design (cf. Hopkins 2008). It aims to mobilise existing change potential in a collaborative process, and emphasises transdisciplinary and reintegration as well as the recontextualisation of knowledge. It is less about having a shaping influence on social phenomena, and more about a deeper understanding of specific environments ("ecosystems"), about the relations between its different parts, what the specific needs are, what works and what does not, and how things could develop in the future (see figure 6).

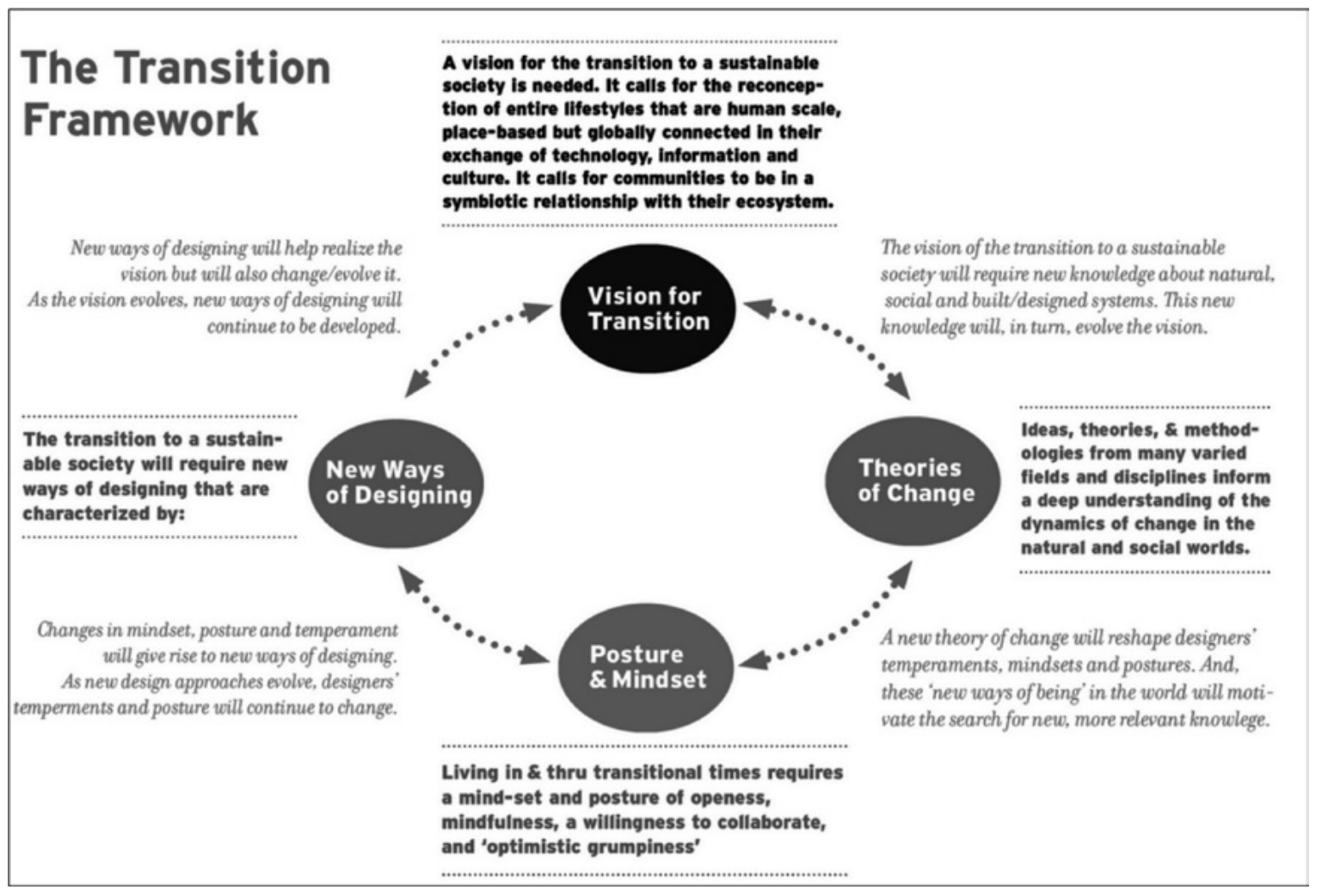

Figure 6. "The Transition Framework" (Irwin et al. 2015, p. 7).

The lack of a social-theory foundation for transformation discourse is also illustrated by the fact that, with regard to social transformation processes, recourse is often made to the multi-level perspective (MLP) (cf. Geels 2006; Geels/Schott 2007) that was developed in socio-technical innovation research, and the governance model of transition management that builds on it (cf. Loorbach 2007). In this perspective, system innovations in social functional areas such as transportation, the energy supply, food, housing, and communication are considered (cf. Geels 2005). These functional areas are characterised by specific socio-technical systems. System innovations emerge from interlinked developments on different levels. Different societal sectors, actors, practices, (learning) processes, routines, abilities, and rules play a role here, but this is always with regard to the question of their influence on the emergence, development and establishment of new technologies, and socio-technical systems or regimes that are shaped as a result.

Despite various criticisms, the approach seems to have lost little of its attractiveness as a theoretical model of the shaping of social transformation processes However, from the point of view of an understanding of social innovation that is grounded in practice theory, it does not offer any suitable basis for an appropriate understanding of social transformation processes. It systematically ignores the change dynamics of social practices and is therefore unable to capture the importance of social innovations in transformation processes (cf. Avelino et al. 2014). 
One prominent application of transition design is the transition town movement (http://www.transition-initiativen.de) initiated by Rob Hopkins, and the embedded transition research network (http://www.transitionresearchnetwork.org), which aims to bring together and promote transition initiatives and transition research. Around the world, some 500 transition initiatives are now registered, and have initiated diverse social innovations at local level (https://www.transitionnetwork.org).

The transition town movement can be interpreted as a concretisation of the post-growth economy and economy for the common good (cf. Pufe 2014, p. 276). Here it is not a question of theories, but of practice which itself "is the intellectual equipment for the process of transforming society as a whole, for an economy and a society that is on its way into and through the 21st century" (ibid., p. 291).

In the German sustainability discussion, the concept of "transformation design" has gained importance in recent years. Transformation design begins with small transformation examples that affect only a limited number of people as exercises in path-changing and inspiration for similar path changes, and is here understood as shaping a necessary process of transformation of the capitalist growth economy, i.e. a change process includes changing social structures together with the corresponding power and control structures (Sommer/Welzer 2014).

\section{CONCLUSION - RETHINKING SOCIAL SCIENCE}

In the increasing discussion on social innovation new participatory concepts for social science research have been developed. While there are a lot of differences with regard to the field of action, their objectives and the addressed problems these approaches are based on the idea of developing research and innovation process with and for society.

In these briefly outlined approaches social sciences are challenged to redefine their functions with regard to innovation and societal transformation. This goes far beyond a better understanding of science or new concepts of transfer, but deeply affects the traditional academic ways of knowledge production. New modes of the production of social science and the social production of science will become necessary. "Mode 2" has been the label tagged to this newly emerging type of knowledge production by Nowotny et al. (2001) mostly referring to natural or engineerial sciences.

There is a large gap between the traditional understanding of social research and science and the new mode of generating socially robust knowledge under the framework conditions as we have outlined them. The new mode of knowledge production will definitely require a thorough review of the classical quality criteria of what is scientific along with the development of new concepts, methods, procedures and organisational structures. The discussion about such an innovative approach to the production of social science as a process of social production could be very valuable for understanding the specific contribution of the social sciences to processes of innovation and societal transformation 4 .

In the past, innovation research in the context of social sciences has contributed to explain the social dimensions, the complexity and paradoxes of innovation processes. Henceforth, much will depend on realigning the range of competencies of social science as well as social scientists by contributing actively to the development and integration of innovations as well as by developing social innovation. The great challenge for contemporary innovation research lies in analysing its potential in the search for new social practices that enable us to secure the future and allow people to live "a richer and more fulfilled human life" (Rorty 2008, p. 191).

Against that background participatory approaches that promote participation and empowerment of civil-society actors are indispensable. The requisite know-how is found not only in the sociology of technology, economic sociology, and organisational sociology (cf. Blättel-Mink 2006) but also in the debate about the importance of stakeholder involvement to increase the impact of the social sciences and humanities (Spaapen/ van Drooge 2011). This also includes the question of new modes of knowledge production and scientific co-creation of knowledge (Nowotny et al. 2001) aiming at an integration of practitioners and social innovators in the innovation processes (Soler Gallart 2017). There is a lot of evidence that social innovation research will become of growing importance not only with regard to social integration and equal opportunities but also with regard to preserving and expanding the innovative capacity of society as a whole.

\section{REFERENCES}

Aderhold, J., Mann, C., Rückert-John, J. and Schäfer, M. (2015). Experimentierraum Stadt: Good Governance für soziale Innovationen auf dem Weg zur Nachhaltigkeitstransformation. Umweltforschungsplan des Bundesministeriums für Umwelt, Naturschutz, Bau und Reaktorsicherheit, TEXTE 04/2015. Retrieved April 8, 2018 from: https://www. umweltbundesamt.de/sites/default/files/medien/378/publikationen/ texte_04_2015_experimentierraum_stadt_good_governance.pdf.

Anderson, M. M., Domanski D. and Howaldt, J. (2018). Social Innovation as a chance and a challenge for Higher Education Institutions. In J. Howaldt, C. Kaletka, A. Schröder and M. Zirngiebl (Ed.), Atlas of Social Innovation. New Practices for a Better Future (pp. 50-53). Dortmund: Sozialforschungsstelle, TU Dortmund University.

Avelino, F., Wittmayer, J., Haxeltine, A., Kemp, R., O'Riordan, T., Weaver, P., Loorbach, D. and Rotmans, J. (2014). Game-changers and Transformative Social Innovation. The Case of the Economic Crisis and the New Economy. TRANS/T working paper. Retrieved April 8, 2018 from: http://www.transitsocialinnovation.eu/content/original/TRANSIT\%20 outputs/91\%20Gamechangers_TSI_Avelino_etal_TRANSIT_workingpaper_2014.pdf.

BEPA (Bureau of European Policy Advisers) (Ed.) (2010). Empowering People, Driving Change. Social Innovation in the European Union. Luxembourg: Publications Office of the European Union. Retrieved April 8, 2018 from: http://www.transitsocialinnovation.eu/resource-hub/empoweringpeople-driving-change-social-innovation-in-the-european-union.

Bjogvinsson, E., Ehn, P. and Hillgren, P.-A. (2012). Design Things and 
Design Thinking: Contemporary Participatory Design Challenges. Design Issues, 28(3), 101-116.

Blättel-Mink, B. (2006). Kompendium der Innovationsforschung. Wiesbaden: VS Verlag für Sozialwissenschaften.

Brand, K.-W. (2010). Social Practices and Sustainable Consumption: Benefits and Limitations of a New Theoretical Approach. In M. Gross and H. Heinrichs (Ed.), Environmental Sociology: European Perspectives and Interdisciplinary Challenges (pp. 217-235). Dordrecht: Springer.

Deserti, A., Rizzo, F. and Cobanli, 0. (2018). From Social Design to Design for Social Innovation. In J. Howaldt, C. Kaletka, A. Schröder and M. Zirngiebl (Ed.), Atlas of Social Innovation. New Practices for a Better Future (pp. 65-68). Dortmund: Sozialforschungsstelle, TU Dortmund University.

Eckhardt, J., Kaletka, C. and Pelka, B. (2017). Inclusion through digital social innovations. Modelling an ecosystem of drivers and barriers. In M. Antona and C. Stephanidis (Ed.), Universal access in human - computer interaction, UAHCI 2017 Proceedings, Part / (pp. 67-84). Springer.

Elsen, S. and Lorenz, W. (2014). Social innovation, participation and the development of society: Soziale Innovation, Partizipation und die Entwicklung der Gesellschaft. Bolzano: University Press.

European Commission (2012). Responsible Research and Innovation. Europe's ability to respond to societal challenges. Retrieved August 10, 2018 from: https://ec.europa.eu/research/swafs/pdf/pub_rri/ KI0214595ENC.pdf.

Franz, H.-W., Hochgerner, J. and Howaldt, J. (2012). Challenge Social Innovation: Potentials for Business, Social Entrepreneurship, Welfare and Civil Society. Berlin, New York: Springer.

Geels, F.W. and Schot, J. (2007). Typology of sociotechnical transition pathways. Research Policy, 36 (3), pp. 399-417.

Geels, F.W. (2005). Process and patterns in transitions and system innovations: Refining the co-evolutionary multi-level perspective. Technological Forecasting and Social Change, 72(6), pp. 681-696.

Geels, F.W. (2006). Multi-level Perspective on System Innovation. In X. Olsthorn and A. J. Wieczorek (Ed.), Understanding Industrial Transformation (pp. 163-186). Dordrecht: Springer.

Giesecke, D. (2014). Postwachstum: Geschichten „zur Senkung der Arbeitsmoral". WSI Mitteilungen 7/2014, 551-554.

Godin, B. (2012). Social Innovation: Utopias of Innovation from c. 1830 to the Present. Project on the Intellectual History of Innovation. Working Paper No. 11. Montreal.

Groß, M., Hoffmann-Riem, H. and Krohn, W. (Ed.) (2005). Realexperimente. Ökologische Gestaltungsprozesse in der Wissensgesellschaft. Bielefeld: Transcript.

Gustavsen, B. (2012). Social Innovation and Action Research. In H.-W.
Franz, J. Hochgerner and J. Howaldt (Ed.), Challenge social innovation: Potentials for business, social entrepreneurship, welfare and civil society (pp. 353-366). Berlin, New York: Springer.

Hargreaves, T., Haxeltine, A., Longhurst, N. and Seyfang, G. (2013). Up, Down, Round and Round: Connecting Regimes and Practices in Innovation for Sustainability. Environment and Planning, 45 (2), 402-420.

Hochgerner, J. (2013). Social Innovation and the Advancement of the General Concept of Innovation. In C. Ruiz Viñals and C. Parra Rodríguez (Ed.), Social Innovation. New Forms of Organisation in Knowledge-based Societies (pp. 12-28). Oxon: Routledge.

Hopkins, R. (2008). The Transition Handbook: from oil dependency to local resilience. White River, VT: Chelsea Green.

Howaldt, J. and Schwarz, M. (2010). Social Innovation: Concepts, Research Fields and International Trends. IMO International Monitoring. Dortmund: Sozialforschungsstelle, TU Dortmund University. Retrieved June 12, 2018 from: http://www.sfs.tu-dortmund.de/odb/Repository/ Publication/Doc/1289/IM0_Trendstudie_Howaldt_Schwarz_englische_ Version.pdf.

Howaldt, J. and Schwarz, M. (2016). SI-DRIVE Report: Social Innovation and its Relationship to Social Change. Dortmund: Sozialforschungsstelle, TU Dortmund University. Retrieved October 24, 2018 from https://www. si-drive.eu/wp-content/uploads/2016/07/SI-DRIVE-D1-3-Social-Changefinal-260416-2.pdf.

Howaldt, J., Butzin, A., Domanski, D. and Kaletka, C. (2014). Theoretical Approaches to Social Innovation: A Critical Literature Review. Dortmund: Sozialforschungsstelle, TU Dortmund University. Retrieved March 29, 2018: http://www.si-drive.eu/wp-content/uploads/2014/11/ D1_1-Critical-Literature-Review_final.pdf.

Howaldt, J., Kaletka, C. and Schröder, A. (2018). Social Innovation on the Rise. Results of the First Global Mapping. In J. Howaldt, C. Kaletka, A. Schröder and M. Zirngiebl (Ed.), Atlas of Social Innovation. New Practices for a Better Future (pp. 12-15). Dortmund: Sozialforschungsstelle, TU Dortmund University.

Howaldt, J., Schröder, A., Kaletka, C., Rehfeld, D. and Terstriep, J. (2016). Comparative Analysis (Mapping 1) - Mapping the World of Social Innovation: A Global Comparative Analysis across Sectors and World Regions (D1.4). Dortmund: TU Dortmund University. Retrieved May 18, 2018 from: https://www.si-drive.eu/wp-content/uploads/2016/07/SIDRIVE-D1-4-Comparative-Analysis-2016-08-15-final.pdf.

Irwin, T., Kossoff, G., Tonkinwise, C. and Scupelli, P. (2015). Transition Design 2015. A new area of design research, practice and study that proposes design-led societal transition toward more sustainable futures. Pittsburgh. Retrieved 0ctober 24, 2018 from: http://design.cmu.edu/sites/default/files/Transition_Design_Monograph_final.pdf.

Klein, J. L., Fontan, J. M., Harrisson, D. and Lévesque, B. (2013). The Quebec Model: a social innovation system founded on cooperation and consensus building. In F. Moulaert, D. MacCallum, A. Mehmood, and A. Hamdouch 
(Ed.), The International Handbook on Social Innovation: Collective Action, Social Learning and Transdisciplinary Research (pp. 371-383). Cheltenham: Elgar.

König, T., Nowotny, H. and Schuch, K. (2018). Impact re-loaded. Pathways to impact from SSH research. Retrieved October 24, 2018 from: https://www.ssh-impact.eu/impact-re-loaded/.

Loorbach, D. and Rotmans, J. (2010). The Practice of Transition Management: Examples and Lessons from Four Distinct Cases. Futures, 42 (3), pp. 237-246.

Loorbach, D. (2007): Transition Management. New mode of governance for sustainable development. Utrecht: International Books.

McGowan, K. and Westley, F. (2015). At the Root of Change: The History of Social Innovation. In A. Nicholls, J. Simon and M. Gabriel (Ed.), New Frontiers in Social Innovation Research (pp. 52-68). London: Palgrave Macmillan.

McKelvey, M. and Zaring, 0. (2017). Co-delivery of social innovations: exploring the university's role in academic engagement with society. Industry and Innovation, 25, 6, pp. 594-611. Abingdon: Routledge.

Melles, G., de Vere, I. and Misic, V. (2011). Socially responsible design: Thinking beyond the triple bottom line to socially responsive and sustainable product design. Codesign-International Journal of Cocreation in Design and the Arts, 7(3-4), 143-154.

Moulaert, F., MacCallum, D., Mehmood, A. and Hamdouch, A. (2013). The International Handbook on Social Innovation. Collective Action, Social Learning and Transdisciplinary Research. Cheltenham, Northhampton: Edward Elgar.

Moulaert, F., MacCallum, D., Mehmood, A. and Hamdouch, A. (2013a). General introduction: the return of social innovation as a scientific concept and a social practice. In F. Moulaert, D. MacCallum, A. Mehmood and A. Hamdouch (Ed.), The International Handbook on Social Innovation. Collective Action, Social Learning and Transdisciplinary Research (pp. 1-6). Cheltenham, Northhampton: Edward Elgar.

Moulaert, F., MacCallum, D. and Hillier, J. (2013b). Social innovation: intuition, precept, concept, theory and practice. In F. Moulaert, D. MacCallum, A. Mehmood and A. Hamdouch (Ed.), The International Handbook on Social Innovation. Collective Action, Social Learnig and Transdisciplinary Research (pp. 13-24). Cheltenham, Northhampton: Edward Elgar.

Moulaert, F., Martinelli, F., Swyngedouw, E. and González, S. (2005). Towards Alternative Model(s) of Local Innovation. Urban Studies, 42(11), 1969-1990.

Mowery, D. C. and Sampat, B. N. (2005). Universities in National Innovation Systems. In J. Fagerberg, D. C. Mowery and R. R. Nelson (Ed.), The Oxford Handbook of Innovation (pp. 209-239). Oxford: University Press.

Nicholls, A., Simon, J. and Gabriel, M. (Ed.) (2015). New Frontiers in Social Innovation Research. London: Palgrave Macmillan.

Nowotny, H., Scott, P. and Gibbons, M. (2001). Re-thinking Science.
Knowledge and the Public in an Age of Uncertainty. Cambridge: Polity Press.

Osterhammel, J. (2011). Die Verwandlung der Welt. Eine Geschichte des 19. Jahrhunderts. München: C. H. Beck.

Pufe, I. (2014). Nachhaltigkeit. 2. Ed. Konstanz: UVK Verlagsgesellschaft $\mathrm{mbH}$.

Rammert, W. (2010). Die Innovationen der Gesellschaft. In J. Howaldt and H. Jacobsen (Ed.), Soziale Innovation. Auf dem Weg zu einem postindustriellen Innovationsparadigma (pp. 21-52). Wiesbaden: VS Verlag für Sozialwissenschaften.

Reckwitz, A. (2003). Grundelemente einer Theorie sozialer Praktiken: Eine sozialtheoretische Perspektive. Zeitschrift für Soziologie, 32 (4), 282-300.

Rorty, R. (2008). Philosophie als Kulturpolitik [Philosophy as Cultural Politics]. Frankfurt am Main: Suhrkamp.

Schaper-Rinkel, P. and Wagner-Luptacik, P. (2014). Design Thinking. In J. Howaldt, A. Butzin, D. Domanski and C. Kaletka (Ed.), Theoretical approaches to social innovation: A critical literature review (pp. 97-103). Dortmund: Sozialforschungsstelle, TU Dortmund University. Retrieved March 29, 2018 from: http://www.si-drive.eu/wp-content/uploads/2014/11/ D1_1-Critical-Literature-Review_final.pdf.

Schneidewind, U. and Singer-Brodowski, M. (2013). Transformative Wissenschaft. Klimawandel im deutschen Wissenschafts- und Hochschulsystem. Metropolis: Marburg.

Schneidewind, U. (2013). Wandel verstehen - Auf dem Weg zu einer „Transformative Literacy“. In H. Welzer and K. Wiegandt (Ed.), Wege aus der Wachstumsgesellschaft (pp. 115-140). Frankfurt am Main: Fischer Taschenbuch.

Sgaragli, F. (2014). Enabling social innovation ecosystems for communityled territorial development. Rom: Fondazione Giacomo Brodolini.

Shove, E. (2003). Comfort, Cleanliness and Convenience: The Social Organization of Normality. Oxford: Berg.

Shove, E., Pantzar, M. and Watson, M. (2012). The Dynamics of Social Practice: Everyday Life and How it Changes. Los Angeles: Sage.

Soler Gallart, M. (2017). Achieving Social Impact. Sociology in the Public Sphere. Cham: Springer.

Sommer, B. and Welzer, H. (2014). Transformationsdesign. Wege in eine zukunftsfähige Moderne. München: oekom verlag.

Spaapen, J. and van Drooge, L. (2011). Introducing 'Productive Interactions' in Social Impact Assessment. Research Evaluation, 20 (3), 211-218.

Tarde, G. (2009). Die sozialen Gesetze. Skizze einer Soziologie (1898). Marburg: Metropolis Verlag.

Von Schomberg, R. (2011). Prospects for Technology Assessment in a 
framework of responsible research and innovation. In M. Dusseldorp and R. Beevroft (Ed.), Technikfolgen abschätzen lehren: Bildungspotenziale transdisziplinärer Methoden. Wiesbaden: VS Verlag.

Wallin, S. (2010). The co-evolvement in local development - From the triple to the quadruple helix model. Paper presented at Triple Helix VIII, Madrid. Retrieved October 24, 2018 from: http://www.leydesdorff.net/th8/ TRIPLE\%2OHELIX\%20-\%20VIII\%20CONFERENCE/PROCEEDINGS/0110_ Wallin_Sirkku_0-104/triple\%20helix\%20Wallin\%20final.pdf.

Zapf, W. (1989). Über soziale Innovation. Soziale Welt, 40 (1-2), 170-183.

\section{AUTHOR}

\section{JÜRGEN HOWALDT}

Sozialforschungsstelle, TU Dortmund University, Evinger Platz 17, Dortmund, 44339 (Germany)

E: Howaldt@sfs-dortmund.de

\section{KEYWORDS:}

Social Innovation, Societal Impact, Societal Transformation, Empowerment, Participatory Approaches, Civil Society 


\title{
THE IMPORTANCE OF SSH RESEARCH IN HORIZON EUROPE'
}

\author{
JOHN STEPHEN BELL \\ DOI: $10.22163 / f t e v a l .2019 .366$
}

\section{INTRODUCTION}

$\mathrm{T}$

his paper is a revision of the proposals for the regulation and specific programme of the forthcoming European Framework Programme for Research and Innovation presented by the European Commission (EC) on 7 June 2018. It presents ideas on how Social Sciences and Humanities (SSH) research could be better integrated and puts forward suggestions for collaborative research and innovation as a main line of engendering change and securing competitiveness. It is crucial for the future of the "European Research Area" to recognise the value and importance of the SSH, including through continued annual SSH Monitoring Reports that have up to now illustrated the lack of progress that the EC has made in integrating SSH in Horizon 2020.

By engaging with the concepts of innovation and impact, the paper promotes an understanding of innovation as a factor to transform society and calls for a conceptualisation of impact that is taking wider social, cultural and political developments into account. Last but not least, this is followed by some practical suggestions for potential missions and ways of implementation.

\section{THE CONCEPTS OF "INNOVATION" AND "IMPACT"}

Horizon Europe brings together the European Union's (EU) research and innovation activities largely under one Framework Programme. But there is always a danger that the emphasis on the contribution of research to economic growth fosters a technocratic paradigm in which the translation of fundamental research into innovative 'products' is seen as the benchmark of success. In the past, the EC has understood the relationship between research and innovation too much in terms of an overly simplistic, linear process in which research is expected to lead to ever higher Technology Readiness Levels (TRLS). The dominance of this paradigm belittles the contribution of Humanities and the Social Sciences. Humanities and Social Sciences have different perspectives on problems, but they contribute to a rounded approach.

What is it that the Humanities contribute to innovation? They offer an ability to challenge present ways of approaching social problems by tes-

ting out alternatives in rigorous, but non-experimental forms. Philosophy offers the chance to think through hypothetical alternatives, whilst literature makes use of imagination to conceive alternative scenarios and to explore them. Historians and archaeologists use the analysis of the past as a form of laboratory of different worlds. Being able to think through "what if?" scenarios deepen one's understanding of the world.

These approaches also bring into consideration the non-material features of our human existence. The quality of life depends not on having new gadgets or new products, but on being able to live a life which has value that may make use of what technology has to offer in a valuable way. Vision, beauty, style, and enjoyment are integral to a valuable human life.

The social sciences offer yet different ways of challenging contemporary norms and traditions of doing things. Techniques such as modelling enable alternative scenarios to be built and tested in a rigorous way without the ability to repeat experiments as in laboratory science. Modelling often reduces complexity by focusing on key features of a situation and then varying them.

Quantification is typically an approach of economics, geography and sociology. Other approaches look at qualitative analysis, scaling up from samples. These social sciences enable us not just to gain information about what might change, but also identify and test our deep values against which to test the social contribution of technological advances.

In our view, any worthwhile science programme for the EU has to harness the potential of all branches of scientific endeavour and to encourage them to work together. This perspective agrees with the view of the Lamy Report: "Innovation is more than technology. EU innovation policy must be based on a definition of innovation that acknowledges and values all forms of new knowledge - technological, but also business model, financing, governance, regulatory and social - which help generate value for the economy and society and drive systemic transformation."

"Innovation" should be redefined and implemented more holistically and openly in order to achieve the aims the EU wishes to support. Innovation is not limited to business and economic opportunities, but it is also fundamentally about transforming the way we live and the things we do, socially and culturally as well as economically. The humanities and social sciences have a very strong contribution to make such transformations happen.

Similarly, "impact" should be conceived in terms of how it affects not only the economy and governmental policies, but also the way social in- 
teractions, culture and ways of thinking are affected. There are good examples of this being recognised in Societal Challenge 6 of Horizon 2020, but the approach is not reflected consistently across the other Challenges. A definition of impact that incorporated the contribution of research and innovation to the wellbeing of society would be a significant and important step in the right direction.

The ambition of European research needs to look at how the individual and collective lives of residents in the EU are improved and how Europe can contribute to the quality of life of other parts of the world. Research needs to look at not only individual situations, but also the features of structures within society - power, institutions, political participation, and new actors in civil society. It explores cohesion and diversity in the way we are living together. It needs to investigate appropriate foundations and ingredients of contemporary democracy to make our societies more sustainable, open and resilient; widen our knowledge on the social and cultural dynamics and effects of (democratic) governance structures as we take advantage of changes that science and technology bring and the new questions they raise. For instance, fields of inquiry and more concrete objectives could include the social application of historical studies.

What is an appropriate balance between individuality and solidarity for modern European societies? Social and cultural diversity are valuable features of life in Europe (as in many other parts of the world). This provides not only a context for research and policy, but it also provides a wonderful resource. For example, if we look at the arts and society, we might ask whether literary models can enhance social cohesion?

Literature and art offer us a laboratory of the future, drawing on our deep sense of identities in the present and related to our past. Developing strategies to foster social access to art history and to critique, and to increase participation in cultural and artistic endeavour have creative potential to contribute to the transformation of society as much as any technological innovation.

\section{THE DESIGN OF THE EUROPEAN RESEARCH AGENDA}

In order to tackle the global challenges of the decades to come and enable European citizens and societies maintain the pace of innovation and social transformation, Europe needs to harness the creative capacities of all its researchers and social actors. This can be only achieved in a joint endeavour, especially by intensified inter- and transdisciplinary cooperation. In order to understand the human dimension of social and technological transformation, it is crucial to look at different perspectives and use the potential of the humanities and social sciences in enabling innovation and reflection. Each branch of science has its own contribution to knowledge and Horizon Europe needs to draw on them all in a holistic way.

This has implications for the drafting of the "Global Challenges" and "Missions". How do we identify the problems which these instruments are designed to solve and the methods appropriate for tackling them? The challenges Europe and its citizens face today and in the decades to come are not merely economic, technological and political, they are also social, cultural, legal and ethical. Challenges such as rising inequalities, nationalism, radicalism and terrorism threaten inclusion, social cohesion and democratic governance all over Europe. Demographic change, migration and digitisation create constant change. These challenges call for a profound and inclusive dialogue between all actors in society.

Technological innovation is obviously necessary to improve many features of the way we live, e.g. in medical interventions for healthcare, in smart systems to improve the quality of life, in ways of reducing threats to the climate and in improved transport. But technologies need to be embedded in an understanding of how we human beings might use them, how lives might adapt to their presence, and whether this would improve the quality of our lives. Scientists understand this well - they are, after all, members of society with a humane interest in living and contributing to good lives. They are keen to involve different branches of knowledge in ensuring that their efforts really do transform the lives of people in society.

From the perspective of the humanities and social sciences communities, these challenges require concerted efforts within and outside Europe, cutting across borders, cultures, languages, disciplines, sectors and institutions. That is why not only the "ALLEA Working Group Horizon Europe" argues for more interdisciplinarity and a bigger and well-defined role of the SSH in design and evaluation of the research which is funded through Horizon Europe. Otherwise the societal challenge to build inclusive, innovative and reflective societies runs the danger of being marginalised by other, more tangible material and technological challenges.

\section{MISSIONS}

The ambition of "Missions" to achieve tangible results within a defined timeframe is laudable in many ways. Nevertheless, again, it is necessary to guarantee interdisciplinarity and a bigger and well-defined role of the SSH in design and evaluation of the missions. In a statement published in cooperation with other stakeholders ${ }^{3}$, the ALLEA Working Group Horizon Europe critically reflects on the type and scope of missions that would adequately respond to the societal challenges Europe faces in the years and decades to come.

The 2030 Agenda of the United Nations (UN) should serve as a framework of inspiration when targeting these challenges through the development of missions. Such "Missions" have a strong potential to bring together researchers from many disciplines as well as political, cultural, economic and social actors and civil society in a common endeavour of ensuring that Europe is at the forefront of research, innovation and smart implementation - and hence well equipped to answer urgent societal questions. Actions should be inter- or multidisciplinary and involve organisations in the cultural, economic or social sectors: Co-creation of research questions will allow the translation of societal needs into research and innovation and facilitate the translation of research results into smart applications and societal uptake.

If it is to achieve worthwhile results, mission-oriented research should thus

(1) be transformative in that it generates new knowledge and understanding,

(2) acknowledge that innovation is more than technology, 
(3) be broad enough and not too constrained on specific end-products through a premature identification of indicators of success or failure,

(4) be open to researchers to come up with projects of all sizes to produce innovative ideas (bottom-up approach),

(5) integrate all countries and regions in order to counter the research and innovation divide in the "European Research Area" and maintain openness towards collaboration with non-EU countries.

Many of these ideas are reflected in the Mazzucato report ${ }^{4}$ on which the "Draft Regulation" now draws. The Mazzucato report sought to find a way in which research and economic growth could be steered. As a result, its second criterion is that missions should be "targeted, measurable" and time-limited (now Article 7(3)(c) of the "Draft Regulation on Horizon Europe", p. 14), and its third criterion is that they should be ambitious, but realistic. That requires careful thought in terms of the design of mission calls. This is a top-down activity by EU institutions. Of course, the final criterion for missions is that they should be open to multiple, bottom-up proposals (now Article 7(3)(f)). That leaves an important scope for the initiative of individual researchers and innovators or groups of them. But those bottom-up proposals will come within a framework. This feature draws out a major area for thought about Horizon Europe - not just the content and the budget, but the process by which it is implemented, particularly at the level of the EC.

\section{IMPLEMENTATION}

Call design: We consider that the drafting of "Work Programmes for Challenges and Missions" should draw on researchers in different disciplines and different methodologies as well as on experts from civil society and the culture and economics sector. The wording of calls should reflect the need to draw on the full range of research capacity within Europe. That has not always been the case in Horizon 2020 (especially outside "Societal Challenge 6" SC6) and this is reflected in the low level of participation by SSH disciplines within those other challenges.

The idea of involving humanities and social sciences in planning is to enable topics to be identified correctly in the first place. Take a current example: In the "Work Programme" of SC4 (Smart, Green and Integrated Transport) for 2018-2020, there is a call in relation to "Harnessing and understanding the impacts of changes in urban mobility on policy making by city-led innovation for sustainable urban mobility" (LC-MG-1-3-2018). The detail of the challenge states that "Urban mobility is in transition. This is a result of, for example, changing user needs; emerging transport technologies; new transport services using new business models; and new institutional and financing structures." (LC-MG-1-3-2018, p.20). Further it states that "Special attention should be paid to the needs of vulnerable groups and users with different cultural backgrounds taking into account gender issues; and to the specific context of areas that are undergoing rapid economic change." (LC-MG-1-3-2018, p.20). Both of these clearly call for a contribution by social scientists and humanities scholars in order to understand the social needs that transport technologies, business models and financing structures are required to serve.
However, compared with the emphasis on data-driven planning, new business models and technology, not much thought has gone into identifying the social phenomena which research in this area should be addressing. It should also be looking at why people are using vehicles, how changes in work patterns (e.g. mobile and home working) affect demands for and timing of vehicle use, and whether the location of schools, leisure and shopping venues make a difference. In a document which runs to over a hundred pages on all the calls, the thought given to the potential contribution of humanities is very limited, and the contribution of social scientists, such as social geographers, is badly under-developed. There are words which have potential, but in comparison with the detail on other matters, they give the impression of being an after-thought.

Horizon Europe should aim to designate broad fields of enquiry which leave substantial flexibility to accommodate the innovative, but unexpected proposal. This means that the drafts of calls should be far less detailed than the current calls for "Societal Challenges" within Horizon 2020.

Emerging priorities: Given the uncertainty about the future and the rapid development of technology, the fields of research identified for "Missions" should not cover the whole of the 2021-2027 period, but should initially be shorter, with the possibility of continuation where they prove fruitful.

Project design: Projects submitted should be broad enough to include, where appropriate, participatory actions (co-design) by non-researchers. For example, research on migrants or elderly people might involve those groups in shaping the design of projects and in selecting the materials to be included as part of the research. It is in these ways that "citizen-led science" is best understood. Such processes of co-creation of knowledge ensure better acceptance and implementation in society and the economy. In many research projects in the humanities, it is common to bring together individuals from communities that are being studied to help design and implement the research through writing, oral history or articulating features of their communities which shape the understandings that are necessary for effective research.

Evaluation: The evaluation of proposals should include representatives of a range of disciplines, including the humanities and social sciences. The diversity of social science and humanities subjects (like the diversity of biological sciences) requires a range of expert evaluators to be involved to reflect the diversity of disciplines (and the emerging new fields generated through inter- and transdisciplinary collaboration). "Ethics Reviews" need informed experts.

Project size: There should be greater flexibility in choosing the size of a project. Projects in the humanities and social sciences typically do not have the need for expensive equipment. There are sometimes good reasons for larger teams, but often close working by smaller teams is the most productive way forward. The permitted size of bids should be smaller than in Horizon 2020. It may be prudent to give smaller amounts of initial funding until the proof of concept stage is reached or potential social or conceptual impact is envisaged. This calls for follow-up funding for promising ideas.

Monitoring: Review criteria should recognise that research/science accept a diversity of good solutions and a complexity of contexts in which solutions achieve results. New indicators for societal and cultural impact need to be developed and used. Assessing the performance of missions 
cannot simply be in terms of success (man on the moon) or failure (no man on the moon). Scholars from the humanities and social sciences need to be integral to the monitoring if the potential for steering research and social development (not just economic growth) is to be realised.

\section{CONCLUSION}

The humanities and social sciences need to be deeply embedded in the research agenda of Horizon Europe. This is because the research endeavour needs them in order to achieve its transformational potential. Researchers in these fields do have different research methods and ambitions compared with engineers and scientists, but this offers the potential for mutual enrichment. Ultimately, we are serving a community of nations within the EU who have the ambition to work together to improve their own quality of individual and community lives as well as those of the wider world. If we fail to do this effectively through Horizon Europe, we let down ourselves as researchers and the people we serve.

\section{REFERENCES}

ALLEA (2017). Developing a Vision for Framework Programme 9. Retrieved October 18, 2018 from: https://www.allea.org/wp-content/uploads/2017/07/ALLEA_Statement_FP9.pdf.

ALLEA et al. (2017). Living Together: Missions for Shaping the Future. Retrieved October 18, 2018 from: https://www.allea.org/wp-content/ uploads/2017/12/Living_Together_Missions_for_Shaping_the_Future_2017.pdf

High Level Group on maximizing the impact of EU Research \& Innovation Programmes (2017). LAB - FAB - APP - Investing in the European future we want, Report of the independent High Level Group on maximising the impact of EU Research \& Innovation Programmes. Retrieved October 18, 2018 from: http://ec.europa.eu/research/evaluations/pdf/ archive/other_reports_studies_and_documents/hlg_2017_report.pdf

LC-MG-1-3-2018, (2018). Harnessing and understanding the impacts of changes in urban mobility on policy making by city-led innovation for sustainable urban mobility: http://ec.europa.eu/research/participants/ data/ref/h2020/wp/2018-2020/main/h2020-wp1820-transport_en.pdf

Mazzucato, M. (2018). Mission-Oriented Research and Innovation in the European Union. Retrieved October 18, 2018 from: https://ec.europa.eu/ info/sites/info/files/mazzucato_report_2018.pdf.

\section{JOHN STEPHEN BELL}

Chair of the ALLEA Working Group Horizon Europe, All European Academies (ALLEA)

Jägerstraße 22/23, Berlin, 10117 (Germany)

E: jsb48@cam.ac.uk

\section{AUTHOR}




\title{
INNOVATION FOR THE REFLECTIVE SOCIETY
}

\author{
RICCARDO POZZO \\ DOI: $10.22163 /$ fteval.2019.367
}

\section{ABSTRACT}

$6 \mathbf{6}$ Cultural innovation" sounds like an oxymoron. It is not, though. It is something real that tops up social and technological innovation. How can we measure "cultural innovation"? The answer is, as a result of co-creation. Items of cultural innovation are: content providers such as museums, science centres and libraries, as well as processes triggered by issues such as cosmopolitanism, inclusiveness, mobility, migration, heritage and creativity. Valuating the impact is fundamental to improve societal acceptance of public investment in culture, because these measurements may provide a basis for aligning research and innovation with the values, needs and expectations of society. In recent years, it has become clear that co-creation plays a central role within open innovation, because a "specific innovation can no longer be seen as the result of predefined and isolated innovation activities but rather as the outcome of a complex co-creation process involving knowledge flows across the entire economic and social environment" (Open Science, Open Innovation, Open to the World. EC 2016, p. 11). The paper offers migration-relate case studies for evaluating the impact of cultural innovation in societies that aim at being innovative, reflective and inclusive.

\section{INTRODUCTION}

"Social and Cultural Innovation" is a syntagma that is receiving increased usage among researchers after it was chosen by the "European Strategy Forum Research Infrastructures" (2016) for the name of the working group that deals with research infrastructures primarily connected with Social Sciences and the Humanities (SSH). Innovation refers to the creation of new products and services by bringing a new idea to the market. Economic growth turns on infrastructures, which provide access to services and knowledge, e.g. by overcoming the digital divide. Globalisation has made it clear that a most urgent objective is to work out policies of social and cultural innovation to the advantage of citizens - policies that aim at achieving changes in the regulatory environment that make societies both inclusive and reflective. Thinking ahead of Horizon Europe, there is some fear the notion of "cultural innovation" might sound like an oxymoron, no doubt. It is not, though. Cultural innovation is something real that tops up social and technological innovation by providing the reflective society with spaces of exchange in which citizens engage in the process of sharing their experiences while appropriating common goods content. We are talking of public spaces such as universities, academies, libraries, museums, science-centres, but also of any place in which co-creation activities may occur, e.g., research infrastructures such as "DARIAH-Digital Research Infrastructure for the Arts and the Humanities". At this level, social innovation becomes reflective and generates cultural innovation.

\section{REFLECTIVE SOCIETY}

The "reflective society" is also a syntagma that has found wide usage among researchers since a precise date, in this case the fall of 2013, when the European Commission introduced it for posting Social Sciences and Humanities related calls within the sixth societal challenge of Horizon 2020, the one about "Inclusive, innovative and reflective societies". The last adjective refers to the role of deliberative communication of citizens in a modern public sphere aiming at mutual understanding and goes back to Immanuel Kant (1790), G.W.F. Hegel (1812-13), Jürgen Habermas (1973), James S. Fishkin (1993), Ulrich Beck, Anthony Giddens and Scott Lash (1996) as well as Alessandro Ferrara (1998). A closer scrutiny reveals that Habermas has applied to society what Hegel had elaborated as the passage from the surface of being to the ground of essence, a passage that takes place, literally, by reflecting into the thing - like reflected light that illuminates something previously invisible, or creates a pattern not previously existing. Insisting on reflexivity helps to raise awareness for the importance of framing issues around engaging with science and society, identifying problems and defining solutions. The "Faro Framework Convention on the Value of Cultural Heritage for Society" of UNESCO (2007) encourages reflection on the role of citizens in the process of defining, creating, and managing a cultural environment in which communities evolve.

It is true, innovation is part of economics, because it is about money generating knowledge. There is, however, innovation in society and in culture. Social and cultural innovation is a fact. While according to the traditional - so-called Mode 1 - knowledge production, which is motivated by scientific knowledge alone (fundamental research) and is neither bothered by the applicability of its findings nor by bridging over to other disciplines, in contemporary research, multidisciplinary teams so-called Mode 2 - are brought together for short periods of time to work on specific problems in the real world for knowledge production. This mode can be explained by the way research funds are distributed among scientists and how scientists focus on obtaining these funds (Gibbons, Limoges, Nowotny, Schwartzmann, Scott and Trow 1994). Relatively recent models, such as triple helix and open innovation have stressed that the collaboration among different institutions is crucial for successful innovation. But only marginally these models have taken into account the actual and potential role that citizens in the reflective society have in shaping the innovation process (Leydesdorff and Etzkowitz 1998; Etzkowitz and Leydesdorff 2000; Carayannis and Campbell 2009; Chesbrough 2003). 


\section{CULTURAL INNOVATION}

What is social innovation we know about: it ought to be the backbone of all European research policies, as Marcelo Rebelo de Sousa, President of the Republic of Portugal, made it clear in the concluding remarks he gave at the Opening up to an Era of Social Innovation Conference in Lisbon on 28 0ctober 2017. We still know little about cultural innovation, though. The syntagma is mentioned at times just to say that culture too needs innovation and in fact produces innovation: museum studies foster innovation in museography; archaeology fosters innovation in data science that becomes data humanities; music and art foster innovation through social networks. There are even studies in the "philosophy of museums", whose items are questions such as "What types of value do museums have? What is the ethically correct stance for a museum to take towards its public? And towards the objects constituting its collection? Should museum exhibits seek to make a claim to objectivity?" (Harrison, Bergqvist and Kemp 2016). In sum, we can talk of "heritage-led innovation", which means that culture fosters technological innovation. Digitisation is in itself innovation. Data science has become data humanities. We still need a great deal of reflection on digitisation. However, we can look at it the other way around. Cultural innovation tops up social innovation, which on its turn relies on technological innovation. Innovation must come to term with social innovation, then, this is a European requirement.

The question is what part of social innovation is cultural innovation and what rights can cultural innovation claim with respect to society (Koefoed 2017)? A preliminary answer is: European cultural heritage marks our cultural identity, which is at the same time cultural diversity. The "European Year of Cultural Heritage" is about identity and diversity, said Jean-Claude Juncker in his opening speech at the European 2017 Culture Forum in Milan on 7 December 2017. But to assess cultural innovation as the value-sensitive integration to technological and social innovation is the great challenge contemporary "Science and Technology Studies" are confronted with and we need to look at it more closely. The new "missions" of the next "European Framework Programme for Research and Innovation" of the multi-annual financial period 2021-2027 will foster research on systemic change in the new generations and contribute to the creation of a cross-border and multi-disciplinary open innovation environments for research data, knowledge and services with engaged stakeholders and organisations. The current migrant crisis has made it clear with extraordinary force that a most urgent objective is to work towards Euro-Mediterranean societies that are inclusive, reflective, and attentive to the impact that migration is having on social and cultural innovation, security and health, environment and biodiversity.

The biggest challenge of this century, which is migration, asks for a new narrative of inclusion and reflection. Kantian philosophy, e.g., has the best chances to provide it. What Kant has written on the right of visit (das Recht eines Fremdlings), on hospitality (hospes) and sovereignty (hostis) is the key to shaping the narrative. The commentary to the third definite article of Perpetual Peace makes it clear that "originally no one has more right [Recht] than another to live on a particular place [Ort] on the earth" (Kant 1795, p. 41). Looking at late eighteenth-century colonialism, Kant envisaged a form of ius cosmopoliticum (Weltbürgerrecht), whose consequence is universal hospitality (allgemeine Hospitalität), which is to be acknowledged as the right of the foreigner (das Recht eines Fremdlings), although hospitality does not entail the right of the foreigner to rob, exploit, and enslave (Kant 1797). In sum, philosophy, in its historical dimension, is able to grant a shared narrative of what has happened, what is happening and what will happen with migration in our globalised world. On the basis of a cross-disciplinary approach, philosophers is to be trusted to achieve what Hanna Arendt (1963) did achieve for the Holocaust and Jürgen Habermas (1991) for citizenship, by pushing forward the recent proposal of Donatella Di Cesare (2017) for a philosophical narrative of migration.

It is now time to examine the role of reflection for rethinking the ways in which culture has been envisioned, particularly to visualise the various ways in which users engage with cultural processes in the past, present, and future. Let me propose a case study. Imagine a second-generation diaspora child (huaqiao 华桥) who attends a human sciences high school in Italy. At a certain point, s/he might be asked to read a text by Plato, possibly the Apology of Socrates (Apologia Sokratous Aпо入оyía $\Sigma$ ¿шко́точ५), first in Italian, then perhaps in the Greek original or in the Renaissance Latin rendering of Marsilius Ficinus. Students today delve easily into multilayered, multilingual hypertexts, and they do so on the basis of the reciprocal guidance made possible by social reading tools. Our student ought to read the same text in modern unified Chinese as well, so that s/he might be able to start a discussion on Socrates in its Chinese-speaking family. Inversely, schoolmates might appropriate, say, the Analects (Lunyu 伦语) of Confucius through the conceptual references indicated by our student. Together they may start thinking on movement (dong 动), rest (jing 静), human being (renji 人际), humaneness (ren 仁), and eventually come to grasp key tenets of Neo-Confucianism, such as the dictum that represents the unity of heaven and human or supernal heaven and humanity (tianrenheyi 天人合一), which amounts to "restoring the Heavenly Principle and diminishing human desires" (Wang 2005, p-320; Ni Peimin 2017).

\section{CONCLUSION}

Rémi Brague (2004) has noted that the Arabic term for dictionary, سوماق (qāmūs), is a translation of the name of the Titan of Greek

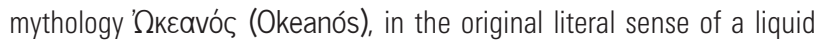
extension that embraces all emerged lands, permitting navigation and hence communication. Leibniz has used the ocean metaphor for an encyclopaedia, which is the very same idea concerning languages that this paper tries to defend. We expect SSH research to trigger a change in the mind-set as regards locating culture for inclusion and reflection in education, life-long learning, healthcare, urban development and regeneration. Culture cannot be but plural, changing, adaptable, constructed. Inclusion and reflection are constructed whenever we are in contact with other human beings, regardless where they come from. This we have to learn.

\section{REFERENCES}

Arendt, H. (1963). 'Eichmann in Jerusalem: A report on the banality of evil'. The New Yorker, 17 May and following

Beck, U., Giddens A. and Lash S. (1996). Reflexive Modernisierung. Frankfurt: Suhrkamp

Brague, R. (2004). 'Langues et traditions constitutives de la philosophie en Europe'. In: Cassin, B. (ed.) Vocabulaire européen des philosophies: Dictionnaire des intraduisibles, edited by, pp. 694-99, Paris: Seuil 
Carayannis, E. and Campbell, D. (2009). "'Mode 3" and "Ouadruple Helix": toward a 21 st century fractal innovation ecosystem', International Journal of Technology Management, 46, 201-34.

Chesbrough, H. W. (2003). Open innovation: The new imperative for creating and profiting from technology. Harvard University Press

DG-RqI (2016). Open science, open innovation, open to the world: A vision for Europe. Brussels: EC

Etzkowitz, E. and Leydesdorff, L. (2000). 'The dynamics of innovation: From national system and "Mode 2" to a triple helix of university-industry-government relations', Research Policy, 29, 109-23

Ferrara, A. (1998). Reflective authenticity. London: Routledge

Fishkin, J. S. (1993). The dialogue of justice: Towards a self-reflective society. Yale University Press

Gibbons, M., Limoges, C., Nowotny, H., Schwartzmann, S., Scott, P. and Trow, M. (1994). The New production of knowledge: The dynamics of science and research in contemporary societies. Thousand Oaks: Sage

Habermas, J. (1973). 'Wahrheitstheorien'. In: Fahrenbach H. (ed.) Wirklichkeit und Reflexion: Walter Schulz zum 60. Geburtstag, pp. 211-265. Pfüllingen: Neske

Habermas, J. (1991). Staatsbürgerschaft und nationale Identität. Frankfurt: Erker

Harrison, V. S., Bergqvist, A. and Kemp, G. (eds. 2016). Philosophy and museums: Essays on the philosophy of museums. Cambridge University Press

Hegel, G.W.F. (1812-13). Wissenschaft der Logik. Nürnberg: Schrag

Kant, I. (1790). Kritik der Urteilskraft. Königsberg: Nicolovius

Kant, I. (1795). Zum ewigen Frieden. Königsberg: Nicolovius

Kant, I. (1797). Metaphysik der Sitten. Königsberg: Nicolovius

Koefoed, 0. (2017). 'Cultural heritage and social innovation: A memory of the future?', Cartaditalia, 1: 417-44

Leydesdorff L. and Etzkowitz, E. (1998). 'The triple helix as a model for innovation studies'. In Science and Public Policy, 25, 3, 195-203 $\mathrm{NI}, \mathrm{P}$. (2018). Understanding the Analects of Confucius: A new translation of Lunyu with annotations, Albany, N.Y.: SUNY PreSS

Pozzo, R. and Virgili, V. (2016). 'Governing cultural diversity: Common goods, shared experiences, Spaces for Exchange', Economia della cultura, 26: 41-47, doi.org/10.1446/84035

Pozzo, R. and Virgili, V. (2017). 'Social and cultural innovation: Research infrastructures tackling migration', Diogenes: International Journal of Human Sciences, 64, doi.org/10.1177/0392192117739822
Wang, R. R. (2005). 'Zhou Dunyi's diagram of the supreme ultimate explained (Taijitu shuo): A construction of the Confucian metaphysics', Journal of the History of Ideas 66 (2005) \#3, pp. 307-323, doi.org/10.1353/ jhi.2005.0047

\section{AUTHOR}

\section{RICCARDO POZZO}

Department of Human Sciences, University of Verona Via San Francesco 22, Verona, 37129 (Italy)

E: riccardo.pozzo@univr.it 


\title{
STRIVING FOR REFLEXIVE SCIENCE
}

\author{
ROGER STRAND \\ DOI: 10.22163/fteval.2019.368
}

\section{ABSTRACT}

$\mathrm{T}$ The value of reflexivity has repeatedly been mobilised in claims for Social Sciences and Humanities (SSH) involvement in Science, Technology, Engineering and Mathematics (STEM) research. In short (if not in caricature), the policy narrative goes like this: Scientists, scientific practices, the governance of science and indeed modern society should become more reflective/reflexive. This can be achieved by involving $\mathrm{SSH}$, which are inherently reflexive.

In this paper, I will follow this narrative from the "Ethical, Legal and Social Implications" (ELSI)/ "Ethical, Legal and Social Aspects of bio-, neuro-, and nanotechnology (ELSA) policies and practices of the 1990s and 2000s, to the concept of "Responsible Research and Innovation" (RRI) and the "need to integrate" SSH with STEM to address societal challenges in Horizon 2020. Drawing upon my experience as an SSH practitioner in ELSA, RRI and societal challenges-focused interdisciplinary collaborations, I shall propose two lessons learnt. One key lesson is the need to go beyond the simple policy narrative "SSH makes science more reflexive" and the many disappointments that it invariably produces. The other key lesson is the need to go beyond simple dichotomies between SSH research and scholarship on one hand and non-SSH research on the other in order to look for meaningful collaborations.

\section{INTRODUCTION: THE REFLEXIVITY POLICY NARRATIVE}

This paper discusses the role and value of the social sciences and the humanities (hereafter abbreviated as SSH) in research endeavours primarily driven by the natural "Science, Technology/Technoscience, Engineering and Mathematics" (hereafter abbreviated as STEM). To the concept of STEM we may also include the main part of medical science, which in its methods and orientation is quite similar to natural sciences. In the abstract of this paper, I claimed the existence of a policy narrative that can be summarised as follows: STEM scientists, scientific practices, the governance of science and indeed the modern, knowledge-based society should become more reflective/reflexive. This can be achieved by involving SSH, which is inherently reflexive.

The narrative is never expressed exactly as such, or with such bluntness, in the European Union Ryl policy documents, which have to balance the argument for SSH with the appropriate tokens of respect for STEM. In a small country such as my own (Norway), however, one can find more direct expressions. The following quote states the mission of the (second) "ELSA funding programme" of the Research Council of Norway (2008-2014; ELSA = Ethical, Legal and Social Aspects of bio-, neuroand nanotechnology):
"An important aim of ELSA is creating societally robust bio-, nano- and neurotechnologies. The programme will work to explore central challenges for governance, risk, regulation, culture, and values connected to these technologies. The programme should contribute to increase reflexivity and promote learning among ELSA researchers as well as scientists."

(Forskningsradet, s. a.)

Likewise, a frequently stated goal for responsible innovation/responsible research and innovation (RRI), is that RqI processes become reflexive/reflective - such as in the " $\mathrm{R}$ " of the British "AREA" framework: "Anticipate, Reflect, Engage and Act"i. While there is no consensus at all on the overall goal and purpose of RRI in the EU - for some, the goal is reflexive practice and governance of science and technology; for others it is "better alignment" between civil society and the Ryl sector - it is interesting to reflect upon the origin of the RRI concept. Except for sporadic and quite unrelated mentions, the term was introduced by philosopher and European Commission (EC) Directorate-General (DG) Research and Innovation (RTD) policy officer René von Schomberg in 2011. Interestingly, he did so with explicit reference to the potential of technology to have negative ethical and social implications:

"[...] we are confronted with the Collingridge dilemma, implying that ethical issues could be easily addressed early on during technology design and development whereas in this initial stage the development of the technology is difficult to predict. Once the social and ethical consequences become clearer, the development of technology is often far advanced and its trajectory is difficult to change."

(von Schomberg 2011, p. 8)

In this regard the so-called Collingridge dilemma is taken to stand for the following: Technologies (created by research and innovation) have negative side-effects (such as risks and hazards), but by the time the side-effects are identified and understood, the technologies have become entrenched in society and infrastructure or otherwise difficult to remove. Neither existing modes of technology assessment, ethics procedures, risk assessment nor market mechanisms have been able to solve this problem. R\&I practice and governance accordingly should become more anticipatory - better able to anticipate and avoid Rgl trajectories that instantiate the dilemma.

This narrative, as well as the accompanying idea that SSH knowledge and practice can contribute in the strive for reflexivity, builds on extensive scholarship - some would say back to Vico (Rommetveit et al. 2013), others to Heidegger and the Frankfurter School, and yet others would make a more easily documented claim that it builds on latter decades' "Science and Technology Studies" (STS), history, philosophy and sociology of science and technology, and related strands of scholarship. Indeed, since the late 1960s, there have been various maxims of critical science, radical science, the science and society movement, technology assessment, post-normal science, socially robust knowledge and finally responsible research and innovation that had similar content (see Sardar 
and van Loon 2012 for an introduction to the history of this development). At times, the call was simply for an awakening of the political and ethical sensibilities of (natural) scientists - get out of the lab and engage in society! - while often this was not seen as enough: There was an implicit diagnosis of political ignorance and social, ethical and epistemological naivety within STEM research cultures that SSH involvement presumptively would correct. Indeed, this resembled a "deficit model", this time the scientists being the empty vessels that should be filled with knowledge from SSH in order to become reflexive. It also followed what would be the most relevant knowledge from SSH: Above all history, philosophy and sociology of science, STS, Wissenschaftstheorie (in the Germanic language area), ethics and philosophy of technology etc - that is, the various strands of scholarship that have science and technology as their object of study.

I write as if I have ironic distance to this narrative. I should immediately admit that I am among its many narrators. For instance, I was among those who strongly argued for the mandatory presence of ELSA in bioand nanotechnology as the Norwegian government revised its research policies in the late 2000s; and the government agreed. The strive for reflexive science also underpinned my and others' efforts to give content to $\mathrm{RRI}$ in the EU context, although the efforts rarely bore the desired fruit (Rip 2016). And I have kept publishing claims to that inverted deficit model, even with a paper entitled "Naivety in the Molecular Life Sciences" (Strand 2000). The need to historicise these claims does not void them of truth value. We should note, however, the speculative nature of the claim for SSH as a means to make STEM practice and governance reflexive. To the extent that the policy narrative has been used to legitimise a space for SSH in funding programmes, it should be admitted that it was not, and could not be, evidence-based. Rather, to apply our own concepts, it was more of an imagined future of a desirable social, scientific and technological order in which the Collingridge dilemma would be solved. In this sense, that is, in the sense of Jasanoff and Kim (2009), our policy narrative of how SSH would be conducive to reflexive science constitutes a sociotechnical imaginary.

\section{ELSI/ELSA AND RRI: OPPORTUNITIES AND DISAPPOINTMENTS}

The many published self-reflection essays from SSH scholars confirm the personal experience of my colleagues and I who have taken part in the various generations of ELSI/ELSA ${ }^{i i}$ and RRI-labelled interdisciplinary collaborations over the latter two decades: While results have been achieved and lessons have been learnt, there are also quite frequent expressions of disappointment.

It is useful to distinguish between two phases of ELSI/ELSA research each with their phases of disappointment. The typical disappointment of first ELSI/ELSA involvements was the lack of impact, which was diagnosed as a lack of true interaction and true interdisciplinarity (Nydal et al. 2011), as well as the lack of critical mass and proper organisation (Kaye et al. 2012). The sociologists, ethicists and philosophers were funded to do ELSI/ELSA research within a larger STEM (typically biotechnology) project but they had too much distance. For instance, at the Research Council of Norway, this diagnosis was explicitly endorsed, and from the mid-2000s ELSA funding was directed towards "integrated ELSA" and "integrated projects" with real and intense interaction between SSH scholars and STEM researchers. Similar developments took place elsewhere, drawing on longer traditions of scholarship of constructive technology assessment (Schot and Rip 1997) and innovative combinations of ethics and ethnographic work (e.g. "Socio-Technical Integration Research", see Fisher and Schuurbiers 2013).

Again, disappointments are well documented, ironically by the socalled post-ELSI manifesto by Balmer et al. (in a British context), later to be elaborated as lessons learnt (Balmer et al. 2016). Also in my own country, self-reflection and self-analysis by these integrated ELSA researchers has had a relatively pessimistic tone (Forsberg 2014, Nydal et al. 2016). Taking one step back from the more immediate concerns raised in these papers, the disappointment appeared to be related to the adjusted role as "integrated" ELSA/SSH scholars on their way into the STEM laboratories. In integrated ELSA, distance was reduced sufficiently for, as it were, CP Snow's famous "Two Cultures" (1959) to clash, that is, between the natural sciences on one hand and the social sciences and the humanities on the other. SSH scholars experienced that they were not taken seriously qua researchers, were perhaps not even welcome, were neither advancing their own careers nor having an impact on society, or generally uncomfortable with finding their role in co-producing the science and technology that they by virtue of their own expertise could not really vouch for in terms of its ethical and social desirability. The scientists, on their side, had problems coming to terms with what exactly they had let into the lab - a sort of spies? Saboteurs? Or just an irrelevant expense, forced onto them by the grant conditions? A number of lessons were drawn, most of them quite commonsensical, such as being reflexive and open to dialogue about our own facts and values; seek out the meaningful collaborative relationships with scientists rather than forcing ELSA down their throats; etc.

In Europe in 2011-2012, ELSA gradually ceded to the new EU policy concept of RRI (Owen et al. 2012). The European Commission (EC) concept was interpreted differently across Europe, and notably also within the European Commission, with the orthodox DG RTD bureaucracy insisting on the five or six "keys" (ethics, gender equality, public engagement, open access, science education (sic!) and sometimes "governance") at the same time as the original von Schomberg definition was implicitly endorsed by most SSH scholars who acted for and interacted with the European Commission. In the UK, the alternative "AREA" framework for Responsible Innovation proved influential well beyond the British Isles. RRI functioned as an umbrella not only for ELSA but also a number of other communities of practice and scholarship, notably those of technology assessment and public engagement. Still, RRI actions and projects recruited quite a few of the same SSH scholars who surfed the ELSA waves. For some of us, RRI gave new promise and new enthusiasm, perhaps primarily because RRI was seen less as an inherent negative response to STEM (in spite of its origin in the governance of the Collingridge dilemma) and also as an opportunity to "promote the good" by steering science and technology towards the common good and a better society. Again, the presumption was that such steering is not only possible but also that SSH scholars hold the expertise that enables us to engage in this steering and identify its goals, this time in active dialogue with civil society. Again, there were lessons and disappointments, often related to RRI practices appearing less than meaningful both to SSH scholars and STEM researchers. To quote a biotech PhD student in one of our RRI courses: "I am still waiting for the moment when you say 
that we have to engage the citizens in our laboratory research and we tell you that it won't work." Indeed, in my own subjective experience, I have witnessed how debates among RRI scholars/practitioners in 201618 appeared quite similar to the ELSA debates $5-10$ years ago, even with and without overlap in the actual people taking part. For instance, at the 2018 international conference of S.Net (the Society for the Study of New and Emerging Technologies) in Maastricht, the difficult convergence worker role of SSH scholars hired to "do RRI" in STEM projects was discussed in several of the sessions.

\section{LESSON 1: BEYOND THE PURE IMAGINARY OF "REFLEXIVE SCIENCE"}

Let us recall for a moment the policy narrative that I claimed to underlie ELSA, RRI and other attempts at integrating SSH into STEM:

STEM scientists, scientific practices, the governance of science and indeed the modern, knowledge-based society should become more reflective/reflexive. This can be achieved by involving $\mathrm{SSH}$, which are inherently reflexive.

Above I have described some experiences of disappointment as ELSI/ELSA and RRI efforts often seemed to have little impact and sometimes were perceived as downright meaningless. Since reflexivity is what we as SSH scholars by assumption are supposed to purport, it seems timely to ask reflexive questions about the disappointment. How may we understand our own role as participants in the strive for reflexive science?

The policy narrative of reflexive science can be seen as a sociotechnical imaginary, that is, a collective vision of good and attainable future science, technology and society. It will be useful to pursue that analytical lens somewhat further in the case of RRI. "Science" means two things in this regard. At the distal pole of the imaginary, it is a vision of coproduced good (i.e., reflexive) STEM science, good (i.e., ethically, socially and environmentally desirable) STEM-based technology and a good society that can benefit from this ethically and socially good STEM science and technology. The programme of action corresponding to this vision, is simply the successful deployment of SSH-informed and SSH-driven RRI practices. However, these RRI practices are also themselves imagined; they are in no way present as off-the-shelf technologies. So at the proximal pole of the imaginary we have the vision of co-produced "science" as SSH-based knowledge on RRI, "technology" as the RRI practices, tools and methods to be applied onto STEM research, and "society" as the research and innovation sector that no longer will give rise to Collingridge dilemmas or otherwise create problems in the world. Programmes of action corresponding to this version of the imaginary include RRI frameworks and funding schemes, such as the Horizon 2020 SwafS (Sciencewith-and-for-Science) programme.

All imaginaries are speculative; this is what makes them imaginaries rather than plans or cost-benefit analyses. Change is generated by imagining the non-existent and agreeing on a programme of action that may bring it into existence. This implies, however, that there can be no guarantee of success. Anything can go wrong in the attempts to realise a sociotechnical imaginary, and the failure may have any type of cause: material, social, epistemic, political.
As for the proximal pole of the imaginary, one assumption stands out in its boldness: The belief that STEM practices will produce substantively "better" technologies (in the sense of their ethical, environmental and social desirability) if these practices become reflexive and so can account for their own value-ladenness and their own context of implication. This assumption seems to be shared in all strives for reflexivity, going back to Marxist and feminist critiques, through radical science, post-normal science and the concept of socially robust technology, all the way to the RRI of the 2010s. The exact mechanism of how this is supposed to happen, varies from quite elitist beliefs in the normative expertise of SSH, ethics, "Technology Assessment" experts and the like, to beliefs in the power of deliberation and democratisation. The latter would entail recommendations of bringing in a range of stakeholders, citizens and social actors in upstream engagement exercises to cancel the tunnel vision of STEM practitioners and/or "align" research agendas with society, that is, steer research funding towards STEM that addresses social needs and concerns.

We do not know if this assumption of the effectiveness of reflexivity holds. It is of course always possible to cherry-pick examples that seem to confirm the assumption; hence the industry of projects that document "best practices" of RRlii. In my experience, many STEM researchers can sympathise with the goals of RRI but they also find the working assumption quite naïve. I quoted above the PhD student who said: "I am still waiting for the moment when you say that we have to engage the citizens in our laboratory research and we tell you that it won't work." Indeed, he expressed the expectation that we were making naïve assumptions about the impact of upstream engagement.

The experience of disappointment with RRI as expressed by SSH scholars engaged in RRI projects and efforts has been connected to more than this problematic (but central) assumption, however. Perhaps above all there has been frustration with practical and organisational issues related to the programmes of action. The RRI frameworks and the SwafS programme have been seen as too superficial and not really embodying the insights of relevant SSH scholarship (see e.g. Rip 2016); research policy-makers don't really understand RRI; even when STEM researchers engage, they might not engage with the level of commitment required; and when research funding organisations require RRI from STEM projects, they may be satisfied with mere tokens and window-dressing, not unlike "Corporate Social Responsibility" at its worst. The pure ideas about reflexive science originating from STS and all the other relevant SSH fields become co-opted, contaminated and perverted.

I suggest that this type of disappointment can be overcome by applying our own scholarship onto our own situation; by an exercise of reflexivity, as it were. Indeed, if our vision was to achieve impact on a large scale, co-producing goodness in STEM and the whole world by first coproducing our own RRI knowledge and technology, then this was a vision of massive upscaling. We know, however, from STS and the history, philosophy and sociology of science and technology, that upscaling processes are open-ended and that they introduce surprise. Above all, other actors who are not trained in SSH have to become enrolled into the programme of action, and they cannot help but make their own sense of these policies and practices. Inside the bureaucracy of the European Commission, for instance, the successful deployment of any policy concept both necessitates and hinges upon the development of numerical indicators and a monitoring system. Otherwise it cannot survive within the institutional logic.

The open-endedness and complexity of such processes also imply that one should not trust one's own assessment and evaluation of 
the process while it is unfolding. It is a paradox that while we as SSH teachers will instruct our students about the virtues of critical distance to the object of study, ELSA and RRI scholars are to the highest degree both participants and observers at the same time; indeed, we seem to be our own chroniclers. This criticism hits the present author as much as anybody else and it also hits several authors in the reference list of this paper. It is a striking feature of SSH scholars who work with STEM that we write quite a lot about ourselves.

None of these analyses proves that RRI or other strives for reflexive sciences are futile or meaningless. The analysis indicates the trivial conclusion that there can be no recipe for success but also the slightly less trivial insight that success may be different from what was imagined and might be identified in hindsight and perhaps by others than the SSH scholars who were involved in the first place. We are reminded of Hegel: "The owl of Minerva spreads its wings only with the falling of the dusk." (Hegel 1972, p. 14)

At the same time, SSH scholars are knowledge workers, and even when consciously involved and engaged in the co-production of society, we are involved with knowledge production. I will end this section by a personal example, taken from my work for the Centre for Digital Life Norway, a national, "virtual" (meaning geographically delocalised) centre for systems biology and biotechnology. The centre and its research projects are funded by the Research Council of Norway, and RRI is a mandatory requirement in all research projects and in the activities of the centre hubiv. I participate as one of the RRI coordinators of this hub. In this capacity I see myself and my colleagues as knowledge workers in three respects. First, we teach and disseminate SSH knowledge to STEM scientists, in particular PhD students and postdoc researchers, but also to some extent the "Principal Investigators" who are ultimately responsible for their own implementation of RRI into their respective research projects. Secondly, we make some effort to attend to the core of the assumption of the RRI imaginary, namely to understand the possible relationship between the many methodological choices in the STEM research and its context of implication. In this effort it has made little sense so far to "bring citizens into the lab". Rather, we work to understand the downstream implications of choosing, say, one type of computational or biological model over another. This is a challenge not so much of participatory technology assessment as of Wissenschaftstheorie, of being able to penetrate deep into the epistemological questions of STEM science, actually deeper than what is normally required for STEM daily practice, to identify sites of de facto politics in the lab. This kind of work depends on combined STEM and SSH knowledge to the extent that it has proven difficult to do without "double competence", that is, persons who are trained in both STEM and SSH.

Finally, we do our own SSH-based research, organised in the recently started Res Publica project, which is led by Dr Heidrun Åm. ${ }^{\vee}$ The Res Publica project will among other issues focus on how the bioeconomy is imagined and attempted to become realised by biotechnology. In other words, the project will not restrict itself to the potential de facto politics of minute methodological decisions in the laboratory but also keep an open eye for the de facto politics of politics itself, in the conventional sense of public decision-making and political institutions. Again, one could imagine a future ex-post assessment of the RRI endeavours of the 2010s to conclude that they had an STS bias and focused too much on the implicit micro-politics at the expense of attention to political economy. Perhaps future historians would identify this bias as part of a larger $\mathrm{SSH}$ trend at the beginning of the $21^{\text {st }}$ century and relate it to increasing differentiation and fragmentation of SSH. Even worse, they might relate the fragmentation of SSH research to how important issues are lost out of sight. They may even connect this to how SSH students and scholars maintained an intersectionalist focus on micro-aggressions in university life in European and North-American countries, while the public sphere in the same countries saw the rise of populism and open threats to democracy. There might be a need for a wake-up call to engage with the big issues and ask what is important.

\section{LESSON 2: GOING FOR WORTHWHILE COLLABORATIONS BEYOND THE INVERTED DEFICIT MODEL}

The big issues do not respect disciplinary borders or even the distinction between nature and culture. For SSH to gain impact, it seems that SSH scholars have to learn about issues outside of their usual scope, which is an excellent motivation for research. This has been a key point from actor-network theory for decades: The development of science and technology (and accordingly its governance) depends on many nonhuman actors: the genetically modified organisms, the nanoparticles, the CRISPR-Cas systems, the plastic in the Pacific Ocean - such things that STEM researchers know much better than us. Here there is a research challenge, not just a challenge to educate STEM researchers and policy-makers with our perfect SSH understanding, and it is a research challenge that is profoundly Mode 2 in the sense that it demands contributions from radically different types of disciplines but also that they leave their comfort zones. I will end this paper with another personal example, not from an RRI project but from the "Horizon 2020 Societal Challenge" project called MAGIC (making GRADE the irresistible choice) ${ }^{\mathrm{v}}$. In MAGIC, we study the science-policy interface for the governance of the water-energy-food nexus with a combination of ecological economics, energetics, biosemiotics, sociology and STS, because this is called for to understand the interactions between the human, social and natural agencies involved, including our own role as change agents. Whereas it is possible to classify the researchers in the project as "mainly STEM" and "mainly SSH", the practice is more usefully described in Germanic languages that have less dualistic concepts for the "Two Cultures": We are all Wissenschaftler. There may be occasions when researchers from one culture, say SSH, have to fill in knowledge gaps left open by STEM and vice versa. However, the interaction goes beyond seeing the others as empty vessels whose knowledge deficit has to be corrected. I interpret the richness of interaction in part as a result of the scope and complexity of the research topic, namely the water-energy-food nexus. In order to understand the biophysical system of, say, a river and the surrounding agriculture, one needs to understand the human, social, cultural and political dimensions of this system. Conversely, in order to understand and interpret the intricacies of policy-making in the field of water governance, one also needs to understand what is at stake in the policy debates, in biophysical terms. What we learn in the MAGIC project, is that SSH methods and theories are not void of implicit assumptions about the nature that humans try to govern, and that STEM methods and theories also hold implicit assumptions about governance and society. Part of the 
research challenge is to discover these implicit layers, explicate them and challenge them. This is quite different from filling deficits.

There is nothing unique in this particular example; there are whole journals that identify features and approaches conducive to meaningful inter- and transdisciplinary work between SSH and STEM. When I invoke the example of MAGIC, it is to make a specific point by contrasting it with features of ELSA and RRI interactions that have created disappointment. I have launched above the idea of going for "big" and "worthwhile" issues; however, with further comment this idea is nothing more than two fine words. Above it was noted how STS may have introduced a bias towards the de facto micro-politics of the STEM laboratory. In some instances this "bias" may be terribly important and exactly what one should focus on; and it was a great achievement of STS to discover the micro-politics through thorough empirical research from the 1970s and onwards. Still, one potential disappointment of the ELSA or $\mathrm{RRI}$ convergence worker is created from the realisation that the actual micro-politics of a particular STEM research project may be quite unimportant or uninteresting, or that it may be important but that there is no willingness, neither in the practice or the governance of the science, to change anything. This is partly why the Res Publica project also will return to the "politics of politics proper", to find other and promising sites for the co-production of the good future. If a STEM project has the express and unshakeable goal of producing a cybernetic soldier or a genetically modified salmon, there may be little use in spending years in the lab to do RRI as a kind of activist ethnographic action researcher. The contrast with the MAGIC project is striking. Although its main part is quantitative biophysical science, its goal is to rethink and help change the science-policy interface in the governance of the nexus. It addresses a "big" issue not by trying to device a technical solution but by creating knowledge that may induce institutional change.

The openness of the MAGIC project to theoretical and institutional change fits SSH really well and in particular the $\mathrm{H}$ for Humanities. SSH rarely sits well in collaborations in which it is relegated to a technical role, defined by STEM; this is seen well in the disappointments described above. Indeed, before the split of the "Two Cultures", the laboratory had to be invented for natural philosophy to become able to solve technical problems. In the example of the MAGIC project, we accordingly see a marker of a worthwhile collaboration: The willingness of all participants to go beyond the technical challenges and engage with theoretical as well as practical-political challenges. However, this marker - indeed a marker of reflexive science already present - is sufficient but not necessary. One could still strive for reflexive science, not necessarily to solve the Collingridge dilemma but to arrive to the point at which the SSH-STEM collaboration becomes meaningful because a shared interest in theoretical and practical-political challenges has been cultivated. Perhaps what has been learned through the successes and failures of ELSA and RRI endeavours is that SSH cannot provide a technical fix to the lack of reflexivity. Rather, it brings a repertoire and knowledge reservoir that may or may not be relevant in the context at hand. Mechanical and mindless deployment of that repertoire may end in disappointment because it tries do what especially the humanities are not at all equipped to do, namely reduce the other human subject (the STEM researcher) to an object. For worthwhile collaborations towards reflexive science to develop, it seems a better strategy to cultivate common intellectual curiosity and engagement towards the big issues across the STEM-SSH divide. Part of that strategy will be to identify contexts in which such commonalities are likely to be possible. This insight reflects back on the policy narrative of reflexive science, however: It might mean that RRI or other SSH interactions with STEM will never come off-the-shelf (Delgado and Åm 2018).

\section{ACKNOWLEDGEMENTS}

The work leading to the present article was performed as part of the projects MAGIC and Res Publica. MAGIC has received funding from the European Union's Horizon2020 Research and Innovation Programme under grant agreement No 689669. Res Publica has received funding from the Research Council of Norway under grant agreement 270623/070. This work reflects the author's view only; the funding agencies are not responsible for any use that may be made of the information it contains. I am most grateful to the editors of the journal for feedback and to Irmelin W Nilsen for careful editing.

\section{REFERENCES}

Balmer, A. S., Calvert, J., Marris, C., Molyneux-Hodgson, S., Frow, E., Kearnes, M., Bulpin, Pablo Schyfter, K., Mackenzie, A. and Martin, P. (2016). Five rules of thumb for post-ELSI interdisciplinary collaborations, Journal of Responsible Innovation, 3, 73-80.

Delgado, A., and Åm, H. (2018). Experiments in interdisciplinarity: Responsible research and innovation and the public good. PLoS biology, 16(3), e2003921. doi:10.1371/journal.pbio.2003921

Fisher, E. and Schuurbiers, D. (2013). Socio-technical Integration Research: Collaborative Inquiry at the Midstream of Research and Development. 97-110.

Forsberg, E. M. (2014). Institutionalising ELSA in the moment of breakdown? Life Science, Society \& Policy, 10, 1-16.

Forskningsradet (s. a.) Ethical, legal and social aspects of biotechnology, nanotechnology and neurotechnology (ELSA), from https://www. forskningsradet.no/en/Funding/ELSA/1253954329471

Hegel, G. F. (1972). Grundlinien der Philosophie des Rechts. Suhrkamp, Frankfurt am Main.

Jasanoff, S., and Kim, S. (2009). Containing the Atom: Sociotechnical Imaginaries and Nuclear Power in the United States and South Korea. Minerva, 47(2), 119-146.

Kaye, J., Meslin, E. M., Knoppers, B. M., Juengst, E. T., Deschênes, M., Cambon-Thomsen, A., Chalmers, D., De Vries, J., Edwards, K., Hoppe, N., Kent, A., Adebamowo, C., Marshall, P. and Kato, K. (2012). Research priorities. ELSI 2.0 for genomics and society. Science, 336, 673-4.

Nydal, R., Myhr, A. I. and Myskja, B. (2011). NANOETHOS. Report to the ELSA programme, the Research Council of Norway, from http://genok.no/wp-content/uploads/2013/03/NANOETHOS_1_1.pdf 
Nydal, R., Myhr, A. I. and Myskja, B. (2015). From ethics of restriction to ethics of construction. ELSA research in Norway, Nordic Journal of Science and Technology Studies, 3, 34-45.

Owen, R., Macnaughten, P. and Stilgoe, J. (2012). Responsible research and innovation: From science in society to science for society, with society. Science and Public Policy, 39, 751-760.

Rip, A. (2016). The clothes of the emperor. An essay on RRI in and around Brussels, Journal of Responsible Innovation, 3, 290-304.

Rommetveit, K., Strand, R., Fjelland, R. and Funtowicz, S. (2013). What can history teach us about the prospects of a European Research Area? Luxembourg: Publications Office of the European Union.

Sardar, Z. and van Loon, B. (2012). Introducing Philosophy of Science. London: Icon Books.

Schot, J. and Rip, A. (1997). The past and future of constructive technology assessment, Technological Forecasting and Social Change, 54, 251-268.

Snow, C. (1959). The Two Cultures. London: Cambridge University Press

Strand, R. (2000). Naivety in the Molecular Life Sciences. Futures, 32, 451-470.

von Schomberg, R. (2011). Introduction. In R. von Schomberg (Ed.), Towards Responsible Research and Innovation in the Information and Communication Technologies and Security Technologies Fields (pp. 7-15), Luxembourg: Publications Office of the European Union.

\section{AUTHOR}

\section{ROGER STRAND}

Senter for vitenskapsteori (Centre for the Study of the Sciences and the Humanities/Zentrum für Wissenschaftstheorie), University of Bergen

Parkveien 9, P.0. Box 7805, Bergen, N-5020 (Norway)

E: roger.strand@uib.no

\section{KEYWORDS:}

Social Innovation, Societal Impact, Societal Transformation, Empowerment, Participatory Approaches, Civil Society 


\title{
SSHA-DRIVEN KNOWLEDGE TRANSFER WITHIN THE THIRD MISSION OF UNIVERSITIES
}

\author{
GEORG RUSSEGGER \\ DOI: 10.22163/fteval.2019.369
}

\section{INTRODUCTION}

$\mathrm{B}$ ased on observations within the structural framework provided by the "Wissenstransferzentrum 0st"1 (Knowledge Transfer Centre East), an inter-university collaboration project of all nine Viennese universities, this contribution is pointing out different elements driven by the sectors of "Social Sciences, Humanities and the Arts" (SSHA) within the paradigm of a "third mission"i of universities. Besides the core functions of teaching and research, university outreach activities have developed different formats to provide services to populations who might not otherwise have access to those services. Historically the field of knowledge transfer has been dominated by valorisation and utilisation methods within the field of technology transfer, which is dealing mainly with the sectors of "Science, Technology, Engineering and Mathematics" (STEM). The SSHA had not been taken into account largely, since the significance for societal development and contribution were obviously integral to a greater good of humankind and by these means seemed obsolete for utilisation. On the contrary the SSHA field is forced to generate a rather radical and new approach of knowledge exchange with a multitude of peers - both within the academic community and with actors and stakeholders from society as well as economy - if they want to be part of the third mission paradigm. The paper elaborates on different approaches, formats and processes, which have been designed and applied within the collaboration project since its start in 2014, to exemplify such willingness. There will be a focus on examples from the knowledge transfer framework focusing on exchange methods from university to society at large and how impact have been catalysed within different levels of implementation. Based on these observations and learnings, the contribution reflects on target groups, such as researchers, alumni and university staff from different disciplines within the SSHA field, with a focus on inter- and trans-disciplinary endeavours. The aim is to figure out the potentials and methodological adaptions for a substantial integration of the SSHA in existing knowledge transfer structures, to describe pathways of impact oriented narratives, give examples from a process oriented and format driven participatory empowerment framework, to strengthen the position of SSHA at large in the field of know- ledge transfer and to describe themes, formats and programme elements to illustrate the role of SSHA sectors within university outreach actions.

\section{KNOWLEDGE TRANSFER - WITH STRINGS ATTACHED FOR SSHA}

Knowledge transfer is often associated with utilisation of intellectual property, patent- or product-centred technical innovations. The so-called TTOs ("Technology Transfer Officers") are responsible to deliver hard evidence for businesses through lawyers and patent offices. Obviously there are several reasons why the SSHA fields cannot be very interested in participating within such kind of transfer business. It is even hard to say that the social sciences, the humanities and the arts are sharing the same viewpoint and goals within the knowledge transfer setup. For people outside these fields, the acronym SSHA is not even graspable and therefore has to be brought into attention by awareness activities and good practice examples. From a critical point of view, plenty of reasons have been brought into discourse about how and why the transfer of knowledge and the economic trigger behind it will corrode the core values of SSHA (e.g. Castells 1997, Lui 2004, Boltanski / Chiapello 2005). Nevertheless, the goal and motivation to create societal impact through knowledge transfer to communities and peers is seen as an important issue. On the one hand, the judgment is often driven by a strong mistrust (e.g. Raunig 2007)2 according to the systematic approach to include neoliberal and entrepreneurial elements into the freedom of sciences and arts. On the other hand, social impact and responsible innovations are identified as core drivers and motivations to contribute academic knowledge and research skills to a broader field of application within society. Still, we see problems on an individual level of precarity and stagnation arising, which have to deal with fundamental issues like "make a living" or feeling valuated for the work done, because academic assessment structures are not aligned to mark and qualify these actions. The claim is: Valuation processes in the SSHA with societal impact are developed; they "just" have to be applied in existing structures of knowledge trans- 
fer and exchange channels.i This cannot be done within a top down super-structure treating all SSHA field and stakeholders with the same policy instruments. It might not be even possible to just apply something in an existing understanding of know-how transfer without sharing the same visions, agendas and tools.

\section{THE CASE - “KNOWLEDGE TRANSFER CENTRES" IN AUSTRIA}

The case is referring to a funding scheme introduced by the Austrian Federal Ministry of Education, Science and Research in 2014 and is carried out by the funding entity "Austria Wirtschaftsservice" till the end of 2018.iii One Module of the funding scheme is dividing Austria in three regional centres (East, West, South). The eastern centre is Vienna with its nine Universities. ${ }^{i v}$ Every centre is receiving about half a million Euro per year to create, develop and carry out knowledge transfer activities addressing society and economy. The important and new element within the action is a special awareness to the SSHA fields, designated through an additional budget to the mentioned funding earmarked for SSHA related activities only and thus the obligation for existing technology and knowledge transfer structures to include SSHA in their developments and actions. This rather new and experimental approach led to the development of several new knowledge exchange formats trying to identify and address new target groups. Including workshops, lectures and seminars to empower researchers, university stuff and doctoral students for participatory methods of knowledge exchange e.g. citizen science, or to develop training formats for alumni based on topics like social entrepreneurship and social business. The impact on this level of development could be described as "impact by design". Pre-existing pathways and approaches mainly focused on exploitation and valorisation of products (e.g. patents, inventions, technologies) instead of the empowerment of certain groups and stakeholders to contribute and distribute their knowledge and skills to society. To channel these actions a second level has to be delineated within an external cooperation framework of the knowledge transfer centre, to ensure external organisation units can receive and apply knowledge on inter- and trans-disciplinary level. By identifying and including external organisations and stakeholders as experts, mentors and partners, awareness raising and dissemination into new areas of application was accomplished. Furthermore a network of partners based on a qualitative cooperation experience can multiply outcomes and foment new actions. The implementation of the knowledge transfer centre showed, if a clear applicable cooperation framework is provided by universities, it is used by the mentioned target groups and partner organisations.

\section{THE CHALLENGE - TAILORING PROCESSES WITHOUT BLUEPRINTS}

The opportunity to create and develop a sustainable framework of SSHA knowledge transfer between all Viennese universities and all regional centres in Austria is an appealing challenge. The establishment of a communication pattern for all responsible university entities and centres is one of them. Another one is to establish a compliant form of interaction to find common grounds and languages of SSHA interests. The key question is about the implementation of support levels in different organisational structures and cultures within the university system. However the project structure given by the funding entity was rather vague and had not foreseen a clear structure of contents and transfer activities within the application. The project partners delivered the conceptual outline and main emphasis on content. The SSHA sector in Vienna is led by the Academy of Fine Arts Vienna dealing with the fact of minor preliminary work done concerning knowledge transfer at the partners, and hardly any structures established in the field. The main aim was to create new, open, flexible and interdisciplinary formats of exchange and transfer to support a bottom up process in the SSHA field based on projects, rather than defining knowledge transfer processes as tailor made blueprints. The organic vision for capacity building in the universities and the identification of cooperative communities in society and with economy still had to be narrowed down, especially concerning the mentioned target groups, contents, and channels to design tools and formats for several different patterns in the SSHA spectrum. For this reason, so called transfer and creativity hubs have been designed to deliver training programmes with certain SSHA relevant topics. One of the most integral parts within the development plan was relying on the empowerment of diverse target groups within the university system, such as university staff and researchers. Additionally, a priority was set on targeting alumni to broaden the responsibility of knowledge transfer and to create a level of exchange with their competences and needs, to offer new connectivity options and peer learning formats for all participants. By these decisions, a trust is laid in identifying unknown and upcoming actors of knowledge transfer within a broader angle of empowerment and knowledge production, in relation to universities but not necessarily only from universities. Kirsten Langkilde describes the interplay between a creative and critical culture and the realm of society as "impact of culture" (Langkilde, 2018, p 32). The process of production is understood as a contribution to society. Knowledge transfer channels can support such elements by tailoring processes to address special interest communities such as social businesses or impact investors to provide a participation or cooperation framework. To intermediate between academic projects and external partners the knowledge transfer centre helped to support on both sides of the spectrum. 


\section{IN THE MAKING - FORMATS AND TOOLS}

First of all a multi-level participatory approach for target groups and cooperation partners was outlined to be adapted by different hosting university and opinion leaders. All of the designed tools and formats of knowledge transfer within the SSHA sector of the Knowledge Transfer Centre East are rather offers than instructions. Since not every partner university in the network is working under the same conditions and is having a similar development status, equally claimed needs had been replaced by offers that can be used, adapted and modified by the universities for their purpose. Working in a network made it easier to exchange offers and apply them within the existing structures. All actions are open to all university partners and free of charge to ensure an interdisciplinary exchange. The offers cover extra curricula courses, workshops and training programmes, called transfer and creativity hubs, to cover topics and aspects of diverse backgrounds identified from the SSHA fields. For example a continuing education programme ${ }^{v}$ with more than forty workshops, lectures and seminars per year is hosted by the Viennese universities. Different locations and topics should help to identify potentials, support projects and catalyse communities around special interest groups. The low-threshold of the formats should raise awareness for upcoming and existing elements of knowledge transfer and attract peers to learn more about contemporary developments and upcoming topics within the SSHA transfer activities and its impact on society. Experts, partners and stakeholders from the region have been included in the activities as speakers and trainers to carry out a large proportion of the education programme, to multiply the channels of dissemination and to recognise new actors. This rather generic approach was driven by the fact that most of the actors within the SSHA field we are reaching are motivated to apply their expertise in meaningful actions, they want to make a living from what they do and are looking for cooperation and communities to expand their sphere of action. Supporting transferable skills and peer learning within a consistent setup of programmes is building trust in sustainable structures where individual know-how and expertise can be adjusted within groups, leading to connections with external partners and assuring the step by step development of a recognised practice for cultural wellbeing as integral part of societal challenges. From a technical level we used the extra curricula courses to identify the interest of participants on a quantitative and qualitative level to decide if a more intensive set up of a creativity and transfer hub is useful.

\section{TRANSFER FOR THE PEOPLE - IT'S ALL ABOUT CONTENT.}

Within the multitude of structures and interaction patterns built to identify and support the needs of transfer cultures in the SSHA and to deliver it to a larger context of society, precise examples and good practices are the key. Knowledge transfer and all its implications, especially in the SSHA fields, have to be channelled in tangible topics to observe a social or societal impact. By this means I want to point out some examples to elaborate on. Within the four-month training programme called "Make yourself an Expert Hub"vi we focused on different topics such as, how to make a living in the arts, how to set up a social business, how to create a crowdfunding campaign. vii The trainings always have been introduced by a call, assessed by a jury, carried out with external stakeholder and cooperation partners. Trainers and experts from the field delivered state of the art examples, helped with the setup of teams and contribute towards project management skills and supporting channels. By the establishment of micro-funding and financial support, structures external funding sources could have been convinced to cooperate, so called "impact investors" have been made sensitive to the contributions of SSHA to society at large and stakeholders had the chance to understand better why SSHA achievements are having a fundamental significance to society, which is under a heavy transformation process.

Using the example of the "Social Business Hub" we worked closely with partners like "magdas hotel" a social business operated in the framework of Caritas. They provide work for refugees from crisis-ridden countries to offer them the chance to demonstrate their abilities and talents. ${ }^{3}$ In addition we included organisations like the "Impact Hub Vienna", the platform "Social City Vienna" and the "Social Entrepreneurship Center of the Vienna University of Economics and Business" to develop a training programme for the "Social Business Hub". Within the hub we called for projects from the university context with social business ideas do co-develop and co-create their ideas. Out of 25 applications ten projects have been selected to bring their ideas to life. After two month of intensive and productive training the projects pitched their ideas in front of the partners, potential investors and stakeholder from the field of social business. ${ }^{4}$ More than $50 \%$ of the supported projects founded a social business. Others got offers to develop further within project cooperation or found partners to go on with.

\section{CONCLUSION}

One of the greatest achievements within the logic of knowledge transfer activities with people from a multi-disciplinary background is the belief to contribute something good and important for people and society. Even if this sounds pathetic, but the credo "to make the world a better place" is enabling a very contemporary culture of knowledge beyond the fact of economical reason. In general social, cultural and 
artistic approaches are often misunderstood as philanthropic, with a minor economical dimension. The mentioned trainings as well as "Artistic Incubators and Cultural Accelerators" as discussed at the "Conference for Knowledge Exchange and Technology Transfer Professionals, Vienna $2018^{\prime \prime 5}$ are dealing with this bias by focusing on substantial transfer and exchange activities based on socio-cultural change and critical reflection to contribute deeply for societal challenges and the contemporary transformation of society. The empowerment of social scientist, artists and cultural workers in collaborative and interdisciplinary developments of new frameworks of action such as artistic incubators and cultural accelerators have led to a unique perspective on economic frameworks having the common good and social impact at stake. For the SSHA sectors and especially for researchers in the field, the described fields of action could be rather seen as a chance than as a burden. Taking these challenges with an understanding of diversity, responsibility, ethical guidelines and sustainability for the whole society into account will as well influence and change encrusted economical driven structures. Despite the commendable fact, monetary aspects cannot be disregarded. Furthermore, a clear vision of financial needs and compensation for these actions has to be developed within the SSHA interests, to deliver a serious approach beyond counter cultures and dependence on third party funding approaches. If the SSHA fields want to succeed in regard of the contemporary societal challenges they are forced to step out of the comfort zone. To help and support this process knowledge transfer between universities and society can be a useful tool.

\section{REFERENCES}

Buckland, M. and Gey, F. (1994). The relationship between recall and precision. Journal of the American Society for Information Science, 45, 12-19.

Boltanski, L. and Chiapello, E. (2005). The New Spirit of Captialism. London: Verso.

Castells, M. (1997). Power of Identity: The Information Age: Economy, Society, and Culture. Cambridge, MA: Blackwell Publishers.

Langkilde, K. (2018). Impact of Cultures. In: Wissenstransferzentrum Ost (Ed.), Wissenstransfer gestalten. Werkzeuge, Formate, Potenziale. Vienna: facultas Verlag. 22-33.
Lui, A. (2004). The Laws of Cool: Knowledge Work and the Culture of Information. Chicago: University of Chicago Press.

Raunig, G. and Wuggenig, U. (2016). Kritik der Kreativität. Vienna: transversal texts.

\section{AUTHOR}

\section{GEORG RUSSEGGER}

Centre for Knowledge Transfer, Project Lead: Knowledge Transfer Centre East, SSHA Secto

Academy of Fine Arts Vienna

Schillerplatz 3, Vienna 1010 (Austria)

E: g.russegger@akbild.ac.at

i Survey by the Institute for Advanced Studies. (2012) (in German language): https://www.wien.gv.at/wirtschaft/standort/pdf/third-mission.pdf (last accessed: 15.06.2018

ii Benneworth B. and Jongbloed B.W. (2009). Who matters to universities? A stakeholder perspective on humanities, arts and social sciences valorisation. (Article is published with open access at: springerlink.com)

iii Funding scheme and funding guidelines (in German language): https://www.aws.at/foerderungen/aws-wissenstransferzentren-und-ipr-verwertungmodul-1a-regionale-wissenstransferzentren (last accessed: 12.05.2018)

iv The nine Viennese universities within the Knowledge Transfer Centre East: Academy of Fine Arts Vienna, University of Applied Arts Vienna, University of Music and Performing Arts Vienna, University of Vienna, Vienna University of Economics and Business, Technical University Vienna, University of Natural Resources and Life Sciences Vienna, Medical University Vienna, University of Veterinary Medicine Vienna.

v Details about the actual education program of the Knowledge Transfer Centre East is available online (in German language): http://www.wtz-ost.at/veranstaltungen (last accessed: 17.06.2018)

vi Video examples of projects and supported projects can be viewed via online streaming video channel: https://vimeo.com/akbild (last accessed: 17.06.1018)

vii See also: http://www.crowdfundershub.at (Website in German language, last accessed 15.06.2018) 


\title{
PANEL COMPOSITION AS PATHWAY TO IMPACT: DO WE NEED STAKEHOLDER EXPERTISE TO SELECT RELEVANT MISSION-ORIENTED PROJECTS?\#
}

\author{
PETER VAN DEN BESSELAAR AND ULF SANDSTRÖM \\ D0I: 10.22163/fteval.2019.370
}

\section{ABSTRACT}

$\mathrm{I}$

$\mathrm{t}$ is often argued that the presence of stakeholders in review panels may improve the selection of societal relevant research projects. In

this paper, we investigate whether the composition of panels indeed matters. More precisely, when stakeholders are in the panel, does that result in more positive evaluation of proposals of relevance to that stakeholder? We investigate this for the gender issues domain, and show that this is the case. When stakeholders are present, the relevant projects obtain a more positive evaluation and consequently a higher score. If these findings can be generalised, they are an important insight for the creation of pathways to and conditions for impact.

\section{INTRODUCTION}

There is an increasing awareness that to generate impact, focus should be on the relations between knowledge producers and knowledge users, on relations that can be seen as the pathways to impact. A main reason for this is it may take many years after R\&D projects have taken place before impact becomes visible. If one wants to evaluate the possible societal impact of research, it makes sense to focus on the conditions that increase the probability of impact. Therefore, more retrospective research is needed to identify the conditions for contemporary impact of research done in the past.

One may argue that the nature of the relations between knowledge producers and knowledge users may help to increase utilisation of knowledge, which in turn may lead to impact. The term "productive interactions" has been suggested for these relations, and one of the objects of research within this topic is to identify the variety of interactions and how they are formed in different knowledge domains. Understanding

those interactions may help policy makers to stimulate the conditions for impact and through this increasingly open up for the probability that research projects do generate not only scholarly but also (and especially) societal impact (Spaapen et al. 2011; Eric 2010).

In the ERiC projecti and in its successor the SIAMPI projecti", the concept of productive interactions was deployed to study research impact in a broad way, including societal impact. The approach was applied in several scientific and technological fields like information and communication technology (De Jong et al. 2014), architecture (De Jong et al. 2011), law (Van Arensbergen et al. 2010), electronic engineering (Propp et al. 2010); mechanical engineering (Van der Meulen et al. 2010), in biomedical fields (Prins 2010), and in the social sciences and humanities (Molas et al. 2011).iii

Many of these productive interactions or "impact pathways" were found when the investigations were focused on the direct or indirect links between users and producers of knowledge. More recently, the concept of co-production of knowledge has become fashionable, pointing at collaboration between researchers and stakeholders in the process of knowledge creation. It is expected that such collaboration, in which stakeholders bring in local knowledge about the topic under study and knowledge about possibilities and constraints of applying knowledge, the dissemination and use of scholarly research output will be more frequent, easier and faster (see among others: Wardenaar 2014; Hegger et al. 2012; Hegger and Dieperink 2014; Djenontin and Meadows 2018). The model of co-production is mainly deployed in studying complex problems such as climate change and environmental studies, and many problems are still to be solved, such as resourcing knowledge co-production and the cultural differences between researchers and stakeholders (Djenontin and Meadows 2018). Furthermore, the role of stakeholders is often not so much in the co-production of the knowledge, but more in the start of the project when the research questions are formulated, and in the end when disseminating the new knowledge (Wardenaar 2014).

However, also other interactions may be relevant. As research is de- 
pendent on increasingly scarce funding, an important pathway to impact may be the selection of the "correct" projects - projects that include the creation of impact pathways as discussed above, but also projects that directly or indirectly focus on the relevant societal issues. It is often claimed that the presence of stakeholders in the panels may increase the relevance and possibly the impact of research: extended peer review (Nowotny et al. 2001) through broadening panel and peer review by including practitioners (Cornell et al. 2013). In this paper we investigate whether this indeed works: does the presence of stakeholders influence the selection process? In this paper we use an innovative method to investigate whether this indeed works: does the presence of stakeholders affect the selection process? We do so for a case where selection panels differ in terms of membership: some include specialists on gender issues, whereas others do not. Are the former panels more positive about proposals that have a gender relevant dimension than the latter?

\section{GENDER ISSUES IN RESEARCH}

Research on gender and science has been focused on the position of women in science, such as on gender bias in grant allocation (Wennerås and Wold 1997; Van den Besselaar et al. 2018) and in academic careers (Brouns 2003; Benschop and Brouns 2003; Van den Brink 2006).

More recently, the effect of underrepresentation of women in science on the content of research has become a prominent issue. Biomedical research is a good example, as it has become clear that in much clinical research only male subjects were included. This has blinded the field for gender differences in symptoms, diagnosis and in medication and treatment (Chapman et al. 2013). At the same time, several studies have shown that female researchers are more inclined to take gender issues into account than male researchers do (Nielsen et al. 2017). If this holds for research and research output, it may also hold for research input: grants. Are female panel members, and - more specifically for this paper - panel members that specialise in gender issues more inclined to select proposals with a gender dimension?

In this paper, we address the question whether the availability of gender expertise in grant selection panels does matter. Do panels with gender specialists and panels without gender specialists look differently at proposals? And, do panels with gender expertise have a more positive report on gender related proposals than panels without gender expertise on board? After having answered this question, the issue comes up in what respect gender expertise is relevant and is influencing the selection process. To answer that, observations of panels would be needed, and that falls outside the scope of this paper. However, the research question is also relevant from a practical perspective. If we find a positive effect of gender expertise on the selection process, this knowledge can be used for composing panels, even if we do not know how it exactly works.

\section{DATA AND METHOD}

\section{DATA}

We use a dataset covering 111 granted project proposals, and all were considered by the applicants as gender-relevant. ${ }^{i v}$ The projects were submitted in various calls in the Horizon 2020 programme, all with a two-stage procedure. Table 1 gives an overview of the calls the proposals were addressing.

Border security and external security (BES)

Table 1. Number of proposals in the sample by call.

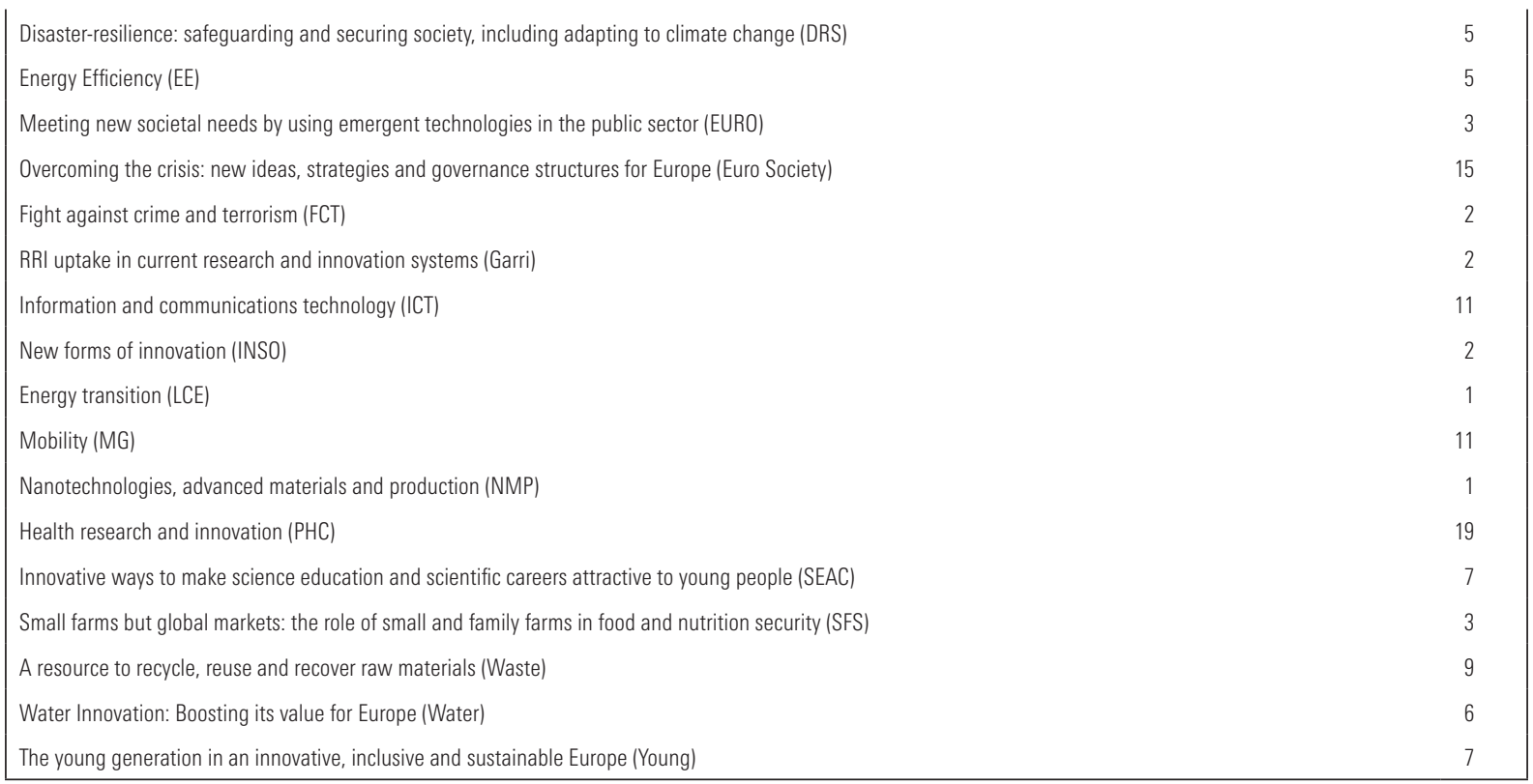

All these projects are "flagged" as gender relevant, which means that the applicants claim that their project has a relevant gender 
dimension. ${ }^{v}$ However, an independent evaluation of the proposals - in terms of the "Cross-Cutting Monitoring Indicator" (CCMI) - indicates whether a gender dimension is really present in the proposals. Of the 110 projects in the sample, 60 get a positive CCMI indicator score by an "independent" project officer. This means that the sample consists of 60 gender relevant applications, and 30 applications that lack gender relevance. For 17 projects, the variable is missing. Obviously, many applicants try to sell their project as gender relevant - even if this is not the case. This is not unexpected, as they may hope that this improves the probability to get funded. .i $^{\text {ii }}$

Apart from information about the gender content of the proposals, we have information about the relevant panels. vii We do know for each of the projects whether at least one expert on gender issues was in the relevant panel - which was the case for 71 of the projects. We also know the scores the proposals have received from the panels. Finally, we have the evaluation reports about the proposals in the second stage of the evaluation. All data were provided by the funder. The information available enables to distinguish four conditions which will be used for the analysis. These are shown in Table 1.

Table 2. Sample: gender expertise and CCMI*

\begin{tabular}{|lll|}
\hline \multicolumn{3}{|l|}{ Gender expert in panel } \\
\hline CCMI & No & Yes \\
\hline No & 22 & 11 \\
\hline Yes & 15 & 45 \\
\hline Missing & 2 & 15 \\
\hline Total & 39 & 71 \\
\hline & * "Cross-Cutting Monitoring Indicator"
\end{tabular}

\section{METHODS}

In order to assess the evaluation of the project proposals, and the influence of (in this case gender) stakeholders in the panel, we perform a linguistic analysis of the review reports. The "Evaluation Summary Reports" (ESR) consist of (i) the project summary produced by the applicants and (ii) the review text (including the scores) produced by the panel. Summary and review were separated for the analysis. We use the summaries to check whether gender is mentioned in the content of the research. The review text was used to investigate whether the reviews explicitly relate to gender, and to analyse whether the reviews have a more positive or negative tone.

The ESR files are in PDF format, and we did split all files in a review part and in a summary part. Then, the PDF files were converted into plain text files and from these files the "standard text" was deleted, such as headings of sections. The remaining parts of the files were imported into the text analysis software CorTex $\top^{1}$ for term extraction. For finding terms referring to gender and gender issues, we used both the summary and the review. For the linguistic analysis we used only the review texts (see below).

Finding terms that refer to gender and gender issues was done by manual inspection of the word lists produced by CorTexT. The review parts were also used for a linguistic analysis in order to distinguish between negative and positive reviews. The latter was done using LIWC2, a tool for linguistic analysis of texts. The tool works with a variety of predefined linguistic categories and has been applied regularly for the analysis of reviews (Kaatz et al. 2014a; Van den Besselaar et al. 2016, 2018b). Each linguistic category consists of a set of words representing that category, which have been validated in other studies (Abele and Wojciszke 2014). The LIWC programme counts for each of the categories how many times a word belonging to that category is present in a review report. As the reports are of different length, normalisation is needed: the number is translated into a percentage. In this case, we start with using those categories that are tested and used in previous studies on grant decision and panel deliberation (Kaatz et al. 2014a; Van den Besselaar et al. 2016, 2018b):

- Ability words, such as gift*, intel/*, skill*;

- Achievement words such as creati*, exce/*, compet*;

- Agentic words such as outspoken, solid, risk;

- Negative evaluation words such as naïve, defect*, lack*;

- Positive evaluation words such as intriguing, compelling, commit*;

- Research words such as laboratory, result*, fund*;

- Standout adjectives such as world class, outstanding, exceptional*.

The term extraction of the review reports resulted in a list of frequently used (stemmed) terms. This list was inspected in order to find additional review terms not included in the above-mentioned linguistic categories. Based on the term extraction, the following additional linguistic categories are added:

- Negating words such as hasn't, don't, can't;

- Negative emotions words such as abuse* ${ }^{*}$, bitter ${ }^{*}$, bad*;

- Positive emotions words such as agreeabel*, benefit, helpful;

- Exclusion words such as but, either, except, just, not;

- Insight words such as define, reflect, idea*;

- Certainty words such as fundamental, commitment, truly.

Why were these additional categories selected? Firstly, as term extraction shows that the categories may play a role given the frequency they appear. For negation words, an additional argument is that the excellent applicants are the norm in science, and the others are measured against those excellent: "not excellent". Exclusion words might be used biased because of the same argument. Positive and negative emotions are relevant to include, as one would want to see how strong sentiments play a role in panel deliberation.

Running LIWC gives for every review the percentage of words belonging to each linguistic category. We can now compare the average frequencies of the linguistic categories between those applications that have a positive CCMI score versus a negative CCMI score, and those evaluated by a panel with gender expertise or by a panel without such expertise. As there are some missing values in the CCMI variable (17) we actually can include 93 projects in the analysis. As we also have the scores the proposals received, we can also compare the scores for the four groups with the results of the linguistic analysis.

\section{RESULTS}




\section{ARE THE SUMMARIES OF THE PROJECTS GENDER RELATED?}

Manually inspecting the words used in the project summaries shows firstly that words like sex, male, and female are hardly used. The word gender is used in the project summaries, in a different way in the four conditions (Table 2). In the two groups with proposals that were flagged as gender relevant, $40 \%$ uses the term gender, whereas this is only the case for $27 \%$ of the non-gender relevant proposals. In the reviews the pattern is similar. Comparing the two sets of proposals that have been evaluated by panels with gender expertise with the other two sets, show that panels with the gender experts more frequently evaluate in terms of gender issues (39\%), whereas the other panels do this in only $14 \%$ of the proposals. As a tentative conclusion, panels including stakeholders do more often evaluate proposals partly from the stakeholders' point of view.

Table 3. "Gender" in the summary and in the review.

\begin{tabular}{|l|lll|}
\hline & \multicolumn{3}{|c|}{ Gender expert in panel } \\
\hline & CCMI & No & Yes \\
\hline "gender" in & No & $5(23 \%)$ & $4(36 \%)$ \\
\hline summary & Yes & $3(20 \%)$ & $21(47 \%)$ \\
\hline "gender" in & No & $2(9 \%)$ & $2(18 \%)$ \\
\hline review $^{*}$ & Yes & $3(20 \%)$ & $20(44 \%)$ \\
\hline
\end{tabular}

* excluding "gender balance" in the team

More sophisticated approaches to this are possible. We only used the term gender, but one could think of producing ontologies (or structured thesauri) describing gender relevant topics in detail, and use these for analysing the content of the proposals (e.g., Van den Besselaar et al., 2017). This approach, however, is outside the scope of this paper.

\section{ANALYSING THE REVIEW REPORTS}

We use a linguistic analysis of the review reports, as described in the methods section. We compare the four groups of proposals, defined by the two core variables: (i) availability of gender expertise in the panel, and (ii) the CCMI score for gender relevance. We use group 4 as reference: gender expertise present and a positive CCMI score.

1. Group 1 (no gender expertise, negative CCMI) versus group 4 (gender expertise, positive CCMI): Compared with Group 4, Group 1 has a significant higher mean score on negative emotions (mean $=1.14$ vs mean $=0.70, p=0.004$ ), agentic language ( mean $=2.96$ vs mean $=2.57, p=0.037)$ and on negative evaluation (mean $=2.13$ vs mean $=1.22, p=0.000$ ), and a significant lower mean score on insight (mean $=2.34$ vs mean $=2.90, p=$ 0.008 ) and on positive evaluation (mean $=8.12$ vs mean $=9.10$, $p=0.070$ ). As these scores are generally not normally distributed, we use next to Analysis of Variance (to compare the means) also a non-parametric test (to compare the mean ranks). This shows that compared with group 4, group 1 has a significant higher mean rank on negative emotions, agentic, and on negative evaluations, and a significant lower mean rank on insight and on positive evaluation. So, both tests give the same results.
2. Group 2 (gender expertise, negative CCMI) versus group 4 (gender expertise, positive CCMI): Compared with group 4, group 2 has a significant higher mean score on negative evaluations (mean $=1.79$ vs mean $=1.22, p=0.061)$, and a significant lower mean score on positive emotions (mean $=2.75$ vs mean $=3.70, p=0.005$ ) and on positive evaluation (mean $=7.57 \mathrm{vs}$ mean $=9.10, p=0.037$ ). Again, as these scores are generally not normally distributed, we use next to Anova also a non-parametric test. Compared with group 4, group 2 has a marginally (non-significant) higher mean rank on negative evaluation, and a significant lower mean rank on positive emotions and on positive evaluation. Both tests give similar results. The marginally/nonsignificance is due to the small number of cases in group 2. The conclusion of this analysis is that panels with gender-specialists are more positive on gender-related projects than non-genderrelated projects.

3. Group 3 (no gender expertise; positive CCMI) versus group 4 (gender expertise, positive CCMI): Compared with Group 4, Group 3 has a non-significant (small N) higher mean score on negative evaluations (mean $=1.61$ vs mean $=1.22, p=0.13$ ), and a significant lower mean score on positive emotions (mean $=3.16$ vs mean $=3.70, p=0.056$ ). As these scores are generally not normally distributed, we use next to Anova also a nonparametric test. Compared with Group 4, Group 3 has a significant higher mean rank on negative evaluations, and a significant lower mean rank on positive emotions. Obviously, both tests give about the same results. The non-significance may also be due to the small $\mathrm{N}$ for group 3. The conclusion is that proposals with a gender dimension are more positively evaluated by panels with gender expertise than by panels without gender expertise.

\section{PANEL SCORES}

Comparing the evaluation scores for the different groups shows that in panels with gender expertise, "gender relevant" proposals (ccmi = yes) get a higher score than the "non-gender relevant" proposals (ccmi $=$ no): 14.0 versus 13.6; and the panels with gender expertise score the gender proposals $(\mathrm{ccmi}=$ yes$)$ higher than the panels without gender expertise do: 14.0 versus 13.1 points.

\section{CONCLUSIONS}

The conclusions are that (i) panels with gender-specialists are more positive about gender-related proposals than about non-gender-related proposals, and (ii) that panels with gender expertise are more positive than panels without gender expertise about proposals with a gender dimension. This difference in evaluation language is also reflected in the scores as we showed above. The overall finding would confirm the strategy of getting stakeholders into panels: it helps getting projects funded that work on issues relevant to the stakeholders. So we can tentatively answer the question whether gender expertise matter or not: our findings indicate that gender expertise in panels matter. If these findings can be generalised, stakeholder representation seems a good way for increasing research project relevance and impact.

Further work is needed, as this paper only is only a first modest step. Several improvements need to be addressed in the future. (i) First of all, 
a better operationalisation is needed of what is "stakeholder relevant research". This asks for ontologies that give a structured representation of the topics relevant to the specific stakeholders. With such ontologies, it becomes easier to identify relevant research but also to assess where there are white spots in the relevant research portfolio. (ii) The analysis was done using only the summary of the proposals. Using the full text may improve the analysis, although it is also more difficult to identify the relevant parts of the proposal and may introduce more noise. (iii) Only granted proposals are taken into account, but the analysis of the nongranted proposals is as important. (iv) Other aspects of the evaluation may be taken into account, such as the scientific quality of the consortium, and earlier work in relation to the stakeholders needs. (v) Since we only have access to projects in gender flagged topics, it is not possible to conduct a more refined analysis that focuses on the differences between flagged and not flagged topics: how good is the identification of stakeholder relevant projects? (vi) Field differences should be taken into account. This could not be done due to the relative small number of proposals. (vii) Last but not least, ex post evaluation is needed too. Do the proposals that were defined as stakeholder-relevant indeed produce more useful and more used output? And what is the quality in other dimensions, such as the scholarly quality?

\section{REFERENCES}

Abele, A.E. and Wojciszke B. (2014). Communal and agentic content in social cognition: A Dual Perspective Model. Advances in Experimental Social Psychology 50, 195-255.

Benschop, Y. and Brouns, M. (2003). Crumbling Ivory Towers: Academic Organizing and its Gender Effects. Gender, Work and Organization, 10 (2), 194-212.

Brouns, M. (2000). The gendered nature of assessment procedures in scientific research funding: the Dutch case. Higher Education in Europe, 25, 193-201.

Chapman, E.N., Kaatz, A. and Carnes, M.(2013), Physicians and implicit bias: how doctors may unwittingly perpetuate health care disparities. Journal of General Internal Medicine 28, 1504-1510.

Cornell, S., Berkhout, F., Tuinstra, W., Tàbara, J.D., Jager J. Chabay I, de Wit, B., Langlais, R., Mills, D., Moll, P., Otto, I.M., Petersen, A., Pohl, C. and van Kerkhoff L. (2013). Opening up knowledge systems for better responses to global environmental change. Environmental Science and Policy 28, 60-70

Djenontin, I.N.S. and Meadow, A. M. (2018). The art of co-production of knowledge in environmental sciences and management: lessons from international practice. Environmental Management 61, 885-903

ERiC (2010). Evaluating the social relevance of academic research: a guide. (In Dutch: ERiC: Evaluatie van de maatschappelijke relevantie van wetenschappelijk onderzoek: handleiding. Den Haag, 2010)

de Jong S., van Arensbergen, P., Daemen, F., van der Meulen, B. and van den Besselaar, P. (2011). Evaluating research in its context: an approach and two cases. Research Evaluation 20 (2011)

de Jong S., Barker, S., Cox, D., Sveinsdottir, T. and van den Besselaar, P. (2014). Understanding societal impact through studying productive interactions. Research Evaluation 23 (2014) 2, 89-102

Hegger, D., Lamers, M., van Zeijl-Rozema, A. and Dieperink, C. (2012). Conceptualizing joint knwledge production in regional climate change adaptation projects: Success, conditions and levers for action. Environmental Science and Policy 18, 52-65

Hegger, D. and Dieperink, C. (2014). Toward successful joint knowledge production for climate change adaptation: lessons from six regional projects in the Netherlands. Ecology and Society 19 (2), 34.

Kaatz, A., Magua, W., Zimmerman, D.R. and Carnes, M. (2015). A quantitative linguistic analysis of NHI R01 application critiques. Academic Medicine 90, 1, 69-75

Knoll, B., de Chevigne, S., Bustelo, M., Engebretsen, E. and Sandström, U. (2017). Interim Evaluation: Gender equality as a crosscutting issue in Horizon 2020. Report of the Expert Group on "the Interim Evaluation of Gender Equality as a crosscutting issue in Horizon 2020". European Commission.

Molas-Gallart, J., Tang, P. (2011). Tracing 'productive interactions' to identify social impacts: an example from the social sciences. Research Evaluation 20, 219-226

Nielsen, M.W., Andersen, J.P., Schiebinger, L. and Schneider, J. (2017). One and a half million medical papers reveal a link between author gender and attention to gender and sex analysis. Nature Human Behaviour 1 (11):791-796.

Nowotny, H., Scott, P. and Gibbons, M. (2001). Re-thinking ScienceKnowledge and the Public in an Age of Uncertainty. Polity Press, Cambridge.

Prins, A. (2010). Evaluation Research in Context: the Mechanical Engineering case. Den Haag, ERiC project

Propp, T. and van der Meulen, B. (2010). Evaluation Research in Context: the Electrical Engineering case. Den Haag, ERiC project

Spaapen, J. and van Drooge, L. (2011). Introducing 'productive interactions' in social impact assessment. Research Evaluation 20, 211-218.

Staw, B.M. and Epstein, L.D. (2000). What bandwagons bring: effects of popular management techniques on corporate performance, reputation and CEO pay. Administrative Science Quarterly 26, 501-524.

Van Arensbergen, P., Pen, M., van Drooge, L., Spaapen, J.and van den Besselaar, P. (2010). Evaluation Research in Context: the law case. Den Haag, ERiC project

Van den Besselaar, P., Stout, L., Gou, X. (2016). A linguistic analysis of peer review reports. In Proceedings of the Science Technology Indicators 
Conference (ST/ 2016), Valencia, September 2016.

Van den Besselaar, P., Schiffbaenker, H., Sandström, U. and Mom, C. (2018). Explaining gender bias in ERC grant selection. Proceedings Science and Technology Indicators (STI 2018). Leiden, September 2018

Van den Besselaar, P., Sandström, U. and Schiffbaenker, H. (2018b). Studying grant decision-making: a linguistic analysis of peer review reports. Scientometrics 117, 313-329

Van den Besselaar, P., Khalili, A. and Sandström, U. (2017). Evaluating research portfolios, a method and a case. Proceedings Science and Technology Indicators (STI 2017), Paris, September 2017.

Van den Brink, M., Brouns, M. and Waslander, S. (2006). Does excellence have a gender? A national research study on recruitment and selection procedures for professorial appointments in The Netherlands. Employee Relations 28, 523-539

Van der Meulen, B., Daemen,F., van Drooge, L., de Jong, S., Spaapen, J., Wamelink, F. and van den Besselaar, P. (2010). Evaluation Research in Context: Architecture. Den Haag, ERiC project

Wenneras, C. and Wold, A. (1997). Nepotism and sexism in peer-review. Nature 387 (6631): 341-343.

Wardenaar, T. (2014). Organizing collaborative research: the dynamics and long term effects of multi-actor research programs (PhD thesis Vrije Universiteit Amsterdam). Den Haag: Rathenau Instituut.

\section{PETER VAN DEN BESSELAAR}

Network Institute \& Department of Organization Sciences, Vrije Universiteit Amsterdam

De Boelelaan 1081, Amsterdam, 1081 HV (Netherlands)

E: p.a.a.vanden.besselaar@vu.nl

Teresa Mom Consultancy Itd

Middenweg 203, Amsterdam, 1098 AN (Netherlands)

E: peter@teresamom.com

\section{ULF SANDSTRÖM}

KTH Royal Institute of Technology, Department of Industrial Economics and Management

Lindstedtsvägen 30, Stockholm, 11428 SE (Sweden)

E: ulf.sandstrom@indek.kth.se

\section{AUTHORS}

Endnotes

ERiC stands for "Evaluating Research in Context". It was a project of the Royal Netherlands Academy of Arts and Sciences, The Netherlands Research Council NWO, the Association of Universities, and the Association of Universities of Applied Sciences. The aim was to develop a method for research evaluation that takes into consideration all relevant quality and impact dimensions, scholarly as well as societal and economic.

The SIAMPI project was funded by the European Commission under grant agreement no 230330. SIAMPI means "Social Impact Assessment Methods for research and funding instruments through the study of Productive Interactions between science and society". Partners were the Royal Netherlands Academy of Arts and Sciences (KNAW), CSIC (Spain), MSH (France) and University of Manchester (UK). The SIAMPI consortium developed methods to assess social impact of research projects, research programmes and research funding instruments.

However, the social impact of the SSH may be strongest in interdisciplinary projects, where the social sciences are an important part as these fields focus often on the conditions under which the larger project can have impact. We cannot go into this issue here.

What counts as gender relevant is not further discussed here: we take the classification of the proposals in terms of gender relevance as it was done by the funding organisation (the European Commission). It is necessary to more detailed define what gender (or other societal) relevance means, as discussed above.

Since we only have access to projects in "gender flagged" topics, it is not possible to conduct a more refined analysis that focuses on the differences between 'flagged' and 'non-flagged' topics.

This is not uncommon, also in other domains. For example, organisations advertise themselves with fashionable labels ("Our company aims to empower the employees") even if they do not anything that could count as empowering. Research shows that this indeed has a positive effect on the reputation of those companies (Staw and Epstein 2000).

As this are all funded projects, it would be possible to include more information about the partners, as these are in the proposals. Characteristics of the applicants, such as their earlier work on (in this case) gender relevant topics, might also influence the discussion and scores by the panel. This extension is also for further research. 


\title{
SENIOR ACADEMICS AS KEY NEGOTIATORS IN THE IMPLEMENTATION OF IMPACT POLICIES IN THE SOCIAL SCIENCES AND HUMANITIES
}

\author{
MARC VANHOLSBEECK, THEODOSIA DEMETRIOU, AGNE GIRKONTAITE, ANDREJA ISTENIC STARCIC, VILLE KEISKI, \\ EMANUEL KULCZYCKI, ELENA PAPANASTASIOU, JANNE PÖLÖNEN, HULDA PROPPE AND MAJA VEHOVEC \\ DOI: $10.22163 / f t e v a l .2019 .371$
}

\begin{abstract}
Acknowledgement: This article is based on work from COST Action 15137 European Network for Research Evaluation in the SSH (ENRESSH) and supported by COST (European Cooperation in Science and Techno-
\end{abstract} logy).

$\mathrm{I}$ n this paper, we pursue two main objectives. First, we review the relevant literature and present it according to a theoretical framework that combines structural perspectives and consideration for individual agency, to allow us a better understanding of the role played by senior academics in the social sciences and humanities (SSH) in the implementation of the different policies that concern the production, the dissemination and the evaluation of research, including impact related policies. Indeed the academics' negotiating power of the impact agenda - as it is currently promoted by European policy makers (see e.g. European Commission 2018) and encompasses the impact on policy making, economy as well as the environment and society - cannot be understood in isolation of their perception and attitudes towards the broader political changes that affect the practice of academic research. Secondly we discuss some preliminary results from the interviews we have conducted in the context of the COST ENRESSH action with 16 European senior sociologists active in eight European countries, focusing here on their perceptions and attitudes towards the impact agenda.

\section{A. CHANGES IN THE RESEARCH POLICY MAKING}

Most current research policies and policy agendas in research can be considered, directly or indirectly, in the perspective of a few tendencies that have been initiated or fostered by research policy makers, both at national and European levels, and that concern STEM (Science, Technology, Engineering and Mathematics) as well as - often with some delay - SSH disciplines. We will distinguish between the tendencies towards internationalisation, digitalisation, managerialism, marketisation and "exoterisation" of research (Vanholsbeeck 2016).

Internationalisation relates to the tendency to encourage the production of research contents that focus on global phenomena, or comparisons of national situations, that are published in international journals - mostly in the English language - communicated at international conferences and imply geographical and/or virtual mobility of the researcher. In some disciplines, internationalisation has antedated policy prescriptions, following epistemological motives.

Digitalisation refers to the use of digital tools and media to produce and disseminate research.

Managerialism mostly consists in the adoption of "New Public Management" (NPM) in the administration of research. NPM relates to the introduction into the public sector of a diversity of managing practices and tools from the private sector, with an emphasis on the notions of efficiency, effectiveness, excellence, accountability and standards of performance (Hood 1995; Deem 1998; Enders et al. 2009; Whitley and Gläser 2014). In regards to human resources management, there is a related tendency - which constitutes one of the most important changes in the governance of research - to favour funding modes that combine recurrent with temporary project based funding, in a context of increasing scarcity of research budgets (Gläser and Laudel 2016: 121-122).

Marketisation relates to the tendency to consider universities, researchers and the research outputs themselves in the quasi-market perspective of a competitive knowledge economy, and to reconsider in this perspective the relations between academia and industries.

By the less usual notion of exoterisation we designate the various processes of opening the production, dissemination and evaluation of research outside (exo) of the disciplinary circles of the academic peers. In that respect, European and national level policies have supported the transfer of knowledge from researchers to non-academic stakeholders - in particular to the industry - as well as, more recently, the cocreation by researchers, policy makers, industries and/or citizens alike of solutions to societal challenges, under the influence of programmatic 
ideas such as "mode 2 of knowledge production" (Gibbons et al. 1994) or - in the context of the preparation of the next "European Research and Innovation Framework Programme "Horizon Europe" (2021-2027) - "missions" (Kattel and Mazzucato 2018). The European Open Science agenda ${ }^{1}$ - including open access to publications, open research data and citizen science - and the impact related policies also align with this tendency to exoterisation. The concept of exoterisation is thus broader than marketisation, since it includes social innovation - which can take non-commercial forms - and relates to the notion of knowledge society rather than to the sole knowledge economy.

If there is some degree of convergence between most of the above mentioned trends, the tendencies towards exoterisation and managerialism of research are not (yet) congruent, since performance indicators that are currently in usage in the management of research do not take into account in any significant way the extra-academic impact of research, nor open science practices (0'Carroll et al. 2017; Vanholsbeeck 2017).

\section{B. INSTITUTIONAL AND INDIVIDUAL PERSPECTIVES ON THE ROLE OF SENIOR ACADEMICS}

The role of senior academics in the implementation of research policies and science related political agendas, including the impact agenda, is better appreciated according to two theoretically different but eventually complementary perspectives: the institutionalist and the comprehensive - in the Weberian meaning of the term - approach. The first focuses on the structural determinants that impact individual behaviours, studying institutional pressures on collective organisations (at a meso-sociological level). The second takes the opposite perspective, focusing on the inner motivations and perceptions of individuals as well as to their agency, and dedicates attention on the impact that individual strategies and subjective interactions may have on organisations (at a micro-sociological level).

\section{INSTITUTIONALIST APPROACH}

Institution is a broad social science concept which, in the context of science sociology, can take two main meanings. It refers indeed to the official organisations in which science is practised (i.e.: universities, research centres, research units, academies, etc.), but also to the specific rules, processes and stable usages that weigh on the beliefs and behaviours of those who practice science (Gingras 2017: 29).

\subsection{INSTITUTION AS SCIENTIFIC ORGANISATION}

Of particular interest while considering changes in scientific organisations, the so-called "neo-institutionalist" school of sociology has renewed organisation theory, by focusing on the supra-individual cognitive and cultural factors that explain the social and organisational phenomena (DiMaggio and Powell 1991). Neo-institutionalists developed the concept of isomorphism, which explains why rational actors increase the similarity of organisations that have emerged as a certain field or domain, while trying to change them (DiMaggio and Powell 1983). Conceptual distinction has been made between coercive isomorphism - involving pressures from other organisations on which the organisation depends as well as social expectations surrounding them - mimetic isomorphism - consisting into an organisation imitating another organisation's structure because of the belief that such imitative process will be beneficial - and normative isomorphic process, relating to professional norms that span organisations belonging to the same field.

These three types of isomorphism are to some degree at work in contemporary academia, fostering similar moves towards internationalisation, marketisation and managerialism.

First, forms of coercive isomorphism can be found in the driving effect that performance quantitative indicators have on the practices they try to measure, in SSH research evaluation like in other areas of social life (as expressed for example in Campbell's or Goodhart's laws, according to which a measure ceases to be a good measure once it becomes a target). In particular, bibliometric indicators are increasingly used, both at European (Vanholsbeeck 2017) and national level (e.g. Hammarfelt et al. 2016; Pölönen and Wahlfors 2016), to benchmark national science systems and universities, but also to assess - and provide funding to - individuals and projects (De Rijcke et al. 2016; Gläser and Laudel 2016). Usually developed by private companies - such as the infamous Impact Factor (now provided by Clarivate Analytics) - bibliometrics is mostly based on international databases of scholarly journals. As such, they directly or indirectly coerce researchers in their publishing habits. They contribute to the rising proportion of the share of SSH publications that take the form of articles published, in English, in international journals (Hammarfelt and de Rijcke 2015; Kulczycki et al. 2018), even if any strictly causal ascription of the effects of a given research policy on research contents has to be considered with caution, because of the many confounding variables which are to consider (Gläser and Laudel 2016)2. Furthermore, some evaluation systems still take books and publications aimed at professional and general audiences into account (GiménezToledo et al. 2016).

Second, world university rankings which are in a significant part based on bibliometric indicators have become increasingly important in the last decade, not least due to their media exposure. Often produced by non-academic organisations, they exert some influence on universities around the world, promoting a global model of "world-class universities" worth following (mimetic isomorphism).

2 For example, it has been shown that the decrease in share of publications published in Finland, which is also partially indicative of publication language, is attested in the national publication statistics since 1994, well before the performance based funding model was established in Finland (Auranen and Pölönen 2014) 
Finally, efforts to standardise higher education - including third cycle and researchers' training - notably via the Bologna Process, contribute to some normative isomorphism within academia.

\section{ORGANISATIONS' REACTION TO INSTITUTIONAL PRESSURES}

In a meta-analysis of neo-institutional approaches and resource dependence theories, Oliver (1991) brought important nuances to the concept of isomorphism, and to the idea that organisations conform to the pressures of their institutional environment, benefitting from adhering to external rules and norms. She proposed a more nuanced typology of strategic responses to institutional process and active organisational behaviours that vary from passive conformity to active resistance, taking the form of acquiescence, compromise, avoidance, defiance or manipulation.

Relying on Oliver's typology and applying it to research organisations, Leisyte (2007) studied the effects of governance models on the research practices of research units in the fields of medieval history and biotechnology. She makes the distinction between three organisational strategies towards managerialism: passive compliance, symbolic compliance - a combination of acquiescence and avoidance consisting in pretension of compliance, but changing nothing to the way research is performed - and proactive manipulation of the rules and norms of the institutional environment.

It has to be noticed that local specificities have to be taken into account while considering the effect of managerialism on organisations and individuals alike (Stöckelová 2012). In particular, in former European socialist countries, the introduction of managerialism has accompanied a process of de- and re-politicisation (Linková and Stöckelová 2012).

\subsection{INSTITUTION AS A SET OF SOCIO-PROFESSIONAL VALUES}

According to the second of the abovementioned institutional definitions, the institution of science designates the specific social system of science. As such scientists are not only exposed to rules, processes and stable usages coming from the non-academic world, but also produce their very own socio-professional values, that span the boundaries of the organisations by whom they are employed.

In this perspective, the professional values of science have been analysed as a potential source of resistance to organisational changes in universities (Chandler et al. 2002; Kirkpatrick and Ackroyd 2003). Indeed, the values to which scientists adhere - such as academic freedom or the ones identified by Merton (1973), of communalism, universalism, disinterestedness and organised scepticism - may diverge from those that are supported by research policies in general, and by new public management in particular.

The discrepancies between the values of the scientific institution and the management of the scientific organisation may even create a clash between (internal) professional accountability, based on professional values, and (external) managerial accountability, based on managerial norms and processes (Linková and Stöckelová 2012). Hence some scientists engage in double allegiance: they "rarely seem to see themse/ves first and foremost as organisational members. Their allegiance is primarily to the disciplines or the institution of science, rather than its organisations. [...] They may even treat the universities, departments and institutes they are part of as irritations, a collection of performance indicators and management demands which threaten to get in the way of real science" (Davies and Horst 2016: 65).

In the same professional perspective, the relationships between senior researchers and PhD candidates - although little research has been carried out that focuses on PhD directors' reactions to the changing context of $\mathrm{PhD}$ education - constitute another place where professional values may conflict with organisational processes (Deuchar 2008; Bøgelund 2015).

It should be noted though that the dominant bibliometric performance indicators that are currently used in the new public management of research are still linked to the primary professional activity of academia. They mostly relate indeed to the production and citation of articles in scientific papers, and not to the engagement of the researchers in their organisation. Hence it can be argued that those indicators, although often criticised, are not entirely foreign to some core academic professional values.

\section{COMPREHENSIVE APPROACH}

Institutionalist perspectives should be combined with the analysis of the perceptions and attitudes of the individual researchers, with due consideration to their agency. Indeed scientific organisations provide individual scholars with a certain power to "negotiate" higher education and research policies (Linkova 2014), not the least because European universities do generally enjoy a high level of institutional autonomy, while the professional norm of academic freedom prevails in European higher education. Furthermore, some academics are active as full or parttime administrators in their institution, without being per se in an administrative career path. Senior academics are also those mostly in charge of leading a research team and training early stage researchers, assuring their professional socialisation.

The "comprehensive" literature that we reviewed in the context of this COST ENRESSH project resorts to a diversity of theoretical frameworks and concepts, some of them even referring to neo-institutionalism (e.g. Lam 2010 or Teelken 2011). Theoretical framing notwithstanding, most results tend to emphasise the ambivalent attitudes of researchers towards the abovementioned tendencies of marketisation, managerialism and exoterisation of research, bringing out at the individual level a similar attitude of "symbolic compliance" to the one that had been observed at the institutional level.

\section{REACTIONS TO MANAGERIALISM}

Most studies we reviewed concentrate on the scholars' reaction to managerialism, with an early focus on the UK situation. Already in 2001, it was contended that managerialism was not entirely embedded in UK universities, and that middle and junior level academics actively keep professional academic values alive and moderate the harsher effects of the changes (Barry et al. 2001). Deem (2003) has argued that the attitude towards managerialism of UK academic administrators varies depending on their intention to return later to teaching and research role. Those who intend to go back to primary academic tasks mitigate the new managerial language and keep some core professional values. Studying the 
negotiation of evolving research policies by UK life scientists, Morris and Rip (2006) similarly underlined that scientists develop more or less proactive strategies to modulate the impact of changing research policies.

Regarding SSH disciplines, Kehm and Leiðytë, on the basis of interviews with researchers in English medieval history units, also showed that they "try to find a balance between their own research agenda and the research priorities of the funding bodies [...]. They do so by following largely symbolic compliance strategies - maintaining their own research lines and at the same time selling their research interests according to the priorities of the external research funders" (Kehm and Leiðytë 2010: 80). Teelken (2011) analysed the individual behaviours of 48 academic and support staff members at ten universities in the Netherlands, Sweden and the UK, in faculties of social sciences and economics/business studies. The research shows that academics dissociate themselves from the managerial prescriptions, and appear to be only loosely coupled from their organisations, even if beside symbolic compliance and professional pragmatism (dealing with the managerial prescriptions "in a critical but serious manner"), an attitude of "formal instrumentality" is also observed (Teelken 2011: 278). Respondents do not consider assessment as such as undesirable, but are critical of the increasingly quantitative and time-consuming performance based assessment, as well as the growing competition for research funding. From interviews conducted with communication scholars in French speaking Belgium, Vanholsbeeck (2012) similarly concluded that those researchers, rather than fully accepting or resisting to the prescriptions that support the publication of (many) papers in international journals, are rather ambivalent towards the prescribed quality requirements. Some of them "tinker" with these prescriptions, trying to publish according to the (perceived) prescriptions, while still allowing time for publishing according to their very own definition of quality.

Focusing on the use of bibliometrics for evaluation purposes in Dutch law faculties, micro-politics of indicator use have also been revealed, through which scholars in advanced administrative positions try to proactively pursue "competing normative and epistemic agendas" rather than passively reacting to externally-imposed administrative procedures (Kaltenbrunner and de Rijcke 2016: 284). Comparably, Finnish and Norwegian universities use the national publication channel-based quality indicator for assessing individual academics, more particularly in the humanities. As such, the indicator is used as a replacement for publication counts, in lack of alternative indicators such as the Impact Factor in the SSH fields (Pölönen and Wahlfors 2016). Also in Finland, research on SSH researchers ( $\mathrm{N}=92$ ) has shown that the introduction of the new performance based funding model has involved what the authors call the "publication laundering" (in Finnish "julkaisupesu"), meaning the manipulation of publication lists to meet the standards, e.g. peer-review, of measured performances (Sivula et al. 2015: 153).

Some authors even contend that there are attitudes of real resistance, and not only of symbolic compliance, from academics to managerialism. Clegg maintains that academics do resist managerialism, albeit passively and individually, by creating spaces for the exercise of "principled personal autonomy and agency" which allow them to develop "their own ways of practising and a personal sphere of meaning" in which they can practise with integrity (Clegg 2008: 343). Similarly, Anderson finds that the resistance of Australian academics takes many forms and follows every day and covert discursive strategies, considering "academics' capacity - indeed, their perceived responsibility - to assess, analyse and criticize" as well as deeming them as particularly "skilled in rebellion and innovation" (Anderson 2008: 256 and 267). On the basis of interviews with Austrian historians, Kehm and Leiðytë (2010) identified a generation gap, senior researchers being more prone to resistance than early career academics who may have been professionally socialised in the new managerial context. Linková, studying the responses of Czech researchers in the humanities, social sciences, and natural sciences to research assessment, found that some academics engage daily in micro-politics of resistance and critiques which "are located within traditional 'Science' values stressing autonomy and peer judgment on the one hand or individual performance, primacy and competitiveness on the other" (Linkova 2014: 85-86). By doing so, they rely on traditional scientific values and stress autonomy and peer judgment, even if, overall, researchers show adaptation to the new types of governmentality.

The last category of articles we reviewed considers on the contrary that academics mostly - and not only symbolically - comply with the new managerial processes, and that resistance, whenever it happens, is essentially ideological or discursive, only a minority resisting actively. Those are the conclusions that Clarke, Knights and Jarvis (2012) have reached on the basis of their 48 interviews with British business school academics. Leathwood and Read (2013), as well as Ylijoki and Ursin (2013), made similar conclusions, respectively in regards to the British and Finnish academics they interviewed. A recent study of the introduction of performance appraisals in a regional Australian university showed little resistance either from academics' side, early career academics being particularly compliant with the new prescriptions. (Kalfa et al. 2018).

\section{MARKETISATION}

In regards to the tendency to the marketisation of research, it has been argued, on the basis of focused interviews with senior researchers in three different types of research settings in Finland (departments of History and Surface Science and Semiconductor Technology; Work Research Centre), that their engagement in "academic capitalism" depends on how close their field is from the market (Ylijoki 2003). The study shows that researchers try to accommodate traditional academic practices and values to more entrepreneurial activities, under the pressure of working increasingly on short-term contracts and projects. Similarly, a study based on 36 interviews and a survey of 734 academic scientists from five UK research universities shows the active agency of academics in the shaping of the relationships between science and business (Lam 2010). Most academics exploit the ambiguities of "boundary work" between academia and industry, rather than being entirely "traditional" or "entrepreneurial".

\section{ATTITUDES TOWARDS EXTRA-ACADEMIC IMPACT}

As far as we know, there is no dedicated research on the researchers' perception of impact policies as such, wherever such policies do exist in an explicit form. In their abovementioned study of the effects of the managerialism on research, Kehm and Leiðytë (2010) showed that the prescriptions to publish for a broader public, combined to the prescriptions in favour of more interdisciplinary research, have affected the research topics on which German medieval historians are working. Furthermore, the findings of Smith (2010) suggest that the growing pressure to 
produce policy relevant research in health inequality is diminishing the autonomy and creativity of sociologists, and is instead promoting the construction of institutionalised and vehicular ideas.

Other studies focus on the perception of scholars on science communication, public engagement and valorisation of research. In their review of past studies and surveys on how scientists view the public, the goals of communication, the performance and impacts of the media, as well as the role of the public in policy decision-making, Besley and Nisbet (2013) have argued that scientists consider the public as generally uninformed about sciences. They are critical of media coverage but believe that interactions with journalists are important for promoting science literacy as well as career advancement, policy makers being considered as the most important external stakeholders to engage with. Furthermore, on the basis of parallel surveys of scientists from multiple scientific societies, the most consistent predictors of willingness to take part in public engagement activities are a belief that the experience will be enjoyable and make a difference, as well as the time available to engage (Besley et al. 2018). Age, sex, scientific field but also the researcher's perception of the public, of her peers and of her personal engagement skills are inconsistent predictors.

Finally, a survey conducted in Belgium on higher education institutions of the Brussels Capital Region ( $N=727)$ showed that one respondent on two has experience in valorisation (Dobbels et al. 2015). The vast majority of respondents in SSH were concerned by social valorisation rather than economic valorisation - which is the main focus of knowledge transfer policies of the Brussels Region - contrarily to their peers in the exact and applied sciences. A majority of researchers agreed that researchers should contribute to valorisation, although $62 \%$ of the respondents consider that academics should remain free to valorise or not. Mentioned obstacles are the lack of time (85\%), lack of skills or dedicated funding (64\%) as well as lack of reward (60\%). Valorisation is perceived like a personal affair, rather than a professional opportunity or necessity.

\section{DISCUSSION OF EXPLORATORY RESULTS}

We conducted 16 semi-structured interviews with senior researchers in sociology, having earned their PhD for at least eight years and active in Belgium, Croatia, Cyprus, Finland, Iceland, Lithuania, Poland and Slovenia. We interviewed them about their perceived roles in the definition, the dissemination and the implementation of the quality criteria and rationales that are to be used in evaluation situations. In particular, we wanted to know to what extent they consider it important that impact is taken into account in the evaluation of SSH research. We had previously agreed on a broad definition of impact, considering it as the result of all kinds of "productive interactions" (Spaapen and Van Drooge 2011) through which researchers engage with all kinds of non-academic publics.

Even if we will bring further nuances and developments to the analysis in a future publication, it is already possible to contend that our results do not essentially contradict the most important conclusions from the studies we reviewed above. In particular we have found that inter- viewees perceive the same isomorphic evolutions of higher education towards managerialism and internationalisation, and many also share to some degree an attitude of symbolic compliance with the related prescriptions.

In regards to our interviewees' perception and attitudes towards the impact agenda, it is quite clear that the impact agenda is not perceived as having currently any direct and significant incidence on their professional life. The real pressure is obviously on producing more papers, in the English language, in international journals, rather than on getting more interactions with the non-academic world. In some countries there is even a recent and very strong focus on the use of bibliometrics in SSH research assessment (e.g. Croatia, Poland), although dedicated funding tools for supporting "impacting" SSH research have also been put in other places (Belgium). Quantitative performance based evaluation of research is mostly perceived as being inconsistent with any stronger engagement in impact related activities, which some respondents associate with local research (and publications in vernacular language) and perceive as harder to properly quantify (Lithuania). In some cases (like in Slovenia), past evaluation processes involving general public in the evaluation process to higher extent may have been associated with more societally impacting research policies than what is currently the case.

However, some interviewees mention that it is still possible to reward - even if slightly - impact in the assessment (like in Finland or in Iceland) or that it may even be feasible in some cases to pursue a "parallel career" in academia, based on media engagement and the conduct of more operational research. "Open Science" (OS) and "Open Access" (OA) are not considered as priorities (at all) and some interviewees even perceive $\mathrm{OA}$ journals as being of a lower quality and/or reputation, or even as fostering the prevailing science system. One Belgian respondent underlines though that institutional OA repositories do allow the dissemination of a diversity of research outputs - beside scholarly articles - including those who may impact society.

In one Belgian researcher's perspective, impact should not be considered only in an instrumental perspective, but relies on the sociologists' duty to "engage in the city" in a scientifically informed but also critical way. An Icelandic respondent considers that interacting with the media is an intrinsic part of his academic job.

Finally, we would like to emphasise that several researchers - in particular those who do not have responsibilities in administrative areas (Cyprus) or do not belong to the new academic generation (Croatia) - wish that assessment takes better impact-related endeavours into account. As one of our Croatian respondents told us: "The responsibility of science is towards society and the community as they are funding us, and not just our personal scientific career or our motives. This is part of our social responsibility of being scientists. Often our scientific results have no impact. Nevertheless, it is our responsibility to interpret social processes even when we feel that our notions have no resonance. It is our responsibility to interpret social processes and try to be convincing, even through nonscientific publications such as policy documents or the like" ${ }^{\text {"3. }}$ 


\section{REFERENCES}

Anderson, G. (2008). Mapping academic resistance in the managerial university. Organization, 15(2), 251-270.

Auranen, 0., and Pölönen, J. (2014). "Julkaisufoorumi-luokitus ja kansallinen julkaiseminen" (Publication Forum rating and national publishing). In R. Muhonen and H.-M. Puuska (Eds.), Tutkimuksen kansallinen tehtävä. Tampere: Vastapaino, 153-175.

Barry, J., Chandler, J. and Clark, H. (2001). Between the ivory tower and the academic assembly line. Journal of Management Studies, 38(1), 87-101.

Besley, J. C., Dudo, A., Yuan, S. and Lawrence, F. (2018). Understanding scientists' willingness to engage. Science Communication, 40(5), 559-590.

Besley, J. C. and Nisbet, M. (2013). How scientists view the public, the media and the political process. Public Understanding of Science, 22(6), 644-659.

Bøgelund, P. (2015). How supervisors perceive PhD supervision-And how they practice it. International Journal of Doctoral Studies, 10(1), 39-55.

Chandler, J., Barry, J. and Clark, H. (2002). Stressing academe: The wear and tear of the new public management. Human Relations, 55(9), 1051-69.

Clarke, C., Knights, D., and Jarvis, C. (2012). A labour of love? Academics in business schools. Scandinavian Journal of Management, 28(1), 5-15.

Clegg, S. (2008). Academic identities under threat?. British Educational Research Journal, 34(3), 329-345.

European Commission (2018). Communication from the Commission to the European Parliament, the Council, the European Economic and Social Committee and the Committee and the Committee of the Regions: Horizon 2020 interim evaluation: maximising the impact of EU research and innovation. 11/01/2018.

Davies, S. R. and Horst, M. (2016). Science Communication: Culture, Identity and Citizenship. Springer.

Deem, R. (1998). 'New managerialism' and higher education: The management of performances and cultures in universities in the United Kingdom. International Studies in Sociology of Education, 8, 47-70.

Deem, R. (2003). "New managerialism in UK universities: manager-academic accounts of change". In H. Eggins (Ed.), Globalization and reform in Higher Education. Berkshire : Open University Press, 55-67.

De Rijcke, S., Wouters, P. F., Rushforth, A. D., Franssen, T. P. and Hammarfelt, B. (2016). Evaluation practices and effects of indicator use - a literature review. Research Evaluation, 26(2), 161-169.

Deuchar, R. (2008). Facilitator, director or critical friend?: Contradiction and congruence in doctoral supervision styles. Teaching in Higher Education, 13(4), 489-500.
DiMaggio, P. J. and Powell, W. W. (1983). The iron cage revisited: institutional isomorphism and collective rationality in organizational fields. American Sociological Review 48(2), 147-160.

DiMaggio, P. J. and Powell, W. W. (Eds.) (1991). The New Institutionalism in Organizational Analysis. Chicago: University of Chicago Press.

Dobbels, J., Kesbeke, W. and Ysebaert, W. (2015). Hoe onderzoekers werkelijk denken over valoriseren. THandMA, 1: 93-97.

Enders, J., De Boer, H. and Leišytè, L. (2009). "New Public Management and the Academic Profession: the Rationalisation of Academic Work Revisited". In J. Enders, and E. de Weert (Eds.), The Changing Face of Academic Life: analytical and comparative perspectives. New York : Palgrave-Macmillan, 36-57.

Gibbons, M., Limoges, C., Nowotny, H., Schwartzman, S., Scott, P. and Trow, M. (1994). The new production of knowledge: The dynamics of science and research in contemporary societies. London: Sage.

Giménez-Toledo, E., Mañana-Rodríguez, J., Engels, T. C. E., Ingwersen, P., Pölönen, J., Sivertsen, G., Verleysen, F. T. and Zuccala, A. A. (2016). Taking scholarly books into account: Current developments in five European countries. Scientometrics, 107(2), 685-699.

Gingras, Y. (2017). Sociologie des sciences. Presses universitaires de France.

Gläser, J. and Laudel, G. (2016). Governing science: how science policy shapes research content. European Journal of sociology/Archives Européennes de sociologie, 57(1), 117-168.

Hammarfelt, B. and de Rijcke, S. (2015). Accountability in context: effects of research evaluation systems on publication practices, disciplinary norms, and individual working routines in the faculty of Arts at Uppsala University. Research Evaluation, 24(1), 63-77.

Hammarfelt, B., Nelhans, G., Eklund, P. and Åström, F. (2016). The heterogeneous landscape of bibliometric indicators. Evaluating models for allocating resources at Swedish universities. Research Evaluation, 25(3), 292-305.

Hood, C. 1995. The 'new public management' in the 1980s: Variations on a theme. Accounting, Organizations and Society, 20, 93-109.

Jiménez-Contreras, E., de Moya Anegón, F. and López-Cózar, E. D. (2003). The evolution of research activity in Spain: The impact of the National Commission for the Evaluation of Research Activity (CNEAI). Research policy, 32(1), 123-142.

Kalfa, S., Wilkinson, A. and Gollan, P. J. (2018). The academic game: Compliance and resistance in universities. Work, Employment and Society, 32(2), 274-291.

Kaltenbrunner, W. and de Rijcke, S. (2017). Quantifying 'Output' for Evaluation: Administrative Knowledge Politics and Changing Epistemic Cultures in Dutch Law Faculties. Science and Public Policy, 44(2), 284-293. 
Kattel, R. and Mazzucato, M. (2018). Mission-oriented innovation policy and dynamic capabilities in the public sector. Industrial and Corporate Change, 27(5), 787-801.

Kehm, B. M. and Leiðytë, L. (2010). “Effects of New Governance on Research in the Humanities -The Example of Medieval History". In D. Jansen (Ed.), Governance and Performance in the German, Public Research Sector. Dordrecht: Springer, 73-90.

Kirkpatrick, I. and Ackroyd, S. (2003). Transforming the professional archetype? The new managerialism in UK social services. Public Management Review, 5(4), 511-531.

Kulczycki, E., Engels, T. C., Pölönen, J., Bruun, K., Dušková, M., Guns, R., Nowotniak, R., Petr, M., Sivertsen, G., IsteniĐ StarĐiĐ, A. and Zuccala, A. (2018). Publication patterns in the social sciences and humanities: evidence from eight European countries. Scientometrics, 116(1), 463-486.

Lam, A. (2010). From "ivory tower traditionalists" to "entrepreneurial scientists"? Academic scientists in fuzzy university-industry boundaries. Social Studies of Science, 40(2), 307-340.

Leathwood, C. and Read, B. (2013). Research policy and academic performativity: compliance, contestation and complicity. Studies in Higher Education, 38(8), 1162-1174.

Lee, A. (2008). How are doctoral students supervised? Concepts of doctoral research supervision. Studies in Higher Education, 33(3), 267-281.

Leisyte, L. (2007). University governance and academic research: Case studies of research units in Dutch and English universities. PhD thesis, University Twente.

Linková, M. (2014). Unable to resist: Researchers' responses to research assessment in the Czech Republic. Human Affairs, 24(1), 78-88.

Linková, M. and Stöckelová, T. (2012). Public accountability and the politicization of science: The peculiar journey of Czech research assessment. Science and Public Policy, 39(5), 618-629.

Merton, R. K. (1973) [1942]. "The Normative Structure of Science". In R.K. Merton, The Sociology of Science: Theoretical and Empirical Investigations. Chicago: University of Chicago Press, 267-278.

Morris, N. and Rip, A. (2006). Scientists' coping strategies in an evolving research system: The case of life scientists in the UK. Science and Public Policy, 33(4), 253-263.

O'Carroll, C., Rentier, B., Cabello Valdès, C., Esposito, F., Kaunismaa, E., Maas, K., Metcalfe, J., McAllister, D. and Vandevelde, K. (Eds.) (2017). Evaluation of Research Careers fully acknowledging Open Science Practices. Rewards, incentives and/or recognition for researchers practicing Open Science. Publication Office of the European Union.

Oliver, C. (1991). Strategic responses to institutional processes. Academy of management review, 16(1), 145-179.
Pölönen, J. and Wahlfors, L. (2016). "Local use of a national rating of publication channels in Finnish universities". Poster presented at the 21st Nordic Workshop on Bibliometrics and Research Policy, Copenhagen, 3.-4.11.2016. URL: https://figshare.com/articles/Local_Use_of_a_ National_Rating_of_Publication_Channels_in_Finnish_Universities_ NWB_2016_poster_/4246541.

Sivula, A., Suominen, J. and Reunanen, M. (2015). A1 alkuperäisartikkeli tieteellisessä aikakauslehdessä. Uusien julkaisukäytänteiden omaksuminen ihmistieteissä 2000-luvulla. Kasvatus and Aika, 9(3), 149-171.

Smith, K. (2010). Research, Policy and Funding - Academic Treadmills and the Squeeze on Intellectual Spaces. The British Journal of Sociology, 61 (1), 176-195.

Spaapen, J. and Van Drooge, L. (2011). Introducing 'productive interactions' in social impact assessment. Research Evaluation, 20(3), 211-218.

Stöckelová, T. (2012). Immutable mobiles derailed: STS and the epistemic geopolitics of research assessment. Science, Technology and Human Values, 37(2), 286-311.

Teelken, C. (2011). Compliance or pragmatism: how do academics deal with managerialism in higher education? A comparative study in three countries. Studies in Higher Education, 37(3), 271-290.

Vanholsbeeck, M. (2012). Entre qualité prescrite et qualité souhaitable. Quaderni, 77, 71-84.

Vanholsbeeck, M. (2016). La notion de "qualité» des publications dans l'évaluation de la recherche et des chercheurs en sciences humaines et sociales: Le potentiel de l'Open Access pour dépasser le paradoxe des prescriptions en matière de qualité et l'ambivalence de leur perception par les chercheurs en sciences de la communication. PhD thesis, Université Libre de Bruxelles.

Vanholsbeeck, M. (2017). La notion de Science Ouverte dans l'Espace européen de la recherche: Entre tendances à l'«exotérisation» et à la "gestionnarisation» de la recherche scientifique. Revue française des sciences de l'information et de la communication, 11.

Weingart, P. (2005). Impact of bibliometrics upon the science system: Inadvertent consequences?. Scientometrics, 62(1), 117-131.

Whitley, R. and Gläser, J. (2014). The impact of institutional reforms on the nature of universities as organisations. In R. Whitley, and J. Gläser (Eds.), Organizational Transformation and Scientific Change: The Impact of Institutional Restructuring on Universities and Intellectual Innovation. Emerald Group Publishing Limited, 19-49.

Ylijoki, 0.-H. (2003). Entangled in academic capitalism? A case-study on changing ideals and practices of university research. Higher Education, 45, 307-35.

Ylijoki, 0.-H. and Ursin, J. (2013). The construction of academic identity in the changes of Finnish higher education. Studies in Higher Education, 38(8), 1135-1149. 


\section{AUTHORS}

\section{MARC VANHOLSBEECK}

Department of Information and Communication Studies, Université Libre de Bruxelles

30 Depage Avenue (level 11), Brussels, 1050 (Belgium)

E: mvholsbe@ulb.ac.be

\section{THEODOSIA DEMETRIOU}

Department of Education, 4

6 Makedonitissas Avenue, CY-2417, P.0.Box 24005, Nicosia, 1700

(Cyprus)

E: demetriou.th@unic.ac.cy

\section{AGNE GIRKONTAITE}

Department of Sociology, Vilnius University

Universiteto str. 9, room 309, Vilnius, 01513 (Lithuania)

E: agne.girkontaite@fsf.vu.It

\section{ANDREJA ISTENIC STARCIC}

University of Primorska, Faculty of Education

Cankarjeva 5, Koper, 6000 (Slovenia)

University of Ljubljana, Faculty of Civil and Geodetic Engineering

Jamova 2, Ljubljana, 1000 (Slovenia)

E: andreja.starcic@gmail.com

\section{VILLE KEISKI}

University of Jyväskylä, Department of Social Sciences and Philosophy, Opinkivi, Keskussairaalantie 2, P0 Box 35, 40014 (Finland)

E: ville.keiski@jyu.fi

\section{EMANUEL KULCZYCKI}

Scholarly Communication Research Group, Adam Mickiewicz University, Szamarzewskiego 89c, Poznań, 60-568 (Poland)

E: emek@amu.edu.pl

\section{ELENA PAPANASTASIOU}

School of Education, Department of Education

46 Makedonitissas Avenue, CY-2417, P.0.Box 24005, Nicosia, 1700

(Cyprus)

E: papanastasiou.e@unic.ac.cy

\section{JANNE PÖLÖNEN}

Publication Forum, Federation of Finnish Learned Societies

Snellmaninkatu 13, Helsinki, 00170 (Finland)

E: janne.polonen@tsv.fi

\section{HULDA PROPPE}

Háskóli Islands / University of Iceland, Gimli

Sæmundargata 2, Reykjavi, 101 (Iceland)

E: hproppe@hi.is

\section{MAJA VEHOVEC}

Institute of Economics

Trg J. F. Kennedyja 7, Zagreb, 10000 (Croatia)

E: mvehovec@eizg.hr 


\title{
INCLUSIVE FUNDING FOR ENHANCED IMPACT OF SOCIAL SCIENCES AND HUMANITIES
}

\author{
MALIN LINDBERG, SVETLANA GROSS, MILDA RÖNN, LISSA NORDIN, JAN SANDRED, LARS WÄRNGÅRD AND \\ CATHARINA NORBERG
}

DOI: $10.22163 /$ fteval.2019.372

\section{ABSTRACT}

I n order to expand the knowledge on how societal impact of social sciences and humanities (SSH) can be enhanced through public funding of research and innovation, a process of designing digitalised tools for inclusive funding is scrutinised, involving four Swedish funding agencies and an Non-Governmental Organisation (NGO) of SSH researchers. The agencies shared the challenge to attract a broader range of SSH researchers to apply for their funding. Excluding and including mechanisms were identified in the interactions, texts and images of the formulation, communication and processing of calls for funding. The developed tools digitally guide the user through queries regarding the present and potential diversity of SSH representation among applicants, reviewers, agency staff, etc. and regarding the formulation and communication of call texts, assessment criteria and reviewer instructions. The tools thus enhance SSH impact by making funding more available, but fail to demonstrate how concrete interaction with societal actors may enhance this.

\section{INTRODUCTION}

Despite the widely acknowledged importance of social sciences and humanities (SSH) for understanding and enhancing societal development, public support structures for knowledge transfer, innovation and impact of research in society have traditionally focused natural sciences, engineering and technology (SET) (Brundenius et al., 2016; Olmos Peñuela et al, 2014; Wutti and Hayden, 2017). This is part of a more encompassing pattern, where also policy and research on innovation and growth have focused industrial, technological and commercial renewal rather than social transformation (cf. Dawson and Daniel, 2010; Godin, 2014; Lindberg, 2012, 2018; van der Have and Rubalcaba, 2016). The importance of SSH research for innovation and impact is however increasingly emphasised in EU policy strategies on "Science with and for Society" (SwafS), "Responsible Research and Innovation" (RRI), "Open Science", etc. (European Union, 2014, 2016, 2017). This is accompanied by a rapidly increasing academic interest in social innovation, with reference to new figurations or combinations of social practices that meet social needs, where SSH knowledge is esteemed as pivotal (Brandsen et al., 2016; Brundenius et al., 2016; Howaldt et al., 2018; Moulaert et al., 2013; Nicholls et al., 2015).
As a consequence of this trend in policy and research, public funding agencies are expected to improve their support of SSH impact. In Sweden, four national funding agencies joined forces with a national NGO of SSH researchers in the design of a set of digital tools for inclusive funding of research and innovation (R\&I). The process that took place 2014-2018, is in this paper used as a springboard for expanding the knowledge on how public Ryl funding may be designed in order to enhance SSH impact. The main research question concerns what excluding and including mechanisms towards SSH researchers that were delineated in the process, and how these mechanisms impacted the design of digital tools for inclusive funding. Previous studies on academic impact support serve to theoretically contextualise these mechanisms and tools. A participatory research approach, where new knowledge is developed jointly by researchers and other societal actors, serves to ensure the social contextualisation of the process and results.

Initially, the theoretical framework of academic impact support is presented. This is followed by an outline of the participatory research design. Subsequently, the results are presented in terms of identified mechanisms of inclusion and exclusion, and their impact on the tool design. Finally, conclusions are drawn regarding how digital tools for inclusive funding may be designed in a way that enhances SSH impact.

\section{IMPACT SUPPORT}

In Sweden and several other European countries, public support services for knowledge transfer, innovation and impact are offered by Ryl funding agencies, university innovation offices, technology transfer offices, academic incubators, science parks, etc. These institutions generally provide financial and/or non-financial support in terms of grants, loans, investments, business counselling, peer-to-peer support, networks, testbeds, training, lectures, etc. As most of these support services traditionally target researchers and innovators in technology, engineering and natural sciences, needs within social sciences and humanities are insufficiently met (cf. Bakhshi et al., 2008; Brundenius et al., 2016; Dawson and Daniel, 2010; Howaldt et al., 2018; Lindberg, 2012, 2018; Lindberg and Nahnfeldt, 2013; Lundström and Zhou, 2011; Muhonen et al., 2018; Olmos Peñuela et al., 2014; Phipps et al., 2012; TEPSIE, 2012, 2014; Wutti and Hayden, 2017). Studies have identified both environmental barriers - e.g. lack of support structures in terms of funding, counselling, 
networks, etc. - and actor-related barriers - e.g. lack of capacities and competencies - for realising and growing innovations with prominent social motives and components (Brandsen et al., 2016; Howaldt et al., 2018; TEPSIE, 2012, 2014). This reflects the "reasonably settled consensus within the innovation community that science, engineering and technology (STEM) research is more 'useful' to societies than other types of research, notably social sciences and humanities (SSH) research", noted by Olmos Peñuela et al. (2014:384). The further note that by seeking to "increase and concentrate (RqI) funding on areas that bring the greatest, narrowly economic return", governments tend to regard SSH research as "not worthy of investment" (ibid:385).

SSH do however matter in societal progress, "because they help us understand and address wicked problems (...) about which there is little agreement on solutions", according to Phipps et al. (2012:167). It is advocated that SSH provide insights into - and innovative solutions to - current societal challenges of poverty, immigration, climate change, security, health, etc. (Bakhshi et al., 2008; Lindberg and Nahnfeldt, 2013; Phipps et al., 2012; Wutti and Hayden, 2017). Traditional impact indicators of patents, licenses and spin-off companies are thus too narrow for estimating SSH impact (Lundström and Zhou, 2011; Muhonen et al., 2018; Olmos Peñuela et al., 2014; Wutti and Hayden, 2017). SSH have a well-documented tradition of engagement practices towards users, thus producing results that these users value, but that may be difficult to measure in terms of macro-economic impact (Olmos Peñuela et al., 2014). A study of 1600 Spanish researchers exposes that SSH achieve its impact not primarily by direct interaction with businesses, but by indirect interaction through creating content for the media, and by cooperating more directly with government and civil society organisations to improve the quality of life (ibid). An Austrian study similarly detects SSH impact in terms of transmission of academic knowledge into professional practice and public spheres, not primarily seeking to obtain profits, but rather raising consciousness (Wutti and Hayden, 2017). Based on a comparative analysis of 60 examples from 16 European countries, Muhonen et al. (2018) proposes a framework for estimating impact that considers both societal interaction and the societal changes it enhances. These examples highlight SSH impact through "social innovation", i.e. the development of new figurations or combinations of social practices that meet social needs (Brandsen et al., 2016; Grimm et al., 2013; Howaldt et al., 2018; Moulaert et al., 2013; Nicholls et al., 2015; Phipps et al., 2012).

The public and academic interest in social innovation has rapidly grown during the last decade, as a way to handle complex societal challenges (Brandsen et al., 2016; Grimm et al., 2013; Howaldt et al., 2018; Moulaert et al., 2013; Nicholls et al., 2015). This has served to consolidate social innovation studies as a multi-disciplinary research field, providing insights into the development of new solutions for improved welfare, wellbeing and relations among various groups and communities, especially those perceived as economically or socially vulnerable (Cajaiba-Santana, 2013; Dawson and Daniel, 2010; Haxeltine et al., 2017; Ionescu, 2015; Pol and Ville, 2009; van der Have and Rubalcaba, 2016). According to several studies, social innovation is characterised by active involvement of those groups that are to benefit from the developed solutions, making individual and collective empowerment a crucial component of such processes (Brandsen et al., 2016; Howaldt et al., 2018; Moulaert et al., 2013; Nicholls et al., 2015). These studies also characterise social innovation processes as complex multi-actor and multi-level endeavours, where public, private and civil sector actors on various organisational and geographical level are forced to interact, in order to pro- perly understand and address complex social systems. Transformation of these systems are dependent upon the interplay between structure and agency, i.e. established institutions, regulations and norms, on the one hand, and individual's capacity to challenge or enforce these structures, on the other hand (Haxeltine et al., 2017; Westley et al., 2017). The complex nature of social innovations and the challenges they address, makes SSH expertise on human relations, social progress and organisational development pivotal in such processes (Brundenius et al., 2016; Grimm et al., 2013; Lundström and Zhou, 2011; Phipps et al., 2012).

A global mapping of over 1000 social innovations expose, however, that researchers and other university officials are involved only in 15 percent of the mapped cases (Butzin and Terstriep, 2018). In contrast, public authorities, civil society organisations and private companies are more frequently involved, amounting to about 40 percent of the cases. This contrasts to the prominent role of universities and researchers in traditional innovation processes, focusing technological and industrial innovation. In social innovation, researchers tend to be replaced as knowledge providers by users, beneficiaries and consultants, in line with the grassroot character of such processes (Butzin and Terstriep, 2018; Domanski and Kaletka, 2018; Sørensen and Torfing, 2015). As noted by Phipps et al. (2012:167-168), "new SSH knowledge that isn't shared cannot contribute to (...) social innovations", calling for improved "knowledge mobilization" to maximise societal impact of SSH, through the use of knowledge brokers and social media. It is predicted that universities will be increasingly inclined to invest in knowledge transfer services to support SSH in the co-production of societally useful knowledge (Lindberg and Nahnfeldt, 2013; Lundström and Zhou, 2011; Phipps et al., 2012). Lundström and Zhou (2011) note the establishment of 'social innovation parks' in various parts of the world, where SSH knowledge either forms the basis for or enriches development of new solutions to societal challenges. Lindberg and Nahnfeldt (2013) discern that public support services could enhance SSH innovation through improved competences regarding how to design, finance and scale social solutions, through revised procedures and tools to fit the needs and prerequisites among SSH researchers/innovators, as well as through alternative words and images - e.g. by referring to "ideas" rather than "innovations" and images of people instead of machinery - to illustrate and inspire academic innovation. Bakhshi et al. (2008) conclude that national funding agencies may enhance wider contributions of SSH to innovation by setting SSH-suitable standards for evaluating good practices of knowledge transfer, by supporting team-based collaboration across disciplines, by facilitating a culture of knowledge transfer with societal actors, and by functioning as active knowledge brokers through face-to-face networking, personal contacts, representation on external boards, panels and steering groups, etc. The role of national funding agencies as knowledge brokers is further scrutinised by de Jong et al. (2016), exposing that despite their efforts to apply impact criteria in their funding, in line with government policies, it remains unclear to many researchers how impact should be organised, presented and assessed.

\section{RESEARCH DESIGN}

In order to expand the knowledge on how public Rgl funding may be designed in order to enhance SSH impact, the study employs a participatory research approach, where new knowledge is developed jointly by researchers and other societal actors (Aagaard Nielsen and Svensson, 
2006; Reason and Bradbury, 2008). The researchers represented a Swedish NGO of SSH researchers (Humsamverkan) that joined forces with representatives from four national R\&l funding agencies (Formas - The Swedish Research Council for Sustainable Development, Forte - The Swedish Research Council for Health, Working life and Welfare, Energimyndigheten - The Swedish Energy Agency, VINNOVA - Sweden's Innovation Agency) - in a process of designing digital tools for inclusive funding of research and innovation. Two researchers from Humsamverkan facilitated the process, while the agency representatives contributed with their practical experiences. During 2014-2018, they incrementally delineated excluding and including mechanisms towards SSH researchers in their processes of formulating, communicating and processing calls for funding. This took place at regular workshops, alternately arranged individually with each agency and collectively with all agencies. Based on the acquired insights, tools were piecewise collectively designed during continued, joint workshops. The tools were publicly launched in the spring of 2018, freely available at www.humsamverkan.se (in Swedish only).

In order to simultaneously expand the academic knowledge on how public Ryl funding may be designed to enhance SSH impact, the participating researchers and agency representatives decided to scientifically analyse the process and results, in line with the participatory research approach of joint knowledge development (cf. Aagaard Nielsen and Svensson, 2006; Reason and Bradbury, 2008). The results of that analysis form the basis for this paper that is co-authored by the main participants. The participatory procedure helps attaining 'socially robust knowledge', as the results are validated through continuous dialogue between those who possess practical experiences and academic knowledge in the studied area (Nowotny et al., 2001). The data informing the study consists of meeting minutes, tool drafts and the finalised tools, collected at the workshops during 2014-2018. The collected data was then analysed in the light of the theoretical framework of academic impact support, as part of the joint writing process. As part of this, a thematic analysis was performed in order to distinguish excluding and including mechanisms towards SSH researchers (cf. Guest et al., 2012).

\section{RESULTS}

Motivated by the rising interest in European policy and research for SSH impact and innovation, the four national funding agencies joined forces with the national NGO of SSH researchers in 2014, in order to delineate excluding and including mechanisms towards SSH researchers in their calls for funding, as a basis for designing digital tools for inclusive RYl funding, which were launched in 2018 (available in Swedish at www.humsamverkan.se). The agencies shared the ambition to promote societal progress through their funding, as well as the challenge to attract a broader range of SSH researchers to apply for their funding. In order to improve their understanding of obstacles and opportunities for inclusive funding, they started off by identifying excluding and including mechanisms towards SSH researchers in their processes of formulating, communicating and processing calls for funding. Firstly, they delineated their call processes, identifying each phase from initial initiative till final funding decisions. Even if each agency had their own, specific routes for initiating and managing calls, common phases included identification of relevant areas or challenges to address in the call, formation of a staff team to manage the call, formulation of the call text, communicating of the call to target groups, reviewing applications and communicating decisions.

In each of the delineated phases, crucial interactions between staff, target groups, intermediaries and other stakeholders were pinpointed. Key texts and images were also identified, including instructions from government and top management, call texts, websites, other marketing material, evaluation instructions, decision letters, etc. The delineated interactions, texts and images were then scrutinised with regard to their potentially excluding or including effects on SSH researchers from various disciplines. In the interactions, such mechanisms were primarily identified in the composition of the staff team, the contact networks with intermediaries for communicating the call, the presentation forms for communicating the call, as well as the composition of - and instructions to - review committees. Excluding or including effects were perceived to be dependent on the representation and application of a variety of competence areas in these interactions, including a variety of SSH-specific ones. In the texts, similar mechanisms were identified primarily in the description of the addressed areas or challenges, in the demanded competence profile, in the assessment criteria, as well as in the headings and structure of power point presentations. Excluding or including effects were perceived to be dependent on the choice and ordering of specific terms and criteria, intentionally or unintentionally linked to specific research disciplines or ideological/political norms. Such links could either be explicit or implicit, concrete or abstract, specific or generic. Both explicitly and implicitly stated disciplines - named directly or implicated through discipline-specific terminology - might give the impression that only researchers in these disciplines are the target group for the call. The order in which certain areas or criteria are presented might also affect who is appealed by the call, where areas/criteria that are presented first often are perceived as the most important. If the most limiting areas/ criteria are presented first, a narrower range of applicants will probably be appealed, than if it is presented last. In the images, excluding and including mechanisms were identified primarily in the illustrations in call texts, websites and power point presentations. The effects were perceived to be dependent on the representation of a variety of researchers, disciplines, areas, etc., in a variety of formats and settings.

The delineation of these excluding and including mechanisms was used as a springboard for designing a set of digital tools for inclusive Ryl funding. The ambition was that the tools would be useful both for the participating agencies, as well as other funding agencies in Sweden, in the strive for improved societal impact through broadened representation of researchers and disciplines. Besides improved SSH representation, the tools might serve to enhance applications from other under-represented groups as well, in regard to gender, origin, age, etc. The designed tools encompass three main entry points: 1) What do we miss out?, 2) What are the reasons? 3) What can we do?. When entering any of these, the user is guided through a set of awareness-raising and practice-oriented queries. Three to four main queries accompany each point, further specified in a number of sub-queries. The user is encouraged to use a previous or potential call for funding as a basis, when responding to the queries. The first entry point - What do we miss out? - is followed by three main queries: 1) Who has applied and been granted funding - and who has not?, 2) What kind of applicants and granted applications would have been desirable, 3) How can the insights into what applicants have been missed out be improved?. Examples of sub-queries are: How well are various disciplines represented among applicants and granted applications?, Are the approval-rates the same for various disciplines?, What 
disciplines ought to be represented in order to properly understand the addressed area?. To enhance the reflections, a list of all existing SSHdisciplines is provided.

The second entry point - What are the reasons? - is followed by four main queries: 1) How is the call text formulated?, 2) What criteria are applied in the call?, 3) How is the call communicated?, 4) How are the applications reviewed?. Examples of sub-queries are: To what extent is a broad variety of disciplines represented among staff, reviewers, applicants, intermediaries and other stakeholders?, What explicit, implicit or absent disciplines are distinguishable in the texts and images of the call, and to what extent do these reflect a broad variety of disciplines?, To what extent do existing criteria qualify or disqualify applicants from a broad variety of disciplines?, Do the established communication channels reach a wide variety of researchers?. The third entry point - What can we do? - is followed by three main queries: 1) Do we need more knowledge?, 2) Do we need to change our routines and frameworks?, 3) Do we need to develop internal routines and support functions?. Examples of sub-queries are: What new knowledge is needed in order to understand and counteract the excluding mechanisms identified in the first and second entry point?, What new instructions, courses, forms or other routines and support functions can be established in order to attain a greater variety of applicants and granted applications?, Who possesses the organisational power to alter comprehensive frameworks or regulations?.

The entry points are complemented by three fictional case-examples, of which one is presented below.

\section{A CALL THAT DOES NOT REACH ITS INTENDED TARGET GROUPS}

A call for funding of research about sustainable transport systems intends to engender new knowledge on how traffic volumes may be reduced by infrastructural planning. The agency esteem that comprehensive studies are needed, spanning from how actors communicate during planning processes to how norms and discourses affect their decisions. The agency therefore wants to attract a variety of applicants to the call, not least from social sciences and humanities.

The call is however formulated in a way that assumes that the reader already possesses expertise in the transport area. It contains, for example, several expressions that are specific to the area. The text thus signals that the call is directed to a specific, narrow group of transport experts. When communicating the call through newsletters and information meetings, the agency makes no attempt to explain the area-specific expressions to a wider audience.

As a result, the financier does not attain the aspired variety of applicants. The few applications received only come from researchers who are already accustomed to applying for funding from transport-specific calls.

\section{CONCLUSIONS}

The joint design of digital tools for inclusive R\&l funding, as depicted in the preceding section, serves to expand the knowledge on how such efforts may enhance SSH impact. Similar to the argumentation in previous studies on academic impact support, the process was motivated by the acknowledged ability of SSH to provide insights into and innovative solutions to complex societal challenges (cf. Bakhshi et al., 2008; Brundenius et al., 2016; Grimm et al., 2013; Lindberg and Nahnfeldt, 2013; Lundström and Zhou, 2011; Phipps et al., 2012; Wutti and Hayden, 2017). In line with previously identified barriers to SSH impact and innovation, the participating funding agencies and SSH researchers perceived the narrow range of SSH disciplines represented among applicants as hampering to such ambitions (cf. Brandsen et al., 2016; Howaldt et al., 2018; TEPSIE, 2012, 2014). The joint ambition was to address both environmental barriers in terms of granted funding and actor-related barriers in terms of improved capacities and competencies to provide such funding (cf. ibid). The process thus acknowledged that transformation of complex social systems requires an interplay between established institutions, regulations and norms, on the one hand, and individuals' capacity to challenge and change such structures, as concluded in previous studies (cf. Haxeltine et al., 2017; Westley et al., 2017).

The first research question, regarding what excluding and including mechanisms towards SSH researchers that are delineated in the process, exposes that such mechanisms are at play through all phases of the call processes, including identification of areas to address, staff team formation, call text formulation, target group communication, application review and decision communication. The interactions, texts and images in each phase are delineated as especially relevant. In the light of SET-related norms of economic and commercial impact, pinpointed in previous studies on academic impact support, the delineated mechanisms mainly concern: 1) the variety of SSH-related competences represented among the agency staff and review committees, 2) the presence of SSH-tailored area descriptions, terms and assessment criteria, in call texts, website information, power point presentations, reviewer instructions, etc. (cf. Bakhshi et al., 2008; Brundenius et al., 2016; Dawson and Daniel, 2010; Howaldt et al., 2018; Lindberg, 2012, 2018; Lindberg and Nahnfeldt, 2013; Lundström and Zhou, 2011; Muhonen et al., 2018; Olmos Peñuela et al., 2014; Phipps et al., 2012; TEPSIE, 2012, 2014; Wutti and Hayden, 2017). The identified excluding mechanisms enforce - just as the including mechanisms challenge - the perception of SET being more useful and investment-worthy for the society (cf. OImos Peñuela et al., 2014).

The second research question, regarding how the identified mechanisms impacted the design of digital tools for inclusive funding, exposes that three main entry points - regarding what is missed out, why this is missed, and what can be changed - were perceived as the most crucial. By guiding the user through queries regarding the present and potential diversity of SSH representation among applicants, granted applications, reviewers, intermediaries, contact networks and agency staff, a pathway to more inclusive funding is established. The path is further clarified by queries regarding the formulation and communication of call texts, assessment criteria and reviewer instructions, as well as organisational routines and support for enhanced SSH impact. The tool design thus concords with conclusions in previous studies, regarding the crucial function of public funding agencies as knowledge brokers, by encouraging 
societal knowledge transfer in general, and tailored tools and criteria, widened communication paths, alternative words and images, etc. in particular (cf. Bakhshi et al., 2008; de Jong et al., 2016; Lindberg and Nahnfeldt, 2013)

By underlining the need for simultaneous changes of practical call design and strategic organisational frameworks, the developed tools reflect the crucial interplay between established procedures/norms and individual/collective empowerment (cf. Haxeltine et al., 2017; Westley et al., 2017). When attempting to make the funding more inclusive towards underrepresented groups, conflicts may arise in relation both to other missions and tasks of the agencies, and to conservative attitudes among agency managers, staff and other stakeholders. A similarly hampering factor is that neither the identified mechanisms nor the designed tools consider how SSH impact may be enhanced through concrete interaction with users and other stakeholders from various societal sectors, highlighted as pivotal in previous studies (cf. Brandsen et al., 2016; Howaldt et al., 2018; de Jong et al., 2016; Moulaert et al., 2013; Muhonen et al., 2018; Nicholls et al., 2015; Olmos Peñuela et al., 2014; Wutti and Hayden, 2017). As social innovation studies underline the importance of active involvement of users and stakeholders in order to enable both individual and collective empowerment, the intended enhancement of SSH impact may have been hampered (cf. Brandsen et al., 2016; Howaldt et al., 2018; Moulaert et al., 2013; Nicholls et al., 2015). This is especially concerning, as researchers tend to be replaced as knowledge providers by users, beneficiaries and consultants in social innovation processes, thus missing out on valuable SSH expertise on human relations, social progress and organisational development (cf. Brundenius et al., 2016; Butzin and Terstriep, 2018; Domanski and Kaletka, 2018; Grimm et al., 2013; Lundström and Zhou, 2011; Phipps et al., 2012; Sørensen and Torfing, 2015).

\section{REFERENCES}

Aagaard Nielsen, K. and Svensson, L. (Eds.) (2006). Action research and participatory research. Maastricht: Shaker Publishing.

Bakhshi, H., Schneider, P. and Walker, C. (2008). Arts and Humanities Research and Innovation. London/Bristol: NESTA and Arts and Humanities Research Council.

Brandsen, T., Cattacin, S., Evers, A. and Zimmer, A. (Eds.) (2016). Social innovations in the urban context. New York: Springer.

Brundenius, C., Göransson, B. and Carvalho de Mello, J. M. (Eds.) (2016). Universities, Inclusive Development and Social Innovation: an international perspective. Cham: Springer.

Butzin, A. and Terstriep, J. (2018). Actors and Roles in Social Innovation. In J. Howaldt, C. Kaletka, A. Schröder and M. Zirngiebl (Eds). Atlas of Social Innovation - New Practices for a Better Future. Dortmund: Sozialforschungsstelle, TU Dortmund University.

Cajaiba-Santana, G. (2013). Social innovation: Moving the field forward. A conceptual framework. Technological Forecasting \& Social Change. 82(1): 42-51.
Dawson, P. and Daniel, L. (2010). Understanding social innovation: a provisional framework. International Journal of Technology Management. 51(1): 9-21.

de Jong, S. P. L., Smit, J., van Drooge, L. (2016). Scientists' response to societal impact policies: A policy paradox. Science and Public Policy. 43(1):102-114.

Domanski, D. and Kaletka, C. (2018). Social Innovation Ecosystems. In J. Howaldt, C. Kaletka, A. Schröder and M. Zirngiebl (Eds). Atlas of Social Innovation - New Practices for a Better Future. Dortmund: Sozialforschungsstelle, TU Dortmund University.

European Union (2017). $\angle A B$ - FAB - APP - Investing in the European future we want. Report of the independent High Level Group on maximising the impact of EU Research \& Innovation Programmes. Brussels: European Commission.

European Union (2016). Open Innovation, Open Science, Open to the World. Brussels: European Commission.

European Union (2014). Science with and for Society. HORIZON 2020 Work Programme 2014-2015. Brussels: European Commission.

Godin, B. (2014). An old word for a new world, or the de-contestation of a political and contested concept. In K-E. Sveiby, P. Gripenberg and B. Segercrantz (Eds.). Challenging the Innovation Paradigm. New York/ London: Taylor and Francis.

Grimm, R., Fox, C., Baines, S. and Albertson, K. (2013). Social innovation, an answer to contemporary societal challenges? Innovation: The European Journal of Social Sciences. 26(4): 436-455.

Guest, G., MacQueen, K. M. and E. E. Namey (2012). Applied thematic analysis. Thousand Oaks: SAGE.

Haxeltine, A., Pel, B., Dumitru, A., Avelino, F., Kemp, R., Bauler, T., Kunze, I., Dorland, J., Wittmayer, J. and Jørgensen, M. S. (2017). Towards a TSI theory: a relational framework and 12 propositions. TRANSIT.

Howaldt, J., Kaletka, C., Schröder, A. and Zirngiebl, M. (2018). Atlas of Social Innovation - New Practices for a Better Future. Dortmund: Sozialforschungsstelle, TU Dortmund University.

Ionescu, C. (2015). About the conceptualization of social innovation. Theoretical \& Applied Economics, Vol. 22 No. 3, pp.53 - 62.

Lindberg, M. (2012). A striking pattern - Co-construction of innovation, men and masculinity in Sweden's innovation policy. In S. Andersson, K. Berglund, J. Thorslund, E. Gunnarsson and E. Sundin (Eds.). Promoting Innovation. Stockholm: VINNOVA, pp. 47-67.

Lindberg, M. (2018). Relating inclusiveness and innovativeness in inclusive innovation. International Journal of Innovation and Regional Development. 8(2): 103-119. 
Lindberg, M. and Nahnfeldt, C. (2013). Commercializing work life balance - outlining a model for analyzing and promoting social science innovation. International Journal of Innovation Science. 5(1): 21-30.

Lundström, A. and Zhou, C. (2011). Promoting innovation based on social sciences and technologies: the prospect of a social innovation park. Innovation - The European Journal of Social Science Research. 24(1-2): 133-149.

Moulaert, F., MacCallum, D., Mehmood, A. and Hamdouch, A. (Eds.) (2013). The international handbook on social innovation. Cheltenham: Edward Elgar.

Muhonen, R., Benneworth, P. S. and Olmos Peñuela, J. (2018). From productive interactions to impact pathways: Understanding the key dimensions in developing SSH research societal impact. CHEPS working paper; Vol. 2018, No. 02. Center for Higher Education Policy Studies (CHEPS).

Nicholls, A., Simon, J. and Gabriel, M. (Eds.) (2015). New Frontiers in Social Innovation Research. New York: Palgrave Macmillan.

Nowotny, H., Scott, P. and Gibbons, M. (2001). Re-thinking science - knowledge and the public in an age of uncertainty. Cambridge: Polity Press.

Olmos-Penuela, J., Benneworth, P., Castro-Martinez, E. (2014). Are STEM from Mars and SSH from Venus? Challenging disciplinary stereotypes of research's social value. Science and Public Policy. 41: 384-400.

Phipps, D. J., Jensen, K. E. and Myers, J. G. (2012). Applying Social Sciences Research for Public Benefit Using Knowledge Mobilization and Social Media. In A. Lopez-Varela Azcárate (Ed.). Theoretical and Methodological Approaches to Social Sciences and Knowledge Management. London: Intech0pen.

Pol, E. and Ville, S. (2009). Social innovation: Buzz word or enduring term?. The Journal of Socio-Economics. 38(6): 878-885.

Reason, P. and Bradbury, H. (Eds.) (2008). The Sage Handbook of Action Research. Participatory Inquiry and Practice. London: SAGE Publications.

Sørensen, E. and Torfing, J. (2015). Enhancing Public Innovation through Collaboration, Leadership and New Public Governance. In A. Nicholls, J. Simon, M. Gabriel (Eds.). New Frontiers in Social Innovation Research. New York: Palgrave Macmillan.

TEPSIE (2012). Barriers to social innovation. Brussels: European Commission.

TEPSIE (2014). Social Innovation Theory and Research - A Guide for Researchers. Brussels: European Commission.

van der Have, R. P. and Rubalcaba, L. (2016). Social innovation research: An emerging area of innovation studies? Research Policy. 45: 1923-1935.
Westley, F., McGowan, K. and Tjörnbo, 0. (Eds.) (2017). The evolution of social innovation: building resilience through transitions. Cheltenham: Edward Elgar Publishing.

Wutti, D. and Hayden, M. (2017). Knowledge Transfer in the Social Sciences and Humanities (SSH) - Definition, Motivators, Obstacles, and Visions. Colloquium: New Philologies. 2(1): 87-101.

\section{AUTHORS}

\section{MALIN LINDBERG}

Professor, Industrial Design, Luleå University of Technology

Luleå, 97187 (Sweden)

E: malin.lindberg@|tu.se

\section{SVETLANA GROSS}

PhD student, Stockholm School of Economics and Humsamverkan Stockholm, 11383 (Sweden)

\section{MILDA RÖNN}

Chair, Humsamverkan

Stockholm (Sweden)

\section{LISSA NORDIN}

Senior Analyst, Formas - Swedish Research Council for Sustainable Development

Stockholm, 11182 (Sweden)

\section{JAN SANDRED}

Project Officer, VINNOVA - Sweden's Innovation Agency

Stockholm, 10158 (Sweden)

\section{LARS WÄRNGÅRD}

Senior Advisor, Forte - Swedish Research Council for Health, Working life and Welfare

Stockholm, 10137 (Sweden)

\section{CATHARINA NORBERG}

Research Officer, Energimyndigheten - The Swedish Energy Agency Eskilstuna, 63104 (Sweden)

\section{KEYWORDS}

funding; humanities; impact; innovation; knowledge transfer, social sciences 


\title{
THE OULU WAY OF STRENGTHENING SOCIAL IMPACT OF SSH SCIENCES: FROM LINEAR MODELS OF IMPACT TO PRODUCTIVE INTERACTIONS AND BEYOND
}

\author{
JUHA TUUNAINEN , RAULI SVENTO , PENTTI HADDINGTON, KIRSI OJUTKANGAS AND SIRPA AALTO
}

DOI: $10.22163 /$ fteval.2019.373

\section{INTRODUCTION}

$\mathrm{I}$ $n$ the recent years, the University of Oulu has taken an active role in supporting its research in social sciences and humanities (SSH). The support has manifested itself in the introduction of new structures such as the "Eudaimonia Institute"1, established in the early 2010s to promote and coordinate multi- and cross-disciplinary research in human sciences. "Eudaimonia" constitutes a community in which researchers are provided a collegial and supportive environment for carrying out research. It also serves as a platform in the new service concept called "Rapid Research Radicals" (3R), which aims to develop collaborative excellence and new openings in research. Connected to this, "Tellus Innovation Arena"2 offers methods and facilitation expertise to support various forms of collaboration. In addition to this, "Oulu Think Tank of Science and Society" ${ }^{3}$, which operates under the auspices of the "Eudaimonia Institute", was established to facilitate the interaction between scientists in SSH and the broader society. The "Oulu Think Tank" aims to produce policy-relevant research of internationally high standard, and to offer its expertise to different parties, such as companies and science policy makers. The SSH community has also been successfully included in the development of the university's strategy ${ }^{4}$ and profiling measures.

\section{BACKGROUND: SOCIAL IMPACT OF SCIENCE THROUGH PRODUCTIVE INTERACTIONS}

Academic research evaluation has seen important changes in the 2000s mainly related to changes in the societal role of university and the transformation of university governance towards increased social responsibility and accountability. The traditional research assessment was based on peer review and, subsequently, the number of scientific publications. In the 1990s, the demand for measuring economic returns from research funding increased and was closely associated with advancement of commercialisation of university research results. In the 2000s, various EU countries started to develop frameworks for analysing wider societal impacts of academic research, a task that was related to the introduction of the third mission of universities. Thus, the focus in the impact assessment shifted to the development of quantitative indicators through which the societal impact could be measured and used in allocating funds for research (Kearnes and Wienroth 2011).

Another development in impact assessment has been the shift from linear to interactive models of science's social impact. The fact that science has become "contextualised" and knowledge "socially robust" (Nowotny et al. 2001) has had implications for research policy with the end result of that being the replacement of linear processes of understanding the social impact with interactive approaches. Thus, instead of seeing science as the fountain of new knowledge, which would unproblematically flow from universities to society, interactive models, such as productive interactions (Spaapen and van Drooge 2011), acknowledge that societal actors other than scientists are increasingly important in creating science's societal impact. 
A common feature of the interactive approaches is their emphasis on situated and negotiated character of science within local social contexts (Haywood and Besley 2014) occupied by heterogeneous groups of stakeholders with specific interests of their own (Michael 2009). The question is thus no more about the unilinear transfer of knowledge to society, but about various ways in which societal actors are engaged in knowledge creation and application (Spaapen and van Drooge 2011). Interactive models therefore involve a more equal and collaborative communication between academics and societal actors, and increased levels of negotiation across the blurred border between science and society (Haywood and Besley 2014). By so doing, they also help us to appreciate how societal stakeholders attribute value to scientific findings and make use of these as a part of their own activities.

Among the most popular interactive concepts of science's social impact is that of productive interactions. The concept was designed to circumvent the problems of attribution, temporality and causality in impact assessment by looking at interactive processes by means of which impacts are created in practice by heterogeneous sets of social actors. The concept seeks thus to address the iterative production of new understanding and mutual learning at the interface of science and society. According to Spaapen and van Drooge (2011: 212), productive interactions can be defined as "exchanges between researchers and stakeholders in which knowledge is produced and valued that is both scientifically robust and socially relevant". In addition, Spaapen and van Drooge (2011: 212) elaborated that the interaction is productive if "it leads to efforts by stakeholders to somehow use or apply research results". Productive interactions are thus preconditions of achieving societal impacts of science or "intermediate indications of de facto social impact" (Spaapen and van Drooge 2011: 216).

In our view, productive interactions are among the most fruitful suggestions for understanding the social impact of science but have limited value in providing differentiated understanding about the various modes of interaction that take place during such mutual involvement. What is needed is a more tangible framework with the help of which one could differentiate between various kinds of productive interactions and articulate the role new scientific understanding has in solving societal problems. For such a framework, we will use a distinction made between three dimensions of societal impact of science, 1) epistemological, 2) artefactual and 3) interactional-institutional foundations of impact (Miettinen et al. 2015). Further, we will illustrate how such an approach could be used to analyse energy research led by scholars working in Oulu Business School, the University of Oulu, Finland. We do so to stimulate self-reflection of research communities and to help them articulate the societal impact of their research whenever it is needed.

\section{BRIGHT CLOUDS - DARK CLOUDS (BCDC) ENERGY CONSORTIUM AS A CASE EXAMPLE}

The research consortium we analyse, "BCDC Energy", was funded by the newly established Strategic Research Council of Finland (SRC), which is set to encourage and enable the discourse between scientific research and society. The major innovation political rationale to precede the creation of SRC was the recognition of how societal decision-making was lacking research-based evidence. To fill this gap, SRC funds highquality research with actual societal impact with an annual budget of around 55 million euros. It encourages researchers to find concrete solutions to so-called grand challenges. Solving grand challenges requires multidisciplinary approaches and active interaction among a very heterogeneous set of societal actors. Thus, an important element of SRC projects is active interaction between those who produce new knowledge and those who use and further elaborate it.

"BCDC Energy", the case example we chose to analyse in this paper, is a large, multidisciplinary and multiorganisational consortium chosen in the first call of the SRC research funds in 2015. The consortium is led by Oulu Business School, the University of Oulu, and the other partners are: the Centre for Wireless Communications and Department of Information and Communication Studies at the University of Oulu, the Finnish Environment Institute (SYKE), the VATT Institute for Economic Research, the Department of Computer Science at University of Helsinki and the Finnish Meteorological Institute (FMI). The aim of the consortium is to develop new types of services to integrate renewable resources into the smart grids in cost efficient ways.

\section{EPISTEMOLOGICAL DIMENSIONS OF ACHIEVING IMPACT IN "BCDC ENERGY"}

The epistemological dimension of productive interactions addresses what new research results and understanding of relevant phenomena have contributed to the solving of technological and societal problems. The basic research question of "BCDC Energy" relates to the intermittency problem created to the energy system through increasing share of renewable energy sources. Wind and solar energy production cannot be dispatched in similar ways than traditional power technologies. This creates new types of uncertainties into the system. "BCDC Energy" search solutions from different types of flexibilities both in supply side and demand side of the system. In supply side, the role of hydro power in compensating intermittency generated by renewables has been studied. It has been shown how hydro power has positive potential in hydro dominated markets like the Nordic electricity market "NordPool" (Huuki et al. 2017). From the demand side, consumer flexibility has been studied by analysing the role of real time pricing (Huuki et al. 2017). It has been shown that real time pricing can play a role in solving the intermittency problem but there are also constraints related to its efficiency (Kuhnlenz et al. 2018). Consumer attitudes have been analysed through large surveys using the choice experiment method. It turns out that consumers are willing to adapt new technologies and contracts if they get acceptable compensations from the disutility that are created to them (Ruokamo et al. 2018, Krishnamurty et al. 2018).

The research community of "BCDC Energy" quite early recognised that without multidisciplinary knowledge the project could easily have focused into narrowly defined dimensional features of the ongoing energy market disruption. Furthermore, the project's understanding of the need to take all market contexts into consideration became obvious in the workshops organised. The project is thus confident that the broadening of its view and research agenda has helped the researchers to communicate their scientific findings with and serve the needs of the energy market stakeholders. "The Finnish Transmission System Operator" (TSO) Fingrid published a working paper and a related report entitled "Electricity market needs fixing - What can we do?" in May 2016. Together with 
the other energy-related SRC consortia, i.e., "Smart Energy Transition" (SET) and "Transition to a resource efficient and climate neutral electricity system" (El-Tran), "BCDC Energy" delivered a joint comment to this initiative. A round-table discussion based on the arguments put forward in that comment was then organised by Fingrid. In addition to this, the energy-related SRC projects published a policy brief emphasising the need to move into coal neutral society ${ }^{5}$ ().

Finally, based on the multidisciplinary research by the BCDC consortium, a novel terminology "Clean Energy Research" was compiled to the Bank of Finnish Terminology in Arts and Sciences hosted by the University of Helsinki (www.tieteentermipankki.fi). In this way, the results of the project can be utilised by wider audiences also after the end of the project. The fact that "The Institute for the Languages of Finland" picked "energiasääennuste" (energy weather forecast) as a new word in their Finnish words database indicates that the project has achieved a significant epistemic outcome and a new concept related to the disruption of the energy markets in Finland. The new term and the related software application (see artefactual dimension below) keep spreading - without any effort from the consortium - to a variety of internet sites (e.g., http:// www.finsolar.net/aurinkoenergia/aurinkoatlas/).

\section{ARTEFACTUAL DIMENSIONS OF ACHIEVING IMPACT IN "BCDC ENERGY”}

A key ingredient in solving the intermittency problem is development of energy related forecasts. This is why meteorologists were included in the consortium from the beginning. Already in the consortium kick-off meeting in January 2016 this proved to be a good choice. During the meeting dinner, business collaborators discussed with meteorologists and economists on how to make energy related weather forecasts more practical. During the discussions an idea of an application showing the forecast of wind and sunshine in energy units popped up. This sounded like a good idea and the development started immediately. In June 2016 the application "Energy Weather Forecast" ${ }^{\text {" }}$ (http://www.bcdcenergia.fi/ en/energy-weather/) was opened in the consortium web page. It is an open access application showing 24 hours hourly forecasts for wind and solar power in $\mathrm{kWh}$. It shows these forecasts for all 200 measurement points of the Finnish Meteorological Institute in Finland. It is scaled for $2.5 \mathrm{~kW}$ solar panels and similar size wind mills so that they are applicable even in detached single household houses. The forecast is updated every hour. The application turned out to be very popular. From its opening it has reached over 12.000 visits. The development process of the Energy Weather Forecast has also been documented (Suorsa et al. 2018).

\section{INTERACTIONAL-INSTITUTIONAL DIMENSIONS OF ACHIEVING IMPACT IN BCDC ENERGY}

The interactional-institutional dimension of productive interactions includes forms and forums of collaboration between scientists and other societal actors. In the BCDC consortium, the involved researchers work in the interface between science and energy markets. Therefore, the companies familiar with the market situation in the energy sector are vital partners in developing the service platform the project aims to build. Consequently, the project's Advisory Board (AB), which involves important societal stakeholders, was formed at the beginning of research. The aim was to use co-creation methods to develop the services with the key players of the energy markets, and to share knowledge of the recent research with them. Interactive events and workshops organised by "BCDC Energy" with the AB have been the means to develop models for new types of service platforms. These means allow multidisciplinary, multi-organisational and multi-professional collaboration. Face-to-face interactions in the workshops enable finding alternative solutions to chicken-and-egg problems typical to two-sided platforms. In addition, the members of the $A B$ have their own online forum, which has been used to share, store, produce and manage information related to the project.

External interaction and communication in BCDC is designed and implemented in collaboration with the communications' units of the partner universities, other organisations and partners involved in the work of the BCDC consortium. The project has managed to utilise the resources lying in the multiorganisational structure of the consortium and developed a fruitful, reciprocal collaboration network with the communication units involved. With the communication specialists of different disciplines, a stronger and wider competence has been in the BCDC community's reach. This interaction network supports managing interdisciplinary and transepistemic issues. The perceptions of the researchers and communication professionals on projects' science communication are also being investigated in a separate work package.

The "BCDC Energy" communication network functions as a meeting point for highly esteemed professionals and colleagues. Face-to-face meetings with a timely agenda and informal in nature, have been held since the beginning of the project with project partners and the "Kaskas Media agency". The communication units design and develop a variety of methods for science communication and the communication specialists provide support and empower the researchers to communicate their research in public and online. This support includes editorial help, repeatedly checked routines and informal discussions regarding communication. In order to open up scientific work and processes of creating new knowledge the BCDC project publishes a blog and tweets. Monthly at least one expert blog is published on the "BCDC Energy" website and shared via the project's communication network. The participation and representation of the "BCDC Energy" consortium in social media is secured via weekly appearances in Twitter, where the researchers also actively follow other peers and stakeholders. The principal investigator of the "BCDC Energy" consortium leads by example and regularly publishes updates of the research progress as BCDC story posts on the BCDC website. 
WHAT CAN THE UNIVERSITY LEARN? CAPITALISING SUCCESS AND INSTITUTIONALISING GOOD PRACTICES IN THE FUTURE

The interactive models of science's social impact, together with the new organisational structures ("Eudaimonia Institute"; "Oulu Think Tank" of Science and Society; "GenZ Hub") provide a solid background to develop, spread and institutionalise the identified good practices for strengthening the societal outreach of SSH research at the University of Oulu. First, the good practices identified in the BCDC project - and other projects - will be spread to the SSH community in Oulu. Second, "Eudaimonia" and "Oulu Think Tank" will collaborate with other focus institutes at the university to introduce SSH research on a regular basis in various events. Third, the identified good practices will be used to establish and strengthen connections to the broader society to promote and add to impact of SSH research. Finally, as a brand new endeavour, they will facilitate the activities of the new profiling effort of the university, "Genzproject", starting in August 20187.

"GenZ" - Generation Z and beyond: Co-evolution of human capabilities and intelligent technologies in the 21 st century - is a cross-disci- plinary project led by SSH-sciences. It explores how human skills and capabilities can be strengthened in the rapidly transforming world that is increasingly driven by new digital and technological solutions. Further it involves ambitious aims to initiate co-creation activities in five faculties within the university, and to facilitate societal outreach and impact (see Figure 1). More specifically, concrete activities to support societal impact of SSH sciences include:

- utilising the university's new research organisation, profiling projects and service structure also for knowledge transfer and societal impact (e.g. as of 2017 each faculty has their own dedicated communication specialist who helps design and implement interaction plans to and with stakeholders);

- reflective and co-creative communicative practices within multiand cross-disciplinary research communities;

- new interactive practices for research groups and communities to make social impact: blogs, encouraging active participation in the social media, and research story posts;

- regular (e.g. annual) graduate school courses, seminars and workshops facilitated by "Eudaimonia", "Oulu Think Tank" and the "GenZ Hub" that provide platforms and forums for SSH scientists and stakeholders to reflect on their practices, identify common interests, share knowledge, and establish possible collaboration across disciplines and interest groups.

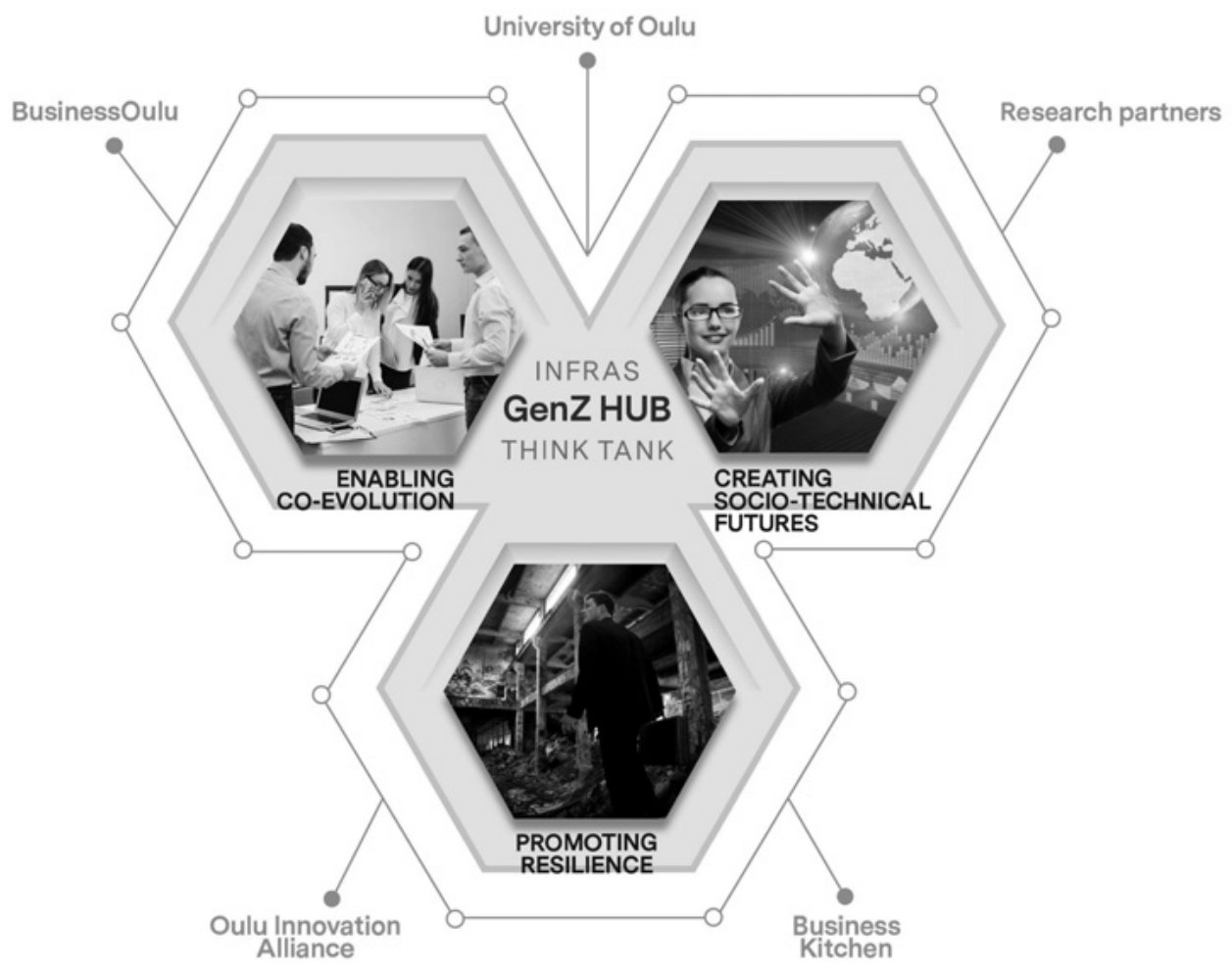

Figure 1: The Oulu way - Facilitating cross-disciplinary research in SSH sciences; responding to a global challenge; strengthening societal outreach and impact. 


\section{REFERENCES}

Haywood, B. K. and Besley, J. C. (2014). Education, outreach, and inclusive engagement: Towards integrated indicators of successful program outcomes in participatory science. Public Understanding of Science 23/1, 92-106.

Huuki, H., Karhinen, S., Kopsakangas-Savolainen, M. and Svento, R. (2017). Flexible Demand and Flexible Supply As Enablers of Variable Energy Integration. https://papers.ssrn.com/abstract=2966053

Kearnes, M. and Wienroth, M. (2011). Tools of the trade: UK research intermediaries and the politics of impacts. Minerva 49: 153-174.

Krishnamurthy, C. K. B., Vesterberg, M., Böök, H., Lindfors, A. V. and Svento, R. (2018). Real-Time Pricing Revisited: Demand Flexibility in the Presence of Micro-Generation. Energy Policy 123, 642-658.

Kuhnlenz, F., Nardelli, P.H.J., Karhinen, S. and Svento, R. (2018). Implementing flexible demand: Real-time price vs. market integration. Energy, 149, 550-565.

Michael, Mi. (2009). Publics Performing Publics: of PiGs, PiPs and Politics. Public Understanding of Science 18/5: 617-630.

Miettinen, R., Tuunainen, J. and Esko. T. (2015). Epistemological, Artefactual and Interactional-Institutional Foundations of Social Impact of Academic Research. Minerva - A Review of Science, Learning and Policy 53/3, 257-277

Ruokamo, E., Kopsakangas-Savolainen, M., Meriläinen, T. and Svento, R. (2018). Towards flexible energy demand - preferences for dynamic contracts, services and emission reductions. Paper presented at the $6^{\text {th }}$ World Congress of Environmental Economics, Gothenburg, June 2018.

Spaapen, J. and van Drooge, L. (2011). Introducing 'productive interactions' in social impact assessment. Research Evaluation 20/3: 211-218.

Suorsa, A., Svento, R., Lindfors, A. and Huotari, M.-L. (2018). Knowledge creation and interaction in an RandD project: the case of the Energy Weather Forecast, Mimeo.

\section{AUTHORS}

\section{JUHA TUUNAINEN}

Oulu Business School, University of Oulu

Pentti Kaiteran katu 1, Oulu, 90570 (Finland)

E: juha.tuunainen@oulu.fi

\section{RAULI SVENTO}

Oulu Business School, University of Oulu

Pentti Kaiteran katu 1, Oulu, 90570 (Finland)

E: rauli.svento@oulu.fi

\section{PENTTI HADDINGTON}

Faculty of Humanities, University of Oulu Pentti Kaiteran katu 1, Oulu, 90570 (Finland)

E: pentti.haddington@oulu.fi

\section{KIRSI OJUTKANGAS}

Research Support Services, University of Oulu Pentti Kaiteran katu 1, Oulu, 90570(Finland)

E: kirsi.ojutkangas@oulu.fi

\section{SIRPA AALTO}

Faculty of Humanities, University of Oulu Pentti Kaiteran katu 1, Oulu, 90570 (Finland)

E: Isirpa.aalto@oulu.fi 


\title{
QRIH: TOWARDS A FITTING SYSTEM FOR HUMANITIES RESEARCH EVALUATION'
}

\author{
AD PRINS, JACK SPAAPEN, THED VAN LEEUWEN AND NELLEKE VAN DEN BROEK-HONINGH \\ DOI: $10.22163 /$ fteval.2019.374
}

\section{INTRODUCTION}

I $n$ this paper we report the design and introduction of a new system for the assessment of "Ouality and Relevance in the Humanities" $(\mathrm{ORiH})$ in the Dutch evaluation context and report also the first experiences of using the system in ongoing evaluations. The design of the "ORiH system" is an attempt to meet two challenges: The first is to find an evaluation method that fits the ways in which humanities researchers communicate with science and society. In many of the current evaluation systems, with the usual attention to metrics fitting the characteristics of research in "Science, Technology, Engineering and Mathematics" (STEM) fields, the research and communication practices in the humanities are hardly acknowledged. The second challenge is to deal with the increasing pressure on researchers, in humanities and all other fields, to demonstrate the societal relevance of research, while at the same time there is a lack of consensus on how to assess research with regard to the societal mandate towards greater attention for knowledge utilisation. We describe specific characteristics of research communication in humanities and address how the communities of researchers and policy makers have been involved in a bottom up development. Also, we will argue that the format of the narrative for self-evaluation addresses the above challenges and gives room to the diversity in the communication outcomes among the research units. The first experiences in using the system are encouraging, but demand sustained attention of panels, researchers and policy makers in making the system valid.

\section{THE CONTEXT IN WHICH QRIH EMERGED}

The report Duurzame Geesteswetenschappen /Sustainable humanities) of the Committee on the "National Plan for the future of the Humanities", also known as the "Committee Cohen" (Committee on the National Plan for the Future of the Humanities 2009), observed that, in terms of research assessment, the humanities are too much at the mercy of models derived from the exact sciences and medicine. The Committee recommended that the humanities develop its own set of assessment standards. In the years that followed, the Royal Netherlands Academy of Arts and Sciences (KNAW) took this task upon itself and tackled this assignment via several studies that culminated in a proposal for an adequate evaluation system for humanities research in 2012 (Quality and relevance in the Humanities 2012). This proposal has been quite influential, as it included as one of the first national systems both the academic and the societal dimension of scholarly activity. Two other sections in the Academy, the fields of design and engineering and the social sciences, developed similar visions on research at the same time. This work was also reported (KNAW 2010 and KNAW 2013) and together these fields influenced to a great extent the model that was adopted in the new "Standard Evaluation Protocol" 2015-2021 (SEP), launched in 2014.

The SEP 2015-2021 enables a balanced assessment of both the academic quality and the relevance to society. These two are the main criteria for the evaluation by an international review committee, which is based on a self-assessment report. SEP Table D1 (table 1) forms the basis for providing evidence in support of the self-assessment report. The indicators in this table determine the content of the self-assessment report and the information gathered for the report forms the basis for the evaluation by the assessment committee.

\begin{tabular}{|c|c|c|c|}
\hline & & \multicolumn{2}{|l|}{ Quality domains } \\
\hline & & Research quality & Relevance to society \\
\hline \multirow{3}{*}{ 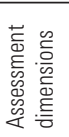 } & Demonstrable products & Research products for peers & 4. Research products for societal target groups \\
\hline & Demonstrable use of products & Use of research products by peers & 5. Use of research products by societal target groups \\
\hline & Demonstrable marks of recognition & Marks of recognition from peers & 6. Marks of recognition by societal target groups \\
\hline
\end{tabular}

Table 1. Quality domains and assessment dimensions of the "Dutch Standard Evaluation Protocol" (SEP 2015 - 2021).The indicators given in the table are given as yet empty categories, which can be used in a field-dependent fashion. Each discipline can bring up indicators that suit best production and communication practices of the field.

7 The authors thank Frank van Vree, chair of the working group, for his energetic and forceful efforts in developing QRiH and in realising the conditions for the working group to do so. 
The KNAW reports, and the subsequent SEP protocol, were published in a wider context of increasing criticism - such as the "San Francisco Declaration On Research Assessment" (DORA), the "Leiden Manifesto", and the "Science in Transition movement" - on the perverse influences of research metrics in the science system, among other the ever-increasing drive to "publish or perish". As a consequence of this criticism, productivity which used to be a main quality criterion, now has been taken out of the SEP 2015-2021, while societal relevance gained an equal status to scientific quality in the evaluation model.

One of the main characteristics of the SEP is that it presents a general framework, but within this framework leaves room for the various disciplines to develop a set of criteria and indicators that fit best the production and communication habits of the field.

In this context a small working group was assigned by the humanities deans to develop a set of assessment standards specifically for the humanities. The working group worked along three lines of activity. First, we researched production and communication practices in the Dutch academic humanities research. Second, we looked at what was happening in a few neighbouring countries, Norway and Flanders ("Current Research Information System in Norway" - CRISTiN and VABB database systems), and the United Kingdom (focusing on the impact pathways developed in the "British Research Excellence Framework" - REF 2014). Third, we developed $\mathrm{QRiH}$ in a way that this would fit into the current national evaluation system for academic research, SEP 2015-2021. And we did this bottom-up that is we engaged the research and policy community in the humanities. The three lines of activity will be explained in more detail in the following paragraphs.

\section{RESEARCH PRODUCTION AND COMMUNICATION IN THE HUMANITIES}

A brief analysis of the production and communication practices of two large faculties of humanities (Leiden and Amsterdam) shows a wide diversity in types of output and use of languages, and also indicates difficulties when relying on resources that are often used in the evaluation of STEM fields, such as Web of Science. The research information systems of these faculties show - in line with other research (Van Leeuwen 2013) - that the largest portion of research output is not in journals but in book chapters, and lists also a wide variety of other types of research outcomes, such as books, professional publications, book reviews, or publications aimed the general public (figure 1).

Also, the output in peer reviewed journals, accounting for $16 \%$ of the total of research output of the two humanities faculties, appears to be represented only to some degree in journals mentioned or listed in "Web of Science" (WoS). The share of "Web of Science" source journals and of journals mentioned in Web of Science also varies according to the domains of scholarly research. In "Economic History", about half of the output in reviewed journals is in WoS journals, whereas in many other fields, such as "Culture Studies", "Religion and Theology Studies" or among researchers from "Archaeology" the share of WoS journals is below $20 \%$. (See figure 2 .)

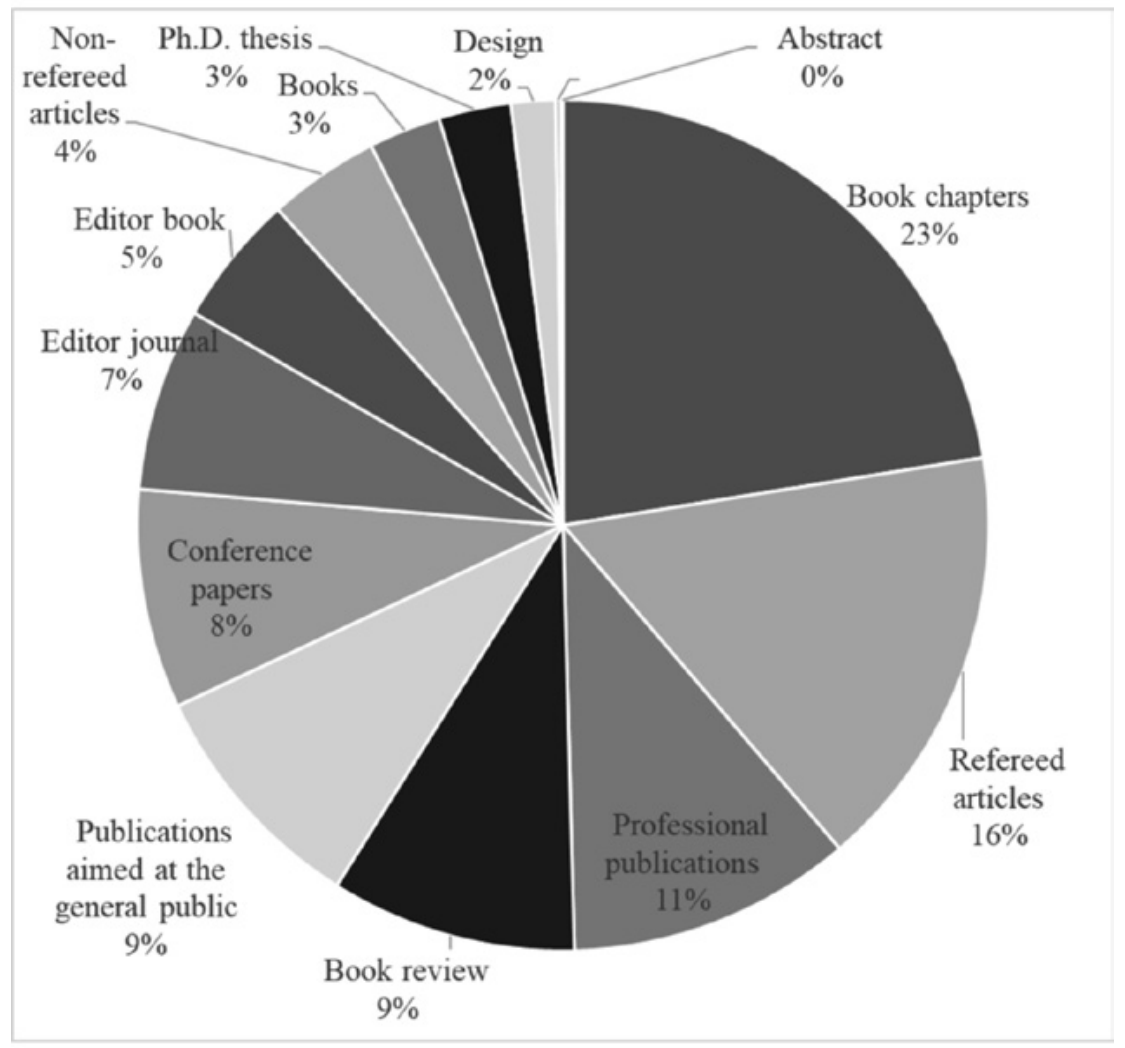

Figure 1. Relative sizes of research output per type, Leiden and Amsterdam Universities. 


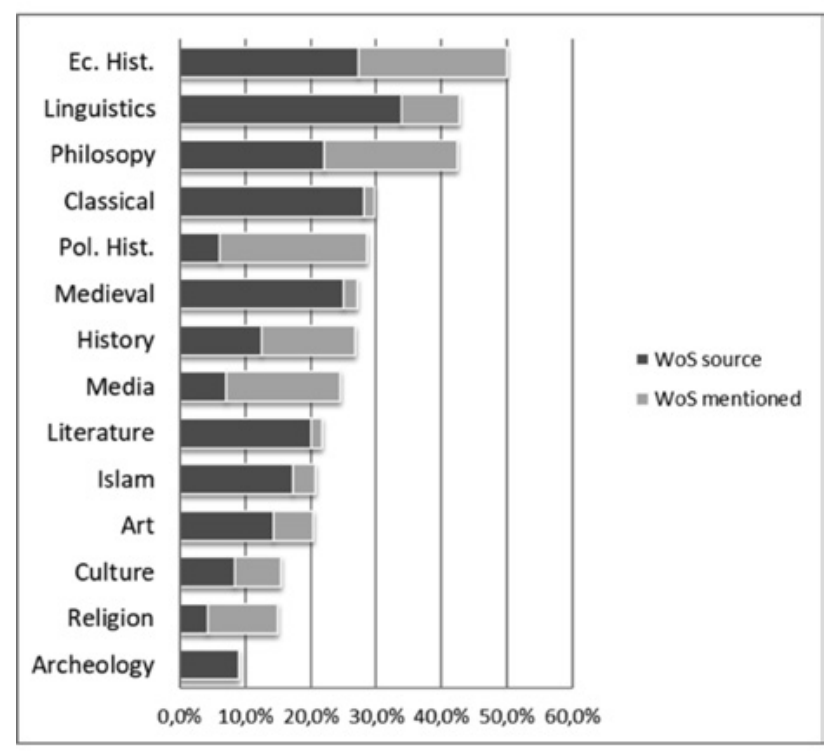

Figure 2. Share of output in journals with WoS source status or mentioned in WoS in Leiden and Amsterdam output. Total of papers in peer reviewed journals (100\%).

Another well-known characteristic of humanities research is the fact that communication occurs in many more languages than English. This characteristic varies across its various domains. Considerable differences occur in the language of journals selected by panels in the field of humanities researchers as prominent publication channels (see also paragraph IV). For example, the selection for digital humanities consists almost completely of journals in the English language oriented, while
"Medieval studies" shows that more than half of the selected journals is in other languages or in Dutch (see figure 3).

These findings clearly indicate that classical bibliometric approaches such as citation analysis or WoS status of journals are insufficient to support research assessment in the humanities, which is consistent with other literature: a similar study of different publication cultures among "Social Sciences and Humanities" (SSH) and STEM researchers, based upon bibliometric analysis of reference behavior by authors publishing in journals covered in the WoS, shows the lesser relevance of journal based assessment in SSH compared to STEM: in SSH, between 10\% and $40 \%$ of all references are addressed to journal literature in WoS, while for STEM this amounts up to $95 \%$ (van Leeuwen 2013). Additionally, taking it from the perspective of output produced by a whole university, classical bibliometric analysis based upon WoS makes the research conducted in most SSH departments nearly invisible, while the internal output registration system clearly shows the presence of a wide variety of scholarly communication types being present (van Leeuwen et al. 2016). This situation disqualifies the existing bibliometric toolbox for SSH and law research assessments, as quantitative analysis only deals with a very small portion of what actually has been produced, across a variety of communication channels.

One of the assumptions at the start of the $\mathrm{ORiH}$ project was that in the humanities, publications for wider audiences and for students can be regarded as a very important expression of societal relevance, even to the point that the line between academic and non-academic publications often is difficult to draw (Sivertsen 2016). The working group took this idea further by proposing the category of "hybrid" publication as a relevant category for humanities, defined as publications with scholarly status also addressing wider audiences of academics and non-academic readers.

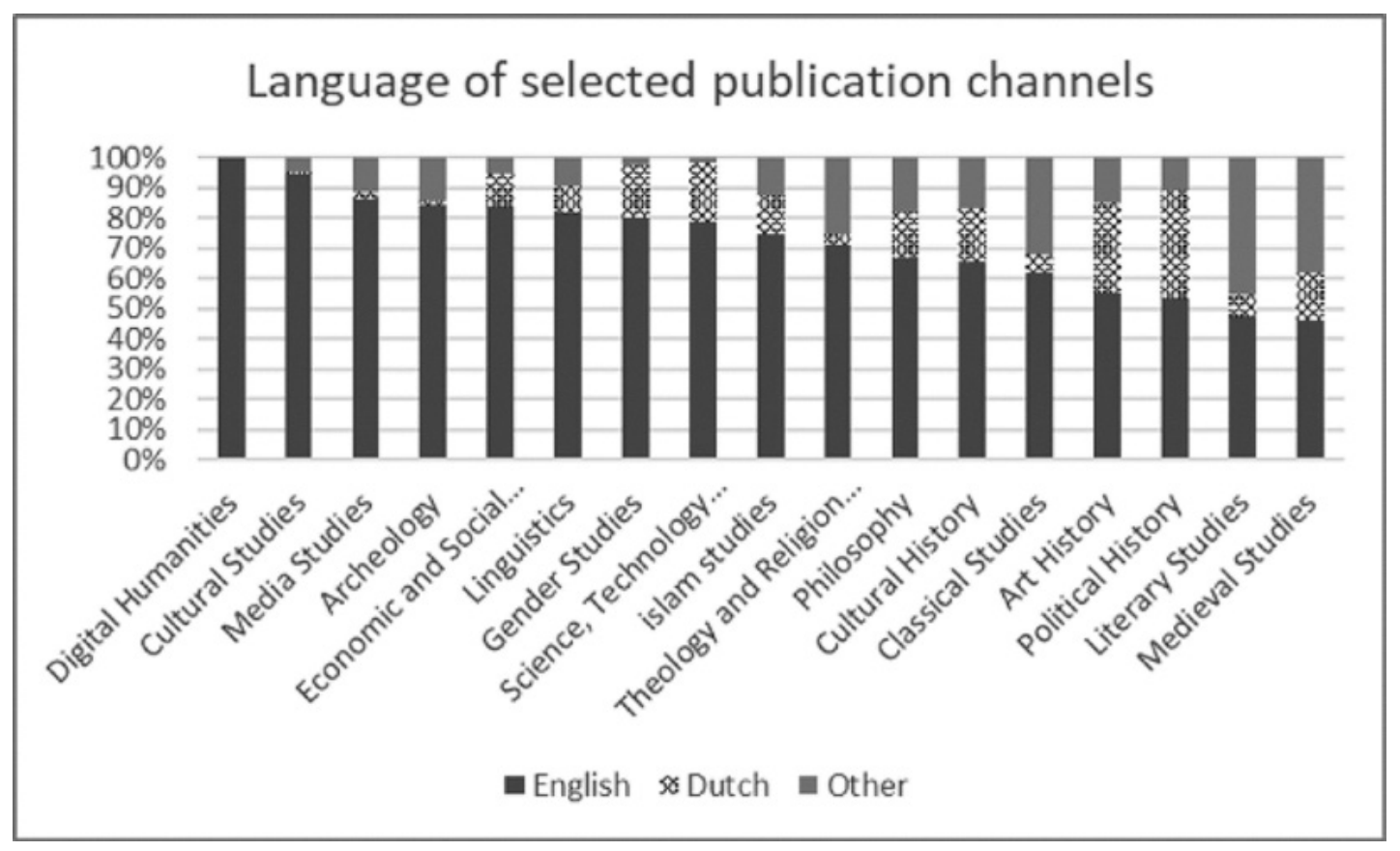

Figure 3. Language orientation of selected journals in 17 humanities fields. 
The claim of the hybrid characteristics of humanities publications can be substantiated. In a questionnaire send out to panels in the field of humanities researchers (see also paragraph V), the various participating panels reacted positively to the request to identify examples of such "hybrids". Several of the suggested works were subsequently analysed for references in scholarly literature (using Google Scholar) and references to be found in non-academic environments using the search engines Google and BING (Prins et al. 2016), demonstrating the actual use in both the scientific and societal sphere.

\begin{tabular}{|l|l|l|}
\hline & $\begin{array}{l}\text { Google } \\
\text { Scholar } \\
\text { cites }\end{array}$ & $\begin{array}{l}\text { \# net societal } \\
\text { stakeholders* }\end{array}$ \\
\hline $\begin{array}{l}\text { Annemarie Mol (2003) The Body } \\
\text { Multiple Duke University Press }\end{array}$ & 3359 & 132 \\
\hline $\begin{array}{l}\text { José van Dijck. The Culture of } \\
\text { Connectivity: A Critical History of Social } \\
\text { Media. Oxford: Oxford UP, 2013. }\end{array}$ & 729 & 132 \\
\hline $\begin{array}{l}\text { James C. Kennedy, Nieuw Babylon } \\
\text { in aanbouw. Nederland in de jaren } \\
\text { zestig (Amsterdam 1995: Boom) }\end{array}$ & 280 & 153 \\
\hline $\begin{array}{l}\text { Ernst van de Wetering. Rembrandt. } \\
\text { The Painter at Work, AUP, 1996. }\end{array}$ & 150 & 170 \\
\hline $\begin{array}{l}\text { Trudy Dehue (2008) De depressie } \\
\text { epidemie, Amsterdam: Augustus }\end{array}$ & 103 & 215 \\
\hline
\end{tabular}

Table 2. Five frequently used humanities publications used both on internet and cited by Google Scholar.

* Net societal stakeholders: Libraries, repositories, web shops and other internet finds not-relevant for meaningful communication are excluded from these results. Also excluded are references from scholarly journals."

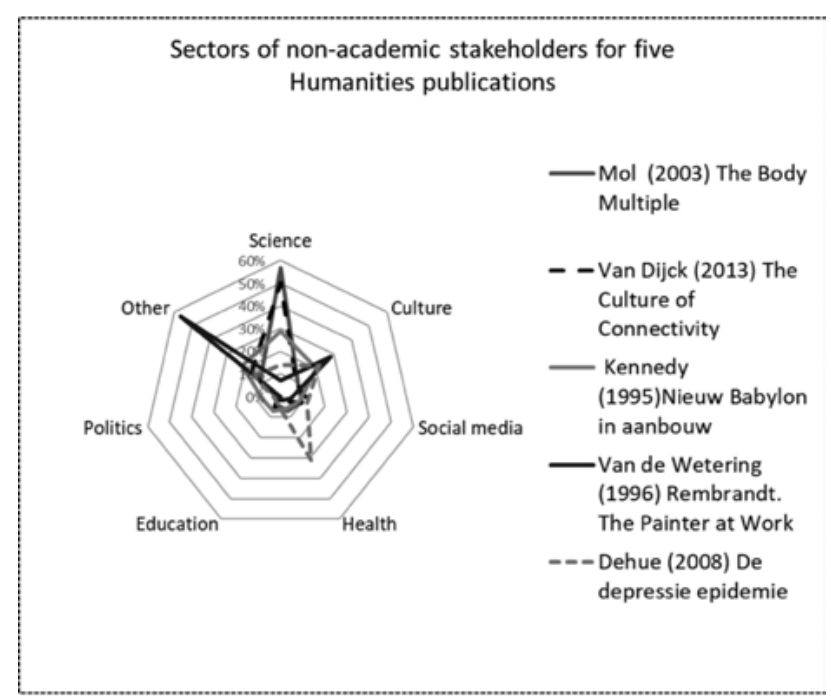

Use of humanities publications by non-academic stakeholders according to their sector. $100 \%=$ total of use by relevant stakeholders.

These examples illustrate the need to develop specific indicators for humanities research. The division between the academic and societal sphere is seemingly less clear or strict in the humanities, meaning that societal production in the humanities is not a spinoff derived from academic production, but can be an intricate outcome of scholarly production. The examples also show the mutual entwinement of academic and societal productivity in the humanities.

\section{LEARNING FROM OTHER RESEARCH EVALUATION SYSTEMS}

Developing the QRiH system also included reviewing comparable systems in some other European countries, such as the impact case studies in the British REF exercise, the publication databases "Current Research Information System in Norway" - CRISTiN (used in Norway) and the Belgian "Vlaams Academisch Bibliografisch Bestand voor de Sociale en Humane Wetenschappen" - VABB-SHW used in Flanders. A comparison between the content of the databases and the ways in which publication and other output data are used in the allocation of funds is useful, even though, unlike the SEP, the indicators derived from the VABB-SHW and CRISTiN are applied in a performance-based research funding system (PRFS) distributing institutional grants to the universities (Ossenblok et al. 2012).

CRIStiN is the national research information system of Norway. It documents all scholarly articles by Norwegian researchers, and complements the library system database BiBSYS, which focuses on books. The Flemish VABB-SHW academic database is developed specifically for the social sciences and the humanities because it is felt that these fields were not adequately represented in WoS database, which serves as the basis for allocating funds among STEM domains in Flanders. Both systems contain several thousands of journal titles and distinguish between them in different ways. The Norwegian system makes a difference between level 2 publications (in international journals) and level 1 publications (other journals, many of them Norwegian, that meet scientific criteria), and level 0 for non-scientific publications. Level 1 and 2 are indirectly tied to financial distribution in the universities. The Flemish system uses a similar distinction between WoS journals and non WoS journals. VABBSHW is directly coupled to allocation of "Bijzondere Onderzoeksfondsen" ("Special Research Fund" - BOF) used to reallocate funding between the universities via points given to 5 different types of publications. Books get 4 points, articles 1 . The policy context of these systems differs from the Dutch policy context the $\mathrm{ORiH}$ system has to operate in. But these systems are worthwhile investigating because they face partly the same problems $\mathrm{QRiH}$ faces. The main issue is how to value publication media that are not part of WoS or other international databases.

In the Dutch SEP evaluation system, a centralised database for journals and publishers or other bibliometric indicators is absent. Also, other than the British REF system (Sivertsen 2016), the evaluation outcomes do not include direct funding consequences between institutions. The implication of financial consequences of systems is that the information is very much focused on competitive elements and comparisons between groups of researchers which may be sensitive of the indicators used (Hammarsfelt et al. 2015). As Ossenblok et al. argued, researchers working in the Flemish VABB-SHW have published increasingly in WoS journals to the detriment of publications in the local language, following its rating system based on the WoS status. By contrast, the share of publications in Norwegian remained stable, occurring in the Norwegian CRISTiN systems that includes a stimulus to publish in the local language next to publishing in WoS journals (Ossenblok et al. 2012).

The Flemish and Norwegian systems also differ from the field orientation of $\mathrm{QRiH}$ to address the specific needs of the humanities only, as 
the VABB-SHW system intends to inform decision making for funding in both the humanities and the social sciences, and the CRISTiN system addresses the entire national field of scientific and scholarly research (Sivertsen 2016). However, there are also similarities between the two: the organisation of domain panels to include specific expertise, a centralised supervising body or authority, and a central collection of information on publications. $\mathrm{QRiH}$ adopts the first two of these similarities between CRISTiN and VABB-SHW.

Another important evaluation system is the 2014 British Research Excellence Framework (REF UK). In REF, experience has been gained of what are called impact case studies. These studies focus on the impact on society and describe, among other things, the project, the participants and their share in the project, the nature and scope of the impact, and what the project actually yields. The case study reports followed a specific structure and were no longer than 5 pages Looking at the UK REF exercise, we focused on these impact case studies that were introduced specifically to evaluate the societal impact of research. Impact was defined rather broadly as research having "an effect on, change or benefit to the economy, society, culture, public policy or services, health, the environment or quality of life, beyond academia" . The REF website has a database with about 7000 impact case studies, all written in the mandatory format. These functioned as a support to specifically underline the societal relevance of the research conducted.

Comparison with these other systems led us to believe that some elements could be very useful in the Dutch situation while other were less suitable. For example, the fact that there is no direct connection between output and funding in the Dutch system we were able to refrain from levels for journals and other kinds of publications. Consequently, we were able to develop lists of journals and publisher without levels and without a metrics system. At the same time, we tried to be selective here and limit the number of entries in the $\mathrm{QRiH}$ database, now including 2210 unique entries for the most important journals and publishers. The selection was given to panels, an element we took from the Flemish and Norwegian systems. Furthermore, we decided to develop indicators for quality and relevance of research bottom up, that is, the research community - via the research schools - was asked to come up with proposals.

\section{BOTTOM UP: THE INVOLVEMENT OF EXPERTISE FROM THE VARIOUS DOMAINS.}

To develop a detailed view on the publication cultures in the different research domains in the humanities, we sent a request to the boards of the 17 research schools in which Dutch humanities is organised to assemble panels of researchers - junior as well as senior and prominent ones -. Over the period of two years, more than 200 researchers have participated in these panels. We have asked the panels to answer a questionnaire about various aspects of their publication culture, including the importance of peer review, typical forms of output, and to list journals and book publishers relevant for the various audiences in their domain, such as publication channels aiming at specialties, disciplinary and multidisciplinary audiences and also de wider context of hybrid audiences (combining both academic audiences and general readership).
Overall, panels have reacted positively to the request but express also concerns that lists of journals and book publishers might lead to the development of a metrics-based system. The question about outcomes of research and communication typical for the scholarly domain has led to listings of various forms of communication usually overlooked in output counting. The lists include catalogues for museum exhibitions, films and documentaries, designs and software programmes and other forms of output.

In the various stages of developing the $\mathrm{ORiH}$ system, we have held meetings with board members and policy makers of Humanities faculties and with the boards of the research schools. Although these meetings have resulted in positive reactions about the involvement of the panels, concerns remained not only over the possibility that $\mathrm{QRiH}$ in the end would lead to a metrics system but also that the outcomes of the work of the panels could be too restrictive in cases of multidisciplinary scholarship, or with respect to domains not covered by the panels. The comments raised during the meetings, and in the numerous talks and phone conversation with policy makers and researchers eventually led to the proposal that the system should be based on the narrative as the leading format for self-evaluations.

\section{ORiH in a nutshell}

The basic structure of $\mathrm{QRiH}$ is the format of the narrative for the self-evaluation of the research unit. The narrative should address both the scientific and the societal mission of the research and be supported by concrete evidence. Indicators in the six cells of table 1 should be elaborated in ways that fit the humanities. The working group decided to publicise the different elements on a website () and use the website as a work in progress. Researchers in the humanities can use the website in SEP evaluations, and at the same time share experiences and do suggestions for improvement. This is what is happening right now because many of the humanities faculties currently are going through a SEP evaluation.

The narrative, much in common with the format developed in the British REF system, should allow the institute, the group or the programme to indicate what the core of the research is, how it should position itself and which strategy is being pursued in order to achieve the objectives and share the research results with the academic world and society, as well as the success of those results. The intention is that claims of productivity, use and recognition put forward in the narrative should be substantiated with evidence that can be derived from indicators proposed by the various domain panels and authorised by a national panel (authorised indicators) or by put forward self-formulated (reasoned) evidence with the help of a broad list of indicator definitions.

As QRiH is to be used in the context of SEP evaluation, its format of the narrative implies a slight but important alteration of the SEP format. The original SEP format consists of 6 cells, in which the various topics of the self-evaluation are to be elaborated (see table 1). Although the SEP format leaves open which kinds of evidence is to be put forward, thus leaving room for variation for the diverse academic disciplines, the format can be read as making a categorical distinction between the spheres of academic research and society, distinguishing Research Quality from Societal Relevance. For many scholarly activities in the humanities, however, this is too restrictive. The narrative of the $\mathrm{ORiH}$ aims to address 
this issue by allowing via the narrative form to demonstrate quality and relevance both in the academic and societal sense either as separate spheres or combined.

The system is sustained by a website ${ }^{8}$ () providing information about authorised and reasoned indicators for each cell in the SEP protocol: there are indicators for products - for peers and for societal parties, indicators of use by peers and in society, and indicators for recognition in both spheres. A template for the narrative can be downloaded to structure the information in the narrative in such way that it will fit the size of maximum 15 pages required according to the SEP protocol. And the website provides various tools, including examples of hybrid publications, domain profiles and lists of journals and publishers selected by the domain panels as exemplary for the communication among specialties, the domain or in multidisciplinary communication.

\section{INTERMEDIARY REVIEW OF ORIH}

In 2018 most of the Dutch humanities research units are to be assessed according to the SEP 2015-2021. The draft version of QRiH was introduced in December 2017 and the working group agreed with the deans of the humanities faculties that research units to be assessed were going to use the $\mathrm{QRiH}$ as a guiding principle. This offered the opportunity to inquire after the first user experiences. For this purpose, we drafted a questionnaire with questions about the usefulness of QRiH in preparing the self-evaluation. Of the twenty units to be evaluated in 2018-2019, so far, seventeen have actually prepared self-evaluations, and possibly have worked with the QRiH system. Fourteen have responded.

The preliminary impressions based on the 14 received and completed questionnaires are that $\mathrm{QRiH}$ is overall appreciated as a tool that gives humanities researchers the opportunity to report in a way that is representative for their activities, especially via the narrative. ORiH appears to be widely known by directors and policy makers; only one policy officer (new at the position) was not familiar with QRiH. Most respondents (11) indicated to have used QRiH (more or less extensively) while writing the self-assessment report. Two respondents indicated that they had not used ORiH because they had started their self-assessment procedure before QRiH was publicised. Most respondents indicated to have used the format of a narrative, which was received with enthusiasm. In general, the set of (qualitative and quantitative) indicators is experienced as helpful.

Some respondents indicate that there are too many different groups of indicators and that indicators for societal productivity, use and recognition should be more specified, preferably also in an authorised form. Also, as QRiH is developed with the help of domain panels from the various research domains, the distinctions among research domains that are visible on the website seem to be confusing for users, especially for research units with a more multidisciplinary focus. It also appeared that the domain profiles developed as a soft benchmarking tool by the domain panels, appeared hardly to be used, because they were not seen as relevant. Arguably, this relates to the fact that many research units cover several research domains.
Remarkably, the lists of journals and publishers, organised per research domain, in the other systems a guiding element, seem to be hardly used by Dutch researchers. The reason for this is not yet clear. It could be due to the grouping of journals and publishers in domains (and research units cover sometimes several domains), but also because not all people find the lists user friendly. Another reason could be that in some domains there is ongoing discussion about the content of the lists.

Additionally, in the contacts with some directors it appears that the distinction of QRiH and the SEP protocol is not yet clear enough, leading some to revert to the SEP protocol. In part it is argued that the SEP protocol is an established format, while others also indicate that the information systems for research output are aligned to the SEP protocol.

Although the first reactions in general are quite positive - in particular with regard to the possibilities offered by the narrative - some specific aspects of $\mathrm{QRiH}$ are hardly used or in need of further elaboration. To get a better sense of why some aspects are hardly used, in-depth interviews will be held in the fall of 2018 .

\section{CONCLUSIONS AND DISCUSSION}

The intention of the QRiH system is to offer an assessment system specifically designed for the humanities, adapted to the specific characteristics of the scholarly work in the humanities and developed with the help of its researchers and policy makers. The system works in the Dutch context of evaluation, meant to function within the broader scope of the SEP evaluation protocol. This SEP protocol is characterised by an equal attention for both the academic and societal aspects of production, use and recognition of research and lacks the linkage of financial consequences that is typical to other systems. Although the Dutch SEP provides a format flexible enough for a wide variety of disciplines, its application for assessing research units in the humanities has lacunae that $\mathrm{QRiH}$ intends to mend. A brief analysis of the characteristics of production and communication of Humanities research reveals not only that the types of communication are far more diverse than journal articles, books and book chapters, or that the communication includes various languages: the distinction between academic and societal communication is often not very relevant, leading to forms of communication distinctly different than in STEM fields such as hybrid publications. The fact that we aim at developing a special indicator for hybrid publications met with considerable enthusiasm in discussions with researchers at the University of Amsterdam. Therefore, QRiH offers the possibility to address the various aspects of quality and relevance both in the scholarly and the societal spheres in a flexible narrative form. The format of the narrative is supported by sets of authorised and reasoned indicators including also lists of prominent channels of communication among specialists, in disciplinary and in multidisciplinary settings.

Developing and implementing the $\mathrm{ORiH}$ system not simply the introduction of a set of indicators to be duly applied by policymakers, researchers and committee members. The development and introduction of QRiH took - and still takes - place in complex sets of contexts each posing constraints and possibilities. The first is the existing structure and 
demands of the protocol for the evaluation of research in the Netherlands, the Standard Evaluation Protocol - SEP. Another highly relevant context are the available evaluation systems and approaches in other countries, such as the British Research Excellence Framework (REF), the Flemish VABB-SHW system, or the Norwegian CRISTiN approach. Yet another is the organisation of the field in universities, faculties, institutes and research schools, and the information available at each level or organisation to sustain and support a specific research evaluation. A most crucial context consisted of the expectations and anxieties of researchers in the field and of the board members of faculties, institutes and schools. The introduction of $\mathrm{ORiH}$ and the idea of a narrative to demonstrate quality and relevance of humanities research could take place by accommodating to each of these, and by challenging these contexts.

The development of $\mathrm{QRiH}$ is a long-term process, for two main reasons. First, both researchers and policy makers should feel as the owners of the system, for which we aimed to have a bottom up process. Second, developing the various parts of the system, in particular the indicators, is a demanding endeavor. From the reactions via the questionnaire and in conversations, it is safe to conclude that we are half way now. QRiH has shown the possibilities to design a system for the evaluation of research in the humanities that does justice to the disciplinary diversity of the field, and to the diversity of its outcomes and ways of communication. Its main characteristic, the possibility to demonstrate the academic and societal quality and relevance of research programmes in a comprehensive way via the narrative, guided by a format and a broad set of well described indicators, authorised or other, is well received among researchers, boards and policymakers.

We have reason to assume that the characteristic of the narrative has contributed to a change in expectations among researchers. At the start of the project, the attempt to formulate indicators for quality and relevance was met with distrust and anxiety among some researchers. In view of the absence of shared views about how research in the humanities is to be publicly accountable, combined with the dispute over research indicators in many countries, this was understandable. The bottom up process proved very valuable: by exchanging experiences and information with researchers and policymakers, and during the various discussions distrust gave way to critical apprehension, but also a raising sense that the new system provides possibilities for the better. Needless to say, the process of development and introduction of $\mathrm{QRiH}$ is still going on. The next steps will be the analysis of the questionnaires that were sent out to all participating research schools and look at the consequences for $\mathrm{ORiH}$. Also, the set of indicators will be elaborated further to strengthen the supporting evidence for the narrative.

\section{REFERENCES}

Commissie Nationaal Plan Toekomst Geesteswetenschappen (Commissie Cohen). (2009). Duurzame Geesteswetenschappen, Amsterdam University Press, Amsterdam

Committee on the National Plan for the Future of the Humanities (Committee Cohen). (2009). Sustainable Humanities, Amsterdam University Press, Amsterdam

Declaration, D. O. R. A. (2012). The San Francisco Declaration on Research Assessment. Putting science into the assessment of research.
Dijstelbloem, H., Huisman, F., Miedema, F. and Mijnhardt, W. (2014). Science in Transition Status Report: Debate, Progress and Recommendations.

Hicks, D., Wouters, P., Waltman, L., Rijcke, S. D. and Rafols, I. (2015). Bibliometrics: the Leiden Manifesto for research metrics. Nature. doi:10.1038/520429a

Koninklijke Nederlandse Akademie van Wetenschappen. (2010). Kwaliteitsbeoordeling in de ontwerpende en construerende disciplines, Amsterdam. [Quality Evaluation in design and engineering disciplines]

Koninklijke Nederlandse Akademie van Wetenschappen (2012). Quality and Relevance in the Humanities. Towards an adequate system for the evaluation of research. Amsterdam: Royal Netherlands Academy of Arts and Sciences.

Koninklijke Nederlandse Akademie van Wetenschappen (2013). Naar een raamwerk voor kwaliteitsbeoordeling van sociaalwetenschappelijk onderzoek, KNAW Amsterdam

Ochsner M., Hug, S. and Galleron, I., (2017). The future of research assessment in the humanities: bottom-up assessment procedures. Palgrave Communications. 3:17020 doi: 10.1057/palcomms.2017.20.

Ossenblok, T. L., Engels, T. C. and Sivertsen, G. (2012). The representation of the social sciences and humanities in the Web of Science - a comparison of publication patterns and incentive structures in Flanders and Norway (2005-9). Research Evaluation, 21(4), 280-290.

Prins, A.A.M. and Spaapen, J. B. (2017). Serving Variegated Audiences: From Ranking Oriented Evaluation to Misssion Oriented Evaluation, fteval Journal for Research and Technology Policy Evaluation, 44, September 2017, pp 42-49

Quality and Relevance in the Humanities. (2017). QRiH, an instrument for describing, systematically, quality and relevance in humanities research in the Netherlands. https://www.qrih.nl/en

Dijstelbloem, H., Huisman, F., Miedema, F. and Mijnhardt, W. (2013). Science in Transition. Why science does not work as it should, and what to do about it, position paper. https://scienceintransition.nl/en/

Spaapen, J.B. and Prins, A. (2016). Contextual evaluation of multi-, inter-, and transdisciplinary research. In: Hubert, B. and Mathieu N. et al. (Eds) Interdisciplinarités entre Natures et Sociétés, Peter Lang, 2016, p. 273-290

Van Leeuwen, T.N. (2013). Bibliometric research evaluations, Web of Science and the Social Sciences and Humanities: a problematic relationship? Bibliometrie - Praxis und Forschung, 2013. 1-18 (http://www. bibliometrie-pf.de/article/viewFile/173/215)

Van Leeuwen, T.N., van Wijk, E. and Wouters, P.F. (2016). Bibliometric analysis of output and impact based on CRIS data: A case study on the registered output of a Dutch university, Scientometrics, 106 (1), 1-16 
VSNU, KNAW and NWO. (2014). Standard Evaluation Protocol 20152021, protocol for research assessments in The Netherlands. Voorburg: VSNU, KNAW \& NWO.

\section{AUTHORS}

\section{AD PRINS}

Support in ResearchManagement

Oosterkade 8b, Groningen, 9711 RS (The Netherlands)

E: info@adprins.nl

\section{JACK SPAAPEN}

Royal Netherlands Academy of Arts and Sciences, Kloveniersburgwal 29, Amsterdam, $1011 \mathrm{JV}$ (The Netherlands)

E: jack.spaapen@knaw.nl

\section{THED VAN LEEUWEN}

Centre for Science and Technology Studies Leiden University

P.0. Box 905, Leiden, 2300 AX (The Netherlands)

E: leeuwen@cwts.leidenuniv.n!

\section{NELLEKE VAN DEN BROEK-HONINGH}

Rathenau Institute

Anna van Saksenlaan 51, The Hague, 2593 HW (The Netherlands)

E: n.vandenbroekhoningh@rathenau.nl 


\section{ETA PROGRAMME}

\section{ARTS, SOCIAL SCIENCES AND HUMANITIES AS AN INTEGRAL PART OF THE INNOVATION ECOSYSTEM OF THE $21^{\text {ST }}$ CENTURY}

\section{ABSTRACT}

$\mathrm{T}$ This article refers to the implementation of the "ETA Programme for Applied Research, Experimental Development and Innovation in Art, Social Sciences and Humanities". The programme addresses dynamic social, economic, globalisation-related, cultural or technological changes of the $21^{\text {st }}$ century with allocation of 92 million EUR of state aid for 6 years, until 2023. The ETA programme introduces the so-called application guarantor, which should both increase applicability of the research results of SSH and broaden the spectrum of R\&D solution users. It is also aimed at supporting the so-called innovation ecosystem of SSH consisting of interdisciplinary collaboration, combination of technical and non-technical research content and usage of basic research discoveries of SSH for application. Several supported research projects will be mentioned as well as points for the ongoing discussion on how to exploit the innovation potential of SSH.

\section{INTRODUCTION}

\section{THE ETA PROGRAMME}

RGD Programme "ETA - Programme for Applied Research, Experimental Development and Innovation in Art, Social Sciences and Humanities" (ETA programme, 2017) was developed and is implemented by the "Technology Agency of the Czech Republic" (hereinafter TACR). Based on findings of the evaluation activities carried out in 2014-2017, the final version of the programme was adopted by the Government of the Czech Republic in January 2017. At present (October 2018), the implementation of the ETA programme is in the mid of its $2 n d$ call for proposals (see also Table 1. "General terms and conditions"). The programme supports social sciences, humanities and art (hereinafter SSH) to address the dynamic social, economic, globalisation-related, cultural or technological changes, which the human and society are currently facing. Other non-SSH fields are also welcomed in the programme mainly for interdisciplinary synergies, but the core of every project must lie in SSH, which are listed under sections 5 and 6 in the "Fields of Research and Development classification" (FORD classification) (OECD, 2015). The principles of applied research and development are promoted in the sense described in the Frascati manual (OECD, 2015), which says the research solution must be practice oriented, novel, creative, uncertain, systematic and reproducible/transferable. The ETA programme is implemented under the Act (Act, 2002), with regard to the Regulation (GBER) (Commission Regulation, 2014) and the "State Aid Framework" (Framework, 2014). The funding is not provided in the de minimis mode.

\begin{tabular}{|c|c|}
\hline Item & Value \\
\hline Programme duration & 2018-2023, 5 calls for proposals \\
\hline Total expenditure & 111 million EUR \\
\hline Public aid (state budget) & 92 million EUR \\
\hline Expected average / max. amount of aid per project & $\begin{array}{l}190 \text { thousand EUR } \\
\text { / } 3 \text { million EUR }\end{array}$ \\
\hline $\begin{array}{l}\text { Funding intensity rates of the } \\
\text { programme / per project }\end{array}$ & up to $80 \%$ / up to $80 \%$ \\
\hline Origin of co-financing & $\begin{array}{l}\text { private and other } \\
\text { public resources }\end{array}$ \\
\hline $\begin{array}{l}\text { Overheads with / without HR } \\
\text { Excellence in Research Award }\end{array}$ & up to $30 \%$ / up to $20 \%$ \\
\hline Min. / expected average / max. duration of the project & $\begin{array}{l}12 \text { months / } 36 \text { months } \\
\text { / } 48 \text { months }\end{array}$ \\
\hline
\end{tabular}

Table 1. General terms and conditions Reference: ETA Programme, 2017. Technology Agency of the Czech Republic

Eligible applicants for funding must have a registered office in the EU, the European Economic Area or the Swiss Confederation and fit to the definition of the following entities:

- Research and knowledge dissemination organisations. The research organisation can be supported up to $100 \%$ of their eli- 
gible expenditure on R\&D activity within the respective project. The co-financing can be ensured from private or other public sources.

- Enterprises. The maximum of the allowable funding intensity is set up with the respect of their size and financial performance according to the Regulation (Commission Regulation, 2014). Companies carrying out the project alone or in collaboration with other participants must demonstrate the ability to cofinance the project only from private sources.

- Other natural and legal persons. For the 1st and 2nd call for proposals, the other natural and legal persons are local authorities or legal entities in which local authorities take part in the role of founders or members. These are mainly municipalities, city quarters, regions, microregions or local action groups (hereinafter local authorities). These entities are considered as eligible for funding only if at least one research organisation or enterprise is among the project applicants. The intensity of support is based on the scheme applicable to enterprises, the maximum reaches up to $80 \%$ of their eligible expenditures. The rest can be added from public or private sources.

\section{MISSION, VISION, OBJECTIVES AND CHALLENGES OF THE $21^{\text {st }}$ CENTURY}

Mission: The mission of the program is to support the application culture of academic staff and other professionals from SSH fields (R\&D solution providers) and to stimulate interest in exploitation of their solutions by SSH application sphere (R\&D solution users, such as ministries, municipalities, health, social or cultural organisations, schools, universities, churches, research organisations, enterprises, NGOs etc.). Vision: The vision of the programme is to encourage research creativity of SSH community, where SSH and non-SSH scientific fields are linked with each other and connected with R\&D solutions users and/or target groups to such an extent, that SSH becomes a fully integrated part of the innovation ecosystem. Objectives: The objective of the programme is to support the involvement of art, social science and humanities in applied research, experimental development and innovation projects and use of their research outputs in the form of new or substantially improved existing products, procedures, processes or services in practice. Challenges of the $21^{\text {st }}$ century: All projects shall be aimed at mitigating threats and exploiting opportunities in the context of the current and the future challenges of the $21^{\text {st }}$ century. Such challenges affect the dynamic transformations of contemporary society, in the areas of:

a. Human and society in the context of dynamic social and technological transformations and challenges of the $21^{\text {st }}$ century: (1) the principles of the Fourth Industrial Revolution; (2) digitisation, virtual reality and artificial intelligence; (3) media and social networks; (4) social services, social work, social housing and social inclusion; (5) family policy; (6) demographic change-aging and fragmentation of society; (7) social insurance schemes; (8) migration and integration; (9) equal opportunities for men and women and principles of non-discrimination; (10) health, psychosocial development and spirituality;

b. Human and the environment for his / her life in the context of sustainable development of the landscape, regions, towns and municipalities and the building culture: (11) globalisation and regionalisation; (12) architecture, urbanism and living space; (13) sustainability and the environment; (14) physical and virtual linking;

c. Human and the economy in the context of discovering new competitive advantages and competence development for the $21^{\text {st }}$ century: (15) educational challenges; (16) employment; (17) health and safety at work; (18) sustainable growth and new competitive advantages; (19) innovative culture, a creative ecosystem; (20) design, design thinking and innovation; (21) new strategic non-material resources; (22) digital and creative economics; (23) media and technology; (24) business creation, business culture and business ethics; (25) clustering and strategic networking;

d. Human and the social system in the context of interaction between the citizen and the state, public policies, governance and citizen-oriented public services. (26) citizen participation in government and community life; (27) protection of intellectual property rights, open innovation, big data; (28) strategic support for research, development and innovation; (29) responsible research, development and innovation and corporate social responsibility; (30) creation and evaluation of public policies and interventions; (31) citizen-oriented public services.

Each project must be focused on at least 1 of the 31 so-called challenges and opportunities of the $21^{\text {st }}$ century.

\section{INNOVATION ECOSYSTEM OF SSH}

In order to foster sustainability of the intervention, the ETA programme also aims at supporting the system in which applied R\&D in SSH takes place. It belongs to crosscutting current and future challenges of the $21^{\text {st }}$ century not only for the human and society, but also for the SSH as such. Three aspects of the so-called innovation ecosystem of SSH have been identified:

1. Interdisciplinarity - breaking down the barriers between disciplines. Support of this aspect should result in a higher permeability of different knowledge of SSH and non-SSH fields and in an increased synergy effect of their innovation potential. Challenges and opportunities of the $21^{\text {st }}$ century are so complex that their solutions often lie beyond the boundaries of various scientific disciplines. In addition, the innovation potential of some fields of SSH can be better exploited in conjunction with other disciplines. Thus, this aspect of the innovation ecosystem of SSH promotes the convergence of knowledge in between of SSH or between SSH and technical, life or natural sciences to acquire new knowledge and ways for applications.

2. Responsibility - producing more responsible research outcomes and innovation. Support of this aspect should minimise the negative undesirable effects of innovation on humans or certain social groups and strengthen the fair distribution of benefits arising from use of the R\&D solutions in practice. Innovations - whether they are products, procedures, processes or services - should be developed with regard to possible side effects they may have on other groups of the population. Without sufficient reflection of their non-technical aspects, research outputs for some social groups may be potentially dangerous or exclude them from use. In addition, linking technical and 
non-technical research content will enable SSH to use technology solutions to deliver their innovative potential to society. In evaluating this aspect, it is necessary to assess whether the project proposal respects the value of social justice and benefits for different target groups. Projects that practically address the overlooked dimension of social responsibility (e.g. integrate knowledge of age, ethnicity, sex, or gender) in the research content are also welcomed.

3. Connectivity - utilisation of innovative potential of the SSH knowledge and discoveries. Support of this aspect shall help to build a bridge between basic and applied research. The innovative potential of discoveries and knowledge of SSH for society often remains latent. Certain outcomes of basic research are not usually used in practice in respective social areas. This aspect will support projects that build up their practical research solution on an existing knowledge from basic research. During the evaluation process, it is necessary to assess whether the use of specific knowledge or discoveries for applications is justified and feasible.

Each project must be based on at least one aspect of the so-called innovation ecosystem of SSH.

\section{PROGRAMME LOGIC MODEL}

The programme logic model is based on the "Evaluation Reference Model" for "TAFTIE's' Taskforce" (Technolopis Group, 2014), which consists of four components: Inputs: To reach the mission, achieve the vison and fulfil the objectives, the Government of the Czech Republic has allocated 92 million EUR which represent up to $80 \%$ of total expenditures of the programme. The other $20 \%$ shall come from other public or private resources. Outputs: Research work - result of the funding will be measured by e.g. number of supported projects, form of collaboration, involvement of organisations in applied R\&D activities or number and type of research results such as comprehensive research reports; certified methodologies, procedures and specialised maps; audiovisual works; organisation of a conference, workshop or exhibition; scientific publications; dictionaries, textbooks, teaching methods and tools, psychodiagnostic methods, mapping and planning studies, evaluation and impact studies, software; data structures and files, hardware prototypes, game simulations and simulators, ICT applications, patent; prototype; functional sample; business creation (start-ups, spin-offs) etc. Outcomes: The immediate benefits for beneficiaries or partners of the supported projects are expected not only in form of innovation coming from usage of research results in practice (innovation of products, procedures, processes or services), but also in form of stronger innovation ecosystem of R\&D solution providers: interdisciplinary collaboration; combining technical and non-technical research content in one R\&D project and more intensive exploitation of outputs from basic research for applications. Impact: If the produced outcomes are made within the sustainable innovation ecosystem of the SSH and used in day-to-day practice of the R\&D solution users, then the positive impact of the innovation potential of the SSH will be achieved as well as new quality of life of human and society: a) Impact on the human and society: quality of human life is improved; sustainable environment for human life is supported; competitiveness of the Czech Republic is improved; efficiency and quality of public policies, public administration and public services is increased.

b) Impact on the SSH innovation ecosystem: boundaries between scientific areas are permeable, research outcomes and innovation are made on the responsible way; innovative potential of the SSH discoveries is practically used.

\section{SPECIFIC TERMS AND CONDITIONS}

\section{APPLICATION GUARANTOR}

One of the main challenges of the ETA programme is to change the way of thinking, that the applied R\&D only takes place between academia and businesses. The ETA programme stresses the relationship between "R\&D solution providers" and "R\&D solution users" in order to advance the existing support structures. In the case of SSH application sphere the natural users of the R\&D solutions may not only be enterprises but any entity in public space. Therefore, the ETA programme introduces the so-called application guarantor, which represents the users of $\mathrm{R} \& \mathrm{D}$ solutions in the project. The main task of the application guarantor is to contribute to making the outcomes of the project fit for use in practice through verifying their reliability and usability. In addition, application guarantor can play an important role in the development of participative research methods through its proximity to the project target group. The relevant application guarantor is an entity that can use the main R\&D outputs for its practice and thus fulfil the project aim. Nevertheless, not all of these entities may be eligible applicants for state aid for research (according to Act Act, 2017), Regulation (Commission Regulation, 2014) and "State Aid Framework" (Framework, 2014). Hence, the ETA programme distinguishes between two kinds of application guarantors (hereinafter $A G)$ :

a. Internal $\mathbf{A G}$ - the entities performing the role of $A G$ can be financially supported if they belong to the eligible applicants (research organisations, enterprises or local authorities). In order not to break the legislation related to the rules of state aid for research, when the AG is an enterprise, it must become an applicant of the project and therefore always act as an internal $A G$.

b. External AG - if $A G$ does not belong among the eligible applicants, it acts as an external $A G$ in the project (e.g. ministries, public authorities, health or social organisations, schools, cultural organisations, Non-Governmental Organisations (NGOs) etc.). Eligible costs of the external AG cannot be covered from the programme resources. Yet, the representatives of the external AG might be employed by the applicants for the R\&D project purposes. Entities in the role of $A G$ in the project must have their registered office in the Czech Republic.

Each project must have at least one relevant $A G$ for the main research outputs, regardless if it's internal or external. 


\section{MARKET-ORIENTED AND PUBLIC-ORIENTED RESEARCH PROJECTS}

SSH can be useful for the society by creating added value of marketoriented products or services by giving them e.g. an element of responsibility or social justice. However certain SSH research solutions cannot, or even should not be delivered to the benefit of human and society through market mechanisms. Thus, the ETA programme supports research solutions both, market-oriented (sell on the market to costumers) or public-oriented (provided free of charge to the target groups). While it is often difficult to separate these two types of projects, as many projects contain both components at once, the project proposals must opt for the predominant component. No priority is given to one of these two types of projects, the market-oriented and public-oriented projects are treated during the evaluation procedure in the same way.

\section{TARGETED INVESTIGATORS AND EQUAL OPPORTUNITY FOR CAREER IN RESEARCH}

The ETA programme seeks to promote the principles of equality, diversity, responsibility or social justice not only via the scale of activities and research results of selected research projects, but also in the way the projects are organised and conducted. The research teams of the ETA programme may consist of scientists and researchers, university teachers, doctoral students, post-docs, artists, designers, architects, employees of municipalities or staff of external application guarantors and other practitioners or experts (see also the figure number 1). Thus, we would like to encourage applicants to pay greater attention to the benefits of diverse research teams, and also to equal opportunities for men and women for the development of their research careers. For those reasons, several rules and recommendation have been developed, published and used for promotion, evaluation and realisation of the projects: e.g. gender diverse team is considered positive in evaluation; research references of the team members have to fit to the project aims, but we do not put any limitation in terms of time when the result was achieved - in order not to disadvantage those, who experienced some career break (maternity leave, parental leave, illness etc.); or higher flat rate for indirect costs (from $20 \%$ up to $30 \%$ ) - which we recommend to spend on activities aiming at the work-life balance of the team members - but for those only who are "HR Excellence in research Award" holders.

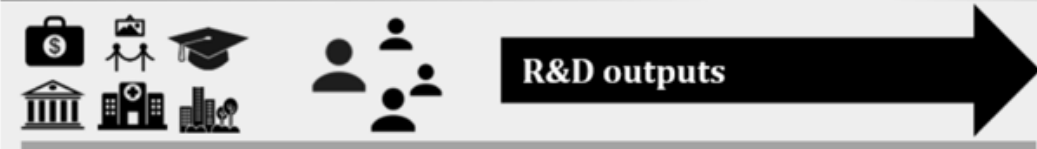

Arts, Social sciences, Humanities

Eligible aplicants

- Universities

- Research institutions

- (some) Hospitals

- (big) Museums

- (big) Galeries

- Big/medium/small Enterprisies

- Cities

- Regions
Team memebres

- Researchers

- Professors

- Students/Ph.D.

- Enterpreneurs

- Artists

- Staff of external AG

- Innovators

- Other relevant practitioners
Innovation ecosystem Outputs/ instruments

1. Interdisciplinarity

2. Resoponsbility

3. Connectivity

- Research report

- Software
- Methodologies/ Maps

- Artistic outputs

- Treatment procedure

- Article, book

- Design

- Conference, workshop

- Exhibition

- ICT application

- Prototype, sample, etc...

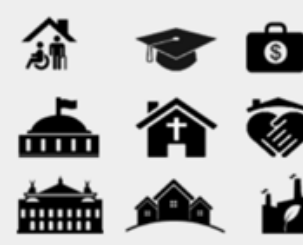

Application guarantor

a) Internal AG: beneficiary $=$ the entity belongs to the eligible applicants

b) External AG: any entity registred in the country, except enterprises

Figure 1: Project logic model. 


\section{RESPONSE AND DISCUSSION}

\section{EXAMPLES OF FUNDED PROJECTS}

In the 1st call for proposals, 306 project were submitted and 94 projects were funded (success rate $32,4 \%$ ). A budget of 18 million EUR was spent, an average of 193.000 EUR per project. The supported projects involved 180 application guaranties (with duplicities) such as ministries, charities, elementary schools, small and medium-sized cities, enterprises, museums, philharmonics, regions, umbrella organisations etc., most of which are external AG. The majority of applicants are research organisations incl. universities (150 participations) and small enterprises (22).
Public-relevant projects dominate. The most frequent main scientific focus of the supported projects is "Management and Administration" (23 projects), "Sociology and Demography" (12 projects) and "Urban, Regional and Transport Planning" (11 projects) (TACR, 2018). The available allocation for the 1st call for proposals allowed to support all projects, which have been evaluated positively. Even though their distribution among the sphere of art, social sciences, and humanities is non-proportional (the majority of funded project belong rather to social sciences, minority to humanities and only a fraction seems to belong to the fields of art), three examples of each sphere were collected (table 2).

\begin{tabular}{|c|c|c|c|c|}
\hline & Scope & Name of the project & Application guarantor(s) & Total costs \\
\hline \multirow{3}{*}{$\stackrel{\longmapsto}{<}$} & Audience value & $\begin{array}{l}\text { Customer lifetime value in the environment } \\
\text { of cultural institutions of live art }\end{array}$ & $\begin{array}{l}\text { Philharmonic orchestra Hradec } \\
\text { Králové; Collegium 1704; Novofest }\end{array}$ & 184.000 \\
\hline & $\begin{array}{l}\text { Big data and } \\
\text { artistic research }\end{array}$ & $\begin{array}{l}\text { Decentralised collection, analysis, visualisation and } \\
\text { interpretation of large data in an artistic practice }\end{array}$ & $\begin{array}{l}\text { Faculty of Fine Arts, Brno } \\
\text { University of Technology }\end{array}$ & 84.000 \\
\hline & Design of smart furniture & $\begin{array}{l}\text { Development of a smart furniture prototype for } \\
\text { the new permanent design collection of the } \\
\text { Museum of Decorative Arts in Prague }\end{array}$ & mmcité1 a.s. & 144.000 \\
\hline \multirow{3}{*}{ 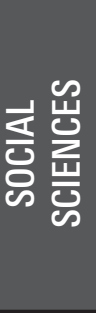 } & $\begin{array}{l}\text { Liveable cities and } \\
\text { communities }\end{array}$ & Guidelines for planning of public space in digital era & Central Bohemian Innovation Center & 153.000 \\
\hline & $\begin{array}{l}\text { Earth protection } \\
\text { from asteroids }\end{array}$ & $\begin{array}{l}\text { A multidisciplinary analysis of planetary defence } \\
\text { from asteroids as the key national policy }\end{array}$ & Ministry of Transport & 392.000 \\
\hline & Environmental education & $\begin{array}{l}\text { Solar energy, water in the countryside, vegetation: a new } \\
\text { methodology of training municipalities and schools }\end{array}$ & $\begin{array}{l}\text { Cities: DaĐice and TĐeboĐ; } \\
\text { Gymnasium Jírovcova and J.V. Jirsíka; } \\
\text { Nerudova Elementary School }\end{array}$ & 139.000 \\
\hline \multirow{3}{*}{$\frac{\text { uㅡㄹ }}{E}$} & $\begin{array}{l}\text { Ethics and autonomous } \\
\text { mobility }\end{array}$ & Ethics of autonomous vehicles & $\begin{array}{l}\text { Prototypum s.r.o.; Keen Software } \\
\text { House a.s.; Ministry of Transport }\end{array}$ & 173.000 \\
\hline & $\begin{array}{l}\text { Industry } 4.0 \text { and } \\
\text { social change }\end{array}$ & $\begin{array}{l}\text { Development of the frameworks for a social change } \\
\text { in the reality of the industry transformation }\end{array}$ & Confederation of Industry of the Czech Republic & 253.000 \\
\hline & Historical literacy & $\begin{array}{l}\text { Historylab: using technology to foster historical } \\
\text { literacy - software for history education }\end{array}$ & Antikomplex (NGO) & 293.000 \\
\hline
\end{tabular}

Table 2. Examples of supported R\&D projects from the 1st call for proposals (total cost in EUR). Reference: Results examples of funded projects in 2018, Technology Agency of the Czech Republic

\section{DISCUSSION}

Based on experience with the designing of the ETA programme, with the implementation of the 1st and 2nd calls for proposals and feedbacks, taking into account types of submitted project proposals and their most frequent weak points and qualities - the following areas remain challenging:

- Structural level: Institutionalisation of drawing on innovation potential of SSH

- Political level: Blindness of legislation and R\&D policy to the needs of SSH for innovation

- Academic level: Innovative mind-set of SSH community

The most frequent reasons for project proposals rejection, is the lack on SSH in the core of the research project. There may be several reasons for this: The common applicants of the TACR's programme portfolio mainly come from the "Science, Technology, Engineering and Mathematics" (STEM) fields or natural sciences. The SSH academic community has been historically supported primarily in the area of basic research. Moreover, the system of knowledge transfer between SSH and application guarantors (municipalities, schools, cultural organisations, NGOs, public administration, enterprises etc.) is still emerging. Even though the third role of universities is recognised as an integral part of their mission, the assessment and institutional financing of SSH are based substantially on their publication performance, not application of their results. Thus, the demand-oriented research attention of the SSH community might be more stimulated. Vice versa, the demand on the R\&D solution side of the application guarantors is rather low, as they might not have enough ca- 
pacity - whether financial, time or human resources related - to innovate. It seems that the higher impact of SSH on human and society hinders the low institutionalisation of the relationship between R\&D solution providers and RGD solution users, and we should ask what does it prevent?

Not all partnerships can be fully developed, since only research organisations or businesses can receive the state aid for research. Many important partners have to play the role of external AGs (without financial support), although it would be more appropriate for them to actively participate on research activities. State aid rules do not have to be applied to many research projects of SSH, however a clear methodology to recognise which project has to and which does not have to is poorly available for SSH. Compliance with the intensity of support applicable to businesses and the need to co-finance from private resources is often unrealistic for many R\&D solution users of SSH. De minimis mode of support might be too dangerous for them. Neither national nor European legislation of the state aid for research is friendly to these new types of partnerships. Furthermore, considering the fields of arts, the ETA programme creates an incentive for an expanded outlook at the artistic research: focus on innovation. But the artistic research has not been fully recognised yet as an integral part of the R\&D policy, much less as a part of innovation policy - neither on national, nor on European level. What is the reason for this omission?

The second most frequent reason for the rejection of the project proposal, is the lack of novelty and innovativeness of the R\&D solutions. It is not clear what novelty or originality means in terms of applied-oriented SSH research: e.g. whether the project aims to move the current practice forward, offers a novel and original R\&D solution that has not been used in practice yet or if it introduces existing concepts into another environment or context? It seems to be difficult to understand, how to build up a background, on which the originality of the new (or substantially improved existing) creative R\&D solutions will be visible and thus better assessable. The willingness to take risk, which consist e.g. of a previously untested interdisciplinary partnership or new research methods or their combining, is still low. Many rejected project proposals have remained in the current borders of the traditional research paradigms, which negatively affected their innovativeness. Is it possible that the reason for this deficiency lies especially in the two previous questions?

\section{CONCLUSIONS}

The ETA programme is a new tool for support of innovation ecosystem through scaling-up SSH pathways in order to boost their positive impact on the human and society. Its novelty lies - at least within the national context - in the fact that, through the role of an application guarantor, it encourages the SSH community to find partners who can use their R\&D solutions. And vice versa, the interest on the side of the R\&D solutions users for cooperation with actors of SSH is stimulated. Even though the programme is at the start of its implementation, first experiences show that it creates an appropriate tool in harnessing the innovation potential of SSH. However, there is a need to deepen the relationship between the SSH community and the funding organisation and to constantly reflect the way the ETA programme is implemented. It should be taken into account that not only organisations, but also members of research teams might be first time applicants to the TACR programme portfolio. An important part of the success of the programme is both the parallel adjustment of legislation and policies on national and EU levels in such a way that better reflects the specific nature of $\mathrm{SSH}$, and structural exploitation of the innovation potential of SSH for society of the $21^{\text {st }}$ century.

\section{REFERENCES}

Act (2002). Act No. 130/2002 on the Support of Research, Experimental Development and Innovation from Public Funds and on the Amendment of Certain Related Acts.

Commission Regulation (2014). No 651/2014 of 17th June 2014 declaring certain categories of aid compatible with the internal market in accordance with Articles 107 and 108 of the Treaty - Official Journal of the European Union L 187, 26th June 2014, in particular Articles 25, 28 and 29.

ETA programme (2017). Funding programme for Applied Research, Experimental Development and Innovation in Social Sciences and Humanities, Technology Agency of the Czech Republic, January 2017.

Framework (2014). Framework for State Aid for Research, Development and Innovation - Official Journal of the European Union C 198, 2014.

Government Office of the Czech Republic (2017). Definition of the results, Separate Annex No. 4 Methodology of evaluation of research organizations and programs of targeted support of research, development and innovation, Ref. No. 26822/2017-0MP. Approved by Resolution of the Government of the Czech Republic on 29th November 2017 No. 837.

OECD (2015): Frascati Manual 2015. Guidelines for Collecting and Reporting Data on Research and Experimental Development, The Measurement of Scientific, Technological and Innovation Activities, OECD Publishing, Paris.

Technopolis Group (2014). Evaluation Reference Model For TAFTIE's Taskforce Benchmarking Impact, Effectiveness and Efficiency of Innovation Instruments. Amsterdam.

TACR (2018). Technology Agency of the Czech Republic, \#TAĐRvDatech, Project ProEval, EU Operational Programme Employment. Retrieved on 7th of October, 2018 from: https://visual.tacr.cz/eta_en.php

\section{AUTHOR}

\section{MARCEL KRAUS}

Department of Strategy and Analysis, Technology Agency of the Czech Republic

Evropská 36, Praha 616000 (Czech Republic)

E:kraus@tacr.cz

\section{KEYWORDS}

art, social sciences, humanities, applied research, innovation, R\&D programme 


\section{THE "6I RESEARCH MODEL": EVOLUTION OF AN INNOVATIVE INSTITUTIONAL STI' POLICY FRAMEWORK AT THE UNIVERSITY OF DEUSTO}

ANTONIA CARO-GONZALEZ

DOI: $10.22163 /$ fteval.2019.376

\section{INTRODUCTION}

\section{6}

current infrastructures dissuade interdisciplinary research" (Moedas, 2017), immersed as they are in the so called "interdisciplinarity paradox" (Woelert and Millar, 2013). Interdisciplinary research is increasingly fostered at a policy level to tackle complex local and/or global problems, but it is, at the same time, poorly rewarded by funding instruments and academic structures (Bromham, Dinnage and Hua, 2016).

Navigating through this paradox, universities are creatively developing ways to integrate the growing demands posed to academic life. These are, at times, conflicting in terms of aims and interests (basic research vs. closer to the market innovations, collaboration vs. competition). In this way, several European higher education institutions have made attempts at enhancing interdisciplinary research through virtual, physical or combined approaches on issues of relevance at a more global level. This is the case at Trinity College Themes; Universitá de Bologna Integrated Research Teams; University of Sussex Strategic Research Programmes and Lund University Strategic Research Areas, to name but a few. In most cases, these new endeavours coexist with more traditional ways of managing research (discipline driven, "Social Sciences and Humanities" (SSH) vs. "Science, Technology, Engineering, Arts and Mathematics" (STEAM), etc.).

The aim of this paper is twofold:

1. Firstly, to introduce the main features and elements of an innovative research management system, the "6i Research Model". Emerging from a bottom-up initiative, the model is the result of our quest for a clear holistic vision to devise a comprehensive research management model, with diverse mechanisms, structures and measurement tools. The "6i Research Model" takes its name from the integration of six elements that are usually managed in a disconnected manner: (1) international, (2) inter- disciplinary, (3) intersectoral, (4) innovative, (5) impactful and (6) inclusive.

2. Secondly, to analyse key aspects of the practical implementation of the model at a higher education institution: in this case the University of Deusto ${ }^{2}$. By analysing process indicators and outcomes, this paper focuses on

a. the evolution of the implementation of the "6i Research Model" over the last decade and how it has been sustained in practice;

b. the results produced; and

c. the changes which the institution has undergone to accommodate and support the evolving model.

Focusing on the implementation of the "6i Research Model" model at the University of Deusto, the second part will respond to the following research questions:

1. How did the "6i Research Model" evolve over time and how has it been sustained?

2. What kind of impact on institutional change did the model involve in terms of structures and resources, mechanisms, initiatives and outputs? and

3. Is Deusto steadily evolving into a research ecosystem for impactful research excellence, while adopting the "6i Research Model"?

Based on lessons learned, we will draw some conclusions for future applications and scaling up the model to other higher education institutions.

\section{A MULTIFACETED MODEL}

Building collaborative inter- and trans-disciplinary communities requires deep reflection and a clear, well-planned strategy. 
With the focus on social impact valuation-driven research, the "6i Research Framework" adopts a system thinking approach and is based on three innovative, interrelated and mutually reinforcing pillars:

- An evolving the "6i Research Model": this is made up of a combination of (i)nternational, (i)nterdisciplinary, (i)ntersectoral, (i)mpact, (i)nnovation and (i)nclusion features and dimensions.

- A self-feeding flexible governance system which integrates top-down and bottom-up uptakes with well-rounded flexible governance support structures and mechanisms.

- A dynamic process which combines competitive and collaborative research endeavours with a focus on excellence and real impact.

Research has shown that a collaborative culture is a strong predictor of creativity (DeCusatis, 2008, Barczak, Lassk and Mulki, 2010) and, according to Waddel and Brown (1997), inter-sectoral partnerships can "help reduce duplication of effort and activity that works at cross-purposes; they can also stimulate innovation and unusually creative solutions if the diverse goals of participants can be addressed" (p. 1). Taking this into account, the "6i Research Model" departs from the firm conviction that interdisciplinarity is absolutely useful for understanding complex problems, such as human mobility or climate change (Repko, 2012). It also assumes that engaging in international interdisciplinary and intersectoral collaborations helps to: a) identify global priorities; b) develop more responsible and accountable research; and c) strengthen the capacities required to be able to tackle global and local challenges.

Since researchers suffer from a number of limitations in terms of their individual agency, career development and stability (i.e. secure funding for research), new forms of researcher collaborations and partnerships with non-academic stakeholders have enormous potential for generating innovative ideas and stronger social impact. Studies also demonstrate that people are inclined to collaborate, provided that there is reciprocity, which is the basis of trust (Thomson, Perry and Miller, 2007). Nevertheless, in order to take interdisciplinarity seriously, each person must be "secure in his or her competence", as being interdisciplinary means being intentional in group formation and decisions, while incorporating different approaches, methodologies and procedures (Hall and Weaver, 2001). Along these lines, creating a collaborative culture requires the cooperation of people at different levels and areas of the organisation and requires trust and leadership, reciprocity, commitment, dialogue and the sharing of ideas and projects that give a sense of belonging, teamwork and result-oriented processes.

In order to provide such basis, the "6i Research Model" proposes putting forward an orchestrated multi-layered and flexible intervention which includes:

- a well-defined vision at a strategic level, integrating targeted initiatives around the $6 i$ axe;

- clear, underlying, governing principles which include (a) a people-centred approach; (b) building trust and (c) having confluent "win-win" goals;

- a number of support structures and mechanisms, put in place to creatively and steadily make progress in the implementation phase with a highly professionalised body of research managers and administrators; and

- a definition and implementation of specific measures to value impact at a project level, with established specific rewarding mechanisms for assessing social impact.

The model also makes use of a dialogical blend of collaboration vs competition to achieve excellence in research. Although perceived as opposites, the 2017 "League of European Research Universities" report (LERU report) argues that both collaboration and competition are necessary to achieve excellence in research and its impact, whenever research excellence and social impact are complementary to, or compete with, each other (Akker and Spaapen, 2017).

A last key element of the "6i Research Model's" engine is the definition of indicators of progress and achievements regarding collaborative endeavours and inter- and trans-disciplinary integration. As with any shared effort and teamwork in general, the objectives of the model and its respective intervention must be clearly defined and mutually agreed by all members, including the quantitative and qualitative indicators that provide an evaluation of achievement.

\section{METHODS}

This research is framed within a broader investigation focused on understanding the multilevel process dynamics, results and impacts of the $6 i$ innovative research management model at higher education institutions. Based on the system thinking approach we have envisioned a model capable of devising holistic and adaptable implementations to the characteristics of each institution; and able to respond to more humanistic and social purposes.

Using a methodological approach that combines a myriad of data collection instruments with quantitative and qualitative methodologies (data and policy analysis, surveys, in-depth interviews, discourse analysis), the "6i Research Model" is being assessed as implemented at the University of Deusto during the period 2010-2018. The combination of data collection instruments, methodologies and triangulation of research results has enabled us to identify and describe the change processes, while understanding them, capturing and reconstructing their meaning.

In order to answer the questions related to the second objective of this paper (which is to analyse the case study of the implementation of the model at the University of Deusto), we have, from the universe of data collection mentioned above, specifically focused on the combination of two variables:

a. The timeline, to analyse the evolution of the "6i Research Model" over time from 2010 to October 2018, and

b. The key enabling elements, such as (b1) the university's strategy and its backing on policies developed for and introduced to drive the different actions, (b2) the supporting structures, (b3) the driving mechanisms, initiatives and instruments, which have been sequentially introduced to generate change and (b4) capacity building, which prepares researchers and research managers to engage in the process. Table 1 shows the second variable containing the main elements intervening in the process, as well as the sources used in order to collect evidence related to each indicator. This paper is focused on the descriptive analysis of the process for which the type of data used is mainly quantitative. 


\begin{tabular}{|c|c|c|}
\hline b1) Policy and strategy & $\begin{array}{l}\text { Institutional policies addressing } \\
\text { management of the } 6 i \text {. }\end{array}$ & $\begin{array}{l}\text {-"Deusto Strategic Plan 2015-2018" including specific "Master Plans" for: a) Internationalisation; } \\
\text { b) "Interdisciplinary and intersectoral collaborations; and c) Social Impact"; }\end{array}$ \\
\hline $\begin{array}{l}\text { b2) Supporting research } \\
\text { structures and staff }\end{array}$ & Deusto Research support structures and staff & $\begin{array}{l}\text {-Records kept by the "International Research Project Office" indicating: } \\
\text { a) The number of support structures created or re-organised by year; } \\
\text { - Annual records kept by the "Human Resources Department" showing the number of employees } \\
\text { hired by the main support structure responsible for channelling the strategy (IRPO); }\end{array}$ \\
\hline $\begin{array}{l}\text { b3) Mechanisms } \\
\text { and initiatives }\end{array}$ & $\begin{array}{l}\text {-International proposals and projects } \\
\text {-Interdisciplinary platforms } \\
\text {-Core groups } \\
\text {-Concerted actions } \\
\text {-DIRSi-COFUND project } \\
\text {-Self-created and external initiatives to } \\
\text { drive innovation and social impact. } \\
\text {-Dissemination initiatives } \\
\text {-"Deusto Social Impact Label", } \\
\text { "Deusto-Santander Award" }\end{array}$ & $\begin{array}{l}\text {-Records kept by the "International Research Project Office" indicating: } \\
\text { a) Number of proposals submitted to international projects and the number of concerted actions (yearly } \\
\text { progress reports to the "Basque Government Framework Programme and Master Programmes"); } \\
\text { b) The analysis of intra-platform dynamics relies on the data collected from two platforms } \\
\text { ("Ageing and Wellbeing", "Gender") since these were the platforms with specific data } \\
\text { available. For each platform, the data included: the year of creation, the number of proposals } \\
\text { submitted in related topics, number of meetings held, number of core groups. } \\
\text { c) Number of topics published for the DIRS-COFUND selection process. } \\
\text { d) Number of COFUNDERs enrolled. } \\
\text { e) Internal initiatives and participation in external initiatives to drive } \\
\text { innovation and social impact as well as dissemination initiatives. } \\
\text { f) Number of actions regarding social impact evaluation and recognition granted per year. }\end{array}$ \\
\hline b4) Capacity building & $\begin{array}{l}\text { Specific 6i-related training provided to } \\
\text { researchers and research managers. }\end{array}$ & $\begin{array}{l}\text { - Records kept by the "International Research Project Office" and the "Human Resources } \\
\text { Department" indicating the number, nature and basic facts about in-house and } \\
\text { external training sessions attended by Deusto researchers and managers. }\end{array}$ \\
\hline
\end{tabular}

Some indicators, such as international proposals and projects, act

Table 1. Data collection and analysis.

i) "Deusto International Research School"

both as process catalysers and results, having an impact on and playing a role in institutional change in a self-feeding mechanism.

\section{THE "6I RESEARCH MODEL": AN IMPLEMENTATION IN MOTION AT UNIVERSITY OF DEUSTO}

The process, as implemented at the University of Deusto, has been studied by combining two analytical variables: a) time; and b) elements intervening in the process. For this reason, data collected under the four elements included in the second variable - b1) policy and strategy; b2) support research structures and staff; b3) mechanisms and initiatives; and b4) capacity building - have been examined longitudinally for the period 2010-2018 to describe the process and the chronological evolution of the "6i Research Model". Figure 1 graphically summarises the aggregated indicators under each variable and element, and results are reported in sequence.

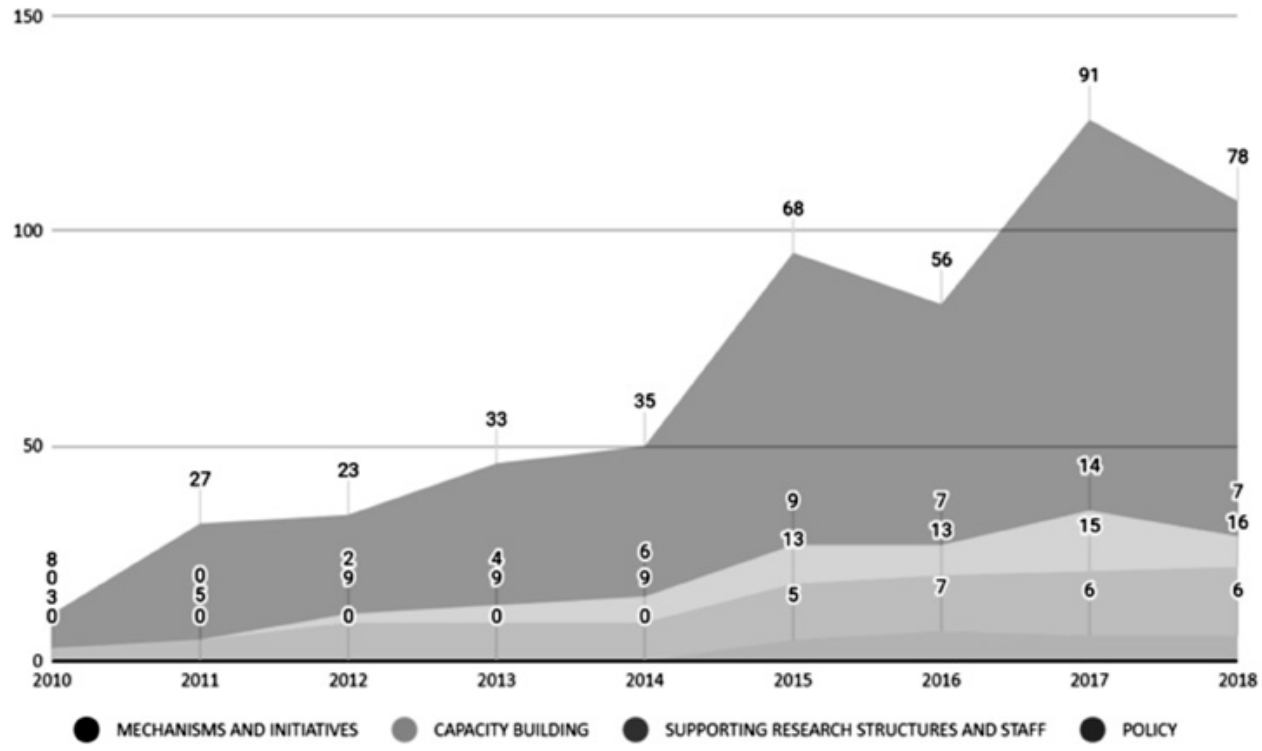

Figure 1. Process evolution of the "6i Research Model" at the University of Deusto Source: prepared by the author based on data gathered. 


\section{0-2011 - BOTTOM-UP INCEPTION}

Policy and strategy. At the start of the decade, research at Deusto was carried out in a disconnected manner and projects gravitated more around the work of individual research interests. We were doing many things related to 6i dimensions, and had been doing so for many years, only we called them different names as they were dissociated from each other and took place in different places.

However, the University of Deusto had a solid base on which to build:

- Over 130 years of history that backed solid relationships with companies, SMEs, regional clusters, entities, policymakers, other academic institutions and social organisations. This has allowed "Deusto Research" to blend competitiveness, innovation, and technology in order to tackle challenges for communities, companies and public bodies in the region.

- A robust number of externally evaluated and accredited researchers, research teams and units at the University with a proven record of research excellence and engagement with society (37 research teams, 9 research institutes, 13 chairs) $)^{3}$;

- A committed senior leadership with a deep knowledge of the institution, the individuals, the system and the internal dynamics. There are three elements providing the driving force for this leadership: firstly, flexibility, with room for manoeuvre in terms of finding solutions, proposing ideas, introducing changes and creatively introducing innovations in research management; secondly, alignment with the defined strategy; and finally, a firm conviction that collaboration is the driving force required to achieve higher scientific competitive levels and closer links with the needs of society.

Therefore, based on intuition and an emerging vision of a more integrated way of managing research, we basically started to join the dots. The first steps were informal meetings with researchers and transfer of knowledge officers working in the field of ageing. We gathered to discuss, meet, take stock (of existing expertise, ongoing projects and publications) and plan the steps forward.

Supporting research structures and staff. In 2011, the International Research Project Office (IRPO) was created. Made up of 3 experienced advisors, the IRPO team was assigned with the task of driving the university's research forward by identifying opportunities to internationalise the university's research and build bridges between the university and stakeholders.

Mechanisms and initiatives. In 2010, despite submitting six proposals, launched by international calls, only one research unit at the university had included international projects in its portfolio. However, by the end of 2011, Deusto had more than tripled its submissions to international projects (21 submissions) and the number of funded projects (3 funded projects). Though these data show the initial results, it was clear from the early phases of the process that both learning how to write proposals and the participation in international projects were key mechanisms for moving the strategy forward.

Furthermore, in 2011, the first interdisciplinary research platform, "Ageing and Wellbeing", emerged as a bottom-up initiative aligned with the "European Innovation Partnership on Active and Healthy Ageing". The "Deusto Interdisciplinary Research Platforms" are flexible mecha- nisms organised around societal challenges for establishing collaborative inter- and trans-disciplinary research partnerships between different research teams and external actors. By gathering researchers from different disciplines to promote active, healthy and meaningful ageing, the "Ageing Platform" paved the way to other interdisciplinary platforms which were to emerge in the following years. The path to constructing the "6i Research Model" was underway.

\section{2-2013 - GROWING STRUCTURES AND BUILDING CAPACITY}

Supporting research structures and staff development. In 2012, with the support of the Vice-Rector for "Research and Transfer of Knowledge", Deusto organised its research structure around the "Deusto Advanced Research Centre" (DARC). This was made up of two support research units: the "DEIKER-Deusto Research Results Transfer Office" and the "IRPO-International Research Project Office". In the same year, IRPO also increased its staff by hiring two more experienced advisors and one junior manager. This was an important increase in resources directed towards the impulse of mechanisms and results.

Mechanisms and initiatives. With less proposals submitted in 2012 than in the previous year (15), the number of international projects funded was higher (5) than previous results, which, in fact, meant an increase in the success rate and having four research units involved in international projects. In 2013, there were more researchers involved in the internationalisation of research $(8$ research units compared to 4 in the previous year). These submitted eleven more proposals than in 2012, three of which were funded. The low success rate was justified due to some units that were just starting to build up their capacity in this field, having had little experience in writing proposals.

2012-2013 was also the period in which the first proposals within the "Ageing and Wellbeing Interdisciplinary Platform" were prepared (2 proposals in 2012 and 5 in 2013). The platform also started to hold two periodic meetings (one every six months). Envisaged as cohesion tools, these meetings facilitated spaces for exchanging ideas, networking and planning between platform members. Once piloted and based on lessons learned, regular general platform meetings were introduced successively over the other interdisciplinary platforms, adjusting the content and dynamics for each specific context and field.

Capacity building. With more staff, the IRPO managed to organise one in-house training session in 2012 and four training sessions in 2013. The focus of these sessions was to instruct researchers on how to apply for international competitive proposals and funding.

\section{4-2015 - GAINING CLARITY: ORGANISING STRUCTURES AND TOP-DOWN SUPPORT}

Policy and strategy. Since 2010, "Deusto Research" had been steadily developing a clearer vision for challenge-driven research aligned with the Europe 2020 and the "Basque Country Smart Specialisation Strategies", with advanced research units and experts contributing to knowledge generation and innovative solutions. Nevertheless, it was in 
2015 that the first four "i's" in the research model (internationalisation, interdisciplinarity, intersectoral and impact) were included in the "Deusto 2018 Strategic Plan" (2015-2018).

With the establishment of these internal policies and recognition mechanisms, the model received backing at the highest institutional level from the rector's team, with:

1. The introduction of the founding principles and governing elements into the agenda and strategy;

2. The development of a valuation system at a research and innovation policy level within the university, including three specific "Master Plans" in the "Deusto 2018 Strategic Plan", creating synergies with other strategic areas of the university, such as a "Commitment to Social Justice";

3. Securing a portion of the research support budget to promote joint participation in international research projects;

4. Setting a flexible structure and support mechanisms to create, develop and establish interdisciplinary platforms; and

5. The definition of progress indicators, against which this multilayered process has been regularly monitored and evaluated.

Supporting research structures and staff. At the end of 2014/beginning of 2015, the "DIRS-Deusto International Research School" was created under the DARC structure to coordinate doctoral training at the university. In the same period, the IRPO hired three more junior advisors.

Mechanisms and initiatives. International proposals continued to be the key mechanism for engaging researchers and units in the " $\mathrm{i}$ strategy". The number of proposals submitted to international calls nearly doubled in 2014 (going from 26 proposals submitted in 2013 to 40 in 2014 -7 of them received funding). This was the result of a good positioning strategy for the initial calls under the Horizon 2020 programme. In 2015, the number of submissions to international projects reached its highest level (53 proposals submitted and 9 projects funded) ${ }^{4}$. Consequently, the number of research units working on international projects literally doubled from 8 in 2014 to 16 in 2015.

A significant event in 2014 was the emergence of a new interdisciplinary platform focused on "Gender issues". Meanwhile, the "Ageing and Wellbeing" platform kept increasing the number of proposals submitted (rising from 5 submissions in 2013 to 14 proposals in 2015). In addition, as a result of the development and approval of the specific "Master Plan" to boost interdisciplinary collaborations, three more platforms emerged in 2015 ("B-Creative-Creative Cultural Industries and Cities"; "Social Justice and Inclusion"; and "Strengthening Participation").

In 2015, the platforms also officially started to unfold into core groups as performing mechanisms for collaborative endeavours. These core groups were smaller groups of experts working together with their local and international peers and stakeholders around specific societal challenges on specific proposals or projects. These had undergone testing during the previous two years and were found to be viable mechanisms for focusing collaboration on:

1. building win-win situations between researchers;

2. tangible work aligned with the agenda, the results expected and the interests of different research units; and

3. creating meeting spaces to build trust and personal relationships.

The data show an increase in the number of active core groups, from a number of timid informal exchanges in 2010 to the current regular, content-specific, ad hoc core group meetings held on the two studied interdisciplinary platforms.

Capacity building. In order to manage the increasing demand and to provide training and support to researchers, the IRPO organised 9 training sessions in 2015 , including in-house and external training.

\section{6-2017 - HARVESTING RESULTS AND BOOSTING MECHANISMS}

Policy and strategy. Internationalisation, interdisciplinary and intersectoral collaboration (the first 3 "i's") were the driving forces that articulated Deusto's research response to societal challenges, and social impact (the 4th I) was incorporated steadily into the research and innovation policy and internal reward mechanisms. In 2016-2017, an evolving multi-layered process of "Social Impact Valuation" was finally in place. The process encompassed progress at four different levels:

1. Reflection and state-of-the-art knowledge production that resulted in the establishment of an evaluation criteria set contrasted with international, national and regional experts;

2. The generation of support units, dependent on the senior manager appointed to the specific "Strategic Master Plan" and two performing bodies: the steering and the evaluation committees in charge of planning, implementing and evaluating progress and results;

3. Training of social impact managers in charge of the everyday implementation of the proposed action plan; and

4. The launch of concrete valuation measures and initiatives: an internal call was developed and launched: the "Deusto Social Impact Briefings". "Deusto Social Impact Briefings" are brief publications to disseminate the research results of projects to specific stakeholders and a wider audience.

Mechanisms and initiatives. In 2016, Deusto achieved its highest number of international funded projects (12) while the "Ageing and Wellbeing" platform managed to submit 12 proposals between 2016 and 2017. In 2017, the "Gender Platform" also started to increase results and presented 4 proposals for international calls.

In the same year, 4 interdisciplinary research areas were identified in alignment with the "Basque Smart Specialisation Strategy" and the intersectoral collaborative framework: "Energy, Territory, Health and Industry 4.0". In addition, specific committees were assigned the responsibility of monitoring the implementation of the action plan envisaged under the "Master Plan" on social impact.

Interdisciplinary co-operation steadily increased within the 5 international interdisciplinary platforms. This process, coordinated by research managers at the "International Research Project Office", crystallised in the creation of a collaborative culture (i.e. exchange of ideas, sharing knowledge, building trust) based on regular formal and informal gatherings, meetings and exchanges (six-monthly general platform meetings and more frequent ad hoc meetings, which were topic-specific or project-based, were held regularly on a demand basis.

Framed within the then $4 \mathrm{i}$ strategy and backed by 42 partner organisations, the University of Deusto received a prestigious Marie-Sklodowska Curie COFUND project. This was led by the Vice-Rector of "Research 
and Transfer" as an institutional project to channel a collaborative culture among PhD programmes, research teams, support structures and interdisciplinary research platforms and areas. It was a challenging proposal to prepare, with multiple negotiations and multilevel coordination. However, it was successfully evaluated and funded and has allowed the university to:

a. Leverage the coordination level between different departments, research support units, and $\mathrm{PhD}$ programmes at the university;

b. Introduce innovations in the selection process and the identification of topics offered in the two call for candidates open under the project; and

c. Offer 8 doctoral positions to attract talented young researchers of excellence to Deusto PhD programmes, teams and platforms.

In terms of social impact, in 2016, we organised the first "Deusto Conference", which, together with non-academic stakeholders participating in research projects, addressed issues related to the social impact of university research. In addition, the university set up two new related initiatives in the period: the "Deusto Research Social Impact Label", which recognises impactful research projects, and, for the first time, the social impact dimension was introduced into the "Deusto-Santander Research Awards". A second "Deusto Conference" was held in March 2017. Furthermore, in 2017, DIRS-COFUND topics evolved and were evaluated using the existing $4 \mathrm{i}$ model. Another 8 positions were published in the second call, resulting in the enrolment of a new cohort of 8 "Early Stage Researchers" (ESRs), who joined the 8 previous ones.
Capacity building. In 2017, the amount of in-house and external training provided to researchers reached its peak, with twice as many sessions held than in the previous year (14 training sessions in 2017 compared to 7 sessions in 2016).

\section{8 - BROADENING THE MODEL}

Policy and strategy. The "6i Research Model" gained its last two "i's" in this year: innovation and inclusion. We are, at present, incorporating innovation and entrepreneurship in a more systematic way. Apart from the existing innovation initiatives ${ }^{5}$, we are devising mechanisms and actions to align innovation within the research strategy.

A fundamental underlying principle of the mission and vision of the University of Deusto is inclusion (the 6th I). We are currently taking stock of the way this dimension is being tackled within the model. A clear example of this is that anyone who wishes to is welcome to contribute to the Deusto interdisciplinary platforms in a variety of different roles (as part of a core group to prepare a proposal, as a representative of the platform at relevant international or local events, etc.). Specific methodologies and indicators are being developed to capture the inclusion of disciplines, roles, researchers within the interdisciplinary platforms, the preparation of international proposals, the implementation of projects, etc.

Figure 2 illustrates the "6i Research Model" and shows the alignment of the university's "Strategic Plan" (in the centre) with the vertical and

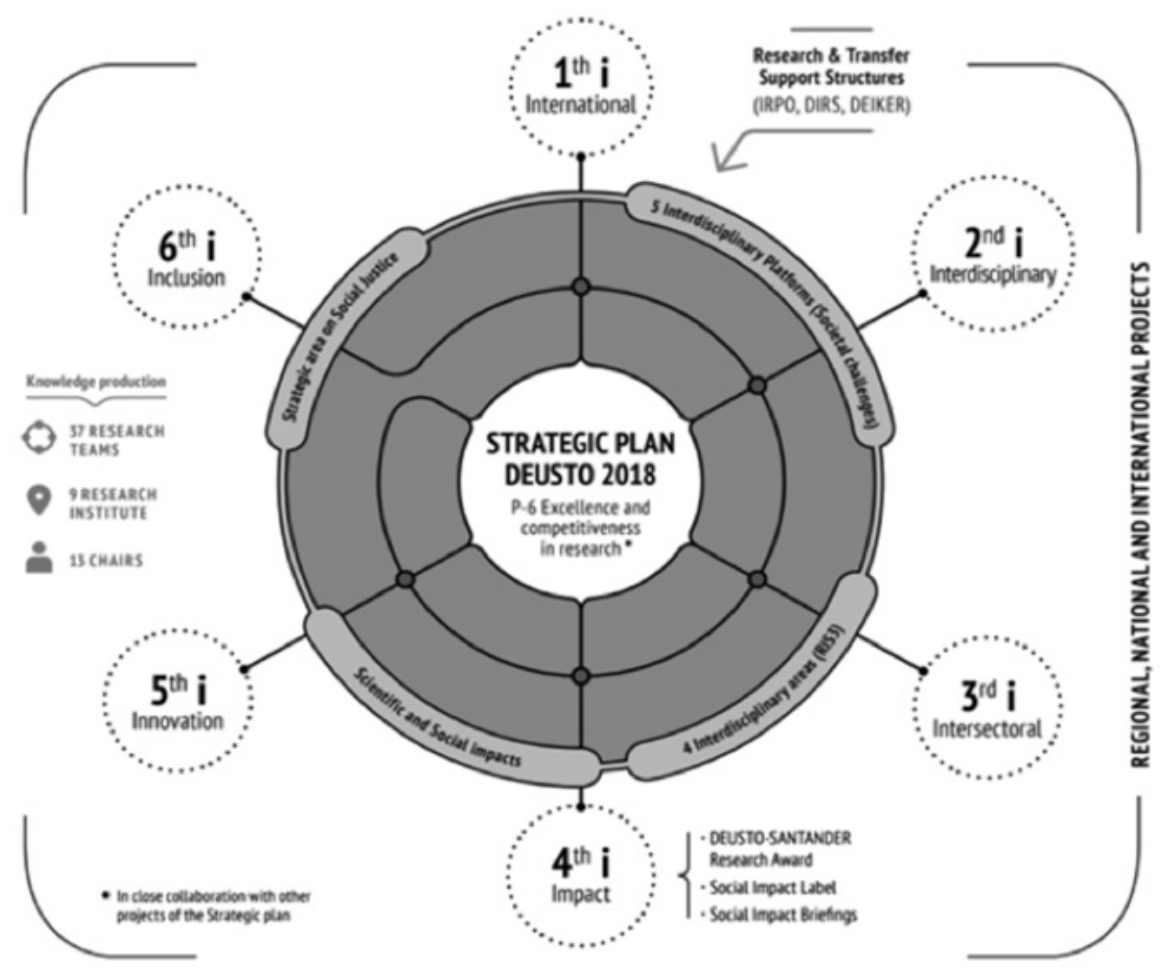

Figure 2. Deusto implementation of the "6i Research Model".

$5 \quad$ There are already a number of outstanding but dissociated innovation initiatives, units and researchers at Deusto. The innovation dimension of the "6i Research Model" will build on this rich body of already existing initiatives, researchers and units. It will figure out suitable collaborative mechanisms to integrate the research-innovation-transfer continuum of knowledge-social impact. 
horizontal interconnections between the levels and elements in a continuously evolving self-feeding process.

Supporting research structures and staff. As of today's date in 0ctober 2018, IRPO staff numbers have been bolstered by the hiring of a project manager and one junior advisor. The increased support structure will make it possible to keep up with the continuous workload:

- providing support to staff in preparing proposals for competitive calls;

- taking on the preparation of ambitious initiatives, such as the coordination of a Hackathon within the AAL Forum 2018 and the Biscay Silver Week held in September;

- capacity building (7 training sessions have been held only this year);

- boosting the action plan for innovation (innovation radar pilots, social impact licensing); and
- improving the communication and dissemination of research results (generation of news for the interdisciplinary platforms, "Deusto Research" website).

Mechanisms and initiatives. The monitoring of the performance indicators for the 3 Master Plans shows the driving force of the strategy in terms of the dynamics generated, blending collaboration and competitiveness. This blend resulted in the participation by DEUSTO in a total of 167 research proposals between 2015 and 2018, with 35 of them being successfully funded under Horizon 2020 and other related programmes. This represents a success rate of over $20 \%$, meaning that the University of Deusto is showing a competitive performance above the national and European average.

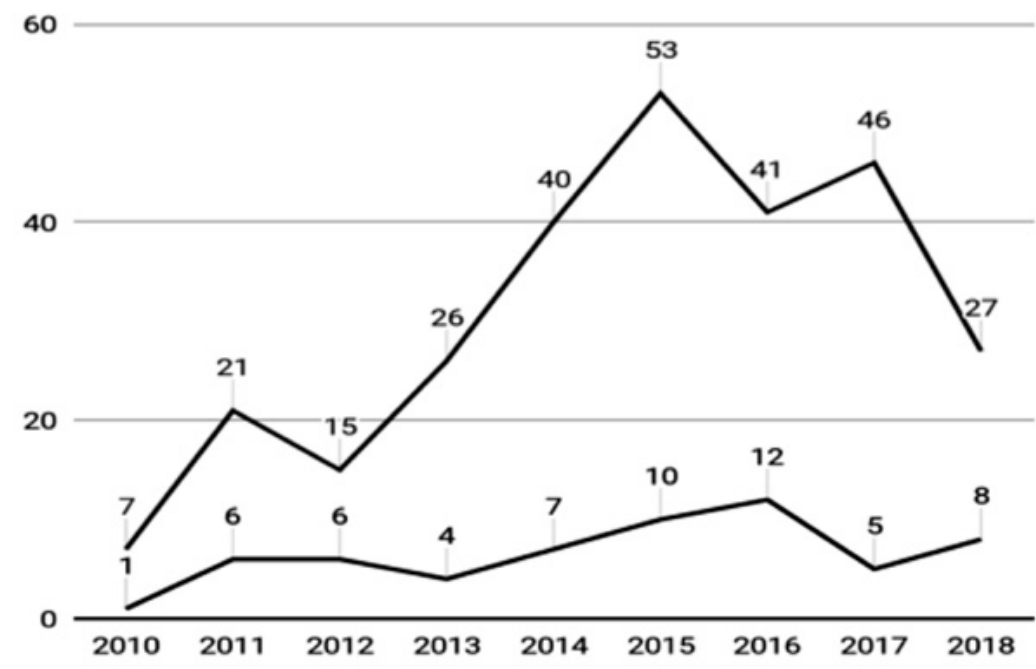
- Proposals submitted to international calls (FP7. $\mathrm{H} 2020$ and brother programmes)
- International projects (FP7-H2020 and brother programmes) which got funded

Figure 3. International proposals submitted and projects funded (FP7, H2020 and related programmes)

For the last two "i's": innovation and inclusion, driven by initiatives from the European Union such as "Innovation Radar", Deusto started to run "Innovation Radar" pilots with selected research projects carried out by the university. It has also collaborated with local industry partners to launch an initiative called the "Social Impact Licensing Strategy", which is aimed at screening technologies and/or services provided by "Deusto Research" to evaluate societal markets.
Finally, in relation to inclusion and aligned with the internationalisation of research, the wider ongoing research project will include initiatives in which Deusto has taken part which are directly related to inclusion (i.e. the "European Science for Refugees initiative", which is aimed at opening doors for refugee scientists to European institutions) ${ }^{6}$. 


\section{CONCLUSION}

This paper had two objectives: the first was to introduce the main features of the "6i Research Model" and give a brief account of its multifaceted composition. The second was to analyse how the model has evolved in practice during its implementation at the University of Deusto in the period 2010-2018.

Firstly, to summarise the model, 6i stands for six research dimensions that are usually managed in a disconnected manner: international, interdisciplinary, intersectoral, innovative, impactful and inclusive. Along these lines, the "6i Research Model" is a multidimensional system that combines key elements in order to sustain a multi-layered intervention that: (1) includes 6i in a well-defined vision and strategy, (2) defines clear governing principles, (3) provides mechanisms and structures to support international, interdisciplinary, intersectoral, impactful, innovative and inclusive collaboration and (4) defines specific measures for evaluating the on-going process.

The combination of a system thinking approach with a hands-on practical implementation, which is embedded in the requisites and assessment mechanisms of university life has helped us envision a model capable of

a. devising more holistic implementations open to future developments and collaborations;

b. being able to adapt to the features, characteristics and everyday business of each institution; and

c. proposing research questions and innovations that respond to more humanistic and social purposes in collaboration with other researchers and stakeholders.

Secondly, by combining two main variables (time and key enabling elements), we have explained the main features and evolution of the process over the period in question. Changes introduced under each of the sub-variables (policy and strategy, support structures, mechanisms and initiatives, and capacity building) have longitudinally generated different institutional responses that accommodate the ever-evolving research management process.

The results obtained from the analysis of the implementation of the model at Deusto show how a process that integrates these 6 usually disconnected elements into an orchestrated strategy can pave the way to growing a robust model. The firm institutional commitment to $6 i$ at Deusto, together with the innovative combination of institutional strengths and elements, demonstrates a complex self-feeding dynamic. In this dynamic, bottom-up initiatives and top-down support combine and drive each other, integrating around the ordinary delivery of research results at academic institutions (i.e. research project funding).

This self-feeding process can be clearly illustrated by the evolution of the "Deusto Interdisciplinary Research Platforms". Emerging as a bottom-up initiative in 2011 to address both the agency of researchers and the university's research management structure, research platforms were backed at the highest level over time and incorporated into the university's strategic plan. In addition, they are steadily becoming a key part of the university's research structure through which to channel international, interdisciplinary and intersectoral collaborations. Together with research excellence units and groups, the platforms are fostering the inclusion and engagement of researchers and stakeholders in impactful research. Specifically, the five "Interdisciplinary Research Platforms" and the four "Interdisciplinary Areas" have helped to aggregate expertise and critical mass to strive for research excellence aligned with the "Europe 2020 Strategy" and the "Basque Smart Specialisation Strategy" (RIS3).

In terms of people management, engaging university staff to work into interdisciplinary communities successfully is a long-term and complex process. Interdisciplinary communities, such as the "Deusto Interdisciplinary Research Platforms", are living, dynamic people-centred systems, with fears and emotions, knowledge and expertise, attitudes and personalities, interests and personal history and relationships within the institution. There is nothing more intricate in an organisation than the people that comprise it, and in general, not enough importance, efforts and resources are dedicated to their development and demands.

By innovatively linking the individual, collective and institutional levels, the evolving "Deusto Research Collaborative Framework" is enabling conditions to overcome barriers and develop successful and sustainable inter and trans-disciplinary, intersectoral collaborations. This is easing the path for delivering indicators of sustainable, real, collaborative efforts, while at the same time moving towards defining meaningful research questions and real impacts.

Finally, one limitation of this work is that, by taking concrete evidence as a reference, this research opts to analyse institutional change from a more "tangible" perspective. To complement this, further studies that are currently in process, as previously mentioned, will broaden the scope and deepen the understanding of the "6i Research Model" from a more sociological approach. Using a combination of quantitative and qualitative datasets and methodologies, the evolution and process will analyse the drivers, the barriers and the role of the agency of individuals and human interaction on the process.

\section{REFERENCES}

Akker, W., and Spaapen, J. (2017). Productive Interactions: Societal Impact of Academic Research in the Knowledge Society. Brussels: LERU.

bmwfw (2016). Hochschulmobilitätsstrategie des BMWFW zur Förderung transnationaler Mobilität an österreichischen Universitäten, Fachhochschulen und Privatuniversitäten. bmwfw.

Barczak, G., Lassk, F., and Mulki, J. (2010). Antecedents of Team Creativity: An Examination of Team Emotional Intelligence, Team Trust and Collaborative Culture. Creativity \& Innovation Management, 19 (4), 332-345.

Bromham, L., Dinnage, R., and Hua, X. (2016). Interdisciplinary research has consistently lower funding success. Nature. Nature. Vol, 534 : 684-687.

Hall, P., and Weaver, L. (2001) Interdisciplinary education and teamwork: a long and winding road. Send to Med Educ. Vol, 35 (9): 867-75.

Moedas, C. (2017). The European Open Science Cloud: The new republic of letters. Speech given at the EOSC Summit.

Waddell, S, and Brown, L. D. (1997). Fostering intersectoral partnering: A guide to promoting cooperation among government, business, and civil society actors (Vol. 13). Institute for development research (IDR). 
Thomson, A., Perry, J., and Miller, T. (2009). Conceptualizing and Measuring Collaboration. Journal of Public Administration Research \& Theory, 19 (1), 23-56.

Woelert, P., and Millar, V. (2013). The 'paradox of interdisciplinarity' in Australian research governance. Higher Eduaction. Vol, 66 (6).

\section{AUTHOR}

\section{ANTONIA CARO-GONZALEZ}

Deusto Advanced Research Centre. International Research Project Office, University of Deusto

Avda Universidades 24, Bilbao, 48007 (Spain)

E: ancaro@deusto.es 


\title{
EVIDENCE-BASED PRACTICE AND POLICES FOR IMPACT ON MENTAL HEALTH OF CHILDREN AND ADOLESCENTS
}

\author{
RAPHAELA E. KAISLER AND JEAN L. PAUL \\ D0I: 10.22163/fteval.2019.377
}

\section{Acknowledgement}

We thank the members of the "Research Group Village" for their contributions in co-creating the research concept and implementation of this work. This group comprises seven academic investigators based within their host institutions:

"Principal Investigator" (PI) Dr. Jean Paul, PhD, BASc, BSc (Hons) ("Mental Health Research Programme" Ludwig Boltzmann Gesellschaft; Medical University of Innsbruck/Innsbruck; Austria), and "Co-Investigators" (Col)) Dr. Ingrid Zechmeister-Koss, MA (Ludwig Boltzmann Institute for Health Technology Assessment/Vienna/Austria), Dr. Melinda Goodyear, PhD, MBSc (Monash University/Melbourne/Australia) Diplom-Kffr. Annette Bauer, MSc (London School of Economics/London/UK), Univ. Prof. Dr. Hanna Christiansen, PhD (Philipps University/Marburg/Germany), Dr. Batool Fatima, Dr.PH, PMDCP, MA (Aga Khan University/Karachi/Pakistan), and Dr. Ingunn Olea Lund, PhD (The Norwegian Institute of Public Health/Oslo/Norway). The team is supported by Dr. Raphaela Kaisler, the research group and relationship manager for the "Mental Health Programme" at the Open Innovation in Science (OIS) centre, Ludwig Boltzmann Gesellschaft (LBG).

"The Village" is a research project funded by the Austrian Federal Ministry of Education, Science, and Research through the Open Innovation in Science Centre at the Ludwig Boltzmann Gesellschaft GmbH, hosted at the Medical University of Innsbruck, with collaboration of Coinvestigator institutions. The study is supported by expertise from Prof. Lesley Stirling (linguistics, The University of Melbourne/Australia) and Prof. Geoff Wong (realist approach, Oxford University/United Kingdom).

Authors would also like to acknowledge the work and contributions of the Innsbruck "Village team": Project coordinator Rosanna Buchauer; PhD students Daniel Purtscheller, Franziska Huck, and Lisa Dobener; and student assistants Julia Kapferer, Elena Seis, Anna-Elena Pinggera, and Johanna Krönner.

The study is performed according to the "Declaration of Helsinki" and its later amendments and was approved by the "Human Research Ethics Committee" of Monash University Melbourne, Australia in addition to internal approval from the London School of Economics, London, UK.

\section{Conflict of interest}

All authors declare no conflict of interest in sense of the "Uniform Requirements of Manuscripts Statement of Medical Journal Editors"1.

\section{INTRODUCTION}

The Ludwig Boltzmann Gesellschaft (LBG), a non-profit research organisation, addresses complex social challenges together with partners, by developing and testing novel forms of cooperation between science and non-scientific actors in a dynamic social environment. In this way, the LBG aims to develop economic and social solutions that positively support social change and can be used directly by civil society, politics and the private sector. LBG's "Research and Innovation Policy" emphasises the targeted and coordinated transgression of the boundaries of organisations, disciplines and systems (Open Innovation in Science) aiming to improve the societal impact of research. Thus, novel forms of engagement increase the opportunity to generate innovative problemsolving approaches.

In this case study, the "Village project", we investigate different measures aiming to drive evidence-based change, towards making a sustainable impact for children that have a parent with a mental illness. First, we introduce an innovative approach to engage the public in generating societal relevant research questions and establishing international and interdisciplinary "Research Groups" on mental health of children and adolescents. Second, we introduce educational programmes for researchers and adolescents to enrich research with meaningful youth engagement and transfer knowledge among different stakeholders and people with lived experience. Last, we focus on community engagement, awareness raising for mental health and working together with people with lived experience as game changers in advocating for informed decision-making on a community and policy level. 


\section{CREATING EVIDENCE-BASED PRACTICE USING A COLLECTIVE IMPACT FRAMEWORK}

Aiming to generate societal impact in the field of "Mental Health of Children and Adolescents", LBG adopted a novel approach towards forming highly interdisciplinary "Research Groups". This aligns to the European Union's Horizon 2020 scheme (European Commission, 2013) to address society's "Grand Challenges" and recognises the central role social sciences and humanities can play through truly collaborative and additive research from multiple paradigms for research to create social impact (Maxwell and Benneworth, 2018). LBG's goal was to engage with different stakeholders and the public throughout the entire research process to develop novel solutions to challenges in the field of mental health, which directly impact society. Therefore, LBG started the "Open Innovation in Science" initiative, with the aim of systematically opening up processes of scientific discovery in an effort to enrich research, through new knowledge drawn from beyond traditional disciplinary boundaries.

\section{ADDRESSING SOCIETAL RELEVANT CHALLENGES THROUGH PUBLIC ENGAGEMENT IN RESEARCH}

Sauermann and Franzoni (2015) showed that user contribution in crowd sourcing is significant in magnitude and speed of crowd-sourcing knowledge. LBG's "Tell Us!"2 was Europe's first crowdsourcing project, generating research questions on mental illness involving patients and family members and healthcare professionals. Four hundred high-quality contributions were analysed and clustered by an expert jury regarding their importance. Out of several important topics, securing mental health for children and adolescents emerged as a key issue. Additional interviews with experts in the field emphasised to focus on "children of mentally ill parents" emerging as the main topic.

Based on this result, LBG announced a research call representing an interactive workshop, "Ideas Lab" ${ }^{3}$, to bring together 30 researchers for a multi-day event, during which researchers were specifically encouraged to think out-of-the box and dissolve disciplinary boundaries. Applicants were asked to complete an application via an online platform comprising six questions with regard to their professional background, expertise and interests contributing to realising the goal of the "Ideas Lab", and approach to team work. In total, 136 researchers applied to participate in the "Ideas Lab", and further assessment by the evaluators consisting of the mentors, an organisational psychologist and the programme mana- ger. Thereof 30 applicants from a diverse range of disciplines had been invited to participate in the "Ideas Lab". During the 5-day event in Vienna, the researchers were supported by mentors, international experts representing a variety of pediatric and adolescent health fields, providing ongoing feedback on the development of project ideas in the "Ideas Lab". The mentors changed their role to become live peer-reviewers for the final presentations and project proposals on the last day of the "Ideas Lab" giving funding recommendations to LBG. Additionally, "provocateurs" or guest speakers, including international mental health researchers and experts by experience (young adults whose parents have a mental illness), were invited to inspire researchers and identify gaps in the mental health service system.

The LBG OIS centre developed novel evaluation criteria for the project proposal that were based on opening up disciplinary boundaries, fostering public engagement in the research process, and establishing new forms of stakeholder interaction and collaboration that lead to interdisciplinary and transdisciplinary research. These following criteria were applied to find innovative solutions to existing challenges in mental health by involving the public in the research process:

1. novelty, revolutionary and high-quality approach to complex challenges,

2. interdisciplinary research,

3. engagement, stakeholder/user engagement throughout the entire research process including dissemination activities and involvement of patients and family members in research activities,

4. feasibility, the capability to deliver their project as a high-quality interdisciplinary activity, provided both through the presentation of their joint proposal and their activity during the "Ideas Lab", and

5. impact, clear relevance to and the potential to make a distinctive and novel contribution towards addressing the research challenges in this area creating added value for society.

As a result of the "Ideas Lab", two "Research Groups", "DOT - Die offene Tür [The open door]" and "Village - How to raise the Village to raise the child" 5 were recommended for funding with a combined budget of EUR 6 million during four years (2018-2021). To ensure public engagement and interdisciplinary research throughout the research process, the "Research Groups" are embedded in a dynamic network working closely with existing networks and patient organisations and are supported by a "Research Group and Relationship Manager" to foster community engagement and collective impact.

\section{"RESEARCH GROUPS'" GOVERNANCE STRUCTURE}

To empower people with lived experience in decision-making, we included their voices in the "Advisory Board" of the "Research Groups", which advises and evaluates the research activities twice a year. The 
"Advisory Board" consists of two academic experts in the field of mental health or specific methodologies within the project, an open innovation expert, a peer PI researcher, and two people with lived-experience. The recommendations of the "Advisory Board" are discussed and agreed upon by the "Steering Committee" including a representative of the LBG and the Medical University of Innsbruck (the "Village project's" university host organisation).

Besides traditional scientific measures, such as peer-review publications, dissemination and outreach activities, we introduced new assessment criteria regarding the meaningful engagement of public in research activities:

1. inclusion of people with 'lived experience' in research activities and community engagement,

2. co-development of interventions with stakeholders,

3. implementation and evaluation of their practice,

4. policy recommendation and engagement of policy makers in research activities,

5. up-scaling strategies for sustainable impacts for children and adolescents.

Additionally, to foster continuous engagement of people with livedexperience in the research process, we established the "Competence Group" as a new advisory body consisting of six young adults with lived experience ("Children of parents with a mental illness" - COPMI). This group consults both "Research Groups" on their research activities on a regular basis. In this way, we ensure the research supports inclusion of expertise based on own experiences. As a next step, governmental funding should be applied to increase awareness of valuable contribution of people with lived experience in research and sustainability of their work.

\section{THE "VILLAGE PROJECT": CO-DEVELOPMENT WITH STAKEHOLDERS TO CHANGE PRACTICE}

"Children of parents with a mental illness" (COPMI) often need additional supports to lead the happy and healthy lives they desire. However, in some cases, those supports are either not available or not found by families, resulting in negative long-term outcomes for these children. The "Village project" aims to increase identification and strengthen formal and informal supports around children when their parents have a mental illness (Christiansen et. al., submitted). This project will be co-developed with stakeholders and will implement and evaluate two practice approaches, focused on the child and on principles of collaborative care. A key challenge is that much of the 'hard' evidence of what works for whom, and what is good value for COPMI is largely lacking. In the light of this lack of evidence, it has been argued (Nicholson, 2009) that the following should be emphasised: involving practitioners and people with livedexperience as equal partners in research; the appropriate application of mixed-methods to explore the issues; and the development and application of appropriate child-specific outcome measures to better understand the needs and impacts on COPMI (focusing on child's self-esteem and resilience). After a scoping phase, synthesising the international evidence on barriers and opportunities for support for
COPMI, we will provide information on the mental health and social services within Tyrol in Austria, the project site.

Continuing public engagement in research to make an impact, the "Village" project aims to improve the situation of children who have mentally ill parents (COPMI) in Tyrol, Western Austria. In order to develop practice approaches to better identify and support these children and their parents, we needed an in-depth understanding of the regional Tyrolean characteristics in terms of existing support structures and the societal context in which they are embedded. This work was led by Col Ingrid Zechmeister-Koss, and the following welfare-state sectors were systematically analysed in terms of potentially relevant benefits: 'Health care', 'children/families', 'social affairs' and 'education'. The information on available benefits was firstly categorised according to welfare state sectors, and then synthesised into an overview of services that could be potentially relevant in the process of identifying and supporting COPMIs and their families (Zechmeister-Koss and Goodyear, 2018).

Tyrol is a region in the Western part of Austria, constituting nine political districts. From roughly 750.000 inhabitants, around 140.000 persons (19\%) are dependent children (0-18 years). The vast majority lives in dual-parent families. Catholic religion plays an important role in Tyrol. $85 \%$ of Tyroleans are Austrian citizens. $50 \%$ of the population is actively working in paid employment, the remainder is either retired $(20 \%)$, in education or in other forms of activity (parental leave, household leading only, military service). Regarding the identified benefits, both in-kind as well as cash-benefits are relevant. While benefits for children/families are mostly cash benefits with only limited publicly funded child-care facilities, in the other sectors, in-kind benefits (e.g. publicly paid health or social care services) are dominant. We identified a broad variety of benefits that may be utilised to identify and support COPMIs and their families. However, only one of the existing services (available in two districts) directly targets COPMIs. In terms of setting, a vast majority of services are office-based and a much smaller proportion of providers offer outreach services (e.g. in families' homes). The available services are characterised by a high proportion of public funding, however, access to publicly funded services may be restricted via gate-keeping (e.g. referrals from child and youth service) or shortage of capacities (e.g. psychotherapy, child care). The existing services show a geographical variation with more (types of) services available in the urban than in the rural regions. Services are characterised by high fragmentation in terms of governance (federal, regional, municipality), financing (taxes: federal, regional; social insurance) and service provision (public and private providers).

These results and a scoping of international best practice examples will inform the co-development phase with stakeholders in Tyrol, which will be made up of six co-design workshops which began in November 2018. During the co-development phase, we will develop practice approaches and tools to identify COPMI and to support them in everyday life by strengthening networks among formal and informal support systems in Tyrol. This will be supplemented with training material for implementing the practice approaches and thirdly, key-indicators for evaluating the practice approaches will be defined. The development of the practice approaches and evaluation indicators will be done in a participatory manner (co-design) involving representatives of stakeholders and particularly including people with lived experience. Community-capacity building approaches, concerned with developing a supportive network of allies around a person, utilising principles of collaboration, personcenteredness, and prevention, can increase resilience at an individual and community level, as well as be cost-effective (Knapp, Bauer, Perkins 
and Snell, 2013; Wistow, Perkins, Knapp, Bauer and Bonin, 2016); how this relates to COPMI is not yet known, and this project will generate evidence to address this gap. A participatory and co-developed approach to the development of screening approaches and collaborative care, that is evidence-informed and evidence-generating, has not yet been implemented for COPMI - neither worldwide, nor in Austria. To this end, we will facilitate a series of design workshops with stakeholders at the study site to develop the components of the practice approaches based on the results from the scoping phase.

Practical efforts to initiate the practice approaches are central to the installation phase of implementation and include activities such as: developing the competence and confidence of staff through training and coaching in the new approach, as well as monitoring progress through regular check-ins and supervision of staff at study sites. The training protocol developed in the workshops will include the theoretical basis and underlying values of the programme, use adult learning theory, introduce components and rationales of key practices, provide opportunities to practice new skills to meet fidelity criteria, and receive feedback in a safe and supportive training environment. The length of training will be determined by the extent of change to the existing programme and practice model, but typically the face-to-face component will run over two days. A significant activity is to support each site in using the new practice approaches, as well as the research protocols. Champions of change will be identified during the workshops. These professionals, "Village facilitators", will be trained and supported to facilitate the formal and informal child-focused support. Once the new practice approaches and associated supportive systems are being used, strategies to promote continuous improvement and rapid-cycle problem solving will be applied. The research team will work with the study sites to use data to assess implementation progress, identify barriers, potential solutions, and drive decision-making.

An additional feature of this project is the central focus of understanding and listening to the 'child voice'. COPMI support in adult focused services has so far been mostly parent-centred, and not likely to identify or develop an evidence-informed support plan that meets the needs and listens to the 'voice' of the child. Incorporating the child's voice in practice approaches is likely to contribute positively to better outcomes, but this knowledge has not yet been developed. The importance of 'assent' and supporting children to develop their own 'voice' in healthcare is becoming increasingly recognised within the broader field of child health research. This follows the "United Nations Convention on the Rights of the Child" (United Nations. General, 1989), acknowledging the ethical imperative and rights for children to be provided with their own health information. Although research in healthcare communication is increasingly recognised as important in improving health outcomes, in both the areas of mental health and paediatrics, rigorous research investigating naturally occurring healthcare interactions involving the child is extremely limited. In particular, good healthcare service delivery is dependent upon clear and open communication between patients and their treating team. Improving communication within healthcare encounters can reduce medical errors, and act as a therapeutic lever to support patient empowerment (Roter and Hall, 2006). Limited research has shown that children retain some information better than their parents, and an increased proportion of doctor-child communication compared to doctor-parent communication can increase parental satisfaction (Pantell, Stewart, Dias, Wells and Ross, 1982). For COPMI, these children may also not have the support of their parents in healthcare interactions. Consequently, supporting health professional-child communication could be argued to be even more important with COPMI to ensure children's concerns, needs, and wishes are discussed. This project provides a unique and valuable opportunity to investigate children's perspectives and interactive capacity within the COPMI setting, and to observe changes over time, in parallel with the broader interventions of this project. This project will importantly address knowledge gaps in this area and drive practice change. Evidence collected during the investigation of the 'child voice' will contribute to training approaches and inform the design of practice changes within the broader project.

\section{EMPOWERMENT OF COMMUNITY AND LEADERSHIP}

To empower patients, family members and the wider public to engage in research, $L B G$ offers a public training programme "SCIENCE4YOU ${ }^{\text {TH" } 6}$ that was launched in September 2018 addressing adolescents and young people with lived experience. This programme aims to train adolescents scientific principles and methods in order to work as a co-researcher in research groups and teams. In a flipped-classroom approach (Moffett, 2015) with interactive video tutorials and quizzes, participants learn about the research process, how to apply open innovation in science (OIS) methods, develop their own research projects and apply their newly gained knowledge working together with the "Research Groups" (internships). Nineteen adolescents form high schools across Austria applied for the programme, thereof 16 females, that are mentored by pre and post doc researchers. Each mentor supports two mentees during the whole programme and development of their own research projects. Additionally, mentees are supported by a buddy system, each adolescent work in tandem with a peer. With this mutual learning approach, potentially new insights on how to actively involve the community in research will be established and the relationship between young people and researchers will be strengthened. These activities aim to empower youth, in order to establish youth leadership in mental health and develop youth partnerships with the government to make informed health decisions and be represented in national decision-making boards drawing on their experience and expertise.

\section{COLLECTIVE IMPACT AND COMMUNITY ENGAGEMENT}

A variety of routes can be applied to create change and impact for mental health practice and policies for children and adolescents. Besides providing rigorous scientific evidence and systematically increasing competences of individuals, it is critical to strengthen advocacy in order to raise awareness, identify and connect advocates and foster decision- 
making processes to steer political change on many levels.

\section{ADVOCACY}

Raising awareness for the topic through multi-channel broadcasting activities is one of the main pillars to increase the potential for successful advocacy. This can be done through a variety of methods; however, crowdfunding is one of the main methods applied in this context that allows simultaneously raising money and awareness. In order to create successful crowdsourcing and crowdfunding campaigns, it is absolutely necessary to transform scientific messages into commonly understandable language with a clear scope and precise call to action. This approach will identify individuals who have not been aware of the topic before and reach individuals who are willing to support the implementation. Additionally, crowdsourcing helps researchers and practitioners to reflect on their own work and allows for new structures and approaches to emerge. Raising money and awareness is a complementary effort that will underpin the basis to strengthen and encourage advocates as a first initial step. Furthermore, creating awareness will lead to the representation of patients and people with (lived) expertise in decision-making boards that influence priority setting, making the topic more pressing and thus, relevant for political agenda setting and decision-making.

We will foster new ways of collaboration and structures among stakeholders that allow a cross-disciplinary exchange of practice and experience. Additionally, possible awareness campaigns in schools will inform and activate students and their families to find peer support providing self-help groups for COPMI, professional support and referral to specific networks. Further, we will engage with policy makers in our research activities early in the process to present evidence-based practice and strategies to upscale the project including people with lived-experience in the exchange.

\section{VALUING COMMUNITY CONTRIBUTIONS}

Engaging the general public in research is crucial to drive practice change to tackle socially relevant challenges. However, it is equally important to value the community's contributions and act on a level playing field to foster sustained engagement and collective impact. We envision capacity building activities that will be rewarded to maintain people's own development. For example, we will provide public space to inform and foster discussion about mental health between the public, researchers and people with lived-experience, create a peer network where people with lived-experience share their expertise, train interested people in research principles and public engagement, and foster community ownership by conducting youth/community-led research initiatives and projects. Close collaboration with stakeholders will be crucial to successfully drive these activities. An initial strategy to this end has already been initiated through the development of an online discussion forum hosted on the "Village project's" website ${ }^{7}$. These initiatives may be supported by additional governmental funding, cooperation with industry and donations.

In conclusion, creating evidence-based practice, using a collective impact framework and community engagement, will foster a sustainable impact on children and adolescents to truly drive system change. These activities will build capacity within a community, national and European level raising awareness of policy-makers on current challenges in mental health. Nevertheless, advocating for change on a community and policy level is key for successful implementation of system change thereby valuing communities' contribution and development in mental health.

\section{REFERENCES}

Christiansen, H., Bauer, A., Fatima, B., Goodyear, M., Lund, I. O., Zechmeister-Koss, I. and Paul, J. L. (submitted). How to raise the village to raise the child. Protocol of a practice approach to improve outcomes for children of parents with a mental illness. Frontiers in Psychiatry.

European Commission (2013). EUR 25271 - The Grand Challenge The design and societal impact of Horizon 2020. Retrieved from Luxembourg: Publications Office of the European Union

Knapp, M., Bauer, A., Perkins, M. and Snell, T. (2013). Building community capital in social care: is there an economic case? Community Development Journal,, 48(2, 1), 313-331.

Maxwell, K. and Benneworth, P. (2018). The construction of new scientific norms for solving Grand Challenges. Palgrave Communications, 4(1), 52.

Moffett, J. (2015). Twelve tips for "flipping" the classroom. Med Teach, 37(4), 331-336.

Nicholson, J. (2009). Building the evidence base for families living with parental mental illness. Australian e-Journal for the Advancement of Mental Health (AeJAMH), 8(3), 222-226.

Pantell, R. H., Stewart, T. J., Dias, J. K., Wells, P. and Ross, A. W. (1982). Physician communication with children and parents. Pediatrics, 70(3), 396-402.

Roter, D., and Hall, J. (2006). Doctors Talking with Patients/Patients Talking with Doctors: Improving Communication in Medical Visits, Second Edition: Santa Barbara: ABC-CLIO.

Sauermann, H. and Franzoni, C. (2015). Crowd science user contribution patterns and their implications. Proc Natl Acad Sci U S A, 112(3), 679-684.

United Nations. General, A. (1989). Convention on the Rights of the Child, 20 November 1989. Annu Rev Popul Law, 16, 95, 485-501.

Wistow, G., Perkins, M., Knapp, M., Bauer, A. and Bonin, E.-M. 
(2016). Circles of Support and personalization: Exploring the economic case. J Intellect Disabil., 20, 194-207.

Zechmeister-Koss, I. and Goodyear, M. (2018). Supporting children who have mentally ill parents in Tyrol: A mapping of existing support structures and epidemiological dimensions. LBI-HTA Projektbericht Nr. 113a, Wien: Ludwig Boltzmann Institut für Health Technology Assessment.

\section{AUTHORS}

\section{RAPHAELA E. KAISLER}

Open Innovation in Science Center, Ludwig Boltzmann Gesellschaft, Nussdorferstrasse 64, Vienna, 1090 (Austria)

E: Raphaela.kaisler@|bg.ac.at

\section{JEAN L. PAUL}

Research Group Village, Ludwig Boltzmann Gesellschaft Anichstrasse 35/2, Innsbruck, 6020 (Austria)

Department of Psychiatry, Psychotherapy and Psychosomatics, Division of Psychiatry I, Medical University of Innsbruck

Vinzenzgebäude - Haus 11 / 2. Stock, Anichstraße 35, Innsbruck, 6020 (Austria)

E: Jean.paul@|bg.ac.at 


\title{
IMPACT OF SOCIAL SCIENCES AND HUMANITIES FOR A EUROPEAN RESEARCH AGENDA - VALUATION OF SSH IN MISSION-ORIENTED RESEARCH ${ }^{1}$
}

\section{RETHINKING SOCIETAL IMPACT - COLLABORATION WITH STAKEHOLDERS}

\author{
BETTINA UHRIG
}

DOI: $10.22163 /$ fteval.2019.378

\section{ABSTRACT}

$\mathrm{P}$ rojects funded by "Framework Programmes for Research and Innovation" should increasingly involve citizens and create societal impact. In particular, Social Sciences and Humanities (SSH) researchers focus on societal challenges and collaborate with different stakeholders. The involvement of stakeholders in proposal development and project implementation is needed to secure citizens involvement. However, successful stakeholder involvement in proposals and projects is time consuming and needs a strong management structure, which should support and monitor activities, which can lead to societal impacts. Furthermore, Horizon 2020 and Horizon Europe indicators should place greater importance on the involvement of stakeholders.

\section{BACKGROUND AND PURPOSE}

Close collaboration with stakeholders has been a demand for "European Framework Programme" projects for many years. The "Fourth Framework Programme" (FP4, 1994 - 1998) already contained a specific programme called "Targeted Socio-Economic Research" (TSER). The TSER programme encouraged the involvement of stakeholders in research projects to achieve a better uptake of project results by policy makers and civil society: "In line with the Commission's White Paper on Growth, Competitiveness and Employment, the research activities aim at rationalising future decision-making at decentralised, national or Community levels in order to develop a shared knowledge base on the socio-economic challen- ges facing Europe." Also in the "Fifth", "Sixth" and "Seventh Framework Programmes" collaboration with civil society was valued.

With Horizon 2020 (FP8), the involvement of stakeholders and the discussions on societal impact of research projects increased. Yet, the interim evaluation of Horizon 2020 (H2020) showed that one main area for improvement is bringing results to citizens and involving them more. "There is a need for greater outreach to civil society to better explain results and impacts and the contribution that research and innovation can make to tackling societal challenges, and to involve them better in the programme co-design (agenda-setting) and its implementation (co-creation)." (EC, DG RTD 2017a, p.21). Also, the report from the "High Level Group on maximising the impact of EU Research \& Innovation Programmes" calls for mobilising and involving citizens through co-design and co-creation of programmes and projects at European, national and regional levels (EC, DG RTD 2017b).

Consequently, Horizon Europe (FP9) will demand even further citizen involvement ${ }^{3}$. However, it is often forgotten that citizens speak different languages and sufficient funding needs to be available for interpretation and translations. Policy reviews, published by the Directorate-General for Research and Innovation, provide tools and analysis to policy makers, but they are often not wide enough distributed and discussed and only available in English.

Close collaboration with stakeholders could be one way of involving citizens. In this regard, it is important to remember that people working with research policies, programmes and projects are citizens, as well.

To increase citizens' involvement in Horizon Europe, a critical reflection on stakeholder involvement in $\mathrm{H} 2020$ projects and a discussion on tools for achieving societal impact is necessary. Here, societal impact is

1 The author acknowledges the STI 2018 Leiden conference, from which this template was adapted.

2 FP4-TSER - Specific programme of targeted socio-economic research, 1994-1998. Retrieved October 25, 2018 from: https://cordis.europa.eu/programme/ rcn/465_en.html.

3 Information on Horizon Europe: https://ec.europa.eu/info/designing-next-research-and-innovation-framework-programme/what-shapes-next-frameworkprogramme_en. 
defined as "social improvements e.g. via the use of project results by policy makers or other societal actors" (Net4Society 2017, no page number). Such 'use' often happens after the end of the project and is very often not part of the project evaluation. For example, "ASIRPA (Asian Society for International Relations and Public Affairs) found that the average time lag for impact that comes from applied research was 19.9 years. For fundamental research, much longer time lags are needed." (Science Europe 2017, p.17)

\section{METHODS AND ACTIONS TO INCREASE STAKEHOLDER INVOLVEMENT}

The following methods and actions have been developed and used by the author and colleagues for drafting proposals and implementing projects since FP4. The focus is on proposal development and project implementation.

\subsection{STAKEHOLDER INVOLVEMENT FROM THE BEGIN- NING AND “CIVIL SOCIETY ORGANISATIONS" AS CON- SORTIUM MEMBERS}

Successful proposals on call topics in H2020 Societal Challenge 6 "Europe in a changing world - Inclusive, innovative and reflective societies" contain a clear description of impact. They outline the project's contribution to the scientific/academic impact, societal (incl. political) impact and economic impact. In order to develop a project proposal that convinces evaluators and, at the same time, is feasible, it is vital to involve stakeholders from the beginning of the proposal development. Discussions with representatives from organisations, which should work with the research results, are needed to develop the research questions, the concept and the work packages to produce the promised outputs and to contribute to the expected impacts, which are described in the call topic text.

In successful research proposals, submitted by the Norwegian Social Sciences research institute (NOVA), "Civil Society Organisations" (CSOs) were involved in the project design and have been members in the project consortium from the beginning of the project. Since H2020, they can be a project partner under the same financial conditions as higher education institutions and research organisations. In most cases, their involvement demands more openness and leads to more discussions during the proposal process and the project implementation. Different ways of working need to be discussed and agreed upon. This influences re- search methods, as well as communication and dissemination activities. Developing the proposal and implementing the project becomes even more time-consuming, but brings the proposal and the projects closer to the expected impacts described in the call topic and enhances the possibilities for the uptake of research outcomes by stakeholders.

\subsection{INVOLVEMENT OF STAKEHOLDERS AT DIFFERENT LEVELS}

In addition to involving CSOs as full consortium members, we contacted possible members for an international or European advisory group supporting the implementation of the project already during the proposal phase. The members came from academia, public, private and social partner organisations, industry or CSOs. Some of them reviewed the proposals before submission and in this way contributed to excellent proposals. If a proposal was approved, members from the advisory group were involved in the implementation of the project, for example in discussions of methods and research questions and in supporting dissemination activities. In most cases, the project covered their travel and hotel costs to attend project meetings (max. twice a year), but did not finance any working time. These limited funding options make it difficult to convince people working at CSOs to join advisory groups at project level. Their involvement needs to be approved by their boards and many board members and directors of CSOs would like to see some financial compensation for their involvement, which makes it less likely for them to approve such involvement.

An even more important tool for stakeholder involvement has been the set-up of stakeholder groups or committees at national levels. The members of these groups can again come from academia, public, private or social partner organisations, industry and CSOs. Their involvement in projects has contributed to more publicity of the research projects. Group members have not only supported dissemination actions; they have also helped in finding interviewees and drafting "Policy Briefs" describing research findings relevant for stakeholders ${ }^{5}$. "Policy Briefs", translated into national languages, have been very useful for the work of CSOs. In all projects, some members from the national stakeholder groups were also invited to project conferences. In projects, coordinated by NOVA, national stakeholder groups have not received any funding, only travel costs and, if necessary, translations were covered by the project budget.

Furthermore, stakeholders can be involved in the research projects though different activities, like advocacy meetings, focus groups and thematic workshops ${ }^{6}$.

\subsection{DEDICATED IMPACT MANAGEMENT}

4 Since 2007, the author has been employed at NOVA, which merged with the Oslo and Akershus University College of Applied Sciences (HiOA) in 2014. In 2018, HiOA was granted the status of a university and changed its name to Oslo Metropolitan University (OsloMet).

$5 \quad$ Examples for Policy Briefs from H2020 SSH projects can be viewed at http://ec.europa.eu/research/social-sciences/index.cfm?pg=libraryglib=policy_briefs.

6 The H2O2O project DANDELION - "Promoting EU funded projects of inclusive, innovative and reflective societies" described several different tools for dissemination and stakeholder involvement, http://www.dandelion-europe.eu/en/infobase/guides-to-maximise-impact-of-ssh-projects/guides-to-maximiseimpact-of-ssh-projects1.html, viewed October 27, 2018. 
Since 2016, we include, an impact manager in the implementation of the $\mathrm{H} 2020$ research projects due to the many project management tasks. We found that it works best if it is already clear during the proposal phase who will have this position in case the proposal is approved. Our experiences show that the involvement of an impact manager can ease the communication between the consortium members and leads to a stronger focus on achieving impact.

\subsection{CASE STUDY DARE - TOOLS FOR ACHIEVING SO- CIETAL IMPACT}

In the ongoing H2020 project "DARE" (Dialogue About Radicalisation and Equality) ${ }^{7}$, the impact manager has been involved from the beginning of the proposal process, which started in the summer of 2016. Together with the coordinator, the impact manager invited CSOs to the project during the proposal development. This affected the project description and implementation in, among others, the following three ways:

1. The "Plan for Exploitation and Dissemination of Results" (PEDR) is very detailed and specific. In the proposal, we already included a detailed plan describing dissemination and exploitation activities in each work package, the target audiences and users, as well as related output and impact measures. The PEDR is regularly updated throughout the project duration (May 2017 - April 2021).

2. The management structure contains an "Impact Sub-Committee" (ISC), which supports and monitors the dissemination, exploitation and impact activities and is chaired by the impact manager. The ISC meets regularly online and approximately three times face-to-face each year. The ISC also writes internal impact reports every nine months.

3. By October 2018, nearly all consortium members had established "National Stakeholder Groups" (NSGs), with whom they discuss the development of the project and which they involve in dissemination activities. For the DARE consortium, it is important that all DARE partners create the NSG they require and meet with their NSG to reflect on their tasks in DARE and their national context when it fits (approximately two times each year). The types of stakeholders and size of the NSG therefore differ, with most NSGs having between six and 12 members. All partners write minutes of their NSG meetings, which are available for all consortium members and which are a very important resource for the impact management and monitoring.

In April 2018, the project published its first "Policy Brief" written by members of the ISC and the coordinator. During the third project meeting in May 2018, the ISC organised an impact workshop for all DARE colleagues discussing their experiences, questions and ideas related to working with societal impact. Already now, it is evident that the involvement of an impact manager and an "Impact Sub-Committee" has created a stronger focus on impact for all consortium members.

\section{RECOMMENDATIONS}

\subsection{IMPACT MANAGEMENT AT ORGANISATION LEVEL}

The good practice example resulting from DARE leads to the question of whether impact management should also have a more prominent role at organisation level: Could an impact manager, employed at the management level of an organisation, ease and enhance the collaboration with citizens, stakeholder involvement in projects and the uptake of research results by individuals, organisations and institutions?

Several universities, especially in the UK, already employ impact managers. Among other tasks, they support and collect the descriptions of impact case studies. Excellent impact case studies can lead to additional funding by national authorities ${ }^{8}$. Impact case studies are used for collaboration with the media and enhance the communication with citizens. Of course, the creation of an impact manager's position requires further personnel resources. Establishing impact management at organisation level would help to advance the project outcomes after the end of the project and would furthermore give time and resources for impact assessments.

\subsection{REVISED INDICATORS FOR SOCIETAL IMPACT}

Involvement of stakeholders in research projects should count not only for evaluators dealing with proposals but also for the overall evaluation of research projects and the programme evaluation.

A public debate on revised indicators for Horizon Europe (EC, DG RTD 2015) is therefore needed. The orientation on the "Technology Readiness Levels" (TRLS) of a project needs to be questioned and broadened. For measuring societal impact, a longer timeframe after the end of a project is needed, and, instead of TRLs, programme evaluators and developers could consider the "Societal Readiness Levels" (SRLS) of a proposal and project. Cooperation with stakeholders could be one indicator for societal impact and be included in the description of the SRLs. This is reflected by the "Innovation Fund Denmark", which has published a description of SRLS. SRLs are already considered for the development of indicators for Horizon Europe (EC, DG RTD, 2018a). Table 1 below examines the differences between TRLs and SRLs. It demonstrates the relevance of SRLS when measuring societal impact.

\section{FINDINGS AND}

7 DARE has received funding from the European Union's Horizon 2020 research and innovation programme under grant agreement No 725349. http://www. dare-h2020.org/.

$8 \quad$ This is for example the case in UK, where "Higher Education Institutions" can receive additional state funding based on their impact cases. Further information can be found on the website of the "Research Excellence Framework": http://www.ref.ac.uk/. 


\begin{tabular}{|l|l|l|}
\hline 1 & Basic principles observed. & Identifying problem and identifying societal readiness. \\
\hline 2 & Technology concept formulated. & $\begin{array}{l}\text { Formulation of problem, proposed solution(s) and potential impact, expected } \\
\text { societal readiness; identifying relevant stakeholders for the project. }\end{array}$ \\
\hline 3 & Experimental proof of concept provided. & Initial testing of proposed solution(s) together with relevant stakeholders \\
\hline 4 & Technology validated in lab. & $\begin{array}{l}\text { Problem validated through pilot testing in relevant environment } \\
\text { to substantiate proposed impact and societal readiness. }\end{array}$ \\
\hline 5 & Technology validated in relevant environment. & Proposed solution(s) validated, now by relevant stakeholders in the area. \\
\hline 6 & $\begin{array}{l}\text { Technology demonstrated in relevant } \\
\text { environment. }\end{array}$ & $\begin{array}{l}\text { Solution(s) demonstrated in relevant environment and in coĐoperation with } \\
\text { relevant stakeholders to gain initial feedback on potential impact. }\end{array}$ \\
\hline 7 & System prototype demonstrated in operational environment. & $\begin{array}{l}\text { Refinement of project and/or solution and, if needed, retesting } \\
\text { in the relevant environment with relevant stakeholders. }\end{array}$ \\
\hline 9 & System complete and qualified. & Proposed solution(s) as well as a plan for societal adaptation. \\
\hline
\end{tabular}

SRLs and stakeholder involvement should be linked to the "United

See EC, DG RTD, 2018a, page 10.

"Innovation Fund Denmark" (n.d.). Societal Readiness Levels (SRL) defined according to Innovation Fund Denmark, Copenhagen Retrieved October 27, 2018 from: https://innovationsfonden.dk/sites/default/files/2018-08/societal_readiness_levels___srl.pdf.

Table 1. Comparison of TRLs and SRLS.

Nations Sustainable Development Goals" (SDGs). In particular, SDG 17 'Strengthen the means of implementation and revitalise the global partnership for sustainable development through capacity building' could be studied to improve stakeholder involvement. ${ }^{9}$

\subsection{FUNDING FOR COMMUNICATION, DISSEMI- NATION AND IMPACT MANAGEMENT AFTER THE END OF A PROJECT}

To secure the focus on project results, future "Framework Programme" projects should receive additional funding after the end of the project to continue with communication and dissemination activities (see also 3.1), which can lead to societal impacts. The interim evaluation of H2020 made clear that "the projected social and economic impacts, for example on the creation of spin offs, on employment or the development of new innovation, are difficult to measure lin terms of causality with the projects financed), in particular because they might happen at a point beyond the lifetime of the project. This needs to be taken into account in future impact evaluations. It is also difficult to predict if stakeholder collaboration across different types of organisations will last beyond the duration of the projects." (EC 2017, page 969).

\subsection{COLLABORATION WITH STAKEHOLDERS AND CITIZENS' INVOLVEMENT}

Citizens' involvement, as demanded by the members of the "High Level Group on maximising the impact of EU Research \& Innovation Programmes" (EC, DG RTD 2017b), should be discussed in detail. Studies published by the European Commission (EC) and academic networks describe many different possibilities involving citizens in EU policies and research programmes (Van den Brande 2017; Science Europe 2018; CIMULACT 2018). Collaboration with stakeholders, as described above, creates several possibilities for citizens' involvement. How this can be organised could be discussed with CSOs, which have experiences with FP projects.

Furthermore, and bearing in mind the rise of populism, it is important to reflect on the challenges created by citizens' involvement. It could be important to agree on joint values before starting any form of collaboration. Here, it could be useful to refer to the fundamental values of the European Union and the Council of Europe: human rights, democracy and the rule of law.

Even though, the EC organised workshops with stakeholders and implemented stakeholder consultations (EC 2018), Horizon Europe is not well known by regional and national CSOs. To change this, Net4Society ${ }^{10}$ could, in close collaboration with for example SDG Watch Europe ${ }^{11}$ and the Directorate-General for Research and Innovation, organise "Future Search Conferences" (Weisbord, M. and Janoff, S. 1999) involving CSOs

$9 \quad$ For closer information on SGDs see: https://sustainabledevelopment.un.org/topics/capacity-building. Furthermore, the H2020 project DANDELION contributed to the discussion of SRLs (Dandelion n.d.) and in June 2018 DG RTD published a detailed description of key societal impact pathways and progress indicators (EC, DG RTD, 2018a).

10 Net4Society is the International network of National Contact Points (NCPs) for Societal Challenge 6 in Horizon 2020, http://www.net4society.eu/.

11 SDG Watch Europe is a European cross-sectoral civil society alliance advocating for the implementation of the SDGs, https://www.sdgwatcheurope.org/ about-us/. 
and other stakeholders to discuss the main societal challenges, which will be important for the design and implementation of Horizon Europe. Public engagement has become one of the main demands for developing missions in Horizon Europe and missions should have societal relevance (Mazzucato 2018; EC, DG RTD 2018b).

\section{CONCLUSIONS}

Rethinking collaboration with stakeholders in H2020 research projects and linking it to citizens' engagement in Horizon Europe, in particular in missions and projects funded under the Global Challenges, could be useful for widening the discussions on the design and implementation of Horizon Europe and the revision of indicators. Professional and clearly defined impact management could ease the collaboration with stakeholders and the work with proposals, projects and project outcomes to achieve societal impacts.

\section{REFERENCES}

CIMULACT (2018). Inspiration Catalogue for consulting different groups. Retrieved October 29, 2018 from: http://www.cimulact.eu/wp-content/ uploads/2018/04/D5.1_Inspiration-Catalogue-for-consulting-differentgroups-compressed.pdf.

DANDELION (n.d.). Inclusive, innovative and reflective societies-sensitive valorisation concept. Retrieved October 27, 2018 from: http://www. dandelion-europe.eu/imagem/IIRS_sensitive_Valorisation_Concept.pdf.

European Commission (EC). (2017). Commission staff working document Interim evaluation of HORIZON 2020. Part 0. Europe in a changing world - Inclusive Innovative and Reflective Societies. Brussels. Retrieved October 27, 2018 from: https://ec.europa.eu/research/evaluations/pdf/ archive/h2020_evaluations/swd(2017)221-annex-2-interim_evaluationh2020.pdf\#view=fitgpagemode=none.

European Commission (EC). (2018). Horizon Europe Stakeholder Consultation. Synopsis Report. Brussels.

European Commission, Directorate-General for Research and Innovation (EC, DG RTD). (2015). Horizon 2020 indicators - Assessing the results and impact of Horizon. Luxembourg: Publications Office of the European Union.

European Commission, Directorate-General for Research and Innovation (EC, DG RTD). (2017a). Key findings from the HORIZON 2020 interim evaluation. Luxembourg: Publications Office of the European Union.

European Commission, Directorate-General for Research and Innovation (EC, DG RTD). (2017b). $\angle A B-F A B-A P P$ - Investing in the European Future we want. Luxembourg: Publications Office of the European Union.

European Commission, Directorate-General for Research and Innovation (EC, DG RTD). (2018a). A NEW HORIZON FOR EUROPE - Impact Assessment of the 9th EU Framework Programme for Research and Innovation. Luxembourg: Publications Office of the European Union.
European Commission, Directorate-General for Research and Innovation (EC, DG RTD). (2018b). Mission-oriented research and innovation - Inventory and characterisation of initiatives. Luxembourg: Publications Office of the European Union.

Mazzucato, M. (2018). Mission Oriented Research \& Innovation in the European Union. Luxembourg: Publications Office of the European Union.

Net4Society (2017). Increasing Impact! Retrieved October 27, 2018 from: http://www.net4society.eu/_media/Net4Society4_D3_1_1_Factsheet_ Impact_final.pdf.

Science Europe (2017). Symposium Report - Building a Scientific Narrative on Impact and Societal Value of Science. Brussels.

Science Europe (2018). Briefing paper on Citizens Science. Brussels.

Van den Brande, L. (2017). Reaching out to the citizens: A new opportunity 'About us, with us, for us'. Luxembourg: Publications Office of the European Union.

Weisbord, M. and Janoff, S. (1999). Future Search - Collaborating for Change. San Francisco.

\section{AUTHOR}

\section{BETTINA UHRIG}

Norwegian Social Research (NOVA), Os/o Metropolitan University

Stensberggata 26, Oslo, 0170 (Norway)

E: Bettina.uhrig@oslomet.no 


\section{THE CHANGING PATTERN OF SOCIAL SCIENCES AND HUMANITIES IN THE EU FRAMEWORK PROGRAMMES RETHINKING SOCIETAL IMPACT - COLLABORATION WITH STAKEHOLDERS}

\section{INTRODUCTION}

$\mathrm{T}$ The European Union EU "Research and Development Framework Programmes" (FPs) had been in operation for ten years before socio-economic research was included under the "Fourth Framework Programme" (1994-1998). It was directly related to the results of the Maastricht Treaty (Reillon, V., 2017). It was a period when the need for "soft power" arose. Joseph Nye's (Nye, J., 1990) "soft power" approach adopted during the fifth enlargement of the EU was considered the EU's most successful foreign policy instrument (Rehn, 0 ., 2007, Tulmets, E., 2008). Certainly, this gave an impetus to the further deepening of the social dimension of the Framework Programme. In the successive frameworks more and more elements of the social sciences and humanities (SSH) research were added (Table 1).
By now, the FP is undoubtedly one of the largest funding instruments for the European SSH scholarships through its various instruments. Research on impact and performance of SSH in FPs have been mainly the task of expert groups set up by the European Commission (Watson, J., et al., 2010, Hetel, L., et al., 2015, Birnbaum, B. I., et al., 2017, Bade Strom, T., et al., 2018, Challis, L., et al., 2003, Cerletti, C., et al., 2001.). In research journals, the approaches have appeared relatively scarcely (Georghiou, L., et al., 2002, Must, Ü., 2010a, 2010b, Schindler-Daniels, A., 2014.). The aim of this paper is to monitor and analyse the evolution (or overlapping) of the SSH thematic pattern through the three framework programmes since 2002.

\begin{tabular}{|l|l|l|}
\hline FP & Period & SSH Work Programme \\
\hline FP4 & $1994-1998$ & Targeted socio-economic research. \\
\hline FP5 & $1998-2002$ & Improving the socio-economic knowledge base. \\
\hline FP6 & $2002-2006$ & Citizens in the knowledge-based society. \\
\hline FP7 & $2007-2013$ & Socio-economic Sciences and Humanities. \\
\hline H2020 & $2014-2020$ & Europe in a changing world - inclusive, innovative and reflective societies. \\
\hline
\end{tabular}

Table 1. EU Framework Programmes with elements of SSH research. 


\section{METHODS}

We used textual analysis for conducting the survey. The set of documents to perform the analysis is based on two sources: a) FP Work Programmes 2002-2020 (Table 2).

\begin{tabular}{|c|c|c|}
\hline Work Programme & Words & $\begin{array}{l}\text { Lexical } \\
\text { density }\end{array}$ \\
\hline $\begin{array}{l}\text { FP6 Specific Programme "Integrating and } \\
\text { Strengthening the European Research Area". } \\
\text { Priority 7: Citizens and Governance in a knowledge- } \\
\text { based society. Work Programme } 2002 \text {-2003. }\end{array}$ & 15,103 & 12,99 \\
\hline $\begin{array}{l}\text { FP6 Specific Programme "Integrating and } \\
\text { Strengthening the European Research Area" } \\
\text { Priority 7: Citizens and Governance in a knowledge- } \\
\text { based society Work Programme } 2004 \text { - } 2006 .\end{array}$ & 12,606 & 13,5174 \\
\hline $\begin{array}{l}\text { FP } 7 \text { Cooperation Work Programme: SSH } \\
\text { Work Programme } 2007 \text { Cooperation. Theme 8: } \\
\text { Socio-economic Sciences and Humanities. }\end{array}$ & 20,943 & 10,3328 \\
\hline $\begin{array}{l}\text { FP } 7 \text { Cooperation Work Programme: SSH } \\
\text { Work Programme } 2008 \text { Cooperation. Theme } 8 \text { : } \\
\text { Socio-economic Sciences and Humanities. }\end{array}$ & 20,726 & 10,3445 \\
\hline $\begin{array}{l}\text { FP } 7 \text { Cooperation Work Programme: SSH } \\
\text { Work Programme } 2009 \text { Cooperation. Theme } 8 \text { : } \\
\text { Socio-economic Sciences and Humanities. }\end{array}$ & 15,014 & 12,9679 \\
\hline $\begin{array}{l}\text { FP } 7 \text { Cooperation Work Programme: SSH } \\
\text { Work Programme } 2010 \text { Cooperation. Theme } 8 \text { : } \\
\text { Socio-economic Sciences and Humanities. }\end{array}$ & 21,558 & 11,2302 \\
\hline $\begin{array}{l}\text { FP } 7 \text { Cooperation Work Programme: SSH } \\
\text { Work Programme } 2011 \text { Cooperation. Theme 8: } \\
\text { Socio-economic Sciences and Humanities. }\end{array}$ & 22,894 & 11,8808 \\
\hline $\begin{array}{l}\text { FP } 7 \text { Cooperation Work Programme: SSH } \\
\text { Work Programme } 2012 \text { Cooperation. Theme 8: } \\
\text { Socio-economic Sciences and Humanities. }\end{array}$ & 26,934 & 10,5332 \\
\hline $\begin{array}{l}\text { FP } 7 \text { Cooperation Work Programme: SSH } \\
\text { Work Programme } 2013 \text { Cooperation. Theme } 8 \text { : } \\
\text { Socio-economic Sciences and Humanities. }\end{array}$ & 26,821 & 10.6446 \\
\hline $\begin{array}{l}\text { HORIZON } 2020 \text { Work Programme } 2014 \text { - } 2015 \\
\text { 13. Europe in a changing world - inclusive, } \\
\text { innovative and reflective societies }\end{array}$ & 52,043 & 7,5956 \\
\hline $\begin{array}{l}\text { HORIZON } 2020 \text { Work Programme } 2016 \text { - } 2017 \\
\text { 13. Europe in a changing world - inclusive, } \\
\text { innovative and reflective societies }\end{array}$ & 59,111 & 8,1711 \\
\hline $\begin{array}{l}\text { HORIZON } 2020 \text { Work Programme } 2018 \text { - } 2020 \\
\text { 13. Europe in a changing world - inclusive, } \\
\text { innovative and reflective societies }\end{array}$ & 31,565 & 9,9034 \\
\hline
\end{tabular}

The text of the work programmes is still changing. As of March 2018, materials have been used for analysis.

Table 2. FP SSH Work Programmes 2002-2020 by Word Count and Lexical Density.

And b) Statistics on funded projects from the CORDIS project database (=Projects) (https://cordis.europa.eu/). We used title and abstract words for textual analysis (Table 3).

\begin{tabular}{|l|l|l|l|}
\hline FP Programme & Projects & $\begin{array}{l}\text { Words } \\
\text { (abstracts, titles) }\end{array}$ & $\begin{array}{l}\text { Lexical } \\
\text { density }\end{array}$ \\
\hline $\begin{array}{l}\text { FP6 Specific Programme } \\
\text { "Integrating and Strengthening } \\
\text { the European Research Area" } \\
\begin{array}{l}\text { Priority 7: Citizens and Governance } \\
\text { in a knowledge-based society }\end{array}\end{array}$ & 166 & 8,415 & 25,1693 \\
\hline $\begin{array}{l}\text { FP 7 Cooperation. Theme 8: } \\
\text { Socio-economic Sciences } \\
\text { and Humanities. }\end{array}$ & 255 & 13,532 & 20,2335 \\
\hline $\begin{array}{l}\text { HORIZON 2020 Societal Challenge } \\
\text { 6. Europe in a changing world - } \\
\text { inclusive, innovative and reflective } \\
\text { societies (as of March 2018) }\end{array}$ & 277 & 14,520 & 22,3898 \\
\hline
\end{tabular}

Table 3. FP SSH funded projects in FPG, FP7 and H202O.

Since the goal was to monitor substantive changes across framework programmes, we cleaned the data of punctuation marks, numeric values, articles (a, the), prepositions (on, at, in), conjunctions (and, or, but) and auxiliary verbs, such as "to be" (am, are, is, was, were, being), "do" (did, does, doing), "have" (had, has, having).

The final analysis and comparisons between different datasets were made on the basis of the 200 most used words 2 .

\section{RESULTS}

\section{LEXICAL DENSITY}

Lexical density is the term most often used for describing the proportion of content words (nouns, verbs, adjectives, and often also adverbs) to the total number of words. By investigating this, we receive a notion of Information packaging; a text with a high proportion of content words contains more information than a text with a high proportion of function words (prepositions, interjections, pronouns, conjunctions and count words). Large majority of the spoken texts have a lexical density of fewer than $40 \%$, while a large majority of the written texts have a lexical density of $40 \%$ or higher (Johannson, V., 2008).

As we see from Figure 1, the lexical density of work programmes of different FPs has declined over the years while in the abstracts and titles of projects it has remained roughly the same and is significantly higher than in work programmes.

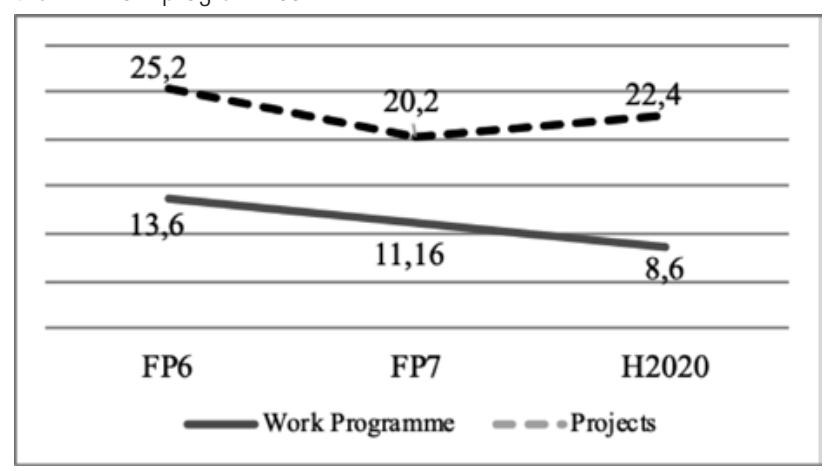

Figure 1. Lexical density of different FP work programmes and projects. 


\section{PATTERN OF WORDS}

We analysed to what extent words overlap in the work programmes of the three successive framework programmes and which unique words characterise specific programmes (Figure 2).

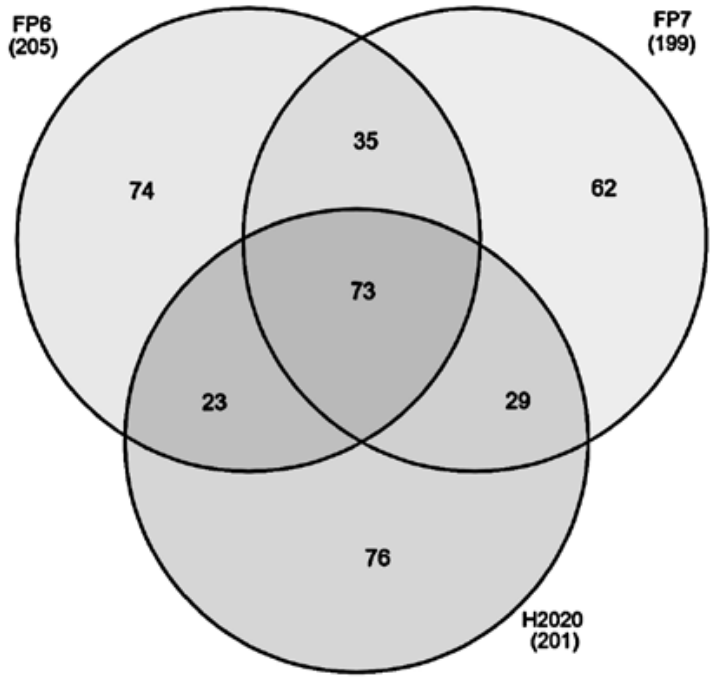

Figure 2. The overlapping of words in FP6, FP7, and Horizon 2020 work programmes.

In $20 \%$ of the cases the words overlap in all three framework programmes. These include words like programme, participant, democracy, public, research, Europe. However, the number of unique words is the same as overlapping words: in FP6 and in H2020 20\%, in FP7 17\%. Some example of unique words: in $\mathrm{H} 2020$ - business, ICT, in FP7 - foresight, emerging, family, in FP6 - associated, target, embryonic. The introduction of new words can also be followed in work programmes. For example, starting from the "7th Framework Programme", the core words introduced crisis, identity, digital, heritage, reflective, urban.

In case of words from projects, the general overlapping occurs in $14 \%$ of cases (Figure 3). Partially words overlap with the same the most overlapping words in the work programmes (programme, research, Europe) but in majority cases the words are different (human, education, approach, engage). In case of projects, the proportion of unique words is much bigger than the proportion of overlapping words: in FP7 and in H2O20 21\%, in FP6 26\%.

Some commonly used words change over time. For example, while radio and television were among the most commonly used words in the "6th Framework Programme", in the H2O2O projects these terms have not occurred and the most widely used words include software, digital, online.

We can also monitor the frequency of usage of words over time. For example, the term "innovation": in the "6th Framework Programme", it ranked the 87th by its use, seventh in the "7th Framework Programme" and second in the $\mathrm{H} 2020$.

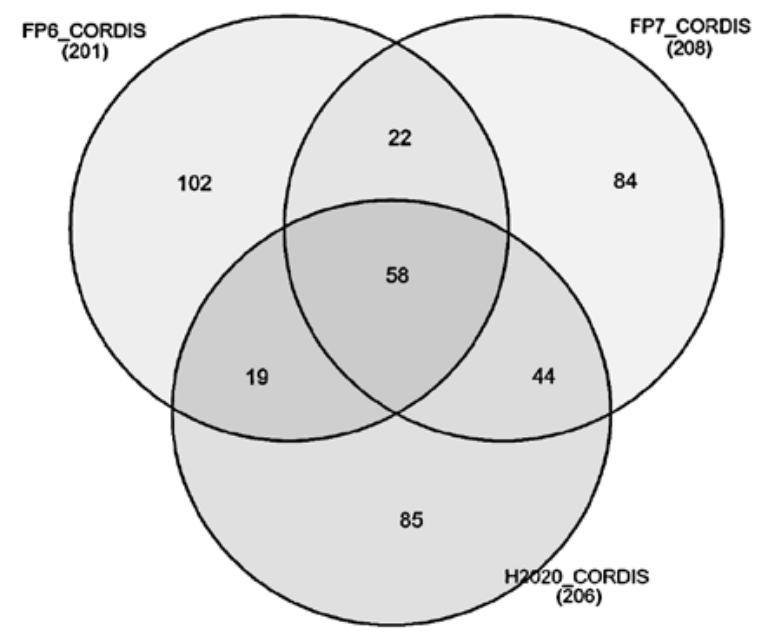

Figure 3. The overlapping of words in FP6, FP7, and Horizon 2020 projects.

When comparing two datasets, we can see that the average proportion between overlapped and unique words in work programmes is rather balanced, but in case of project words the situation is different - the majority of words are unique. At the same time, the analysis of FP project and work programmes texts with two overlaps indicates that there is continuity between successive framework programmes. For example, FP6 project words are overlapping with FP7 to an extent of 58.8\% (in work programmes $57,1 \%$ ), the words of $\mathrm{H} 2020$ projects overlap with FP7 to an extent of $55.3 \%$ (in work programmes even $65,6 \%$ ).

The subject we were examining was how much the words of work programmes and projects overlap (Figure 4).

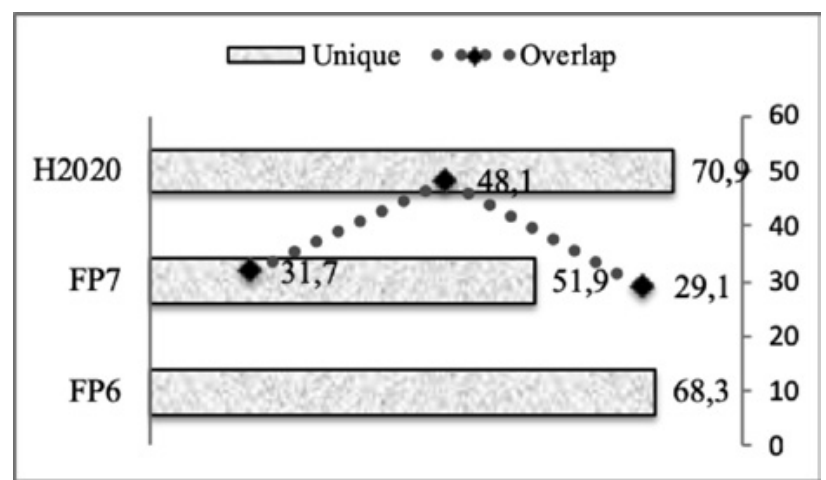

Figure 4. The overlapping of words between work programmes and projects.

As we see from Figure 4, the texts of work programmes and projects were the most in line during the $7^{\text {th }} \mathrm{FP}(48,1 \%$ overlapping), the picture is different in $6^{\text {th }} \mathrm{FP}$ (unique words constitute $68,3 \%$ ), and in $\mathrm{H} 202 \mathrm{O}$ (unique words constitute $70,9 \%)$. On the basis of the existing material, it seems that in majority cases there is no overlap between work programmes and project texts (titles and abstracts). We can only assume that the results could be different if to use the full texts of the proposals. 


\section{CONCLUSIONS}

Textual analysis is one way to track the changes in framework programmes over time. On the one hand, it shows that the language is a living entity that changes over time. On the other hand, the terminology shows the priorities existing in the given period.

Some results:

a. Lexical density of work programmes of different "Framework Programmes" has declined over the years. It has to be studied in more detail whether this is due to the addition of a greater number of non-lexical words to the text or due to the change in the language use of the text writers;

b. Overlapping words reflect the core vocabulary which does not change over time, and we can monitor the frequency of its use. Also, the introduction of new words/terms into work programmes can be monitored;

c. The words used more often in work programmes and projects generally do not coincide. At the same time, it can be observed that there is continuity between successive framework programmes.

\section{REFERENCES}

Bade Strom, T., Lemaire, C., Zacna, J., Arango Montanez, J. and Birnbaum, B. I. (2018). Integration of Social Sciences and Humanities in Horizon 2020: Participants, Budget and Disciplines 3rd Monitoring report on SSH flagged projects funded in 2016 under the Societal Challenges and Industrial Leadership priorities. Luxembourg: Publications Office of the European Union, $58 \mathrm{pp}$.

Birnbaum, B. I., Keraudren, P., Strom, T. and Vavikis, T. (2017). Integration of Social Sciences and Humanities in Horizon 2020: Participants, Budget and Disciplines. 2nd Monitoring report on SSH-flagged projects funded in 2015 under the Societal Challenges and Industrial Leadership priorities. Luxembourg: Publications Office of the European Union, 64 pp.

Cerletti, C., Gaune-Edscard, M., Gordillo, V., Krull, W., Taylor-Gooby, P. and Vanek, T., (2001). 2000 External Monitoring Report on the Specific Programme for Research and Technological Development in the Field of Improving the Human Research Potential and the Socioeconomic Knowledge Base. $30 \mathrm{pp}$.

Challis, L., Montes Ponce de Leon, J., Runeberg, K. and Walsh, J. (2003). 2002 Specific Monitoring Report on the Specific Programme for Research and Technological Development in the field of Improving Human Potential and the Socio Economic Knowledge Base. 55 pp.

Georghiou, L., Rigby, J. and Cameron, H. (2002). Assessing the Socioeconomic Impacts of the Framework Programme. Policy Research in Engineering Science and Technology PREST, University of Manchester, England. PREST, 369 pp.

Hetel, L., Møller, T-E. and Stamm, J. (2015). Integration of Social Sciences and Humanities in Horizon 2020: Participants, Budget and Disciplines. Monitoring report on SSH-flagged projects funded in 2014 under the
Societal Challenges and Industrial Leadership. Luxembourg: Publications Office of the European Union, 2015. 46 pp.

Johansson, V. (2008). Lexical diversity and lexical density in speech and writing: a developmental perspective. Lund University, Dept. of Linguistics and Phonetics Working Papers 53, 61-79.

Must, Ü. (2010a). Collaboration of the social science researchers of Central and Eastern European countries in European research programmes during the period 1994-2006. In: Internationalisation of Social Sciences in central and Eastern Europe. The "catching up" - a myth or a strategy? London, New York, Routledge, 99-114.

Must, Ü. (2010b). Collaboration in EU Framework Programme - the case of the social sciences and humanities. Innovation - The European Journal of Social Science Research, 23 (1), 79-83.

Nye, J. (1990). Bound to Lead: The Changing Nature of American Power. New

York: Basic Books. 336 pp.

Rehn, 0. (2007). The EU's soft power and the changing face of world politics. Euroklubi

Helsinki, 20 April 2007. SPEECH/07/245.

Reillon, V. (2017). EU framework programmes for research and innovation. Evolution and key data from FP1 to Horizon 2020. European Parliamentary Research Service. 38 pp.

Schindler-Daniels, A. (2014). Shaping the Horizon: social sciences and humanities in the EU framework programme "Horizon 2020". Zeitschrift fur Erziehungswissenschaft, 17 (6), 179-194.

Smith, J. (2002). Implementation of the European Research Area in the Social and Human Sciences, especially as regards the coordination and opening-up of national programmes Discussion Paper. Luxembourg: Office for Official Publications of the European Communities, 78 pp.

Tulmets, E. (2008). The European Union: A "Soft Power" with Civilian Means? Kultura i Polityka, 2-3, 60-74.

Watson, J., Kitchener, M., Gutheil, M., Ward, B., Zadrozny, T., Ackers, L. and Harrap, K. (2010). Evaluation of the impact of the Framework Programme on the formation of the ERA in Social Sciences and the Humanities (SSH). Brussels: European Union, 2010, 152 pp.

\section{AUTHOR}

\section{ÜLLE MUST}

Archimedes Foundation

Väike-Turu 8, Tartu, 51013 (Estonia)

E: ulle.must@archimedes.ee 


\section{SPECIFIC SSH RESEARCH AND GENERAL RESEARCH POLICY INSTRUMENTS. THE CASE OF THE NATIONAL RESEARCH PROGRAMMES AND “NEED DRIVEN SSH RESEARCH" IN LITHUANIA}

RŪTA PETRAUSKAITÉ

DOI: 10.22163/fteval.2019.380

\section{INTRODUCTION}

$\mathrm{S}$ cientific research is confronted with a number of oppositionbased choices: interdisciplinary or disciplinary, fundamental (basic) or applied, academic research or innovation, blue-sky or mission (policy, agenda) driven research aiming more at either advancement of knowledge or practical societal impact. The choices are made even more complicated by the traditional duality of Social Sciences and Humanities (SSH) and Science, Technology, Engineering and Mathematics (STEM) research deeply ingrained into their methodologies and abilities to serve the most urgent needs of society. However, the essence of any research, cutely summed up by the initiators of the conference Impact of Social Sciences and Humanities for a European Research Agenda Valuation of SSH in mission-oriented research, is as follows: "Scientific research is about transformation - how to enable it, or how to avoid it." (König et al. 2018: 4). The transformative power of research and its mission to influence society and to be influenced by its needs has been widely discussed by politicians and researchers, especially in the case of SSH research. Growing push for transparency of public funds and accountability coming from citizens combined with criticisms against SSH for being socially inefficient, ideologically biased and living in an ivory tower caused activities directed towards the improvement of societal impact.

Societal impact of the SSH research is a frequently used but insufficiently conceptualised notion. Hence a bad need to define it from two different perspectives: usage or bottom-up approach that helps to identify its present most widely spread senses and connotations and top-down approach aiming at re-thinking the transformative relationship between science and society and re-defining the notion of impact. The same holds for the related notions of social and political impact, social benefits, impact evaluation or valorisation, etc. Any attempts prior to re-defining SSH impact to measure and account for social or societal impact (let alone to provide indexing systems) are deemed for vague and imprecise outcomes.

Whatever the notion of impact nowadays may be, research funding organisations on both national and transnational level usually prioritise policy driven and mission-oriented research. Blue-sky research is wel- come on the condition of clear commitments and evidence for a measurable societal impact. A closer look at the variety of SSH research policy and funding instruments might reveal their impact driven orientation and funders' attempts to lure researchers out of their ivory towers. It is worthwhile to observe how researchers respond to research policy push on behalf of research funders. In that respect Lithuania can provide a good case study for various types of research instruments and their uptake by SSH researchers as the government aims at financing research for the sake of a better societal impact. However, it is obvious that without a clearer understanding of what research impact is expected to be and without a specified notion of the impact of the SSH research, the aim cannot be achieved.

\section{SSH RESEARCH POLICY IN LITHUANIA: BACKGROUND AND LANDSCAPE}

A large-scale funding of competitive research (alongside with the basic funding of academic institutions) was introduced in 2008 by the Research Council of Lithuania that was made up of two committees, the Committee of Natural and Technical Sciences, and the Committee of the Social Science and Humanities. Both committees participated on equal bases in initiating calls for proposals and their evaluation procedures for the main instrument of blue-sky research, the so-called "Projects of Collaborative Research". This activity was based on a bottom up approach allowing researchers to choose for any topic they prefer. In the case of initiating policy driven research instruments, such as national research programmes, the committees acted within the remits of their respective research areas. By now the SSH committee has participated in all stages of the life cycle of two finalised and two on-going national programmes, mostly meant for either social sciences or humanities with a possible mixed approach. Thematic areas of the national programmes were quite 
specific, chosen by a special commission out of numerous suggestions by research communities due to their importance to the state and its society. The names of the national research programmes in SSH illustrate their specificity and national importance as expressed by two programmes in the humanities: "State and Nation: Heritage and Identity, and Modernity in Lithuania". As for the social sciences, the programmes differ in their thematic scope. "Social Challenges for the National Security" is narrower than "Welfare Society"

One programme of a different type (no matter that is has the word national in its title) following the state priority of the Lithuanian studies is the "National Programme of the Lithuanian Studies". It could be placed in between freely chosen and prescribed thematic areas. It was limited from the point of view of the object rather than the topic of research and confined to the investigation of specific topics. The topics had to be related to the "past and present of the state of Lithuania, its society and culture as well as the development and present state of the Lithuanian language and nation" (2006: 2), as prescribed by "The Programme of the Research Priority of the Lithuanian Studies 2007-2008" (2006:), allowing researchers to investigate their specifically chosen topics within this area.'

The most relevant research funding instrument impact-wise at the Research Council of Lithuania is a national research programme. Despite research area specific programmes the overall description of the national programme as an instrument meant to be universal and equally suitable for all fields of research. Its most distinctive feature related to the impact of the programme as a whole is presented in its aim. National research programmes are meant "to solve problems, crucial for the state and its society, with the help of research" (Procedural description 2012: 2). Moreover, in the procedural description of the initiation of a national programme it is stated that "the problem meant to be solved by the national programme should be such that it could be solved by Lithuanian researchers within a period of 3-7 years. "(Procedural description 2012: 3). The latter requirement implies that the problem has to be well-defined and concrete, a demand feasible exclusively for the natural and technical sciences. Social sciences and humanities, no matter that they comprise a wide range of fields and subfields from the point of view of their research objects, methods and approaches, cannot formulate any problem of social relevance that could be solved in such a short period of time. The titles of the SSH national programmes and their aims are clear manifestations of a different, i.e. more broad, reflective and descriptive approach to the important issues of the society than a purely instrumental user-oriented problem-solution approach. The academic and societal outcomes of the SSH programmes need an ex-post evaluation but even a fast glance at the outcomes reveals the traditional academic result, i.e., an advancement of knowledge and its transfer in the form of academic publications and conferences. Rare attempts to provide recommendations and to influence political activities of the state institutions, mostly made by social scientists, cannot be seen as very effective or impactful.

Thus impact-oriented requirements of the national SSH programmes are hardly met by researchers as the impact is not yet an essential part of their research design. Awareness of the core evaluation criteria that include "potential impact through the development, dissemination and use of project results" (Procedural description 2012: 12) does not inspire researchers to devote their research to social challenges. The gap between SSH research communities and state institutions still exists, depriving the latter of the possibility to make research based political decisions. Direct societal impact of policy driven research due to its purely academic nature is also hardly visible and measurable except for the result dissemination activities and popularisation of the most prominent research. It can be stated that policy driven research instruments that prevail in the country do not provide satisfactory feedback to the policy itself.

\section{COMPETITIVENESS OF THE SSH RESEARCH FUNDING INSTRUMENTS}

Four problem oriented SSH national research programmes and two state priority programmes with object-limited research as compared to the only one instrument of blue-sky research provide a scale of possibilities for the Lithuanian SSH researchers to choose from. Their willingness to compete is reflected in the success rates of seven programmes calculated for all the calls of proposals of each finalised and on-going programme.

\begin{tabular}{|l|l|l|l|l|}
\hline \multicolumn{1}{|c|}{ Type } & \multicolumn{1}{c|}{ Main area } & \multicolumn{1}{c|}{ Duration } & \multicolumn{1}{c|}{ Name } & $40,13 \%$ \\
\hline National research programme & Humanities & $\begin{array}{l}2010 \\
2013\end{array}$ & "State and Nation: Heritage and Identity" & $39,28 \%$ \\
\hline National research programme & Social Sciences & $\begin{array}{l}2010 \\
2013\end{array}$ & "Social Challenges for the National Security" & $33,87 \%$ \\
\hline National research programme & Humanities & 2017 & "Modernity in Lithuania" & $12,83 \%$ \\
\hline National research programme & Social Sciences & 2022 & & $46,28 \%$ \\
\hline State programme & Humanities & 2020 & "Welfare Society" & $30,68 \%$ \\
\hline State programme & Humanities & 2015 & "National Programme of the Lithuanian Studies" & $26,62 \%$ \\
\hline Blue-sky research & 2024 & "The State Research and Dissemination & Programme of the Lithuanian Studies" \\
\hline
\end{tabular}

Table 1. Success rates of the policy-oriented programmes and blue-sky research. 
Success rates should be interpreted on the bases of the type and timing of the programmes. The main split between policy driven or state contracted research and blue-sky research (six programmes versus one) gives the ratio of $33,85 \%$ to $26,62 \%$ in favour of blue-sky research as more preferred by researchers. However, one national programme „Welfare state" provides an exception as it has a comparatively low success rate and high competitiveness due to its broad thematic scope and openness for interdisciplinary approaches. Previous national research programmes were less popular in comparison with the present ones due to the fact that at the time of their initiation competitive and collaborative research was still in its infancy. On the bases of success rates of the above programmes supplemented by information provided by individual researchers it can be stated that blue-sky research or broad scope policy driven research is more preferred by the Lithuanian SSH community than specific agenda driven research. Needless to say that more competitive research is more promising quality-wise.

\section{“NEED DRIVEN SSH RESEARCH”}

All the above presented state contracted and policy driven SSH research instruments has a double purpose to promote academic outputs and impact on decision makers at the state institutions and broader society. Usually, research output and its possible impact are needed faster than project duration and the life cycle of a programme would allow. A state demand for prompt solutions in case of burning issues of national cyber security, informational wars, waste of food, refugee integration, to mention a few, caused appearance of a new instrument, the so-called "Need Driven Research". The new instrument was initiated on requests for specific applied research from the government, ministries and other state institutions. It was meant to be more flexible time-wise as the duration of a project was shortened up to 1-2 years and calls for proposals organised every year starting with 2015.

The major distinction of the "Need Driven Research" is the list of topics suggested by ministries or any other state institutions and approved by the committees of the Research Council of Lithuania as suitable for research and experimental development. The committees pay attention to all the evaluation criteria for the approval of the topics suggested, however, the most important criterion is the necessity for the research or experimental development to meet social challenges and to solve practical problems. A possibility to investigate a problem named by state institutions applying methods of research is one of the most frequent bottlenecks for the approval of the topic by the SSH committee. It is hard for the governmental institutions to formulate the topic of research and research questions in a scientific way. Moreover, sometimes they need a more modest outcome, such as a set of recommendations or a feasibility study, instead of a full-fledged research project.

Every topic suggested by the government, its ministries or any other state institutions has to be judged whether:

a. it has a strategic value and importance for the state and society,

b. the problem has to be solved urgently,

c. its solution requires methodology of research or experimental development,

d. the results of research will substantially contribute to the solution of the problem, e. the planned research does not overlap with any other previously financed research.

(Procedural description 2016: 2)

The most valued outputs of the "Need Driven Research" are different if compared to the national or any other research programmes. Apart from publications, they include special applied outputs such as practical recommendations, new methods, evaluative methodologies, new technologies, networks, forecasts and scenarios of the activities planned, or anything else that can have, according to the contractor's view, a prompt impact for the state institutions and society at large. No matter that "Need Driven Research" is a general research policy instrument, SSH related topics prevail (71\%) as they turn out to be of paramount importance for the state affairs, especially for its policies.

"Need Driven Research" as a research policy instrument cannot be easily compared with the other instruments from the point of view of its success rates as it is based on a two-step procedure. Pre-proposals are evaluated by a joint commission of social partners and experts from the Research Council of Lithuania, the most suitable proposals from the point of view of evaluation criteria (such as feasibility of the project, competences of the researchers, and socio-economical, political or any other impact) are suggested for a full proposal phase where they are re-evaluated by experts and the joint commission. Therefore there are two success rates: those of pre-proposals and full proposals. The pre-proposal success rate $(17,28 \%)$ is fairly low in comparison with the success rates of other research policy instruments, however, it increases up to $33,85 \%$ for the full proposal submissions. In general, on the scale of research instruments ranging from free topic blue-sky research to a limited topic choice research instruments, "Need Driven Research" is situated at the far end of the research policy. In order to prove the value of the research stakeholders have to report to the Research Council implementation of its outputs.

\begin{tabular}{|l|l|l|l|}
\hline Blue-sky research & $\begin{array}{l}\text { Policy driven } \\
\text { research } \\
\text { programmes }\end{array}$ & $\begin{array}{l}\text { Policy driven } \\
\text { research } \\
\text { programmes }\end{array}$ & $\begin{array}{l}\text { Policy driven } \\
\text { research }\end{array}$ \\
\hline $\begin{array}{l}\text { Projects of } \\
\text { collaborative } \\
\text { research } \\
\text { on researchers' } \\
\text { initiative }\end{array}$ & $\begin{array}{l}\text { State programmes } \\
\text { on Lithuanian } \\
\text { studies }\end{array}$ & $\begin{array}{l}\text { National research } \\
\text { programmes }\end{array}$ & $\begin{array}{l}\text { "Need Driven } \\
\text { Research" }\end{array}$ \\
\hline
\end{tabular}

Table 2. The scale of research instruments from free to limited choice of topics.

The instrument of Need Driven Research is fairly new therefore hard to evaluate, nevertheless, it looks quite promising from the point of view of its societal impact of SSH research. Its main drawback is the same as in case of national programmes, i.e. top down approach to specific problems and their solution leaving SSH researchers with even less time and more stringent requirement for practical outputs. 


\section{CONCLUDING REMARKS}

Impact-oriented research policy executed by the Research Council of Lithuania has its pluses and minuses. It is praiseworthy to promote the idea of mission-oriented and transformative research, to raise awareness among researchers and to support the culture of societal research impact. However, it is obvious that policy driven research instruments, especially of a general type, are not always suitable for the SSH research. Traditional impact (both academic and societal) assessment methods do not reveal multilateral impact of the SSH research that remains to be re-defined taking into account possible side effects and unintended consequences. Bottom up approaches, such as blue-sky research, could be a better alternative for society-oriented research provided its impact is conceived, defined and assessed in multiple ways. In any case, impact, especially societal impact, of the SSH research has not only to be carefully planned before made during the process of research but also identified, reflected and evaluated from a long-term perspective. No one could do it better than SSH researchers themselves. In spite of all highly appreciated attempts to serve the state and society, policy driven research instruments deprive SSH community of its blue-sky research as well as of a chance for uncertain but high-gain opportunities and a long-term impact on society that is hard to measure and to evaluate immediately (Nowotny 2016).

\section{REFERENCES}

König, T., Nowotny, H., Schuch, K. (2018). Impact Re-Loaded. In: Pathways to impact from SSH research. Impact of Social Sciences and Humanities for a European Research Agenda. Valuation of SSH in missionoriented research. Programme booklet, 28-29 November, 2018 Vienna. P. 4, https://www.ssh-impact.eu.

Nowotny, H. (2016). The Cunning of Uncertainty, Cambridge: Polity.

Procedural Description of the Initiation and Execution of the Projects of the National Research Programmes. (2012). Retrieved October 31, 2018 from https://e-seimas.Irs.It/portal/legalAct/lt/TAD/ TAIS.416490?jfwid=-9dzqnu3vw.

Procedural Description of the Initiation and Execution of the Projects of the Need Driven Research. (2016). Retrieved October 31, 2018 from (https://www.e-tar.It/portal/lt/legalAct/9bf9aba0296711e6acf89da936 cb7409.

The Programme of the Research Priority of the Lithuanian Studies 2007-2008. (2006). Retrieved October 31, 2018 from https://e-seimas. Irs.It/rs/legalact/TAD/TAIS.284561.

\section{AUTHOR \\ RŪTA PETRAUSKAITE் \\ Research Unit (Lab or Department), Vytautas Magnus University \\ K. Donelaičio g. 58, Kaunas, LT-44248 (Lithuania) \\ E: ruta.petrauskaite@vdu.lt}




\title{
GHENT UNIVERSITY'S INTERDISCIPLINARY SSH-CONSORTIA -
}

\author{
A STRATEGY TO ENHANCE THE \\ SOCIETAL IMPACT OF RESEARCH
}

NOËL KLIMA, STEFAN MEYSMAN, JULIE CARLIER, ALEXIS DEWAELE AND ESTHER DE SMET

DOI: 10.22163/fteval.2019.381

\section{INTRODUCTION}

$\mathrm{G}$ hent University is one of the biggest Universities in Belgium with 11 faculties, 117 departments and 650 research institutes containing around 9000 employees and 41.000 students (Ghent University, 2016). Ranked best Belgian University on 61 in the Shanghai ranking in 2018 Ghent University is home to 17 highly cited authors and more than 55 grantees since the start of the European Research Council (ERC) funding scheme. In such a large organisation, Ghent University considers the potential for top-down steering of research strategy limited. Therefore, Ghent University applies a range of decentralised research strategy initiatives including SSH-focused ones.

Due to a national and regional focus on "objective" distribution of research funds and a willingness to become a world-renowned knowledge economy, performance indicators are often limited to quantitative and individual output and traditional figures such as number of publications, PhD's and citations. When compared to the STEMM (Science, Technology, Engineering, Mathematics and Medicines) disciplines, this system is widely known to disadvantage the Social Sciences and Humanities (SSH) in part because of a different research and publication culture. In measuring performance, traditionally less attention is given to indicators such as (interdisciplinary) cooperation or service to society while many SSH disciplines show especially here a strong potential and some already well-developed good practices. In addition, characteristics such as a high level of individuality of researchers, less "big" funding due to smaller research groups as well as a high teaching load and lack of (planned) societal value creation in SSH, urged Ghent University's Research Department to support joint initiatives in SSH to strengthen research excellency and impact through the stimulation of cooperation.

One of these initiatives entailed the set-up of interdisciplinary SSH research consortia. The SSH research consortia may be considered the counterpart of Ghent University's business development centers as funded by the "Industrial Research Fund" (IOF)' which were installed to bridge the gap between strategic fundamental research, industrial cooperation and technological innovation. The SSH-consortia are complementary to these STEMM initiatives and have the purpose to bridge the gap between SSH (fundamental) research, interdisciplinary cooperation and societal impact.

\section{PILOT PHASE OF THEMATIC SSH-CONSORTIA}

Within the framework of a new research policy in 2012 (DOZA, 2012a), Ghent University launched a competitive call for consortia to strategically support several SSH disciplines. A pilot funding of five years was granted to five consortia including a strengthening of the middle management through a coordinator on a postdoctoral level. All five consortia received a "carte blanche" to develop the consortium and to define and focus on specific priorities within broader missions of interdisciplinarity, internationalisation, academic excellence and societal value creation. It was important in this initiative that the consortia could start to operate from scratch and develop their own aims, mission, and eventually output. The five consortia were funded through the university internal "Special Research Fund" (BOF) and hosted at different faculties.

\section{“CRIME, CRIMINOLOGY AND CRIMINAL POLICY" (CCCP)}

At the Faculty of Law and Criminology the consortium "Crime, Criminology and Criminal Policy"2 has been installed. The consortium deals with the domain of deviance and its (policy) response, as well as in the areas of crime and security. The consortium brings together 16 scholars from different disciplines in six departments working inter-disciplinary on security, crime and deviance related topics in local, national, European and international contexts. The range of topics covers e.g. research into vulnerable groups in detention, policing and police mobility, desistance from crime and drug use, (youth) crime prevention, but also other complex cross-border phenomena such as cybercrime, terrorism and organised crime, or privacy, information exchange, big data, law enforcement responses, policies and laws. The consortium fosters knowledge translation and exchange, strengthening societal value creation leading to societal impact and stimulates synergies and cooperation with external academic, policy and practice partners from different disciplines. 


\section{PIRENNE CONSORTIUM FOR MEDIEVAL STUDIES}

The Pirenne Consortium for Medieval Studies ${ }^{3}$, primarily based at the Faculty of Arts and Philosophy fosters cross-disciplinary research into the medieval period and advances knowledge exchange between these different fields of study, as well as with societal partners and the general public. The consortium brings together all medievalists at Ghent University under the promotor-board of 29 senior scholars. It draws more than 100 members from four faculties and ten departments, covering both Science, Technology, Engineering and Mathematics (STEM) and SSH. Research on original medieval heritage such as texts, images, maps, artefacts and sites is the core business, including the development and integration of "Digital Humanities" methods. However, the consortium also houses expertise in collaboration with archives, libraries, museums, and other cultural heritage institutions, as well as with several societal sectors such as education, policy and tourism.

\section{GHENT CENTRE FOR GLOBAL STUDIES}

The Ghent Centre for Global Studies ${ }^{4}$ is hosted at the Faculty of Political and Social Sciences. As an interdisciplinary research platform it unites scholars from Social and Economic Geography; International, EU and Conflict and Development Studies; Economics, Sociology, Global History and Ethics; Human Rights Law and Intercultural Pedagogy. With a total of 11 research groups from six different faculties the consortium focuses on the critical study of globalisation, with special attention to the interaction of global and local processes. With its interdisciplinary research and education - on urbanisation, rural transformations, economic governance and migration - the Centre aims to contribute to the societal debate on, and evidence-based policy-making and development cooperation for, sustainable development.

\section{"WORKING TOGETHER FOR MENTAL HEALTH" - PSYNC}

"Working Together for Mental Health" - PSYNC 5 refers to "psychology' and 'synchronise". This research consortium is housed within the Faculty of Psychology and Educational Sciences. Its objective is to develop a common strategy to translate clinically relevant research to the clinical field and to the broader society. The consortium is dedicated to improving the mental health of all citizens, running research projects in close collaboration with diverse stakeholder groups, with a clear focus on generating real world impact and providing societal innovation. PSYNC's main goals are reaching vulnerable groups, stressing the importance of lifecycle perspective on mental health, increasing mental health literacy and health promotion, safeguarding ethical perspectives, and developing innovations in the treatment of mental health disorders.

\section{“INNOVATION AND ALL INCLUSIVE GROWTH" - CIG}

The consortium "Innovation and All Inclusive Growth" - CIG6 found its base at the Faculty Economics and Business Administration. The consortium's goal is to act as an economic and scientific base for everything which concerns innovation, entrepreneurship and all-inclusive growth at Ghent University. Research focuses on different topics from different angles such as technological innovation and entrepreneurship, "Corporate Social Responsibility" (CSR), corporate and entrepreneurial finance, business architecture and modelling, innovation and growth at macro level. This consortium decided not to continue its work after the pilot phase and therefore was not part of some of the later mentioned evaluation mechanisms after the five year pilot phase.

All consortia have created their own strategic plan and modus operandi, and developed their own support structure in line with their thematic focus and their members who are researchers from different faculties and departments. The consortia and how they operated have been evaluated on different occasions and from different angles. Before it was decided to provide continued funding, four out of five consortia were evaluated on three criteria that were discipline-specific, consortiumspecific and coordinator-specific.

\section{EXTERNAL PEER EVALUATION (DISCIPLINE-SPECIFIC)}

In 2016, the consortia received a first evaluation through an external discipline-specific peer review evaluation coordinated by the Ghent University Research Department in collaboration with the faculties of Arts and Philosophy, Faculty of Law and Criminology, Faculty of Economics and Business Administration, Faculty of Psychology and Educational Sciences and the Faculty of Political and Social Sciences. This evaluation was not consortium specific but rather discipline specific. However, the consortia have been considered being part of the respective faculties which also received the attention of the evaluators. The consortia and their role have been evaluated as valuable parts of the relevant faculties and disciplines. The focus on interdisciplinary cooperation was considered an asset in the faculty structures.

\section{STAKEHOLDER PEER EVALUATION (CONSORTIUM- SPECIFIC)}

In 2017, a new targeted call was launched for the continuation of the five pilot consortia. For this evaluation the panel consisted of members not only from the University Research Council, but also of individuals from non-academic stakeholder groups. External members came from the public sector, civil society and international organisations. All consortia have been evaluated in a two-fold manner. First, each consortium was considered retrospectively by evaluating the outputs and outcomes with regard to the former "Ghent University Research Policy Plan" (2012 
- 2016) under which the consortia were installed (DOZA, 2012). Second, the consortia were assessed from a future-oriented perspective. The panel looked into the ambition, the organisation, the strategy of the consortium and its match with the current "Ghent University Research Policy Plan" (DOZA, 2017).

As a result, the decision was made to continue funding for four of the five consortia and structurally embed the role of the research coordinator as a shared position with a long-term contract. The major strengths of the four were the stimulation of interdisciplinary research in each thematic area but also the stimulation on a cross-consortium level such as e.g. joint events, projects, knowledge exchange which was considered a major added value for the research and impact agenda. Also, the involvement of the coordinators in several central University research policy working groups was a positive outcome of the organic bottom-up development of the consortia during the pilot period.

As a consequence, the objective was to build on the developed strengths and particularities of each consortium and the naturally grown cooperation between them through knowledge sharing and research policy involvement. With a consolidation of the existing consortia new opportunities with regard to interdisciplinarity7 and societal value creation8 leading to societal impact would be created. The consortia will be evaluated every five years on their organisation and management, their interdisciplinarity through cooperation and joint initiatives, their societal impact through impact case studies and the planning of the future five year period.

\section{INTERNAL EVALUATION (COORDINATOR-SPECIFIC)}

During the pilot phase, the coordinators in some of the consortia changed due to staff turnover. After the decision to fund the existing consortia permanently, the acting coordinators have been evaluated separately by a Ghent University panel including members from the consortia, the Research Department and the Ghent University Research Council. This evaluation examined the coordinator's profile, approach and strategy to manage the consortium for the next five years. The panel gave positive advice to extend their contracts towards indefinite appointments. All four coordinators could show the relevant thematic expertise and management skills to coordinate the consortia on a permanent basis. All have also built up a close collaborative relationship with various policy officers within the Research Department and strengthened the information flow and the cooperation between the central university level and the consortium researchers from the different departments. The profile, skills and approaches of the coordinators will also be used to define the requirements for the recruitment of future coordinators for additional consortia.

\section{CONSORTIA ARE EMBEDDED IN THE BIGGER RESEARCH POLICY AGENDA}

The SSH-consortia are embedded in the general research policy and were also part of Ghent University's policy initiative focusing on the ex- cellence in the humanities, social and behavioural sciences. This specific initiative brought together different incentives which were targeted specifically at the faculties Law and Criminology, Arts and Philosophy, Psychology and Educational Sciences, and Political and Social Sciences and was intended to strengthen research quality and research strategy, taking into account the idiosyncrasies of research in these fields (DOZA, 2012b). Ghent University's intention to enhance research excellence through higher research quality, visibility and recognition accompanies the initiated SSH initiatives. Next to the SSH consortia, budgets were reserved for additional professor and tenure track positions and a reform of the sabbatical rules (DOZA, 2012b). From other research policy initiatives, such as the "research spearheads", also known as the MRP initiative ("Multidisciplinary Research Partnerships") (DOZA, 2010), Ghent University learned about the demand to develop methods and incentives relevant for SSH with regard to the bibliometric bias, the high individuality of researchers and less "big" funding due to smaller groups as well as the high teaching load and the lack of (focused) societal value creation. At the same time, a new policy plan on societal value creation called "IM-pact" was developed by the Research Department wherein the SSH-consortia and their structural embedment plays one of the key roles to stimulate interdisciplinary cooperation and enhance societal value creation of research (DOZA, 2015).

The experiences from the pilot led Ghent University to consider the SSH-consortia as a good practice and led to a wish to expand the initiative across the university. The focus, the working and the development of the four SSH-consortia are considered to be an inspiration for other new interdisciplinary consortia working in other research areas. Ghent University intends to extend the initiative with six more consortia to strengthen its general profile with regard to interdisciplinarity and societal impact.

\section{CONSORTIUM COORDINATOR WITH A PERMANENT ASSIGNMENT}

The structural embedment and long term vision requires the sustainable position of the coordinator. Against common university customs fixed term assignments would in this case weaken the position of the coordinator and hamper the working and development of the consortium. Interdisciplinary cooperation and societal impact creation take time and require consolidated and sustainable relationships both with and between researchers and non-academic stakeholders. To limit the risk of a high fluctuation of staff and related loss of expertise, Ghent University decided to provide fixed contracts for the coordinators.

The coordinators are knowledge brokers who promote collaboration and networking within the consortium, between the consortia and with the Research Department. They develop expertise in facilitating, promoting and appreciating interdisciplinarity and take initiatives to support internal interdisciplinary cooperation. Within and across the consortia and in collaboration with the Research Department the coordinators elaborate generic and thematic initiatives and share knowledge with regard to research policy, interdisciplinarity and social impact.

The coordinators are also monitoring the sustainability and long-term strategy of the consortium and optimise the involvement and commitment of the researchers in the consortium. The coordinators assist the 
researchers during the idea phase for acquiring external financing. They follow and influence the European research policy, both generically and thematically for the consortium and for Ghent University.

The coordinators also manage societal value creation and societal impact activities. Therefore, they develop expertise in a number of value creation and impact related topics relevant to the consortium. As antennae, the coordinators are in contact with several stakeholders playing an active role in the expansion of the stakeholder network of the consortium, e.g. by setting up a structural advisory board of societal stakeholders. Finally, the coordinators develop and use models for the design of value creation and impact processes and for the evaluation of impact, in line with their research expertise.

Each of the coordinators has generic expertise within the research areas of the consortium at PhD level and is able to assess strategically the potential of projects and other initiatives with respect to scientific and societal impact. They are knowledge brokers, provide technical assistance and safeguard the pathways to impact, defined by each of the consortia. The coordinators receive an annual lump-sum bench fee that can be used to support their work agenda and where coordinators themselves act as budget holders.

\section{JOINT CONSORTIA PATHWAYS TO IMPACT - AN EXTRA DIMENSION}

The work of a coordinator in the thematic consortium makes around $70-80 \%$ of the total workload. The other $20-30 \%$ are dedicated in crossconsortium activities and generic Ghent University work. Besides the interdisciplinarity within each of the consortia the collaboration between the coordinators and with the central Research Department brings an extra dimension. Within this extra dimension of interdisciplinarity, impact, relevant outputs and outcomes as well as knowledge and information exchange could be generated. This concerns university-wide initiatives in function of knowledge sharing, expertise building and training cooperation with the Research Department and other Ghent University partners in the area of interdisciplinarity, societal value creation, impact and research policy, including prospecting funding opportunities and promotion of best practices.

All coordinators are members of the "Impact Task Force" and the Alpha-EU working group at central University level, participate in writing of position papers ${ }^{9}$ (e.g. Ghent University, 2017a; 2017b; 2018), organise joint workshops ${ }^{10}$, information sessions ${ }^{11}$, lectures ${ }^{12}$, participate in joint projects ${ }^{13}$ and plan to organise an interdisciplinary impact award. All joint activities generate outputs feeding the common objectives to increase the societal impact of Ghent University SSH research. Also, it adds to the optimisation of the impact research policy and evaluation at Ghent University and to the defence of Ghent University's interests at European level e.g. through input on the development of EU Framework Programmes. This exchange on Ghent University's (EU) research policy is highly valued by all involved actors and shows already a range of tangible results.

\section{LESSONS LEARNED}

The pilot experiment of the SSH-consortia brought a range of positive experiences to the surface but also points of potential improvement in the future. The SSH-consortia were established in an environment where interdisciplinarity and openness for other disciplines is necessary, but not self-evident. The structural support for researchers in view of interdisciplinary collaboration showed positive effects. The coordinators stimulate researchers towards more cooperation and collaboration within their consortia but also with external parties. The organisation of interdisciplinarity requires good leadership by the coordinators but also from the professors and researchers involved. The different consortium pilot tracks showed that just a coordinator in a group of researchers is not sufficient to gain effects from a consortium. Dedication and commitment is required from coordinators and the professors and researchers to be able to reach another level of cooperation.

An important step in the process was the decision to make the coordinator position fixed term and extract them from the "usual" academic career track of a research oriented postdoc. Some of the consortia lost their coordinators during the pilot phase and even one consortium stepped out during the pilot phase. Some researchers left for a fixed term position elsewhere or followed their regular research track on top of their consortium management duties. A safe position with an autonomous budget from the start prevents a high level of fluctuation among the coordinators, which goes along with a loss of the acquired expertise. This kind of initiative should not just be a "stepping stone" for postdocs on their jump to the next project contract or the next step on their way towards a professorship. Nevertheless, teaching and research activities can be of added value to stay in touch with academic expertise, and to disseminate the coordinator's expertise on interdisciplinarity and impact related topics. The profile of the consortium coordinators requires expertise in research and topic knowledge, but in addition (research) management and policy expertise and expertise on societal value creation and research funding. This position is different from a pure research position and should be filled with people motivated to build the relevant expertise as a pivot point between research, research policy, funding, outreach and management.

In addition, a well organised research information system is required in each consortium but also on central level to avoid the loss of information and knowledge. Information management and data exchange still depended very much on individual researchers. Therefore, incentives are needed for participating researchers to value their engagement and commitment within a consortium might help to convince researchers much quicker to dedicate more effort and energy in interdisciplinary cooperation and societal value creation of their research. This could be done e.g. through including open science incentives in their personal career goals.

It will take time until the results from interdisciplinary collaboration develop effect. The five-year period has shown that this is a process of building trust, dedication and commitment. This needs also to be created and maintained between coordinators, researchers and central university research policy departments. An interaction on regular basis in structural working groups raises the tonus of joint actions between the three parties. Mutual recognition and understanding is important to fruitfully bring together the different working levels.

Starting with a pilot on a small scale has proven to be the right way. The learning effect from the pilot evaluations puts the Research Depart- 
ment in a position to immediately call for permanent consortia building on the structures and cooperation grown during the pilot phase.

A strong asset in the process has been the bottom-up approach in the development of the structure and working of the consortia. The researchers do not consider the consortia an extra institution with heavy administrative burden which operates independent from the other existing structures such as research institutes, departments or faculties. The bottom-up approach made it possible to fully adapt the consortia to the needs of the researchers and to build a complementary structure that is intertwined with all other structures. A fully functioning consortium brings assets to the central research policy level of the University.

The boon or bane of the bottom-up approach was the diversity of the consortia and their working which is difficulty to measure and compare according to strict and measurable indicators. Ghent University has chosen for panels to evaluate the work individually. Clear guidelines on how the consortia will be evaluated periodically are necessary. Ghent University decided to focus on four domains: the organisation of the consortium and internal procedures, the interdisciplinarity of the working, impact case studies and the future planning.

The SSH-consortia were inspired and considered complementary to the "Industrial Research Funds" (IOF) business development centres that were established over a decade ago. However the bonds and cooperation between both initiatives are developing very slowly. The same applies for the exchange and cooperation with the University technology transfer office which was not fully exploited during the pilot phase. In the case of Ghent University's pilot bottom-up approach, an exchange with STEMM disciplines was in some cases existent based on single projects or individual collaboration moments. This might be taken away for the next cohort of interdisciplinary consortia at Ghent University but also for Universities that want to start with such an initiative. It is certainly recommended to engage immediately and structurally with STEMM researchers that do have relevant connection with the topic. In some cases this is not possible or relevant. However, it will help to open silo researching and opens borders for new cooperation ventures.

\section{FUTURE PERSPECTIVES OF THE SSH-CONSORTIA}

The four SSH-consortia will continue on permanent basis embedded in the research policy structure of Ghent University. In some consortia (where relevant) exchange and cooperation with STEMM researchers will be further stimulated and extended especially with regard to the next "European Framework Programme Horizon Europe". The consortia will work through a range of specific pathways to impact and also a range of joint ones. The initiatives will inter alia cover the enhancement of impact literacy among researchers and informing research policy at Ghent University level and EU level. A new call will make the number of consortia grow from four to ten which also will lead to new challenges. The cooperation and exchange infrastructure built during the pilot phase provides a situation where new consortia with their new coordinators are able to be immediately integrated. Finally, the consortia and the Research Department will continue to exchange knowledge on impact measurement.

\section{REFERENCES}

DOZA (2010). Strategisch Speerpuntenbeleid Onderzoek. Ghent: Ghent University. [eng. Strategic Spearheads Policy Research]

DOZA (2012a). Ghent University - Research Policy Plan 2012 - 2016. Ghent: Ghent University.

DOZA (2012b). Een hefboom naar excellentie in de humane, sociale en gedrags- wetenschappen. Ghent: Ghent University. [eng. A lever for excellence in the human, social and behavioral sciences]

DOZA (2015). Strategisch project maatschappelijke valorisatie van onderzoek - IM-pact. Ghent: Ghent University. [eng. Strategic project societal value creation of research - IM-pact]

DOZA (2017). Ghent University - Research Policy Plan 2017 - 2021. Ghent: Ghent University.

Ghent University (2016). Focus on Ghent University. Retrieved September 10, 2018 from: http://unigentdemo.online-magazine.nl/en/magazine/11623/815119/focus_on_ghent_university-_cover.html.

Ghent University (2017a). Societal impact of SSH research: perspectives on co-creation and working with societal readiness levels. Retrieved September 15, 2018 from: https://www.ugent.be/en/research/positionpapers

Ghent University (2017b). Position Paper IE H2020 The 'SSH embedding Challenge'. Retrieved September 15, 2018 from: https://www.ugent.be/ en/research/position-papers.

Ghent University (2018). Call on the European Union to create highquality dedicated and embedded SSH research opportunities in FP9. Retrieved September 15, 2018 from: https://www.ugent.be/en/research/ position-papers.

\section{AUTHORS}




\section{NOËL KLIMA}

IDC Crime, Criminology and Criminal Policy, Ghent University

Universiteitstraat 4, Ghent, 9000 (Belgium)

E: noel.klima@ugent.be

\section{STEFAN MEYSMAN}

IDC Pirenne Consortium for Medieval Studies, Ghent University

Sint-Pietersnieuwstraat 35, Ghent, 9000 (Belgium)

E: stefan.meysman@ugent.be

\section{JULIE CARLIER}

IDC Ghent Centre for Global Studies, Ghent University

Universiteitstraat 8, Ghent, 9000 (Belgium)

E: julie.carlier@ugent.be

\section{ALEXIS DEWAELE}

IDC PSYNC - Working Together for Mental Health, Ghent University

Henri Dunantlaan 2, Ghent, 9000 (Belgium)

E: alexis.dewaele@ugent.be

\section{AND ESTHER DE SMET}

Research Department, Ghent University

Sint-Pietersnieuwstraat 25, Ghent, 9000 (Belgium)

E: esther.desmet@ugent.be

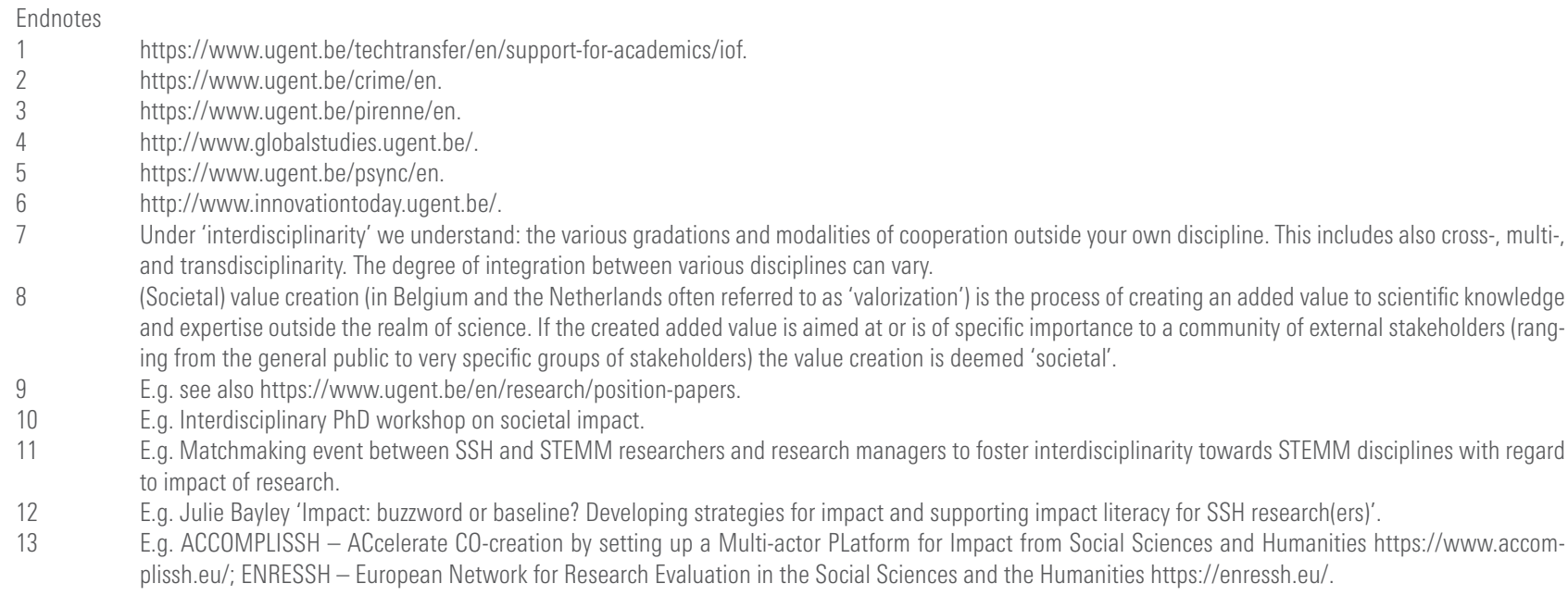

$7 \quad$ Under 'interdisciplinarity' we understand: the various gradations and modalities of cooperation outside your own discipline. This includes also cross-, multi-, and transdisciplinarity. The degree of integration between various disciplines can vary.

8 (Societal) value creation (in Belgium and the Netherlands often referred to as 'valorization') is the process of creating an added value to scientific knowledge and expertise outside the realm of science. If the created added value is aimed at or is of specific importance to a community of external stakeholders (ranging from the general public to very specific groups of stakeholders) the value creation is deemed 'societal'.

$9 \quad$ E.g. see also https://www.ugent.be/en/research/position-papers.

$10 \quad$ E.g. Interdisciplinary PhD workshop on societal impact.

11 E.g. Matchmaking event between SSH and STEMM researchers and research managers to foster interdisciplinarity towards STEMM disciplines with regard to impact of research.

12 E.g. Julie Bayley 'Impact: buzzword or baseline? Developing strategies for impact and supporting impact literacy for SSH research(ers)'.

13 E.g. ACCOMPLISSH - ACcelerate CO-creation by setting up a Multi-actor PLatform for Impact from Social Sciences and Humanities https://www.accomplissh.eu/; ENRESSH - European Network for Research Evaluation in the Social Sciences and the Humanities https://enressh.eu/. 


\title{
EVALUATION OF SOCIETAL IMPACT IN NORWEGIAN SSH EVALUATIONS
}

\author{
JON HOLM AND ANETTE ASKEDAL
}

DOI: 10.22163/fteval.2019.382

\section{INTRODUCTION}

$\mathrm{I}$ n this paper we present how evaluation of societal impact of research was introduced in national research evaluations in Norway within social sciences and the humanities through an adaptation of the "Research Excellence Framework" (REF) 2014 impact case method. We focus on the practical aspects of this introduction, the processes of evaluation and the impact of the impact evaluation on the discourse on societal benefits of "Social Sciences and Humanities" (SSH) research. Finally, we discuss the limitations of the impact case method and indicate some possible ways forward.

\section{THE INCLUSION OF SOCIETAL IMPACT}

The Research Council of Norway (RCN) has been performing nationwide research evaluations for over 20 years. The interval of these evaluations is approximately 10 years which means that nearly all academic subjects have now been evaluated twice. The aim of the subject-specific evaluations is to provide a critical review of the Norwegian research system in an international perspective, and to provide recommendations on measures to encourage increased quality and efficiency of research. The evaluations help to ensure that the RCN has the necessary information on which to base its strategic research funding and efforts vis-à-vis public bodies. The evaluations also serve as a tool for the institutions themselves in their ongoing efforts to refine their own strategic and scientific framework. There is no direct link to funding.

Traditionally, the national research evaluations have focused on the quality and efficiency of research activities at the national, institutional and group level. As a response to the political expectations of harvesting societal benefit from increased investments in research, the RCN decided to include societal impact as a dimension of the latest evaluations of the humanities (2017) and social sciences (2018). The large majority of researchers in Norway within the relevant disciplines were included in the two evaluations.

The main method used to assess societal impact in the two evaluations was borrowed from the "2014 Research Excellence Framework" in the UK. The method was chosen for two main reasons: 1) It was well documented, tested and evaluated2, and 2) the definition of impact used in the REF was judged to be sufficiently broad to include most of the expected societal benefits from SSH research: "an effect on, change or benefit to the economy, society, culture, public policy or services, health, the environment or quality of life, beyond academia"s

In contrast to more traditional methods for measuring societal and economic benefit, like counting patents or spin-off companies, we saw the REF definition as more open to disciplinary differences and compatible with the multitude of pathways to impact documented in empirical studies. ${ }^{4}$

\section{INTRODUCING SOCIETAL IMPACT TO THE SSH INSTITUTIONS}

Choosing an existing method to assess societal impact made the task of introducing a new evaluation dimension to the national evaluation system in Norway more manageable. The main effort of the RCN then went into convincing the Norwegian higher education institutions that the REF impact case template could actually be used to document the societal benefits resulting from SSH research in a meaningful way.

When planning the evaluation of humanities research in 2013 , the international debate on the public value of the humanities was making its waves felt also in Norway. There was a strong resistance in academia against thinking of humanities research in terms if usefulness. At the same time, proclamations on the essential role of the humanities for the development of society were manifold. In other words, there was a discrepancy between the feeling of importance in academia and the ability to document how research results had been put into use and to point out the actual beneficiaries.

The impact case method also received various types of criticism from the researcher community. The most common objections were that the cases only covered a small part of the societal relevance of an institution, they implied a linear relationship between research and impact, they were not reflecting the complexities of researcher - user relations and not covering the important impacts taking place within academia.

With this in mind, the RCN invited representatives from institutions that took part of the evaluation to an impact-workshop. The aim of the workshop was to explore how the institutions could use the REF impact case template to describe the pathways from research to societal impact according to the REF definition. The participants were introduced to the REF case-model by Professor Helen Small - a literary scholar and from Cambridge University - who had had a leading role in her faculty's impact case submissions to the REF. 
During the workshop, many participants took the opportunity to discuss how they could use the REF impact template to describe specific societal benefits from research at their institutions. In this way, the workshop produced a change in the discourse from an essentialist question of what impact is to a pragmatic question on how to document the societal benefits of research. This change in attitude was crucial for the success of the evaluation exercise. There is a fundamental difference between the effort of understanding and conceptualising a certain phenomenon like the societal benefit from research to the task of actually establishing a new practice of documenting societal impact. The debate on how to document and assess the societal benefits from research should thus not be limited to a discussion of the meaning of a certain concept or theory on the role of science in society. In order to inform policy, the debate should also take into account how political expectations for societal benefits from investments in research are implemented through evaluation exercises or regulatory regimes.

An interesting example of this implementation perspective is given by Marta Natalia Wróblewska, who in a recent PhD-theses investigates the process of constructing the notion of impact in the British REF. Inspired by Michel Foucauld's theory of governmentality, Wróblewska (2018) argues that the resulting understanding and practices of societal impact "is a response to a set of struggles over issues related to selecting a new direction for the economic development (knowledge-based economy), reshaping the role of universities in society /third mission, entrepreneurial university), as well as class issues and tensions between particular aca-

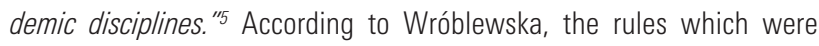
introduced with the REF guidelines could be considered as empty and unfinalised before they were taken into use and translated into concrete practices at the research institutions and thus forming an impact infrastructure consisting of professional roles, teaching frameworks and specified procedures and timeframes.

\section{THE EVALUATION PROCESS}

The RCN collected a total of 404 impact cases from the participating institutions and research groups for the evaluations of humanities and social sciences (170 cases were submitted to humanities evaluation and 234 cases to social sciences evaluation). The submission of impact cases was optional and for that reason the number of impact case per researcher varied a lot among institutions with an average of one case per 13 researchers.

For both evaluations the RCN carried out a brief descriptive analysis of the categories of impact that was reported in the impact cases. The purposes of these analyses were not to evaluate the cases, but to describe trends in the submitted material. The analysis showed that research leading up to the reported impact was commonly conducted in groups, that the geographical reach was national, and that the most common channel from research to impact was user-oriented dissemination. For the social science cases, the most common beneficiary of the impact were political institutions, and the principal registered effect was political. The general public was the most common beneficiaries for the cases within humanities, and the principal effect registered was cultural.

The RCN also did a mapping of the impact cases onto the thematic priorities within Horizon 2020 (H2020) societal challenges and those indicated by the Norwegian government's long-term plan for research and higher education. The somewhat surprising result was that there was a greater match with the European priorities than with the Norwegian priorities. This was to a great extent due to the presence to the $\mathrm{SSH}$-related theme "Europe in a changing world" in H2O2O.

The evaluation of the impact cases was carried out by the same international peers who evaluated the quality of Norwegian research. The evaluation panels found several good and varied examples of societal impact among the submitted cases. In the humanities evaluation the committee was "favourably impressed with the range and depth of societal impacts from the Humanities", and in the evaluation of social sciences the evaluators found that the research had "considerable relevance to a large range of public and private societal actors and activities" . The evaluators highlighted 64 cases as examples of good practice. These were cases that documented concrete and significant proof of impact on society.

\section{CHALLENGES}

Despite this, the evaluators experienced a number of difficulties when trying to assess societal impact in the two evaluations, and the evaluation task was described as "particularly challenging"8 in the evaluation of social sciences. The evaluators found that there was an uneven understanding of the meaning of impact among the participating institutions and research groups. A majority of the submitted impact cases merely described communication activities, rather than providing documentation of societal impact. For this reason, the panels found it difficult to assess several of the submitted impact cases, and they recommended that the institutions developed a more strategic approach to impact, and also that the difference between impact and engagement was better defined for the institutions. In addition, the evaluators recognised that there were many methodological difficulties linked to the assessment of societal impact, and they saw a need for further development of the methods for assessing and evaluating societal impact, and also for more sophisticated tools for gathering and articulating evidence of impact.

The RCN has used impact case descriptions as the main source for evaluating societal impact also in other recent evaluations (including evaluations of research institutes and thematic evaluations). The reported difficulties have been the same in most of these evaluations. In many cases the distinction between societal impact and dissemination is not clear. We take this as an indication that researchers and institutions have not fully understood the expectations embedded in the REF impact case genre. The different interpretation of impact, and also the failure to document actual change, made it difficult for the experts to assess a number of cases.

The RCN recognises, in order to make robust assessments of the societal impact of research, that there is a need to combine different methods. For that reason user-surveys and interviews were included in some of RCN's recent evaluations in order to add a users' perspective to the assessment of societal impact. It was however problematic to use the result of the surveys in most of the evaluations. The response rate was sometimes very low, and the internal response rate varied between the different sets of questions. As a result, the evaluators placed more emphasis on impact cases than on survey results when assessing the societal impact of an institution. 


\section{THE IMPACT OF THE IMPACT EXERCISE}

Despite the many methodical challenges in impact evaluations, the RCN has received positive feedback from the institutions and researchers on the usefulness of the impact-exercise. Several of the impact cases produced for the evaluations have been used by the institutions and researchers themselves e.g. published on the institutions websites or included in the researcher's CV. We also see signs of a more systematic approach in the institutions in identifying and documenting the (potential) societal impact of research.

The impact case method has also given valuable new knowledge in the variety of ways in which SSH research creates societal benefits. We have thus moved from a situation with a rather vague discourse on SSH-research as a general societal good to a collection of concrete evidence that could be used in a debate on how research funding should be attributed in order to obtain specific societal (or commercial) aims. As an example, the impact cases from the humanities were used in policyadvice to the government related to the white paper on the humanities that was launched during the evaluation. ${ }^{9}$ It is however important to note that a collection of 404 impact cases cannot give a representative picture of the societal impact of SSH research in Norway.

\section{THE WAY FORWARD}

So, where do we go from here? There is a rising demand from policymakers and funders that potential societal benefit should be considered through the whole life cycle of the research process onto the application of results. In this perspective, the difficulties reported by the evaluation committees in assessing the actual impact of Norwegian SSH research is a cause of concern. Based on our experience with the recent evaluations in the RCN we would argue that there are two aspects that needs to be addressed in the time to come:

- further development of the impact infrastructure at the institutions,

- further development of the methods for assessing and evaluating societal impact;

\section{FURTHER DEVELOPMENT OF THE IMPACT INFRASTRUCTURE:}

The evaluation committees' recommendation to the institutions to take a more strategic approach to impact documentation is in our view a result of an underdeveloped impact infrastructure at the institutions. This is not only a problem for policy-makers and funders searching for a return on their investments in research. It is also a problem for the academic institutions themselves that are confronted with an expectation to document societal benefits from their research, but lacking the impact infrastructure that will help them to identify, document and learn from how research produced at their institutions in the past have led to positive (or negative) effects in society.

\section{FURTHER DEVELOPMENT OF THE IMPACT METHODOLOGY:}

The evaluation committees also calls for further development of the methods for assessing and evaluating societal impact, and for more sophisticated tools for gathering and articulating evidence of impact. As earlier noted, the RCN recognises, in order to make robust assessments of the societal impact of research, that there is a need to combine different methods. In addition to this, it might also be useful to change the focus. In a recent report by two Norwegian evaluation experts on the concept and practice of societal impact ${ }^{10}$, it is argued that the object of evaluation should shift from the research results and their dissemination towards the process of interaction between researchers and users. They also argue that the evaluation of impact needs to be related to the actual goals of the research performing institutions.

The RCN is currently investigating the possibility of creating a national evaluation protocol in Norway that will allow the higher education institutions to take a larger responsibility for the evaluation of their own activities as it is done under the Dutch "Standard Evaluation Protocol"." Our hypothesis is that evaluation results will be more relevant for the strategic development of each institution if the evaluation criteria are aligned with their strategic goal. Giving the higher education institutions a greater responsibility for the evaluation of their own activities, will probably also tie the evaluation processes more closely to the research processes, creating loops of feedback from evaluation results to the managers of research projects, groups and departments.

\section{CONCLUSION}

One of the main lessons of the recent evaluations of SSH in Norway is how a pragmatic approach to assessing societal impact contributed to a change in the way that academics and institutional leaders talk about the societal benefits from research in Norway. Although better definitions and conceptualisations of evaluation criteria - such as societal impact - are always welcome, our experience is that the evaluation process in itself created a new understanding of the phenomenon to be evaluated.

Recommendations provided by evaluation experts based on the recent evaluations in Norway and cases of international best practice, could indicate that future evaluation exercises in Norway - including societal impact - should be more closely linked to the purposes and strategic goals of the research organisations in order to allow these organisations to experiment with different kinds of evaluations methods and processes that are more in tune with the actual research processes and the multitude of ways that researchers interact with partners outside of academia.

So far, the national research evaluations in Norway have served an important function in the implementation of national policies for research and higher education. The impact of the latest evaluations in SSH - changing the way that societal impact of SSH research is conceived and discussed - is an example of this transformative role. In the choice of future model for research assessment in Norway, there is thus a balance to be struck between the need for a better adaptation of evaluation criteria to the strategic goals of each institution and the use of research evaluations as a policy instrument at the national level. It remains to be seen if it will be possible to move the evaluation processes and stewardship closer to the research institutions, while assuring at the same time that such institutional evaluations respond to national policy needs. 


\section{REFERENCES}

Manville, C., Guthrie, S., Henham, M-L., Garrod, B., Sousa, S., Kirtley, A., Castle-Clarke, S. and Ling, T. (2015). Assessing impact submissions for REF 2014: An evaluation. RAND Corporation, Santa Monica, Calif. And Cambridge, UK.

Gulbrandsen, M. and Sivertsen, G. (2018). Impact i anvendt forskning: begrepsavklaring og praksis. NIFU Report [Impact in applied research concepts and practice. Forthcomming]

Ministry of Education and Research (2017). Humaniora i Norge [The Humanities in Norway] (Meld. St. 20 2016-2017)). Oslo.

REF2014 Reserach Excellence Framework (2011). Assessment framework and guidance on submissions, Bristol

The Research Council of Norway (2017). Evaluation of the Humanities in Norway, Oslo.

The Research Council of Norway (2018). Evaluation of the Social Sciences in Norway, Oslo.

Thune, T. et al. (2014). Noder i kunnskapsnettverket: Forskning, kunnskapsoverføring og eksternt samarbeid blant vitenskapelig ansatte i UHsektoren. [Internal and external cooperation patterns among academic staff in the Higher Education sector in Norway] NIFU Report 2014:23. Oslo.

VSNU, KNAW and NWO (2014). Standard Evaluation Protocol 2015 2021, Voorburg.

Wróblewska, M. N. (2018). The making of the Impact Agenda: A study in discourse and governmentality governmentality (PhD Thesis Synopsis, p.7). Warwick University, Coventry, United Kingdom, Retrieved from: https://tinyurl.com/y2nkhlwe 25 February 2019

\section{AUTHORS}

\section{JON HOLM}

The Research Council of Norway

Drammensveien 288, 0283 0slo (Norway)

E: jon.holm@rcn.com

\section{ANETTE ASKEDAL}

The Research Council of Norway

Drammensveien 288, 0283 Oslo (Norway)

E: anette.askedal@rcn.com

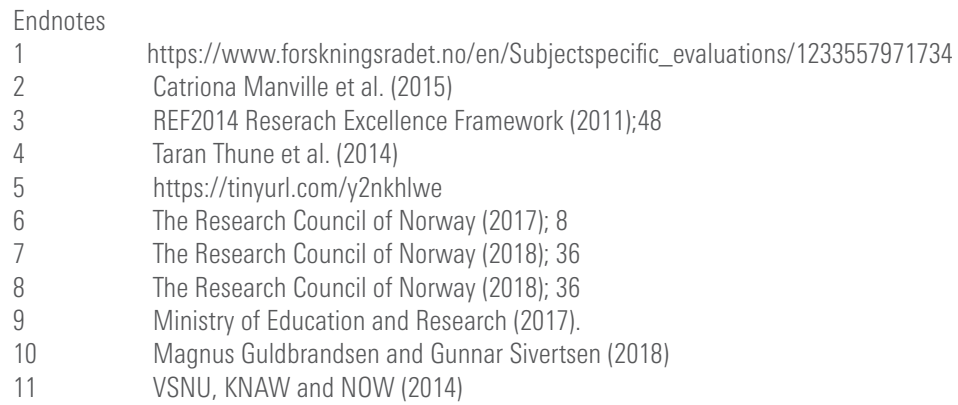




\title{
STAKEHOLDERS' ROLE TO PRODUCE IMPACT FROM SOCIAL SCIENCE RESEARCH: WHAT LESSONS FOR EVALUATION? ${ }^{1}$
}

\author{
EMANUELA REALE, SERENA FABRIZIO AND LUCIO MORETTINI \\ DOI: 10.22163/fteval.2019.383
}

\section{INTRODUCTION}

$\mathrm{T}$ he aim of the paper is twofold: a) to analyse the ways in which stakeholders are involved in social science research (SS) funded under European research projects, in order to identify elements -organisational characteristics of the project, types of stakeholders, type of involvement, that can increase the likelihood of producing an impact on policy and society; b) to discuss consequences of the empirical evidences for research evaluation both at the ex-ante level (elements characterising the design of the project) and ex-post level (achievements and practices indicating that an impact is produced or signalling that an impact might occur).

The paper deals with social impact, which is mostly defined as an effect that research could produce beyond the academic context in terms of benefits on societal and institutional challenges, including also impact on the political side (Penfield et al., 2014; Reale, Primeri, Fabrizio, 2017). The interest to deepen issues of social impact in SS derives from the limitations of using the traditional approach based on input-output-outcome measurements; SS are characterised by effects that are more difficult to be singled out than those produced in other areas of science, and measurements provide very poor and often biased understanding of the phenomenon (Reale et al., 2017). The paper follows the theoretical approaches focusing on research process (Spaapen and van Drooge, 2011) and contribution to the impact generation (Mayne, 2012), instead of attribution of impact to research activities; in this respect it is of crucial importance to shed light about the generating mechanisms that transform knowledge into actionable goods, and the network of actors involved (Joly et al., 2015).

Social impact could be strengthened by participatory involvement of different social actors through productive interactions (Molas-Gallart, 2012; Weik et al. 2014); the positive effects of these interactions are closely related to the ways in which researchers and stakeholders communicate about research, its goals and societal demand (Molas-Gallart, 2012). Thus, social impact is pointed out as a consequence of a process in which knowledge and expertise circulates to achieve specific objectives that are relevant for the progress of society (Spaapen and Van Drooge, 2011). A participatory approach could deeply affect the sustainability of research so it must be implemented since the beginning of projects (Talwar, 2011). Under a slightly different conceptualisation, social impact is generated through translation of actors involved in the process (Joly et al., 2015), which co-define their interests along the so-called impact pathway (Walker et al., 2008; Joly et al. 2015). In both cases, the role of stakeholders is at the core of impact production, and understanding features affecting their involvement is still a low explored issue. This paper contributes to demonstrate key determinants of impact in the different types of interactions with stakeholders, discussing what implications this can have on evaluation criteria and methods of research projects.

\section{THEORETICAL BACKGROUND}

\section{AND CONCEPTUAL FRAMEWORK}

Participation of stakeholders in research activities gained a momentum from the launch of the actions on science and society and science with society within the "European Framework Programmes" (EUFP); studies developed on this topic figured out the importance of involving non-academic partners in research projects to improve the likelihood to produce an impact from research activities (Lang et al. 2012; Reale et al., 2017). Participation of stakeholders could allow the extension of research results towards a practical path but in a broader perspective they can provide to the project a general insight focused to the problem field. This means that stakeholders invited to collaborate with researchers should be those i) more affected by the challenge faced by the research project, and ii) more stimulated to offer their knowledge to define a range of options for results implementations (Wiek, 2014). The cooperation with the stakeholders includes the possibility to keep in touch with each of the categories, placing as unique point of reference the competences necessary to reach the project's aim in the best possible way. This means that research cooperation is open to actors from public institutions, corporate sector, and not-for- profit organisations (Lang et al., 2012). 
Interactions and collaborations between researchers and stakeholders take different forms. The literature outlined several types of stakeholders' involvement, which can be typified around three main categories of contribution, namely: i) co-creation of knowledge between academics and non-academics (Weik et al., 2014; Edelnbons et al., 2011; Spaapen and van Drooge, 2011; de Jong et al., 2013); ii) unpacking the research objectives into sub-task that are more manageable for producing usable results (Bell et al., 2012); iii) discussion and dissemination of research results after their production in order to facilitate generating an impact (Spaapen and Van Drooge, 2011; Weik et al., 2014). It is worth to recall also the work of Muhonen et al., (2018), which developed 60 case studies on social sciences and humanities pathways to societal impact by paying attention not only to productive interactions but also to the changes they mediate. Based on the empirical results, the authors developed a typology of four pathways to societal impact, articulated in twelve models, which starts from the classical pipeline model, of results transferring from research to society. The models are presented in hierarchical progression, according to the deviation from the base model: as high are the level of complexity in terms of interaction between research, society and intermediating institutions as high will be the deviation from the pipeline model. The pathway models belong to four general typologies, namely dissemination, co-creation, reacting to social change and driving social change.

Projects can have one or more types of stakeholders' involvement but direct participation indicates the goal of a social effect of research, an element to assess with instruments other than standard academic indexes (Penfield et al., 2014; Weik et al., 2014). In the same line, Talwar et al. (2011) distinguish between two main categories: a) unidirectional approach, when social actors are involved in the final phases of the project, for a weak support in results consolidation and/or a consultation with researchers to implement results in an applicative way; b) interactive approach, when stakeholders are involved also in the early phases of the project and contribute to define the research goal and/or to design the research strategy. While in the first approach, contribution of stakeholders is basically limited to elaborate a tool to use research results, in the second one, stakeholders are invited to provide their expertise to broaden the knowledge base useful to define all aspects of the problem, beyond the scientific analysis, and implementing the usability of results throughout all the phases of the project.

However, the advantage to have a relevant applicative core in a project could expose the research to the risk that pursuing applicative results become prevalent with respect to the achievement of high-quality scientific outputs. In a more general way, several contributions underline that a large involvement of stakeholders in a research project could focus the analysis on solving a single problem (or a restricted range of problems), channelling research efforts to a punctual objective at the expense of results of general application, also relevant for other cases (Talwar et al., 2011; Lang et al., 2012).

We assume a link between the degree and the way of stakeholders' involvement in the project and the emergence of social impact of research. We thus consider that one of the key features for generating impact is the capability of the project to build a common language between the different actors, scholars and non-scholars. Under this condition, exchanges are able to create new knowledge and mutual understanding, which is likely to produce transformative changes. Also, we consider that the mentioned result can be achieved when continuous involvement of stakeholders is at stake, in the different phases of the project, and sta- keholders show concrete willingness and interest to contribute in a substantial way to the research achievements. We analyse the relevance of role held by stakeholders, respect to the researchers, and how they jointly contribute to the research activity, with the expectation that a more extensive and effective co-participation in research creates the conditions both for dissemination of results in broad and articulated terms, over the original boundaries, and for generating impact pathway.

\section{METHODOLOGY AND DATA}

The paper is based on two in depth case studies of the projects "Strategies for inclusion and social cohesion from education in Europe" - INCLUD-ED and "Making Persons with Disabilities Full Citizens" - DISCIT, funded respectively under the "European Framework Programmes" FP6 and FP7 in social sciences, where a social impact became visible just after the project completion. The case studies selected are two out of 22 top success stories developed under the "Evaluating the impact and outcomes of EU SSH research" project (IMPACT-EV), which are illustrative examples of successful modes for stakeholders' involvement in research actions. Cases follow a standardised structure, developed though triangulation of information from different sources, namely information from documentary analysis (characteristics of the call under which the project has been funded, reports and deliverables produced, other administrative documents), data and indicator on research outputs (bibliometrics and other web-based resources), interviews with researchers, coordinators, and stakeholders involved in the activities. Four aspects of actors' involvement have been considered:

- Modalities and communications - projects' organisational features;

- Timing - timely interactions during the project and after the project completion determining the impact pathway;

- Language - capability to develop mutual understanding between researchers and stakeholders;

- Outcomes - co-creation of results with transformative effects on science and society.

The projects analysed both present a broad involvement of stakeholders in order to maximise the impact in political and social terms.

INCLUD-ED emphasises the role of the dialogic and participative collaboration among researchers and stakeholders (end-users, local institutions) in the development of educational strategies for the social inclusion of vulnerable groups (IMPACT-EV, 2017b). The project focused on strategies that could contribute to social inclusion of vulnerable people, deciding about key elements and actions to improve social and educational policies. "Successful Educational Actions" (SEAs) - thus evidencebased solutions able to achieve good results in many diverse contexts, were identified as examples of positive achievement in the inclusion of vulnerable groups; SEAs were transferred to other communities and contexts to improve social cohesion. INCLUD-ED produced significant achievements on educational practices, decreasing the rates of school failure and improving the families' involvement. The project put forward the hypothesis that social exclusion is more a consequence of actions implemented than an effect deriving from the social characteristics of the context (IMPACT-EV, 2017b). The consortium was composed by an interdisciplinary research team covering anthropology, economics, history, research methods, political sciences, linguistics, sociology and educa- 
tion, comprising fifteen academic organisations from fourteen different European countries. The organisational structure includes ten "Working Groups" (WGs), different "Free Task Oriented Groups" (FTOGs), and a "Panel of Experts" (POE), which supported the consortium, and an "Advisory Committee" (AC) composed of representatives of vulnerable groups. The project also included horizontal type of actions and structures, whose aim was to monitoring and harmonising the activities, solving problems that might emerge, and combining the results and progresses made by the different groups.

DISCIT main goals were a) to help definition of a new "European Social Model" of inclusion and cohesion through the analysis of political and institutional instruments existing in the countries involved, and b) to indicate a way to remove and prevent physical, attitudinal, social and organisational barriers to a full and effective participation to the society of persons with disabilities (IMPACT-EV, 2017a). To achieve its goals, DISCIT considered different forms of stakeholders' engagement. Eight research institutions, from eight different countries, and two international organisations of disabled people's rights - "The European Disability Forum and The Mental Disability Right Initiative", composed the consortium. Organisations contributed to the drafting of the project and helped the consortium to set up the analysis in general terms without make the differences between types of disabilities irrelevant. Furthermore, two associations of disabled people helped to identify the space of intervention of the project among the different social areas, contributing decisively to define the change of perspective that characterises DISCIT: the idea that disability is not a particular case of each area of intervention but it is a unique topic with several articulations.

The consortium was supported by one "European Stakeholder Committee" and nine "National Stakeholder Committees", one for each Country involved in the project. These committees included members of "Disabled people's organisations" (DPOs) and representatives of general directorates (limited to the "European Committee"), administrative and political institutions at national and local level. Committees contributed in different ways: providing information about social and regulatory peculiarities within countries and commonalities between countries, refining the documentary and empirical survey tools of the project, helping in sample selection for the interviews, and proposing themselves as intermediaries between the researchers and the disabled people interviewed, in order to help the latter to overcome any embarrassment.

Periodical forum at international and national level were organised to facilitate mutual exchanges between researchers and stakeholders, discussing research development and incentivising dissemination of policy briefs based on research results. All in all, these forums produced more results than expected, favouring a harmonisation of language between different groups of stakeholders (representatives of associations and institutions) and facilitating the creation of networks for the exchange of information and best practices at international level between DPOs.

\section{FINDINGS}

The case studies highlighted that both projects show significant evidences related to the three dimensions of stakeholders' involvement investigated in the paper; however, differences emerged from the analysis, which are related to the organisational and structural features of the projects.

\section{INCLUD-ED}

Stakeholder involvement was a key objective from the beginning of the project, affecting the methodologic approach, shaping the research questions and the architecture of the whole research activities. The collaboration between researchers and stakeholders concerned both the knowledge-exchange dimension and the concrete implementation in specific social contexts through specific sub-task. However, the most significant evidence was the long-term impact of the model implemented, through a constant dissemination of main results deeply involving also a large network of stakeholders (IMPACT-EV, 2017; Reale et al., 2017b).

\section{MODALITIES AND COMMUNICATIONS}

Diverse voices-associations of vulnerable groups, families, teachers, local decision makers enforced the validity and rigorousness of the scientific process thus contributing to the co-production research results.

"I remember that it was very egalitarian collaboration because they were first of all introducing each of us, at each meeting we were the first who were talking in the centered explaining each community we were representing and I remember being very diverse, so people, researchers, but also women, immigrants or people with disabilities, so the meeting was very diverse and there were the researchers were presenting the results or part of the results corresponding to the part we were supposed to discuss, and they were asking maybe questions or maybe very open debate on what do we think or what do we believe that concrete strategies they were presenting may affect our community or not."

(End User)

"From my point of view is exactly the same methodological structure of the entire project that eases the portability, because it is based on the communicative theory of Habermas, this means that every time we simply did the interviews, as you are doing with me, stakeholders, etc., we are focused on the one hand to receive the information and on the other hand to give ourselves a contribution, support for change precisely"2

(Researcher) 


\section{TIMING}

Main events occurred over the project life and beyond, documented on the official website ${ }^{3}$ :

- 10 technical meetings with "Advisory Committee" (each meeting consisted of a presentation of the results of different projects/subtasks and a discussion and reflection between representatives of different vulnerable groups), meetings with the panel of experts and members of research team;

- 15 dissemination events around Europe like Conferences, Congresses, public meetings and launch of project website;

- 7 institutional events mainly attended by representatives from the European Commission - Directorate General for Research, representatives of Member State governments, social actors, researchers and scholars;

- 13 training seminars attended by members of the research community, government representatives and Non-Governmental Organisations (NGOs).

These events have been scheduled during each year because they had different purposes and involved diverse types of stakeholders in order to discuss steadily short and medium-term achievements of the project, to share different points of view on the methodological approach, and to implement the model through specific training seminars. Thus, the work was basically devoted to follow a path to gain impact on interested communities.

"We were meetings twice a year, at the meetings we were discussing the results of the project, they were making right, so I remember that researchers from INCLUD-ED project were presented us the results or the development of the project and then we were discussing about that." (End User)

"I remember that we had, a year if I'm not long we meet with the expert group and the advisory committee every year and we were presenting [...] all day presenting the results, they had them in a bag but of course some people might not read report, so we synthesised the main points, and we were discussing with them, the $A C$, the Advisory Committee, and expert group. The contributions from the expert group were not that different from the one's that we could come up as research consortium, even they were a lot of policy makers at high level impact." (Researcher)

Language

INCLUD-ED put into action the critical communicative research methodology (Flecha and Soler, 2014) which was crucial for the project success, because it allows integrating and including knowledge from different disciplines and orientations, using both quantitative and qualitative methods and techniques to analyse data; furthermore, the communicative methodology allowed researchers to apply mixed-methods approach to pursue impact. "While the voices of vulnerable groups have traditionally been excluded from research, the communicative methodology depends on the direct and active participation of the individuals observed throughout the research process." (INCLUD-ED website ${ }^{6}$ ).

"I remember that main researcher of INCLUD-ED it was talking and he was very interested on our opinion, we felt that, we felt that we are not attending the meeting because this is part of the project but because they wanted to know what we think what we believe and what we disagree with them. I remember they were asking all the time to criticize them, to disagree with them because this is good for improving and in the way that we felt that they were taking our opinion into account. ${ }^{4}$ (End User)

".... I have often also found critical points of view that are not even critical in dialectical sense; in other cases I have found resistance also to the type of interview because being a dialogic interview when the other dialogues must give you his time not only to answer but also to listen to you, and it is not said that everyone wants of this thing because you already put yourself in a very strong relationship, it is more challenging, not just intellectually as time, it is really challenging from a relational point of view. ${ }^{5}$ (Researcher)

\section{Outcomes}

The members of the Advisory Committee had access to the INCLUDED results and met periodically with the coordination team to discuss the research. More important, they suggested recommendations on how the findings could be used to have a greater social and political impact; those recommendations were problematised with the researchers.

"They were very motivated because they really give importance to our words, and then in further meetings we could see during the years of the project, during the different meetings we have we could see also the improvement they were achieving they were explaining that. [...] / remember a concrete neighbourhood in Spain they were telling us about and that people who never have a job before they are now getting jobs or starting to organise themselves and I remember that for me was important." (End User)

Stakeholders played a further important role with respect to the political dimension of impact, since policy makers were well attentive to the instance of changing coming from society testifying the goodness of the transformations suggested through evidences of SEAs:

"If we make lobbing with policy makers, we don't get results. If we get social impact and social actors who are beneficiaries of social impact go to policy makers with us, this has political impact. Even with friends, even with policy makers that are friends of mine... "you are very nice and..." but nothing. We will remain friends. ... Do not ask to policy makers what are thinking, because they think "Well, they are researchers, they are coming here for resources, for applying". (Researcher)

3 http://creaub.info/included/ Last access: 20/06/2018

4 http://creaub.info/included/

$5 \quad$ English translation from the Italian original: "[...]spesso ho trovato anche punti di vista critici, anche critici in senso dialettico; in altri casi ho trovato in effetti delle resistenze anche alla tipologia di intervista perché essendo un'intervista dialogica nel momento in cui dialoghi devi dare all'altro il suo tempo non solo per risponderti ma anche per ascoltarti, e non è detto che tutti abbiano voglia di questa cosa perché già ti poni in una relazione molto forte, è più impegnativa, non solo intellettualmente come tempo, è proprio impegnativa dal punto di vista relazionale".

6 English translation from the Italian original: "Mentre il progetto era ancora in itinere, abbiamo organizzato delle presentazioni a livello locale, presso presidi sanitari e amministrazioni locali. Non so dire se abbiamo avuto un impatto politico o se abbiamo avuto un effetto sulle loro pratiche con questi incontri ma posso dire che abbiamo avuto la possibilità di presentare il nostro approccio ad unità di base del servizio sanitario e della pubblica amministrazione, avendo con loro un proficuo scambio di opinioni sulla metodologia e sul linguaggio da utilizzare. Abbiamo avuto la possibilità di esportare un po' del progetto nei posti dove vorremmo che fosse applicato tutti i giorni". 
The effects in terms of political impact were in fact remarkable. INCLUD-ED findings have been applied on European resolutions, communications and recommendations; the SEAs were transferred through across Europe, producing in most of the cases positive effects. However, in some cases institutional barriers emerged that constrained the possibility of research to produce an impact in specific national contexts. The effects produced at the European political level were very important: three resolutions by European institutions on early school leaving were approved, mentioning results obtained through INCLUD-ED. Furthermore, two resolutions by European Union on the themes of social and educational inclusion of children of migrants and Roma people were also implemented, using evidences from INCLUD-ED. Other evidences related to national context concern: recommendations of the "Education Ministry on Education and Formation Strategy 2020" in which the development of SEAs in the Basque Country 'appears as a practice to follow'; evidences from state and regional legislation, and finally 8 agreements with public administrations for implementing of SEAs in different countries (IMPACTEV, 2017b).

\section{DISCIT}

DISCIT is characterised by collaborative efforts involving researchers and stakeholders associations, representative groups of disabled, decision makers that helped to define the problems related to disability as a common area of intervention, with several articulations. This change of perspective allowed to calibrate as best as possible the instruments of direct investigation and to define the structure of results in order to make it easier to propose their integration in institutional settings. At the same time, the project created a stable forum for the interactions between researchers, institutions and organisations of people with disabilities, to discuss the problems of the disabled people, to encourage the exchange and dissemination of good practices and to create common understandings between institutions and associations (IMPACT-EV, 2017a).

\section{MODALITIES AND COMMUNICATIONS}

Stakeholders' involvement was directly related to the theoretical framework used for analysing "Active Citizenship" (EC-EESC, 2012), which was articulated in three steps: a) to review the initial conditions of the disability policy system and their configuration with respect to individuals with disability, their families and their inclusion in local communities, in job market, and social and civil activities; b) to analyse the effective implementation of the measures in daily life of persons with disabilities; c) to figure out how the results of the mentioned analysis interacts with respect to the three pillars of the Active Citizenship action, namely Security, Autonomy and Influence (EC-EESC, 2012).

As to the first point, stakeholders gave relevant feedbacks on the effective application of laws, highlighting the levels of protection for the various groups of disabled people. This helped the researchers to have a more complete vision of the state of the art. The effects of this approach are reported in the interview to the representative of one of the two DPOs included in the consortium:

"I think that one specific thing that my organisation bring to the consortium was this specific knowledge of the positions of rights of persons with mental disease that we discussed with other partners of the consortium. I have also a background as researcher at the university and I was a legal advisory of the organisation during the project but it was obvious that the project itself, all the other partners, at the very beginning needed this input from this specific area, it is not easily deducible from the official documents, because the attribution of rights for some categories of people is different from the prescriptions of the law." (Stakeholder)

The second part of the DISCIT research concerned the investigation of the conditions of people with disabilities through a data collection based on interviews with a large audience of disabled people. In this phase, the stakeholders involved in the project provided their contribution to the questionnaire on which the interviews were based:

"I had the opportunity to talk with the stakeholders about the questionnaire. Feedback used to correct the methodological part were greater in the qualitative part, but in general it was a useful debate because it allowed me to focus on the types of indicators used subjects other than researchers, giving me a more balanced view of the problem." (Researcher).

Also, stakeholders actively participated in the interviews, proposing themselves as intermediates between researchers and interviewees and helping the latter to overcome the embarrassment of talking to strangers about their condition of a disabled person.

Members of the DISCIT consortium paid particular attention to the organisation of meetings with social, political and research institutions to discuss the new point of view from which the project aimed to address the issue of disability. The effects of these meetings were double: on the one hand, the principles underlying the approach were disseminated independently of the results, laying the foundations for a discussion on disability in discontinuity with respect to the past; on the other hand, the members of the consortium could gather tips to correct some elements of their methodology of analysis. According to the members of the project, DISCIT organised or has been involved in the organisation of more than 60 international initiatives over the three years of the project. In addition, the national groups have taken charge of organising meetings of the same type with local institutions to allow widespread communication:

"While the project was still in progress, we organised local presentations in hospitals and local administrative offices. I cannot say whether we have had a political impact or if we have had an effect on their practices with these meetings but I can say that we have had the opportunity to present our approach to basic units of health service and public administration, having with them a fruitful exchange of opinions on the framework and the language to be used. We had the opportunity to export some of the project to places where we would like it to be applied every day." (Researcher)

7 English translation from the Italian original: "Mentre il progetto era ancora in itinere, abbiamo organizzato delle presentazioni a livello locale, presso presidi sanitari e amministrazioni locali. Non so dire se abbiamo avuto un impatto politico o se abbiamo avuto un effetto sulle loro pratiche con questi incontri ma posso dire che abbiamo avuto la possibilità di presentare il nostro approccio ad unità di base del servizio sanitario e della pubblica amministrazione, avendo con loro un proficuo scambio di opinioni sulla metodologia e sul linguaggio da utilizzare. Abbiamo avuto la possibilità di esportare un po' del progetto nei posti dove vorremmo che fosse applicato tutti i giorni". 


\section{TIMING}

Collaborations between researchers and stakeholders were implemented through several meetings organised over the project duration, open to the network of actors involved. The project calendar included three plenary meetings. All the representatives of the stakeholder committees were invited to participate in order to discuss the progress of the project with the researchers and to propose initiatives to disseminate the results. Plenary meetings were interspersed with national group meetings. In addition, along the project meetings were organised between members of the consortium and representative of institutions and associations external to the project both at European and national level. In addition to the official meetings, the stakeholders have been constantly involved with requests for active collaboration, especially for data collection and discussion of the results. The constant demand for active participation was particularly appreciated by stakeholders:

"The request for participation was perfect, neither too much nor too little. We were asked to give our opinion on several points, but these requests were not concentrated in specific moments of the project development, as happened in previous experiences. "ㅇ (Stakeholder)

\section{LANGUAGE}

Language harmonisation was one of the most significant and difficult result to achieve, the one that produced the most recognisable social impact.

First the exchange of information between researchers and stakeholders over the project duration was crucial. As reported by several interviews, these two groups started from different definition of "disability" and the difference in definition involved a series of divisions that could generate misunderstandings; the consequence of which would be the failure of research in terms of social and political effects. DISCIT actions helped to disentangle these differences, prompting researchers to assimilate the language of associations in order to increase the likelihood of results to be implemented in other areas than research. Within the project, the interactions between stakeholders and researchers were also useful to overcome the differences in language between different countries.

A second important interaction was with organisations and institutions external to the project. In these occasions, a common language was agreed in order to avoid misunderstanding between researchers, associations and institutions when disability was represented, and this result improved substantially translation of research findings into appropriable goods. In fact, the most interesting element was overcoming cultural gaps between different stakeholders as to the definition of disability, a change that produced effects beyond the aims of DISCIT:

"A problem is what really disability means. There are two understandings of disability. One is the sense of disability as marker of marginalised group of population. But there is another sense of disability which is a phenomenon that people indeed experience during their life, namely some sort of limitation in functional ability. This second sense of disability is more a universal sense of disability but does not involve marginalisation of groups. People tend to define themselves in one of the two groups on the basis of a sort of self-definition, with respect to the impact that the limitation they experience has on their everyday life. [...] The lack of skills in a particular context does not nullify the person as a whole, so it is necessary to rethink the concept of disability, bringing it closer to the most universal sense to prevent policies to support people with disabilities become a way to marginalise a part of the population and deprive them, in fact if not legally, of some rights as human beings." (Researcher)

\section{OUTCOMES}

DISCIT set out to promote the implementation of the Convention on the Rights of Persons with Disabilities in European and national legislation (UNCRPD). Despite translation of research findings into the political processes took longer than the duration of a project, some elements of impact on European and local measures have been observed directly during the activity of DISCIT.

At the European level, the components of the DISCIT research team "Active Citizenship through the use of New Technology" were involved during the drafting of the European Directive "European Accessibility Act" ${ }^{\prime \prime}$, prepared by the Directorate General for Employment, Social Affairs and Inclusion. Furthermore, DISCIT researchers were invited to be part of the High-Level Group on Disability ${ }^{10}$, composed by European and national experts chosen from policymakers and stakeholders, in charge of define the strategies for implementation of the UNCRPD. Other political effects were observed at national level, for instance with the involvement of the Italian research group in the preparatory work of the law of the Tuscany Region for support to families of disabled persons and the audition at the National Observatory for Disabilities of the Ministry of Labour and Social Policies. The Irish research team participated in a national task force that launched a trial of supportive policies for the disabled on more inclusive bases with respect to current legislation. Finally, the Swedish research group elaborated some guidelines, adopted by institutions like the Swedish Agency for Participation. Interviews demonstrated that a new point of observation was developed precisely through the dialogue between researchers and stakeholders on which the project was based:

"I think that this project has broaden the research community knowledge because it has forced the academics to discuss their approach with organisations and to consider this information." (Stakeholder)

"During the international meetings / had the opportunity to meet responsible of associations that work in community living sector from other countries, in particular I was positively impressed by the practices used in Sweden [...] I proposed to use some of these ideas, in experimental way, in order to test if they fit with our social context, and some preliminary results seem to be positive." (Stakeholder)

\section{DISCUSSION}

\footnotetext{
8 English translation from the Italian original: "La richiesta di partecipazione è stata perfetta, né troppo né troppo poco. Ci è stato chiesto di esprimere il nostro parere su diversi punti, ma queste richieste non erano concentrato in momenti specifici dello sviluppo del progetto, come accaduto in precedenti esperienze".

$9 \quad$ https://eur-lex.europa.eu/legal-content/EN/TXT/?uri=COM:2015:0615:FIN

$10 \quad$ http://ec.europa.eu/social/main.jsp?catld=1137\&langld=en
} 
Both the illustrative case studies on projects carried out under the European Framework Programmes show that stakeholders generate a different approach to the social problem addressed; the main features of the stakeholders' interactions with researchers are summarised in Table 1.

\begin{tabular}{|c|c|c|c|c|c|}
\hline & $\begin{array}{l}\text { Modalities and } \\
\text { communications }\end{array}$ & Timing & Language & Outcomes & $\begin{array}{l}\text { Model of interactions } \\
\text { (Muhonen et al. 2018) }\end{array}$ \\
\hline DISCIT & $\begin{array}{l}\text { Circular exchange } \\
\text { of information } \\
\text { Diffusion and discussion } \\
\text { of the methods of analysis } \\
\text { with external stakeholders }\end{array}$ & $\begin{array}{l}\text { Intense involvement in the } \\
\text { review and investigation } \\
\text { phases, partial discussion } \\
\text { of policy proposals }\end{array}$ & $\begin{array}{l}\text { Harmonisation } \\
\text { between countries } \\
\text { and areas of interest }\end{array}$ & $\begin{array}{l}\text { Formal involvement in } \\
\text { policy making process } \\
\text { Exchange of best practices } \\
\text { Cultural impact: new } \\
\text { idea of disability }\end{array}$ & $\begin{array}{l}\text { Collaboration model } \\
\text { Research engagement } \\
\text { Knowledge "creeps" } \\
\text { into society model }\end{array}$ \\
\hline INCLUD-ED & $\begin{array}{l}\text { Dedicated events for targeted } \\
\text { stakeholders (training for } \\
\text { teachers, dissemination for } \\
\text { scholars, political meetings for } \\
\text { institutional representatives) }\end{array}$ & $\begin{array}{l}\text { Continuous involvement of } \\
\text { all stakeholders along the } \\
\text { five years of the project }\end{array}$ & $\begin{array}{l}\text { Communicative } \\
\text { methodology }\end{array}$ & $\begin{array}{l}\text { Formal stakeholders' } \\
\text { involvement in } \\
\text { knowledge creation } \\
\text { Replicability of the outputs } \\
\text { in different national and } \\
\text { institutional contexts }\end{array}$ & $\begin{array}{l}\text { Collaboration model } \\
\text { Public engagement model } \\
\text { Mobility model }\end{array}$ \\
\hline
\end{tabular}

Using the Muhonen and colleagues (2018) typology, INCLUD-ED developed interactions with stakeholders that mainly belong to the co-

Table 1. Comparison of projects on typologies of stakeholders involvement.

creation typology, and the activities adopted elements that relate to collaboration, public engagement and mobility models. DISCIT had a more hybrid structure, which belong to the co-creation typology - through collaboration, and driving social change typology - through activities that featured the research engagement and the knowledge "creeps into society" models ${ }^{11}$. In this respect, typologies aimed at understanding changes produced through the involvement of stakeholders in research actions is a helpful tool for comparing different configurations of the relationships within the network of actors involved, which can also support a more precisely tracing of the translational effects generated.

The co-development of a new language and harmonised wording produced a cultural impact which was extremely important and took a long time. However, it is a type of impact difficult to single out through empirical observations related to measurable items; furthermore, also the impact at policy level took a long time to emerge (beyond the project time limit) and it was in both cases a direct consequence of the cultural transformation. In this respect, stakeholders are key carriers for social impact in SS research.

The transformative effects on society were linked to the co-production of knowledge that is used by societal actors but, in turn, the coproduction of knowledge needed the development of an appropriate communication to deconstruct the content, organisational features and knowledge carriers. The formal and informal confrontation between re- searchers and stakeholders - when it is a recurrent mechanism of networking rather than an endogenous event for them showed enormous potential for producing translational effects. However, the sustainability of the transformations produced through the projects is an element that went beyond the effort of the research teams. The duration of the project and the resources have not been entirely sufficient to have the chance that effects could remain over time, especially when institutional barriers appeared.

How these results are relevant for the evaluation of research projects? Some general advantages of stakeholders' participation can be outlined. On the one hand, it helps to figure out at certain extent problems of attribution of impacts produced by the project, and this is an important support to figure out the presence of causal linkages between project outcomes and effects on society. On the other hand, stakeholders helped to follow effects derived from the project for a longer period after the project completion. It is more difficult to understand how the characteristics of the project organisations and the modes of interactions between researchers and stakeholders can be assessed through specific criteria respectively at ex-ante and ex-post level. Here it is important to highlight two main elements in common of the illustrative case studies analysed.

First, in both cases the scientific quality of the outputs was very good. Bibliometric indicators and web-based indicators show that the scientific gained through open access ideology and through interdisciplinary or transdisciplinary approach. In the "Public engagement model" "results of research are taken into action by using society as a laboratory. Publicity is a necessity for impact.". In the "Mobility model" "knowledge and skills of a researcher are taken into use in a new context". Research engagement "increases awareness of the topic at hand. Targets of the study get recognition and sense of empowerment through the research process". In the model, knowledge 'creeps' into society's daily life's and political arena changes are produced "later on in relation to public opinion or legislation". 
value of the projects research outputs (papers in international indexed journals, books, book chapters, etc.) were well recognised in the field community. This element produced a positive feeling about the capability of the project to realise sound research results, despite some criticisms emerged in the interviews about the possibility that taking on-board considerations coming from the interactions with stakeholders is likely to decrease the originality of the research effort, impeding very innovative results.

Second, both cases are examples of projects pursuing impact using a theory-based approach: INCLUD-ED used the Habermas' theory of agents of social change and the critical communication methodology; DISCIT used the Active Citizenship approach. The effect was that impact was fully integrated in the theoretical framework of the research projects, driving the subsequent phases of the design and implementations of stakeholders' participation. Also, the interactions between researchers and stakeholders were implemented according to conceptual frameworks that included the stakeholders -either they were partners of the consortium or external to the project - as main actors to achieve the intended objectives. This element reduced some very well-known shortcomings generally linked to stakeholders' interactions due to low commitment and contribution to research activities over the project duration. Finally, building common harmonised languages in different contexts of application emerged as the most important element to generate impact under a co-creation model; however, this result can be achieved through dedicated efforts, and it cannot be considered as a taken for granted element.

In sum, theory-based approaches of stakeholders' involvement, building a common language, in combination with organisational features and careful timing of the interactions are all important elements to be considered in ex-ante evaluation. The presence of them in the design of the project should improve the likelihood that an impact might occur. In the same vein, the mentioned items should be assessed over the project implementation in order to understand whether the research activities were properly developed to achieve the objective of producing an impact. Also, in an ex-post assessment the linkage between scientific outputs and impact is an issue that deserve attention in order to avoid a trade-off between pursuing an impact and the quality of the research outputs.

\section{CONCLUSIONS}

Stakeholders' participation to research efforts is definitely an important element to reach social impact. For research in social science, stakeholders are key carriers for translating research results into cultural changes, which are likely to enable transformative effects of society. Furthermore, stakeholders represent the interests of society and this might empower them to mediate research outcomes to policy makers better than researchers themselves.

In this paper we deepened two cases related to a specific context of application, that is the development of research projects under the funding of European Framework Programmes; the analysis shows that organisation and communication, timing and language are key items to realise fruitful interactions that can produce - or contribute to produce - an impact, translating scientific knowledge into appropriable goods.

We also pointed out some items that should be considered in the evaluation of research projects, both at ex-ante and ex-post level, chan- ging to some extent criteria and methods of impact assessment in SS research. However, how this could be realised in concrete terms is definitely an open question that needs more research effort.

\section{REFERENCES}

Bell, S., Morse, S. and Shah, R. A. (2012). Understanding stakeholder participation in research as part of sustainable Development. Journal of Environmental Management 101, pp. 13-22

de Jong, S., Barker, K., Cox, D., Sveinsdottir, T. and Van den Besselaar, P. (2013). Understanding societal impact through studying productive interactions, Rathenau Instituut Working paper n. 1304.

EC-EESC (2012). Active Citizenship. For a Better European Society. EC, European Economic and Social Committee, Brussels.

Edelenbos, J., van Buuren, A. and van Schie, N. (2011). Co-producing knowledge: joint knowledge production between experts, bureaucrats and stakeholders in Dutch water management projects. Environmental science \& policy 14 (2011): $675-684$.

Flecha, R. and Soler, M. (2014) Communicative Methodology: Successful actions and dialogic democracy. Current Sociology Monograph, 62(2): 232-242

IMPACT-EV (2017a). Descriptive report of the case study on project "DISCIT - Making persons with disabilities full citizens"; IMPACT-EV, IRCrES - CNR

IMPACT-EV (2017b). Descriptive report of the case study on project "INCLUD-ED: Strategies for inclusion and social cohesion in Europe from education". IMPACT-EV Consortium, IRCRES CNR.

Joly, B., Gaunand, A., Colinet, L., Laredo, P., Lemarié, S., Matt, M. (2015). ASIRPA: A comprehensive theory-based approach to assessing the societal impacts of a research organization. Research Evaluation, 24, 440-453

Lang, D.J. and Wiek, A., Bergmann, M. et al. (2012). Transdisciplinary Research in Sustainability Science: practices, principles and challenges. Sustain Sci, 7(Suppl 1): 25-43

Mayne, J. (2012). Contribution Analysis: Coming of Age? Evaluation, 18(3), 270-280

Molas-Gallart, J. and Tang, P. (2011). Tracing "productive interactions" to identify social impacts: An example from the social sciences. Research Evaluation, 20(3): 219-226

Muhonen, R., Benneworth, P. and Olmos-Peñuela, J. (2018). From productive interactions to impact pathways. Understanding the key dimensions in developing SSH research societal impact. CHEPS Working Papers 02/2018 available at: http://www.utwente.nl/bms/cheps/

Ozanne, J. L., Davis, B., Murray, J. B., Grier, S., Benmecheddal, A., Downey, H. and Veer, E. (2016). Assessing the Societal Impact of Re- 
search: The Relational Engagement Approach. Journal of Public Policy and Marketing. DOI: 10.1509/jppm.14.121

Penfield, T., Baker, M.J., Scoble, R. and Wykes, M.C. (2014). Assessment, evaluations, and definitions of research impact: A review. Research Evaluation 23, pp. 21-32 doi:10.1093/reseval/rvt021

Reale, E., et al. (2017). A review of literature on evaluating the scientific, social and political impact of social sciences and humanities research. Research Evaluation, 11(1) Spec Issue, onlinefirst, https://academic.oup. com/rev/article/doi/10.1093/reseval/rvx025/3978693/A-review-of-literature-on-evaluating-the?guestAccessKey=ea3f8277-caee-4e3b-854a06d9d3e939de

Reale, E., Primeri, E. and Fabrizio, S. (2017). Assessing social research impact: exploiting productive interactions and dialogic learning. Paper presented at the CHER $30^{\text {th }}$ Conference, Javaskyla, 28-30 August.

Spaapen, J. and van Drooge, L. (2011). Introducing 'productive interactions' in social impact assessment. Research Evaluation 20(3): 211-218.

Talwar, S., Wiek, A. and Robinson, J. (2011). User engagement in sustainability research. Science and Public Policy, 38(5), June 2011, pages 379-390, DOl: 10.3152/030234211X12960315267615; http://www.ingentaconnect.com/content/beech/spp

Walker T., Maredia, M., Kelley, T., Rovere, R., Templeton, D., Thiele, G., Douthwaite, B. (2008). Strategic Guidance for Ex Post Impact Assessment of Agricultural Research. Science Council Secretariat, Rome, Italy. Report prepared for the Standing Panelof Impact Assessment, CGIAR Science Council

Wiek A., Talwar, S., O'Shea, M. and Robinson, J. (2014). Toward a methodological scheme for capturing societal effects of participatory sustainability research. Research Evaluation, 23, pp. 117-132 doi:10.1093/ reseval/rvt031

Zardo P, Barnett, A.G., Suzor, N. and Cahill, T. (2018). Does engagement predict research use? An analysis of The Conversation Annual Survey 2016. PLoS ONE 13(2): e0192290. https://doi.org/10.1371/journal. pone. 0192290 .

\section{EMANUELA REALE}

IRCRES - Research Institute for Sustainable Economic Growth, National Research Council

19 Via dei Taurini, Roma, 00139 (Italy)

E: emanuela.reale@ircres.cnr.it

\section{SERENA FABRIZIO}

IRCRES - Research Institute for Sustainable Economic Growth, National Research Council

19 Via dei Taurini, Roma, 00139 (Italy)

E: serena.fabrizio@ircres.cnr.it

\section{LUCIO MORETTINI}

IRCRES - Research Institute for Sustainable Economic Growth, National Research Council

19 Via dei Taurini, Roma, 00139 (Italy)

E: lucio.morettini@ircres.cnr.it

\section{AUTHORS}




\title{
ASSESSING THE IMPACT OF SSH- RRI APPROACH ON ICT RESEARCH \& INNOVATION: THE HUBIT PROJECT
}

\author{
TAL SOFFER, RUTH ZUZOVSKY, OLENA NEDOZHOGINA AND EMANUELE BARDONE \\ DOI: $10.22163 /$ fteval.2019.384
}

\section{ABSTRACT}

$\mathrm{T}$ The development of information and communication technologies (ICT) introduces radical changes in our lives. These technologies provide answers to a multitude of people needs, but at the same time they increase the concerns about their actual threats and societal impacts. This calls for adopting a responsible research and innovation perspective in the process of developing ICT solutions. This paper presents preliminary results of the "Social Impact Assessment" (SIA) plan and tools that were developed within the EU-funded HubIT project. The study employed both quantitative and qualitative ethnographic tools (e.g. survey questionnaire and observations), in order to address the challenge of conducting a "Responsible Research and Innovation" (RRI) assessment of a European project, focusing on promoting RRI. The project aims at creating an ecosystem that encourages interactions between ICT developers and "Social Sciences and Humanities" (SSH) researchers to ensure responsibility in ICT research. First results indicate an increase in understanding and awareness of the SSH-RRI approach among SSH and ICT researchers and an increase of future plans for collaborations between these two groups. Conclusions are made as to how these results can be fed back into the HublT project, as well as serve as a basis for the policy recommendations to European and national bodies.

\section{INTRODUCTION}

The development of ICT introduces radical changes in our lives. These technologies provide answers to a multitude of people needs, while at the same time increasing concern about their threats and societal impacts. This calls for adopting a "Responsible Research and Innovation" (RRI) perspective in the process of developing ICT solutions. The core of this approach is creating a mutual dialog between SSH researchers and ICT researchers and developers. Indeed, in the year 2012 the European Commission adopted the SSH-RRI approach and defined it as a continuous engagement of societal actors during the whole research and innovation process in order to better align both the process and the outcomes of their research with the values, needs and expectations of "European Society" (European Commission, 2012). Further on, RRI was introduced as a cross-cutting political aim in the "7th Framework Programme of the European Union" and it continues to be a key concept in the current "Horizon 2020 Programme".
The HubIT project, funded under the topic "Boosting inclusiveness of ICT-enabled research and innovation" (REV-INEQUL-09-2017) is part of the overall SSH-RRI approach. It aims to bring together ICT developers, SSH researchers and other stakeholders (NGOs, citizens and users) across H2020 ICT-related projects and beyond, in order to attune ICT development with societal needs and foster the SSH-RRI approach.

\section{THE CONCEPT OF "RESPONSIBLE RESEARCH AND INNOVATION" (RRI)}

One of the more widely accepted definitions of RRI that emphasises the role of SSH researchers, was developed by Von Schomberg (2013). According to this definition "Responsible Research and Innovation is a transparent, interactive process by which societal actors and innovators become mutually responsive to each other with a view to the (ethical) acceptability, sustainability and societal desirability of the innovation process and its marketable products." (Von Schomberg, 2013, p.19).

Further elaboration of these ideas by the appointed European Commission (EC) expert group described six major dimensions of RRI that signify the importance of keeping to the norms of responsible research and innovation that considers different societal needs. Among them are: public engagement, gender equality, science education, open access, ethics, governance. Two additional dimensions, sustainability and social justice, overlap with the previously named ones (Strand et al., 2015). All these dimensions require the involvement of SSH experts in the process of ICT development.

Embedding SSH researchers into ICT research and innovation is a challenge. The integration of the SSH-RRI perspective into ICT research and development is accompanied by specific problems. Jirotka (2017) identified the following: First, the difficulty to predict potential uses of ICT research outcomes since uncertainties in this field are socially shaped and fixed rather than scientific and not fixed. A second difficulty stems from the difference in the quicker "rhythm" of ICT development compared to other fields, as software may be developed and potentially go viral in the same day. Third, there is a problem stemming from different disciplinary languages involve in ICT research, that makes interdisciplinary work more difficult. 
These difficulties created the need to consider social aspects in the process of ICT development and led, among other things, to initiate the HublT project. The HublT project (runtime: 2017-2020) aims at activating a constructive interaction between SSH researchers and ICT developers, in order to implement a socially responsible approach to research and innovation in ICT projects. This approach - termed the SSH-RRI approach - is at the centre of the assessment activities of the HublT project.

\section{ASSESSING THE SSH- RRI APPROACH}

For assessing the SSH-RRI approach in the HublT project, the "Social Impact Assessment" (SIA) methodology was adopted. This methodology is defined as "the process of identifying the future consequences of current or proposed actions, which are related to individuals, organizations and social macro-systems". (Becker, 2001, p. 312). Becker describes this methodology as having two phases: a) An initial phase, including an analysis of the problem. In the case of the HublT project, identifying some negative consequences of ICT development, system analysis and project design; and b) A main phase, including scenario planning, strategic design and an assessment of impacts. Vanclay et al. (2015) followed this scheme and prepared a guide to social impact assessment. The guide included 26 tasks that are divided into four phases: 1 . Understand the issue; 2. Predicting the likely impact; 3 . Developing and implementing strategies to mitigate negative societal consequences; 4. Design and implementing monitoring programmes. Since many of the tasks specified by Vanclay et al. (2015) can be found within the HublT project activities, the assessment plan focused on these tasks. These activities have specific formats (e.g. workshops, conferences, hackathons etc.), target different audiences and lead to different outputs (e.g. an online platform, visual materials, reports or policy briefs). The variability of the activities dictates different tools and evaluation criteria needed for the assessment.

\section{THE DESIGN OF THE ASSESSMENT PLAN INCLUDES THREE STAGES:}

The first stage was to map out the characteristics of each activity i.e. specifying the main objectives, expected outcomes and relevance of the RRI dimensions which are part of each activity.

The second stage focused on the identification of the relevant types of indicators, measures and questions that tackle each of the six RRI dimensions. This stage started with a comprehensive review of the RRIrelated evaluation efforts conducted by other projects, such as "Doing It Together-Science" (DITOs), "Monitoring the evolution and benefits of Responsible Research and Innovation in Europe" (MoRRI), RRI Tools, etc., as well as with the review of the more theoretical studies (Blonder, Rap, Zemler and Rosenfeld, 2017; Von Schomberg, 2011) and several reports from the European Commission $(2012,2013,2015)$ on RRI. Consequently, a bank of assessment measures and questions was created.

The third stage involved a round table discussion (called the "HublT game") where the partners, responsible for certain tasks, were asked to discuss and select from the bank of assessment measures and questions with respect to those that cover the relevant RRI dimensions that appear in those specific tasks. Based on the results from the discussions held in the groups, specific tools were designed for assessing the implementation of the HublT events.

In addition, the SIA methodology included a qualitative evaluation part that focused on the narratives that accompany the interaction between SSH-ICT researches during the activities. The need for a more qualitative approach arose already at the literature review stage. It became evident that a certain dissonance between the current state of the art in the field of RRI impact assessment and the actual evaluation practices exist. Evaluation practices, promoted by the funding bodies, national and supranational authorities, provide encouragement to be accountable (tick the boxes), but are not always responsible (reflexive, oriented towards strategic societal goals) in the meaning of being accepted in the RRI research community. Current forms of evaluations mainly do not look at the process, and the evaluation is conceptualised as something "outside" of the project, while in reality it is usually deeply embedded in the project practice and is conducted by project partners. This can be connected to the recent findings of Felt (2016), who warned about the danger of the emphasis on RRI and other SSH-related practices in science and innovation turning into a simple "annex ritual to be perform at the beginning and at the end of the project" (Felt 2016:15), encouraging accountability, but not reflectivity.

In this way, by employing process oriented ethnographic methods, the evaluation efforts became also partially shaped by the community, surrounding the project, and partially driven by a desire to comprehend and improve transdisciplinary and responsibility of the project. In this sense, a community was formed around the evaluation activities, actively engaging partners and stakeholders in the process of assessment.

\section{THE ASSESSMENT ACTIVITY}

Assessment activities that were enacted in the first two projectspecific events are in the focus of the following section. These events were meant to bring together members of the ICT and SSH communities, public sector representatives, policy makers and other stakeholders. The events intended to present the concept of RRI, the HublT project and the "European Framework Model" (a platform that was developed and presents the various resources and activities of the project). The events also aimed at identifying societal needs that are associated with technological developments and supported matchmaking between ICT developers and SSH researchers.

The assessment activities were conducted during a national workshop in Slovakia in May 2018 and a triple event (annual conference, workshop for social scientists and speed-dating) in Tartu in September 2018. The aims of the national workshop, as well as the Tartu events, were to raise awareness and understanding of the role of the SSH-RRI approach and to boost collaboration between SSH and ICT research communities. The workshop event in Slovakia included 27 participants. 20 out of them responded to an online questionnaire that dealt with the above explained aims. In total, 64 persons participated in the events organised in Tartu (Annual Conference, SSH workshop, networking session). Again, 20 participants responded to the questionnaire.

The evaluation activities meant to answer the following questions:

1. To what extent did the event succeed in targeting members of the ICT and SSH communities? 
2. To what extent did the event contribute to mutual understanding of the ICT and SSH communities in the benefits of bridging between them?

3. To what extent did the event contribute to identifying societal problems that stem from ICT development?

4. To what extent did the event contribute to collaborative teamwork of ICT and SSH researchers?

5. To what extent did the event contribute to the acceptance of the RRI approach along its six dimensions?

The assessment tools that were generated at this stage of the project represented two modes of assessment: a quantitative tool, which includes an online questionnaire with 20 items, and a qualitative tool, which includes an observation guide for outside observers. The observation activities focused not only on the overall organisation and implementation of the event, but also on the dynamics of interaction between SSH and ICT communities, as well as on the narratives, surrounding RRI. Observations also included ethnographic notes taken by the project partners during the events, based on participants' discussions (as each event devoted a significant amount of time to world café style discussions). The main aim of the qualitative evaluation activities was to collect and analyse the narratives, surrounding the concepts of RRI, research inclusiveness and, especially SSH-ICT interaction. These narratives allowed identifying possible weak points of the project structure and unforeseen challenges that the project needs to address, as well as recent developments in the discourse of RRI.

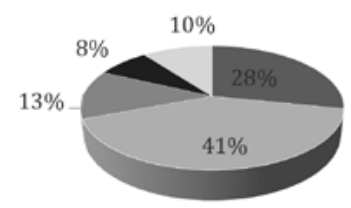

- Information and communications technology

- Social sciences

- Humanities

- Public administration / decision making

Figure 1. Distribution of participants in the events by discipline.

\section{RESULTS}

\section{SURVEY RESULTS}

Figure 1 presents the distribution of the respondents who participated in the two events according to their discipline or field of activity ( $\mathrm{N}=39$ ).

Most of the participants represented social sciences (41\%) and humanities (13\%), mainly because these two events specifically focused on this target group. However, the amount of involved ICT researchers and specialists is still high (28\%). The number of public officials and decision makers is relatively small, and will increase in future events.

\section{THE BENEFIT OF BRIDGING BETWEEN THE TWO COM- MUNITIES IN SUPPORT OF AN RRI APPROACH IN ICT DEVELOPMENT.}

Figure 2 presents respondents' perceptions regarding the interaction between SSH and ICT in support of RRI approach. The highest level of support is related to the statement about the usefulness of SSH collaboration in ICT development (Range: Likert scale from 0 to 5; Median (M) $=4.3$, Standard Deviation $(S D)=0.66)$, while the lowest level of support is connected to the perception that SSH is a burden to ICT research $(M$ $=1.70, S D=0.983$ ). Despite the fact that the national workshop and the events in Estonia had somewhat differing target audiences and distribution of participants by discipline (national workshop was focused on a more diverse audience, while the Tartu events focused specifically on SSH researchers), results do not show major discrepancies between attitudes and perception of participants.

\section{AWARENESS OF THE CONTRIBUTION OF SSH IN- VOLVEMENT IN ICT DEVELOPMENT TO THE IDENTIFI- CATION OF SOCIETAL PROBLEMS}

Figure 3 presents the respondents' awareness of the contribution of SSH-ICT collaboration to the identification of societal needs and problems, as well as the production of solutions to these problems. The respondents found that participation in the workshop helped them on a medium to high level in terms of three aspects: learning about societal needs, identifying societal problems that can be solved by cooperation between ICT and SSH communities and finding partners for future collaborations.

\section{PERCEIVED OPTIONS AND WILLINGNESS FOR ICT - SSH COLLABORATION}

Based on the two events, most of the participants (80\%-83\%) foresee future engagement in cooperation with people from the other fields (ICT or SSH), and most of them (77\%) found that the workshop event was very useful ( $M=4.03)$. Additionally, based on the speed-dating event evaluation, $77 \%$ of participants foresee engagement with ICT researchers, $33.3 \%$ have already contacted a person they matched during networking and $55 \%$ plan to do so. 


\section{To what extent do you agree or disagree with the statements regarding the interaction between $\mathrm{SSH}$ and ICT communities in support of RRI?}

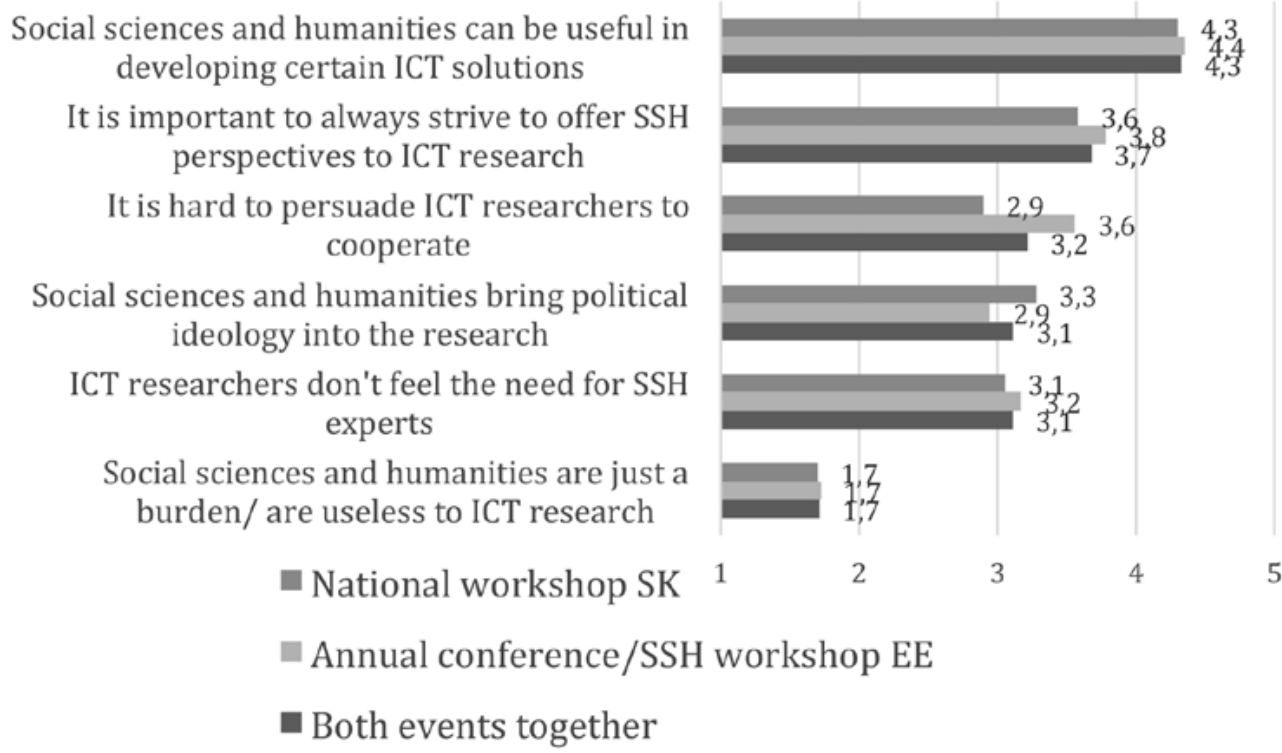

Figure 2. The interaction between SSH and ICT in support of the RRI approach during events in Slovakia (SK) and Estonia (EE), on a scale from 0 to 5.

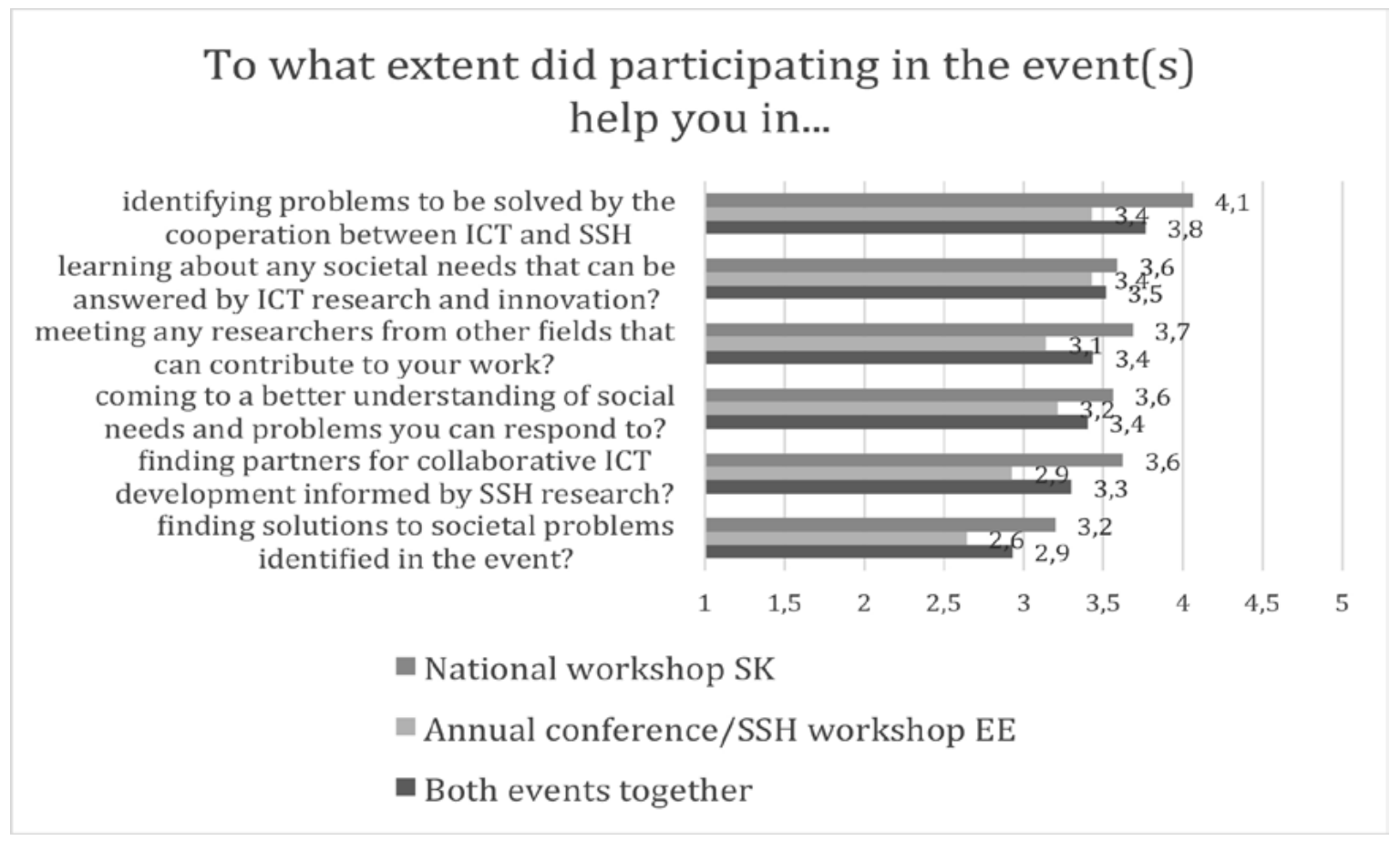

Figure 3. Contribution of SSH involvement in ICT development. 


\section{To what extent do you agree with the following statements regarding RRI dimensions?}

Scientists should spend part of their research budget to present their research online, in a free and open

All researchers should receive mandatory training on scientific research ethics (e.g. on privacy, animal

Research organizations should have an official ethics committee or an equivalent.

Official policies promoting open access data and publications encourage research and innovation in

RRI components should appear in statutory documents of research organizations.

Responsible research and innovation should be enforced from within the organization

It is important to have national/European level policies of responsible research and innovation Public engagement elements/possibilities should be a specific criterion when considering new projects or Gender equality should be a specific criterion when considering new events, projects or collaborations. Research organizations should dedicate resources from its budget specifically for public engagement.

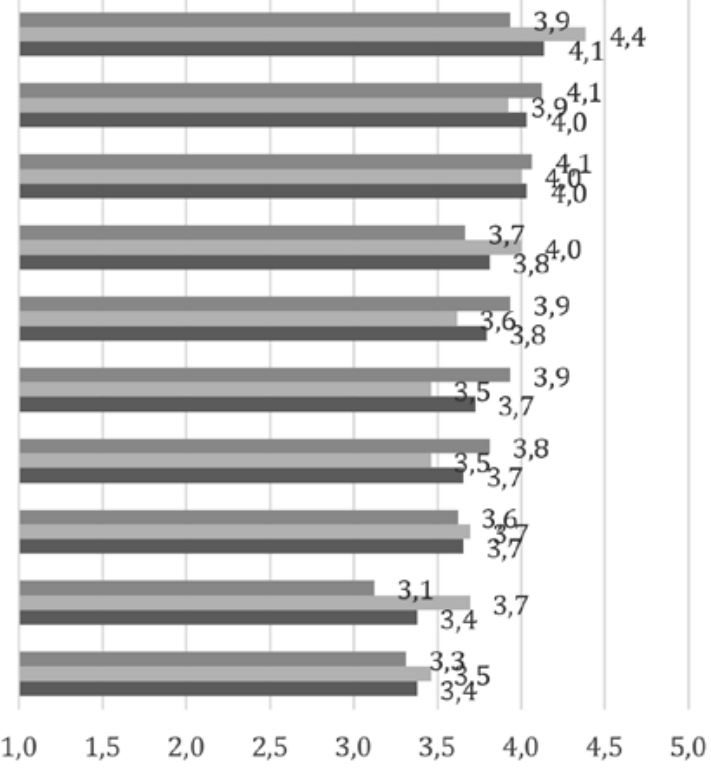

- National workshop SK

- Annual conference/SSH workshop EE

Both events together

Figure 4. Perception regarding the six dimensions of RRI.

\section{UNDERSTANDING AND ACCEPTING THE CONCEPT OF RRI}

Concerning understanding of the concept of the SSH-RRI approach, most of the respondents (62\%) indicated that they improved their understanding of this approach to a high or very high extent. Significant differences were found between the two events: for the national workshop $M=4.17, S D=0.85$ and for the annual conference/SSH event $M=$ $3.00, S D=1.6$.

The participants' agreement with statements reflecting attitudes towards the RRI various dimensions ranged from a medium to a high level (see figure 4 below). Specifically, those related to open science and ethics dimensions, which focused on the need for official ethics committees in organisations and mandatory training on research ethics.

As concerns the national workshop in Slovakia, the respondents indicated that the six RRI dimensions were addressed exceptionally well during the workshop, especially the dimensions of: public engagement $(M=4.00, S D=0.78)$, gender equality $(M=4.33, S D=0.9)$, open access $(M=4.00, S D=1.1)$, and governance $(M=4.06, S D=0.97)$. The annual conference event in Tartu had more moderate scores $M=3.31, S D=1.6$ to $M=3.77, S D=1.16$ ) for the different dimensions.

To sum up, the results from the two events indicate the success of this type of event in raising understanding and awareness of an SSH-RRI approach and initiating future collaboration between actors, representing SSH and ICT.

Based on the qualitative part of the assessment the following narratives were identified:

\section{QUANTIFICATION OF "RESPONSIBILITY” AND THE TICK-BOXING LOGIC}

"I feel that RRI is a fancy term that European Commission has come up with that is more often than not used as an empty signifier (i.e. it is just put into documents without following the principle)" (Participant, expert workshop in Rome)

One of the first events organised by the HublT project - the expert workshop in Rome - brought forward concerns that would accompany project discussions from this point forward. The issue of quantification of responsibility - that RRI can be reduced to a simple list of quantifiable key performance indicators - was discussed at length. Later, this narrative was echoed during the SSH workshop in Tartu: fear that RRI is just something that needs to "checked", but not followed in spirit, was brought forward from the comment section of the event report to the questionnaire (part of event evaluation).

However, we interpreted this narrative as an opportunity to improve our own HublT practices, which prompted efforts to add a qualitative/ 
ethnographic component to the HublT evaluation activities - to continuously engage with the qualitative data, to collect as much observations as possible and to be flexible in the implementation of project activities. Additionally, it was decided that each activity within the HubIT project will undergo an ethnographic qualitative process, especially the planned events, which constitute the core of the HublT project. Thus, evaluation efforts shifted the focus to narratives, open-ended questions in surveys, discussion note-taking, "ethnographic" analysis of event artefacts such as posters and observations notes.

\section{"SSH BRINGS IDEOLOGY AND POLITICS INTO SCIENCE."}

An unexplored topic emerged from the participants:

"I became aware that PC [political correctness culture] would creep into ICT research" (Participant, expert workshop in Rome)

"I am aware of the EC research ideology..." (Participant, expert workshop in Rome)

We provide an interpretation of the examples above in two ways: on the one hand, ethics sometimes is perceived as a complicating factor for research (often its bureaucratic and forceful nature is cited). On the other hand, it can be speculated that no representative of the modern research community would argue against the following ethical guidelines and the spirit of responsibility at their universities. RRI though, as a relatively new term coming from the European Union, a supranational structure, does not carry the same degree of legitimacy, which would explain the conceptual linkage that respondents made between RRI and the ideology of political correctness (avoidance of expressions that might negatively impact marginalised groups), currently associated with the political left. Additionally, the processes of globalisation of information flows and mediatisation (dominant role of (digital) media in framing the discourse) have definitely contributed to the polarisation of societies and rise of populism worldwide. RRI ideally should not be seen as a right/left issue, but as an objective need to consider societal considerations in formulating and implementing research ideas. However this suggests that we might be faced with a reality of politicisation (attribution of political agenda) of the term.

\section{GENDER EQUALITY}

Moreover, some additional insights came from event observations (which complemented open-ended questions of the surveys):

"ICT representatives were mainly men while the SSH area was represented mainly by women. This provides the feeling that SSH is something that women fight for. For instance, in the conference panel men (ICT) formulated their messages softer, while the woman speaker (SSH) was more a "right-fighter"." (Observation, Slovakia national workshop)

Observations of both events proved that often some aspects of event implementation go unnoticed by organisers. Gender equality is the dimension of RRI that, in the experience of the HubIT project, is most visible and causes the most debate. External observers feedback pointed out the imbalance in the presenters (male over female), while also emphasising that female participants make up an active part of the audience, often bringing up the value of the diverse perspectives in ICT product development and the importance of considering gender aspects in some research problems.

\section{THREATS VERSUS OPPORTUNITIES}

The following quotation highlights the tension between SSH and ICT researchers:

"ICT representatives talked rarely to the SSH people lexperience based on one table)...ICT people seemed to be more involved (engaged) in the threats discussion, while SSH more in the opportunities." IObservation, national workshop in Slovakia)

While there is a general presupposition that SSH researchers emphasise responsibility, risks and threats when discussing innovation, and ICT researchers look more into opportunities, this particular example showed an opposite picture. It might simply reflect the current state of the general discourse on innovation and global development: while the backlash against "irresponsible" ICT innovation has made more ICT researchers aware of the risks and pitfalls they might face, the strengthening narrative of "SSH inclusion" has encouraged social scientists to approach the issue of ICT/SSH cooperation more proactively.

\section{CONCLUDING REMARKS}

The assessment activities that were carried out in the first quarter of the project's life-time were mostly focused on the Vanclay et al. (2015) first and second Social impact Assessment (SIA) evaluation phases (e.g. learning and understanding the project). Even in these relatively early stages of the HublT project some specific characteristics of the HublT assessment activities emerged. First, due to a predefined responsible assessment strategy decided by all partners, the assessment activities were found to be deeply embedded in the project. Full engagement of all partners was therefore achieved. Second, the assessment activities are an ongoing process which will evolve in accordance with the progress of the project. This allows for continuous adjustment of the project activities. Third, in the course of the evaluation activities, the importance of interactions with the transdisciplinary community of experts from SSH and ICT became evident. This suits the project's goal to form a community around the evaluation activities, actively engaging partners and stakeholders from different fields in the process of assessment.

General event dynamics hinted that in discussing RRI two main associations appear: societal good (e.g. challenges of privacy, Artificial Intelligence, robotics, etc.) and inclusion (especially gender topics). The analysed narratives suggest that some RRI dimensions might carry more "pressure" than others, and that RRI in itself, should not be treated as a neutral concept. Conversely, it can be presupposed that there is a power struggle involved - even in the light of the increasing pressure to ensure responsibility of research and innovation. Especially in the field of ICT, the discourse of RRI is sometimes interpreted as a discourse of dominance, exerted by the social sciences over other disciplines. An important take-away is to ensure that the "responsibility" and ethics are not seen as special dimensions, monopolised by the social sciences. Rather, the discourse of RRI should be a space for reflection, where multiple ideas and perspectives are welcome.

During the evaluation process a need was identified to complement the survey type of assessment with a more ethnographic type of assessment through observations. This was done through introducing and emphasising open-ended questions in surveys, discussion note-taking during events and ethnographic observations. Based on the narratives extracted it was concluded that the inclusion of SSH perspectives into 
ICT research, as well as encouraging ICT-SSH cooperation has gained momentum at the backdrop of societal calls for more responsibility and reflexivity in handling ICT innovation - challenges of data security, algorithms, and information flows are on everyone's mind. However, the main hurdles to transdisciplinary cooperation have to do with the following: social sciences and "responsibility in research" seem to be tightly linked, to the point where there is a risk that the value of engaging SSH perspective in ICT is not seen beyond the areas of RRI and ethics. Moreover, there is a risk that forcing "responsibility" into some disciplines might only lead to further quantification of RRI and an escape from the need to reflect. Further interactions with the ICT/SSH community in the context of HublT endeavours to build transdisciplinarity are expected to outline directions of future work. There is a need to develop and communicate new evaluation practices, and this presupposes a new view of RRI and the role of social sciences, as well as the way they are presented and promoted by national and European bodies.

The HublT evaluation activities are still in progress. Different evaluation activities will take place and more insight will be available in the future. Further interactions with the SSH and ICT community are expected to contribute towards the directions of future work.

\section{REFERENCES}

Becker, H.A. (2001). Social Impact Assessment European Journal of Operational Research, 128(2), 311-321.

Blonder, R., Rap. S., Zemler, E. and Rosenfeld, S. (2017). Assessing attitudes about responsible research and innovation (RRI): the development and use of a questionnaire. SISYPHUS Journal of Education, 5(3) (pp 122-156).

DITOs Consortium (2016b). Doing It Together science: Phase 2 project evaluation. UCL, London

Felt, U. (2016). "Response-able Practices" or "New Bureaucracies of Virtue": The Challenges of Making RRI Work in Academic Environments. 10.1007/978-3-319-64834-7_4.

European Commission (2010). Eurobarometer 340 annexes. http:// ec.europa.eu/commfrontoffice/publicopinion/archives/ebs/ebs_340_ anx_en.pdf Accessed 14.11.2017.

European Commission (2012). Responsible Research and Innovation: Europe's ability to respond to societal challenges, DG Research and Innovation.

Van den Hoven, J. and Jacob, K. (2013). Options for strengthening Responsible Research and Innovation - Report of the Expert Group on the State of Art in Europe on Responsible Research and Innovation, DG Research and Innovation, European Union.

European Commission (2015). Monitoring the Evolution and Benefits of Responsible Research and Innovation (MoRRI): Analytical Report on the Dimensions of Research and Innovation Governance. Sub-task 2.5, deliverable D2.4.2.
Jirotka, M., Grimpe, B., Stahl, B., Eden, G. and Hartswood, H. (2017). Responsible research an innovation in the Digital Age. Communication of the ACH Vol 60 N05 (pp 62-68).

Owen, R., Macnaghten, P. and Stilgoe, J. (2012). Responsible research and innovation: From science in society to science for society, with society. Science and public policy, 39(6), (pp 751-760).

Strand, R., Spaapen, J., Bauer, M. W., Hogan, E., Revuelta, G., Stagl, S. and Guimaraes Pereira, A. (2015). Indicators for promoting and monitoring Responsible Research and Innovation: Report from the Expert Group on Policy Indicators for Responsible Research and Innovation. Luxemburg.

Vanclay, F., Esteves, A. M., Aucamp, I. and Franks, D. M. (2015). Social Impact Assessment: Guidance for assessing and managing the social impacts of projects.

Von Schomberg, R. (2011). Towards responsible research and innovation in the information and communication technologies and security technologies fields.

Von Schomberg, R. (2013). A vision of responsible research and innovation. Responsible innovation: Managing the responsible emergence of science and innovation in society, 51-74.

\section{AUTHORS}

\section{TAL SOFFER}

Unit for Technology and Society Foresight, School of Education Tel-Aviv University, Tel-Aviv, 69978 (Israel)

E: talsofer@tauex.tau.ac.il

\section{RUTH ZUZOVSKY}

Unit for Technology and Society Foresight, School of Education Tel-Aviv University, Tel-Aviv, 69978 (Israel)

E: ruthz@tauex.tau.ac.il

\section{OLENA NEDOZHOGINA}

Faculty of Social Sciences and Humanities, University of Tartu Ulikooli 14, Tartu, 51003 (Estonia)

E: olena.nedozhogina@ut.ee

\section{EMANUELE BARDONE}

Faculty of Social Sciences and Humanities, University of Tartu Ulikooli 14, Tartu, 51003 (Estonia)

E: emanuele.bardone@ut.ee

\section{KEYWORDS:}

SSH-RRI approach, Social Impact Assessment, ICT 


\title{
RETHINKING RESEARCH \\ IMPACT ASSESSMENT: A
}

MULTIDIMENSIONAL APPROACH

\author{
SERGIO MANRIQUE, MARTA NATALIA WRÓBLEWSKA AND BRADLEY GOOD
}

DOI: $10.22163 /$ fteval.2019.385

\section{ABSTRACT}

$\mathrm{A}$ interest in the evaluation of research impact - or the influence of scientific research beyond academia - has been observable worldwide. Several countries have introduced national research assessment systems which consider this new element of evaluation. So far, research on this practice has focused mainly on the practicalities of the different existing policies: the definition of the term 'research impact', different approaches to measuring it, their relative challenges and the possible use of such evaluations. But the introduction of a new element of evaluation gives rise not only to challenges of a practical nature, but also to important ethical consequences in terms of academic identity, reflexivity, power structures, distribution of labour in terms of workloads etc. In order to address these questions and the relevant needs of researchers in this paper, we propose a multidimensional model that considers different attributes of research impact: Responsiveness, Accessibility, Reflexivity, Ecology and Adaptability. This holistic, multidimensional model of evaluation, designed particularly for self-assessment or internal assessment, recognises the qualities a project has on these different scales in a broader perspective, rather than offering a simple and single numerical evaluation. This model addresses many of the ethical dilemmas that accompany conducting impact-producing research. To exemplify the usefulness of the proposed model, the authors provide real-life research project assessment examples conducted with the use of the Multidimensional Approach for Research Impact Assessment (MARIA Model).

\section{CONTENT}

1. Introduction: The challenges of research impact evaluation $\quad 160$

2. Literature Review

3. Context: Existing systems of research impact evaluation 162

3.1 United Kingdom 162

3.2. The Netherlands 162

3.3. Norway 163

3.4. European Union 163

4. "Multidimensional Approach for Research Impact Assessment" (MARIA model)

4.1. Dimensions of research impact 164

Responsiveness 164

Accessibility 164

Reflexivity 165

Ecology 165

Adaptability $\quad 166$

4.2. The model in practice 166

5. Self-assessment examples 167

6. Discussion 167

7. Conclusion 168

Acknowledgements 168

8. References 168

Ex-ante research impact self-assessment example (Good, 2018)

Mid-term research impact self-assessment e

xample (Manrique, 2018)

Ex-post research impact self-assessment example (Wróblewska, 2018) 


\section{INTRODUCTION}

\section{THE CHALLENGES OF RESEARCH IMPACT EVALUATION}

An interest in the evaluation of research impact - or the influence of scientific research beyond academia - has been observable worldwide (Grant, Brutscher, Kirk, Butler and Wooding, 2009; Wróblewska, 2017a, p.162). Several countries have introduced national research assessment systems which consider this new element, such as the UK, the Netherlands, Norway, Australia (Australian Research Council, 2018), Hong Kong (Hong Kong University Grants Committee, 2018) and Japan (NIAD-OE, 2018), among others. The element of 'impact' is also present in the evaluation of research projects in international contexts, such as certain EU programmes, and several other countries are currently debating the possibility of introducing an impact component into their research evaluation systems. The appearance of a new element of academic evaluation has inspired much scholarship which focuses on the practicalities of the policy itself. However, this introduction gives rise to practical challenges as well as ethical consequences. More qualitatively-oriented studies and reports have pointed to impact evaluation implications in terms of academic identity and ethos, emotion, academic values, and power structures. (Bacevic, 2017; Chubb, 2017). Presently, it seems that many researchers are ill-equipped for dealing with these new and complex issues, often resulting in feelings of frustration, confusion or resentment towards the assessment exercise or impact-related activities (Chubb, Watermeyer and Wakeling, 2016).

Existing systems of evaluation seem to suffer from several shortcomings. Firstly, they mostly take a top-down approach, which does not account for the nuances of academic knowledge production. Secondly, they do not always offer a space to reflect on the ethical side of impact generation, often leaving those assessed feeling alienated. Thirdly, they do not attend to the processual nature of impact evaluations, focusing just on the final effect of research in the form of 'change or benefit to the society'. Fourthly, they often tend towards a 'one size fits all' model aimed a final numerical assessment producing measurable, quantifiable scores which can later be operationalised and ranked, often for funding considerations. Fifthly, they are often time-consuming and cumbersome for the assessed academic.

We believe this quantitatively-oriented, 'numerocratic' perspective on research assessment can result in disregarding less measurable implications of research. To account for the reality of research in its breadth and depth, evaluation systems should recognise these qualitative features and their relative challenges. The lack of recognition of this complex nature of impact-lending science leads to an overly simplified vision of research and contributes to frustration with the exercise, which is seen as not adequately representing the reality of impactful scientific work. To address these questions and the relevant needs of researchers who conduct impactful work, as well as individuals who are in charge of research evaluation (policy-makers, academic managers), we propose a multidimensional model of research impact. A holistic model of assessment enables recognising the qualities a given project might have in different areas, rather than offering a simple numerical assessment. To address the above-mentioned issues, we propose a multidimensional approach, which 1) is created with self-assessment in mind, 2) should stimulate a reflection on the ethical aspects of achieving impact, 3) would ideally be conducted at different stages of the research project to account for the progress of work and the emergent challenges, 4) recognises the strengths of a research project in terms of impact and points to its weaknesses, rather than offering a single score, 5) is a light-touch assessment, which can be as short as one sheet of paper. Our model aims at widening the currently prevalent measurement-oriented and metrics-oriented perspective by promoting a critical and comprehensive assessment of research impact, both individually and institutionally. Through our contribution, we hope to advance the cause of building research impact literacy (Bayley and Phipps, 2017).

The model we put forward has been designed particularly with selfassessment or internal assessment in mind. We do not propose a model for assessment of research ethics, but rather a model for 'ethical assessment of research impact'. The criteria of assessment we propose are: Responsiveness, Accessibility, Reflexivity, Ecology and Adaptability, which we recognise as attributes of impactful research in all scientific disciplines in our "Multidimensional Approach for Research Impact Assessment" (MARIA Model).

\section{LITERATURE REVIEW}

The introduction of exercises of impact evaluation can be placed in a wider perspective of changes affecting academia, and thus the topic can be related to several bodies of literature, drawing from fields as different as philosophy and sociology of science, economics and management as well as the specialised fields of science and technology studies, higher education studies, valuation and evaluation studies, etc. Below, we briefly present the relevant main strands of research for the proposed model, signalling how our proposal compliments the existing literature and addresses gaps.

In a very broad context, the introduction of the impact assessment as part of national or international research evaluation systems can be perceived as part of a wider change affecting the position of universities and scholars in societies. Universities have always been embedded in their local contexts while at the same time guarding their autonomy - a situation of performing 'balancing acts' between 'pure' autonomy and 'impure' social relevance (Hamann and Gengnagel, 2014). Against this background, individual researchers and academic environments have taken various positions towards what is now called 'outreach and engagement'. We can recall the 'public intellectuals' of the post-war era (Baert, 2015), technocratic experts and entrepreneurs who put their knowledge at the service of market-oriented and governmental activities (Spiel and Strohmeier, 2012; Ritter, 2015), as well as researchers functioning in a critical capacity as activists and social engineers, questioning and subverting existing social and economic relations (Maxey, 1999; Pereira, 2016).

In recent decades, the relationship between academia and the surrounding environment has seen a transformation, partly in response to broad political and economic initiatives targeting universities' involvement with society, such as the rise of the so-called 'knowledge-based economy' (Jessop, Fairclough, and Wodak, 2008) which sees the universities as strategic 'knowledge-brokers' (Lightowler and Knight, 2013). Hence social, political, or economic engagement, previously perceived as an additional activity to the 'core business' of research, became incorporated into the definition of what it means to 'do' science. In consequence, there has been an observable increase in the symbolic importance of applied scientific disciplines and collaborations of scholars which their soci- 
al and economic environment (E3M, 2012; European Commission, 2003), often dubbed - particularly in a regional context - as the universities' 'Third Mission' (Brundenius and Göransson, 2011). Numerous initiatives aimed at linking universities with external partners have been launched, focusing on two areas: firstly, enhancing individual academics' autonomy and responsibility in conducting entrepreneurial activities (for an analysis of this process in the British context see: McGettigan, 2013) and secondly, valorising the growing role of universities as business undertakings as well as instruments in national policy agendas, crucially in contributing to the national economy (Gornitzka and Maassen, 2007). The emergent tendency of requiring tangible effects of research conducted within universities can become especially problematic in Social Sciences and Humanities (SSH), where measurable monetary effects beyond academia, such as patents and licenses, are uncommon research outputs. In the context of a growing tension between SSH and Science, Technology, Engineering and Mathematics (STEM) disciplines, often exacerbated by the demands of the performative, metrics-driven academy, our proposal offers a more nuanced, process-oriented evaluation model which would still preserve 'entrepreneurial' research impact, while recognising the specific contribution and public value of SSH disciplines (Benneworth, Gulbrandsen, and Hazelkorn, 2016).

With a growing focus on incentivising university engagement, outreach and impact came a demand to measure such factors, much in line with the managerial approach to governing higher education institutions - sometimes dubbed 'academic capitalism' - which has been on the rise in the last few decades (Münch, 2014; Slaughter and Leslie, 1997; Slaughter and Rhoades, 2004). Numerical indicators - both 'traditional' bibliometrics and metric-based rankings (Hood and Wilson, 2001), as well as newer forms of scientometrics or alt-metrics (Priem et al., 2012; Galligan and Dyas-Correia, 2013) - have been eagerly implemented by the administration of many universities, grant distributors and governments, prompting the metaphor of a growing 'metric tide' (Wilsdon et al., 2015) and academic 'numerocracy' (Angermuller, 2013). At the same time an unproblematic reliance on metrics and rankings continues to be widely contested by researchers in the field of higher education and evaluation (Szadkowski, 2015) and academic communities worldwide (see for instance the DORA declaration: American Society for Cell Biology, 2012).

When reflecting on the emergence of 'research impact' as a new academic value, one can draw important lessons from evaluation and valuation studies. Scholars in this area have argued that new practices of valuation (for instance new sports or culinary practices) are likely to give rise to 'heterarchies' or 'plurarchies' of values, a state where several values can persist and be appreciated at the same time, rather than the often reductionist 'hierarchies', characterised by one scale (Lamont, 2012, p. 212). Given that impact evaluation is a new area of valuation, and that research impact constitutes a complex activity which can be assessed from varied perspectives (the economic, the developmental, the ethical, and the axiological, among others), we put forward our multidimensional model as an attempt to promote an open, multi-levelled approach to research impact recognition.

There have been valuable contributions in the literature towards a better understanding and assessment of research impact. Firstly, the context-based perspective of research assessment (Spaapen et al., 2007) portrays a more comprehensive method for assessing the quality and relevance of scientific research, based on the relationship (mutual transactions) between researchers and their relevant environment, fo- cusing on how "researchers manage to connect to themes in that environment, and on the ways in which this environment absorbs ('uses') and further develops the results of the research" (p. 89). Secondly, the 'productive interactions' concept (Spaapen and van Drooge, 2011) arises as an alternative for overcoming the difficulties of measuring and evaluating the social impact of research, focusing on the personal, indirect (through texts or artefacts) and financial (through money or 'in kind' contributions) interactions between researchers and other actors as a transparent proxy of the process from research to impact. Such a concept has been further developed, bringing more attention to the governance, evaluation and monitoring of "Transdisciplinary Collaborations" (TDCs) addressing societal challenges, as a fruitful - bottom-up or stakeholder oriented - approach for valorising socially robust knowledge (van Drooge and Spaapen, 2017). Thirdly, and in line with the approaches previously mentioned, the need for a more holistic view in the observation and monitoring of interdisciplinary research (Anzai et al., 2012) has been addressed in Japan as an attempt towards research valorisation. Finally, a fairer treatment of SSH in research impact assessment (Benneworth et al., 2016) has been pointed out as a necessity in the discussion on the value, impact and benefit of publicly-funded research.

There are also representative cases of research movements or projects attempting to influence research policy in Europe, specifically in terms of research impact evaluation. Since 2006, all the major science policy organisations in the Netherlands joined the project "Evaluating Research in Context" (ERiC), aimed at addressing the debate and the methodological development of research evaluation in a wider perspective that includes European and international participation (Spaapen et al., 2007). ERiC project promotes a broader discussion and approach for conducting a comprehensive research evaluation in terms of societal quality and valorisation. This societal orientation of research has brought together the major organisation in Dutch science policy around the need for methodological progress and (inter)national attention on this issue. With a stakeholders' approach, the evaluation method considers the construction of a "Research Embedment and Performance Profile" (REPP) that provides a wider societal reference group for a scientific project (embedment) and the degree in which this project serves the interests of a wider reference group (performance), considering a contextbased research impact. In analysing the possibilities of impact evaluation, it is important to reflect on the role the proposed evaluation system will have in this wider panorama of rather tense and polarised attitudes and on how the results of evaluation may be used for managerial aims.

With the proposed approach (MARIA model) we do not seek to create yet another tool aimed at fine-tuning academics' performance through top-down, number-driven assessments. On the contrary, in line with a growing request for responsible evaluation (Hicks et al., 2015), we wish to offer an alternative by arguing for a researcher-centred, multi-dimensional model of self-evaluation, which could not only offer a 'profile' of an assessed research project, but might also serve as an iterative tool for fostering ethical reflection in the new and often challenging field of generating 'research impact' (Chubb and Watermeyer, 2017). While the use of such a model might be rather limited in the framework of large performance-based research funding systems (Hicks, 2012), we argue that it could be valuable as an additional way of reflecting on the research of individuals, research teams, departments etc., in an iterative, qualitative way, in effect advancing the case for responsible, reflexive research impact. 


\section{CONTEXT: EXISTING SYSTEMS OF RESEARCH IMPACT EVALUATION}

Creating an approach to research impact evaluation is a challenge, given that the assessment of academic work has long rested on factors internal to academia: above all the quality (or quantity) of research outputs but also the quality of graduate teaching, research environment, grant funding, international mobility of scholars etc. There certainly seems to be a tension between more qualitative and quantitative approaches to impact evaluation (Donovan, 2017) and depending on the strategic goals handed down to education by the government, academic traditions, prevailing political options, and often several contingent factors (Wróblewska, 2018) impact evaluation strategies vary greatly from country to country.

Below we present the most important points of reference for research impact evaluation. While research agencies in several countries have introduced elements of impact evaluation, particularly in the areas of technology, engineering and medicine (Buxton and Hanney, 1996; Canadian Academy of Health Sciences, 2009), we focus here particularly on the examples of the UK, Netherlands and Norway as the systems which take a most comprehensive approach in assessing impact across all the disciplines according to the same criteria. Apart from the approaches implemented by particular states or organisations there exist various frameworks put forward by scholars. In this context, the most influential and noteworthy, also for our own proposal, is that of 'productive interactions' (Spaapen and van Drooge, 2011) which advocates a process-oriented approach to impact valuation, in line with the approaches of context-based assessment (Spaapen et al., 2007) and TDC's (van Drooge and Spaapen, 2017) introduced in the previous section. Such approaches valorise the pathway from research to practice (impact), transcending the focus on research outputs themselves by considering the different sources and expressions of impact during the whole research process.

\subsection{UNITED KINGDOM}

UK's "Research Excellence Framework" (REF) with its "Impact Agenda" is perhaps the most well-known and influential system of impact evaluation (Khazragui and Hudson, 2015), and surely the first to implement impact evaluation on such a large scale and with a rigorous methodology. The REF was introduced in 2014 to replace the "Research Assessment Exercise" (RAE) which, since its introduction in the 1980s, had grown into a cumbersome, time-consuming exercise. The debate which proceeded the introduction of the REF neatly illustrates the tension between qualitative (peer-review-based) and quantitative (metrics-based) approaches, which we have pointed to above. The REF was initially conceived as a light-touch, metrics-lead exercise which would reduce the burden to assessed departments, while providing evidence as to the return on the government's investment in science. However, this concept was abandoned after the failure of the pilot of the metrics-based approach (HEFCE, 2009) and the "Impact Agenda" was put forward as a replacement for metrics (Sayer, 2015). In its final shape, the REF, run by the British research councils every 5-6 years (the first edition took place in 2014 and the following one was announced for 2021), includes 'impact' as one of the three assessed elements, alongside outputs and environment. Impact is defined as "an effect on, change or benefit to the economy, society, culture, public policy or services, health, the environment or quality of life, beyond academia", assessed on its 'reach' and 'significance' and accounts for 20\% (in 2014) or 25\% (in 2021) of the final result of the assessed unit (HEFCE, 2011, p. 26; HEFCE, 2016). Expert panels evaluate impact in a process of peer review based on 'impact case studies' submitted by university departments, but the results are only published in an aggregated manner, i.e. for entire submissions, not for individual case studies.

The REF has been instrumental in increasing awareness of research impact in the UK (Donovan, 2017) and beyond, indeed becoming the model for impact evaluation in other counties such as Sweden, Norway or Poland (Wróblewska, 2017b). Advantages of the system include being based on and accompanied by several thorough commissioned reports (King's College London and Digital Science, 2015; Manville et al., 2015; Manville et al., 2014), the use of a broad definition of impact, which is likely to be broadened still (Stern, 2016) and the accessibility of both impact case studies and (aggregated) results of the evaluation through online resources. Weaknesses of the REF approach to impact, in our view, include a focus on the 'effects' of impact-related activities, rather than on the processual aspect and intermediate consequences thereof - as advocated by the productive interactions approach (Spaapen and van Drooge, 2011). Furthermore, the impact case study template did not encourage a reflection on the ethical aspect of impact generation, while the performance-oriented character of the evaluation, as well the onus placed on the results lead academics to present often unrealistic, idealised and exaggerated accounts of impact (Derrick, 2018; Wróblewska, 2018). These are all shortcomings which we wish to address with our multi-dimensional model.

\subsection{THE NETHERLANDS}

The "Standard Evaluation Protocol" (SEP) - a system of research evaluation adopted by the Association of Universities in the Netherlands (VSNU), the Netherlands Organisation of Scientific Research" (NWO) and the Royal Academy of Arts and Sciences (KNAW) in 2015 - incorporates 'relevance to society' as one of the evaluation criteria (alongside research quality and viability. 'Relevance to society' is defined as "contributions to economic, social and cultural groups and to public debate" (VSNU, NWO and KNAW, 2016, p. 7). Research conducted in Dutch higher education institutions is evaluated by external assessment committees for each unit or institute once every six years on a rolling schedule. This assessment concerns the research that the unit has conducted in the evaluated period as well as the strategy the unit will pursue in the next period. Each research unit conducts a self-assessment and provides additional documents (including a report of indicators and strengths, weaknesses, opportunities and threats analysis (SWOT) and benchmarking analyses), which are considered, together with interviews by the unit's representatives, the external committee, basing its judgment on international trends and developments in science and society. The exercise concludes with a report in which the external committee offers an assessment both in text (qualitative) and in four possible quantitative categories (excellent, very good, good and unsatisfactory), accompanied by recommendations for the future. PhD programmes, research integrity 
and diversity are also considered in the assessment. The assessment report, together with a response position document by the university, is published in the end.

ERiC project, referred previously, has targeted some of the possible flaws of the Dutch SEP, which, similar to the REF, ignores to some extent the processual nature and intermediate achievements of research activity. A 'one size fits all' model groups diverse research to be assessed within the same basket - or research unit - and by the same committee can ignore the variety of interactions among researchers, their environment and other stakeholders, which are valuable sources of impact. Additionally, the scale 'unsatisfactory-good-very good- excellent' may neglect a number of moderate - but still relevant - impact studies.

\subsection{NORWAY}

The Research Council of Norway has introduced an element of assessment very closely modelled on the British REF in its cyclical evaluation of scientific disciplines. The first disciplines to be evaluated in terms of impact were the Humanities in 2015-2017 (Research Council of Norway, 2017, pp. 36-37), followed by the Social Sciences in 2017-2018 (Research Council of Norway, 2018). The Norwegian evaluation adopted the definition of impact, the peer-review approach and indeed the impact case study template from the REF, hence it might inherit some of the REF weaknesses portrayed in section 3.1. The Norwegian approach differs from the British model in that it is not tied to distribution of funding and, at least in the case of the exercises carried out to date, the exact scores attributed to impact cases were not made public, even in an aggregated manner. Instead descriptive feedback was given on the overall 'impact culture' of a submitting faculty, in some cases referring to individual cases fields (e.g. for the Humanities see Research Council of Norway, 2017, p. 36-41). While this choice promotes a more light-touch approach to impact, without generating excessive anxiety about the exercise, it may be less conducive to improvement in the area of impact creation. Furthermore, the subject-specific evaluations carried out by the Research Council of Norway can either tangle or neglect the assessment of transdisciplinary research, affecting the valorisation of 'productive interactions' and transdisciplinary collaborations, relevant aspects of research impact introduced in section 2 (but note that submissions could point out an additional, secondary panel for references).

\subsection{EUROPEAN UNION}

Horizon 2020 (H2020), the EU's research and innovation framework programme, include ex ante and ex post assessments of research and innovation projects, where impact on regions is a relevant criterion. Applications for funding in the EU's research and innovation programme (H2020 until 2020, and Horizon Europe in the next budgetary period) sets some expected impacts at individual, institutional and systemic levels. Marie Skłodowska-Curie actions, for instance, assess impact, together with excellence and implementation, as criteria for awarding funding. Impact assessment, with a weight of 30\% (2017 call), considers the impact on researchers' future career as well as the strengthening of human resources regionally, nationally and internationally. It also considers and promotes transdisciplinary collaborations between academic and regional partners, as well as the communication and dissemination of research in society. Additionally, and beyond H2020, the EU supports projects related to research and innovation with societal impacts through "Cohesion Policy" (CP) and its "Research and Innovation Strategies for Smart Specialisation" (RIS3). CP is the core of EU's strategy for territorial development of regions, especially less favoured regions (European Commission, 2014). The impact criterion has entered the research assessment exercises conducted by the European Commission in order to fund and monitor research projects. There is a whole range of types of projects and funding calls tackling different aspects and themes in society, encouraging collaborations between academic and non-academic regional partners. The EU is covering different expressions of research impact through their variety of funded programmes, for which the assessment protocols vary too. However, there seems to be a wider focus on ex-ante assessments for allocating funds, and the tracking of research impact at the research projects implementation might not be receiving enough attention.

Other countries in which impact has been introduced somehow in the research assessment exercise include:

- Australia: "Engagement and impact assessment" (EI) in the framework of "Excellence in Research Australia" (ERA) (Australian Research Council, 2018).

- Canada: "Payback System" (Buxton and Hanney, 1996; "Canadian Academy of Health Sciences, 2009).

- Hong Kong: "Research Assessment Exercise 2020" (Hong Kong University Grants Committee, 2018)

- Sweden: Swedish Agency for Innovation Systems (VINNOVA) (Jacob, 2006; Lundequist and Waxell, 2010).

- Japan: National Institution for Academic Degrees and Quality Enhancement of Higher Education (NIAD-OE, 2018)

In addition to the above, also some research institutions have introduced their own approaches to research impact evaluation (for an overview of approaches taken by three European research institutes see: Gulbrandsen and Sivertsen, 2018, pp. 36-42).

Peer-reviewing seems to be the most common methodology for assessing the societal impacts of research, especially in ex ante assessments (Holbrook and Frodeman, 2011), which puts in evidence the importance of qualitative consideration in exercises of research impact assessment. Nevertheless, the different assessment systems described above ignore - to different extents - the multidimensional nature of research impact and do not pay sufficient attention to certain attributes of impactful research, which this paper takes charge of in the model described in the next section.

\section{4. “MULTIDIMENSIONAL APPROACH FOR RESEARCH IMPACT ASSESSMENT" (MARIA MODEL)}

Given the increasing pressure on considering research impact when assessing research activity, it is important to put forward systems which achieve this in broader and accurate way, going beyond (but without dismissing) the measurable effects of research. In alignment with 1) 
the context-based perspective of research assessment (Spaapen et al., 2007), 2) the transdisciplinary collaborations and 'productive interactions' concepts in research evaluation and monitoring (Spaapen and van Drooge, 2011; van Drooge and Spaapen, 2017), 3) the need for a more holistic view in the observation and monitoring of interdisciplinary research (Anzai et al., 2012), and 4) the need for a fairer treatment of SSH in research impact assessment (Benneworth et al., 2016), this paper is an effort for joining and contributing to the ongoing learning process in research impact agenda, by proposing a multidimensional and flexible approach towards this issue. The MARIA model is described in this section.

\subsection{DIMENSIONS OF RESEARCH IMPACT}

We propose a model which indicates six main dimensions of impactful research. These dimensions are attributes of research which may be considered in the assessment process of any research project at any stage: ex ante, mid-term and ex post. The order in which these dimensions are presented does not represent their relevance or weighting within the model. This model is specifically designed with self-assessment in mind. We believe carrying out such exercise would be useful for scholars wanting to reflect on the 'impact' aspect of their work in considering the advantages and possible drawbacks, as indeed it has been for us (see section 5).

\section{RESPONSIVENESS}

\section{"Authentic thinking, thinking that is concerned about reality, does not take place in ivory tower isolation, but only in communication." Paolo Freire (2000)}

Impactful research should be responsive to real problems and issues in society. The isolation of academia from society leads to research which is not rooted in real-world challenges. Hence, research should target societal needs and face these problems in dialogue with affected stakeholders. Following Owen's et al. (2012) idea of policy responsiveness, impactful research should aim at: 1) anticipation, foreseeing topics and issues worth studying for their importance in society's future, 2) reflection, considering the real problem instead of what audiences want to hear or read about, and 3) deliberation, planning conscientious actions to respond to real needs through research. All three of these issues can be summarised in the concept of dialogue and external mediation, which have a critical role to play, especially in an academic environment, where internal thought processes are often prioritised over external responsiveness. Paolo Freire, in Pedagogy of the Oppressed, takes this one step further in discussing the importance of dialogic education as a way to create meaningful, equitable, and transformative educational experiences (Freire, 2000); we extend this paradigm to research practice, by positioning responsiveness as the main requirement for dialogic research.

Impactful responsive research should be realistically ambitious too, by aspiring to make clear, specific and valuable contributions to current public debates and/or to the resolution of needs in society and industry. Ambitious research tackles issues at different levels in terms of geography, disciplines or actors, among others. The pursuit of ambitious research can take place in different ways: by engaging with global or long-term issues, involving stakeholders more integrally, embracing interdisciplinary, implementing collaboration with actors outside academia (e.g. industry, citizens), and in general, performing actions to generate a greater impact. Research should be ambitious and open-minded while remaining realistic and testable. Responsiveness, as a dimension of impactful research, must contribute to achieving "Responsible Research and Innovation" (RRI). Therefore, responsive research should also be responsible "in the context of research and innovation as collective activities with uncertain and unpredictable consequences" (Owen et al., 2012). Ex ante, mid-term and ex post assessments of research responsiveness can revise how the researchers argue, consider or take care of current needs and/or real problems in society and how this is - planned to be - achieved.

Responsiveness example: The body of knowledge on environmental sustainability and clean energies responds to the global warming and pollution problem that threatens society and which has been on the increase during the last two decades (0strom, 2009); this growing research stream is responsive to a relevant issue in current society. The environmental problem that society faces has been studied by several researchers from different disciplines within natural sciences and engineering but also within social sciences and humanities, trying to contribute to the understanding and solution of global warming and pollution generation from different bodies of knowledge and with different perspectives.

\section{ACCESSIBILITY}

"Making research results more accessible to all societal actors
contributes to better and more efficient science, and to innovation
in the public and private sectors."

\section{European Commission (2018)}

Impactful research should be accessible to stakeholders and society in general, within the limits of feasibility. This includes its communication and dissemination both within and outside the academy, ideally allowing all stakeholders to access and engage in the research. Accessibility among the general public is also important but may be limited, depending on research scope. The dimension of accessibility assesses how the research is planned to involve or be communicated to academic and non-academic stakeholders and the general public (ex-ante assessment) and how it ends up involving or being effectively communicated to both groups (ex-post assessment).

One example of accessibility includes public academics. Using Michael Burawoy's definition, public academics are communicative in knowledge production, derive legitimacy from their relevance, are held accountable by the designated publics they interact with, and engage in public political dialogue (Burawoy, 2004). However, the challenge of being a public academic is to also ensure that research is reliable and consistent with all ethical standards. The recent case of Brian Wansink at Cornell University illustrates the damage that can be done when accessibility is valued too heavily. Wansink led the prestigious "Cornell Food and Brand Lab", which was known for its revolutionary and highly accessible studies on the intersection of food consumption and psychology. This research lab regularly grabbed newspaper headlines in the United States with easily reportable headlines, mostly focused on ways humans can be psychologically queued to eat more or less food. These findings were 
regularly reported in magazines, newspapers, on the "Food and Brand Lab's" website, and in Wansink's mass market paperback books. However in 2017, four early career researchers poured through Wansink's publications and created the Wansink Dossier: a list of over 50 publications with "minor to very serious issues" (Zee, 2017, website - no page number available) that eventually resulted in an investigative journalist report (Lee, 2018) and Wansink's eventual resignation from Cornell for data manipulation and tampering (Rosenberg and Wong, 2018). His case makes clear how an extreme drive for accessibility while neglecting ethical standards can significantly damage research aims. For this reason, our overall model is holistic and includes other elements of research impact.

Accessibility may also link to the Open Science movement, a "movement to make scientific research and data accessible to all" (UNESCO, website - no page number available). This movement has most recently been typified by The Amsterdam Call for Action on Open Science which calls for open access for scientific publications, data sharing and reuse, alignment of best practices and policies, and, most notably, "new assessment, reward, and evaluation systems" (Ministry of Education, Culture, and Science, The Netherlands, 2016, pg. 3). Accessibility refers to this type of focus, which does not just encourage openness in research communication/dissemination but proactively pursues it.

Accessibility example: "Why We Post - Social Media through the Eyes of the World" is a collaborative effort from nine anthropologists "researching the role of social media in people's everyday lives" (University College London, website - no page number available). The most extraordinary part of their research was how they communicated findings. The researchers created multiple free eBooks, made an entirely free MOOC (Massive Open Online Course) through the digital education platform FutureLearn, kept a blog throughout the course of the research, had social media presences on Facebook, Twitter, and YouTube, and created a thoroughly interactive website with simplified discoveries, stories, videos, and interactive maps. This is in addition to the book chapters and journal articles published. Furthermore, "Why We Post" also ensured that these materials were accessible in the languages of the countries where they conducted research, ensuring translation in English, Portuguese, Spanish, Italian, Turkish, Chinese, Tamil, and Hindi. "Why We Post" is an extreme but also important example of accessibility.

\section{REFLEXIVITY}

"Train PhD students to be thinkers not just specialists. . put the philosophy back into the doctorate of philosophy."

\section{Gundula Bosch (2018)}

Most of the people who conduct research in academia are PhD students or graduates. In this sense, it is important to remember that $\mathrm{PhD}$ stands for Doctor of Philosophy, and beyond being experts or specialists in a given field, researchers should be, by definition, thinkers and theorisers (Bosch, 2018). To this end, reflexivity is concerned with critical reflection. In this dimension, the researcher may ask: 'has the process of theorising and research design been comprehensive, well-planned, ethical, and critical?', 'have the research theories and conclusions been thoroughly broken down, evaluated, and critiqued?'. Impactful research should incorporate conscious and deep reasoning on the conducted research's objective, methodology and results, in order to understand how it contributes to certain body of scientific knowledge and to public debates. In this sense, the building of theory and analysis of research results is especially relevant for understanding the gap between intention and what has really been achieved (implications) in the conducted research.

While analysis and reflection are important, there is also a need to reflect critically. Brookfield (2000) points out that critical reflection involves a power analysis of the situation or context. This type of reflexivity is necessary from an ethical and even ecological perspective, to ensure that the research itself is not contributing to inequality. While critical reflection is important, it is also necessary to then act upon that reflection, not treating it simply as an academic exercise but one which encourages true change in the research design and otherwise. Critical reflection without social action can be seen as a "self-indulgent form of speculation that makes no real differences" (Cranton, 2006, pg. 45). This leads research impact back to the external focus of responsiveness, the first dimension in this model. Research activity can be critical and reflexive without diminishing its scientific value.

Reflexivity examples: Within the paper "Designs and (Co)|ncidents: Cultures of Scholarship and Public Policy on Foreigners/Minorities in the Netherlands" (Essed and Nimako, 2006), the authors argue for an increased level of reflexivity on "Race Critical Perspectives" in the Dutch academic community. They contend that these frameworks on race and power hierarchies have been disregarded in favour of what they term 'minority research'. Due this focus on ethnic minorities, an institutional culture of problematisation of the 'other' has developed. This example of meta-analysis is most prevalent within "Critical Theory" perspectives but can be incorporated into any discipline.

\section{ECOLOGY}

"What can be studied is always a relationship or an infinite regress of relationships. Never a 'thing.".

\section{Gregory Bateson (2000)}

We believe impactful research should be ecological, not only in its environmental conception, but also socially, culturally and economically (Scoones, 1999). An ecological approach to research is a holistic and intersectional one that considers and is aware of the relationships among different types of agents in the research activity, in the pathway from research to practice and in the implications for researchers themselves. In terms of impact, ecological research should consider not just the possible benefits for the affected community, but also the possible disadvantages which they may suffer in a short and long run. In a broader perspective, ecological research would favour a holistic orientation, which Deshler and Selener (1991) see as one of the primary indicators that the conducted research will be transformative or have impact. While researchers are often encouraged to focus on the micro or minutiae of a topic, a larger understanding of the overall research landscape in a particular field and of the interconnectivity among academic disciplines is essential for research to be deemed 'ecological'.

An ecological mind-set in research should also encourage collegiality, bearing in mind its effect on researchers and research stakeholders. Being collegial refers to being open to other researchers, supporting more junior colleagues, treating people in a non-instrumental way, and in general, considering the well-being of others, enabling and strengthe- 
ning 'learning on the job' (Little, 1982) for academics at universities. In this sense, we think that impactful research should support the position of the field and its impact on external communities, and ideally it should encourage and/or be the result of collaborative work.

Finally, the concept of ecological impact may refer to the position of outreach and dissemination in the researcher's own career plan and broader life perspective. We see increasingly how academic activities aimed at complying with governmental policies or preparation to evaluation exercises takes away valuable time from research itself. This can lead to impact activities becoming 'instrumental' - i.e. the impact itself is secondary to the advantage it generates in terms of research funding, assessment scores etc. Therefore, it is always worth reflecting on whether the paperwork connected to documenting impact is not driving us away from the 'core business' of academic work, and if it is not affecting in a negative way our relationships with the stakeholders.

Ecology example: An impact case study submitted to the British REF (CS1698, Electropalatography (EPG) to Support Speech Pathology Assessment, Diagnosis and Intervention, Queen Margaret University) described a situation in which scholars working on a speech therapy device had too many volunteers for the experimental treatment. In order not to disappoint potential patients who would have to be turned away, the scholars decided not to publicise the experimental treatment at the current stage, despite the fact that this could limit their 'claim to impact', possibly resulting in a lower score in the REF evaluation (Wróblewska, 2018). This illustrates how dimensions which are not accounted for in existing models of evaluation can be reflected in the multidimensional model we propose.

\section{ADAPTABILITY}

"Being open to the possibility that our understanding or definition of a research problem may be inappropriate or partial."

Maureen G. Reed and Evelyn J. Peters (2014)

We argue that impactful research is adaptable to different contexts and stakeholders. This dimension of research impact refers to the usability of the different research components, such as methods and data, in further studies or across different samples (Hill et al., 1997), looking for possibilities for research impact. In view of the permanent development of research infrastructures (Ribes and Polk, 2014), together with the evolution of research objects and researchers themselves, there is a need for research activity to be more adaptive and resilient. Adaptive and resilient research methods "embrace the uncertainty and partiality of knowledge creation as well as the dynamism of the research process" (Reed and Peters, 2014, pg. 19). Accordingly, research resilience should be understood as its ability to absorb perturbations (anticipation) and adapt to change (plan for change), in line with the responsiveness dimension. Adaptable research must take care of recording and reporting methods and data appropriately (Mesirov, 2010). Potential for adaptations of research can be assessed 1) ex ante, by ascertaining how the thesis, hypothesis, methods and analysis meant to be used have the potential to be applied in different contexts and how data and methods are planned to be tracked and recorded, and 2) ex post, by revising executed or planned adaptations of the research, and watching the accuracy in the track and record of extant data and methods.

The adaptability of research can be purposeful and serviceable, as it allows keeping research relevant and strengthening research-policy dialogue in the face of the changing needs of decision-makers in different scenarios. Impactful research can be reused or adapted in numerous occasions, achieving various impacts, or it can bring questions that must be answered several times in different contexts, with different stakeholders, serving different audiences. Consequently, we think that impactful research should be clear about its limitations, potential future research opportunities (including adaptations/reproductions) and unanswered or emerging questions that can lead to further research impact elsewhere. Impactful research can be stimulating both in the questions it answers and in the new questions it rises.

Adaptability example: The "Blue Ocean Strategy", formulated by Kim and Mauborgne (2004), is a marketing theory that transcended academy and has been followed by many firms and entrepreneurs around the world. Such strategy proposes, in general terms, that firms aiming at developing strong competitive advantages should look for unexploited market spaces, avoiding competition and focusing on new innovative applications that generate new customers. This work has also inspired many research pieces including empirical applications or studies and further theoretical developments on organisational strategy.

\subsection{THE MODEL IN PRACTICE}

The MARIA model which we put forward here is primarily designed for qualitative self-assessment by researchers. While this paper discusses other types of national assessment models, it is important to note that this proposal is not meant to be used by third parties, specifically in relation to funding decisions. While qualitative assessment is important, for a simpler visualisation to assist researchers, these dimensions can be operationalised, and the assessment quantified if necessary. Again, the meaning of these numerical values can and should be assigned in a way that is most meaningful to the individual researcher. Hence, we have not provided any recommendations for scale meaning, beyond the basic focus on a numerical (1-5) scale. Having looked at the different research impact dimensions separately, any research can be represented through a pentagonal figure - the "MARIA pentagon" - showing the grades given to the research in the different dimensions, as exemplified in Figure 1. Note that a similar radar representation has been used in the impact assessment of the French National Institute for Agricultural Research (INRA, 2018), although there it represented different areas of impact (e.g. health, economy etc.). 

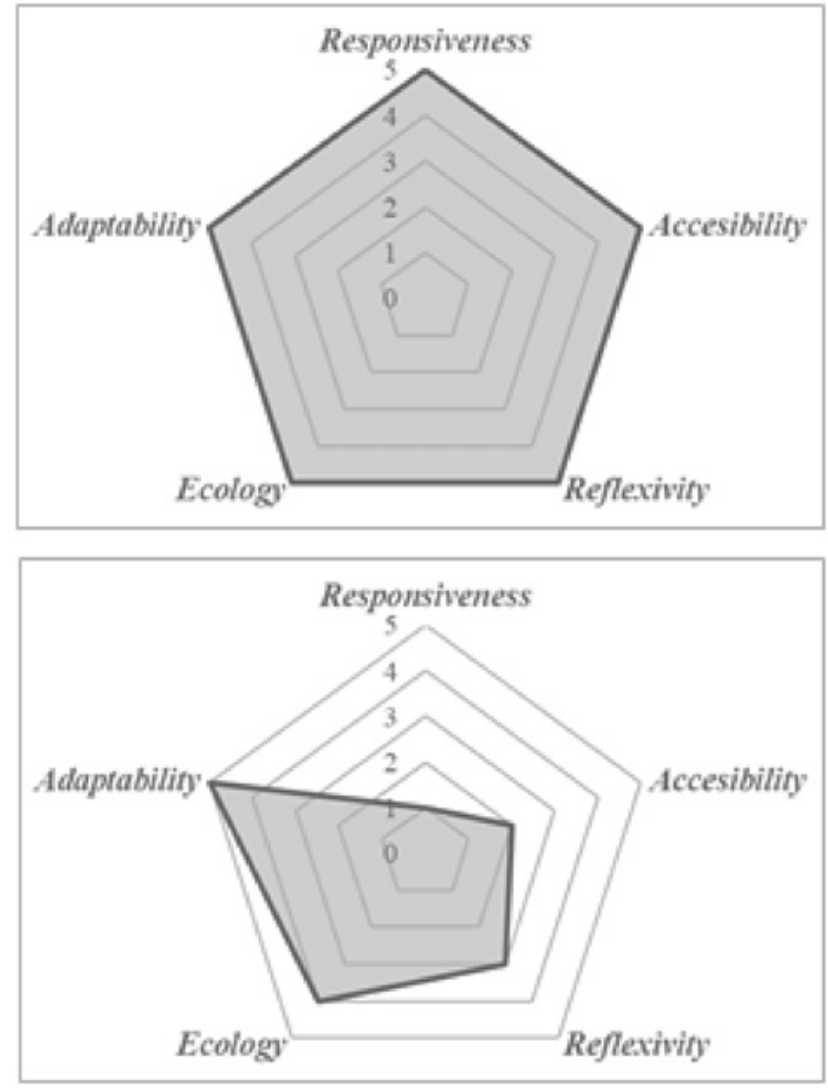

Figure 1 - MARIA Pentagon

The MARIA pentagon of the left represents a hypothetical situation in which the self-assessed research is totally successful in all its dimensions, while the pentagon of the right represents a research assessment that goes in an ascending clockwise order from responsiveness. The next section provides real examples of self-assessments using the model.

\section{SELF-ASSESSMENT EXAMPLES}

We as authors of the MARIA model have put under consideration our own PhD projects using the self-assessment sheets found in this paper's Annex. Ex ante (dissertation in formulation), mid-term (dissertation in progress) and ex post (dissertation finalised) research impact selfassessments were conducted by Bradley Good (2018), Sergio Manrique (2018) and Marta N. Wróblewska (2018) respectively.

\section{DISCUSSION}

Each author of a self-assessment has offered an outline on the experience using the MARIA model to assess research impact:

- Bradley Good: "Last year I underwent a major funding application with the Irish Research Council which contained elements of research impact and encouraged me to reflect on this issue. However, the treatment of this aspect seemed cursory and primarily focused on narrative utilisation rather than a systematic treatment of this issue. I found that utilising this more concrete approach gave my research planning additional focus and provided easily understandable ways that I could improve my project. Specifically, accessibility was lower than I would have anticipated which now provides me with extra incentive to do more outreach and promote my research publicly. This exercise was incredibly helpful, and I plan to incorporate my self-assessment as an official part of my PhD eight-month proposal".

- Sergio Manrique: "I had been exposed to assessment exercises at project/institutional levels, but those really didn't allow me to reflect on my individual research impact. This exercise has brought issues I was not really aware of and might route my future actions towards developing the dimensions that can boost the impact of my research on my stakeholders but also on the general public. This self-assessment has also allowed me to realise that research impact isn't achieved only through the research outputs themselves (publications, reports, patents, etc.), as impact can be generated by taking actions during the research process itself, actions that slip pass in the day-to-day of a researcher".

- Marta Wróblewska: "In theory, every researcher wants to produce research which is reflexive, accessible, adaptable etc., but we rarely take the time to actually evaluate what we have done so far. This is also due to the continuous nature of scientific work: there is always that one more article to write, one more seminar to get to, one more dissemination activity before we can 'wrap up' and evaluate our current project. In this sense, approaching the self-assessment was an incentive to take a step back and reflect on what has been achieved and what still requires work. The most interesting discovery for me would have to do with the 'serendipity' of impact - the areas where my research has been influential are not necessarily the ones where I planned to have impact".

Overall, we found that the utilisation of our model to be simple and effective, with enough data visualised for researchers to know where to improve while keeping the process unencumbered by lengthy narrative or complex metrics. With this initial 'field test' a success, our next step is to acquire feedback and continue to improve our operationalisation, eventually distributing and testing it within a broader demographic of SSH researchers.

Future research opportunities within this paradigm are abundant but of primary importance are the consideration of additional research impact dimensions, exploring links and correlations between these dimensions, studying the operationalisation of this model in different contexts, and identifying potential discipline-specific weighting configurations. In addition, other possibilities include refining specific dimensional indicators, providing further comparison to national systems of evaluations, and examining any differences in use and user experience between STEM and SSH researchers. The usability and usefulness of the model would ideally be tested empirically, for instance within one department or research project over a period of time - the authors intend to pursue opportunities of carrying out such a case study. Regardless, one must bear in mind that this model is in the theoretical stages of development, primarily utilised for self-assessment rather than an institutional focus. It might however be implemented as part of internal assessments (one of the authors of this study intends to implement it in this way - see above) and included as a supplementary document, even in more qualitative 
exercises (to account for the ethical dimension of impact. As mentioned before, this model proposal is a suggestion for broadening the debate on the existent research assessment systems and how these should be enhanced, tasks in which more insights and theoretical and empirical contributions are needed.

\section{CONCLUSION}

The inclusion of research impact criterion within the research assessment exercise in several national systems represents a relevant development in the valuation of research activity. However, the assessment and measurement of research impact is an ongoing process. A heavy focus on quantitative assessment, specifically for funding and allocation of other opportunities, can lead to a neglect of important qualitative factors. To provide an accurate depiction of research impact, recognition and understanding of these attributes must be encouraged. To this end, this research paper proposes and explains a Multidimensional Approach for Research Impact Assessment (MARIA Model), highlighting five impactful attributes of research: Responsiveness, Accessibility, Reflexivity, Ecology, and Adaptability. These dimensions are presented as attributes of impactful research conducted in any area or discipline. However, this multidimensional model explicitly looks for a fairer treatment of SSH in the assessment of research impact. The operationalisation of this multidimensional model has also been explained. To this end, a set of scales is proposed for self-assessing each of the dimensions, and a tool suggested to represent the general impact of a research: The MARIA Pentagon, which could be useful in collective exercises of research assessment where rankings and thresholds are required. Rather than suggesting a fixed model for research impact assessment, this paper aims at evidencing the existence of further impactful attributes that the research impact agenda might have been neglecting. The assessment of research impact can't avoid the qualitative implications of science, as reducing research value to its measurable effects would not be coherent with the nature of research practice, and therefore it would be recommendable to consider a broader perspective in the assessment exercise, like the one proposed in this work.

While there are several developed systems for external assessment of impact, we believe that what is lacking in the panorama of research evaluation is 1) a framework to systematically reflect on the impact of one's own work (self-assessment) 2) a multi-levelled model which recognises the complexity of any impactful work, 3) a model which explicitly recognises the ethical aspect of conducting impactful research and offers a clear framework for reflection on these issues. The model we propose aims to address the above-mentioned gaps. Finally, our model considers the serendipitous nature of research impact generation (Derrick and Samuel, 2016). It could be argued that a research project could fare very highly in the MARIA model scale, without actually realising a 'change or benefit' to society (as the REF definition of impact has it), for instance due to lack of uptake of a potentially impactful innovation, lack of financing for implementation or many other factors which are beyond the academics' control. While this is a real possibility, we would stress that the MARIA model looks at the process of generating impact, rather than the final effects thereof. We would argue that a project which considers the five dimensions is very likely to produce research impact, doing so in a sustainable and ethically-aware way.

Our proposal contributes to the ongoing learning process of research impact, in alignment with the context-based perspective of research assessment (Spaapen et al., 2007) and in recognition of the need for a more holistic view in the observation and monitoring of interdisciplinary research (Anzai et al., 2012). Rather than suggesting a fixed model for research impact assessment, this paper aims at evidencing the existence of additional aspects of conducting impactful research that existing research assessment systems do not fully recognise or represent.

\section{ACKNOWLEDGEMENTS}

- ENRESSH Winter School: The authors thank EU COST action ENRESSH ${ }^{1}$ (European Network for Research Evaluation in the Social Sciences and the Humanities) for the opportunity to participate in its first training school, held in February 2018 in Zagreb, Croatia, where this paper's proposal emerged. Special thanks are due to ENRESSH working group 2 leader Dr Paul Benneworth (University of Twente, NL), and to Dr Leonie Van Drooge (Rathenau Instituut, NL), Xeni Kechagioglou (Università degli Studi di Cagliari, IT) and Eloïse Germain-Alamartine (Linköping University, SE) for their contribution to the teamwork during this training school, which helped to start shaping this paper's contribution.

- PhD funding: Sergio Manrique is a PhD fellow of RUNIN project², a European Training Network for Early-Stage Researchers, funded by EU's Horizon 2020 research and innovation programme under Marie Skłodowska-Curie grant agreement \# 722295.

\section{REFERENCES}

American Society for Cell Biology. (2012). San Francisco Declaration on Research Assessment. Putting science into the assessment of research. Retrieved from: http://www.ascb.org/wp-content/uploads/2017/07/ sfdora.pdf

Angermuller, J. (2013). Discours académique et gouvernementalité entrepreneuriale. Des textes aux chiffres. In M. Temmar, J. Angermuller and F. Lebaron (Eds.), Les discours sur l'économie (p. 71-84). Paris: PUF. Retrieved from: http://www.septentrion.com/fr/livre/?GCOI=27574100482170

Anzai, T., Kusama, R., Kodama, H. and Sengoku, S. (2012). Holistic observation and monitoring of the impact of interdisciplinary academic research projects: An empirical assessment in Japan. Technovation, 32(6), 345-357. DOl: https://doi.org/10.1016/j.technovation.2011.12.003

Association of Universities in the Netherlands (VSNU), the Netherlands Organisation for Scientific Research (NWO) and the Royal 
Netherlands Academy of Arts and Sciences (KNAW). (2016). Standard Evaluation Protocol 2015-2021. Voorburg: KNAW, VSNU and NWO. Retrieved from: https://www.knaw.nl/nl/actueel/publicaties/standardevaluation-protocol-2015-2021

Australian Research Council. (2018). Engagement and Impact Assessment. Retrieved from: https://www.arc.gov.au/engagement-andimpact-assessment

Bacevic, J. (2017). Beyond the Third Mission: Toward an Actor-Based Account of Universities' Relationship with Society. In H. Ergül and S. CoĐar (Eds.), Universities in the Neoliberal Era: Academic Cultures and Critical Perspectives (pp. 21-39). London: Palgrave Macmillan UK. DOI: https:// doi.org/10.1057/978-1-137-55212-9_2

Baert, P. (2015). The existentialist moment: the rise of Sartre as a public intellectual. Cambridge: Polity Press.

Bateson, G. (2000). Steps towards an ecology of mind. Collected Essays in Anthropology, Psychiatry, Evolution and Epistemology. Chicago: University of Chicago Press. D0l: https://doi.org/10.7208/chicago/9780226924601.001.0001

Bayley, J. and Phipps, D. (2017). Building the Concept of Research Impact Literacy. Evidence and Policy: A Journal of Research, Debate and Practice (in press). DOl: https://doi.org/10.1332/17442641 7X15034894876108

Benneworth, P., Gulbrandsen, M. and Hazelkorn, E. (2016). The impact and future of arts and humanities research. London: Palgrave Macmillan. DOl: https://doi.org/10.1057/978-1-137-40899-0

Bosch, G. (2018). Train PhD students to be thinkers not just specialists. Science, 554(277). DOI: https://doi.org/10.1038/d41586-018-01853-1

Brundenius, C. and Göransson, B. (2011). The Three Missions of Universities: A Synthesis of UniDev Project Findings. In B. Göransson, and C. Brundenius (Eds.), Universities in Transition. The Changing Role and Challenges for Academic Institutions (pp. 329-352). New York: Springer. DOI: https://doi.org/10.1007/978-1-4419-7509-6_16

Burawoy, M. (2004). Public Sociologies: Contradictions, Dilemmas, and Possibilities. Social Forces, 82(4), 1603-1618. D0I: https://doi. org/10.1353/sof.2004.0064

Buxton, M. and Hanney, S. (1996). How Can Payback from Health Services Research Be Assessed? Journal of Health Services Research and Policy, 1(1), 35-43. DOI: https://doi.org/10.1177/135581969600100107

Canadian Academy of Health Sciences. (2009). Making an Impact: A Preferred Framework and Indicators to Measure Returns on Investment in Health Research. Ottawa: CAHS. Retrieved from: http://www.cahsacss.ca/wp-content/uploads/2011/09/ROI_FullReport.pdf

Chubb, J. (2017). Instrumentalism and epistemic responsibility: researchers and the impact agenda in the UK and Australia. University of York. Retrieved from: http://etheses.whiterose.ac.uk/18575/
Chubb, J. and Watermeyer, R. (2017). Artifice or integrity in the marketization of research impact? Investigating the moral economy of (pathways to) impact statements within research funding proposals in the UK and Australia. Studies in Higher Education, 42(12), 2360-2372. DOI: https://doi.org/10.1080/03075079.2016.1144182

Chubb, J., Watermeyer, R. and Wakeling, P. (2016). Fear and loathing in the Academy? The role of emotion in response to an impact agenda in the UK and Australia. Higher Education Research and Development, 36(3), 555-568. D0I: https://doi.org/10.1080/07294360.2017.1288709

Cranton, P. (2006). Understanding and Promoting Transformative Learning: A Guide for Educators of Adults. 2nd Ed. The Jossey-Bass Higher and Adult Education Series. San Francisco: Jossey-Bass. Retrieved from: https://www.wiley.com/en-us/Understanding+and+Promoting+Transfo rmative+Learning\%3A+A+Guide+for+Educators+of+Adults\%2C+2nd +Edition-p-9780787976682

Derrick, G. (2018). The evaluators' eye: Impact assessment and academic peer review. London: Palgrave Macmillan. DOI: https://doi. org/10.1007/978-3-319-63627-6

Derrick, G. and Samuel, G. N. (2016). The Evaluation Scale: Exploring Decisions about Societal Impact in Peer Review Panels. Minerva, 54(1), 75-97. DOl: https://doi.org/10.1007/s11024-016-9290-0

Deshler, D. and Selener, D. (1991). Transformative Research: In Search of a Definition. Convergence. 24(3): 9. Retrieved from: https://search.proquest.com/openview/039909077fd7c8ac9743831a0de569d6

Donovan, C. (2017). For ethical 'impactology'. Journal of Responsible Innovation. DOI: https://doi.org/10.1080/23299460.2017.1300756

E3M. (2012). Fostering and Measuring 'Third Mission' in Higher Education Institutions (Green Paper). Retrieved from: http://e3mproject.eu/ Green paper-p.pdf

Essed, P., and Nimako, K. (2006). Designs and (Co)Incidents: Cultures of Scholarship and Public Policy on Immigrants/Minorities in the Netherlands. International Journal of Comparative Sociology, 47(3-4), 281-312. DOl: https://doi.org/10.1177/0020715206065784

European Commission. (2003). The Role of Universities in the Europe of Knowledge. Retrieved from http://eur-lex.europa.eu/legal-content/EN/ TXT/?uri=LEGISSUM:C11067

European Commission. (2014). Cohesion Policy Frequently Asked Questions. Retrieved from: http://ec.europa.eu/regional_policy/en/faq/

European Commission. (2017). What is Horizon 2020? Retrieved from: https://ec.europa.eu/programmes/horizon2020/what-horizon-2020

European Commission. (2018). Horizon 2020: Open Science. Retrieved from: https://ec.europa.eu/programmes/horizon2020/en/h2020-section/ open-science-open-access 
Freire, P. (2000). Pedagogy of the oppressed (30th anniversary Ed.). New York: Continuum. Retrieved from: http://commons.princeton.edu/ inclusivepedagogy/wp-content/uploads/sites/17/2016/07/freire_pedagogy_of_the_oppresed_ch2-3.pdf

Galligan, F. and Dyas-Correia, S. (2013). Altmetrics: Rethinking the Way We Measure. Serials Review, 39(1), 56-61. D0I: https://doi.org/10.1080/ 00987913.2013 .10765486

Good, B. (2018). Teaching critical perspectives: The transformative learning potential of diversity courses within Dutch higher education (Ongoing unpublished PhD thesis). Vrije Universiteit Amsterdam, Amsterdam, the Netherlands.

Gornitzka, Å. and Maassen, P. (2007). An instrument for national political agendas: the hierarchical vision. In P. Maasen and J.P. Olsen (Eds.), University dynamics and European integration (Vol. 19, pp. 81-98). Dordrecht: Springer. DOI: https://doi.org/10.1007/978-1-4020-5971-1_4

Grant, J., Brutscher, P. B., Kirk, S., Butler, L. and Wooding, S. (2009). Capturing Research Impacts. A review of international practice. Retrieved from: http://www.hefce.ac.uk/media/hefce/content/pubs/2009/ rd2309/rd23_09.pdf

Gulbrandsen, M. and Sivertsen, G. (2018). Impact i anvendt forskning: begrepsavklaring og praksis. NIFU (Nordisk institutt for studier av innovasjon, forskning og utdanning) Arbeidsnotat, 10. Retrieved from: https://brage.bibsys.no/xmlui/handle/11250/2570526

Hamann, J. and Gengnagel, V. (2014). The Making and Persisting of Modern German Humanities. Balancing Acts between Autonomy and Social Relevance. In J. Maat, R. Bod and T. Weststeijn (Ed.), The Making of the Humanities (Vol. III The Modern Humanities, pp. 641-654). Amsterdam: Amsterdam University Press. D0l: https://doi. org/10.1515/9789048518449-043

HEFCE. (2009). Report on the pilot exercise to develop bibliometric indicators for the Research Excellence Framework. Retrieved from http:// webarchive.nationalarchives.gov.uk/20120716093655/http://www.hefce.ac.uk/pubs/year/2009/200939/

HEFCE. (2011). Assessment framework and guidance on submissions. Retrieved from https://www.ref.ac.uk/2014/media/ref/content/pub/assessmentframeworkandguidanceonsubmissions/GOS\%20including\%20 addendum.pdf

HEFCE. (2016, 6 May 2016). How we fund research. Retrieved from http://www.hefce.ac.uk/rsrch/funding/

Hicks, D. (2012). Performance-based university research funding systems. Research Policy, 41(2), 251-261. D0I: https://doi.org/10.1016/j. respol.2011.09.007

Hicks, D., Wouters, P., Waltman, L., de Rijcke, S. and Rafols, I. (2015). Bibliometrics: The Leiden Manifesto for research metrics. Nature, 520, 429-431. DOI: https://doi.org/10.1038/520429a
Hill, C. E., Thompson, B. J. and Williams, E. N. (1997). A guide to conducting consensual qualitative research. Counselling Psychologist, 25(4), 517-572. DOI: https://doi.org/10.1177/0011000097254001

Holbrook, J. B. and Frodeman, R. (2011). Peer review and the ex ante assessment of societal impacts. Research Evaluation, 20(3), 239-246. DOI: https://doi.org/10.3152/095820211X12941371876788

Hong Kong University Grants Committee. (2018). Research Assessment Exercise 2020. Draft General Panel Guidelines. Retrieved from: http://www.ugc.edu.hk/doc/eng/ugc/rae/2020/draft_gpg_feb18.pdf

Hood, W. W. and Wilson, C. S. (2001). The literature of bibliometrics, scientometrics, and informetrics. Scientometrics, 52(2), 291-314. D0l: https://doi.org/10.1023/A:1017919924342

INRA. (2018). Translating research into impacts: 30 case studies. Retrieved from: http://www.inra.fr/en/Scientists-Students/Economics-and-social-sciences/All-the-news/Translating-research-into-impacts-30-casestudies

Jacob, M. (2006). Utilization of social science knowledge in science policy: Systems of innovation, triple helix and VINNOVA. Social Science Information, 45(3), 431-462. D0l: https://doi.org/10.1177/0539018406066535

Jessop, B., Fairclough, N. and Wodak, R. (2008). Education and the knowledge-based economy in Europe. Rotterdam: Sense Publishers. Retrieved from: https://www.sensepublishers.com/media/303-educationand-the-knowledge-based-economy-in-europe.pdf

Khazragui, H. and Hudson, J. (2015). Measuring the benefits of university research: impact and the REF in the UK. Research Evaluation, 24, 51-62. DOI: https://doi.org/10.1093/reseval/rvu028

Kim, W. C. and Mauborgne, R. (2004). Blue Ocean Strategy. Harvard Business Review, 82(10), 76-84. Retrieved from: https://hbr.org/2004/10/ blue-ocean-strategy

King's College London and Digital Science. (2015). The nature, scale and beneficiaries of research impact: An initial analysis of Research Excellence Framework (REF) 2014 impact case studies. Retrieved from: http://dera.ioe.ac.uk/22540/1/Analysis_of_REF_impact.pdf

Lamont, M. (2012). Toward a Comparative Sociology of Valuation and Evaluation. Annual Review of Sociology, 38(21), 201-221. D0l: https:// doi.org/10.1146/annurev-soc-070308-120022

Lee, S. M. (2018, February 25). Sliced and Diced: The Inside Story of How an Ivy League Food Scientist Turned Shoddy Data into Viral Studies. Retrieved from: https://www.buzzfeednews.com/article/stephaniemlee/ brian-wansink-cornell-p-hacking

Lightowler, C. and Knight, C. (2013). Sustaining Knowledge Exchange and Research Impact in the Social Sciences and Humanities: Investing in Knowledge Broker Roles in UK Universities. Evidence and Policy: A Journal of Research, Debate and Practice, 9(3), 317-334. D0l: https://doi. org/10.1332/174426413X662644 
Little, J. W. (1982). Norms of Collegiality and Experimentation: Workplace Conditions of School Success. American Educational Research Journal, 19(3), 325-340. DOl: https://doi.org/10.3102/00028312019003325

Lundequist, P. and Waxell, A. (2010). Regionalizing "Mode 2"? The adoption of centres of excellence in Swedish research policy. Geografiska Annaler: Series B, Human Geography, 92(3), 263-279. D0I: https://doi. org/10.1111/j.1468-0467.2010.00352.x

Manrique, S. (2018). Assessing the Impact of University-Firm Collaboration on Firm Performance and Regional Development (Ongoing unpublished PhD thesis). Universitat Autònoma de Barcelona, Barcelona, Spain.

Manville, C., Guthrie, S., Henham, M.-L., Garrod, B., Sousa, S., Kirtley, A., Castle-Clarke, S. and Ling, T. (2015). Assessing impact submissions for REF 2014: an evaluation. Retrieved from: https://www. rand.org/content/dam/rand/pubs/research_reports/RR1000/RR1032/ RAND_RR1032.pdf

Manville, C., Jones, M. M., Frearson, M., Castle-Clarke, S., Henham, M.-L., Gunashekar, S. and Grant, J. (2014). Preparing impact submissions for REF 2014: An evaluation. Findings and observations Retrieved from: https://www.rand.org/pubs/research_reports/RR726.html

Maxey, I. (1999). Beyond boundaries? Activism, academia, reflexivity and research. AREA, 31(3), 199-208. DOl: https://doi. org/10.1111/j.1475-4762.1999.tb00084.x

Mesirov, J. P. (2010). Computer Science. Accessible Reproducible Research. Science, 327(5964), 415-416. D0I: https://doi.org/10.1126/science. 1179653

McGettigan, A. (2013). The great university gamble: money, markets and the future of higher education. London: Pluto Press. Retrieved from: https://www.plutobooks.com/9780745332932/the-great-universitygamble/

Ministry of Education, Culture, and Science, The Netherlands. (2016). Amsterdam Call for Action on Open Science. Amsterdam, the Netherlands: Government of the Netherlands. Retrieved from: https://www. government.nl/documents/reports/2016/04/04/amsterdam-call-for-action-on-open-science

Münch, R. (2014). Academic capitalism: universities in the global struggle for excellence. Abingdon, Oxon: Routledge. DOI: https://doi. org/10.4324/9780203768761

NIAD-OE (2018). National Institution for Academic Degrees and Quality Enhancement of Higher Education. Tokyo, Japan. Retrieved from: https://www.niad.ac.jp/english/unive/

Ostrom, E. (2009). A General Framework for Analyzing Sustainability of Social-Ecological Systems. Science, 325(5939), 419-422. D0l: https://doi. org/10.1126/science.1172133
Owen, R., Macnaghten, P. and Stilgoe, J. (2012). Responsible research and innovation: From science in society to science for society, with society. Science and Public Policy, 39, 751-760. D0I: https://doi.org/10.1093/ scipol/scs093

Pereira, M. (2016). Struggling within and beyond the Performative University: Articulating activism and work in an "academia without walls". Women's Studies International Forum, 54, 100-110. D0l: https://doi. org/10.1016/j.wsif.2015.06.008

Priem, J., Groth, P. and Taraborelli, D. (2012). The Altmetrics Collection. PLoS ONE, 7(11), E48573. DOl: https://doi.org/10.1371/journal. pone.0048753

Reed, M. and Peters, E. (2014). Using an Ecological Metaphor to Build Adaptive and Resilient Research Practices. ACME: An International Journal for Critical Geographies, 3(1), 18-40. Retrieved from: https://acmejournal.org/index.php/acme/article/view/724

Research Council of Norway. (2017). Evaluation of the Humanities in Norway. Report from the Principal Evaluation Committee. Retrieved from https://www.forskningsradet.no/en/Article/Evaluation_of_humanities_ research_in_Norway/1254012498362 


\section{EX-ANTE RESEARCH IMPACT SELF- ASSESSMENT EXAMPLE (GOOD, 2018)}

\section{ACCESSIBILITY}

Are my research outputs accessible to different stakeholders and society in general? Do I communicate and disseminate them broadly and effectively?

My research outputs will primarily exist in the form of journal articles and potential policy documents with direct access available to all participating stakeholders.

Grade: 2.0/5.0

\section{REFLEXIVITY}

Do I reflect on how comprehensive, well-planned, ethical and critical my research is? Have I evaluated and critiqued my theories and analyses?

I regularly revise and update my research plan in accordance with new literature and theories. My analysis itself is based on a theoretical frame that encourages deconstruction and critical analysis.

Grade: $4.0 / 5.0$

\section{ECOLOGY}

Does my research consider the relationships and connections among stakeholders and subjects? Was I collegial while conducting this research?

My research subjects are also some of my most important stakeholders as improving their educational opportunities benefits them, as well as their instructors and institutions.

Grade: $3.0 / 5.0$

\section{ADAPTABILITY}

Is my research impact usable in different contexts and among different stakeholders? Am I aware of the limitations, and unanswered or emerging questions from my research?

While my research focuses on a Dutch context, it could be adaptable to other higher education cultures in the future but only after multiple studies. This is due to the limited sample size and time constraints.

Grade: 3.0/5.0

\section{RESEARCH DESCRIPTION}

Title: Teaching critical perspectives - The transformative learning potential of diversity courses within Dutch higher education.

Type: $\mathrm{PhD}$ thesis.

Dates: September 2018 - Present.

Objective: Studying to what degree diversity education courses in The Netherlands successfully meet course objectives, incorporate critical perspectives, and reduce racist behaviours while encouraging further exploration of these issues beyond the classroom.

Author: Bradley Good.

Institution: Vrije Universiteit Amsterdam (NL).

Status: Formulation.

\section{SELF-ASSESSMENT CONCLUSIONS}

Research Impact Pentagon

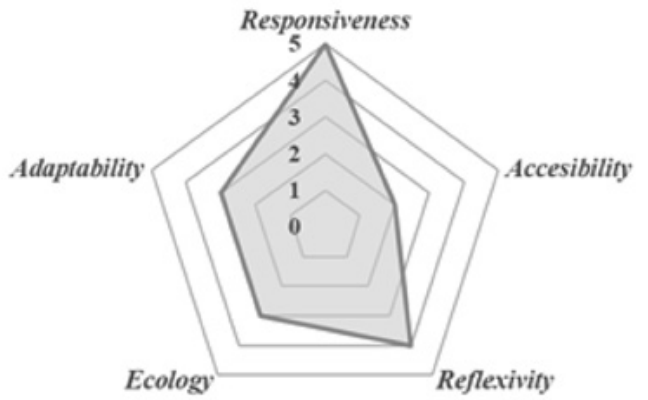

\section{ASSESSMENT CONCLUSIONS}

Overall my research seems to be successfully planned for moderate research impact. However, accessibility could greatly improve, with a secondary emphasis on ecology. While adaptability does not have a high score, this is primarily due to the limited scope of research, which is unavoidable. 


\section{MID-TERM RESEARCH IMPACT SELF-ASSESSMENT EXAMPLE (MANRIQUE, 2018)}

\section{RESPONSIVENESS}

Does my research respond to real problems and needs in society? Am I contributing to current public debates?

University-firm collaboration can be a powerful tool for the performance of firms and for the development of regions, which can indirectly end up benefitting citizens. However, my research is primarily focused on the economic impact on industry.

Grade: 4.0/5.0

\section{ACCESSIBILITY}

Are my research outputs accessible to different stakeholders and society in general? Do I communicate and disseminate them broadly and effectively?

Research in my project is meant to be published in Open Access outlets. I am active in attending conferences and workshops to communicate and disseminate my findings. Work in progress and other research outputs (blog posts, reports) are publicly available at the project website.

Grade: $4.5 / 5.0$

\section{REFLEXIVITY}

Do I reflect on how comprehensive, well-planned, ethical and critical my research is? Have I evaluated and critiqued my theories and analyses?

PhD topics within this Horizon 2020 project were mostly fixed. I have, however, spent a significant amount of time planning the methods and data I should use. In the end, I incorporated a qualitative approach to a project which was planned to be quantitative, and now I am conducting mixed methods research. Grade: $1.5 / 5.0$

\section{ECOLOGY}

Does my research consider the relationships and connections among stakeholders and subjects? Was I collegial while conducting this research?

I make part of a team of junior and senior researchers as well as regional and non-academic partners. My project is one piece in the larger RUNIN proposal. Grade: $3.0 / 5.0$

\section{ADAPTABILITY}

Is my research impact usable in different contexts and among different stakeholders? Am I aware of the limitations, and unanswered or emerging questions from my research?

What I am doing using Spanish data can be readapted using data from other countries and regions, and for phenomena beyond university-firm collaboration. I always state research limitations in my publications.

Grade: 3.0/5.0

\section{RESEARCH DESCRIPTION}

Title: Assessing the impact of university-firm collaboration on firm performance and regional development (part of a horizon 2020 training network).

Type: PhD thesis.

Dates: February 2017 - Present.

Objective: Assessing the impact of university-firm collaboration on firms' innovation capacity and economic performance, and exploring how such impact translates into economic growth and social development in the regions where the interaction takes place.

Author: Sergio Manrique.

Institution: Universitat Autònoma de Barcelona (ES)

Status: In execution.

More info at: https://runinproject.eu/sergio-andres-manrique-garzon/

\section{SELF-ASSESSMENT CONCLUSIONS}

Research Impact Pentagon

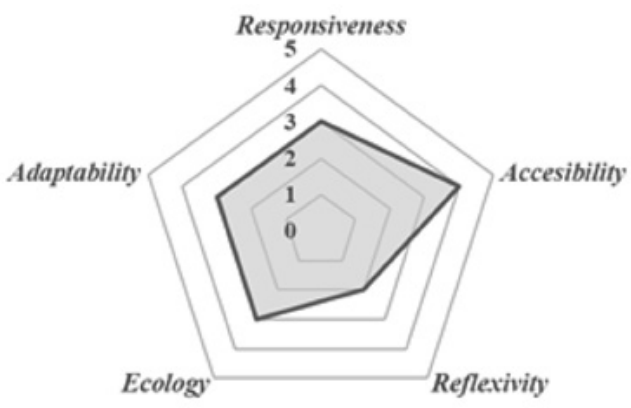

\section{ASSESSMENT CONCLUSIONS}

My PhD project's impact is boosted by being part of a Horizon 2020 training network, through which accessibility of research outputs is facilitated. Additionally, I make part of an established network of academics and regional stakeholders, which contribute to shaping my research in a responsive manner. However, by being a project planned in advance (before recruiting researchers), the range of action on the research design is limited, and there hasn't been too much focus on critical thinking. 


\section{EX-POST RESEARCH IMPACT SELF-ASSESSMENT EXAMPLE (WRÓBLEWSKA, 2018)}

\section{RESPONSIVENESS}

Does my research respond to real problems and needs in society? Am I contributing to current public debates?

My study of the Impact Agenda responds to a need of academics and policymakers to tackle the question of impact evaluation, focusing on the understudied aspect of language change and self-representation. Since I started my PhD, systems of impact evaluation have been adopted in several countries, generating considerable interest in my work's practical implications, particularly in the linguistic aspect of editing impact case studies.

Grade: 4.0/5.0

\section{ACCESSIBILITY}

Are my research outputs accessible to different stakeholders and society in general? Do I communicate and disseminate them broadly and effectively?

I have drafted an 'executive summary' of the findings from my PhD work and shared it with the study's respondents and stakeholders. The reach of my findings remains limited, but I am seeking funding for a practice-oriented publication, ideally in open access.

Grade: 2.0/5.0

\section{REFLEXIVITY}

Do I reflect on how comprehensive, well-planned, ethical and critical my research is? Have I evaluated and critiqued my theories/ analyses?

Reflexivity and ethics were at the core of my study. Still I question to what degree my critical standpoint is influenced by my academic background - one needs to be critical of 'critical theory' too!

Grade: 4.0/5.0

\section{ECOLOGY}

Does my research consider the relationships and connections among stakeholders and subjects? Was I collegial while conducting this research?

To a large degree my work was solitary and individualistic. I might not have fully used the potential present in my research team. I also worry about the control I have over the application of my findings by stakeholders.

Grade: 3.0/5.0

\section{ADAPTABILITY}

Is my research impact usable in different contexts and among different stakeholders? Am I aware of the limitations, and unanswered or emerging questions from my research?

I've engaged with stakeholders in other countries (Poland, Norway) pointing to opportunities and challenges related to adapting impact evaluation. In this sense my research is adaptable, but the question remains to what degree can a scholar influence policy?

Grade: 4.0/5.0

\section{RESEARCH DESCRIPTION}

Title: The making of the Impact Agenda - A study in discourse and governmentality.

Type: PhD thesis.

Dates: October 2014 - September 2018.

Objective: Examining the change in academic discourse engendered by the introduction of the Impact Agenda and its link to practices of subjectivation (work upon one's 'self').

Author: Marta Natalia Wróblewska.

Institution(s): University of Warwick (UK)

Status: Concluded (now in dissemination phase)

More info at: https://warwick.ac.uk/mnwroblewska

\section{SELF-ASSESSMENT CONCLUSIONS}

Research Impact Pentagon

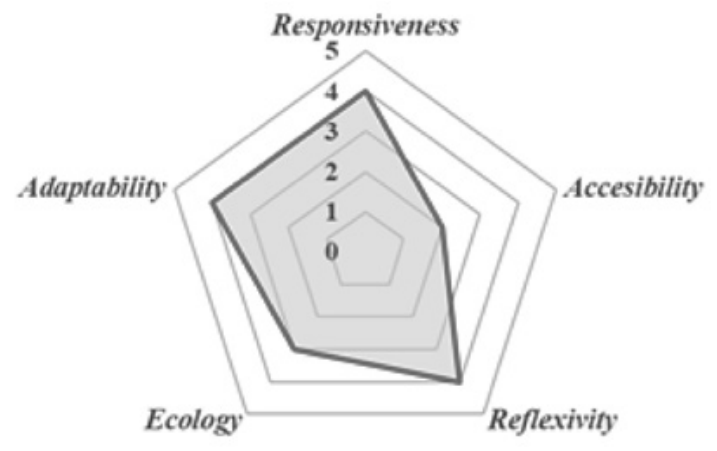

\section{ASSESSMENT CONCLUSIONS}

Given that my work focused on the rise of 'impact evaluation', I was constantly questioned by audiences about the impact of my own work. This incentive, combined with resources offered by my institution for fostering responsible outreach, account for the fact that I have reflected on and pursued impact in my PhD project. The weakness of my project seems to be accessibility of findings and so I resolved to focus on creating open-access publications on the practical elements of my research findings, which would improve my score in this area. 


\section{AUTHORS}

\section{SERGIO MANRIQUE}

Department of Business, Universitat Autònoma de Barcelona

Plaça Cívica, Barcelona, 08193 (Spain)

E: sergioandres.manrique@uab.cat

\section{MARTA NATALIA WRÓBLEWSKA}

Centre for Applied Linguistics, University of Warwick

S.1.74 Social Sciences Building, Coventry, CV4 7AL (UK)

E: m.n.wroblewska@warwick.ac.uk

\section{AND BRADLEY GOOD}

Department of Sociology, Vrije Universiteit Amsterdam

De Boelelaan 1105, Amsterdam, 1081 HV (The Netherlands)

E: b.d.good@vu.nl

\section{KEYWORDS:}

Research Impact, Research Evaluation, Self-Assessment, Research Ethics. 


\title{
EVALUATIVE INQUIRY: ENGAGING RESEARCH EVALUATION ANALYTICALLY AND STRATEGICALLY
}

\author{
SARAH DE RIJCKE, TJITSKE HOLTROP, WOLFGANG KALTENBRUNNER, JOCHEM ZUIJDERWIJK, ANNE BEAULIEU, \\ THOMAS FRANSSEN, THED VAN LEEUWEN, PHILIPPE MONGEON, CLIFFORD TATUM, GOVERT VALKENBURG AND \\ PAUL WOUTERS \\ DOI: 10.22163/fteval.2019.386
}

\section{ABSTRACT}

$\mathrm{T}$ raditional frameworks for academic evaluation are focused on registering the achievements of research units' academic and societal achievements. These frameworks and the ways they are usually carried out are built on a few dichotomies: academic versus societal spheres, quantitative versus qualitative approaches, and representative versus intervening analyses. We argue that these dichotomies contribute to a notion of academic achievement that is unrealistic, in a normative and descriptive sense. The concept of the "evaluative inquiry," as proposed here, amends the linear and individualised notion of academic work and its evaluation and discusses the implications of these moves for the work of the analyst. We suggest instead to understand academic achievement as distributed over a host of academic and non-academic participants to be studied by means of a portfolio approach. This approach to research evaluation requires a more engaged analyst who takes evaluation seriously as both an analytical and a strategic project.

\section{INTRODUCTION}

This paper introduces the evaluative inquiry, an approach that aims to challenge several dimensions of the current science system and the organisation of research evaluation, most notably its understandings of academic achievement, impact, and the ways these should be measured. It contributes to a "re-loading" of the term impact, drawing on the methodological and conceptual approaches of the social sciences and humanities in particular, and all the sciences in general (König et al., 2018). We propose a distributive understanding of academic achievement, thereby recognising the contributions of both academics and nonacademics. In addition, we put forth a portfolio approach to evaluation in order to detect the multiple realities that go into academic quality and in order to inspire conversations about these. Lastly, we move beyond approaches that claim to neutrally represent quality and relevance, by offering a style of strategic and collaborative intervention. We hope these three moves will help identify paths to reform and revitalise the science system and the normative, unilateral, and dichotomous ideals of excellence and impact.
The evaluative inquiry was proposed by Fochler and De Rijcke (2017) as a way to contribute to ongoing discussions about quality and relevance of research. Our team at CWTS (Leiden University, The Netherlands) has since then put the evaluative inquiry into practice in several projects, and this work informs this paper. Our ambition with these experiments in research evaluation is, in essence, to enable better conversations about academic value and its beneficiaries and rewards, rather than to further encourage "accounting for impact" (Rushforth and De Rijcke, 2015) by way of standardised formats and rankings.

\section{1) THE DUTCH CONTEXT}

To situate our approach, a few words are in order on the main characteristics of the Dutch higher education and science governance system. Like many other European countries, the Dutch higher education system distinguishes between two types of higher education institutions: universities on the one hand, and institutions for higher vocational education - so-called hogescholen - on the other. As a general rule, science governance instruments since the early 1980s have been built around a principle of "steering at a distance". The Netherlands in fact operate with what Richard Whitley (2007) has called a "weak" system of research evaluation, meaning that assessment results have no direct consequences for the distribution of funding to universities (in contrast to, for example, the UK). Rather, the principal strategy is to use formal evaluation as opportunities for self-reflection and organisational learning (see also Youtie and Corley, 2011; Hansson and Monsted, 2012). Conceptually, institutional research evaluation systems can serve three main purposes: a distributive, an improvement, and a controlling use (Molas-Gallart, 2012). In the Dutch context, the purpose of evaluation is clearly focused on improvement, and an evaluation can also spark organisational change. As Molas-Gallart puts it, "[a]n improvement use will focus on deriving lessons from the past experience to adapt the activities conducted to what evaluation studies will conclude is better practice. The improvement purpose is therefore relying on the existence of feedback mechanisms and the operational flexibility needed to function as a learning organization." (ibid, 589) We would suggest that an improvement-oriented evaluation system like the Dutch one provides particular opportunities for experimenting with evaluative inquiries. 
The Netherlands introduced a formal evaluation system as early as 1982. All research units at Dutch universities (as well as the institutes of the Royal Netherlands Academy of Arts and Sciences and the Netherlands Organisation for Scientific Research) are required to undergo an assessment in six-yearly intervals (see figure 1). The assessment consists of a peer review procedure by an external committee, involving personal site visits, interviews, and a review of research output and other activities. Halfway between the 6-yearly national research assessments, research units are moreover required to conduct a mid-term evaluation. The results of a self-evaluation serve as input for the assessment, and are also meant to encourage continuous self-monitoring of individual institutions. Another important change introduced in 2015 was that organisational responsibility for evaluation was decentralised and delegated to individual institutions, thus providing them with a greater degree of administrative discretion.

The exact modalities of assessment are outlined in the so-called "Standard Evaluation Protocol" (SEP). According to the SEP, institutions are required to provide a range of materials as input for the assessment, including inter alia a formal documentation of output and "performance indicators" (e.g., a complete list of publications, number of successfully defended $\mathrm{PhD}$ theses etc.), a description of the financing of a given research unit, and a qualitative narrative summarising the results and societal relevance of the research (see table 1 below). Whereas evaluation has traditionally placed an important emphasis on quantity of output and the perceived prestige of publications and research grants, the 2015 iteration of the SEP introduces a stronger emphasis on "societal relevance" of research, i.e. the engagement with non-academic audiences and partners. Academic excellence and societal relevance are however kept largely separate in the evaluative framework. Research units are ultimately graded according to a four-tiered scale (from "world-leading" to "unsatisfactory").

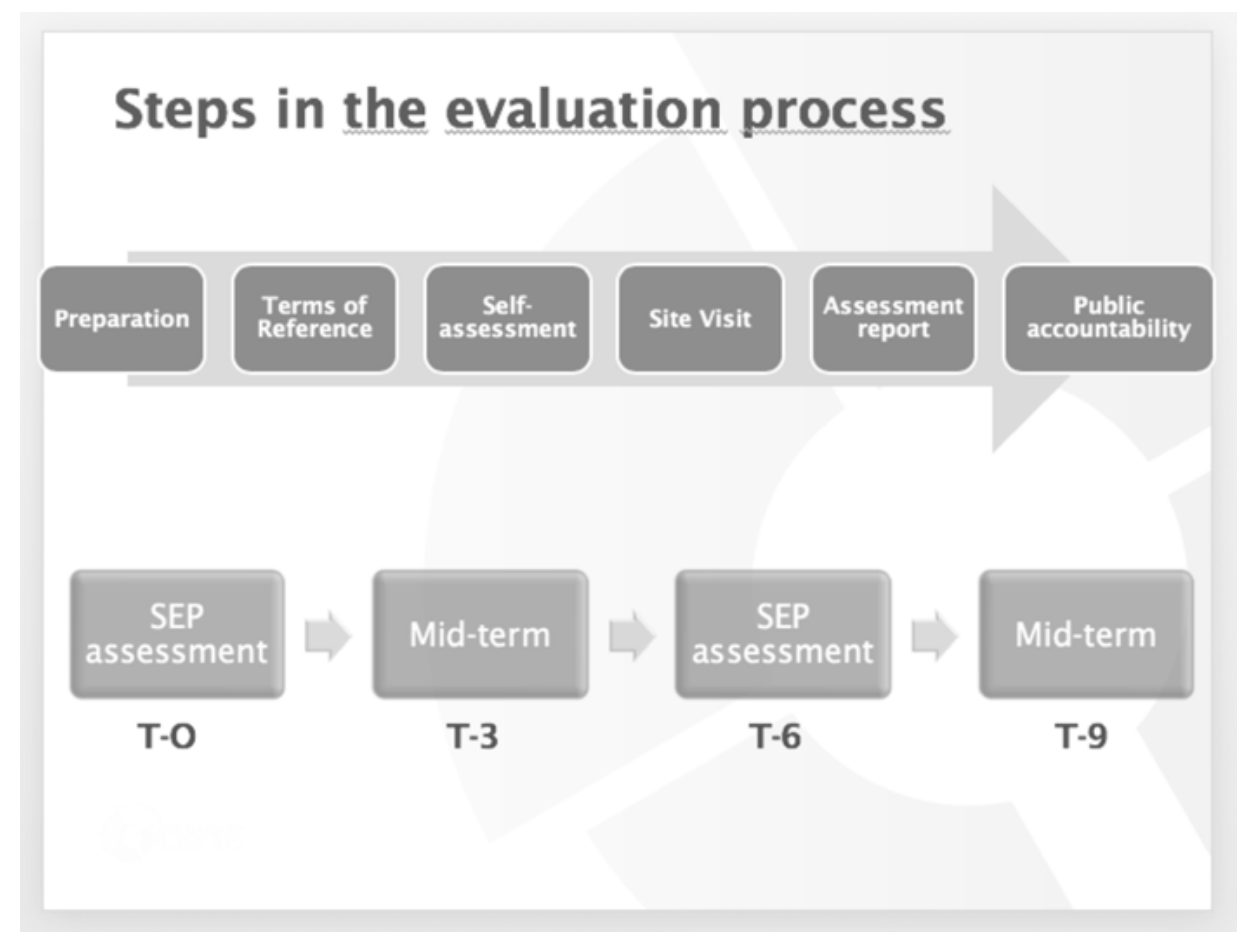

Figure 1. Steps in the Dutch evaluation process.

\begin{tabular}{|l|l|}
\hline $\begin{array}{l}\text { Description of unit's } \\
\text { organisational structure. }\end{array}$ & $\begin{array}{l}\text { Most important (and relevant) } \\
\text { performance indicators. }\end{array}$ \\
\hline Description of unit's financing. & $\begin{array}{l}\text { Results research and societal relevance } \\
\text { past } 6 \text { years (latter in a narrative). }\end{array}$ \\
\hline Strategy past 6 years. & $\begin{array}{l}\text { Link results to SEP criteria (quality, } \\
\text { relevance, viability). }\end{array}$ \\
\hline $\begin{array}{l}\text { Targets past } 6 \text { years (research, } \\
\text { societal relevance). }\end{array}$ & Strategy and targets next 5-10 years. \\
\hline
\end{tabular}

\begin{tabular}{|l|l|}
\hline $\begin{array}{l}\text { Relevant environmental factors } \\
\text { and developments past six years. }\end{array}$ & PhD Programme(s) \\
\hline $\begin{array}{l}\text { SWOT (Strengths, Weaknesses } \\
\text { Opportunities, Threats) analysis } \\
\text { and benchmarking. }\end{array}$ & Research integrity \\
\hline
\end{tabular}

Table 1: Formal requirements for self-assessment report (SEP 20152021). 


\section{2) EVALUATION AS A KNOWLEDGE MAKING PROJECT}

The evaluative inquiry was first introduced as a prompt to more enabling rather than reductive accounts of assessment by Fochler and de Rijcke (2017). The evaluative inquiry understands academic work as a process in which a variety of actors (including non-academic ones) are part of the sociotechnical networks through which knowledge is generated. In emphasising process and engagement rather than rating and ranking, it wants to bring to light the way quality is created and negotiated among multiple participants and amidst multiple epistemic commitments, rather than attributing it to individuals' actions and intentions who are subsequently compared. As such it is aligned with scholarly work that is interested in academic work and quality as it comes into being in interactions between values and networks of people, outputs, and resources (e.g., Bozeman and Sarewitz, 2011; de Jong et al., 2014; Matt et al., 2016; Prins and Spaapen, 2017).

Our team at CWTS is putting the evaluative inquiry into practice in several projects. On the basis of this work, we further specify the inquiry in relation to three contentious issues within the current science system and its evaluation: the much-debated dichotomy between academic and societal realms, the distinction between quantitative and qualitative approaches, and the allegedly invisible and neutral evaluating analyst.

The first issue we identify is the underlying divide in many evaluation frameworks between the academic and the societal. The inclusion of broader impacts into frameworks that originally put most emphasis on academic work has done justice to the interconnections between science and society that are especially strong for the social sciences and humanities. However, the way this relation is often imagined is problematic (cf. Calvert, 2006; de Jong et al., 2014; Felt and Wynne, 2007; Nowotny et al., 2001). It is often envisioned as a linear model of scientific knowledge production that starts with fundamental research and discovery, and ends with innovations that are beneficial for society through translational and applied research. Within this model, the influence of knowledge in society could be traced back to original inventions, the ultimate value of which can then be established. A problem with this model is that the individual (scholar or research institute) remains the locus of both value and responsibility now not only for academic publications, but also for producing societal relevance (Holtrop, forthcoming). Rather than realising that academic work frequently entails engagement with societal actors - and therefore one could argue that both relevance and quality originate in that interaction - one now has to write excellent papers and perform in societally relevant ways as well. The evaluative inquiry problematises the notion of a passive public audience that reaps the benefits of academic expertise, and instead highlights the "productive interactions" (Spaapen and van Drooge, 2011) between diverse stakeholders, and the distributed nature of academic achievement more generally. Regarding the assessment of impact, this would at least entail that audiences are seen not only as (co)producers of knowledge and its impact, but also as (co)producers of the criteria by which such impact is to be evaluated.

The unhelpful divide between spheres and stakeholders is perpetuated by another unhelpful divide: the one between quantitative and qualitative evaluation methods. We recognise the work done in academic and professional environments to problematise reliance on metrics and citation scores alone, and that argues that quantitative and qualitative methods are implicated in one another (cf Callon and Law, 2005). Moreover, initiatives such as the "Leiden Manifesto" (Hicks et al., 2015), the "Metric Tide" (Wilsdon et al., 2015) and "DORA", have presented careful responses and suggestions for next steps. We feel akin to these initiatives, and wish to stay away from the unproductive dichotomy of quantitative and qualitative methods. In our contribution, we move from a fixation on "getting it right" in evaluations, to an approach that presents research numerically, verbally, and/or visually in ways that make visible the complexity of actual practice and its engagements (Fochler and De Rijcke, 2017). This means that evaluative inquiry treats knowledge production as heterarchical (Stark, 2011): it sees phenomena as amenable to multiple orders of worth, rather than as connected to one rank order with clear winners and losers.

Our understanding of the enterprise of academic evaluation changes while we move from a linear model of academic achievement evidenced by individual actions and intentions to an understanding of academic value as situated within multiple epistemic commitments and relations between many actors. Evaluations are now no projects that look into academic worlds from the outside while taking stock of the valuables. Instead they are themselves knowledge producing endeavors, transforming evaluators and analysts into collaborators alongside evaluees. This is the third dichotomy that the evaluative inquiry wants to unsettle: the one between a detached analyst doing representations objectively on the outside and an engaged analyst located within. We build on a previous work that problematises the claim to detachment, objectivity, and neutrality that has characterised dominant modes of research evaluation (Candea et al., 2015; Daston and Galison, 2007). Instead, we take seriously that the act of representing quality is also an intervention (De Rijcke and Rushforth, 2015).

\section{3) THE INQUIRY}

Central to the evaluative inquiry is an understanding of academic achievement as distributed over a host of academic and non-academic participants. These achievements are to be studied by means of a coproduced portfolio approach, tailored to specific research units and evaluation purposes. Each method has its own strong points when it comes to detecting and amplifying reality (Law, 2004). Rather than advocating a combination of methods with the purpose of coming to more accurate representations of academic work, we argue that co-production and multiplication of methods allows for more interesting conversations about academic quality, and offers points of departure for strategically addressing all too real issues of power, money and reputation that are part of academic evaluation. Though the inquiry remains concerned with reaching an adequate understanding of academic achievement (or quality) in the analysis, the approach actively seeks to avoid reproducing the familiar role of the analyst as a detached accountant. Recognising evaluation itself as both an analytical and a strategic project, the analyst thus moves from objective observer into the role of an engaged evaluation expert, not only engaging in the analysis of quality but also in the analysis of the broader political projects of accountability with which it is intertwined. More than working towards a definitive report where research units are assessed on the basis of a predefined set of characteristics, the inquiry is set up to study, map and trace the research themes, pathways and productive interactions around the research unit through 
a portfolio of methods. Practically, the inquiry takes the form of three consecutive phases (see table 2).

\begin{tabular}{|l|l|}
\hline Phases & Approach \\
\hline 1. Exploration & $\begin{array}{l}\text { What is at stake in the assessment? } \\
\text { Questions addressed in document analyses } \\
\text { and interviews with management. }\end{array}$ \\
\cline { 2 - 3 } & $\begin{array}{l}\text { Design of research approach, choice } \\
\text { of combination of methods. }\end{array}$ \\
\hline 2. Data collection and Analysis & $\begin{array}{l}\text { \# (e.g.) "Contextual Response Analysis" (Prins, n.d.); } \\
\text { contextual scientometrics (Waltman and van Eck, } \\
\text { 2016); bibliographic coupling; co-citation analyses; } \\
\text { "Area Based Connectedness" (Noyons, 2018) } \\
\text { \# Interviews with researchers and stakeholders } \\
\text { regarding organisational and academic themes, } \\
\text { operationalisation, outputs and impacts. }\end{array}$ \\
\cline { 2 - 3 } & $\begin{array}{l}\text { Workshops - to test hypotheses, present themes } \\
\text { and pathways, collect more input for SWOT-analysis. }\end{array}$ \\
\hline 3. Reporting & $\begin{array}{l}\text { Analysis in terms of organisational issues } \\
\text { and academic ambitions and themes. SWOT. } \\
\text { Suggestions as to how to write the self-evaluation. }\end{array}$ \\
\hline
\end{tabular}

Table 2: Phases in an evaluative inquiry.

An evaluative inquiry is geared towards detecting lively interactions and outcomes, which can be enveloped in themes and 'pathways' (loosely based on Matt et al., 2016), thereby connecting academic and societal domains. In past projects, we have built these themes and pathways by using a combination of quantitative methods, interviews and workshops to collect information about the themes people work on, the resources and people that are mobilised in their research projects, the highly varied outputs that are generated as a result, and the way these outcomes travel elsewhere into other academic, professional or societal realms (see table 2). Though the inquiry allows for different combinations of methods, one form this combination can take is that of a generative dialogue. Interviews and quantitative analyses are first used to gather information, for example with regards to themes that researchers and stakeholders consider central to the work and the variable audiences that it reaches (or fails to reach). The outcomes of this first round of inquiry are used to identify a first set of possible themes and pathways, which are then presented back to the research unit, for example in a collective workshop. The presentation is meant to elicit further response, and allows those involved to think with and elaborate on the first results in a collective setting with colleagues, stakeholders and the analysts. The final report is subsequently written in terms of the organisational issues and the academic ambitions and themes that have emerged. This could for example include an interactive analysis and visualisation of prevalent themes and ambitions, their operationalisation, the people and resources that are mobilised, the outputs this generates and the way these are cited, used, and travel further into the world. The outcomes of this type of detailed interaction with individual researchers, research leaders and their work can be added to the information that is gathered in the analysis of organisational issues represented in a SWOT. Organisational documents and data can be combined with insights gained in individual interviews or workshops, adding additional depth and possibly room for creative synergy between people and data. Crucially, these processes and roles are scripted together, so as to enable both a highly rigorous and a highly grounded analysis.
The subsequent self-assessment document is authored by the research unit itself. Our report is written in such a way that it can be a conversation piece and offers openings for discussion - internally, and with other academic institutes, science policy environments and stakeholders interested in academic quality. The inquiry can, but not necessarily does, fix the state of the object of evaluation in a definitive account. Moreover, the outcomes of the inquiry are in this sense not limited to the report. Individual elements of the evaluative inquiry itself, like the workshop, are excellent tools to bring the organisation and/or stakeholders together and collaboratively identify problems, make tough decisions, work on solutions, or plan for the future. They trigger meaningful conversations about how to deal with pressing challenges such as the increasing roles and demands of peer communities, professional and societal partners, government or industry while building on individual and institutional strengths. The plurality of actors involved can take on a variety of roles throughout the inquiry. Staff members and stakeholders can be consciously drawn into the production process, being in some ways the experts and authoritative analysts on the values and interrelations of the work. The analysts, in turn, are more than outsiders who merely "run" pre-set quantitative or qualitative analyses: they become active co-producers of the inquiry.

\section{DISCUSSION: EVALUATION AS KNOWLEDGE PRODUCTION}

The notion of "impact" suggests an interaction of which the source, target and content are clearly identifiable. Traditional frameworks for evaluation tend to focus on whether and to what extent such impact is achieved. The concept of evaluative inquiry, as developed in this paper, revises this linear notion of impact as the central precept of research evaluation. The framework builds on work by e.g. Matt et al. (2016), Spaapen and Van Drooge (2011), and Prins and Spaapen (2017), by conceptualising scholarly work not in terms of a linear diffusion of knowledge, but rather as an emergent effect of an unfolding, multidirectional research process. Evaluative inquiry reveals the epistemic commitments and community values of local practices. It thus essentially approaches evaluation as a knowledge production process. From this starting point, our approach to evaluation sees the relevance of scientific work as an unfolding process, in which a variety of academic and non-academic actors are involved. This approach emphasises process and engagement rather than accounting and ranking. Crucially, evaluative inquiry identifies values, networks of people, and resources as collectives. It helps articulate how "worlds" are created and negotiated in relation to these values.

With evaluative inquiry, we thus move away from evaluations as detached, clear delineations of academic value. Researchers obviously do not just produce excellent research or articles in journals, or even knowledge that is of use for society at large. More than that, they are prominent world-makers, and their knowledge has consequences for the world they and others inhabit as well as their experiences in it. This suggests that diverse (relational, communicative, organisational) values, activities and outcomes have to be taken into account in evaluations. Conventional approaches to research assessment treat these values hierarchically. This works well within accountability systems that embed actors and actions in fixed and calculable value regimes. Both bibliometrically framed assessments and assessments of societal impact operate through a quite 
similar logic of "return-on-investment". An evaluative inquiry, in contrast, is not meant to result in one definitive document that "makes up the account". In the mode of evaluative inquiry, standardisation is less relevant than staying close to the epistemic missions, frictions and resonances of academic work. The approach understands academic performance or impact as an effect of translations within and between networks of actors that make up academic research and its environments (cf. Matt et al., 2016). The aim is to find out what are the central issues or ambitions, how they are operationalised, what kind of outcomes this yields and where the outcomes travel to. Evaluative inquiries broaden our understanding of what counts as academic achievement. They afford a greater inclusivity of research settings, which ideally means a more meaningful treatment of the social sciences and humanities. As such, our contribution is meant to give space to, and reinforce the greater role to be played by, the social sciences and humanities, including the fields of research evaluation and scientometrics. The social sciences and humanities have the conceptual tools to enrich the methodological portfolio for gathering information about the worlds that academics inhabit and contribute to. A pluralisation of perspectives and methods enriches the inquiry by opening up what can be talked about in evaluations. This pluralisation should not happen in secluded spaces such as scientometric labs, but with the participation of stakeholders, so as to take in consideration their contexts (cf. Rafols, 2018). Furthermore, evaluative inquiry is sensitive to how value systems might differ across teams an organisations, and evolve over time. This approach makes it possible to articulate positions, roles and values that are subordinate to dominant currents in academic practice and that are often silenced in traditional evaluations.

In conclusion, with the evaluative inquiry we fully subscribe to the call for "re-loading" the notion of impact (König et al., 2018), a notion with problematic ballistic connotations. We hope our contribution feeds ongoing discussions among academics, policy-makers, and other stakeholders about the fault lines between forms of value, the uncertainties in evaluating, and the politics of formats, protocols and endings. Our contribution is a strong plea to create more room for experiments in research evaluation (and it is clear that we are not done experimenting ourselves). We think this simultaneously entails: 1) advocating and conducting rigorous analytical work; 2) a willingness of those under assessment to be open to more engaged modes of assessment; 3 ) using the full potential of the form(s) evaluation can take; and 4) using quantitative methods in much more interesting ways. Rather than taking an a-priori, reductive approach to what counts in research evaluation - think of the proliferation of publication lists and performance metrics - it is much more useful to produce and present the multiple meanings and purposes of research. Evaluative inquiry takes evaluation itself as a deliberative, generative process of knowledge production in its own right. In doing so, it opens up more than one way for empirical data, evaluators, and other actors to be implicated in the evaluation. The generative capacity of the inquiry is partially built on keeping more open the roles of the various coproducers, and the evaluative criteria that may be generated from their variable positions. This also means that the legitimacy of the evaluation is not solely based on the analyst's correct implementation of criteria, but much more so on the degree to which co-producers think that the process and results do justice to their joint work.

\section{REFERENCES}

Association of Universities in the Netherlands (VSNU), the Netherlands Organisation for Scientific Research (NWO) and the Royal Netherlands Academy of Arts and Sciences (KNAW). Standard Evaluation Protocol (SEP) 2015-2021. Protocol for Research Assessments in the Netherlands.

Bozeman, B. and Sarewitz, D. (2011). Public Value Mapping and Science Policy Evaluation. Minerva, 49(1), 1-23.

Calvert, J. (2006). What's Special about Basic Research? Science, Technology \& Human Values, 31(2), 199-220.

Callon, M. and Law, J. (2005). On Qualculation, Agency and Otherness. Environment and Planning D: Society and Space, 23(5), 717-33.

Candea M., J. Cook, C. Trundle and Yarrow T. (Ed.) (2015). Detachment: Essays on the Limits of Relational Thinking. Manchester: Manchester University Press.

Daston, L. and Galison, P. (2007). Objectivity. Cambridge, The MIT Press.

De Jong, S., Barker, K., Cox, D., Sveinsdottir, T. and van den Besselaar, P. (2014). Understanding societal impact through productive interactions: ICT research as a case. Research Evaluation, 23(2): 89-102.

De Rijcke, S. and Rushforth, A. (2015). To intervene or not to intervene, is that the question? $0 \mathrm{n}$ the role of scientometrics in research evaluation. JASIST, 66 (9), 1954-1958.

Felt, U. and Wynne, B. (2007). Taking European Knowledge Society Seriously. Report of the Expert Group on Science and Governance to the Science, Economy and Society Directorate, Directorate-General for Research, European Commission. Luxembourg: Office for Official Publications of the European Communities. Retrieved November 1st, 2018 from: https://ec.europa.eu/research/science-society/document_library/ pdf_06/european-knowledge-society_en.pdf

Fochler, M. and De Rijcke, S. (2017). Implicated in the Indicator Game? An Experimental Debate. Engaging Science, Technology, and Society, 3, 21-40.

Hansson, F. and Monsted, M. (2012). Changing the peer review or changing the peers - Recent developments in assessment of large research collaborations. Higher Education Policy, 25(3): 361-379.

Holtrop, T.J. (forthcoming). The Evaluative Inquiry: A New Approach to Research Evaluation. Blogpost, forthcoming at http://www.cwts.nl/blog.

König, T., Nowotny, H. and Schuch, K. (2018). Impact Re-loaded. Pathways to impact from SSH research. CfP SSH Impact Conference Vienna. Retrieved 2 November, 2018 from: https://www.ssh-impact.eu/ wp-content/uploads/2018/06/Impact_Re-loaded_180614.pdf

Law, J. (2004). After Method: Mess in Social Science Research. London: Routledge.

Matt, M., Gaunand, A., Joly, P.-B. and Colinet. L. (2016). Opening the black box of impact - Ideal-type impact pathways in a public agricultural 
research organization. Research Policy, 46, 207-218.

Molas-Gallart, J. (2012). Research Governance and the Role of Evaluation: A Comparative Study. American Journal of Evaluation, 33(4), 583-98.

Molas-Gallart, J., D'Este, P., Llopis, 0. and Rafols, I. (2015). 'Towards an alternative framework for the evaluation of translational research initiatives', Research Evaluation, 25(3), 235-243.

Nowotny, H., Scott, P. and Gibbons, M. (2001). Rethinking science: knowledge in an age of uncertainty. Cambridge: Polity.

Noyons, E. (2018). Monitoring how science finds its way into society: measuring societal impact through area-based connectedness (ABC). Blogpost. Retrieved November 1st, 2018 from: https://www.cwts.nl/ blog?article $=n-r 2 u 264 \&$ title $=$ monitoring-how-science-finds-its-wayinto-society-measuring-societal-impact-through-area-based-connectedness-abc

Prins, A. A. M. and Spaapen, J. (2017). Serving variegated audiences: From ranking oriented evaluation to mission oriented evaluation. fteval Journal for Research and Technology Policy Evaluation, 44 (September), $42-49$.

Prins, A. (n.d.). Contextual Response Analysis. Retrieved November 1st, 2018 from http://www.adprins.nl/index.php?id=box2

Rabinow, P. (2011). The Accompaniment: assembling the contemporary. Chicago: University of Chicago Press.

Rafols, I. (2018). S\&T indicators 'in the wild': contextualisation and participation for responsible metrics. Blogpost. Retrieved November 3rd, 2018 from: https://www.cwts.nl/blog?article=n-r2u2548title=st-indicatorsin-the-wild-contextualisation-and-participation-for-responsible-metrics

Rushforth, A.D. and De Rijcke, S. (2015). Accounting for Impact? The Journal Impact Factor and the Making of Biomedical Research in the Netherlands. Minerva, 53, 117-139.

Spaapen, J. and Van Drooge, L. (2011). Introducing Productive Interactions in Social Impact Assessment. Research Evaluation, 20 (3), 211-18.

Stark, D. (2011). The Sense of Dissonance: Accounts of Worth in Economic Life. Princeton, NJ: Princeton University Press

Waltman, L., and Van Eck, N. J. (2016). The Need for Contextualized Scientometric Analysis: An Opinion Paper. In I. Ràfols, J. Molas-Gallart, E. Castro-Martínez, and R. Woolley (Eds.), Proceedings of the STI Conference 2016. Peripheries, Frontiers and beyond (pp. 1-9). Valencia, Spain: Universitat Politècnica de València.

Youtie, J. and Corley, E. (2011) Federally Sponsored Multidisciplinary Research Centers: Learning, Evaluation, and Vicious Circles. Evaluation and Program Planning, 34, 13-20.

Whitley, R. (2007). Changing Governance of the Public Sciences. In R. Whitley and J. Glaeser (Eds), The Changing Governance of the Sciences.
Sociology of the Sciences Yearbook (pp. 3-27). Dordrecht: Springer.

\section{AUTHORS}

[Numbers] CASRAI CRediT taxonomy for contributor roles (https://casrai. org/credit/)

\section{SARAH DE RIJCKE $[1,6,7,10,12,13,14]$}

Centre for Science and Technology Studies (CWTS), Leiden University P.0.Box 905, Leiden, 2300 AX (The Netherlands)

E: s.de.rijcke@cwts.leidenuniv.nl

\section{TJITSKE HOLTROP $[1,5,6,13,14]$}

Centre for Science and Technology Studies (CWTS), Leiden University P.0.Box 905, Leiden, 2300 AX (The Netherlands)

E: t.j.holtrop@cwts.leidenuniv.nl

\section{WOLFGANG KALTENBRUNNER ${ }^{[13,14]}$}

Centre for Science and Technology Studies (CWTS), Leiden University P.0.Box 905, Leiden, 2300 AX (The Netherlands)

E:w.kaltenbrunner@cwts.leidenuniv.nl

\section{JOCHEM ZUIJDERWIJK ${ }^{[5,6,13,14]}$}

Centre for Science and Technology Studies (CWTS), Leiden University P.0.Box 905, Leiden, 2300 AX (The Netherlands)

E: j.b.zuijderwijk@cwts.leidenuniv.nl

\section{ANNE BEAULIEU ${ }^{[13]}$}

University of Groningen, Campus Fryslan (CF)

Sophialaan 1, 8911 AE, Leeuwarden (The Netherlands) and

CWTS, Leiden University

P.0.Box 905, Leiden, 2300 AX (The Netherlands)

E: j.a.beaulieu@rug.nl

E: j.a.beaulieu@cwts.leidenuniv.nl

\section{THOMAS FRANSSEN $[5,6,13]$}

Centre for Science and Technology Studies (CWTS), Leiden University P.0.Box 905, Leiden, 2300 AX (The Netherlands)

E: t.p.franssen@cwts.leidenuniv.nl

\section{THED VAN LEEUWEN ${ }^{*}[5,6,13]$}

Centre for Science and Technology Studies (CWTS), Leiden University P.0.Box 905, Leiden, 2300 AX (The Netherlands)

E: leeuwen@cwts.leidenuniv.nl

\section{PHILIPPE MONGEON ${ }^{[13]}$}

Danish Centre for Studies in Research and Research Policy, Aarhus

University

Bartholins Allé 7

DK-8000, Aarhus, C, Denmark and

CWTS, Leiden University

P.0.Box 905, Leiden, 2300 AX (The Netherlands)

E:philippe.mongeon@ps.au.dk

E: p.mongeon@cwts.leidenuniv.nl

\section{CLIFFORD TATUM[1, 13]}

Centre for Science and Technology Studies (CWTS), Leiden University 
P.0.Box 905, Leiden, 2300 AX (The Netherlands)

E: c.c.tatum@cwts.leidenuniv.nl

\section{GOVERT VALKENBURG ${ }^{[13]}$}

Department of Interdisciplinary Studies of Culture, Norwegian University of Science and Technology (NTNU)

N0-7491 Trondheim, Norway

E: govert.valkenburg@ntnu.no

\section{PAUL WOUTERS ${ }^{[13]}$}

Centre for Science and Technology Studies (CWTS), Leiden University P.0.Box 905, Leiden, 2300 AX (The Netherlands)

E: p.f.wouters@cwts.leidenuniv.nl

\section{KEYWORDS:}

Research evaluation; societal relevance; intervention; mixed-methods; engagement; heterogeneity 


\title{
VALUATION OF SSH RESEARCH FOR A TRANSFORMATIVE EUROPEAN RESEARCH AGENDA - A FEW CLOSING WORDS
}

\author{
KLAUS SCHUCH \\ DOI: 10.22163/fteval.2019.387
}

\section{INTRODUCTION}

A s called upon by the promoters of the Austrian Presidency of the EU Council Conference on 'Impact of Social Sciences and Humanities for a European Research Agenda - Valuation of SSH in mission-oriented research' it is time to re-load the notion of impact of Social Sciences and Humanities (SSH) and to shift away from the traditional pre-dominant defensive stance which SSH research often articulates in the discussion about the impact of research. Doubtlessly, most arguments raised by critical SSH researchers are relevant such as the too narrow focus on economically relevant technologies and innovation, but a defensive stance not accompanied by positive propositions would inevitably lead to a marginalised position which is sometimes already now met with suspicion from policy-makers, but also from fellow colleagues of the so called 'hard sciences'. Thus, instead to dwell on the embodiment of 'integrating' SSH into dominantly technologicallyminded projects too long, SSH researchers should shift the notion and the promotion to equally valuated contributions of SSH to transformative inter-disciplinary research with SSH at eye-level.

This also means to push forward SSH into a driver-seat in addressing grand challenges and in implementing mission-oriented research in Horizon Europe. The challenges are grand because they concern our societies and cultures. Challenges can and should not only be met by providing technological fixes, but by investigating their socio-economic and cultural embedding and structural fundaments and by aiming to identify solutions which address, reflect, reframe and eventually also challenge and change these underlying structures. By doing so, SSH research can provide disruptive contributions to break-up with traditional ways of doing things. The political economy in any grand challenge can become scrutinised, but it also needs to be addressed in calls launched under transformative research agendas. This is e.g. true for the political economy of climate change, or the political economy of transportation or of health research. The often raised differentiation between an instrumental understanding of $\mathrm{SSH}$ and a reflexive understanding of SSH is not helpful in this respect and has to be overcome in transformative research because both aspects ('instrumental' and 'reflexive') are important. Finally it also needs to be repeated, that innovation and value creation is not just the scope of R\&D, sales and marketing (or of Pillar 3 in Horizon Europe), but a social process with various social implications that can be addressed by fields such as anthropology, cultural studies, education, sociology or human and economic geography.

\section{A LOOK BACK ON SSH IN HORIZON 2020}

Around the peak of the financial and economic crisis around 10 years ago, national research budgets were cut due to financial constraints in several countries (Schögler and König, 2017; EUA, 2011;). These cuts were often also addressing the Social Sciences and Humanities (SSH) (Marimon et al, 2011, Papanagnou, 2011). Moreover, in the dawn of Horizon 2020, the EU's 8th Framework Programme for RTD, the role of Social Sciences and Humanities (SSH) within the world's largest competitive research programme was downgraded too (see König, 2019 in these proceedings). It was politically intended to mainstream SSH across the entire Horizon 2020 (European Parliament and Council, 2013) with the consequence of abolishing the specific sub-programme dedicated to SSH topics. These attempts, however, met resistance from parts of the SSH communities because 'mainstreaming' often results in 'ceding'. An Open Letter was signed by almost 26,000 people (Rammel et al., 2017), and some of the research ministers from the EU member states were successfully mobilised to express their concerns against this 'mainstreaming', which was frequently perceived as 'downsizing' of SSH (see König, 2019 in these proceedings).

The protest was relatively successful. SSH research is within Horizon 2020 now covered by six panels in the European Research Council, has a dedicated slot within the Societal Challenges Pillar of Horizon 2020 (however with a pitying marginal budget) and is more or less (with emphasis on 'less') sufficiently mainstreamed across the Societal Challenges Pillar.

Also the overall mind-set seems to have changed because the inclusion of SSH with a dedicated topical niche within the next edition of the European Framework Programme for Research and Innovation (in addition to its place in the ERC and the still valid mainstreaming approach) was politically almost not contested anymore. This mind-set change, however, does not materialise in a considerable larger SSH budget for its topical niche, but might rather be an indication of a more sober expectation management by Ryl policy makers in that sense, that technological fixes without proper consideration of human conditions are not sufficient for tackling grand challenges and inducing transformational changes.

The idea behind this observable mind-change seems to refer primarily to attributing an enhanced support or leverage function of SSH to a more 'society-ready' technological development, in order to avoid waste of re- 
sources and idle capacities. In this line of argument, the focus remains on the cooperation of SSH with technology-oriented disciplines rather than on strengthening genuine SSH topics in Horizon Europe, the EU's next European Framework Programme for RTD (2021-2027). The narrative about the potential leveraging function of SSH was already promoted in Horizon 2020 in line with the 'integration/mainstreaming' approach. In fact, and this should be appreciated, Horizon 2020 was a pioneer in this respect, while most national technology-oriented programmes still lack a clear commitment to include SSH research strategically, although one can frequently identify SSH related methods, RRI aspects, and claims of social challenges as well as impacts subcutaneously in industry-oriented applied R\&D programmes too as evidenced by a study about SSH aspects in projects funded by the Austrian Research Promotion Agency (Sturn and Schuch, 2018).

The popular narrative of the auxiliary function or contribution of SSH to technology-based innovation processes is often framed in the context of inter- and trans-disciplinary challenges (see Graf, 2019 in these proceedings). Especially trans-disciplinarity, which features outreach to and inclusion of non-academic stakeholders and of non-formalized knowledge, is a competence which is sometimes credulously assigned to SSH researchers because of their perceived proximity to social spheres. This cross-academic approach is strongly featured in the pillar "Global Challenges and Industrial Competitiveness" of Horizon Europe, which should 'encourage cross-disciplinary, cross-sectoral, cross-policy and cross-border collaboration in pursuit of the UN SDGs and the competitiveness of the Union's industries therein.' (European Commission, 2018; p. 17).

This understanding of the leverage function of SSH requires that SSH researchers are capable and professional in meeting and applying state of the art involvement tools. The contribution of SSH to more technological oriented projects and its peculiar value is basically perceived as a project steering and outreach competence, especially if issues of the normal course of life and/or the inclusion of non-academic audiences (e.g. stakeholders, users) are concerned. This understanding became a partially shared reality in many Horizon 2020 projects. In certain research fields (such as "Public Health and Sustainable Development") the use of transdisciplinary tools is daily business. Often social scientists are charged with engagement processes by applying a variety of process tools such as design-thinking, participatory technology development or multistakeholder workshops.

\section{FROM INSTRUMENTAL TO COMPREHENSIVE SSH CONTRIBUTIONS IN MISSION- ORIENTED RESEARCH}

Clearly, many SSH researchers regard this overall approach to treat SSH research as an auxiliary (or 'instrumental') resource for technological projects which address the grand challenges, often as an improper reduction of SSH. This view is not far-fetched, because the grand challenges are grand since they concern human societies and cultures, the ways how we humans interact with each other but also with our environment, how we produce and consume, how we construct meaning and judgement to our actions, and how we reproduce our societies and cultures but also how we change them and our behaviour. Before asking how SSH can mitigate the effort of technological adaptations to social conditions, needs and wants, thus contributing to an innovation race which continuously seems to pick up pace, SSH should also be employed to frame and analyse the wicked problems before a technological solutionism approach (Morozov, 2013) is taken. Unfortunately, calls for proposals seldom ask for this. Especially in Horizon 2020 the scope and the expected impacts of the topics called for, are usually very specifically described in detail and often more oriented towards an end (i.e. a specific output, solution or impact) than a proper problem analysis. Although it could be argued, for instance, that any topic addressed under Horizon 2020 (from "A" like agriculture, "B" like bio-economy, "C" like climate change to "Z" like zero-waste) would at least deserve a proper analysis of the political economy underlying these topics.

Instead, technological solutionism promises quick results and profits and is positively connoted with an attractive entrepreneurial 'hooray - let's go for it' image, which has undermined and captured research policy-making since more than 30 years and which led to the "holy duality" of research and innovation. The concept of "societal readiness levels' is fitting this auxiliary understanding of SSH to leverage the social acceptance of technologies. It should absolutely not be denied that SSH can be very useful in this respect. On the contrary, usage of novelty and accompanying market pervasion (which is the economic essence of innovation) is a social process with various social implications. Innovation research thus can be a subject of business economics, but also of anthropology, cultural studies, political sciences, sociology, economic and human geography and so forth.

Innovation is basically anything new that creates some form of value; and there is no value creation without some sort of uptake. Value can be an economic one but it should not be limited to it. Thinking about innovation should not be reduced to its technical substance, which is often associated with the notion of innovation per se, often in combination with economic value creation, which belittles the contribution of SSH (Bell, 2019 in these proceedings). In fact, these days we are more and more used to think about different types of innovation such as business-model innovations, organisational innovations, public sector innovations, and social innovations. And we know since the fundamental works of Schumpeter, that (some) innovations have the potential to transform the way we live and the things we do, socially and culturally as well as economically.

After some naive R\&I policy 'gold rush years', characterised by a simplistic understanding of the relationship between research and innovation as linear process in which research is expected to lead to ever higher Technology Readiness Levels (Bell, 2019), there is also increasing awareness that the idea of public support for 'research and innovation' should be to support the right innovations and not innovations per se. What "right" means depends on a plethora of views, principles and beliefs and should never be decided in isolation. The important thing here is to understand, that innovation is not only the business of business, but also the business of society. And as a business of society it also becomes a business of SSH research. Bell (2019) calls in this respect for a genuine and broad added-value of SSH for transformative research, starting with the "what if" question, constructing alternative scenarios and by considering also the non-material features of human existence. He furthermore claims that SSH can provide strong contributions to make transformations happen.

The orientation on grand challenges, energised by the 'missions concept' in Horizon Europe (Mazzucato, 2018) can be regarded as an indica- 
tion of the updated emphasis on the directionality of R\&l policies to tackle the 'right' issues (e.g. connected with sustainability and/or inclusiveness concerns) with the intention of selecting the adequately 'right' R\&I projects in service of society. The Lamy-Report (2017) stipulated the need to develop adequate impact oriented RTI policy designs and made the claim that SSH should also act as driver for some missions of the next Framework Programme for RYl (and as contributor to others). In line with this, the European Parliament (2017) argued for a broader and clearer definition of impact by raising awareness on societal, cultural and long-term impacts, while the Estonian EU Council Presidency (2017) urged in general for a more sophisticated and dynamic approach to impact assessment. These political claims (and others) were taken-up and addressed during the Austrian Council of the EU Presidency Conference on the 'Impact of SSH for a European Research Agenda - Valuation of SSH in mission-oriented research', which was organised by the author of this article in Vienna end of November 2018 (König, Nowotny and Schuch, 2019 in these proceedings; Reiter-Pazmandy, 2019 in these proceedings).

During the conference it became clear that there is widespread accord among the SSH communities that the impact of SSH research is more direct on society than from other research disciplines, although not necessarily more evident or tangible. This seems to be a basic contradiction, which should be solved to overcome disaccord and resistance. The impact of SSH research is more direct because the social subsystems 'Culture', 'State', and 'Market' are very often in the focus of SSH research. SSH researchers thus sometimes claim that they, by purpose, are closer to issues such as societal impacts, structuring impacts on policy-making and policies (i.e. political impact) as well as impacts on innovation and economy (see among others Flecha, Soler-Gallart, and Sordé, 2015; Brewer, 2013). For the sake of orientation, Reale, E. et al. (2017) provide a definition of scientific, social and political impact, stating that "SSH research generates scientific impact when it influences the production of further research outputs following new approaches for analysis or based on new results. Changes related to social impact affect the cultural, economic, and social life of individuals, organizations, and institutions. Political impact incorporates the contents of research into political decisions, and motivations and rationales for political action and priority setting."

\section{TRACING AND MEASURING IMPACT OF SSH RESEARCH}

Impact tracing, however, is a complex exercise, because it is contextspecific and there are different understandings of valuation narratives and theories of change of SSH research, which by themselves request thorough understanding of processes and methods in the phases of knowledge production (e.g. co-design and co-creation; inter- and transdisciplinary approaches; citizen science;), knowledge dissemination (e.g. tailor-made transfer mechanisms and formats; media engagement) and knowledge usage (e.g. social innovation; policy advice; evaluation and accompanying scientific research; research integrated road mapping; [public] service engineering etc.) with all their particular challenging aspects. Beck and Bonß (1989) even claimed that interpretation offers provided by social sciences are practically most successful, when the seemingly vanish without trace in the consciousness of everyday life and policy. What makes impact measurement of SSH research even more complex is the fact that standardised indicators of usage beyond the sci- entific impact dimension are missing. For instance, SSH research does hardly result in patent applications. So far, existing assessments of the social impact of RTI political interventions are often only contextual and specific as well as qualitative and anecdotal in nature (Van den Besselaar et al, 2018; Raua, Gogginsb and Fahyb, 2018; Barré, 2010).

Also the judgments on the value-for-society vary (Reale et al., 2017). Despite the fact that SSH scholarship is often committed to do research for the good of society, the interest of researchers is often not oriented towards producing usable results, but rather to raise awareness and influence society to create capabilities of self-understanding in different contexts (Reale et al., 2017; Benneworth, 2015; Nussbaum, 2010).

Social impact measurement, which - and this is important to note concerns all scientific disciplines and sciences - remains an unresolved issue in technical terms too. The complexity goes far beyond monetarised approaches such as SROI (Social Return on Investments) or SMEV ('Socially Modified Economic Valuation' approach). Tracing, assessment and measuring (centred on the core question 'What is the evidence for observed effects?') is not only complex but also exacerbated by metric problems (which apparently materialise at the level of indicators and data basis). Social impact assessments thus focus often more on processes than on results (e.g. engagement with business, government, the third sector, and the public via the media as a proxy for social impact) (Bastow, Dunleavy, Tinkler; 2014). Moreover, there is often a falsified equation of social impact with dissemination or transfer, to which most of the socalled alternative metrics (altmetrics) focus. In Horizon Europe, mostly process and output indicators will be applied to trace the societal impact and policy impact (Van den Besselaar et al., 2018).

Particular challenges for the development of appropriate indicators to measure societal impact include firstly, that the time taken to achieve the actual impact on society is longer than the achievement of concrete results; secondly, that the assignment of social changes is more difficult than the assignment of scientific references or economic attributes. And, thirdly, that the availability and comparability of data to track social and political impacts is severely limited. So far, however, the tracing, assessment and metric question of social and political impact seems to be more discussed and forwarded by the domains of research policy and evaluation research while most SSH researchers from academia have only partially adopted it as their own.

\section{CLOSING WORDS}

The five missions announced by the European Commission in summer 2019 do not indicate a big change. They all relate to important challenges, for which SSH can make contributions, but not in the driver seat. On the other hand, however, SSH research will also have to keep its promise in thousands of ways to find a new level of interaction with society. This refers to the claim made by König, Nowotny and Schuch, 2019 in these proceedings) as starting point for organising the Austrian Council of the EU Presidency conference on 'Impact of Social Sciences and Humanities for a European Research Agenda - Valuation of SSH in mission-oriented research', that 'transformative science must be transformative in a double sense: wanting to exert influence in society but also open to be influenced by society and its needs.'

Such an exercise is not easy and will demand a lot of efforts and creativity. There are for instance still several areas, where the relation of SSH to society is less expressed than one would assume. Such shortcomings 
can for instance be found with respect to citizen science or social innovation (Howaldt, 2019 in these proceedings; Anderson, Domanski and Howaldt, 2018; Howaldt et al. 2016), although we find a long tradition of action research which stimulated social action (Gustavsen, 2012), which, however, should not be equalized with social innovation.

These shortcomings, however, also have structural reasons, such as the comparatively low funding fondness towards SSH-driven citizen science projects. Also in the area of social innovation, one can hardly find material or immaterial professional structures within most higher education and non-university research organisations for supporting social innovation. Examples like the "6l research model" at the University of Deusto (Caro-Gonzalez, 2019 in these proceedings) or the Knowledge Transfer Centre for SSH in Austria (Russegger, 2019 in these proceedings) are still the exception and not the rule.

\section{LITERATURE}

Anderson, M. M., Domanski D. and Howaldt, J. (2018): Social Innovation as a chance and a challenge for Higher Education Institutions. In J. Howaldt, C. Kaletka, A. Schröder and M. Zirngiebl (Ed.), Atlas of Social Innovation. New Practices for a Better Future (pp. 50-53). Dortmund: Sozialforschungsstelle, TU Dortmund University.

Bastow, S., Dunleavy, P. and Tinkler, J. (2014): The Impact of the Social Sciences. Los Angeles-London-New Dehli-Singapore-Washington DC: SAGE.

Beck, U. / Bonß, W. (Hrsg), 1989: Weder Sozialtechnologie noch Aufklärung? Analysen zur Verwendung sozialwissenschaftlichen Wissens. Frankfurt: Suhrkamp.

Bell, J. S. (2019): The importance of SSH research in Horizon Europe. Fteval Journal for Research and Technology Policy Evaluation, Issue 48, June 2019, pp. 48-51. D0I: 10.22163/fteval.2019.366

Benneworth, P. (2015): Tracing How Arts and Humanities Research Translates, Circulates and Consolidates in Society. How Have scholars Been Reacting to Diverse Impact and Public Value Agendas? Arts and Humanities in Higher Education, 14/1: pp. 45-60.

Brewer, J. (2013): The Public Value of Social Sciences. An Interpretative Essay. Bloomsbury.

Caro-Gonzalez, A. (2019): The "6l Research Model": Evolution of an Innovative Institutional STI Policy Framework at the University of Deusto. Fteval Journal for Research and Technology Policy Evaluation, Issue 48, June 2019, pp. 104-112. DOI: 10.22163/fteval.2019.376.

Estonian Presidency of the Council of the EU (2017): Tallinn Call for Action 2017. Seize the opportunity now: research and innovation matter for the future of Europe. Statement of the Estonian Presidency of the Council of the EU.

EUA (2011): Impact of the economic crisis on European universities. European University Association, January 2011.
European Commission (2018): Proposal for a Regulation of the European Parliament and of the Council Establishing Horizon Europe (COM(2018) 435 final, 2018/0224 (COD)), 17 (URL: https://ec.europa.eu/commission/ sites/beta-political/files/budget-may2018-horizon-europe-regulation_ en.pdf, accessed on 31.10.2018.

European Parliament (2017): Report on the assessment of Horizon 2020 implementation in view of its interim evaluation and the Framework Programme 9 proposal (2016/2147(INI)).

European Parliament and Council (2013). Regulation (EU) No 1291/2013 of 11 December 2013 establishing Horizon 2020, Annex I, 121 (URL: https://eur-lex.europa.eu/LexUriServ/LexUriServ.do?uri=0J:L:2013:347:010 4:0173:EN:PDF, accessed on 31.10.2018.

Flecha, R., Soler-Gallart, M. and Sordé, T. (2015): Social Impact: Europe Must Fund Social Science. Nature, 528: 193. Doi: 10.1038/528193d.

Graf, J. (2019): Bringing Concepts Together: Interdisciplinarity, Transdisciplinarity and SSH Integration. Fteval Journal for Research and Technology Policy Evaluation, Issue 48, June 2019, pp. 32-35. D0I: 10.22163/ fteval.2019.364

Gustavsen, B. (2012). Social Innovation and Action Research. In H.-W. Franz, J. Hochgerner and J. Howaldt (Ed.), Challenge social innovation: Potentials for business, social entrepreneurship, welfare and civil society (pp. 353-366). Berlin, New York: Springer.

Howaldt, J. (2019): New Pathways to Social Change - Creating Impact through Social Innovation Research. Fteval Journal for Research and Technology Policy Evaluation, Issue 48, June 2019, pp. 36-47. D0I: 10.22163/fteval.2019.365

Howaldt, J., Kaletka, C., Schröder, A., Rehfeld, D. and Terstriep, J. (2016). Mapping the World of Social Innovation. Key Results of a Comparative Analysis of 1.005 Social Innovation Initiatives at a Glance. SI-Drive Project. https://www.si-drive.eu/?p=2283; accessed on 30 June 2019.

König, T. (2019): SSH-Imapct Pathways and SSH-Integration in EU Research Framework Programmes. Impact re-loaded. Fteval Journal for Research and Technology Policy Evaluation, Issue 48, June 2019, pp. 7-8. DOI: 10.22163/fteval.2019.362

König, T., Nowotny, H. and Schuch, K. (2019): Impact re-loaded. Fteval Journal for Research and Technology Policy Evaluation, Issue 48, June 2019, pp. 7-8. DOl: 10.22163/fteval.2019.361

Lamy, P. et al. (2017): Lab, Fab, App. Investing in the European future we want. Report of the independent High Level Group on maximising the Impact of EU Research and Innovation Programmes. European Commission (the so called 'Lamy-Report').

Marimon, R., Guardiancich, I., Mariathasan, M. and Rossi, E. (2011): Survey on Research Funding for the Social Sciences in Europe. European University Institute. 
Mazzucato, M. (2018). Mission-Oriented Research and Innovation in the European Union. Retrieved October 18, 2018 from: https://ec.europa.eu/ info/sites/info/files/mazzucato_report_2018.pdf.

Morozov, E. (2013): To Save Everything, Click Here: Technology, Solutionism, and the Urge to Fix Problems that Don't Exist, London.

Nussbaum, M. (2010): Not for Profit. Why Democracy Needs the Humanities. Princeton: Princeton University Press.

Papanagnou, G. (ed) (2011): Social Science and Policy Challenges. Democracy, Values and Capacities. UNESCO.

Rammel, S., Hoffmann, A. and Halbmayr, B. (2017): ERA Thematic Dossier on Social Sciences and Humanities (SSH) in Horizon 2020. FFG: Austrian Research Promotion Agency.

Raua, H., Gogginsb, G. and Fahyb, F (2018): Form invisibility to impact: Recognising the scientific and societal relevance of interdisciplinary sustainability research. Research Policy, 47, pp 266-276. D0I: 10.1016/j. respol.2017.11.005.

Reale, E. et al. (2017): A review of literature on evaluating the scientific, social and political impact of social sciences and humanities research. Research Evaluation 2017, 1-11, doi: 10.1093/reseval/rvx025.

Reiter-Pázmándy, M. (2019): Foreword. Fteval Journal for Research and Technology Policy Evaluation, Issue 48, June 2019, pp. 5-6. DOI: 10.22163/fteval.2019.360.

Russegger, G. (2019): SSHA-Driven Knowledge Transfer Within the Third Mission of Universities. Foreword. Fteval Journal for Research and Technology Policy Evaluation, Issue 48, June 2019, pp. 61-64. D0I: 10.22163/ fteval.2019.369

Schögler, R. and König, T. (2017): Thematic Research Funding in the European Union: What is Expected from Social-Scientific Knowledgemaking? Serendipities 2.2017 (1): 107-130 | D0I: 10.25364/11.2:2017.1.7

Sturn, D. and Schuch, K. (2018): Geistes-, sozial- und kulturwissenschaftliche Aspekte in FFG geförderten Innovationen. Report for the Austrian Research Promotion Agency (not published).

Van den Besselaar, P., Flecha, R. and Radauer, A. (2018): Monitoring the Impact of EU Framework Programmes. Expert Report. Publications Office of the European Union: Luxembourg.

\section{AUTHOR}

\section{KLAUS SCHUCH}

Zentrum für Soziale Innovation GmbH - Centre for Social Innovation (ZSI) Linke Wienzeile 246, Vienna, 1150 (Austria)

E: $\underline{\text { schuch@zsi.at }}$ 


\title{
SCIENTIFIC COMMITTEE OF THE CONFERENCE: \\ IMPACT OF SOCIAL SCIENCES AND HUMANITIES FOR A EUROPEAN RESEARCH AGENDA - VALUATION OF SSH IN MISSION-ORIENTED RESEARCH
}

\author{
HELGA NOWOTNY \\ Chair of the ERA Council Forum Austria and Member of the Austrian Council for Research and Technology Development, Former \\ President of the European Research Council (Chair of the Scientific Committee)
}

\section{PAUL BENNEWORTH}

Senior researcher at the Center for Higher Education Policy Studies at the University of Twente, Netherlands

OLIVIER BOUIN

Director of the Foundation-Excellence Laboratory "Network of French Institutes for Advanced Study"

ULRIKE FELT

University of Vienna, Professor of Science and Technology Studies (Vice chair of the Scientific Committee)

YVES GINGRAS

Scientific Director of the Observatory of Science and Technology (OST), Montréal, Canada

POUL HOLM

Professor of Environmental History, Trinity College Dublin, Ireland

\section{JÜRGEN HOWALDT}

Director of the Social Research Centre at TU Dortmund University and Professor at the Faculty of Economics, Dortmund, Germany

CARINA KESKITALO

Member of High Level Group on Scientific Advisors of the EC, Professor at Umeå University, Umeå, Sweden

KIRSTEN M. LANGKILDE

Director of the University of Applied Sciences and Arts Northwestern Switzerland, Academy of Art and Design, Basel, Switzerland

\section{STEFANIA MILAN}

Associate Professor of New Media and Digital Culture at the University of Amsterdam and of Media Innovation at the University of Oslo, Amsterdam/Oslo, Netherlands/Norway

\section{ANDREA PETŐ}

Professor in the Department of Gender Studies at Central European University, Budapest, Hungary

CLAUDIO M. RADAELLI

Professor of Public Policy, University College London, Department of Political Science, United Kingdom

EMANUELA REALE

Senior Researcher at IRCRES (Research Institute on Sustainable Economic Growth) - CNR (National Research Council), Roma, Italy

\section{SARAH DE RIJCKE}

Professor of Science and Evaluation Studies and deputy director at CWTS, Leiden, Netherlands

\section{EVELYN RUPPERT}

Professor at the Goldsmiths University of London, London, United Kingdom

\section{KLAUS SCHUCH}

Scientific Director of the Centre for Social Innovation, Vienna, Austria

\section{MARTA SOLER}

Professor at the University of Barcelona (Spain) and Director of CREA, Community of Research on Excellence for All

JACK SPAAPEN

Emeritus senior policy advisor at the Royal Netherlands Academy of Arts and Science, Amsterdam, Netherlands

TEREZA STÖCKELOVA

Researcher and editor in chief at the Institute of Sociology of the Czech Academy of Sciences, Prague, Czech Republic

\section{JOHANNES VOGEL}

General Director of the Museum for Natural History Berlin and Chair of the European Citizen Science Association, Berlin, Germany

\section{MILENA ZIC-FUCHS}

Professor at the University of Zagreb, Croatia, Fellow of the Croatian Academy of Sciences and Arts, Member of Academia Europaea 
POSTERS

POSTER 1 / PAGE 190

Cultivating Europe: Democratic Europe - Social Europe

- Educated Europe

Authors: Katharina Berghöfer, Nicole Birkle, Geny Piotti,Stefanie

Preuss, Ursula Schlichter, Christian Veldman, Kristina Wiege.

DOI: 10.22163/fteval.2019.388

POSTER 2 / PAGE 192

Structural Interdisciplinary Cooperation On Crime And Security Research

Authors: Noël Klima

DOl: 10.22163/fteval.2019.389

POSTER 3 / PAGE 194

Inclusive Employment Of Deaf People

Authors: Monika Haider

DOI: 10.22163/fteval.2019.390

POSTER 4 / PAGE 196

Collaborative engagement on societal issues

Author: Alessia Smaniotto

DOl: 10.22163/fteval.2019.391

POSTER 5 / PAGE 198

Generating Impact in Transnational Humanities

Research: HERA 2009-2018

Authors: Joanna Sofaer, Wojciech Sowa, Tony Whyton

DOl: 10.22163/fteval.2019.392

POSTER 6 / PAGE 200

Generating Impact in Transnational Humanities

Research: HERA 2009-2018

Authors: Joanna Sofaer, Wojciech Sowa, Tony Whyton

DOl: 10.22163/fteval.2019.393

POSTER 7 / PAGE 202

CLARIN - Transcending Scientific Boundaries across SSH Research focusing on Parliamentary Data

Authors: Franciska de Jong, Darja Fišer, Maria Eskevich, Karolina

Badzmierowska

DOI: 10.22163/fteval.2019.394

POSTER 8 / PAGE 204

Rethinking Research Impact Assessment: A multdimensional Approach

Authors: Sergio Manriqe, Marta Natalia Wróblewska, Bradley Good DOI: 10.22163/fteval.2019.395
POSTER 9 / PAGE 206

SIOR Contributing to the scientific research transparency by evaluating the social impact of sciences

Authors: Joan Cabré, Vladia Ionescu, Gisela Redondo, Teresa Sordé DOI: 10.22163/fteval.2019.396

POSTER 10 / PAGE 208

Are Humanities failing to generate Impact?

Author: Dorothea Sturn

DOI: 10.22163/fteval.2019.397

POSTER 11 / PAGE 210

Ethics \& economics regarding access to healthcare for marginalised groups

Authors: Ursula Trummer, Sonja Novak-Zezula, Nadav Davidovitch, Nora Gottlieb

DOI: 10.22163/fteval.2019.398 


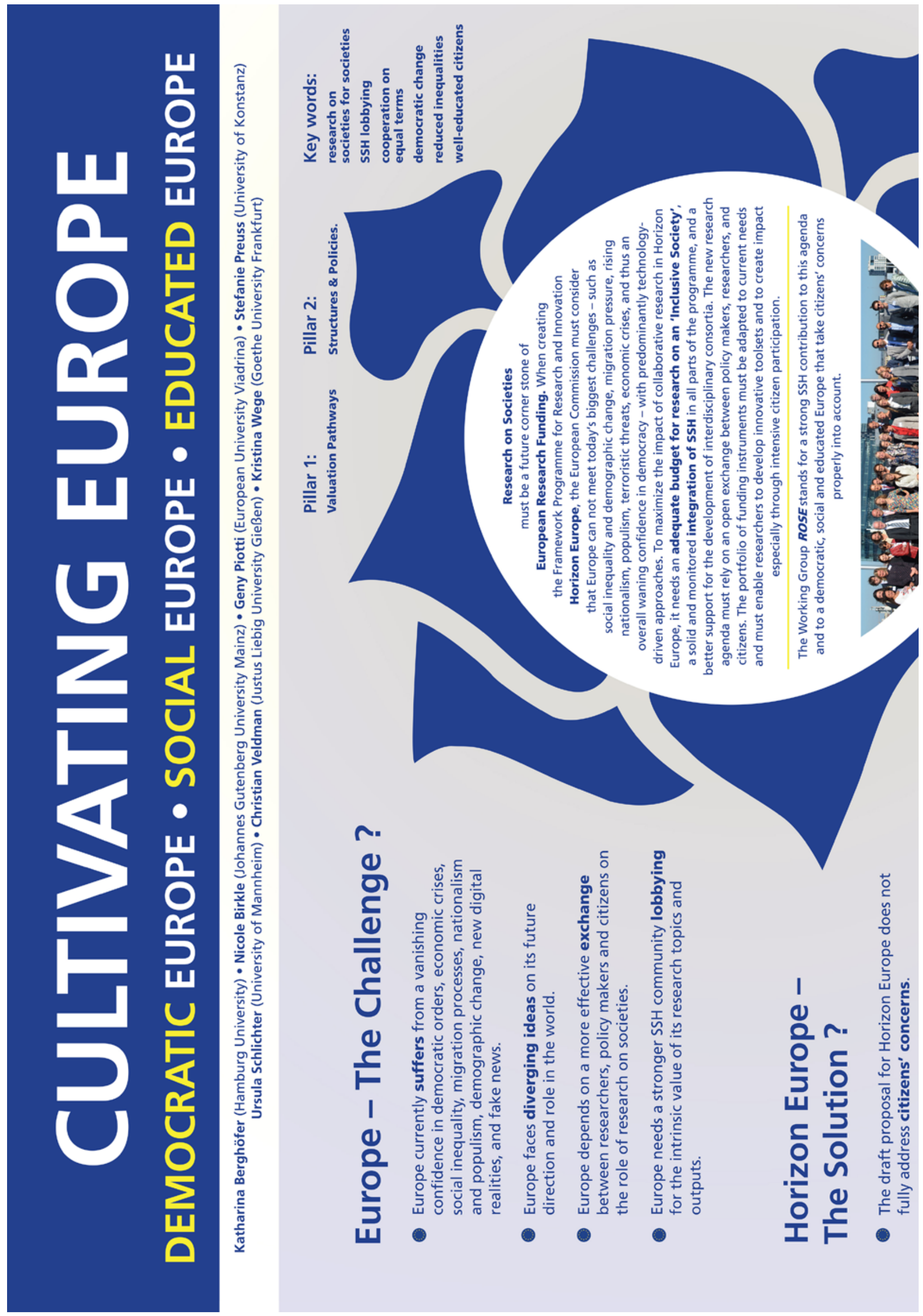




\section{$\Delta$}
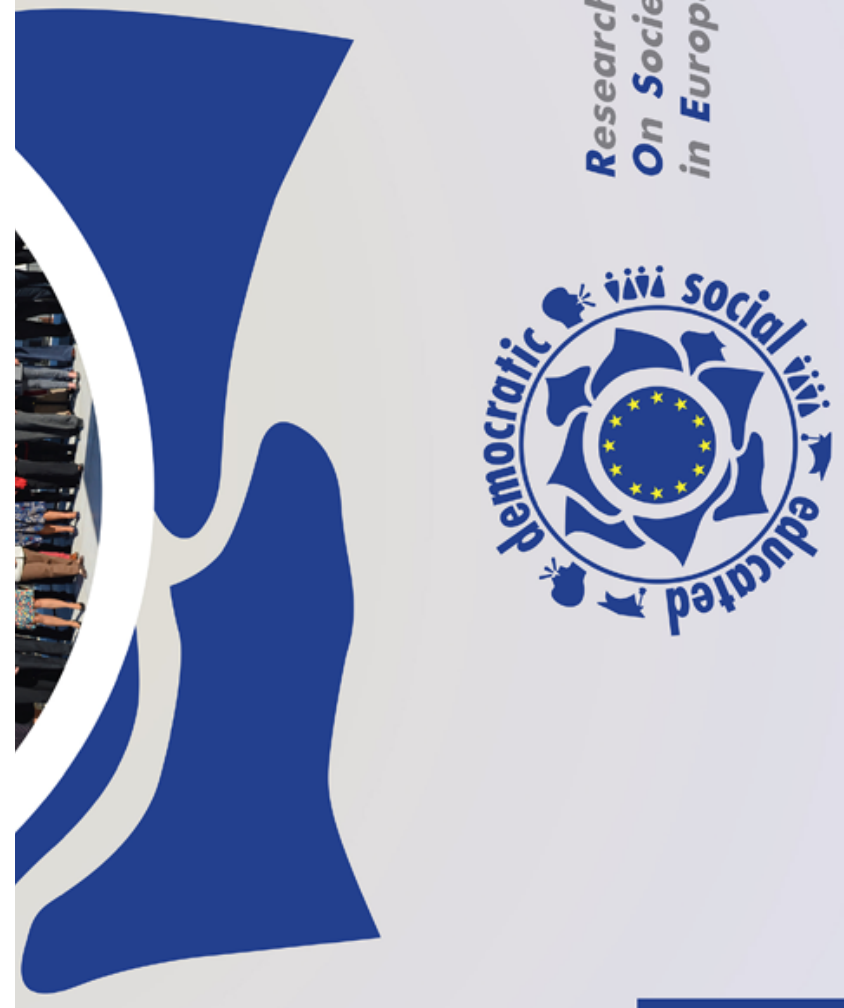

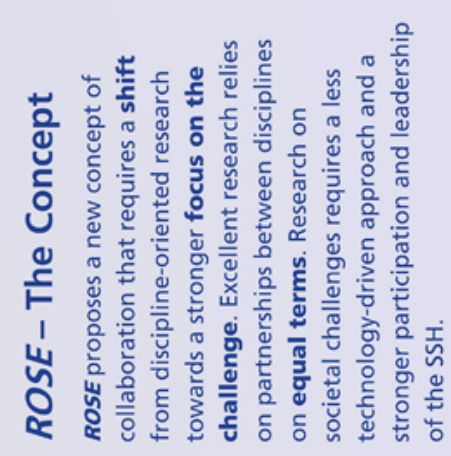

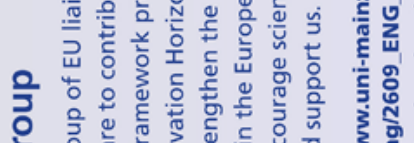
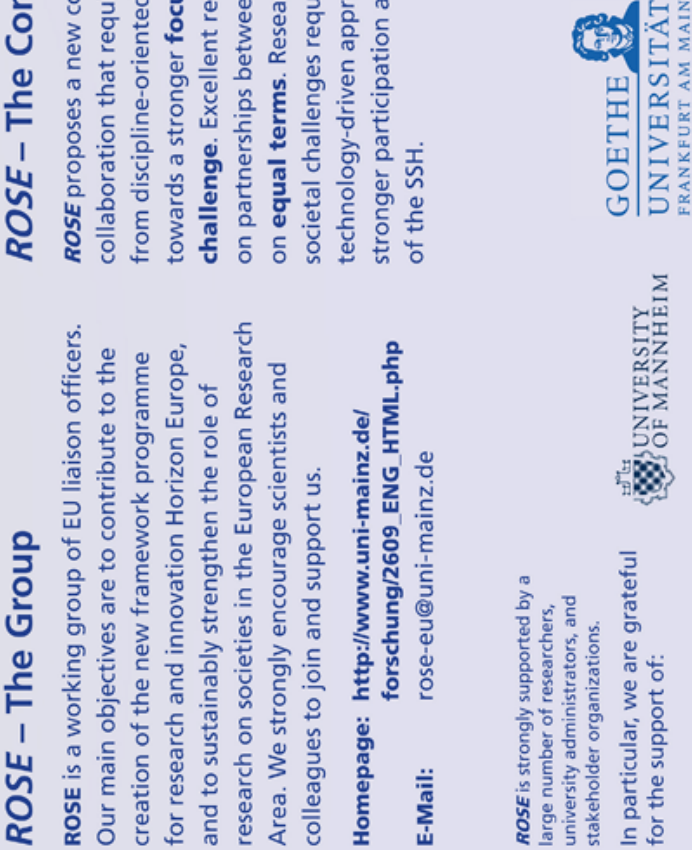
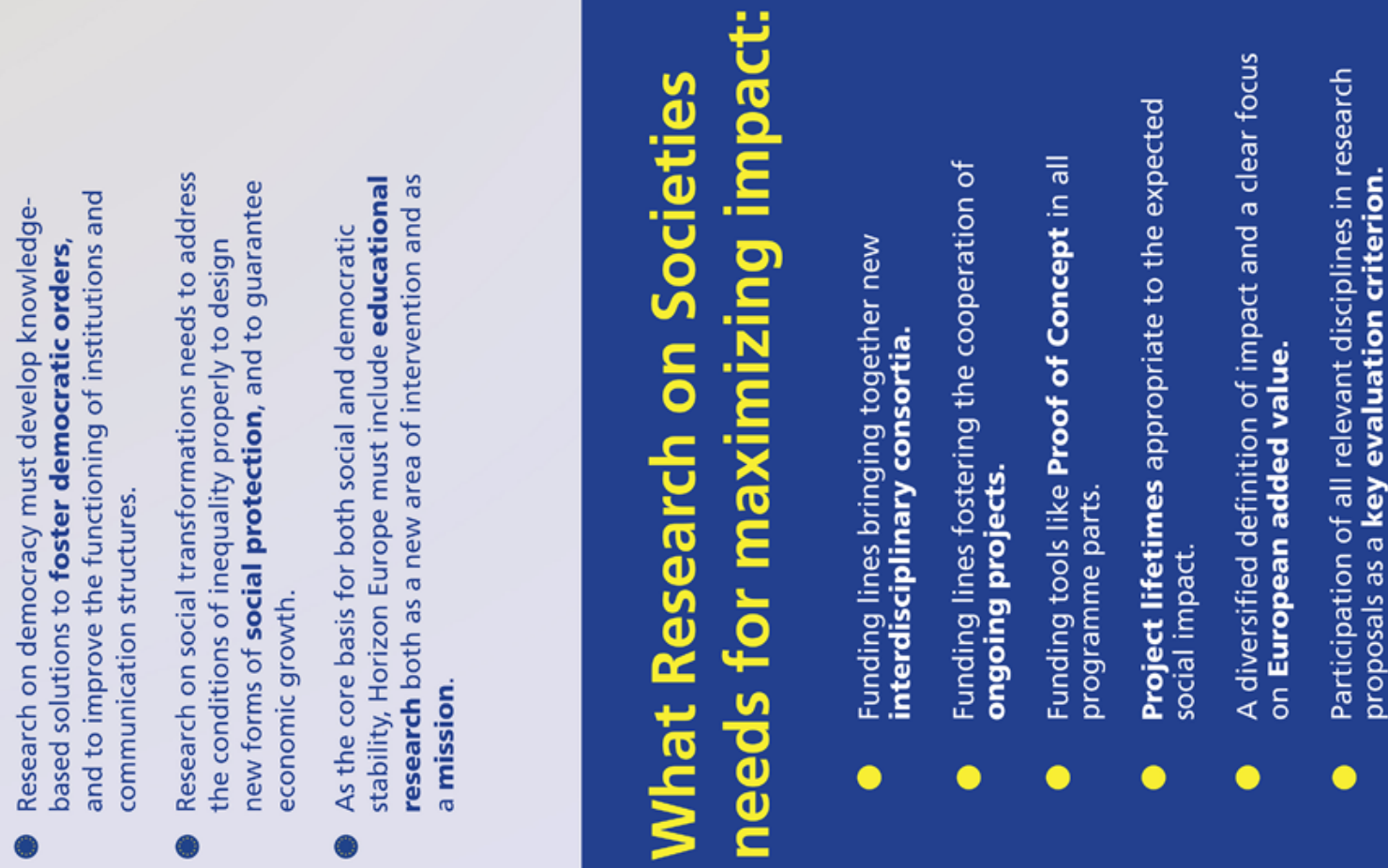


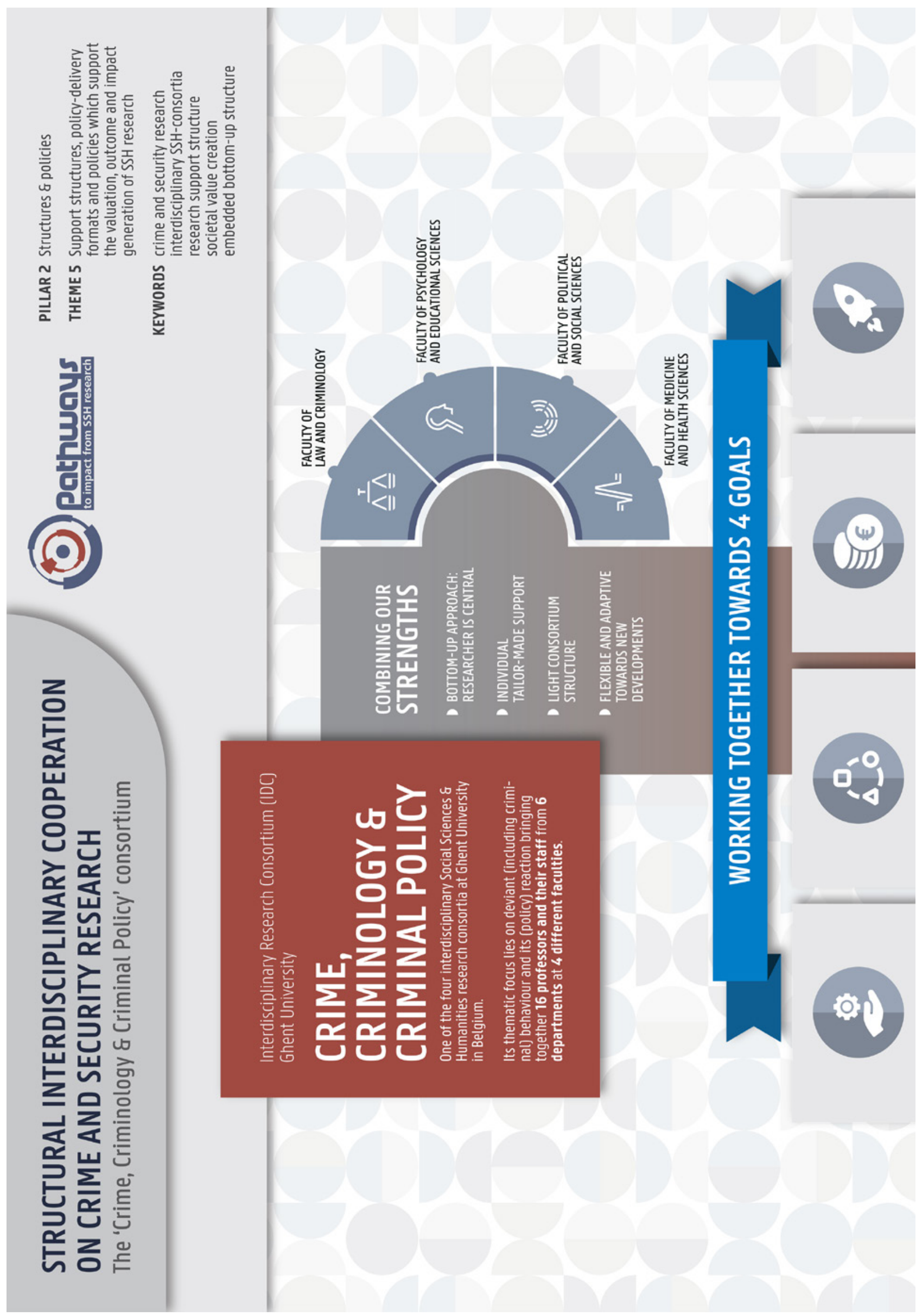




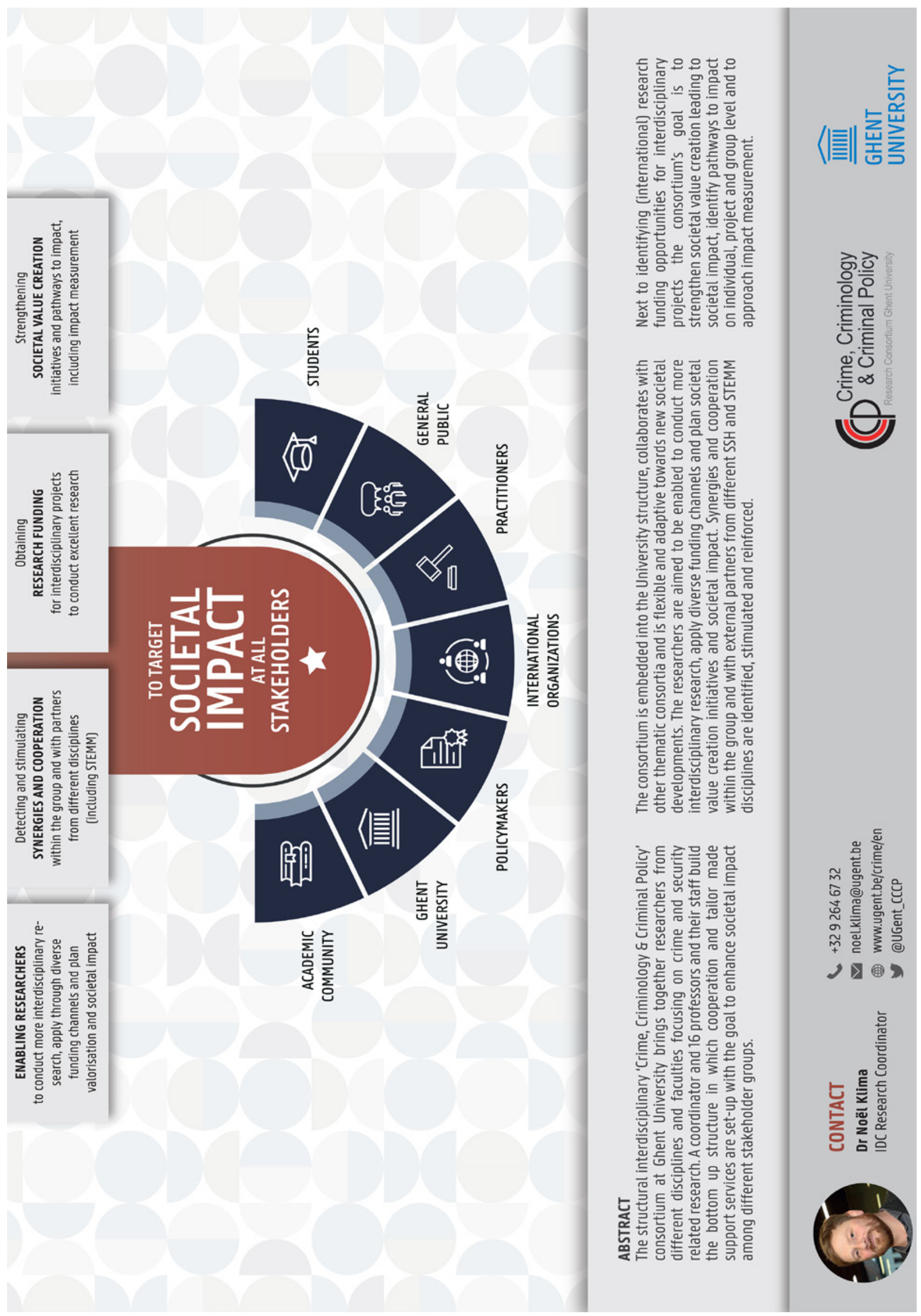


$\rightleftarrows$
$\frac{N}{N}$
$\frac{\pi}{0}$
$\frac{0}{0}$
(1)

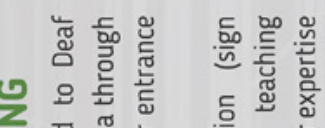

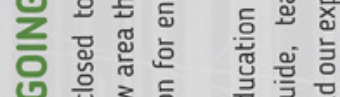

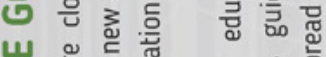

山

《 흐음

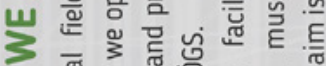

山

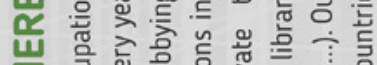

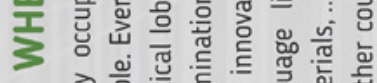

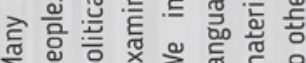

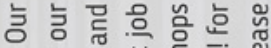

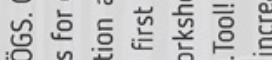

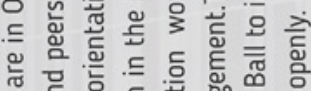

柁塎

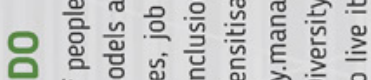

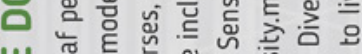

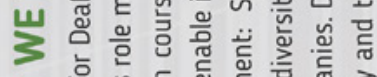

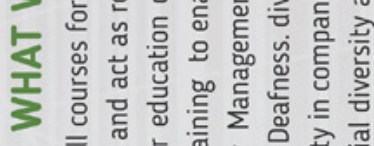

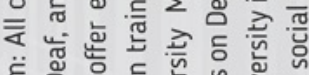

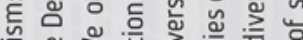

ฉ $\frac{1}{\alpha}$

$4 \leftarrow$

$<v$

분

อ 岌

山旨

○ $\frac{1}{\mathrm{Z}}$

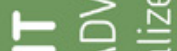

$z \frac{2}{2}$

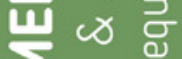

एँ

6 过。

넙

尺实

$\sum \frac{1}{2}$

ய 픔

$\geqslant v i \frac{\pi}{1}$

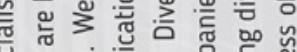

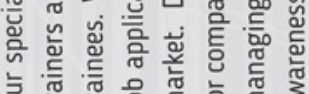

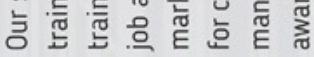

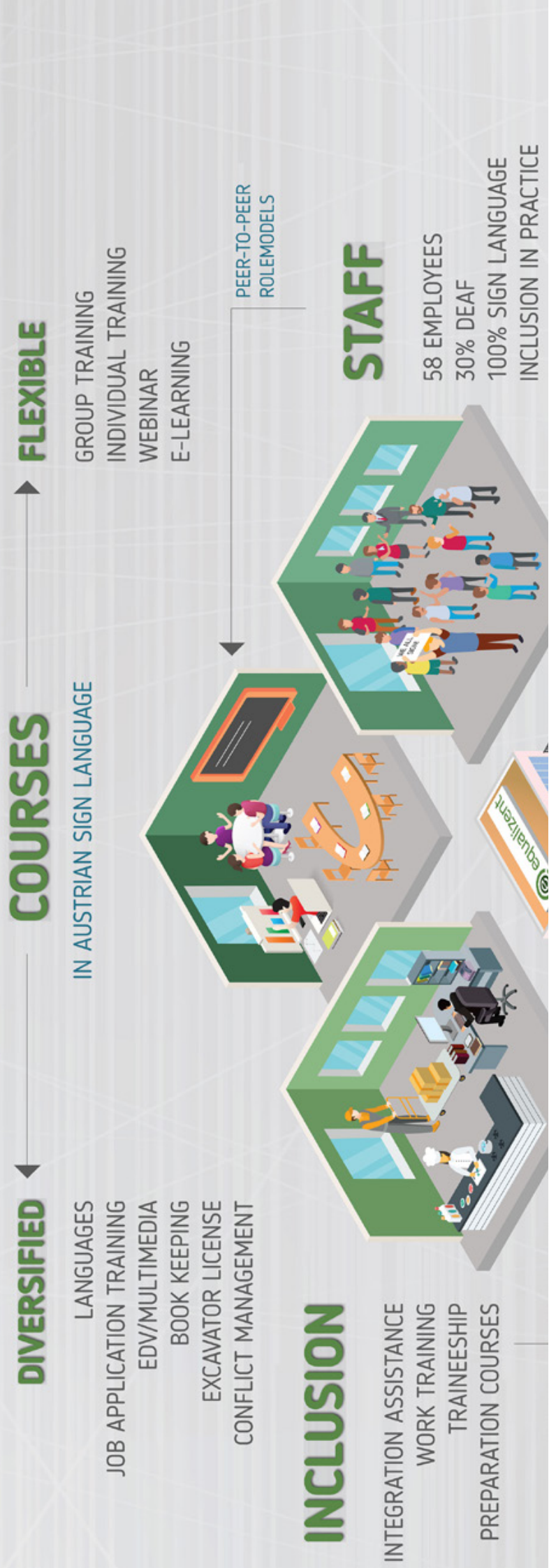

$\geqslant$ 剀龸

ง

$\exists \underset{*}{E} \sum_{\pi}$

ט

运岳

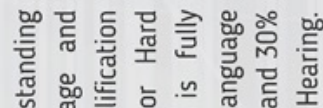

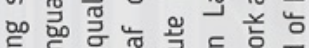

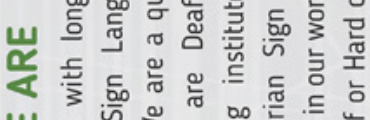

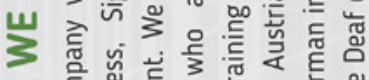

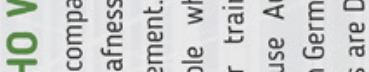

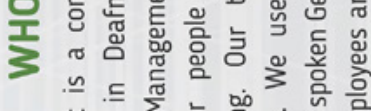

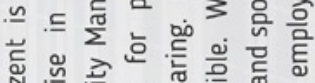

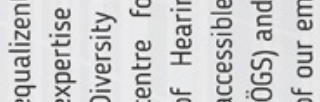




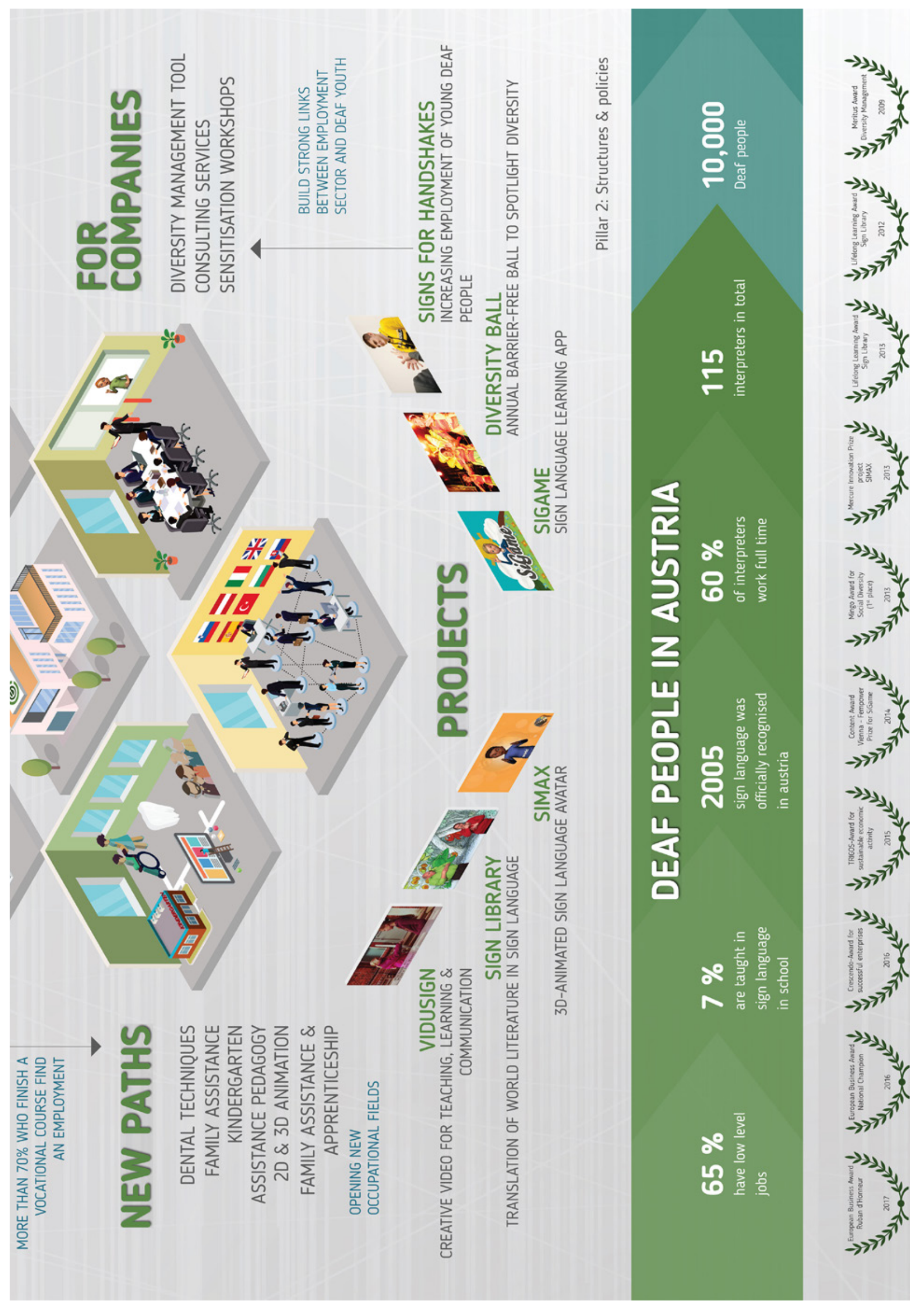




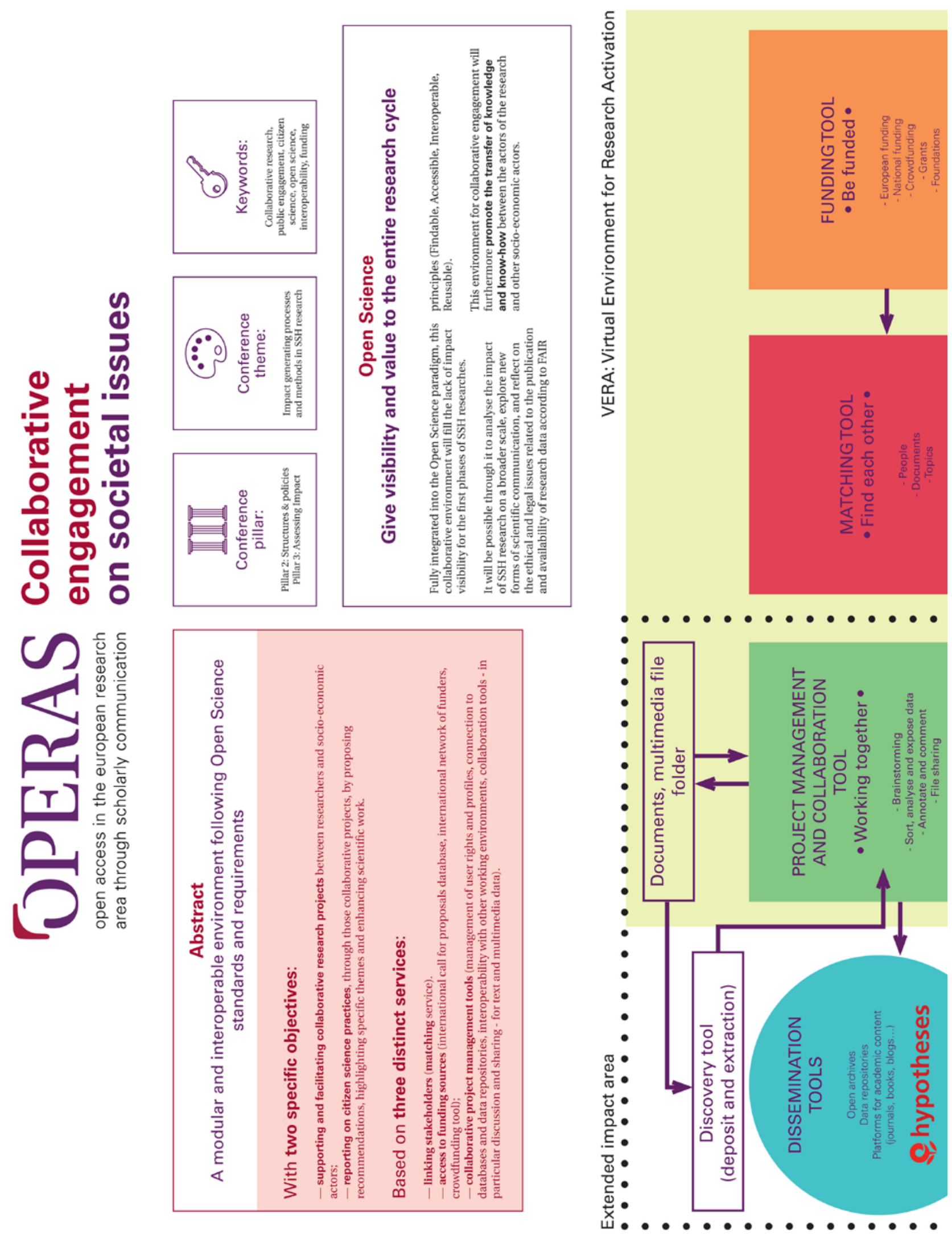



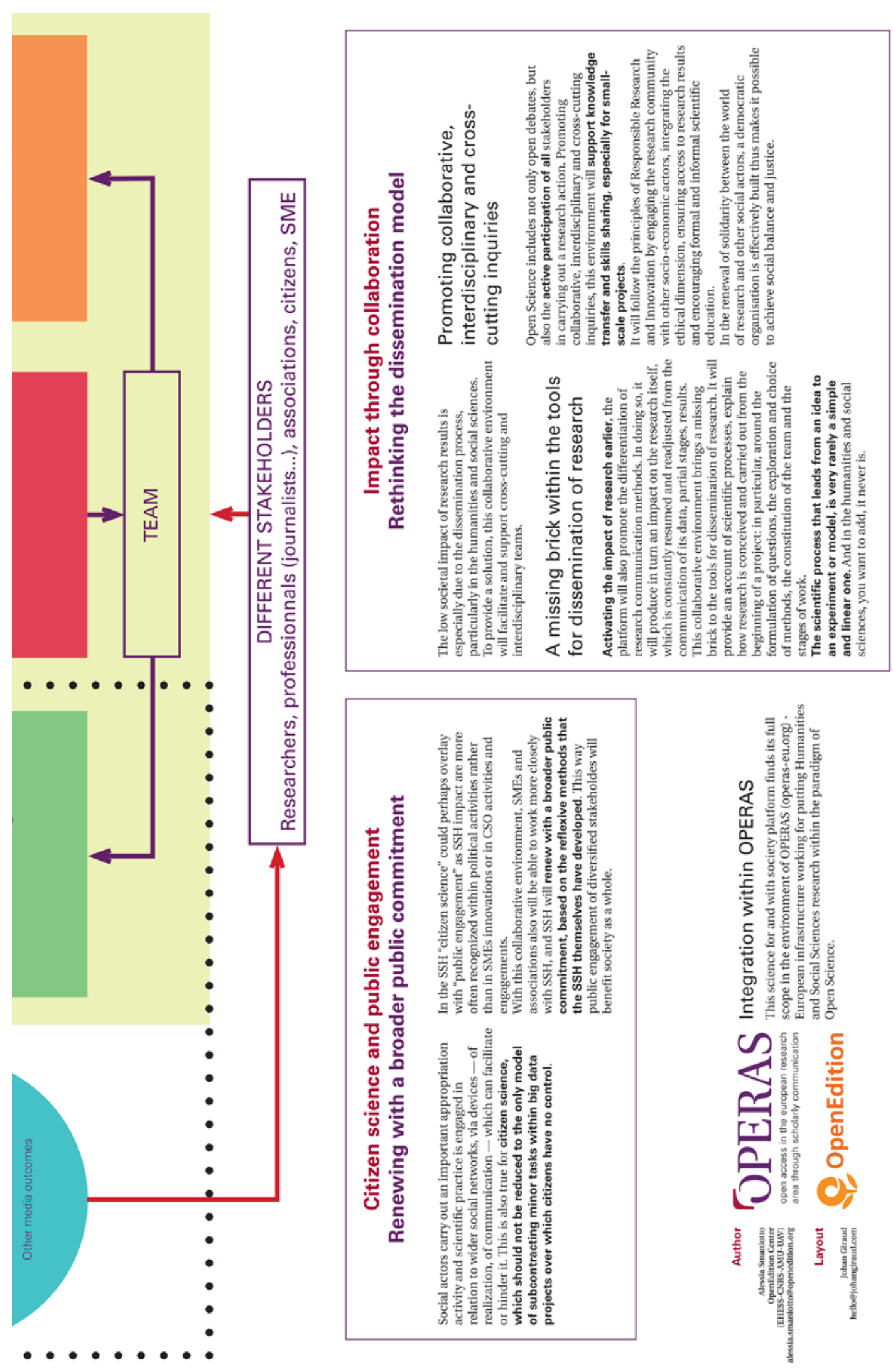


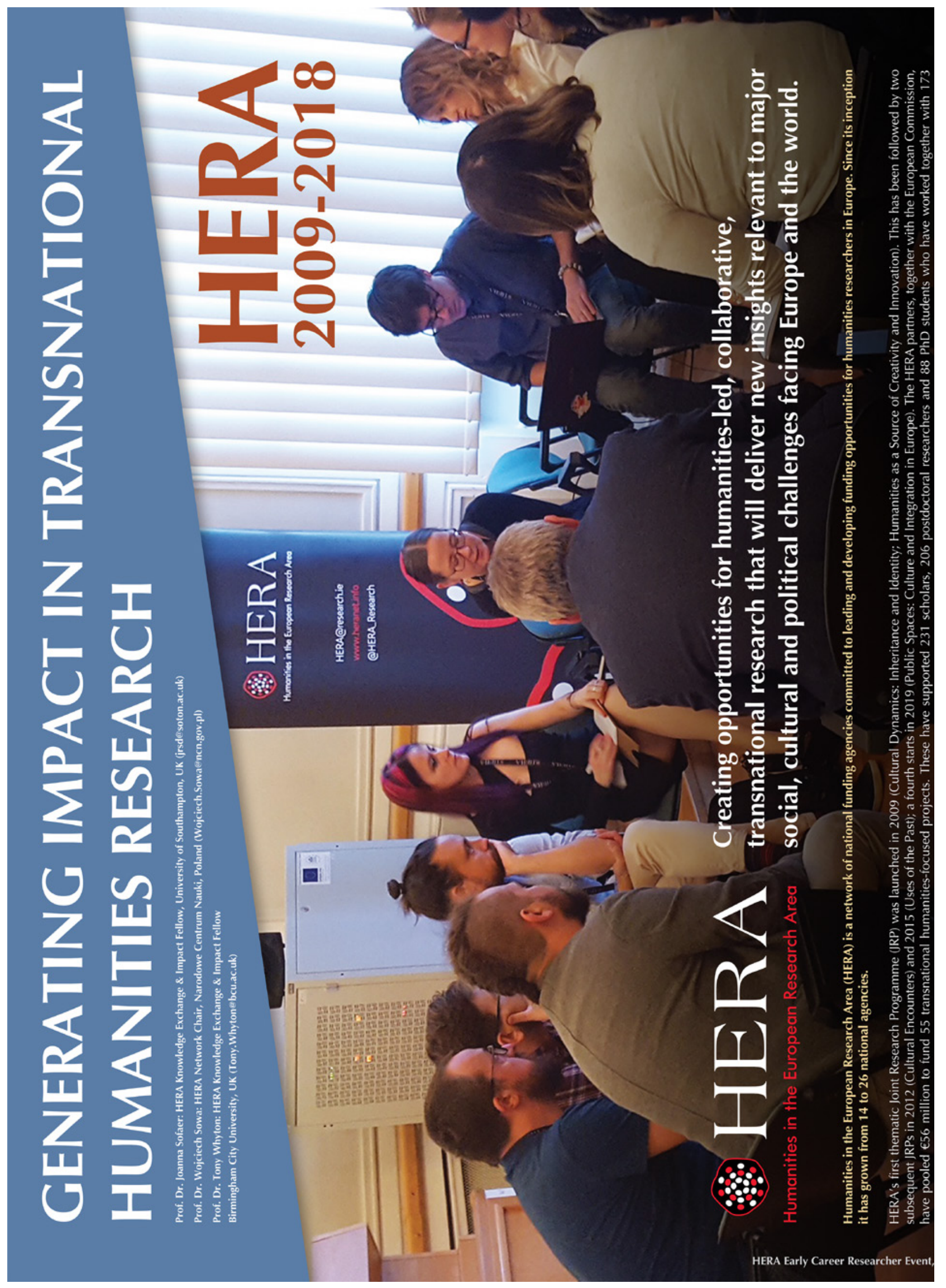




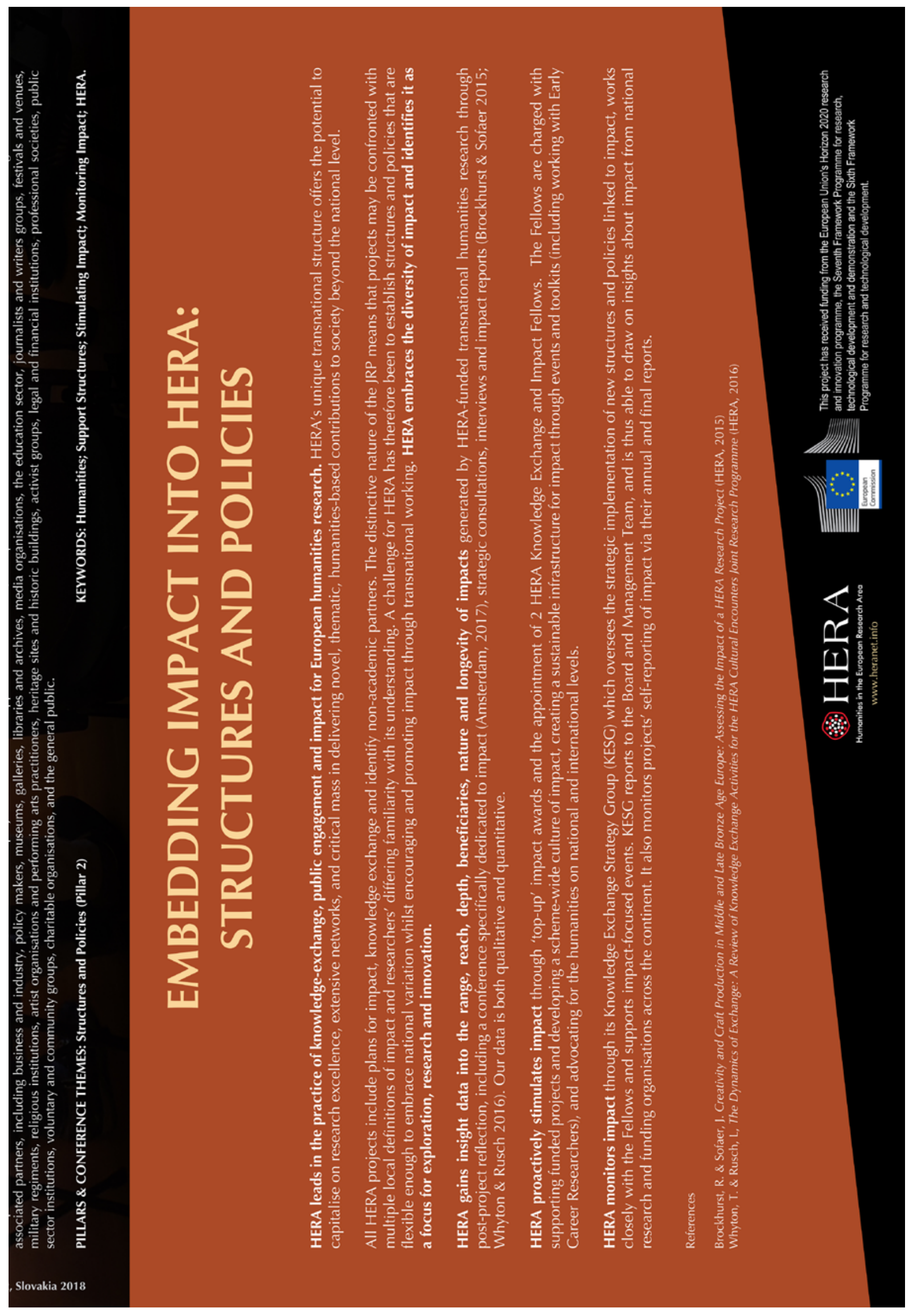




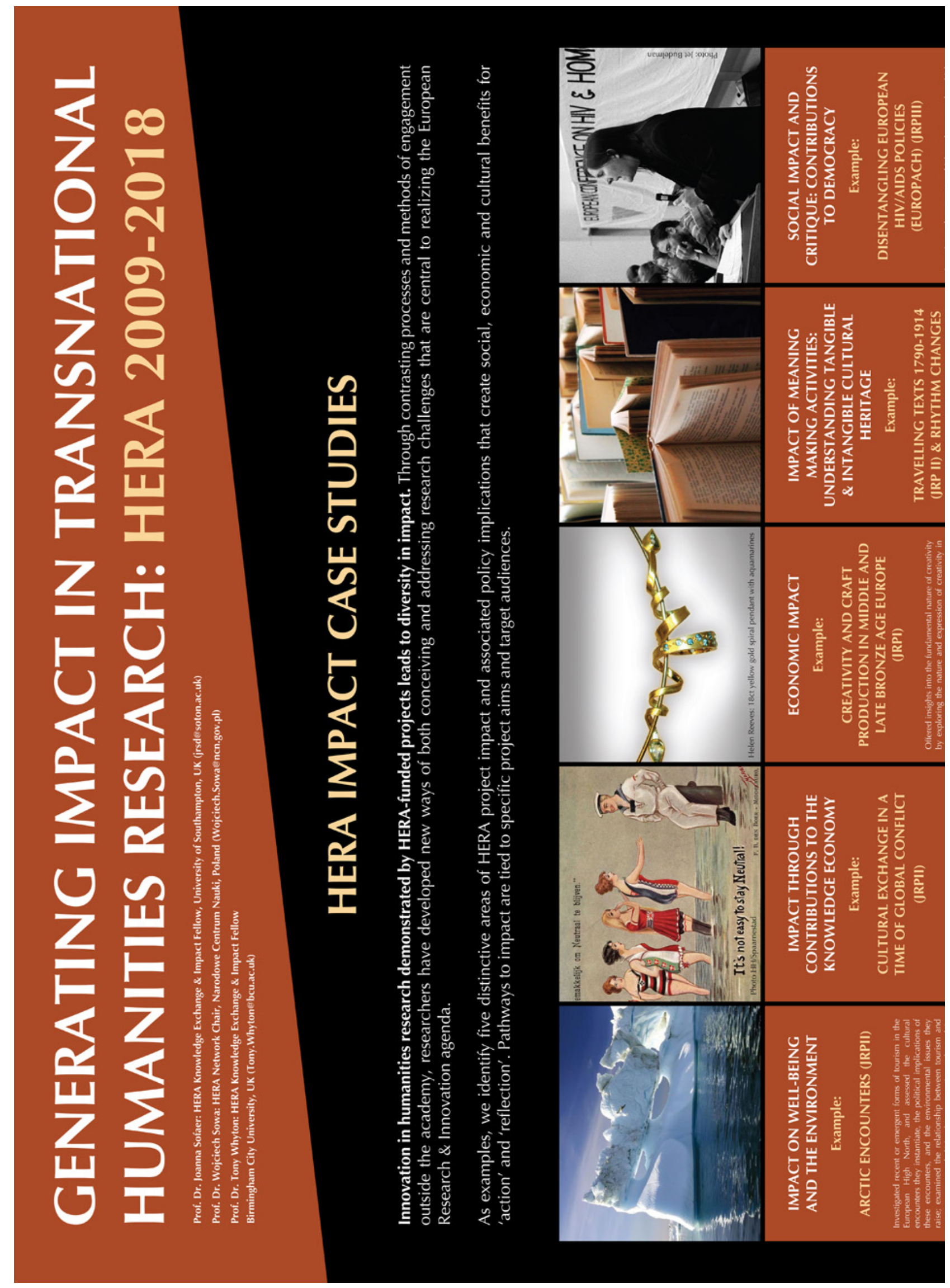




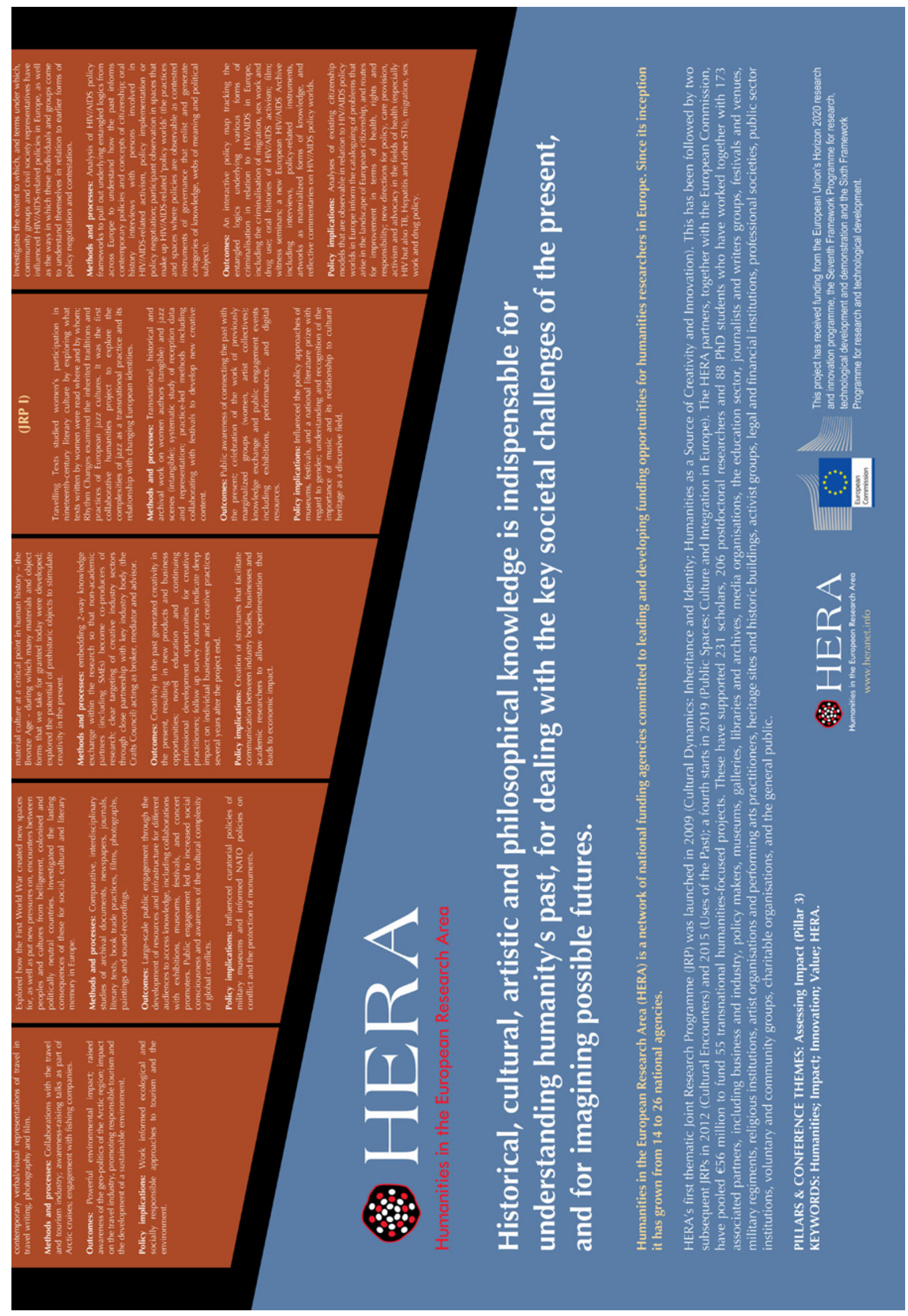




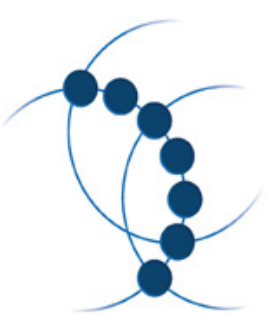

(1)
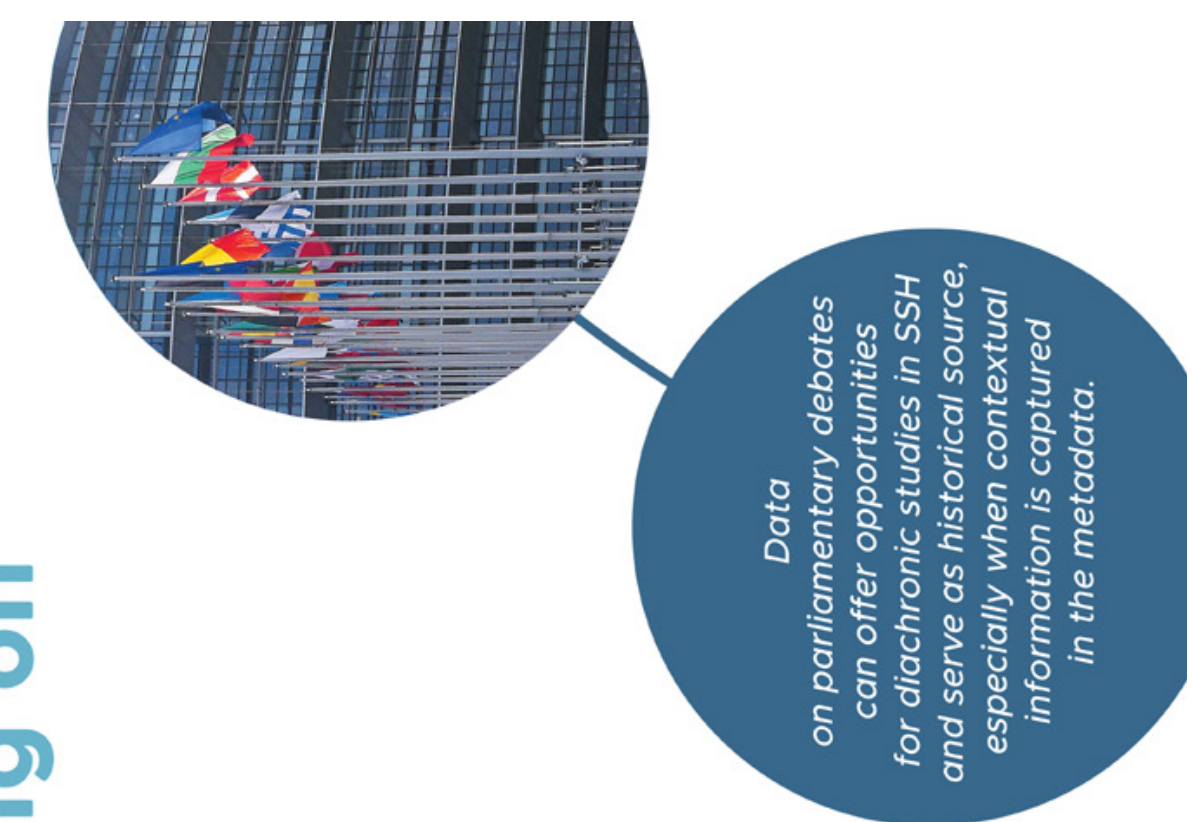

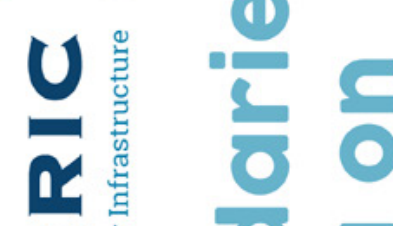

II

Z

준

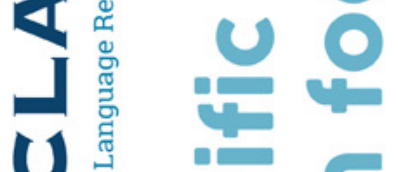

U

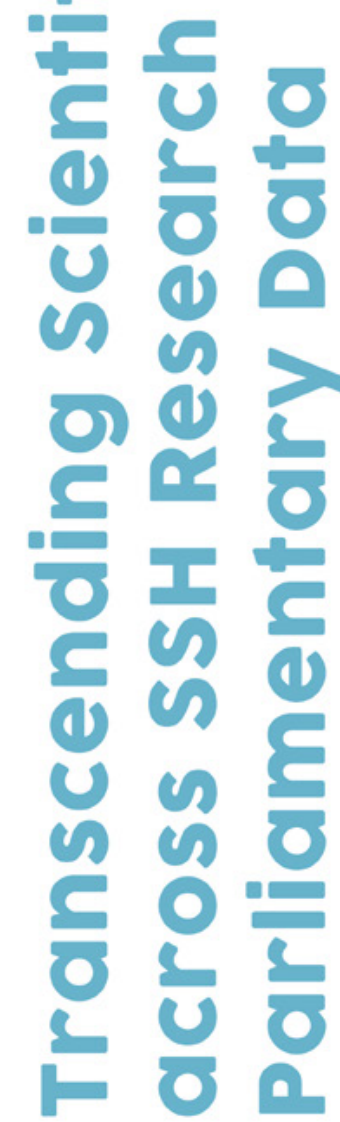
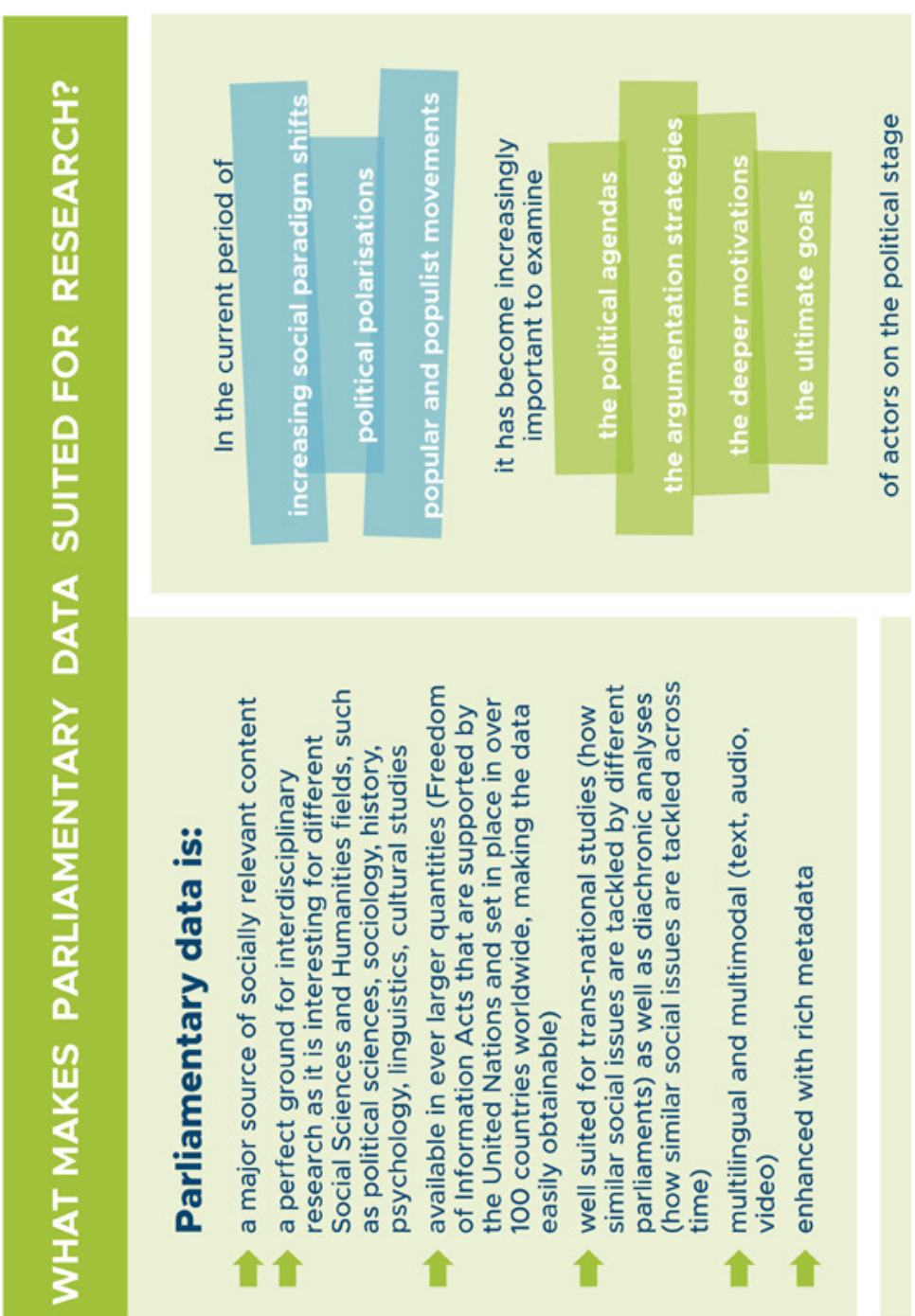


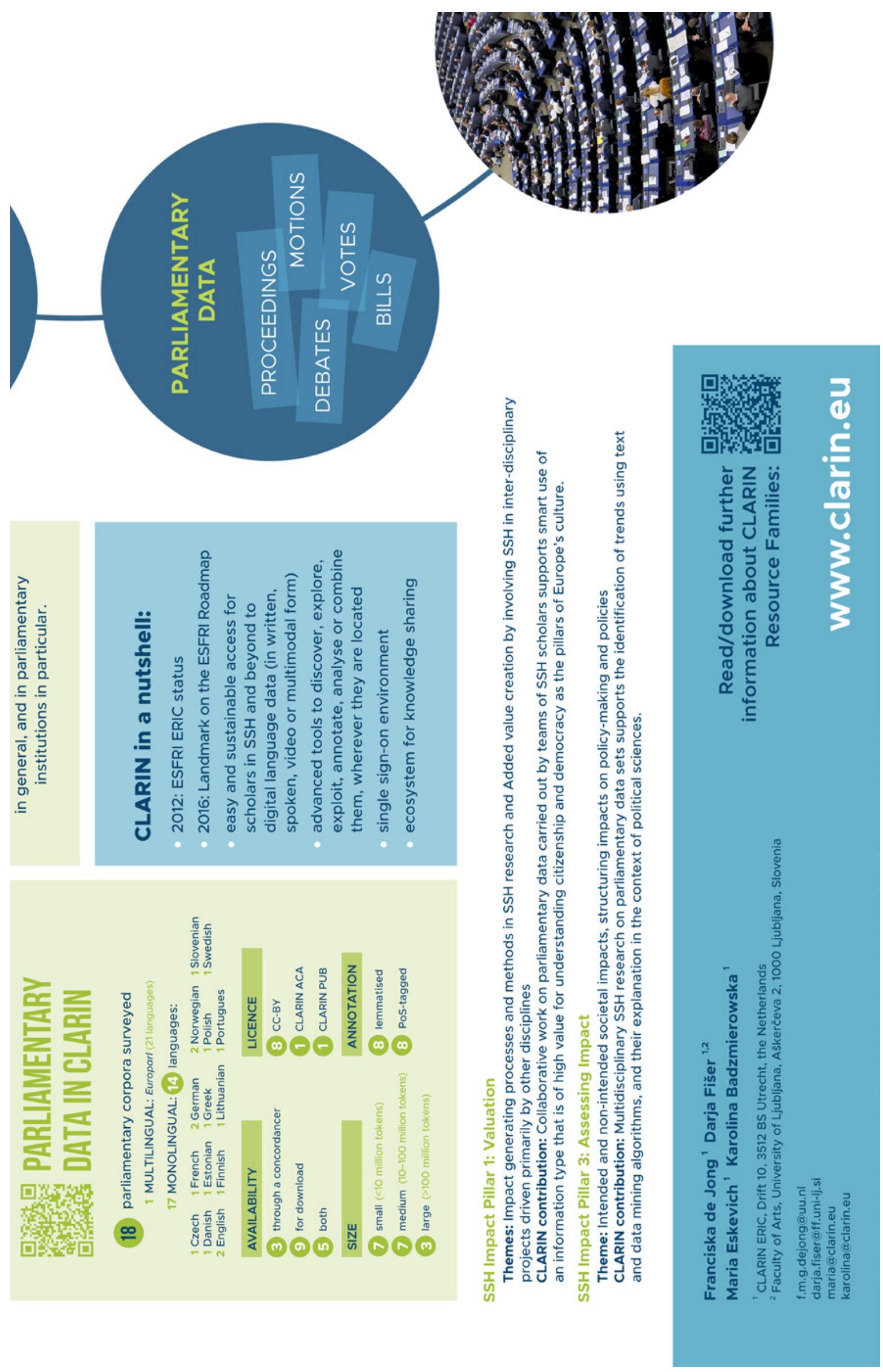




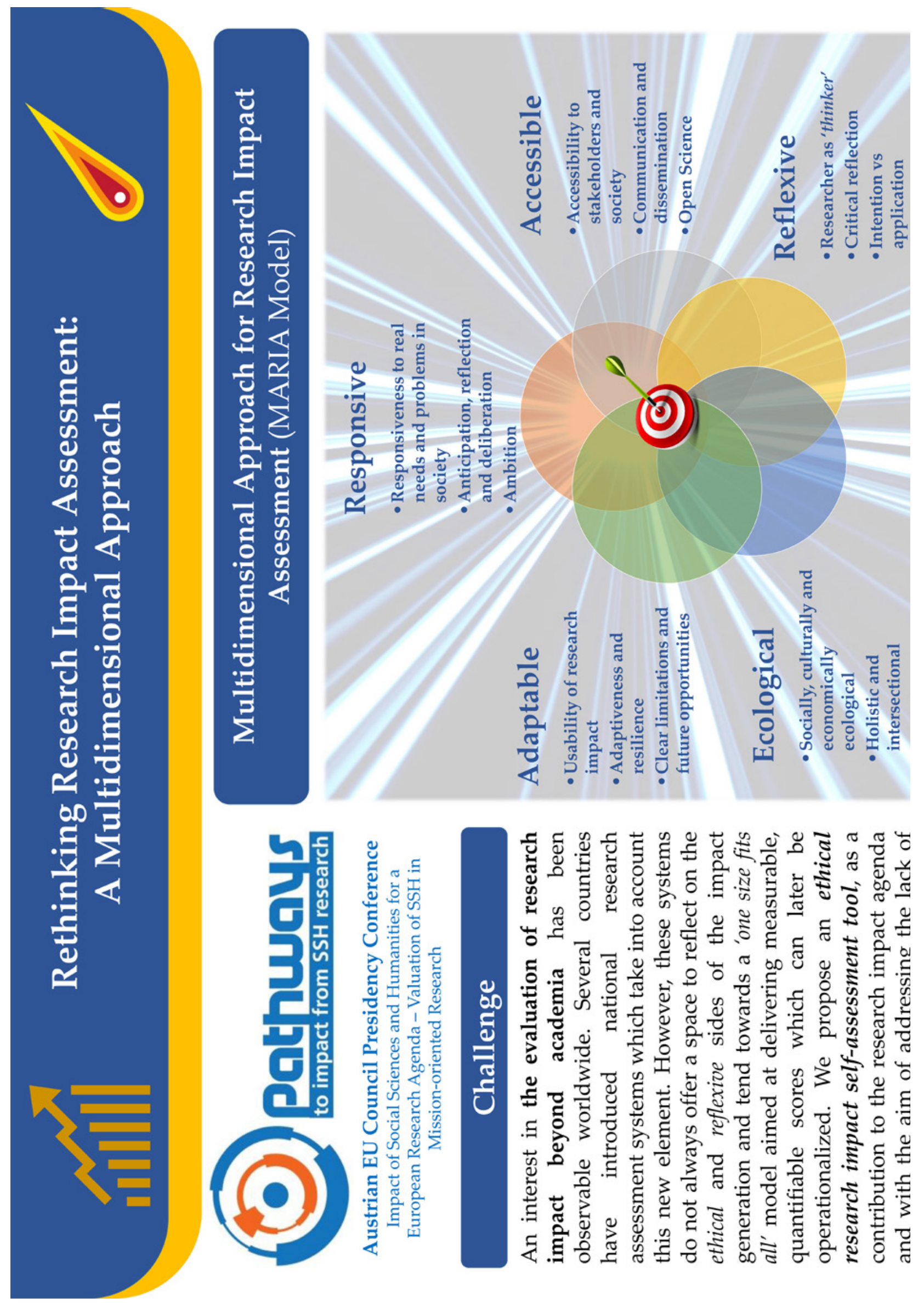




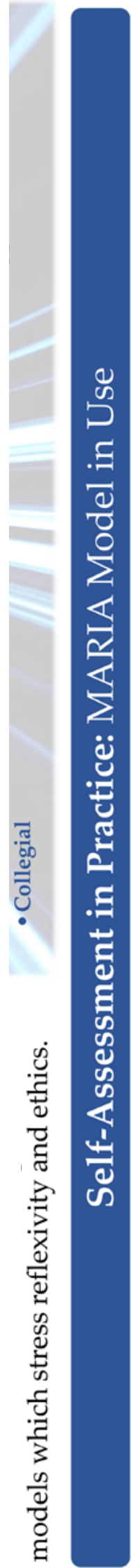

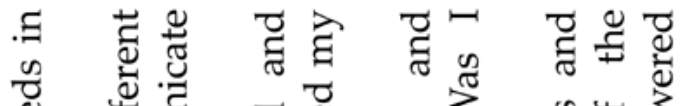

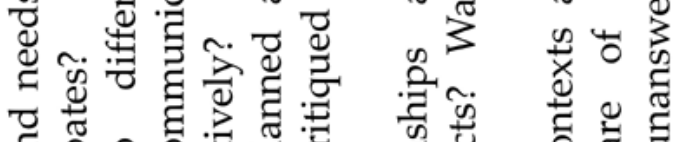

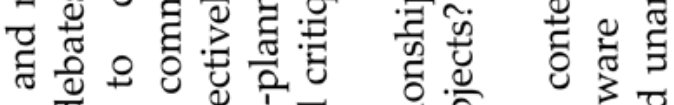

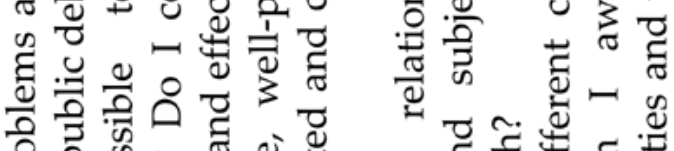
을

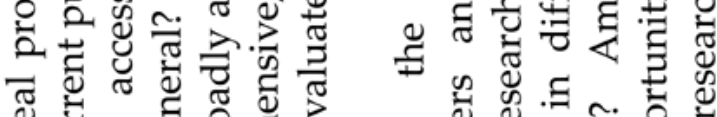
『ٓ

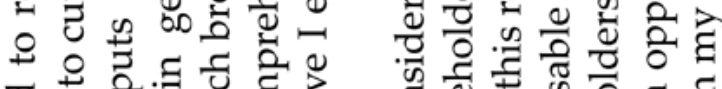
T

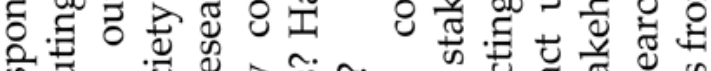
के

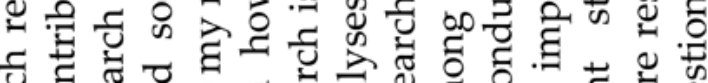

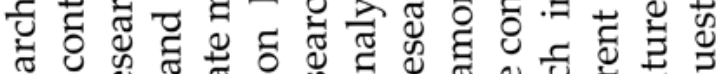
๘

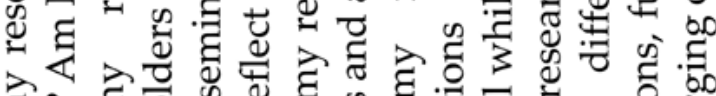

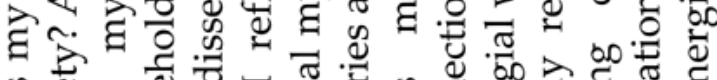
\&

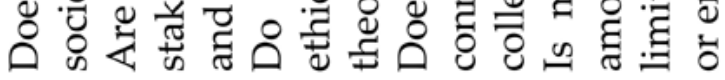

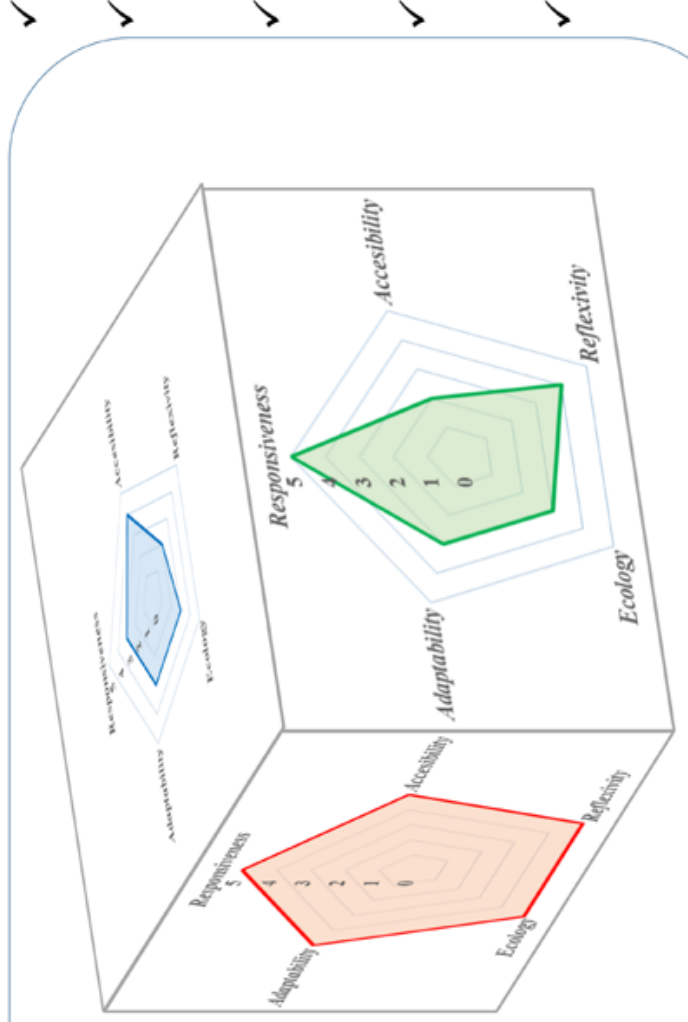

范

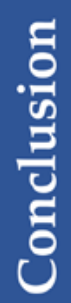

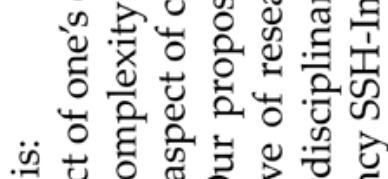

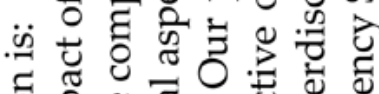

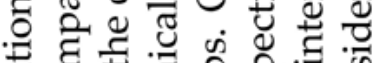

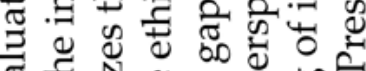
సี Е

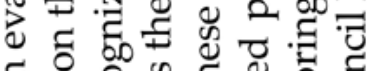

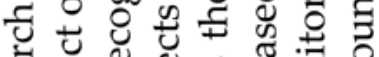

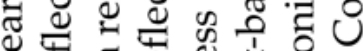

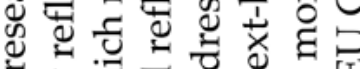
记

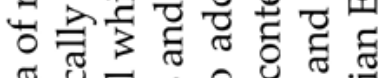

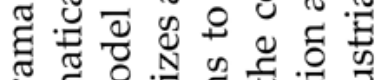
สี

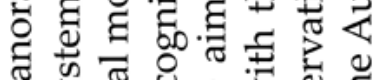
施导范范

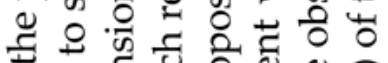

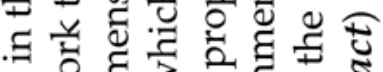
$003 . \exists 30.50$

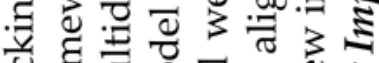
త్త

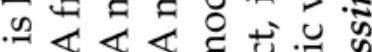

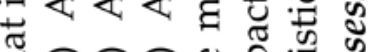

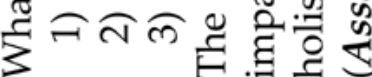

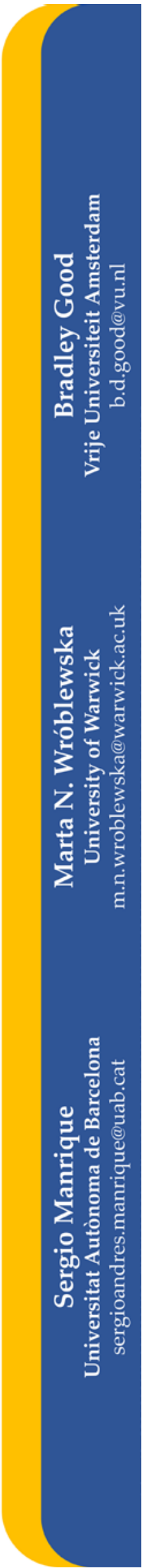



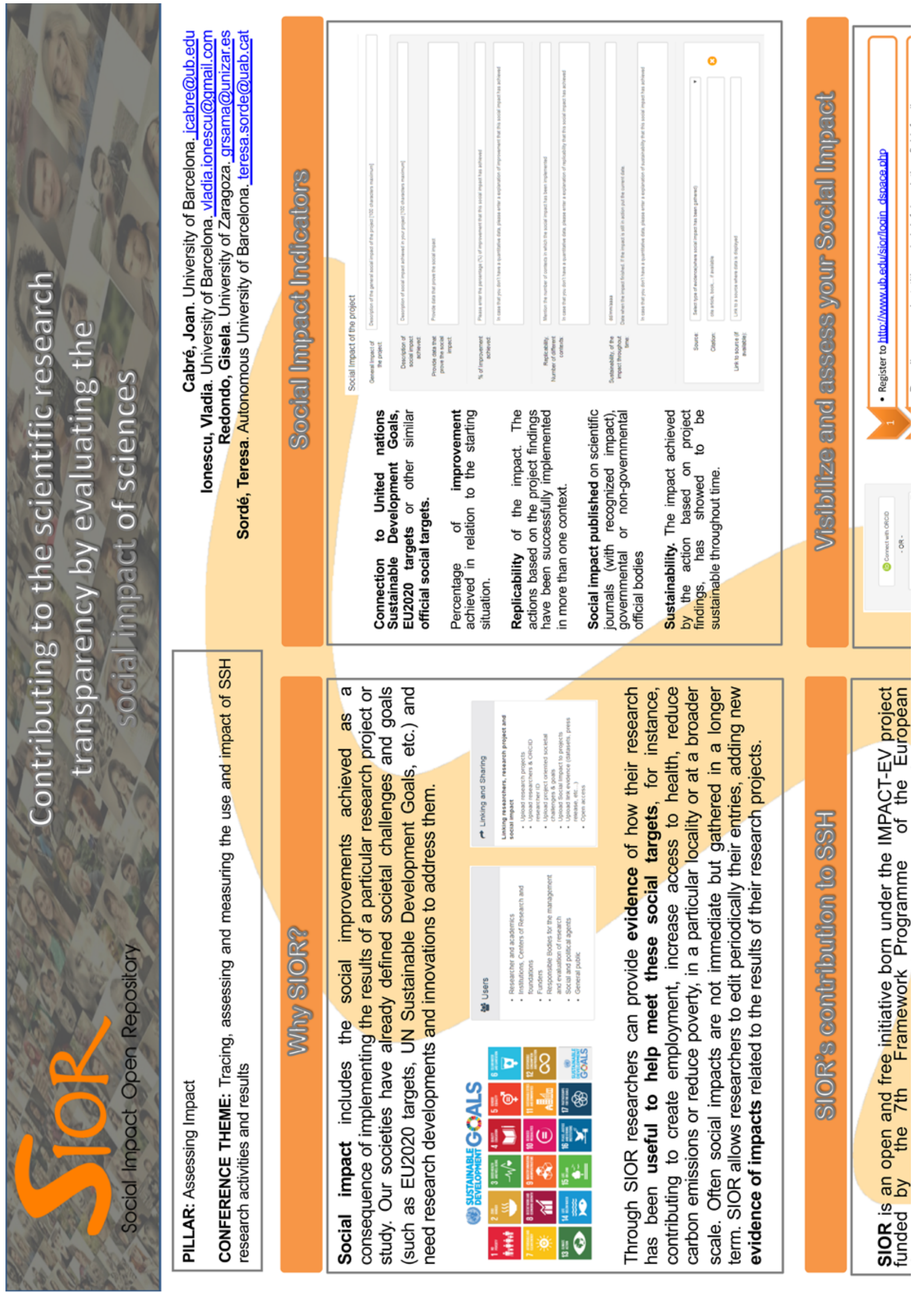

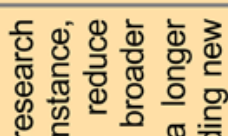
这.

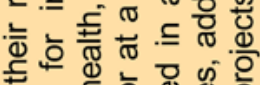

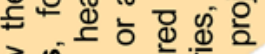

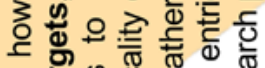

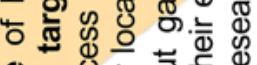
\&

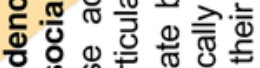
응

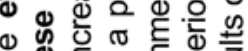
음 政

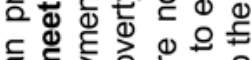

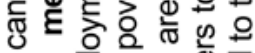

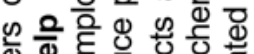

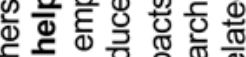

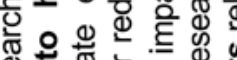

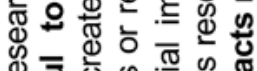

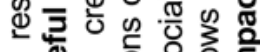

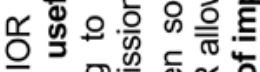
क o.

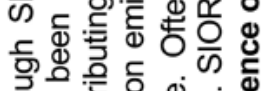

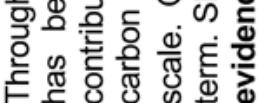

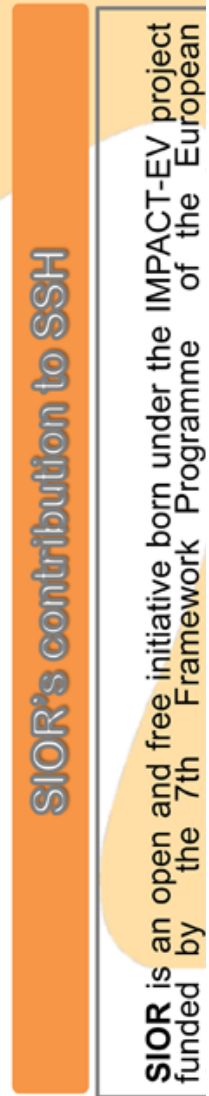




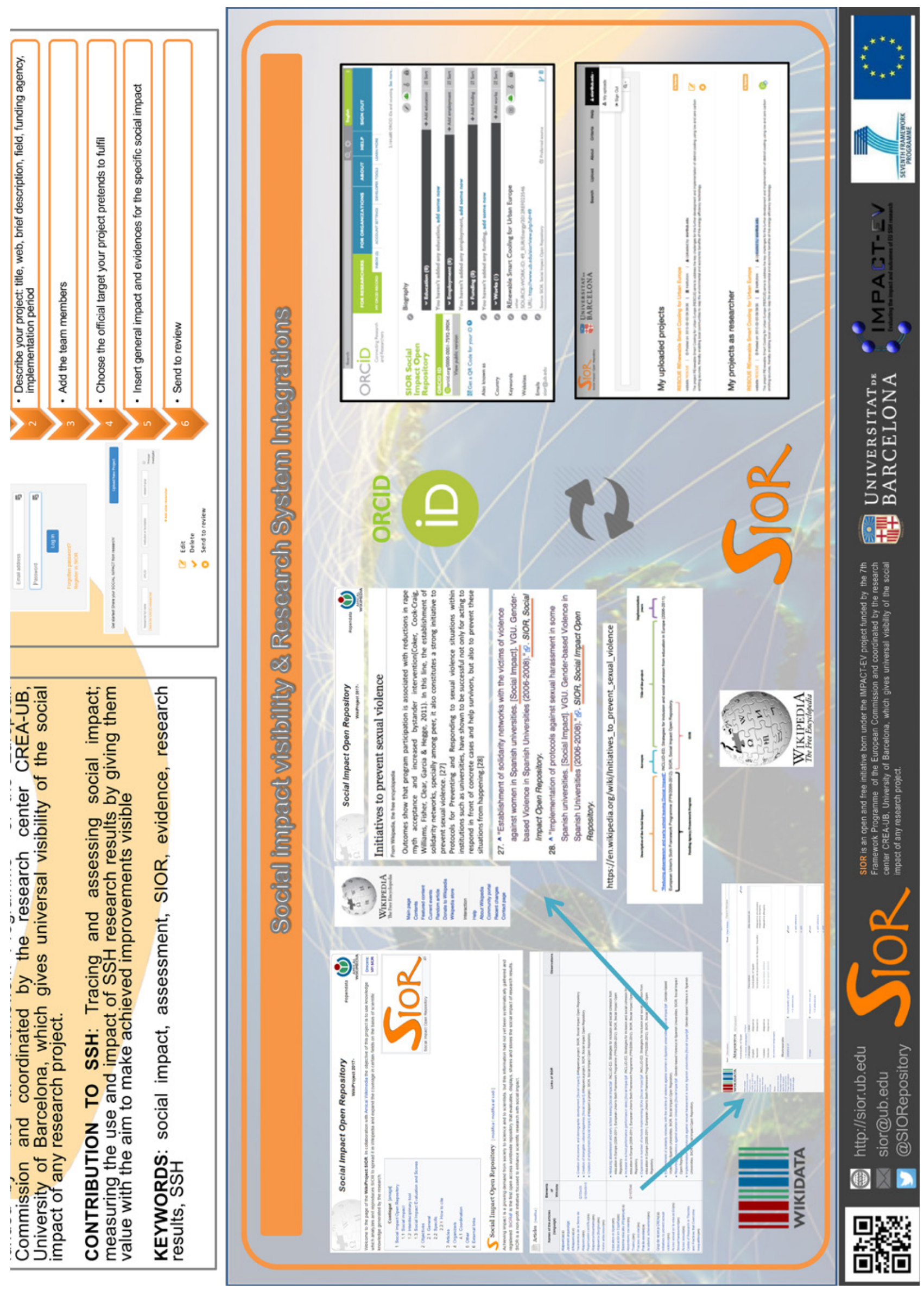



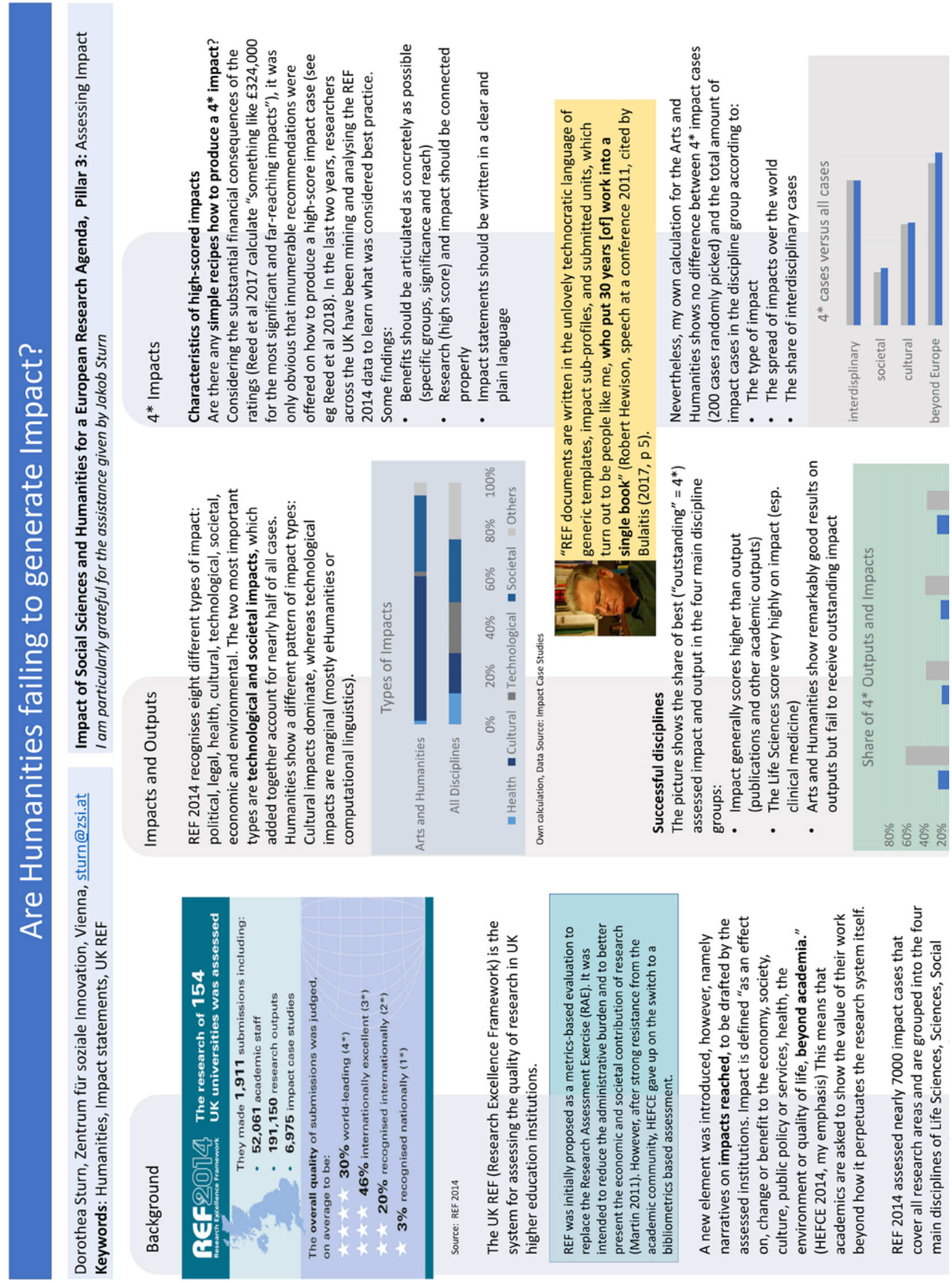

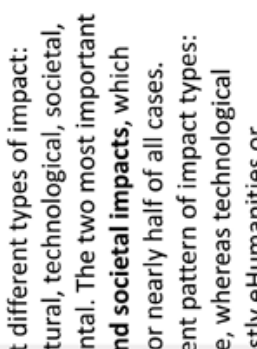
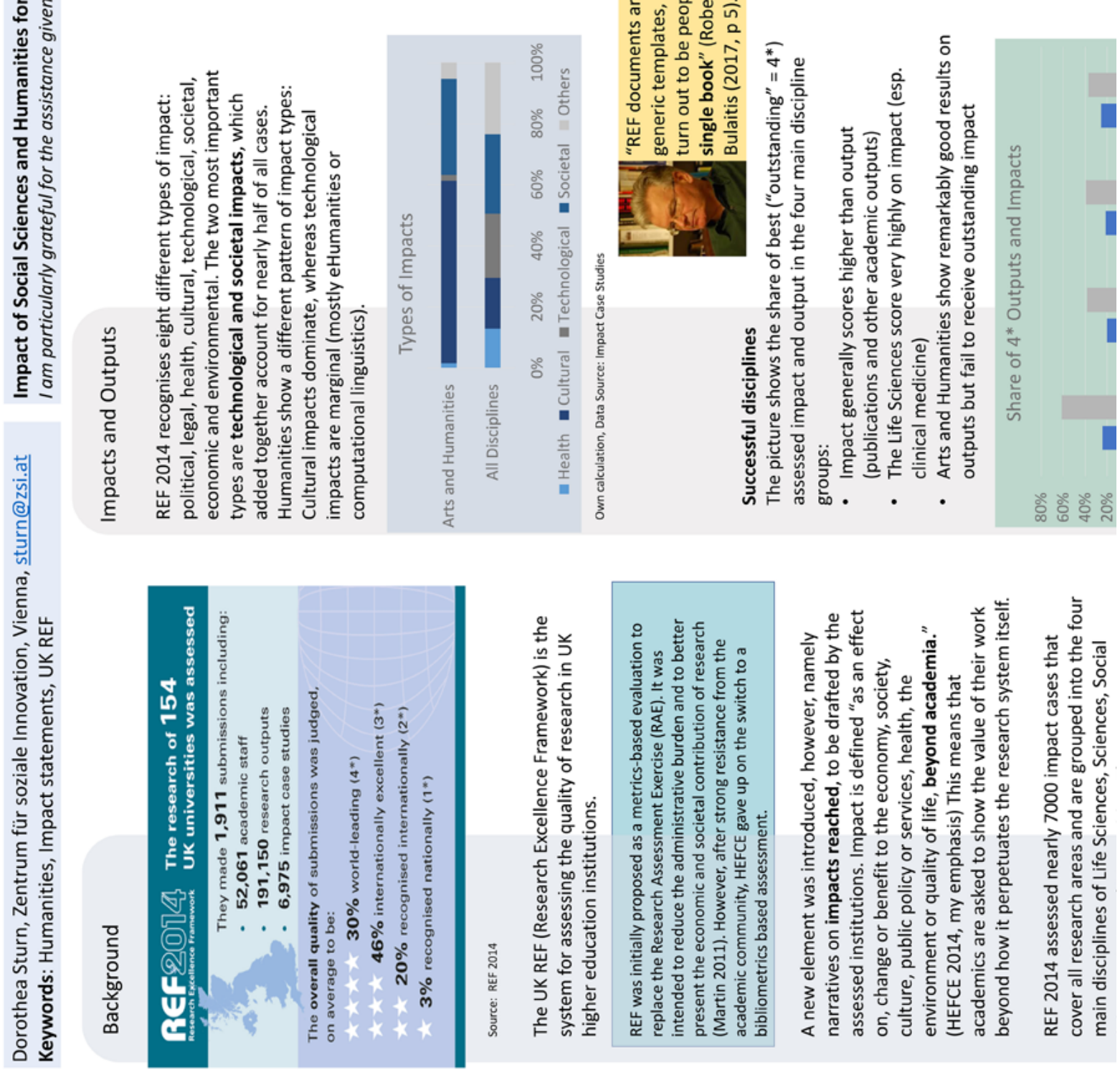

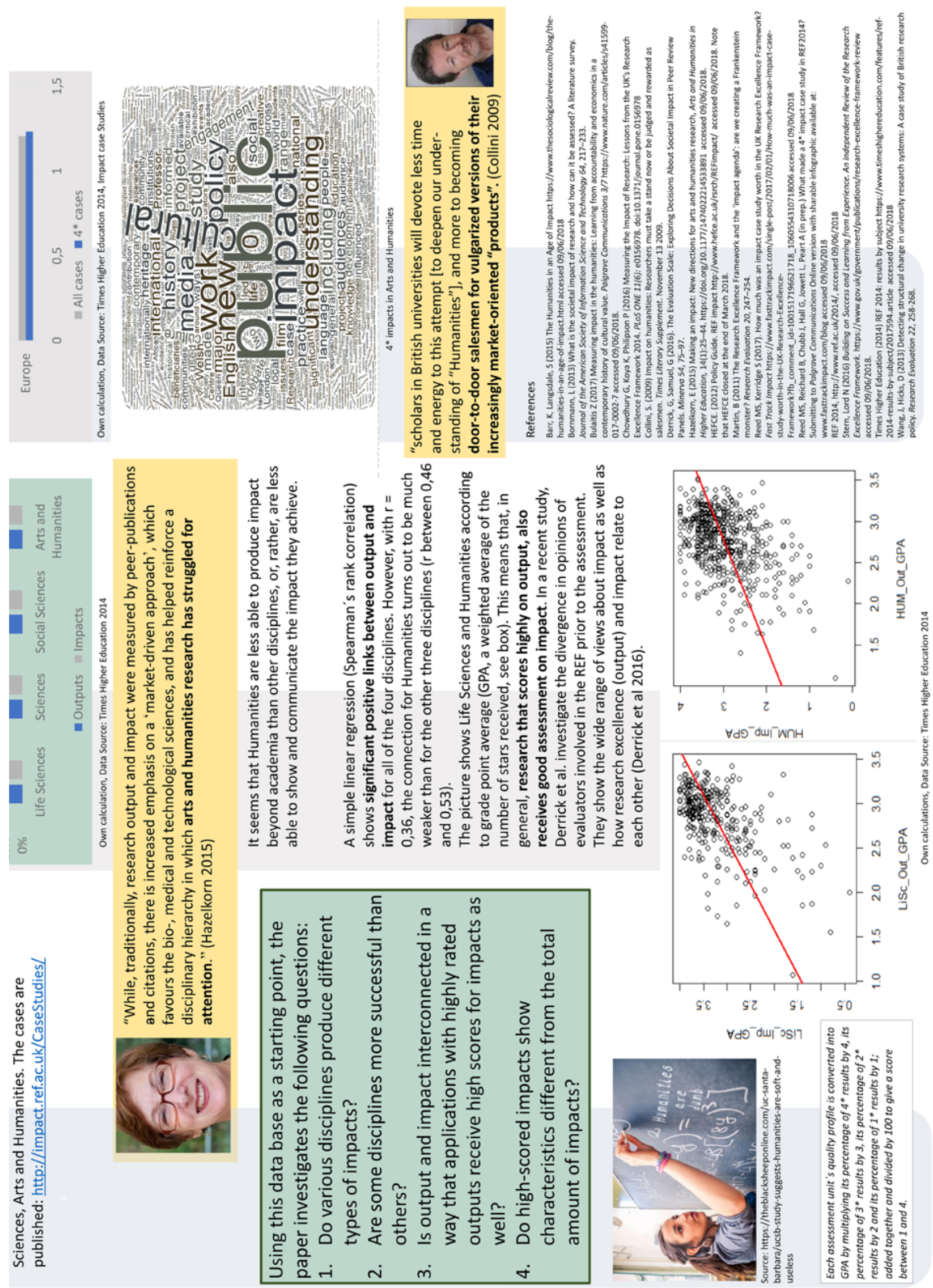


\section{Ethical commitments}

WHO Constitution (1946): "...the highest attainable standard of health as a fundamental right of every human being."

WHO Sustainable Development Goals (2015) No. 3.8. Achieve universal health coverage, including financial risk protection, access to quality essential healthcare services and access to safe, effective, quality and affordable essential medicines and vaccines for all."

EU Charter of Fundamental Rights (2012) Art.35: "Everyone has the right of access to preventive health care and the right to benefit from medical treatment under the conditions established by national laws and practices."

\section{Economic considerations}

Can societies afford equitable healthcare for all? Should nation states allow access to a full range of services also for people who reside without authorization on their territory? Or should irregular migrants' access be restricted to emergency care, as is common in most European countries? Resolution 1946 (2013) - Parliamentary Assembly - Council of Europe on Equal Access to Health Care:

"3. [...] inequalities in access to care, induding mental health care, particularly affect vulnerable groups, [...] especially those in an irregular situation [....]. These inequalities lead to a phenomenon of non-recourse or delayed recourse to care, which conld have disastrous implications for both individual and public health and lead in the long term to an increase in health expenditure."

\section{Evidence on costs of care}

Using different methodological approaches and data sources, recent studies come to similar conclusions: it is not cost saving to restrict access to health care to emergency treatment.

An economic model to calculate costs for two medical conditions - hypertension and prenatal care - was applied to three EU Member States: Germany, Greece and Sweden. It indicated that providing access to regular preventive healthcare for migrants in an irregular situation would be cost-saving for healthcare systems.

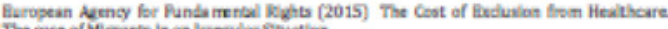
The case of Migats in an irrequie stiation.

A quasi-experimental study in Germany using data from 1994-2013 demonstrated that restrictions on asylum seekers' and refugees' healthcare entitlements ultimately incur higher costs than regular access to care.

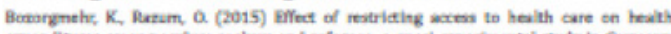

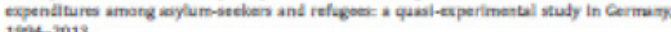

A vignette study using a micro-costing approach in four European countries (AT, BE, ES, IT) analysing 6 primary care sensitive medical conditions (asthma, depression, diabetes, epilepsy, hyptertension, TB) shows the cost-saving potential of timely treatment in primary care settings.

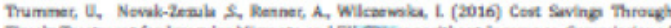

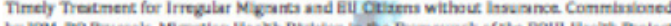

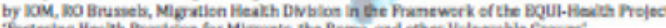

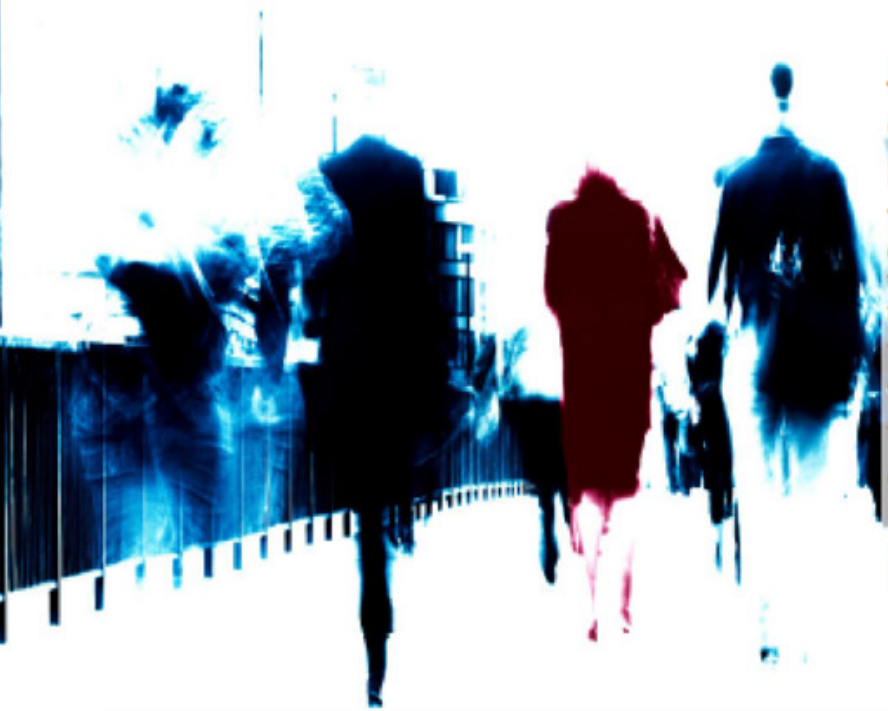

Join the EUPHA working group on economic arguments in migrant health policy-making!

The working group provides a forum for discussions around ethics, economics, and deservingness; e.g, what does ultimately tip the scales in decision-making processes on migrants' health entitlements, value-based or evidence-based ? arguments?

Contact:

the_economic_argument@c-hm.com

office@c-hm.çom

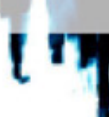

\section{Ethics \& economics regarding access to healthcare for marginalised groups}

Ursula Trummer'1, Sonja Novak-Zezula1, Nadav Davidovitch ${ }^{2}$, Nora Gottlieb ${ }^{3}$ ' Center for Heath and Migration, Vienna ${ }^{2}$ Ben-Gurion University of the Negev, Beer Sheva ${ }^{3}$ Technical University Berin

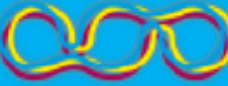

CENTER FOR HEALTH AND MIGRATION Research for Practice
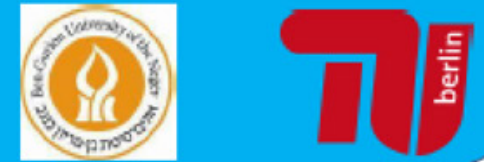


\section{NOW AVAILABLE!}

LATEST EDITION OF THE

'EVALUATION STANDARDS

FOR RESEARCH, TECHNOLOGY

AND INNOVATION POLICY'

OF THE AUSTRIAN PLATFORM FOR RESEARCH

AND TECHNOLOGY POLICY EVALUATION

ÖSTERREICHISChE PLATTFORM
für Forschungs- und Technologiepolitikevaluierung

STANDARDS DER EVALUIERUNG IN DER FORSCHUNGS-, TECHNOLOGIE-, UND INNOVATIONSPOLITIK MÄRZ 2019

DOl: 10.22163/teval 2019.310
AUSTRIAN PLATFORM f

for Research and Technology Policy Evaluation

EVALUATION STANDARDS

FOR RESEARCH, TECHNOLOGY AND INNOVATION POLICY

MARCH 2019

OD: $10.22163 /$ tereal. 2019.344

\section{Download in German:}

fteval.at/content/home/standards/fteval_standards/
Download in English:

fteval.at/content/home/standards/fteval_standards/index.jsp?langld=2 


\section{FOR RESEARCH AND TECHNOLOGY POLICY EVALUATION}

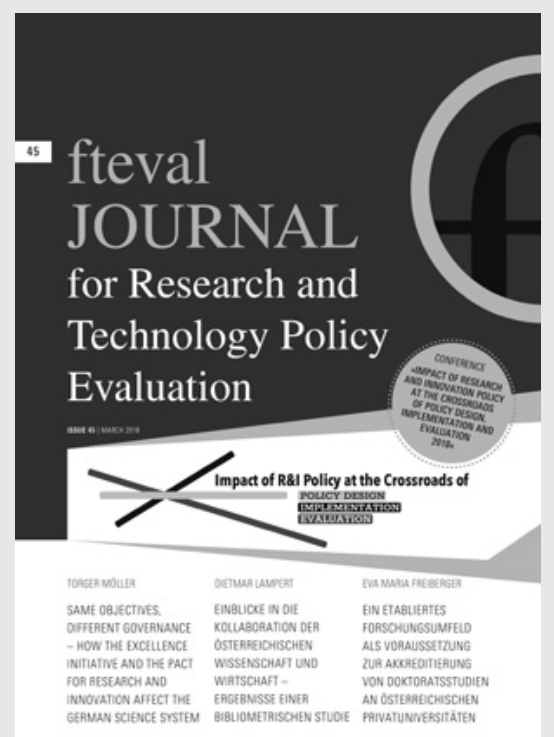

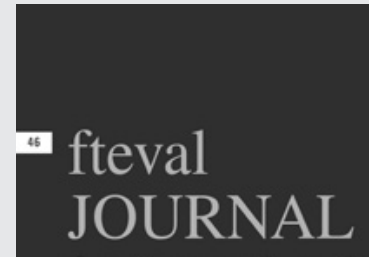

for Research and

Technology Policy

Evaluation
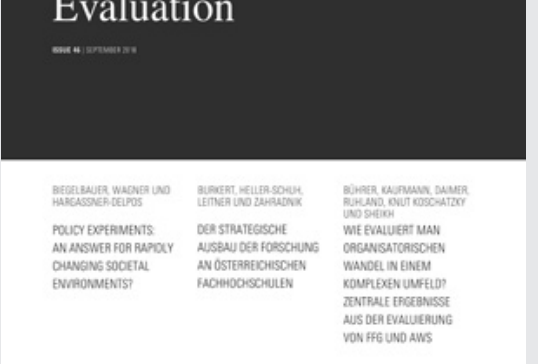

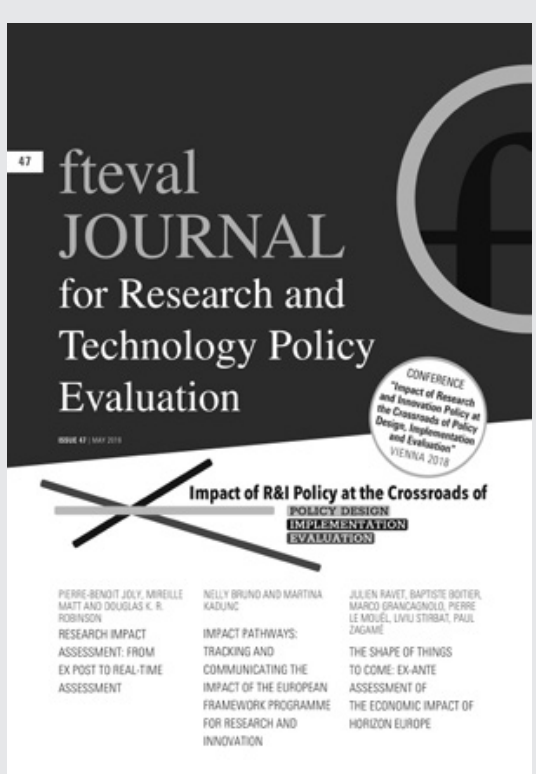

for Research and

Technology Policy

Evaluation

\section{... RTI POLICY EVALUATION AT THE INTERFACE BETWEEN PRACTICE AND ACADEMIA ...}

The 'fteval Journal for Research and Technology Policy Evaluation' contributes in a quality assured way to exchange between various stakeholder groups in the area of science, research, technology and innovation policy evaluation. It addresses policy-makers, practitioners, evaluators and the academic community. Thematic editions alternate with thematically open issues. By now 48 editions were published, which can be accessed and downloaded from the fteval-repository and the fteval homepage. The fteval Journal is open access. The journal and each published paper receive a separate DOI. All paper contributions are quality controlled, but not subject to peer-review in the strict academic sense.

Researchers and practitioners from the RTI policy domain are invited to send contributions to the editor of the 'fteval Journal for Research and Technology Policy Evaluation' (office@fteval.at). The contributions can be submitted either in German or English.

If you want to receive the 'fteval Journal for Research and Technology Policy Evaluation' free of charge, please fil out this form and send it to office@fteval.at or drop it into the infobox at one of our conferences and events.

first name

last name

institution

address

postal code

city/place

country

e-mail

By filling in this information you agree to* receive the fteval Journal by mail (free of charge)

* to receive the electronic newsletter of fteval, which informs about pertinent issues of RTI evaluation in Austria and the EU via email (such as calls for contributions for the next fteval Journal) and thus you entitle the fteval to process your indicated personal data for the purpose of transmission of information to you.

You can revoke consent at any time by informing us via eMail (office@fteval.at). 
EDITORIAL BOARD

Rebecca Allinson, Technopolis UK; Balázs Borsi, Eszterházy Károly College; Elke Dall, Centre for Social Innovation; Michael Dinges, Austrian Institute of Technology; Leonid Gokhberg, National Research University Higher School of Economics; Wolgang Polt, Joanneum Research; Andreas Reinstaller, WIFO; Klaus Schuch, fteval (chief editor); Michael Stampfer, WWTF; Lena Tsipouri, University of Athens

\section{AUSTRIAN PLATFORM FOR RESEARCH AND TECHNOLOGY POLICY EVALUATION (fteval)}

c/o ZSI - Centre for Social Innovation GmbH

Linke Wienzeile 246, A-1150 Vienna

T $\quad+4314950442-79$

F +43149504 42- 40

E office@fteval.at

W www.fteval.at

\section{DESIGN}

W carotte.at

E caroline@carotte.at

\section{PRINT}

agensketterl Druckerei, Bad Vöslau

Gedruckt auf PEFC ${ }^{\mathrm{TM}}$-zertifiziertem Papier.

\section{AUSTRIAN PLATFORM FOR RESEARCH AND TECHNOLOGY POLICY EVALUATION}

\section{Platform fteval members:}

Austrian Federal Ministry of Education, Science and Research (bmbwf), Austrian Federal Ministry for Digital and Economic Affairs (bmdw), Austrian Federal Ministry for Transport, Innovation and Technology (bmvit), Austrian Cooperative Research (ACR), Austrian Council for Research and Technology Development, Austrian Institute of Technology (AIT), A0 Austria - Agency for Quality Assurance and Accreditation Austria, Austria Wirtschaftsservice (AWS), Christian Doppler Research Association (CDG), convelop Cooperative Knowledge Design GmbH, Austrian Research Promotion Agency (FFG), Austrian Science Fund (FWF), Institute for Advanced Studies (IHS), Industriewissenschaftliches Institut (IWI), Joanneum Research Forschungsgesellschaft mbH, Austrian Institute for SME Research (KMFA), Ludwig Boltzmann Society (LBG), Austrian Academy of Science (OEAW), Technopolis Group Austria, Vienna Business Agency A service offered by the City of Vienna, Austrian Institute of Economic Research (WIFO), WPZ Research GmbH, Vienna Science and Technology Fund (WWTF), Centre for Social Innovation (ZSI) 


\section{A U S I R I A N P L A I F O R M
for Research and Jechnology Policy Evaluation}

c/o ZSI - Centre for Social Innovation GmbH Linke Wienzeile 246, A-1150 Vienna

T $\quad+4314950442-79$

F $\quad+4314950442-40$

E_office@fteval.at

W www.fteval.at
The fteval Journal for Research and Technology Policy Evaluation is a forum to discuss current evaluation practices in the field of research, technology and innovation policy.

\section{ZVR-Zahl:}

ISSN-Nr.

(C) Vienna 2019
937261837

$1726-6629$ 